\title{
Selective Difunctionalization of Unactivated Aliphatic Alkenes Enabled by a Metal-Metallaaromatic Catalytic System
}

Fei-Hu Cui, ${ }^{\dagger, \perp}$ Yuhui Hua $,{ }^{\dagger, \downarrow}, \perp$ Yu-Mei Lin,${ }^{\dagger *}$ Jiawei Fei, ${ }^{\dagger}$ Le-Han Gao, ${ }^{\dagger}$ Xiaodan Zhao, ${ }^{\#}$ Haiping $\mathrm{Xia}^{\dagger+, * *}$

†State Key Laboratory of Physical Chemistry of Solid Surfaces, College of Chemistry and Chemical Engineering, Xiamen University, Xiamen, Fujian 361005, China \#Shenzhen Grubbs Institute, Department of Chemistry, Southern University of Science and Technology, Shenzhen 518055, China

\#School of Chemistry, Sun Yat-Sen University, Guangzhou 510275, China

${ }^{\perp}$ These authors contributed equally to this work

*Corresponding Author: linyum@xmu.edu.cn (Yu-Mei Lin); hpxia@xmu.edu.cn (Haiping Xia) 


\section{Table of Contents}

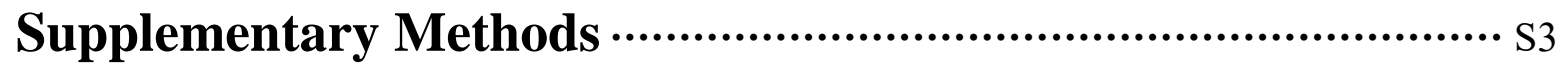

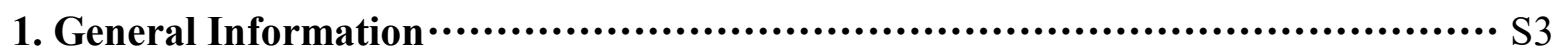

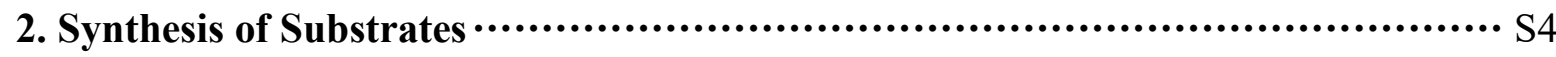

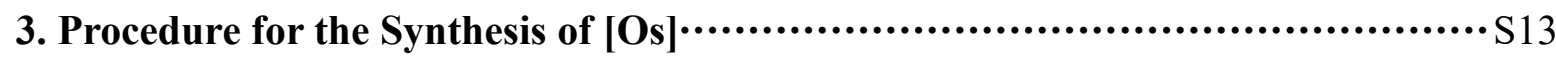

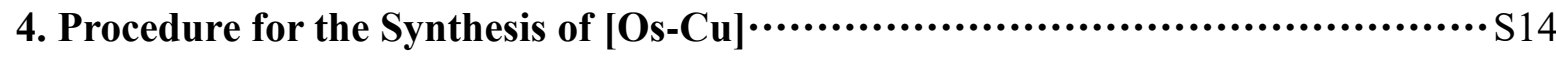

5. Selective Difunctionalization of Unactivated Alkenes …............................ S15

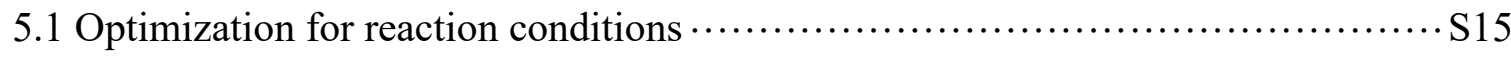

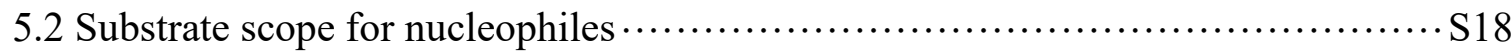

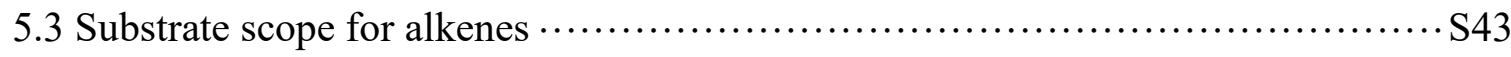

5.4 Synthetic utility of the method ……....................................... S70

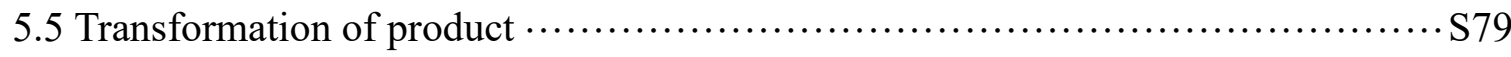

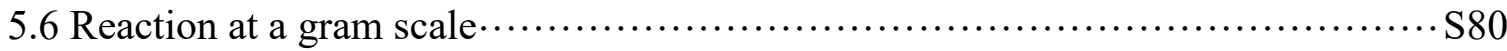

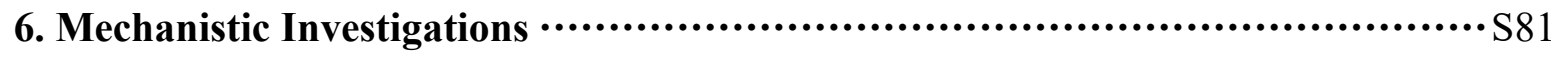

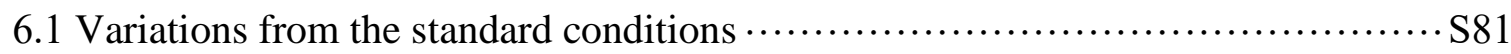

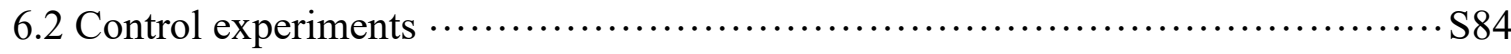

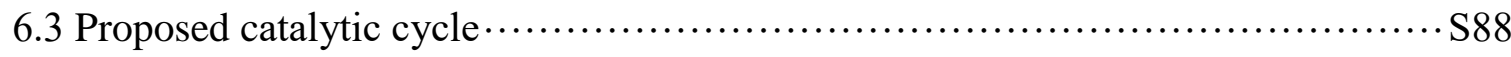

7. Single-Crystal X-Ray Diffraction ……............................................ S89

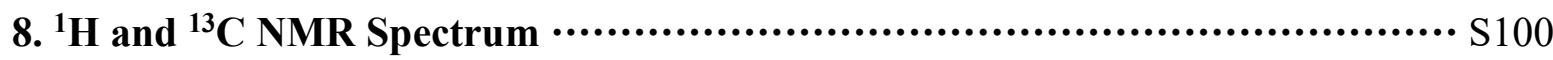

Supplementary References …............................................. S200 


\section{Supplementary Methods}

\section{General Information}

Visible-light-induced catalytic reactions were performed in 10 or $50 \mathrm{~mL}$ Schlenk tubes at the indicated temperature under an atmosphere of oxygen and under irradiation with a $24 \mathrm{~W}$ blue LEDs lamp $\left(\lambda_{\max }=450 \mathrm{~nm}\right.$; commercial supplier: Hong Chang Lighting Co., Ltd., website: http://hongchang-led.taobao.com). Solvents were distilled under argon from calcium hydride $\left(\mathrm{CH}_{2} \mathrm{Cl}_{2}\right)$, or sodium/benzophenone (THF). Alkene substrates and catalysts $[\mathbf{O s}],{ }^{1}[\mathbf{O s}-\mathbf{A}],{ }^{2}[\mathbf{O s}-\mathbf{B}],{ }^{3}$ and $[\mathbf{O s}-\mathbf{C u}],{ }^{4}$ were synthesized according to the published procedures. All others reagents were purchased from commercial suppliers (TCI, Aldrich, Alfa and J\&K) and used without further purification. Flash column chromatography was performed with silica gel (300-400 mesh, $\mathrm{pH}=6.7-7.0) .{ }^{31} \mathrm{P}$ NMR, ${ }^{1} \mathrm{H}$ NMR and ${ }^{13} \mathrm{C}$ NMR spectra were recorded on a Bruker AM (400 MHz) or Bruker AM (500 MHz) spectrometer at ambient temperature. NMR standards were used as follows: $\mathrm{CDCl}_{3}=7.26 \mathrm{ppm}\left({ }^{1} \mathrm{H}\right.$ NMR), 77.0 ppm $\left({ }^{13} \mathrm{C} \mathrm{NMR}\right) ;\left(\mathrm{CD}_{3}\right)_{2} \mathrm{CO}=2.05 \mathrm{ppm}\left({ }^{1} \mathrm{H} \mathrm{NMR}\right), 29.7$ and $206.4 \mathrm{ppm}$ $\left({ }^{13} \mathrm{C}\right.$ NMR). High-resolution mass spectra were recorded on a Bruker En Apex Ultra 7.0 T FT-MS or UPLC-QTOF instrument using ESI technique. IR spectra were recorded on a Nicolet Avatar 330 FT-IR spectrophotometer. 


\section{Synthesis of Substrates}

\subsection{General procedure for the synthesis of substrates 1q-1s, 1za-1zb, and 1zn-1zp}
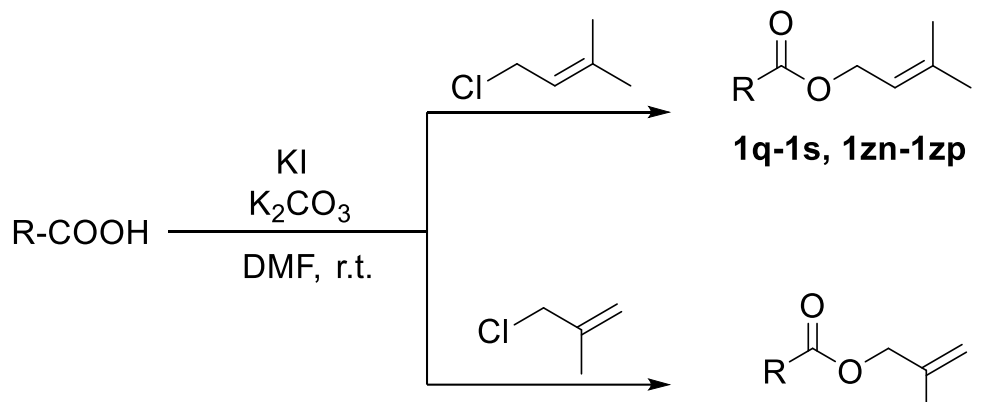

1q-1s, 1zn-1zp

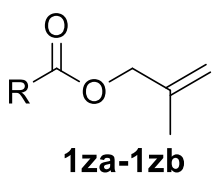

General procedure. A round-bottom flask was charged with 3-chloro-2-methylprop-1-ene or 1-chloro-3-methylbut-2-ene (5.00 mmol), carboxylic acid $(6.00 \mathrm{mmol}), \mathrm{K}_{2} \mathrm{CO}_{3}(828 \mathrm{mg}, 6.00 \mathrm{mmol}), \mathrm{KI}(83 \mathrm{mg}, 0.50 \mathrm{mmol})$ and DMF $(25 \mathrm{~mL})$. The progress of the reaction was monitored by TLC. After the reaction was completed, $\mathrm{H}_{2} \mathrm{O}(50 \mathrm{~mL})$ was added and the resulting mixture was extracted with ethyl acetate $(3 \times 50 \mathrm{~mL})$. The combined organic layers were washed with $\mathrm{H}_{2} \mathrm{O}$, dried over anhydrous $\mathrm{Na}_{2} \mathrm{SO}_{4}$ and concentrated under reduced pressure. The residue was subjected to silica gel chromatography (petroleum ether/ethyl acetate) to afford product.

\section{3-methylbut-2-en-1-yl benzoate (1q)}<smiles>CC(C)=CCOC(=O)c1ccccc1</smiles>

According to the general procedure, substrate 1q $(883 \mathrm{mg}, 4.65 \mathrm{mmol}$, yield: $88 \%)$ was synthesized as a colorless oil. 
${ }^{1}$ H NMR (500 MHz, CDCl $) \delta 8.11-8.04(\mathrm{~m}, 2 \mathrm{H}), 7.56(\mathrm{t}, J=7.4 \mathrm{~Hz}, 1 \mathrm{H}), 7.44(\mathrm{t}, J$ $=7.7 \mathrm{~Hz}, 2 \mathrm{H}), 5.50(\mathrm{t}, J=7.2 \mathrm{~Hz}, 1 \mathrm{H}), 4.85(\mathrm{~d}, J=7.2 \mathrm{~Hz}, 2 \mathrm{H}), 1.81(\mathrm{~s}, 3 \mathrm{H}), 1.79(\mathrm{~s}$, $3 \mathrm{H})$.

${ }^{13}$ C NMR (126 MHz, CDCl3) $\delta$ 166.7, 139.1, 132.8, 130.5, 129.6, 128.3, 118.7, 61.9, $25.8,18.1$.

HRMS (ESI, m/z) calcd for $\mathrm{C}_{12} \mathrm{H}_{14} \mathrm{NaO}_{2}(\mathrm{M}+\mathrm{Na})^{+}:$213.0886, found: 213.0882 .

\section{3-methylbut-2-en-1-yl 4-methoxybenzoate $(1 r)^{6}$}

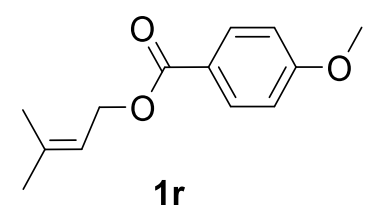

According to the general procedure, substrate 1r (1001 mg, $4.55 \mathrm{mmol}$, yield: 91\%) was synthesized as a colorless oil.

${ }^{1}$ H NMR (500 MHz, CDCl 3$) \delta 8.01(\mathrm{~d}, J=8.9 \mathrm{~Hz}, 2 \mathrm{H}), 6.92(\mathrm{~d}, J=8.9 \mathrm{~Hz}, 2 \mathrm{H})$, $5.48(\mathrm{t}, J=7.1 \mathrm{~Hz}, 1 \mathrm{H}), 4.81(\mathrm{~d}, J=7.1 \mathrm{~Hz}, 2 \mathrm{H}), 3.86(\mathrm{~s}, 3 \mathrm{H}), 1.80(\mathrm{~s}, 3 \mathrm{H}), 1.78(\mathrm{~s}$, $3 \mathrm{H})$.

${ }^{13}$ C NMR (126 MHz, $\left.\mathbf{C D C l}_{3}\right) \delta 166.4,163.3,138.8,131.6,123.0,118.9,113.5,61.6$, $55.4,25.8,18.1$.

HRMS (ESI, m/z) calcd for $\mathrm{C}_{13} \mathrm{H}_{13} \mathrm{NaO}_{3}(\mathrm{M}+\mathrm{Na})^{+}:$243.0992, found: 243.0992 .

\section{3-methylbut-2-en-1-yl 4-(trifluoromethyl)benzoate (1s)}

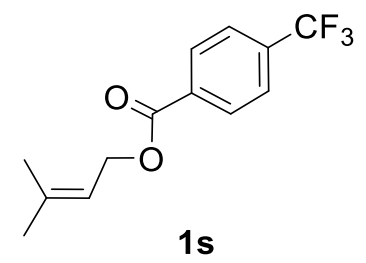

According to the general procedure, substrate 1s (1440 mg, $5.58 \mathrm{mmol}$, yield: 93\%) was synthesized as a colorless oil. 
${ }^{1} \mathbf{H}$ NMR (500 MHz, CDCl $) \delta 8.15(\mathrm{~d}, J=8.1 \mathrm{~Hz}, 2 \mathrm{H}), 7.68(\mathrm{~d}, J=8.2 \mathrm{~Hz}, 2 \mathrm{H})$, $5.47(\mathrm{t}, J=7.2 \mathrm{~Hz}, 1 \mathrm{H}), 4.85(\mathrm{~d}, J=7.2 \mathrm{~Hz}, 2 \mathrm{H}), 1.78(\mathrm{~d}, J=7.5 \mathrm{~Hz}, 6 \mathrm{H})$.

${ }^{13}$ C NMR (126 MHz, CDCl $) \delta 165.4,139.7,134.3(\mathrm{q}, J=32.7 \mathrm{~Hz}), 133.8,130.0$, $125.3(\mathrm{q}, J=3.7 \mathrm{~Hz}), 122.0$ (q, $J=274.1 \mathrm{~Hz}), 118.3,62.4,29.7,25.7,18.1$.

HRMS (ESI, m/z) calcd for $\mathrm{C}_{13} \mathrm{H}_{13} \mathrm{~F}_{3} \mathrm{NaO}_{2}(\mathrm{M}+\mathrm{Na})^{+}$: 281.0760, found: 281.0761.

\section{2-methylallyl benzoate (1za) $)^{7}$}

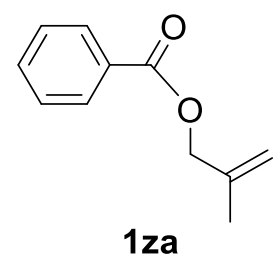

According to the general procedure, substrate 1za (783 mg, $4.45 \mathrm{mmol}$, yield: $89 \%$ ) was synthesized as a colorless oil.

${ }^{1} \mathbf{H}$ NMR (500 MHz, CDCl 3$) \delta 8.08(\mathrm{~d}, J=7.7 \mathrm{~Hz}, 2 \mathrm{H}), 7.55(\mathrm{t}, J=7.4 \mathrm{~Hz}, 1 \mathrm{H}), 7.44$ (t, $J=7.7 \mathrm{~Hz}, 2 \mathrm{H}), 5.08(\mathrm{~s}, 1 \mathrm{H}), 4.99(\mathrm{~s}, 1 \mathrm{H}), 4.75(\mathrm{~s}, 2 \mathrm{H}), 1.84(\mathrm{~s}, 3 \mathrm{H})$.

${ }^{13}$ C NMR (126 MHz, CDCl 3$) \delta$ 166.2, 140.0, 133.0, 130.3, 129.6, 128.4, 112.9, 68.1, 19.6 .

HRMS (ESI, m/z) calcd for $\mathrm{C}_{11} \mathrm{H}_{11} \mathrm{NaO}_{2}(\mathrm{M}+\mathrm{Na})^{+}$: 199.0730, found: 199.0729.

\section{2-methylallyl 1-naphthoate (1zb)}

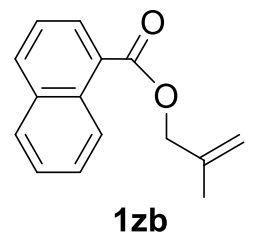

According to the general procedure, substrate 1zb (1051 mg, $4.65 \mathrm{mmol}$, yield: 93\%) was synthesized as a white solid.

${ }^{1} \mathbf{H}$ NMR (500 MHz, CDCl $) \delta 8.95(\mathrm{~d}, J=8.6 \mathrm{~Hz}, 1 \mathrm{H}), 8.24(\mathrm{dd}, J=7.3,1.2 \mathrm{~Hz}$, $1 \mathrm{H}), 8.03(\mathrm{~d}, J=8.2 \mathrm{~Hz}, 1 \mathrm{H}), 7.89(\mathrm{~d}, J=8.1 \mathrm{~Hz}, 1 \mathrm{H}), 7.62(\mathrm{ddd}, J=8.5,6.8,1.4 \mathrm{~Hz}$, 1H), $7.57-7.48(\mathrm{~m}, 2 \mathrm{H}), 5.14(\mathrm{~s}, 1 \mathrm{H}), 5.03(\mathrm{~s}, 1 \mathrm{H}), 4.85(\mathrm{~s}, 2 \mathrm{H}), 1.90(\mathrm{~s}, 3 \mathrm{H})$. 
${ }^{13}$ C NMR (126 MHz, CDCl 3$) \delta 167.2,140.1,133.9,133.5,131.5,130.2,128.6,127.8$, $127.1,126.2,125.8,124.5,113.2,68.3,19.7$.

HRMS (ESI, m/z) calcd for $\mathrm{C}_{15} \mathrm{H}_{14} \mathrm{NaO}_{2}(\mathrm{M}+\mathrm{Na})^{+}:$249.0884, found: 249.0886.

\section{3-methylbut-2-en-1-yl benzo[d][1,3]dioxole-5-carboxylate (1zn)}

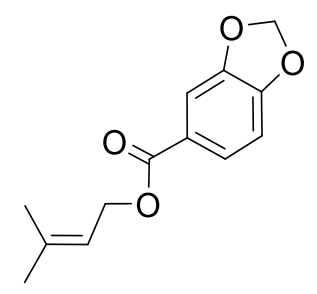

$1 \mathrm{zn}$

According to the general procedure, substrate $1 \mathrm{zn}$ (1076 mg, $4.60 \mathrm{mmol}$, yield: 92\%) was synthesized as a colorless oil.

${ }^{1}$ H NMR (500 MHz, CDCl 3$) \delta 7.68(\mathrm{dd}, J=8.2,1.6 \mathrm{~Hz}, 1 \mathrm{H}), 7.49(\mathrm{~d}, J=1.6 \mathrm{~Hz}$, 1H), $6.85(\mathrm{~d}, J=8.2 \mathrm{~Hz}, 1 \mathrm{H}), 6.05(\mathrm{~s}, 2 \mathrm{H}), 5.47(\mathrm{t}, J=7.2 \mathrm{~Hz}, 1 \mathrm{H}), 4.80(\mathrm{~d}, J=7.2$ $\mathrm{Hz}, 2 \mathrm{H}), 1.81(\mathrm{~s}, 3 \mathrm{H}), 1.78(\mathrm{~s}, 3 \mathrm{H})$.

${ }^{13}$ C NMR (126 MHz, CDCl 3$) \delta 166.0,151.5,147.7,139.1,125.3,124.5,118.8,109.6$, $107.9,101.8,61.8,25.8,18.1$.

HRMS (ESI, m/z) calcd for $\mathrm{C}_{13} \mathrm{H}_{14} \mathrm{NaO}_{4}(\mathrm{M}+\mathrm{Na})^{+}:$: 257.0784, found: 257.0782.

\section{3-methylbut-2-en-1-yl (R)-2-(6-methoxynaphthalen-2-yl)propanoate (1zo)}

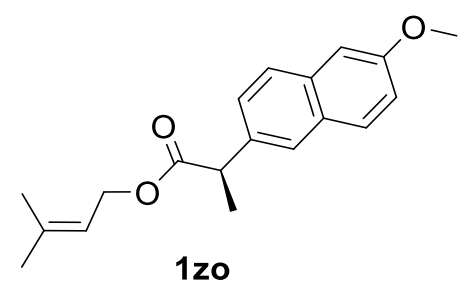

According to the general procedure, substrate 1 zo (1341 mg, $4.50 \mathrm{mmol}$, yield: $90 \%$ ) was synthesized as a white solid.

${ }^{1}$ H NMR (500 MHz, CDCl 3$) \delta 7.70(\mathrm{dd}, J=8.6,3.5 \mathrm{~Hz}, 2 \mathrm{H}), 7.66(\mathrm{~d}, J=1.7 \mathrm{~Hz}$, 1H), $7.44-7.37(\mathrm{~m}, 1 \mathrm{H}), 7.17-7.10(\mathrm{~m}, 2 \mathrm{H}), 5.29(\mathrm{~s}, 1 \mathrm{H}), 4.62(\mathrm{~d}, J=8.4 \mathrm{~Hz}, 1 \mathrm{H})$, 
$4.52(\mathrm{~d}, J=8.2 \mathrm{~Hz}, 1 \mathrm{H}), 3.92(\mathrm{~s}, 3 \mathrm{H}), 3.85(\mathrm{qd}, J=7.1,4.1 \mathrm{~Hz}, 1 \mathrm{H}), 1.72(\mathrm{~s}, 3 \mathrm{H})$, $1.64(\mathrm{~s}, 3 \mathrm{H}), 1.57$ (d, $J=3.5 \mathrm{~Hz}, 3 \mathrm{H})$.

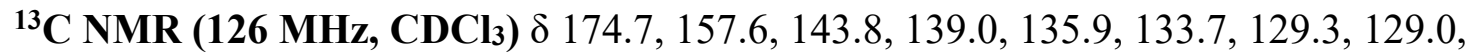
$127.1,126.3,125.9,118.9,118.6,105.6,61.8,55.3,45.5,25.7,18.7,18.0$.

HRMS (ESI, m/z) calcd for $\mathrm{C}_{19} \mathrm{H}_{22} \mathrm{NaO}_{3}(\mathrm{M}+\mathrm{Na})^{+}: 321.1461$, found: 321.1459 .

3-methylbut-2-en-1-yl-2-(1-(4-chlorobenzoyl)-5-methoxy-2-methyl-1H-indol-3-yl) acetate (1zp)

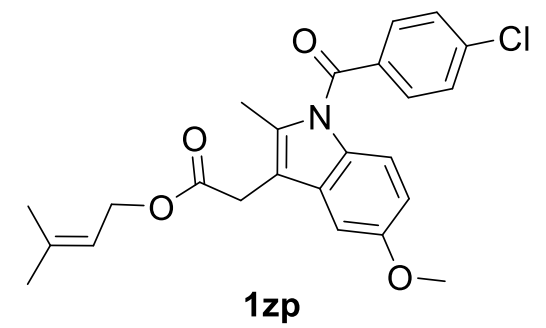

According to the general procedure, substrate 1zp (1934 mg, $4.55 \mathrm{mmol}$, yield: 91\%) was synthesized as a white solid.

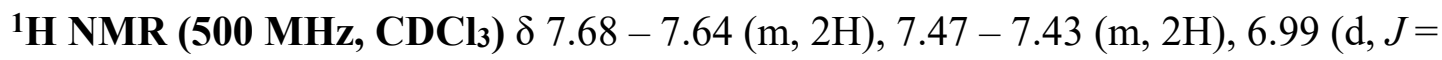
$2.5 \mathrm{~Hz}, 1 \mathrm{H}), 6.90(\mathrm{~d}, J=9.0 \mathrm{~Hz}, 1 \mathrm{H}), 6.67(\mathrm{dd}, J=9.0,2.5 \mathrm{~Hz}, 1 \mathrm{H}), 5.37(\mathrm{t}, J=7.1$ $\mathrm{Hz}, 1 \mathrm{H}), 4.63$ (d, J=7.2 Hz, 2H), 3.83 (s, 3H), 3.67 (s, 2H), 2.39 (s, 3H), 1.77 (s, 3H), $1.70(\mathrm{~s}, 3 \mathrm{H})$.

${ }^{13}$ C NMR (126 MHz, CDCl3) $\delta$ 170.8, 168.2, 156.1, 139.2, 139.1, 135.9, 134.0, 131.2, $130.8,130.7,129.1,118.6,114.9,112.8,111.7,101.4,61.9,55.6,30.4,25.8,18.0$, 13.4 .

HRMS (ESI, m/z) calcd for $\mathrm{C}_{24} \mathrm{H}_{24} \mathrm{ClNNaO}_{4}(\mathrm{M}+\mathrm{Na})^{+}: 448.1286$, found: 448.1291 . 


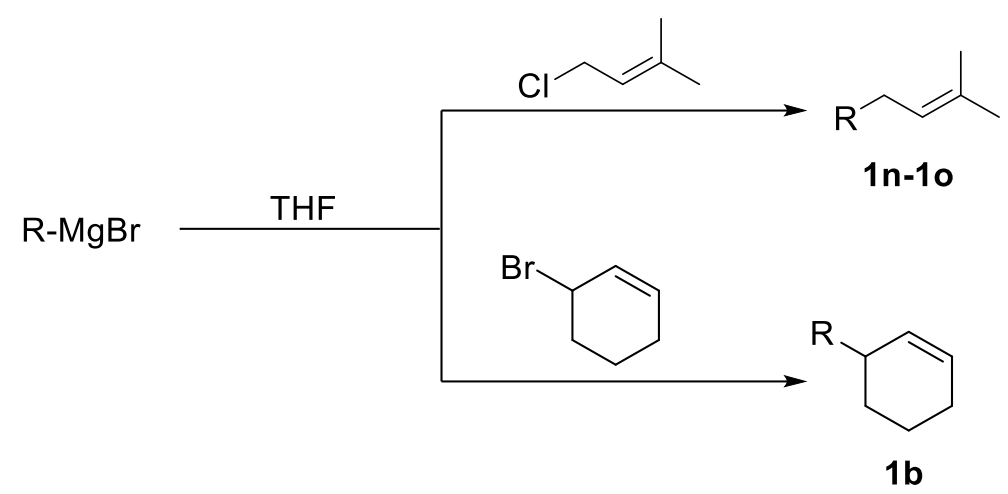

General procedure. To a stirred solution of the 1-chloro-3-methylbut-2-ene or 3-bromocyclohex-1-ene $(5.00 \mathrm{mmol})$ in anhydrous THF $(25 \mathrm{~mL})$ was added $\mathrm{RMgBr}$ (1.2 equiv., $1.0 \mathrm{M}$ in THF) under nitrogen atmosphere at $0{ }^{\circ} \mathrm{C}$. Then the mixture was warmed up to room temperature slowly and stirred until complete consumption of the starting material. The crude reaction mixture was then quenched by $\mathrm{NH}_{4} \mathrm{Cl}$ (sat.) and extracted with ethyl acetate (three times). The combined organic layers were dried over $\mathrm{Na}_{2} \mathrm{SO}_{4}$ and concentrated in vacuum. The product was isolated by flash column chromatography $\left(\mathrm{SiO}_{2}\right.$; hexane).

\section{(5-methylhex-4-en-1-yl)benzene $(1 \mathrm{n})^{8}$}

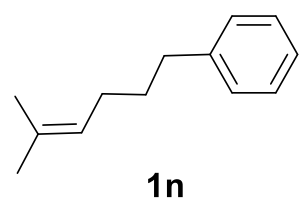

According to the general procedure, substrate 1n (714 mg, $4.20 \mathrm{mmol}$, yield: $84 \%)$ was synthesized as a colorless oil.

${ }^{1}$ H NMR (500 MHz, CDCl $) \delta 7.29(\mathrm{dd}, J=10.6,4.2 \mathrm{~Hz}, 2 \mathrm{H}), 7.19(\mathrm{t}, J=7.6 \mathrm{~Hz}$, $3 \mathrm{H}), 5.17(\mathrm{t}, J=7.1 \mathrm{~Hz}, 1 \mathrm{H}), 2.63(\mathrm{t}, J=7.7 \mathrm{~Hz}, 2 \mathrm{H}), 2.04$ (q, $J=7.1 \mathrm{~Hz}, 2 \mathrm{H}), 1.72(\mathrm{~s}$, $3 \mathrm{H}), 1.70-1.64(\mathrm{~m}, 2 \mathrm{H}), 1.61(\mathrm{~s}, 3 \mathrm{H})$.

${ }^{13} \mathbf{C}$ NMR (126 MHz, $\left.\mathbf{C D C l}_{3}\right) \delta$ 142.8, 131.8, 128.5, 128.2, 125.6, 124.4, 35.5, 31.6, 27.7, 25.8, 17.8. 
HRMS (ESI, m/z) calcd for $\mathrm{C}_{13} \mathrm{H}_{18} \mathrm{Na}(\mathrm{M}+\mathrm{Na})^{+}: 197.1301$, found: 197.1311.

(4-methylpent-3-en-1-yl)benzene (10) ${ }^{9}$

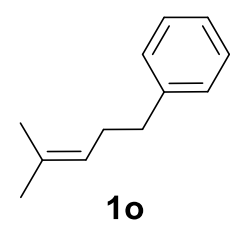

According to the general procedure, substrate $10(680 \mathrm{mg}, 4.25 \mathrm{mmol}$, yield: $85 \%)$ was synthesized as a colorless oil.

${ }^{1}$ H NMR (500 MHz, CDCl 3$) \delta 7.32-7.27(\mathrm{~m}, 2 \mathrm{H}), 7.23-7.18(\mathrm{~m}, 3 \mathrm{H}), 5.20(\mathrm{t}, J=$ $7.1 \mathrm{~Hz}, 1 \mathrm{H}), 2.65$ (t, $J=7.8 \mathrm{~Hz}, 2 \mathrm{H}), 2.32(\mathrm{dt}, J=15.3,7.5 \mathrm{~Hz}, 2 \mathrm{H}), 1.71(\mathrm{~s}, 3 \mathrm{H})$, $1.59(\mathrm{~s}, 3 \mathrm{H})$.

${ }^{13}$ C NMR (126 MHz, $\left.\mathbf{C D C l}_{3}\right) \delta 142.4,132.2,128.5,128.2,125.7,123.8,36.2,30.1$, 25.7, 17.7 .

HRMS (ESI, m/z) calcd for $\mathrm{C}_{13} \mathrm{H}_{18} \mathrm{Na}(\mathrm{M}+\mathrm{Na})^{+}: 197.1301$, found: 197.1311 .

(cyclohex-2-en-1-ylmethyl)benzene $(1 \mathrm{~b})^{10}$

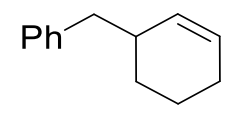

$1 b$

According to the general procedure, substrate 1 b $(765 \mathrm{mg}, 4.45 \mathrm{mmol}$, yield: $89 \%)$ was synthesized as a colorless oil.

${ }^{1}$ H NMR (500 MHz, CDCl3) $\delta 7.32-7.27$ (m, 2H), $7.21(\mathrm{dd}, J=12.0,4.6 \mathrm{~Hz}, 3 \mathrm{H})$, $5.71(\mathrm{ddd}, J=9.7,5.9,3.5 \mathrm{~Hz}, 1 \mathrm{H}), 5.62-5.57(\mathrm{~m}, 1 \mathrm{H}), 2.65(\mathrm{dd}, J=13.3,7.2 \mathrm{~Hz}$, 1H), $2.56(\mathrm{dd}, J=13.3,8.2 \mathrm{~Hz}, 1 \mathrm{H}), 2.45-2.34(\mathrm{~m}, 1 \mathrm{H}), 2.03-1.97$ (m, 2H), $1.79-$ 1.67 (m, 2H), $1.55-1.48(\mathrm{~m}, 1 \mathrm{H}), 1.34-1.21(\mathrm{~m}, 1 \mathrm{H})$.

${ }^{13}$ C NMR (126 MHz, CDCl 3$) \delta 140.9,131.4,129.2,128.2,127.4,125.8,42.8,37.28$, $28.98,25.48,21.3$.

HRMS (ESI, m/z) calcd for $\mathrm{C}_{13} \mathrm{H}_{18} \mathrm{Na}(\mathrm{M}+\mathrm{Na})^{+}:$197.1301, found: 197.1311. 


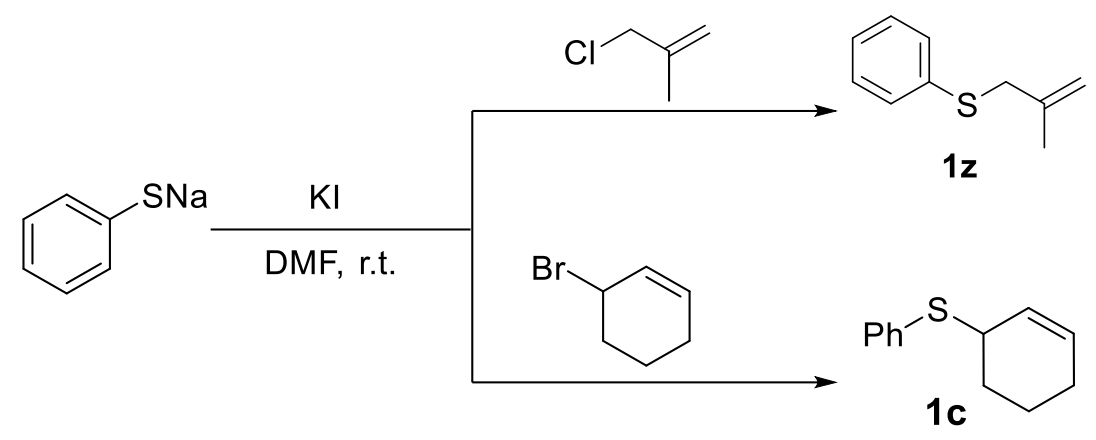

General procedure. A round-bottom flask was charged with 3-chloro-2-methylprop-1-ene or 3-bromocyclohex-1-ene $(5.00 \mathrm{mmol})$, sodium benzenethiolate ( $660 \mathrm{mg}, 5.00 \mathrm{mmol}), \mathrm{KI}(83 \mathrm{mg}, 0.50 \mathrm{mmol})$ and DMF (20 mL). The progress of the reaction was monitored by TLC. After the reaction was complete, $\mathrm{H}_{2} \mathrm{O}$ $(50 \mathrm{~mL})$ was added and the resulting mixture was extracted with ethyl acetate $(3 \times 50$ $\mathrm{mL}$ ). The combined organic layers were washed with $\mathrm{H}_{2} \mathrm{O}$, dried over anhydrous $\mathrm{Na}_{2} \mathrm{SO}_{4}$ and concentrated under reduced pressure. The residue was subjected to silica gel chromatography (petroleum ether/ethyl acetate) to afford product.

(2-methylallyl)(phenyl)sulfane (1z) $)^{11}$

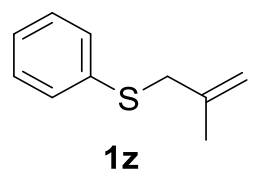

According to the general procedure, substrate $\mathbf{1 z}(705 \mathrm{mg}, 4.30 \mathrm{mmol}$, yield: $86 \%)$ was synthesized as a colorless oil.

${ }^{1}$ H NMR (400 MHz, CDCl 3$) \delta 7.40(\mathrm{dd}, J=9.3,1.9 \mathrm{~Hz}, 2 \mathrm{H}), 7.35-7.28(\mathrm{~m}, 2 \mathrm{H})$, $7.25-7.18(\mathrm{~m}, 1 \mathrm{H}), 4.91-4.83(\mathrm{~m}, 2 \mathrm{H}), 3.57(\mathrm{~s}, 2 \mathrm{H}), 1.91(\mathrm{~s}, 3 \mathrm{H})$.

${ }^{13}$ C NMR (101 MHz, CDCl $) \delta$ 140.9, 136.6, 130.0, 128.8, 126.3, 114.0, 42.0, 21.2.

HRMS (ESI, m/z) calcd for $\mathrm{C}_{10} \mathrm{H}_{12} \mathrm{NaS}(\mathrm{M}+\mathrm{Na})^{+}: 187.0552$, found: 187.0549 . 
cyclohex-2-en-1-yl(phenyl)sulfane $(1 \mathrm{c})^{12}$

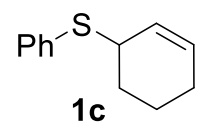

According to the general procedure, substrate 1c (808 mg, $4.25 \mathrm{mmol}$, yield: $85 \%)$ was synthesized as a colorless oil.

${ }^{1} \mathbf{H}$ NMR (500 MHz, CDCl $) \delta 7.43(\mathrm{~d}, J=7.5 \mathrm{~Hz}, 2 \mathrm{H}), 7.30(\mathrm{t}, J=7.6 \mathrm{~Hz}, 2 \mathrm{H}), 7.22$ $(\mathrm{t}, J=7.3 \mathrm{~Hz}, 1 \mathrm{H}), 5.85(\mathrm{~d}, J=10.3 \mathrm{~Hz}, 1 \mathrm{H}), 5.81-5.74(\mathrm{~m}, 1 \mathrm{H}), 3.87(\mathrm{~s}, 1 \mathrm{H}), 2.11-$ $2.01(\mathrm{~m}, 2 \mathrm{H}), 1.99-1.88(\mathrm{~m}, 2 \mathrm{H}), 1.81(\mathrm{ddd}, J=17.7,6.7,3.9 \mathrm{~Hz}, 1 \mathrm{H}), 1.64-1.58$ $(\mathrm{m}, 1 \mathrm{H})$.

${ }^{13}$ C NMR (126 MHz, CDCl $) \delta$ 135.9, 131.3, 130.4, 128.7, 127.0, 126.6, 43.9, 28.8, $25.0,19.5$.

HRMS (ESI, m/z) calcd for $\mathrm{C}_{12} \mathrm{H}_{14} \mathrm{NaS}(\mathrm{M}+\mathrm{Na})^{+}:$213.0708, found: 213.0708.

\subsection{Procedure for the synthesis of substrates $1 \mathrm{zq}$}

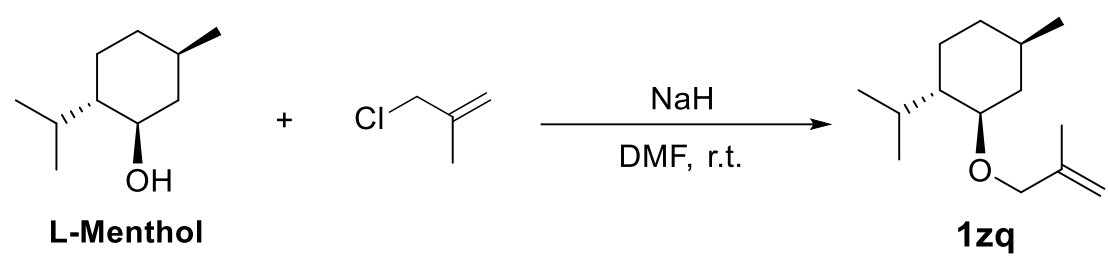

To a solution of L-Menthol (780 mg, $5.00 \mathrm{mmol})$ in anhydrous DMF (20 mL) was added $\mathrm{NaH}(60 \%$ dispersion in mineral oil, $240 \mathrm{mg}, 6.00 \mathrm{mmol})$ at $0{ }^{\circ} \mathrm{C}$ under nitrogen atmosphere. The mixture was stirred for $30 \mathrm{~min}$. 3-chloro-2-methylprop-1-ene (480 $\mu \mathrm{L}, 450 \mathrm{mg}, 5.00 \mathrm{mmol})$ and $\mathrm{NaI}(74.5 \mathrm{mg}, 0.50$ mol) were then added to the mixture. The reaction was stirred at room temperature for $8 \mathrm{~h}$, then quenched with $\mathrm{H}_{2} \mathrm{O}(50 \mathrm{~mL})$ and extracted with ethyl acetate. The combined organic layer was dried over anhydrous $\mathrm{Na}_{2} \mathrm{SO}_{4}$ and concentrated under reduced pressure. The residue was subjected to silica gel chromatography (eluted with petroleum ether: ethyl acetate $=10: 1)$ to afford product $1 \mathbf{z o}(956 \mathrm{mg}, 4.55 \mathrm{mmol}$, yield: $91 \%$ ) as a colorless oil. 
(1S, 2R, 4R)-1-isopropyl-4-methyl-2-((2-methylallyl)oxy)cyclohexane (1zq) ${ }^{13}$

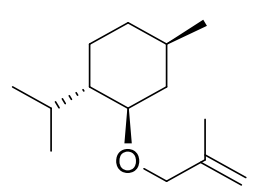

$1 z q$

${ }^{1}$ H NMR (500 MHz, CDCl 3$) \delta 4.96(\mathrm{~d}, J=0.8 \mathrm{~Hz}, 1 \mathrm{H}), 4.84(\mathrm{~s}, 1 \mathrm{H}), 4.00(\mathrm{~d}, J=$ $11.9 \mathrm{~Hz}, 1 \mathrm{H}), 3.75(\mathrm{~d}, J=11.9 \mathrm{~Hz}, 1 \mathrm{H}), 3.06(\mathrm{td}, J=10.5,4.1 \mathrm{~Hz}, 1 \mathrm{H}), 2.26(\mathrm{dtd}, J=$ 14.0, 7.0, $2.7 \mathrm{~Hz}, 1 \mathrm{H}), 2.10(\mathrm{ddd}, J=12.1,5.4,3.8 \mathrm{~Hz}, 1 \mathrm{H}), 1.75$ (s, 3H), $1.67-1.59$ $(\mathrm{m}, 2 \mathrm{H}), 1.36-1.30(\mathrm{~m}, 1 \mathrm{H}), 1.27-1.22(\mathrm{~m}, 2 \mathrm{H}), 1.01-0.94(\mathrm{~m}, 1 \mathrm{H}), 0.91(\mathrm{~d}, J=$ $6.6 \mathrm{~Hz}, 3 \mathrm{H}), 0.89$ (d, $J=7.1 \mathrm{~Hz}, 3 \mathrm{H}), 0.83(\mathrm{dd}, J=4.2,2.1 \mathrm{~Hz}, 1 \mathrm{H}), 0.76$ (d, $J=7.0$ $\mathrm{Hz}, 3 \mathrm{H})$.

${ }^{13}$ C NMR (126 MHz, CDCl3) $\delta 143.0,111.8,78.9,72.6,48.4,40.4,34.6,31.6,25.5$, 23.3, 22.4, 21.0, 19.7, 16.1.

HRMS (ESI, m/z) calcd for $\mathrm{C}_{14} \mathrm{H}_{26} \mathrm{NaO}(\mathrm{M}+\mathrm{Na})^{+}:$233.1876, found: 233.1878 .

\section{Procedure for the synthesis of $[\mathrm{Os}]^{3}$}

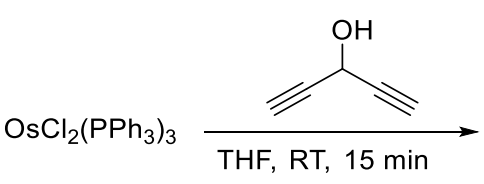

(I)

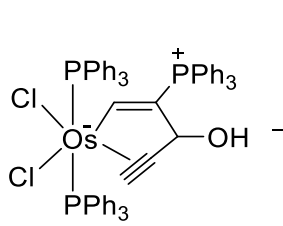

2

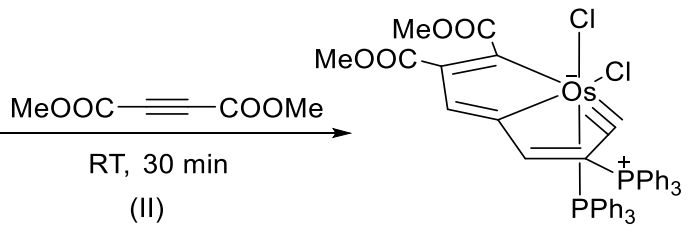

[Os]

For (I): To a green solution of $\mathrm{OsCl}_{2}\left(\mathrm{PPh}_{3}\right)_{3}(1.20 \mathrm{~g}, 1.14 \mathrm{mmol})$ in THF $(15 \mathrm{~mL})$ was slowly added a THF solution $(12 \mathrm{~mL})$ of $\mathrm{HC} \equiv \mathrm{CCH}(\mathrm{OH}) \mathrm{C} \equiv \mathrm{CH}(101 \mathrm{mg}, 1.26 \mathrm{mmol})$. The reaction mixture was stirred for $15 \mathrm{~min}$. to give a brownish-yellow suspension. The yellow solid was collected by filtration, washed with THF $(5 \times 2 \mathrm{~mL})$ and then dried under vacuum to to afford compound 2 as a yellow solid $(790 \mathrm{mg}, 0.71 \mathrm{mmol}$, yield: $62 \%)$.

For (II): MeOOCC $\equiv$ CCOOMe $(258 \mu \mathrm{L}, 2.05 \mathrm{mmol})$ was added to a suspension of compound 2 (790 mg, $0.71 \mathrm{mmol})$ in $\mathrm{CH}_{2} \mathrm{Cl}_{2}(40 \mathrm{~mL})$. The mixture was stirred at 
room temperature for $3 \mathrm{~h}$ to give a yellow solution. The solution was evaporated under vacuum to a volume of ca. $5 \mathrm{~mL}$, then diethyl ether $(30 \mathrm{~mL})$ was added to the solution. The yellow precipitate was collected by filtration, washed with diethyl ether $(2 \times 10 \mathrm{~mL})$ and dried under vacuum to give [Os] (546 mg, yield: 79\%) as a yellow solid.

\section{Procedure for the synthesis of catalyst $[\mathrm{Os}-\mathrm{Cu}]^{4},[\mathrm{Os}-\mathrm{Cu}-\mathrm{A}]$ and $[\mathrm{Os}-\mathrm{Cu}-\mathrm{B}]$}
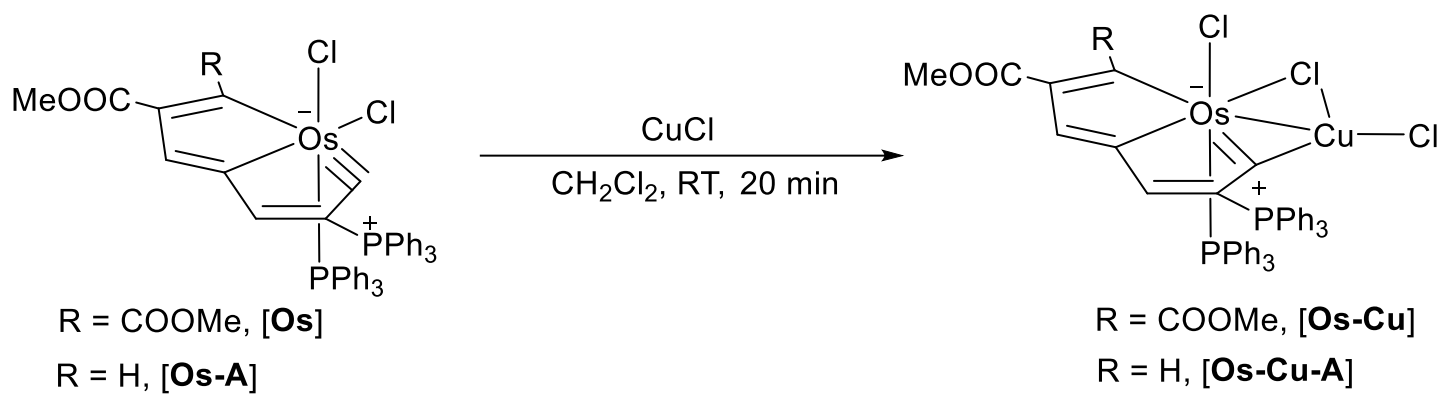

$\mathrm{R}=\mathrm{H},[\mathrm{Os}-\mathbf{A}]$

$\mathrm{R}=\mathrm{COOMe},[\mathrm{Os}-\mathrm{Cu}]$

$\mathrm{R}=\mathrm{H},[\mathrm{Os}-\mathrm{Cu}-\mathrm{A}]$

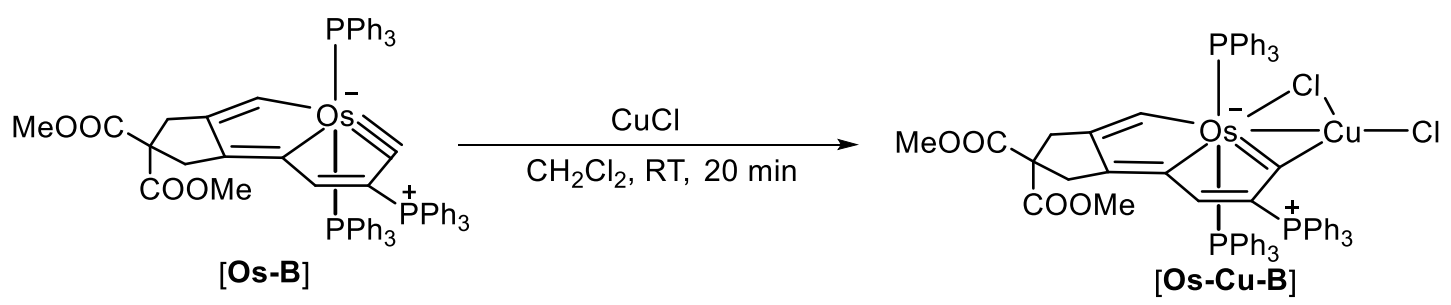

General Procedure. For [Os-Cu], a mixture of [Os] (200 mg, $0.20 \mathrm{mmol})$ and cuprous chloride $(198 \mathrm{mg}, 2.00 \mathrm{mmol})$ in $\mathrm{CH}_{2} \mathrm{Cl}_{2}(10 \mathrm{~mL})$ was stirred at room temperature for 20 min to give a brown solution. The excess cuprous chloride was removed by filtration. The filtrate was concentrated to ca. $2 \mathrm{~mL}$, the residue was purified by column chromatography (silica gel, 200-300 mesh, eluent: dichloro methane/acetone $=10: 1)$ to afford [Os-Cu] $(209 \mathrm{mg}$, yield: $96 \%)$ as a yellow solid.

General Procedure. For [Os-Cu-A]and[Os-Cu-B] , a mixture of [Os-A] or [Os-B] (0.20 mmol) and cuprous chloride $(198 \mathrm{mg}, 2.00 \mathrm{mmol})$ in $\mathrm{CH}_{2} \mathrm{Cl}_{2}(10 \mathrm{~mL})$ was stirred at room temperature for 20 min to give a brown solution. The excess cuprous chloride was removed by filtration. The filtrate was concentrated to ca. $2 \mathrm{~mL}$, and diethyl ether $(20 \mathrm{~mL})$ was added to the solution. The precipitate was collected by 
filtration, washed with diethyl ether $(2 \times 10 \mathrm{~mL})$ and dried under vacuum to give[Os-Cu-A] or [Os-Cu-B] as a yellow solid..

\section{Selective Difunctionalization of Unactivated Alkenes}

\subsection{Optimization for reaction conditions}

A dried $10 \mathrm{~mL}$ Schlenk tube was charged with 1a $(0.20 \mathrm{mmol}), 3 a(0.10 \mathrm{mmol})$, catalysts $(0.01 \mathrm{mmol}), \mathbf{2 a}(0.40 \mathrm{mmol})$, and $\mathrm{CH}_{2} \mathrm{Cl}_{2}(4.0 \mathrm{~mL})$. The Schlenk tube was positioned approximately $3 \mathrm{~cm}$ away from a $24 \mathrm{~W}$ blue LEDs lamp $\left(\lambda_{\max }=450 \mathrm{~nm}\right)$. After being stirred at $25{ }^{\circ} \mathrm{C}$ for the indicated time, the reaction mixture was concentrated to dryness. The reaction mixture was purified by silica gel chromatography to provide products.

Table S1. Optimization for reaction conditions
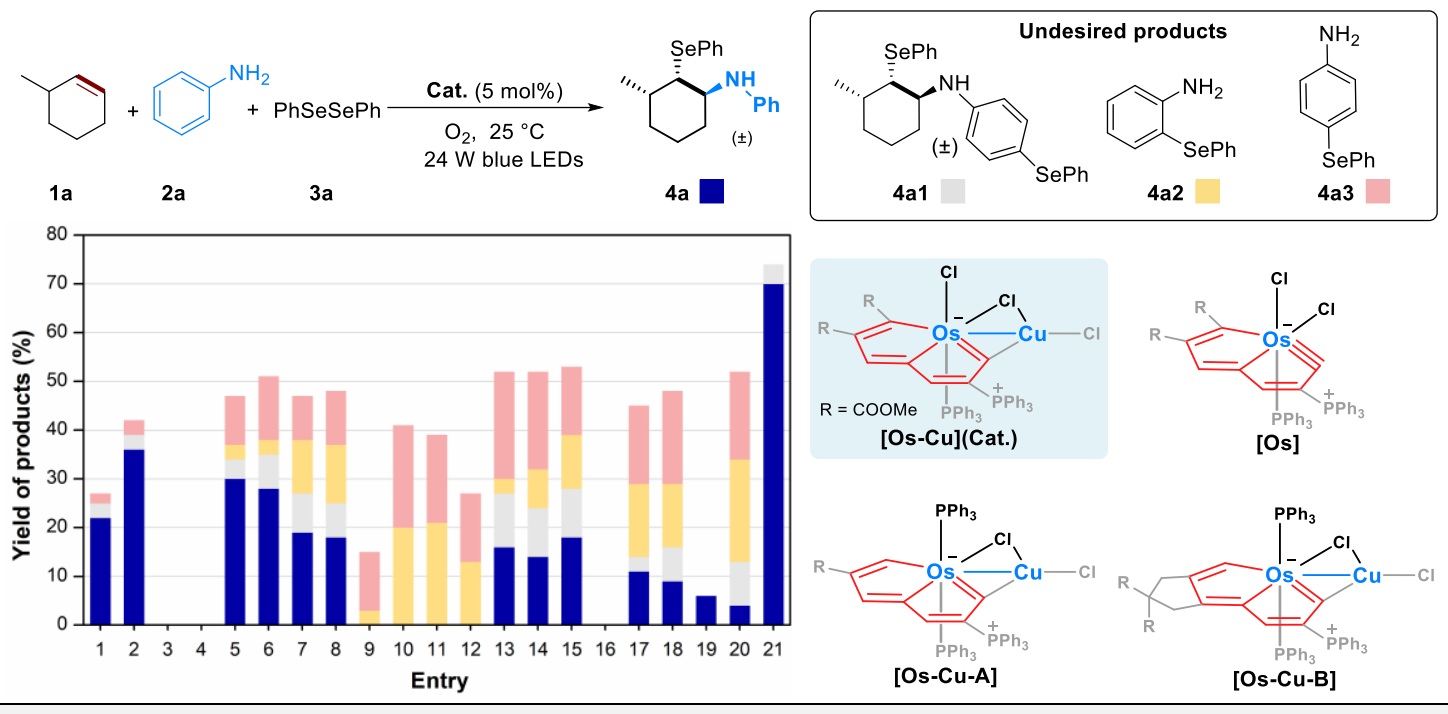

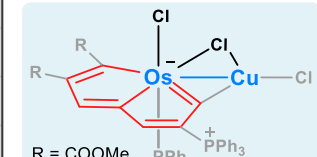

[Os-Cu](Cat.)
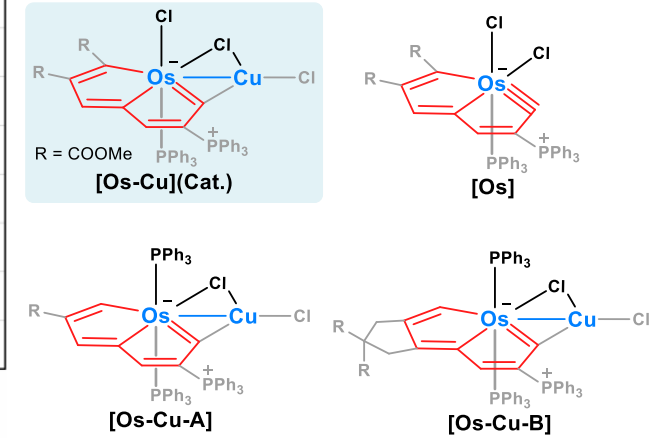

[Os-Cu-A]

Yield (\%)

\begin{tabular}{cccccccc}
\multirow{2}{*}{ Entry } & \multirow{2}{*}{ Catalyst or additive } & \multirow{2}{*}{ Solvent } & \multirow{2}{*}{ Atmosphere } & \multicolumn{5}{c}{ Yield $(\%)^{\mathbf{b}}$} \\
\cline { 5 - 8 } & & & & $\mathbf{4 a}$ & $\mathbf{4 a 1}$ & $\mathbf{4 a 2}$ & $\mathbf{4 a 3}$ \\
\hline 1 & {$[\mathbf{O s}-\mathbf{C u}]$} & $\mathrm{CH}_{2} \mathrm{Cl}_{2}$ & air & 22 & 3 & 0 & 2 \\
2 & {$[\mathbf{O s}-\mathbf{C u}]$} & $\mathrm{CH}_{2} \mathrm{Cl}_{2}$ & $\mathrm{O}_{2}$ & 36 & 3 & 0 & 3 \\
3 & {$[\mathbf{O s}-\mathbf{C u}]$} & $\mathrm{CH}_{2} \mathrm{Cl}_{2}$ & $\mathrm{~N}_{2}$ & 0 & 0 & 0 & 0 \\
$4^{\mathbf{c}}$ & {$[\mathbf{O s}-\mathbf{C u}]$} & $\mathrm{CH}_{2} \mathrm{Cl}_{2}$ & $\mathrm{O}_{2}$ & 0 & 0 & 0 & 0 \\
5 & $\mathrm{CuCl} /[\mathbf{O s}]$ & $\mathrm{CH}_{2} \mathrm{Cl}_{2}$ & $\mathrm{O}_{2}$ & 30 & 4 & 3 & 10 \\
6 & $\mathrm{CuBr} /[\mathbf{O s}]$ & $\mathrm{CH}_{2} \mathrm{Cl}_{2}$ & $\mathrm{O}_{2}$ & 28 & 7 & 3 & 13 \\
7 & {$[\mathbf{O s}-\mathbf{C u}-\mathbf{A}]$} & $\mathrm{CH}_{2} \mathrm{Cl}_{2}$ & $\mathrm{O}_{2}$ & 19 & 8 & 11 & 9 \\
8 & {$[\mathbf{O s}-\mathbf{C u}-\mathbf{B}]$} & $\mathrm{CH}_{2} \mathrm{Cl}_{2}$ & $\mathrm{O}_{2}$ & 18 & 7 & 12 & 11 \\
9 & {$[\mathbf{O s}]$} & $\mathrm{CH}_{2} \mathrm{Cl}_{2}$ & $\mathrm{O}_{2}$ & 0 & 0 & 3 & 12
\end{tabular}




\begin{tabular}{lccccccc}
10 & $\mathrm{Na}_{2}-\mathrm{E}$ osin $\mathrm{Y}$ & $\mathrm{CH}_{2} \mathrm{Cl}_{2}$ & $\mathrm{O}_{2}$ & 0 & 0 & 20 & 21 \\
11 & $\mathrm{Ru}(\mathrm{bpy})_{3}\left(\mathrm{PF}_{6}\right)_{2}$ & $\mathrm{CH}_{2} \mathrm{Cl}_{2}$ & $\mathrm{O}_{2}$ & 0 & 0 & 21 & 18 \\
12 & & $\mathrm{CH}_{2} \mathrm{Cl}_{2}$ & $\mathrm{O}_{2}$ & 0 & 0 & 13 & 14 \\
13 & $\mathrm{CuCl}$ & $\mathrm{CH}_{2} \mathrm{Cl}_{2}$ & $\mathrm{O}_{2}$ & 16 & 11 & 3 & 22 \\
14 & $\mathrm{CuCl} / \mathrm{PPh}_{3}$ & $\mathrm{CH}_{2} \mathrm{Cl}_{2}$ & $\mathrm{O}_{2}$ & 14 & 10 & 8 & 20 \\
15 & $\mathrm{CuCl}\left(\mathrm{PPh}_{3}\right)_{3}$ & $\mathrm{CH}_{2} \mathrm{Cl}_{2}$ & $\mathrm{O}_{2}$ & 18 & 10 & 11 & 14 \\
16 & $\mathrm{CuCl} / \mathrm{OsCl}_{2}\left(\mathrm{PPh}_{3}\right)_{2}$ & $\mathrm{CH}_{2} \mathrm{Cl}_{2}$ & $\mathrm{O}_{2}$ & 0 & 0 & 0 & 0 \\
$17^{\mathrm{d}}$ & $\mathrm{PhI}\left({\mathrm{OAc})_{2}}_{18^{\mathrm{e}}}\right.$ & $\mathrm{CH}_{2} \mathrm{Cl}_{2}$ & $\mathrm{O}_{2}$ & 11 & 3 & 15 & 16 \\
$19^{\mathrm{f}}$ & $\mathrm{K}_{2} \mathrm{~S}_{2} \mathrm{O}_{8}$ & $\mathrm{THF}$ & $\mathrm{O}_{2}$ & 9 & 7 & 13 & 19 \\
$20^{\mathrm{g}}$ & $\mathrm{FeBr}_{3}$ & $\mathrm{EtOAc}^{\mathrm{h}}$ & $\mathrm{O}_{2}$ & 6 & 0 & 0 & 0 \\
$21^{\mathrm{h}}$ & - & $\mathrm{CH}_{2} \mathrm{Cl}_{2}$ & $\mathrm{O}_{2}$ & 4 & 9 & 21 & 18 \\
\hline
\end{tabular}

${ }^{a}$ Reaction conditions: 1a $(0.20 \mathrm{mmol}), \mathbf{2 a}(0.40 \mathrm{mmol}), 3 \mathbf{a}(0.10 \mathrm{mmol})$, catalyst $(0.010 \mathrm{mmol}$, $5 \mathrm{~mol} \%$ ), solvent $(4.0 \mathrm{~mL}), 25^{\circ} \mathrm{C}$, a $24 \mathrm{~W}$ blue LEDs lamp $\left(\lambda_{\max }=450 \mathrm{~nm}\right)$, under an oxygen atmosphere, $24 \mathrm{~h} .{ }^{b}$ Isolated yield. ${ }^{c}$ Under ambient light. ${ }^{\mathrm{d}}$ Reaction conditions: $1 \mathrm{a}(0.25 \mathrm{mmol})$, 3a $(0.25 \mathrm{mmol}), \mathrm{PhI}(\mathrm{OAc})_{2}(0.25 \mathrm{mmol}), \mathrm{PhNH}_{2}(1.0 \mathrm{mmol})$, and $\mathrm{CH}_{2} \mathrm{Cl}_{2}(2.0 \mathrm{~mL})$ in an open flask at room temperature under ambient light for $20 \mathrm{~h}$. ${ }^{\mathrm{e}}$ Reaction conditions: $1 \mathrm{a}(0.5 \mathrm{mmol})$, 2a $(0.55 \mathrm{mmol}), \mathrm{K}_{2} \mathrm{~S}_{2} \mathrm{O}_{8}(1.0 \mathrm{mmol}), 3 \mathrm{a}(0.5 \mathrm{mmol})$ in $2 \mathrm{~mL}$ THF, stirred at $80{ }^{\circ} \mathrm{C}$ under ambient light for $12 \mathrm{~h} .{ }^{\mathrm{f}}$ Reaction conditions: $\mathbf{1 a}(0.1 \mathrm{mmol}), \mathbf{2 a}(0.15 \mathrm{mmol}), \mathbf{3 a}(0.06 \mathrm{mmol})$, $\mathrm{FeBr}_{3}(10 \mathrm{~mol} \%)$, and EtOAc $(1.0 \mathrm{~mL})$, in air, at room temperature, under the irradiation of 24

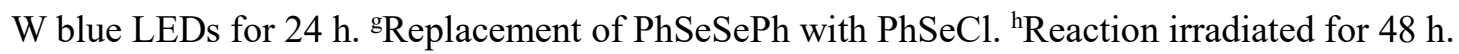

\subsubsection{Characterization of $4 \mathrm{a} 1,4 \mathrm{a} 2,4 \mathrm{a} 3$}

N-((1S,2S,3S)-3-methyl-2-(phenylselanyl)cyclohexyl)-4-(phenylselanyl)aniline (4a1)

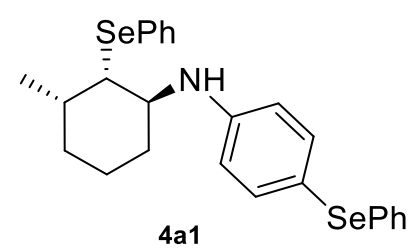

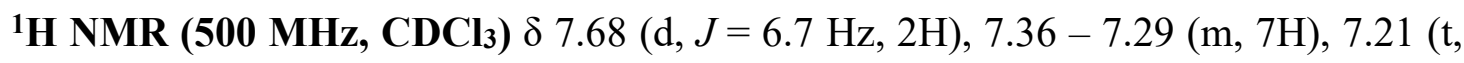
$J=7.3 \mathrm{~Hz}, 2 \mathrm{H}), 7.19-7.14(\mathrm{~m}, 1 \mathrm{H}), 6.24(\mathrm{~d}, J=8.4 \mathrm{~Hz}, 2 \mathrm{H}), 4.16(\mathrm{~s}, 1 \mathrm{H}), 3.84$ (d, $J$ $=3.3 \mathrm{~Hz}, 1 \mathrm{H}), 3.49(\mathrm{~s}, 1 \mathrm{H}), 2.31(\mathrm{dd}, J=17.2,7.8 \mathrm{~Hz}, 1 \mathrm{H}), 2.16-2.03(\mathrm{~m}, 1 \mathrm{H}), 1.81$ 
- $1.68(\mathrm{~m}, 1 \mathrm{H}), 1.59-1.49(\mathrm{~m}, 3 \mathrm{H}), 1.33(\mathrm{dd}, J=13.1,8.4 \mathrm{~Hz}, 1 \mathrm{H}), 1.14(\mathrm{~d}, J=6.7$ $\mathrm{Hz}, 3 \mathrm{H})$.

${ }^{13}$ C NMR (126 MHz, CDCl $) \delta 147.0,142.3,137.3,135.4,134.4,129.9,129.2$, 129.0, 127.8, 125.9, 114.4, 113.6, 55.3, 52.8, 31.5, 31.0, 27.4, 20.7, 20.6.

IR (film): v (cm-1) 3389, 2976, 1451, 1380, 1067, 816, 692.

HRMS (ESI, m/z) calcd for $\mathrm{C}_{25} \mathrm{H}_{27} \mathrm{NNaSe}_{2}(\mathrm{M}+\mathrm{Na})^{+}$: 524.0336, found: 524.0371.

2-(phenylselanyl)aniline (4a2)

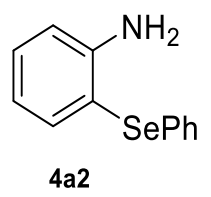

${ }^{1}$ H NMR (500 MHz, CDCl 3$) \delta 7.61(\mathrm{dd}, J=7.6,1.2 \mathrm{~Hz}, 1 \mathrm{H}), 7.29-7.18(\mathrm{~m}, 6 \mathrm{H}), 6.83$ $(\mathrm{d}, J=8.0 \mathrm{~Hz}, 1 \mathrm{H}), 6.73(\mathrm{t}, J=7.4 \mathrm{~Hz}, 1 \mathrm{H}), 4.32(\mathrm{~s}, 2 \mathrm{H})$.

${ }^{13}$ C NMR (126 MHz, CDCl 3$) \delta$ 148.6, 138.6, 131.7, 131.1, 129.4, 129.3, 126.2, 118.8, 115.0, 112.8 .

IR (film): $v\left(\mathrm{~cm}^{-1}\right)$ 3389, 1066, 743, 698.

HRMS (ESI, m/z) calcd for $\mathrm{C}_{12} \mathrm{H}_{12} \mathrm{NSe}(\mathrm{M}+\mathrm{H})^{+}: 250.0135$, found: 250.0129 .

4-(phenylselanyl)aniline (4a3)

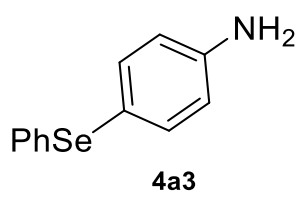

${ }^{1}$ H NMR (500 MHz, CDCl 3$) \delta 7.43-7.39$ (m, 2H), 7.29 (ddd, $J=4.5,3.6,1.9 \mathrm{~Hz}$, 2H), 7.21-7.13 (m, 3H), 6.67-6.61 (m, 2H), $3.78(\mathrm{~s}, 2 \mathrm{H})$.

${ }^{13}$ C NMR (126 MHz, CDCl 3$) \delta$ 146., 137.1, 134.1, 130.2, 129.0, 126.0, 116.5, 116.0. IR (film): $v\left(\mathrm{~cm}^{-1}\right) 3393,1068,851,700$.

HRMS (ESI, m/z) calcd for $\mathrm{C}_{12} \mathrm{H}_{12} \mathrm{NSe}(\mathrm{M}+\mathrm{H})^{+}:$250.0135, found: 250.0129. 


\subsection{Substrate Scope for Nucleophiles}

General Procedure. For 4a-4zb, a dried $10 \mathrm{~mL}$ Schlenk tube was charged with 1a (0.20 mmol), 2a-2zb (0.40 mmol), 3 (0.10 mmol), [Os-Cu] (10.9 mg, $0.01 \mathrm{mmol})$, and $\mathrm{CH}_{2} \mathrm{Cl}_{2}(4.0 \mathrm{~mL})$. The Schlenk tube was positioned approximately $3 \mathrm{~cm}$ away from a $24 \mathrm{~W}$ blue LEDs lamp $\left(\lambda_{\max }=450 \mathrm{~nm}\right)$. After being stirred at $25{ }^{\circ} \mathrm{C}$ for the 48 $\mathrm{h}$ under an $\mathrm{O}_{2}$ (balloon) atmosphere, the reaction mixture was concentrated to dryness. The residue was purified by flash chromatography (petroleum ether/ethyl acetate) to give the desired product; For $\mathbf{4 z c}-\mathbf{4 z g}$, a dried $10 \mathrm{~mL}$ Schlenk tube was charged with 1a (0.20 mmol), 2zc-2zg (0.50 mL), 3a (0.10 mmol), [Os-Cu] (10.9 mg, $0.01 \mathrm{mmol})$, and $\mathrm{CH}_{2} \mathrm{Cl}_{2}(3.5 \mathrm{~mL})$. The Schlenk tube was positioned approximately $3 \mathrm{~cm}$ away from a $24 \mathrm{~W}$ blue LEDs lamp $\left(\lambda_{\max }=450 \mathrm{~nm}\right)$. After being stirred at $25{ }^{\circ} \mathrm{C}$ for the 48 $\mathrm{h}$ under an $\mathrm{O}_{2}$ (balloon) atmosphere, the reaction mixture was concentrated to dryness. The residue was purified by flash chromatography (petroleum ether/ethyl acetate) to give the desired product.<smiles>CC1C=C(P)CCC1</smiles>

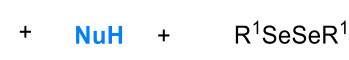

2

3

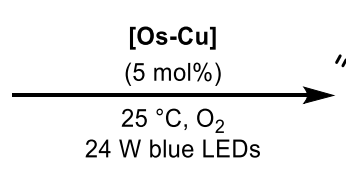

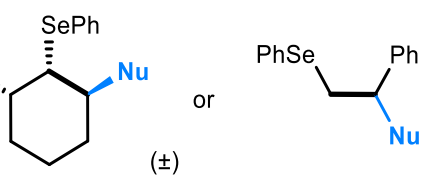

$( \pm)$

$\mathrm{R}=\mathrm{H}, \mathbf{4 a}-\mathbf{4 g}, \mathbf{4 0 - 4 z g}$

\subsubsection{Experimental details and characterization data}

\section{N-((1S, 2S, 3S)-3-methyl-2-(phenylselanyl)cyclohexyl)aniline (4a)}

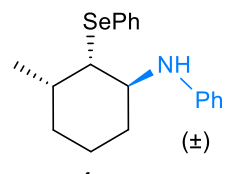

A dried $10 \mathrm{~mL}$ Schlenk tube was charged with 1a $(24 \mu \mathrm{L}, 19.2 \mathrm{mg}, 0.20 \mathrm{mmol})$, phenylamine (37 $\mu \mathrm{L}, 37.3 \mathrm{mg}, 0.40 \mathrm{mmol}), 3 \mathbf{3}(31.2 \mathrm{mg}, 0.10 \mathrm{mmol})$, [Os-Cu] (10.9 mg, $0.01 \mathrm{mmol})$, and $\mathrm{CH}_{2} \mathrm{Cl}_{2}(4.0 \mathrm{~mL})$. The Schlenk tube was positioned approximately $3 \mathrm{~cm}$ away from a $24 \mathrm{~W}$ blue LEDs lamp $\left(\lambda_{\max }=450 \mathrm{~nm}\right)$. After being stirred at $25{ }^{\circ} \mathrm{C}$ for the $48 \mathrm{~h}$ under an $\mathrm{O}_{2}$ (balloon) atmosphere, the reaction mixture 
was concentrated to dryness. The residue was purified by flash chromatography (petroleum ether/ethyl acetate $=40: 1)$ to afford product 4a as a yellow oil (48.3 mg, 0.140 mmol, yield: $70 \%$ ).

${ }^{1}$ H NMR (500 MHz, CDCl3) $\delta 7.73-7.59$ (m, 2H), 7.35 - 7.27 (m, 3H), $7.10-6.98$ $(\mathrm{m}, 2 \mathrm{H}), 6.69-6.58(\mathrm{~m}, 1 \mathrm{H}), 6.30(\mathrm{~d}, J=7.7 \mathrm{~Hz}, 2 \mathrm{H}), 4.01(\mathrm{~s}, 1 \mathrm{H}), 3.84(\mathrm{dd}, J=7.7$, $3.7 \mathrm{~Hz}, 1 \mathrm{H}), 3.50(\mathrm{t}, J=3.5 \mathrm{~Hz}, 1 \mathrm{H}), 2.30-2.21(\mathrm{~m}, 1 \mathrm{H}), 2.05(\mathrm{dtt}, J=13.6,6.7,3.4$ Hz, 1H), 1.69 (ddd, $J=12.8,8.4,4.1 \mathrm{~Hz}, 1 \mathrm{H}), 1.58-1.50(\mathrm{~m}, 3 \mathrm{H}), 1.35-1.29(\mathrm{~m}$, $1 \mathrm{H}), 1.09(\mathrm{~d}, J=6.8 \mathrm{~Hz}, 3 \mathrm{H})$.

${ }^{13}$ C NMR (126 MHz, CDCl 3$) \delta 146.6,135.3,130.2,129.3,129.1,127.6,117.1,112.8$, $55.3,52.9,31.4,31.0,29.7,20.8$.

IR (film): $v\left(\mathrm{~cm}^{-1}\right)$ 3396, 2956, 2850, 1460, 1379, 1076, 748, 712.

HRMS (ESI, m/z) calcd for $\mathrm{C}_{19} \mathrm{H}_{24} \mathrm{NSe}(\mathrm{M}+\mathrm{H})^{+}:$346.1068, found: 346.1063 .

\section{2,6-dimethyl-N-((1S, 2S, 3S)-3-methyl-2-(phenylselanyl)cyclohexyl)aniline (4b)}

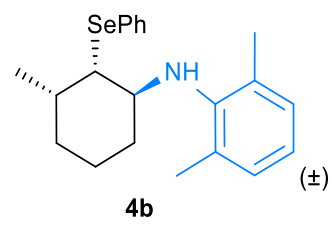

A dried $10 \mathrm{~mL}$ Schlenk tube was charged with 1a $(24 \mu \mathrm{L}, 19.2 \mathrm{mg}, 0.20 \mathrm{mmol})$, 2,6-dimethylaniline ( $49 \mu \mathrm{L}, 48.5 \mathrm{mg}, 0.40 \mathrm{mmol}), 3 \mathbf{3}$ (31.2 mg, $0.10 \mathrm{mmol}$ ), [Os-Cu] (10.9 mg, $0.01 \mathrm{mmol}$ ), and $\mathrm{CH}_{2} \mathrm{Cl}_{2}(4.0 \mathrm{~mL})$. The Schlenk tube was positioned approximately $3 \mathrm{~cm}$ away from a $24 \mathrm{~W}$ blue LEDs lamp $\left(\lambda_{\max }=450 \mathrm{~nm}\right)$. After being stirred at $25{ }^{\circ} \mathrm{C}$ for the $48 \mathrm{~h}$ under an $\mathrm{O}_{2}$ (balloon) atmosphere, the reaction mixture was concentrated to dryness. The residue was purified by flash chromatography (petroleum ether/ethyl acetate $=40: 1)$ to afford product $\mathbf{4 b}$ as a colorless oil ( $45.5 \mathrm{mg}$, 0.122 mmol, yield: $61 \%$ ).

${ }^{1}$ H NMR (500 MHz, CDCl 3$) \delta 7.48(\mathrm{dd}, J=8.1,1.3 \mathrm{~Hz}, 2 \mathrm{H}), 7.25-7.18(\mathrm{~m}, 3 \mathrm{H})$, $6.94(\mathrm{~d}, J=7.5 \mathrm{~Hz}, 2 \mathrm{H}), 6.78(\mathrm{t}, J=7.4 \mathrm{~Hz}, 1 \mathrm{H}), 3.53(\mathrm{dd}, J=7.9,3.8 \mathrm{~Hz}, 3 \mathrm{H}), 2.12$ (s, 6H), $2.08(\mathrm{dd}, J=5.9,4.1 \mathrm{~Hz}, 1 \mathrm{H}), 1.71-1.47(\mathrm{~m}, 5 \mathrm{H}), 1.38(\mathrm{ddd}, J=12.9,9.4$, $5.7 \mathrm{~Hz}, 1 \mathrm{H}), 1.13(\mathrm{~d}, J=6.8 \mathrm{~Hz}, 3 \mathrm{H})$. 
${ }^{13}$ C NMR (126 MHz, CDCl 3 ) $\delta$ 144.9, 134.4, 130.3, 129.0 (3C), 127.2, 121.4, 57.1, $56.3,31.8,31.2,20.9,20.1,19.0$.

IR (film): $v\left(\mathrm{~cm}^{-1}\right)$ 3383, 2970, 2855, 1532, 1463, 1377, 1066, 809, 698.

HRMS (ESI, m/z) calcd for $\mathrm{C}_{21} \mathrm{H}_{27} \mathrm{NNaSe}(\mathrm{M}+\mathrm{Na})^{+}: 396.1206$, found: 396.1211 .

\section{4-fluoro-N-((1S, 2S, 3S)-3-methyl-2-(phenylselanyl)cyclohexyl)aniline (4c)}

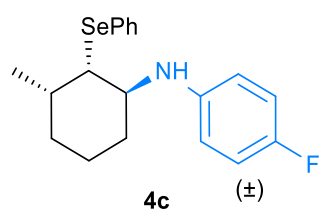

A dried $10 \mathrm{~mL}$ Schlenk tube was charged with $1 \mathrm{a}(24 \mu \mathrm{L}, 19.2 \mathrm{mg}, 0.20 \mathrm{mmol})$, 4-fluoroaniline (38 $\mu \mathrm{L}, 44.5 \mathrm{mg}, 0.40 \mathrm{mmol}), 3 \mathbf{a}(31.2 \mathrm{mg}, 0.10 \mathrm{mmol})$, [Os-Cu] (10.9 $\mathrm{mg}, \quad 0.01 \mathrm{mmol})$, and $\mathrm{CH}_{2} \mathrm{Cl}_{2}(4.0 \mathrm{~mL})$. The Schlenk tube was positioned approximately $3 \mathrm{~cm}$ away from a $24 \mathrm{~W}$ blue LEDs lamp $\left(\lambda_{\max }=450 \mathrm{~nm}\right)$. After being stirred at $25{ }^{\circ} \mathrm{C}$ for the $48 \mathrm{~h}$ under an $\mathrm{O}_{2}$ (balloon) atmosphere, the reaction mixture was concentrated to dryness. The residue was purified by flash chromatography (petroleum ether/ethyl acetate $=40: 1)$ to afford product $4 \mathbf{c}$ as a white solid $(60.0 \mathrm{mg}$, 0.154 mmol, yield: $77 \%$ ).

${ }^{1} \mathbf{H}$ NMR (400 MHz, CDCl 3$) \delta 7.68-7.59(\mathrm{~m}, 2 \mathrm{H}), 7.35-7.27(\mathrm{~m}, 3 \mathrm{H}), 6.78-6.70$ (m, 2H), $6.23-6.17(\mathrm{~m}, 2 \mathrm{H}), 3.87(\mathrm{~s}, 1 \mathrm{H}), 3.74(\mathrm{q}, J=3.9 \mathrm{~Hz}, 1 \mathrm{H}), 3.45(\mathrm{t}, J=3.6$ $\mathrm{Hz}, 1 \mathrm{H}), 2.25$ (ddt, $J=14.9,12.6,4.4 \mathrm{~Hz}, 1 \mathrm{H}), 2.04$ (dtt, $J=13.4,6.7,3.3 \mathrm{~Hz}, 1 \mathrm{H}$ ), $1.67(\mathrm{ddd}, J=8.7,6.4,3.4 \mathrm{~Hz}, 1 \mathrm{H}), 1.55-1.50(\mathrm{~m}, 2 \mathrm{H}), 1.34-1.24(\mathrm{~m}, 2 \mathrm{H}), 1.09(\mathrm{~d}$, $J=6.8 \mathrm{~Hz}, 3 \mathrm{H})$.

${ }^{13}$ C NMR (101 MHz, CDCl 3$) \delta 155.6(\mathrm{~d}, J=234.9 \mathrm{~Hz}), 142.9(\mathrm{~d}, J=1.8 \mathrm{~Hz}), 135.3$, 130.1, 129.1, 127.6, $115.6(\mathrm{~d}, J=22.2 \mathrm{~Hz}), 113.6$ (d, $J=7.3 \mathrm{~Hz}), 55.3,53.6,31.4$, $31.1,29.7,27.5,20.7$.

IR (film): $v\left(\mathrm{~cm}^{-1}\right)$ 3497, 3960, 2926, 1458, 1383, 1107, 1066, 841, 733.

HRMS (ESI, m/z) calcd for $\mathrm{C}_{19} \mathrm{H}_{23} \mathrm{FNSe}^{+}(\mathrm{M}+\mathrm{H})^{+}: 364.0974$, found: 364.0969 .

\section{4-chloro-N-((1S, 2S, 3S)-3-methyl-2-(phenylselanyl)cyclohexyl)aniline (4d)}




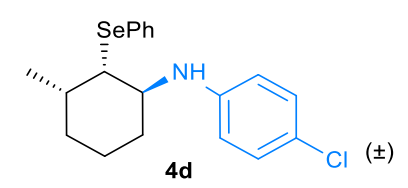

A dried $10 \mathrm{~mL}$ Schlenk tube was charged with 1a $(24 \mu \mathrm{L}, 19.2 \mathrm{mg}, 0.20 \mathrm{mmol})$, 4-chloroaniline (51.0 mg, $0.40 \mathrm{mmol}), \mathbf{3 a}(31.2 \mathrm{mg}, 0.10 \mathrm{mmol})$, [Os-Cu] (10.9 mg, $0.01 \mathrm{mmol})$, and $\mathrm{CH}_{2} \mathrm{Cl}_{2}(4.0 \mathrm{~mL})$. The Schlenk tube was positioned approximately 3 $\mathrm{cm}$ away from a $24 \mathrm{~W}$ blue LEDs lamp $\left(\lambda_{\max }=450 \mathrm{~nm}\right)$. After being stirred at $25{ }^{\circ} \mathrm{C}$ for the $48 \mathrm{~h}$ under an $\mathrm{O}_{2}$ (balloon) atmosphere, the reaction mixture was concentrated to dryness. The residue was purified by flash chromatography (petroleum ether/ethyl acetate $=40: 1)$ to afford product $\mathbf{4 d}$ as a brown oil $(54.6 \mathrm{mg}, 0.144 \mathrm{mmol}$, yield: $72 \%)$.

${ }^{1}$ H NMR (500 MHz, CDCl $)$ ) $7.71-7.59$ (m, 2H), $7.37-7.28(\mathrm{~m}, 3 \mathrm{H}), 7.05-6.92$ (m, 2H), $6.20-6.13(\mathrm{~m}, 2 \mathrm{H}), 3.99(\mathrm{~s}, 1 \mathrm{H}), 3.75(\mathrm{~s}, 1 \mathrm{H}), 3.43(\mathrm{t}, J=3.7 \mathrm{~Hz}, 1 \mathrm{H}), 2.27$ (ddt, $J=13.8,11.5,4.2 \mathrm{~Hz}, 1 \mathrm{H}), 2.02(\mathrm{dtt}, J=13.7,6.8,3.4 \mathrm{~Hz}, 1 \mathrm{H}), 1.73-1.65(\mathrm{~m}$, $1 \mathrm{H}), 1.53(\mathrm{ddd}, J=12.1,11.3,5.4 \mathrm{~Hz}, 3 \mathrm{H}), 1.33-1.26(\mathrm{~m}, 1 \mathrm{H}), 1.10(\mathrm{~d}, J=6.8 \mathrm{~Hz}$, $3 \mathrm{H})$.

${ }^{13}$ C NMR (126 MHz, CDCl 3$) \delta 145.2,135.4,130.0,129.1$ (2C), , 127.8, 121.6, 113.8, 55.1, 53.0, 31.4, 31.0, 27.4, $20.7(2 \mathrm{C})$.

IR (film): $v\left(\mathrm{~cm}^{-1}\right)$ 3399, 2955, 2926, 1465, 1376, 1058, 841, 754, 711.

HRMS (ESI, m/z) calcd for $\mathrm{C}_{19} \mathrm{H}_{22} \mathrm{ClNNaSe}(\mathrm{M}+\mathrm{Na})^{+}: 402.0504$, found: 402.0502 .

\section{4-bromo-N-((1S, 2S, 3S)-3-methyl-2-(phenylselanyl)cyclohexyl)aniline (4e)}

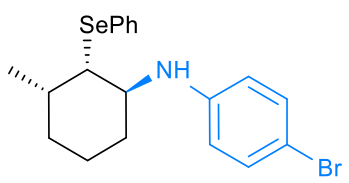

$4 \mathrm{e}$

$( \pm)$

A dried $10 \mathrm{~mL}$ Schlenk tube was charged with 1a $(24 \mu \mathrm{L}, 19.2 \mathrm{mg}, 0.20 \mathrm{mmol})$, 4-bromoaniline (68 mg, $0.40 \mathrm{mmol}), 3 \mathrm{a}$ (31.2 mg, $0.10 \mathrm{mmol})$, [Os-Cu] (10.9 mg, $0.01 \mathrm{mmol})$, and $\mathrm{CH}_{2} \mathrm{Cl}_{2}(4.0 \mathrm{~mL})$. The Schlenk tube was positioned approximately 3 $\mathrm{cm}$ away from a $24 \mathrm{~W}$ blue LEDs lamp $\left(\lambda_{\max }=450 \mathrm{~nm}\right)$. After being stirred at $25{ }^{\circ} \mathrm{C}$ for the $48 \mathrm{~h}$ under an $\mathrm{O}_{2}$ (balloon) atmosphere, the reaction mixture was concentrated 
to dryness. The residue was purified by flash chromatography (petroleum ether/ethyl acetate $=40: 1)$ to afford product $4 \mathbf{e}$ as a brown oil $(62.6 \mathrm{mg}, 0.148 \mathrm{mmol}$, yield: $74 \%)$.

${ }^{1}$ H NMR (500 MHz, CDCl $) \delta 7.71-7.62(\mathrm{~m}, 2 \mathrm{H}), 7.37$ - $7.28(\mathrm{~m}, 3 \mathrm{H}), 7.16-7.02$ (m, 2H), $6.16-6.06(\mathrm{~m}, 2 \mathrm{H}), 4.01(\mathrm{~s}, 1 \mathrm{H}), 3.75(\mathrm{~d}, J=3.8 \mathrm{~Hz}, 1 \mathrm{H}), 3.43(\mathrm{t}, J=3.7$ $\mathrm{Hz}, 1 \mathrm{H}), 2.27$ (ddt, $J=15.8,11.6,4.2 \mathrm{~Hz}, 1 \mathrm{H}), 2.06-1.98(\mathrm{~m}, 1 \mathrm{H}), 1.68(\mathrm{ddd}, J=$ $13.4,8.8,4.4 \mathrm{~Hz}, 1 \mathrm{H}), 1.56-1.45(\mathrm{~m}, 3 \mathrm{H}), 1.34-1.26(\mathrm{~m}, 1 \mathrm{H}), 1.09$ (d, $J=6.8 \mathrm{~Hz}$, $3 \mathrm{H})$.

${ }^{13}$ C NMR (126 MHz, CDCl 3$) \delta 145.6,135.4,131.9,129.9,129.1,127.8,114.3,108.5$, $55.1,52.9,31.5,31.0,27.4,20.7(2 \mathrm{C})$.

IR (film): v (cm-1) 3390, 2965, 2926, 1467, 1386, 1606, 861, 751, 694, 532.

HRMS (ESI, m/z) calcd for $\mathrm{C}_{19} \mathrm{H}_{22} \mathrm{BrNNaSe}(\mathrm{M}+\mathrm{Na})^{+}$: 445.9993, found: 445.9995 .

\section{N-((1S, 2S, 3S)-3-methyl-2-(phenylselanyl)cyclohexyl)-4-(trifluoromethyl)aniline} (4f)

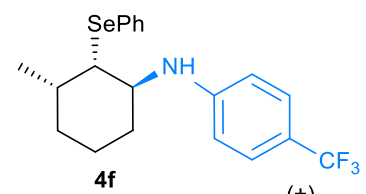

$( \pm)$

A dried $10 \mathrm{~mL}$ Schlenk tube was charged with 1a $(24 \mu \mathrm{L}, 19.2 \mathrm{mg}, 0.20 \mathrm{mmol})$, 4-(trifluoromethyl)aniline (50 $\mu \mathrm{L}, 64.4 \mathrm{mg}, 0.40 \mathrm{mmol}), 3 \mathrm{a}(31.2 \mathrm{mg}, 0.10 \mathrm{mmol})$, [Os-Cu] (10.9 mg, $0.01 \mathrm{mmol})$, and $\mathrm{CH}_{2} \mathrm{Cl}_{2}(4.0 \mathrm{~mL})$. The Schlenk tube was positioned approximately $3 \mathrm{~cm}$ away from a $24 \mathrm{~W}$ blue LEDs lamp $\left(\lambda_{\max }=450 \mathrm{~nm}\right)$. After being stirred at $25{ }^{\circ} \mathrm{C}$ for the $48 \mathrm{~h}$ under an $\mathrm{O}_{2}$ (balloon) atmosphere, the reaction mixture was concentrated to dryness. The residue was purified by flash chromatography (petroleum ether/ethyl acetate $=40: 1$ ) to afford product $\mathbf{4 f}$ as a yellow oil (61.9 mg, $0.150 \mathrm{mmol}$, yield: 75\%).

${ }^{1} \mathbf{H}$ NMR (500 MHz, CDCl 3$) \delta 7.66(\mathrm{dd}, J=8.1,1.3 \mathrm{~Hz}, 2 \mathrm{H}), 7.41-7.29(\mathrm{~m}, 3 \mathrm{H})$, $7.24(\mathrm{~d}, J=8.5 \mathrm{~Hz}, 2 \mathrm{H}), 6.23(\mathrm{~d}, J=8.5 \mathrm{~Hz}, 2 \mathrm{H}), 4.30(\mathrm{~s}, 1 \mathrm{H}), 3.88-3.77(\mathrm{~m}, 1 \mathrm{H})$, $3.43(\mathrm{t}, J=3.7 \mathrm{~Hz}, 1 \mathrm{H}), 2.31$ (ddt, $J=15.7,11.5,4.2 \mathrm{~Hz}, 1 \mathrm{H}), 2.05$ (ddt, $J=13.4,6.6$, 
$3.3 \mathrm{~Hz}, 1 \mathrm{H}), 1.75-1.68(\mathrm{~m}, 1 \mathrm{H}), 1.58-1.50(\mathrm{~m}, 3 \mathrm{H}), 1.35-1.28(\mathrm{~m}, 1 \mathrm{H}), 1.11(\mathrm{~d}, J$ $=6.8 \mathrm{~Hz}, 3 \mathrm{H})$.

${ }^{13}$ C NMR (126 MHz, $\left.\mathbf{C D C l}_{3}\right) \delta 149.1,135.5,129.8,129.2,127.9,126.6$ (q, $J=3.8$ Hz), 124.9 (q, $J=272.0 \mathrm{~Hz}), 118.5$ (q, $J=32.7 \mathrm{~Hz}), 111.7,55.1,52.6,31.6,31.0,20.7$, 20.5 .

IR (film): v (cm-1) 3395, 2974, 2862, 2920, 1467, 1381, 1259, 1110, 1066, 821, 741. HRMS (ESI, m/z) calcd for $\mathrm{C}_{20} \mathrm{H}_{22} \mathrm{~F}_{3} \mathrm{NNaSe}(\mathrm{M}+\mathrm{Na})^{+}$: 436.0762 , found: 436.0765 .

\section{N-((1S, 2S, 3S)-3-methyl-2-(phenylselanyl)cyclohexyl)-3-(trifluoromethyl)aniline}

$(4 \mathrm{~g})$

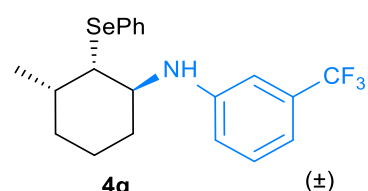

A dried $10 \mathrm{~mL}$ Schlenk tube was charged with 1a $(24 \mu \mathrm{L}, 19.2 \mathrm{mg}, 0.20 \mathrm{mmol})$, 3-(trifluoromethyl)aniline $(50 \mu \mathrm{L}, 64.4 \mathrm{mg}, 0.40 \mathrm{mmol}), 3 \mathrm{a}(31.2 \mathrm{mg}, 0.10 \mathrm{mmol})$, [Os-Cu] (10.9 mg, $0.01 \mathrm{mmol})$, and $\mathrm{CH}_{2} \mathrm{Cl}_{2}(4.0 \mathrm{~mL})$. The Schlenk tube was positioned approximately $3 \mathrm{~cm}$ away from a $24 \mathrm{~W}$ blue LEDs lamp $\left(\lambda_{\max }=450 \mathrm{~nm}\right)$. After being stirred at $25{ }^{\circ} \mathrm{C}$ for the $48 \mathrm{~h}$ under an $\mathrm{O}_{2}$ (balloon) atmosphere, the reaction mixture was concentrated to dryness. The residue was purified by flash chromatography (petroleum ether/ethyl acetate $=40: 1$ ) to afford product $\mathbf{4 g}$ as a yellow oil (64.4 mg, $0.156 \mathrm{mmol}$, yield: 78\%).

${ }^{1} \mathbf{H}$ NMR (500 MHz, $\left.\mathbf{C D C l}_{3}\right) \delta 7.73-7.62(\mathrm{~m}, 2 \mathrm{H}), 7.36-7.28(\mathrm{~m}, 3 \mathrm{H}), 7.11(\mathrm{t}, J=$ $7.9 \mathrm{~Hz}, 1 \mathrm{H}), 6.86(\mathrm{~d}, J=7.6 \mathrm{~Hz}, 1 \mathrm{H}), 6.59(\mathrm{~s}, 1 \mathrm{H}), 6.39$ (dd, $J=8.2,2.1 \mathrm{~Hz}, 1 \mathrm{H})$, $4.19(\mathrm{~s}, 1 \mathrm{H}), 3.86(\mathrm{dd}, J=7.1,3.8 \mathrm{~Hz}, 1 \mathrm{H}), 3.46(\mathrm{t}, J=3.8 \mathrm{~Hz}, 1 \mathrm{H}), 2.31-2.23(\mathrm{~m}$, $1 \mathrm{H}), 2.06(\mathrm{ddq}, J=13.5,6.7,3.2 \mathrm{~Hz}, 1 \mathrm{H}), 1.74-1.67(\mathrm{~m}, 1 \mathrm{H}), 1.59-1.51(\mathrm{~m}, 3 \mathrm{H})$, $1.39-1.30(\mathrm{~m}, 1 \mathrm{H}), 1.10(\mathrm{~d}, J=6.8 \mathrm{~Hz}, 3 \mathrm{H})$.

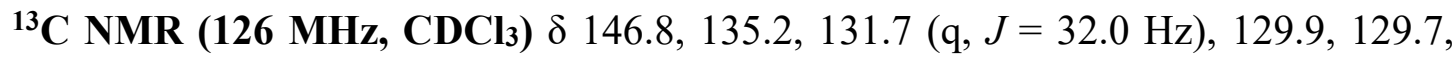
129.2, 127.8, $123.2(\mathrm{q}, J=271.1 \mathrm{~Hz}), 115.7,113.4(\mathrm{q}, J=4.0 \mathrm{~Hz}), 108.9(\mathrm{q}, J=3.8$ Hz), 55.0, 52.9, 31.5, 30.9, 27.5, 20.6, 20.5. 
IR (film): $v\left(\mathrm{~cm}^{-1}\right)$ 3393, 2978, 2856, 1457, 1384, 1243, 1128, 1110, 1066, 789, 693.

HRMS (ESI, m/z) calcd for $\mathrm{C}_{20} \mathrm{H}_{23} \mathrm{~F}_{3} \mathrm{NSe}(\mathrm{M}+\mathrm{H})^{+}$: 414.0942, found: 414.0948 .

\section{4-nitro-N-(1-phenyl-2-(phenylselanyl)ethyl)aniline (4h)}

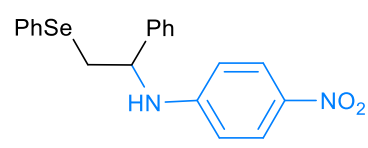

$4 h$

A dried $10 \mathrm{~mL}$ Schlenk tube was charged with styrene $(23 \mu \mathrm{L}, 20.8 \mathrm{mg}, 0.20 \mathrm{mmol})$, 4-nitroaniline (55.2 mg, $0.40 \mathrm{mmol}), 3 \mathbf{a}(31.2 \mathrm{mg}, 0.10 \mathrm{mmol})$, [Os-Cu] (10.9 mg, $0.01 \mathrm{mmol})$, and $\mathrm{CH}_{2} \mathrm{Cl}_{2}(4.0 \mathrm{~mL})$. The Schlenk tube was positioned approximately 3 $\mathrm{cm}$ away from a $24 \mathrm{~W}$ blue LEDs lamp $\left(\lambda_{\max }=450 \mathrm{~nm}\right)$. After being stirred at $25{ }^{\circ} \mathrm{C}$ for the $48 \mathrm{~h}$ under an $\mathrm{O}_{2}$ (balloon) atmosphere, the reaction mixture was concentrated to dryness. The residue was purified by flash chromatography (petroleum ether/ethyl acetate $=40: 1)$ to afford product $4 \mathrm{~h}$ as a yellow oil $(50.1 \mathrm{mg}, 0.126 \mathrm{mmol}$, yield: $63 \%)$.

${ }^{1}$ H NMR (400 MHz, CDCl $) \delta 8.00-7.91(\mathrm{~m}, 2 \mathrm{H}), 7.58-7.49(\mathrm{~m}, 2 \mathrm{H}), 7.36-7.26$ (m, 8H), $6.39-6.32(\mathrm{~m}, 2 \mathrm{H}), 5.20(\mathrm{~d}, J=4.9 \mathrm{~Hz}, 1 \mathrm{H}), 4.53(\mathrm{dt}, J=9.3,4.8 \mathrm{~Hz}, 1 \mathrm{H})$, $3.40(\mathrm{dd}, J=12.9,4.6 \mathrm{~Hz}, 1 \mathrm{H}), 3.21(\mathrm{dd}, J=12.9,8.9 \mathrm{~Hz}, 1 \mathrm{H})$.

${ }^{13}$ C NMR (101 MHz, CDCl3) $\delta$ 152.2, 140.9, 138.6, 133.9, 129.5, 129.1, 128.6, 128.1, $128.0,126.1(2 \mathrm{C}), 112.2,57.6,35.7$.

IR (film): $v\left(\mathrm{~cm}^{-1}\right)$ 3411, 2933, 2861, 1345, 1525, 1471, 1069, 873, 737 .

HRMS (ESI, m/z) calcd for $\mathrm{C}_{20} \mathrm{H}_{18} \mathrm{~N}_{2} \mathrm{NaO}_{2} \mathrm{Se}(\mathrm{M}+\mathrm{Na})^{+}: 421.0431$, found: 421.0431 .

\section{dimethyl 5-((1-phenyl-2-(phenylselanyl)ethyl)amino)isophthalate (4i)}

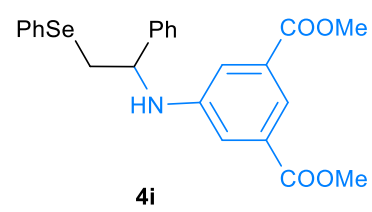

A dried $10 \mathrm{~mL}$ Schlenk tube was charged with styrene $(23 \mu \mathrm{L}, 20.8 \mathrm{mg}, 0.20 \mathrm{mmol})$, dimethyl 5-aminoisophthalate $(83.7 \mathrm{mg}, 0.40 \mathrm{mmol}), 3 \mathrm{a}(31.2 \mathrm{mg}, 0.10 \mathrm{mmol})$, 
[Os-Cu] (10.9 mg, $0.01 \mathrm{mmol})$, and $\mathrm{CH}_{2} \mathrm{Cl}_{2}(4.0 \mathrm{~mL})$. The Schlenk tube was positioned approximately $3 \mathrm{~cm}$ away from a $24 \mathrm{~W}$ blue LEDs lamp $\left(\lambda_{\max }=450 \mathrm{~nm}\right)$. After being stirred at $25{ }^{\circ} \mathrm{C}$ for the $48 \mathrm{~h}$ under an $\mathrm{O}_{2}$ (balloon) atmosphere, the reaction mixture was concentrated to dryness. The residue was purified by flash chromatography (petroleum ether/ethyl acetate $=10: 1$ ) to afford product $4 \mathbf{i}$ as a colorless oil (59.2 mg, $0.122 \mathrm{mmol}$, yield: 61\%).

${ }^{1} \mathbf{H}$ NMR (400 MHz, CDCl 3$) \delta 7.99(\mathrm{t}, J=1.4 \mathrm{~Hz}, 1 \mathrm{H}), 7.56-7.50(\mathrm{~m}, 2 \mathrm{H}), 7.43-$ $7.33(\mathrm{~m}, 6 \mathrm{H}), 7.32-7.26(\mathrm{~m}, 4 \mathrm{H}), 4.86(\mathrm{~d}, J=3.8 \mathrm{~Hz}, 1 \mathrm{H}), 4.64-4.55(\mathrm{~m}, 1 \mathrm{H}), 3.88$ (s, 6H), $3.41(\mathrm{dd}, J=12.7,4.5 \mathrm{~Hz}, 1 \mathrm{H}), 3.25(\mathrm{dd}, J=12.7,8.9 \mathrm{~Hz}, 1 \mathrm{H})$.

${ }^{13}$ C NMR (101 MHz, CDCl $) \delta$ 166.6, 147.3, 141.7, 133.5, 131.2, 129.4, 129.1, 129.0, $127.9,127.7,126.3,119.9,118.5,57.7,52.2,36.2$.

IR (film): $v\left(\mathrm{~cm}^{-1}\right)$ 3393, 2927, 2852, 1769, 1472, 1074, 836, 741 .

HRMS (ESI, m/z) calcd for $\mathrm{C}_{24} \mathrm{H}_{23} \mathrm{NNaO}_{4} \mathrm{Se}(\mathrm{M}+\mathrm{Na})^{+}: 492.0690$, found: 492.0693 .

\section{2,5-dibromo-N-(1-phenyl-2-(phenylselanyl)ethyl)aniline (4j)}

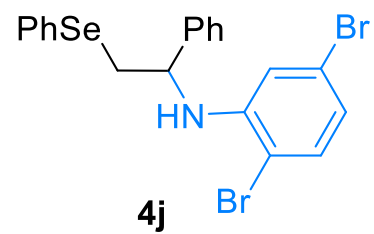

A dried $10 \mathrm{~mL}$ Schlenk tube was charged with styrene $(23 \mu \mathrm{L}, 20.8 \mathrm{mg}, 0.20 \mathrm{mmol})$, 2,5-dibromoaniline (99.5 mg, $0.40 \mathrm{mmol}), 3 \mathbf{3}(31.2 \mathrm{mg}, 0.10 \mathrm{mmol})$, [Os-Cu] (10.9 $\mathrm{mg}, \quad 0.01 \mathrm{mmol})$, and $\mathrm{CH}_{2} \mathrm{Cl}_{2}(4.0 \mathrm{~mL})$. The Schlenk tube was positioned approximately $3 \mathrm{~cm}$ away from a $24 \mathrm{~W}$ blue LEDs lamp $\left(\lambda_{\max }=450 \mathrm{~nm}\right)$. After being stirred at $25{ }^{\circ} \mathrm{C}$ for the $48 \mathrm{~h}$ under an $\mathrm{O}_{2}$ (balloon) atmosphere, the reaction mixture was concentrated to dryness. The residue was purified by flash chromatography (petroleum ether/ethyl acetate $=40: 1)$ to afford product $\mathbf{4} \mathbf{j}$ as a colorless oil $(79.4 \mathrm{mg}$, $0.156 \mathrm{mmol}$, yield: $78 \%$ ).

${ }^{1}$ H NMR (400 MHz, $\left.\mathbf{C D C l}_{3}\right) \delta 7.59(\mathrm{ddd}, J=5.4,4.0,2.1 \mathrm{~Hz}, 2 \mathrm{H}), 7.41-7.35(\mathrm{~m}$, 4H), $7.35-7.27(\mathrm{~m}, 5 \mathrm{H}), 6.70(\mathrm{dd}, J=8.4,2.2 \mathrm{~Hz}, 1 \mathrm{H}), 6.48(\mathrm{~d}, J=2.2 \mathrm{~Hz}, 1 \mathrm{H}), 5.34$ 
$(\mathrm{d}, J=5.3 \mathrm{~Hz}, 1 \mathrm{H}), 4.52(\mathrm{dt}, J=8.4,5.1 \mathrm{~Hz}, 1 \mathrm{H}), 3.45(\mathrm{dd}, J=12.7,4.8 \mathrm{~Hz}, 1 \mathrm{H})$, $3.34(\mathrm{dd}, J=12.7,8.4 \mathrm{~Hz}, 1 \mathrm{H})$.

${ }^{13}$ C NMR (101 MHz, CDCl 3$) \delta$ 145.0, 141.2, 133.6, 133.3, 129.4, 129.1 (2C), 128.1, $127.7,126.3,122.1,121.1,115.5,108.8,57.5,36.2$.

IR (film): $v\left(\mathrm{~cm}^{-1}\right)$ 3386, 3058, 1959, 1472, 1084, 911, 737, 630, 560, 546.

HRMS (ESI, m/z) calcd for $\mathrm{C}_{20} \mathrm{H}_{17} \mathrm{Br}_{2} \mathrm{NNaSe}(\mathrm{M}+\mathrm{Na})^{+}:$531.8791, found: 531.8795 .

\section{4-methyl-N-(1-phenyl-2-(phenylselanyl)ethyl)aniline (4k)}

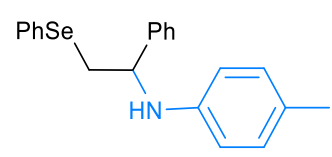

4k

A dried $10 \mathrm{~mL}$ Schlenk tube was charged with styrene $(23 \mu \mathrm{L}, 20.8 \mathrm{mg}, 0.20 \mathrm{mmol})$, p-toluidine (43 $\mu \mathrm{L}, 42.8 \mathrm{mg}, 0.40 \mathrm{mmol}), \mathbf{3 a}(31.2 \mathrm{mg}, 0.10 \mathrm{mmol})$, [Os-Cu] (10.9 $\mathrm{mg}, \quad 0.01 \mathrm{mmol})$, and $\mathrm{CH}_{2} \mathrm{Cl}_{2}(4.0 \mathrm{~mL})$. The Schlenk tube was positioned approximately $3 \mathrm{~cm}$ away from a $24 \mathrm{~W}$ blue LEDs lamp $\left(\lambda_{\max }=450 \mathrm{~nm}\right)$. After being stirred at $25{ }^{\circ} \mathrm{C}$ for the $48 \mathrm{~h}$ under an $\mathrm{O}_{2}$ (balloon) atmosphere, the reaction mixture was concentrated to dryness. The residue was purified by flash chromatography (petroleum ether/ethyl acetate $=20: 1)$ to afford product $\mathbf{4 k}$ as a yellow oil (39.6 mg, 0.108 mmol, yield: $54 \%$ ).

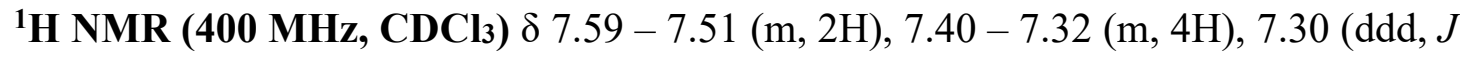
$=6.6,5.0,2.4 \mathrm{~Hz}, 4 \mathrm{H}), 6.92(\mathrm{~d}, J=8.0 \mathrm{~Hz}, 2 \mathrm{H}), 6.42(\mathrm{~d}, J=8.4 \mathrm{~Hz}, 2 \mathrm{H}), 4.48(\mathrm{dd}, J$ $=8.9,4.6 \mathrm{~Hz}, 1 \mathrm{H}), 4.39(\mathrm{~s}, 1 \mathrm{H}), 3.39(\mathrm{dd}, J=12.5,4.6 \mathrm{~Hz}, 1 \mathrm{H}), 3.24(\mathrm{dd}, J=12.5$, $8.9 \mathrm{~Hz}, 1 \mathrm{H}), 2.23(\mathrm{~s}, 3 \mathrm{H})$.

${ }^{13}$ C NMR (101 MHz, CDCl $) \delta$ 144.8, 142.9, 133.5, 129.6, 129.5, 129.3, 128.8, 127.5(2C), 127.0, 126.4, 113.9, 58.1, 36.6, 20.4.

IR (film): $v\left(\mathrm{~cm}^{-1}\right)$ 3399, 2918, 2855, 1473, 1360, 1095, 854, 730, 690.

HRMS (ESI, m/z) calcd for $\mathrm{C}_{21} \mathrm{H}_{21} \mathrm{NNaSe}(\mathrm{M}+\mathrm{Na})^{+}: 390.0737$, found: 390.0739 .

\section{3-methyl-N-(1-phenyl-2-(phenylselanyl)ethyl)aniline (4I)}




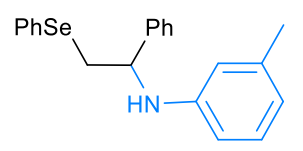

41

A dried $10 \mathrm{~mL}$ Schlenk tube was charged with styrene $(23 \mu \mathrm{L}, 20.8 \mathrm{mg}, 0.20 \mathrm{mmol})$, $m$-toluidine (43 $\mu \mathrm{L}, 42.8 \mathrm{mg}, 0.40 \mathrm{mmol}), 3 \mathbf{a}(31.2 \mathrm{mg}, 0.10 \mathrm{mmol})$, [Os-Cu] (10.9 mg, $0.01 \mathrm{mmol})$, and $\mathrm{CH}_{2} \mathrm{Cl}_{2}(4.0 \mathrm{~mL})$. The Schlenk tube was positioned approximately $3 \mathrm{~cm}$ away from a $24 \mathrm{~W}$ blue LEDs lamp $\left(\lambda_{\max }=450 \mathrm{~nm}\right)$. After being stirred at $25{ }^{\circ} \mathrm{C}$ for the $48 \mathrm{~h}$ under an $\mathrm{O}_{2}$ (balloon) atmosphere, the reaction mixture was concentrated to dryness. The residue was purified by flash chromatography (petroleum ether/ethyl acetate $=20: 1)$ to afford product $\mathbf{4 1}$ as a yellow oil $(36.0 \mathrm{mg}$, 0.098 mmol, yield: 49\%).

${ }^{1}$ H NMR (500 MHz, (CD) 2 CO) $\delta 7.58-7.48(\mathrm{~m}, 2 \mathrm{H}), 7.47-7.43(\mathrm{~m}, 2 \mathrm{H}), 7.29(\mathrm{tt}$, $J=5.9,4.4 \mathrm{~Hz}, 5 \mathrm{H}), 7.22(\mathrm{t}, J=7.3 \mathrm{~Hz}, 1 \mathrm{H}), 6.87(\mathrm{t}, J=7.8 \mathrm{~Hz}, 1 \mathrm{H}), 6.45-6.35(\mathrm{~m}$, 2H), $6.33(\mathrm{dd}, J=8.1,1.6 \mathrm{~Hz}, 1 \mathrm{H}), 5.38(\mathrm{~s}, 1 \mathrm{H}), 4.69-4.57(\mathrm{~m}, 1 \mathrm{H}), 3.39$ (dd, $J=$ 12.2, 8.6 Hz, 1H), 3.34 (dd, $J=12.2,5.4 \mathrm{~Hz}, 1 \mathrm{H}), 2.12$ (s, 3H).

${ }^{13}$ C NMR (126 MHz, (CD))2CO) $\delta$ 147.7, 143.5, 138.1, 132.6, 130.5, 129.2, 128.7, $128.5,127.2,126.9,126.6,117.8,114.1,110.5,57.8,35.4,20.8$.

IR (film): $v\left(\mathrm{~cm}^{-1}\right)$ 3399, 2918, 2852, 1463, 1370, 1072, 999, 770, 691.

HRMS (ESI, m/z) calcd for $\mathrm{C}_{21} \mathrm{H}_{21} \mathrm{NNaSe}(\mathrm{M}+\mathrm{Na})^{+}$: 390.0737, found: 390.0735 .

\section{9-(1-phenyl-2-(phenylselanyl)ethyl)-9H-carbazole (4m)}

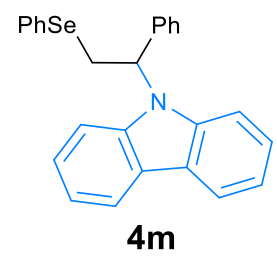

A dried $10 \mathrm{~mL}$ Schlenk tube was charged with styrene $(23 \mu \mathrm{L}, 20.8 \mathrm{mg}, 0.20 \mathrm{mmol})$, carbazole (66.8 mg, $0.40 \mathrm{mmol}), 3 \mathrm{a}(31.2 \mathrm{mg}, 0.10 \mathrm{mmol})$, [Os-Cu] (10.9 mg, 0.01 mmol), and $\mathrm{CH}_{2} \mathrm{Cl}_{2}(4.0 \mathrm{~mL})$. The Schlenk tube was positioned approximately $3 \mathrm{~cm}$ away from a $24 \mathrm{~W}$ blue LEDs lamp $\left(\lambda_{\max }=450 \mathrm{~nm}\right)$. After being stirred at $25^{\circ} \mathrm{C}$ for the $48 \mathrm{~h}$ under an $\mathrm{O}_{2}$ (balloon) atmosphere, the reaction mixture was concentrated to 
dryness. The residue was purified by flash chromatography (petroleum ether/ethyl acetate $=20: 1)$ to afford product $\mathbf{4 m}$ as a colorless oil $(37.6 \mathrm{mg}, 0.088 \mathrm{mmol}$, yield: $44 \%)$.

${ }^{1} \mathbf{H}$ NMR (500 MHz, CDCl 3$) \delta 8.09$ (d, $\left.J=7.7 \mathrm{~Hz}, 2 \mathrm{H}\right), 7.35-7.27$ (m, 9H), $7.24-$ $7.20(\mathrm{~m}, 3 \mathrm{H}), 7.16(\mathrm{~d}, J=8.2 \mathrm{~Hz}, 4 \mathrm{H}), 6.10(\mathrm{dd}, J=9.0,6.2 \mathrm{~Hz}, 1 \mathrm{H}), 4.08(\mathrm{dd}, J=$ $12.8,6.1 \mathrm{~Hz}, 1 \mathrm{H}), 4.01(\mathrm{dd}, J=12.8,9.1 \mathrm{~Hz}, 1 \mathrm{H})$.

${ }^{13}$ C NMR (126 MHz, CDCl 3$) \delta 140.0,139.0,133.3,129.1,129.0,128.8,127.8,127.4$, $126.9,125.5,123.5,120.3,119.2,110.2,57.6,29.9$.

IR (film): $v\left(\mathrm{~cm}^{-1}\right)$ 2910, 2841, 1477, 1069, 954, 750, 690.

HRMS (ESI, m/z)HRMS (ESI, m/z) calcd for $\mathrm{C}_{26} \mathrm{H}_{21} \mathrm{NNaSe}(\mathrm{M}+\mathrm{Na})^{+}:$450.0737, found: 450.0778 .

\section{N'-benzylidene-4-methyl-N-(1-phenyl-2-(phenylselanyl)ethyl)}

benzenesulfonohydrazide (4n)

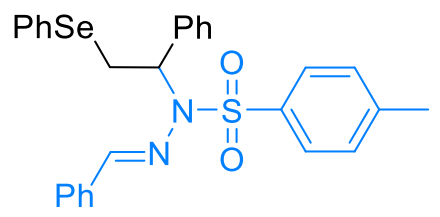

$4 n$

A dried $10 \mathrm{~mL}$ Schlenk tube was charged with styrene $(23 \mu \mathrm{L}, 20.8 \mathrm{mg}, 0.20 \mathrm{mmol})$, benzaldehyde $p$-toluenesulfonylhydrazon (109.6 mg, $0.40 \mathrm{mmol}), 3 \mathbf{a}(31.2 \mathrm{mg}, 0.10$ mmol), [Os-Cu] (10.9 mg, $0.01 \mathrm{mmol})$, and $\mathrm{CH}_{2} \mathrm{Cl}_{2}(4.0 \mathrm{~mL})$. The Schlenk tube was positioned approximately $3 \mathrm{~cm}$ away from a $24 \mathrm{~W}$ blue LEDs lamp $\left(\lambda_{\max }=450 \mathrm{~nm}\right)$. After being stirred at $25{ }^{\circ} \mathrm{C}$ for the $48 \mathrm{~h}$ under an $\mathrm{O}_{2}$ (balloon) atmosphere, the reaction mixture was concentrated to dryness. The residue was purified by flash chromatography (petroleum ether/ethyl acetate $=20: 1$ ) to afford product $4 \mathbf{n}$ as a colorless oil (74.8 mg, $0.140 \mathrm{mmol}$, yield: 70\%).

${ }^{1}$ H NMR (500 MHz, CDCl 3$) \delta 8.48(\mathrm{~s}, 1 \mathrm{H}), 7.64(\mathrm{dd}, J=7.5,1.9 \mathrm{~Hz}, 2 \mathrm{H}), 7.48-$ $7.44(\mathrm{~m}, 2 \mathrm{H}), 7.43-7.38(\mathrm{~m}, 3 \mathrm{H}), 7.36-7.32(\mathrm{~m}, 4 \mathrm{H}), 7.25-7.19(\mathrm{~m}, 6 \mathrm{H}), 7.01(\mathrm{~d}$, $J=8.3 \mathrm{~Hz}, 2 \mathrm{H}), 5.53(\mathrm{t}, J=7.7 \mathrm{~Hz}, 1 \mathrm{H}), 3.40(\mathrm{qd}, J=12.5,7.7 \mathrm{~Hz}, 2 \mathrm{H}), 2.30(\mathrm{~s}, 3 \mathrm{H})$. 
${ }^{13}$ C NMR (126 MHz, CDCl 3$) \delta 156.7,143.8,138.9,135.2,134.3,133.7,130.9,129.8$, 129.3, 129.1, 128.8, 128.3, 128.2, 128.0(2C), 127.9, 127.4, 64.8, 32.0, 21.5.

IR (film): $v\left(\mathrm{~cm}^{-1}\right)$ 2928, 2889, 1616,1452, 1071, 986, 841, 743.

HRMS (ESI, m/z)HRMS (ESI, m/z) calcd for $\mathrm{C}_{28} \mathrm{H}_{26} \mathrm{~N}_{2} \mathrm{NaO}_{2} \mathrm{SSe}(\mathrm{M}+\mathrm{Na})^{+}$: 557.0778, found: 557.0782.

\section{N-((1S,2S,3S)-3-methyl-2-(p-tolylselanyl)cyclohexyl)-4-(trifluoromethyl)aniline}

(40)

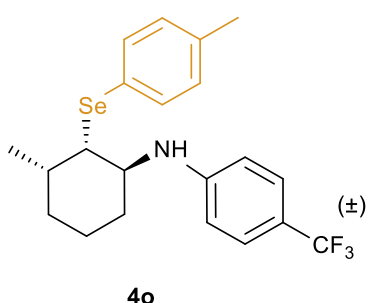

A dried $10 \mathrm{~mL}$ Schlenk tube was charged with 1a $(24 \mu \mathrm{L}, 19.2 \mathrm{mg}, 0.20 \mathrm{mmol})$, phenylamine (37 $\mu \mathrm{L}, 37.3 \mathrm{mg}, 0.40 \mathrm{mmol}$ ), (4-Me)PhSeSePh(4-Me) (34.2 mg, 0.10 mmol), [Os-Cu] (10.9 mg, $0.01 \mathrm{mmol})$, and $\mathrm{CH}_{2} \mathrm{Cl}_{2}(4.0 \mathrm{~mL})$. The Schlenk tube was positioned approximately $3 \mathrm{~cm}$ away from a $24 \mathrm{~W}$ blue LEDs lamp $\left(\lambda_{\max }=450 \mathrm{~nm}\right)$. After being stirred at $25{ }^{\circ} \mathrm{C}$ for the $48 \mathrm{~h}$ under an $\mathrm{O}_{2}$ (balloon) atmosphere, the reaction mixture was concentrated to dryness. The residue was purified by flash chromatography (petroleum ether/ethyl acetate $=40: 1$ ) to afford product 40 as a yellow oil (53.8 mg, $0.126 \mathrm{mmol}$, yield: 63\%).

${ }^{1}$ H NMR (500 MHz, CDCl 3$) \delta 7.54(\mathrm{~d}, J=7.9 \mathrm{~Hz}, 2 \mathrm{H}), 7.23(\mathrm{~d}, J=8.6 \mathrm{~Hz}, 2 \mathrm{H})$, $7.14(\mathrm{~d}, J=7.8 \mathrm{~Hz}, 2 \mathrm{H}), 6.23$ (d, $J=8.6 \mathrm{~Hz}, 2 \mathrm{H}), 4.29$ (s, 1H), 3.80 (s, 1H), 3.38 (t, $J$ $=3.8 \mathrm{~Hz}, 1 \mathrm{H}), 2.38(\mathrm{~s}, 3 \mathrm{H}), 2.34-2.27(\mathrm{~m}, 1 \mathrm{H}), 2.03(\mathrm{dtd}, J=13.3,6.5,3.2 \mathrm{~Hz}, 1 \mathrm{H})$, $1.77-1.63(\mathrm{~m}, 2 \mathrm{H}), 1.54-1.49(\mathrm{~m}, 2 \mathrm{H}), 1.36-1.30(\mathrm{~m}, 1 \mathrm{H}), 1.10(\mathrm{~d}, J=6.7 \mathrm{~Hz}$, $3 \mathrm{H})$.

${ }^{13}$ C NMR (126 MHz, CDCl 3$) \delta 149.1,138.0,135.7,130.0,126.6$ (q, $\left.J=3.7 \mathrm{~Hz}\right)$, 126.0, 119.4, 118.4 (q, $J=32.6 \mathrm{~Hz}), 111.7,55.0,52.6,31.6,31.0,29.7,21.2,20.7$, 20.5 .

IR (film): v (cm $\left.{ }^{-1}\right)$ 3393, 2978, 1457, 1384, 1243, 1128, 1110, 1066, 789, 660. 
HRMS (ESI, m/z)HRMS (ESI, m/z) calcd for $\mathrm{C}_{21} \mathrm{H}_{24} \mathrm{~F}_{3} \mathrm{NNaSe}(\mathrm{M}+\mathrm{Na})^{+}: 449.3744$, found: 449.3752 .

\section{N-((1S,2S,3S)-3-methyl-2-(o-tolylselanyl)cyclohexyl)-4-(trifluoromethyl)aniline}

\section{(4p)}

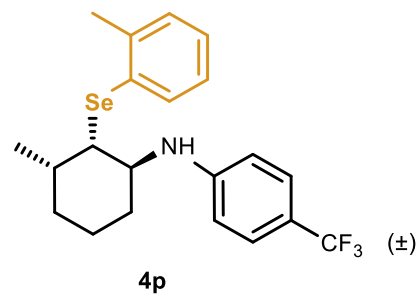

A dried $10 \mathrm{~mL}$ Schlenk tube was charged with $1 \mathrm{a}(24 \mu \mathrm{L}, 19.2 \mathrm{mg}, 0.20 \mathrm{mmol})$, phenylamine (37 $\mu \mathrm{L}, 37.3 \mathrm{mg}, 0.40 \mathrm{mmol}),(2-\mathrm{Me}) \mathrm{PhSeSePh}(2-\mathrm{Me})(34.2 \mathrm{mg}, 0.10$ mmol), [Os-Cu] (10.9 mg, $0.01 \mathrm{mmol})$, and $\mathrm{CH}_{2} \mathrm{Cl}_{2}(4.0 \mathrm{~mL})$. The Schlenk tube was positioned approximately $3 \mathrm{~cm}$ away from a $24 \mathrm{~W}$ blue LEDs lamp $\left(\lambda_{\max }=450 \mathrm{~nm}\right)$. After being stirred at $25{ }^{\circ} \mathrm{C}$ for the $48 \mathrm{~h}$ under an $\mathrm{O}_{2}$ (balloon) atmosphere, the reaction mixture was concentrated to dryness. The residue was purified by flash chromatography (petroleum ether/ethyl acetate $=40: 1$ ) to afford product $4 \mathbf{p}$ as a yellow oil (46.1 mg, $0.108 \mathrm{mmol}$, yield: 54\%).

${ }^{1}$ H NMR (500 MHz, CDCl $) \delta 7.67(\mathrm{~d}, J=7.7 \mathrm{~Hz}, 1 \mathrm{H}), 7.29(\mathrm{dd}, J=5.2,1.7 \mathrm{~Hz}$, 2H), $7.21(\mathrm{~d}, J=8.6 \mathrm{~Hz}, 2 \mathrm{H}), 7.17-7.12(\mathrm{~m}, 1 \mathrm{H}), 6.14(\mathrm{~d}, J=8.5 \mathrm{~Hz}, 2 \mathrm{H}), 4.26(\mathrm{~s}$, 1H), $3.74(\mathrm{~s}, 1 \mathrm{H}), 3.39(\mathrm{t}, J=3.7 \mathrm{~Hz}, 1 \mathrm{H}), 2.51(\mathrm{~s}, 3 \mathrm{H}), 2.45-2.36(\mathrm{~m}, 1 \mathrm{H}), 2.06(\mathrm{dtd}$, $J=10.1,6.7,3.2 \mathrm{~Hz}, 1 \mathrm{H}), 1.75-1.70(\mathrm{~m}, 1 \mathrm{H}), 1.52(\mathrm{ddd}, J=15.8,11.6,4.9 \mathrm{~Hz}, 3 \mathrm{H})$, $1.36(\mathrm{ddd}, J=18.2,12.4,6.1 \mathrm{~Hz}, 1 \mathrm{H}), 1.12(\mathrm{~d}, J=6.8 \mathrm{~Hz}, 3 \mathrm{H})$.

${ }^{13}$ C NMR (126 MHz, CDCl $) \delta 149.1,141.8,136.4,130.5,130.2,128.3,126.6$ (q, $J$ $=3.4 \mathrm{~Hz}), 126.0,123.6,118.4(\mathrm{q}, J=31.2 \mathrm{~Hz}), 111.6,53.8,52.4,31.7,31.0,27.4$, $23.2,20.8,20.5$.

IR (film): v (cm $\left.{ }^{-1}\right)$ 3393, 2978, 1457, 1384, 1243, 1128, 1110, 1066, 800, 693.

HRMS (ESI, m/z) calcd for $\mathrm{C}_{21} \mathrm{H}_{24} \mathrm{~F}_{3} \mathrm{NNaSe}(\mathrm{M}+\mathrm{Na})^{+}$: 449.3744, found: 449.3760 . 


\section{N-((1S,2S,3S)-2-((4-bromophenyl)selanyl)-3-methylcyclohexyl)-4-(trifluoromethy}

l)aniline (4q)

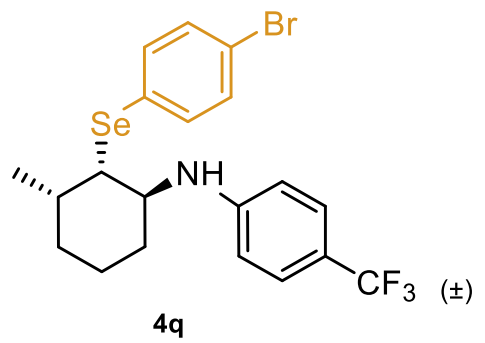

A dried $10 \mathrm{~mL}$ Schlenk tube was charged with $1 \mathrm{a}(24 \mu \mathrm{L}, 19.2 \mathrm{mg}, 0.20 \mathrm{mmol})$, phenylamine (37 $\mu \mathrm{L}, 37.3 \mathrm{mg}, 0.40 \mathrm{mmol}),(4-\mathrm{Br}) \mathrm{PhSeSePh}(4-\mathrm{Br})(34.2 \mathrm{mg}, 0.10$ mmol), [Os-Cu] (10.9 mg, $0.01 \mathrm{mmol})$, and $\mathrm{CH}_{2} \mathrm{Cl}_{2}(4.0 \mathrm{~mL})$. The Schlenk tube was positioned approximately $3 \mathrm{~cm}$ away from a $24 \mathrm{~W}$ blue LEDs lamp $\left(\lambda_{\max }=450 \mathrm{~nm}\right)$. After being stirred at $25{ }^{\circ} \mathrm{C}$ for the $48 \mathrm{~h}$ under an $\mathrm{O}_{2}$ (balloon) atmosphere, the reaction mixture was concentrated to dryness. The residue was purified by flash chromatography (petroleum ether/ethyl acetate $=40: 1$ ) to afford product $\mathbf{4 q}$ as a colorless oil (50.0 mg, $0.102 \mathrm{mmol}$, yield: 51\%).

${ }^{1}$ H NMR (500 MHz, CDCl3) $\delta 7.46(\mathrm{dd}, J=29.6,8.4 \mathrm{~Hz}, 4 \mathrm{H}), 7.29(\mathrm{~d}, J=8.5 \mathrm{~Hz}$, 2H), $6.29(\mathrm{~d}, J=8.6 \mathrm{~Hz}, 2 \mathrm{H}), 4.25(\mathrm{~s}, 1 \mathrm{H}), 3.82(\mathrm{~s}, 1 \mathrm{H}), 3.41(\mathrm{t}, J=4.0 \mathrm{~Hz}, 1 \mathrm{H}), 2.23$ (ddd, $J=14.2,7.3,4.2 \mathrm{~Hz}, 1 \mathrm{H}), 2.04$ (ddd, $J=10.1,6.7,3.6 \mathrm{~Hz}, 1 \mathrm{H}), 1.75-1.67$ (m, $1 \mathrm{H}), 1.56-1.46(\mathrm{~m}, 3 \mathrm{H}), 1.33(\mathrm{~s}, 1 \mathrm{H}), 1.08(\mathrm{~d}, J=6.8 \mathrm{~Hz}, 3 \mathrm{H})$.

${ }^{13}$ C NMR (126 MHz, CDCl 3$) \delta 149.0,136.8,132.3,128.8,126.7$ (q, $J=3.8 \mathrm{~Hz}$ ), $125.97,122.2,118.7$ (q, $J=32.7 \mathrm{~Hz}), 55.5,52.7,31.6,31.0,29.7,22.7,20.5$.

IR (film): $v\left(\mathrm{~cm}^{-1}\right)$ 3393, 2978, 2856, 1457, 1384, 1243, 1128, 1066, 769, 667.

HRMS (ESI, m/z) calcd for $\mathrm{C}_{20} \mathrm{H}_{21} \mathrm{BrF}_{3} \mathrm{NNaSe}(\mathrm{M}+\mathrm{Na})^{+}$: 513.9872, found: 513.9769.

N-((1S,2S,3S)-2-((4-fluorophenyl)selanyl)-3-methylcyclohexyl)-4-(trifluoromethyl )aniline (4r) 


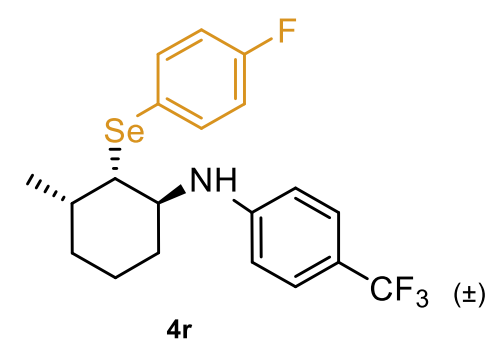

A dried $10 \mathrm{~mL}$ Schlenk tube was charged with $1 \mathrm{a}(24 \mu \mathrm{L}, 19.2 \mathrm{mg}, 0.20 \mathrm{mmol})$, phenylamine (37 $\mu \mathrm{L}, 37.3 \mathrm{mg}, 0.40 \mathrm{mmol}),(4-\mathrm{F}) \mathrm{PhSeSePh}(4-\mathrm{F})(34.2 \mathrm{mg}, 0.10$ mmol), [Os-Cu] (10.9 mg, $0.01 \mathrm{mmol})$, and $\mathrm{CH}_{2} \mathrm{Cl}_{2}(4.0 \mathrm{~mL})$. The Schlenk tube was positioned approximately $3 \mathrm{~cm}$ away from a $24 \mathrm{~W}$ blue LEDs lamp $\left(\lambda_{\max }=450 \mathrm{~nm}\right)$. After being stirred at $25{ }^{\circ} \mathrm{C}$ for the $48 \mathrm{~h}$ under an $\mathrm{O}_{2}$ (balloon) atmosphere, the reaction mixture was concentrated to dryness. The residue was purified by flash chromatography (petroleum ether/ethyl acetate $=40: 1$ ) to afford product $\mathbf{4 r}$ as a yellow oil (42.2 mg, $0.098 \mathrm{mmol}$, yield: 49\%).

${ }^{1} \mathbf{H}$ NMR (500 MHz, CDCl 3$) \delta 7.67-7.61(\mathrm{~m}, 4 \mathrm{H}), 7.28(\mathrm{t}, J=4.2 \mathrm{~Hz}, 4 \mathrm{H}), 7.05(\mathrm{t}$, $J=8.7 \mathrm{~Hz}, 4 \mathrm{H}), 6.28(\mathrm{~d}, J=8.6 \mathrm{~Hz}, 4 \mathrm{H}), 4.30(\mathrm{~s}, 2 \mathrm{H}), 3.84(\mathrm{~s}, 2 \mathrm{H}), 3.39$ (t, $J=3.9$ $\mathrm{Hz}, 2 \mathrm{H}), 2.34-2.25(\mathrm{~m}, 2 \mathrm{H}), 2.05(\mathrm{ddd}, J=10.3,7.1,3.7 \mathrm{~Hz}, 3 \mathrm{H}), 1.72(\mathrm{td}, J=9.3$, $4.7 \mathrm{~Hz}, 3 \mathrm{H}), 1.54(\mathrm{ddd}, J=6.5,5.8,4.2 \mathrm{~Hz}, 6 \mathrm{H}), 1.35(\mathrm{dd}, J=10.5,5.2 \mathrm{~Hz}, 2 \mathrm{H}), 1.12$ $(\mathrm{d}, J=6.8 \mathrm{~Hz}, 6 \mathrm{H})$.

${ }^{13}$ C NMR (126 MHz, CDCl $) \delta 163.8,161.9,145.0,137.7$ (d, $\left.J=8.0 \mathrm{~Hz}\right), 126.6(\mathrm{q}, J$ $=3.8 \mathrm{~Hz}), 125.9124 .3,118.7(\mathrm{~d}, J=32.7 \mathrm{~Hz}), 116.4(\mathrm{~d}, J=21.4 \mathrm{~Hz}), 111.7,55.6,52.6$, $31.6,30.9,29.7,22.7,20.6)$.

IR (film): $v\left(\mathrm{~cm}^{-1}\right)$ 3393, 2978, 2856, 1457, 1384, 1128, 1066, 770, 640.

HRMS (ESI, m/z) calcd for $\mathrm{C}_{20} \mathrm{H}_{21} \mathrm{~F}_{4} \mathrm{NNaSe}(\mathrm{M}+\mathrm{Na})^{+}$: 454.0673, found: 454.0669 .

\section{N-((1S,2S,3S)-2-(benzylselanyl)-3-methylcyclohexyl)-4-(trifluoromethyl)aniline}

(4s)

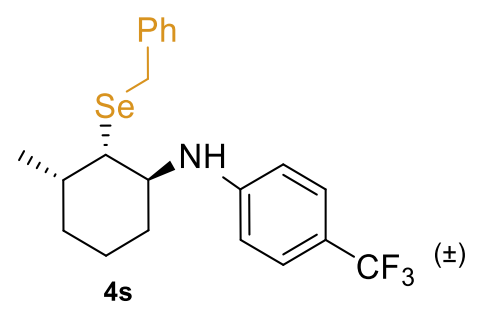


A dried $10 \mathrm{~mL}$ Schlenk tube was charged with 1a $(24 \mu \mathrm{L}, 19.2 \mathrm{mg}, 0.20 \mathrm{mmol})$, phenylamine ( $37 \mu \mathrm{L}, 37.3 \mathrm{mg}, 0.40 \mathrm{mmol}$ ), $\mathrm{PhCH}_{2} \mathrm{SeSeCH}_{2} \mathrm{Ph}$ (34.2 mg, $0.10 \mathrm{mmol}$ ), [Os-Cu] (10.9 mg, $0.01 \mathrm{mmol})$, and $\mathrm{CH}_{2} \mathrm{Cl}_{2}(4.0 \mathrm{~mL})$. The Schlenk tube was positioned approximately $3 \mathrm{~cm}$ away from a $24 \mathrm{~W}$ blue LEDs lamp $\left(\lambda_{\max }=450 \mathrm{~nm}\right)$. After being stirred at $25{ }^{\circ} \mathrm{C}$ for the $48 \mathrm{~h}$ under an $\mathrm{O}_{2}$ (balloon) atmosphere, the reaction mixture was concentrated to dryness. The residue was purified by flash chromatography (petroleum ether/ethyl acetate $=40: 1$ ) to afford product $\mathbf{4 s}$ as a yellow oil (64.1 mg, $0.150 \mathrm{mmol}$, yield: $75 \%)$.

${ }^{1}$ H NMR (500 MHz, CDCl $) \delta 7.34-7.31(\mathrm{~m}, 3 \mathrm{H}), 7.30-7.22(\mathrm{~m}, 4 \mathrm{H}), 6.22(\mathrm{~d}, J=$ $8.5 \mathrm{~Hz}, 2 \mathrm{H}), 4.24(\mathrm{~s}, 1 \mathrm{H}), 3.80(\mathrm{dd}, J=31.2,12.2 \mathrm{~Hz}, 2 \mathrm{H}), 3.73(\mathrm{~d}, J=3.6 \mathrm{~Hz}, 1 \mathrm{H})$, $3.00(\mathrm{~s}, 1 \mathrm{H}), 1.97-1.85(\mathrm{~m}, 2 \mathrm{H}), 1.61(\mathrm{ddd}, J=12.4,8.2,3.9 \mathrm{~Hz}, 2 \mathrm{H}), 1.50-1.42$ $(\mathrm{m}, 2 \mathrm{H}), 1.38-1.30(\mathrm{~m}, 1 \mathrm{H}), 1.00(\mathrm{~d}, J=6.7 \mathrm{~Hz}, 3 \mathrm{H})$.

${ }^{13}$ C NMR (126 MHz, $\left.\mathbf{C D C l}_{3}\right) \delta 149.1,139.5,129.2,128.7,126.9,126.7$ (q, $J=3.8$ Hz), 125.0 (q, $J=261.4 \mathrm{~Hz}), 118.4(\mathrm{q}, J=32.8 \mathrm{~Hz}), 111.8,53.8,49.2,31.3,30.7,28.7$, $28.1,20.4,20.1$.

IR (film): $v\left(\mathrm{~cm}^{-1}\right)$ 3393, 2978, 2856, 1457, 1384, 1243, 1110, 1066, 790, 693.

HRMS (ESI, m/z) calcd for $\mathrm{C}_{21} \mathrm{H}_{24} \mathrm{~F}_{3} \mathrm{NNaSe}(\mathrm{M}+\mathrm{Na})^{+}: 450.0924$, found: 450.0911 .

\section{(1S, 2S, 3S)-3-methyl-2-(phenylselanyl)cyclohexyl pentanoate (4t)}

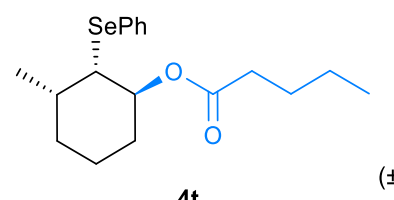

A dried $10 \mathrm{~mL}$ Schlenk tube was charged with $1 \mathrm{a}(24 \mu \mathrm{L}, 19.2 \mathrm{mg}, 0.20 \mathrm{mmol})$, valeric acid (44 $\mu \mathrm{L}, 40.9 \mathrm{mg}, 0.40 \mathrm{mmol}), 3 \mathbf{a}(31.2 \mathrm{mg}, 0.10 \mathrm{mmol})$, [Os-Cu] (10.9 $\mathrm{mg}, \quad 0.01 \mathrm{mmol})$, and $\mathrm{CH}_{2} \mathrm{Cl}_{2}(4.0 \mathrm{~mL})$. The Schlenk tube was positioned approximately $3 \mathrm{~cm}$ away from a $24 \mathrm{~W}$ blue LEDs lamp $\left(\lambda_{\max }=450 \mathrm{~nm}\right)$. After being stirred at $25{ }^{\circ} \mathrm{C}$ for the $48 \mathrm{~h}$ under an $\mathrm{O}_{2}$ (balloon) atmosphere, the reaction mixture was concentrated to dryness. The residue was purified by flash chromatography (petroleum ether/ethyl acetate $=40: 1)$ to afford product $\mathbf{4 t}$ as a colorless oil $(50.9 \mathrm{mg}$, 0.144 mmol, yield: $72 \%$ ). 
${ }^{1} \mathbf{H}$ NMR (500 MHz, $\left.\mathbf{C D C l}_{3}\right) \delta 7.64-7.58(\mathrm{~m}, 2 \mathrm{H}), 7.30-7.26(\mathrm{~m}, 3 \mathrm{H}), 5.27(\mathrm{dd}, J$ $=7.6,4.5 \mathrm{~Hz}, 1 \mathrm{H}), 3.48(\mathrm{t}, J=4.1 \mathrm{~Hz}, 1 \mathrm{H}), 2.31-2.26(\mathrm{~m}, 2 \mathrm{H}), 2.14(\mathrm{dtt}, J=13.8$, 6.8, 3.4 Hz, 1H), $2.08-2.00(\mathrm{~m}, 1 \mathrm{H}), 1.66-1.60(\mathrm{~m}, 4 \mathrm{H}), 1.58-1.50(\mathrm{~m}, 1 \mathrm{H}), 1.42$ $-1.23(\mathrm{~m}, 4 \mathrm{H}), 1.10(\mathrm{~d}, J=6.8 \mathrm{~Hz}, 3 \mathrm{H}), 0.93(\mathrm{t}, J=7.3 \mathrm{~Hz}, 3 \mathrm{H})$.

${ }^{13}$ C NMR (126 MHz, CDCl 3$) \delta 173.1,133.5,130 ., 129.1,127.1,73.6,54.1,34.4$, $31.8,30.8,27.1,26.8,22.3,20.4,20.2,13.7$.

IR (film): $v\left(\mathrm{~cm}^{-1}\right)$ 2961, 2856, 1738, 1460, 1388, 1066, 750, 700.

HRMS (ESI, m/z) calcd for $\mathrm{C}_{18} \mathrm{H}_{26} \mathrm{NaO}_{2} \mathrm{Se}(\mathrm{M}+\mathrm{Na})^{+}: 377.0990$, found: 377.0997 .

(1S, 2S, 3S)-3-methyl-2-(phenylselanyl)cyclohexyl 2-chloropropanoate (4u)

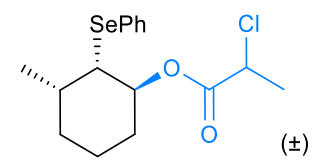

4u

$1: 1 \mathrm{dr}$

A dried $10 \mathrm{~mL}$ Schlenk tube was charged with $1 \mathrm{a}(24 \mu \mathrm{L}, 19.2 \mathrm{mg}, 0.20 \mathrm{mmol})$, 2-chloropropionic acid (37 $\mu \mathrm{L}, 43.2 \mathrm{mg}, 0.40 \mathrm{mmol}), 3 a(31.2 \mathrm{mg}, 0.10 \mathrm{mmol})$, [Os-Cu] (10.9 mg, $0.01 \mathrm{mmol})$, and $\mathrm{CH}_{2} \mathrm{Cl}_{2}(4.0 \mathrm{~mL})$. The Schlenk tube was positioned approximately $3 \mathrm{~cm}$ away from a $24 \mathrm{~W}$ blue LEDs lamp $\left(\lambda_{\max }=450 \mathrm{~nm}\right)$. After being stirred at $25{ }^{\circ} \mathrm{C}$ for the $48 \mathrm{~h}$ under an $\mathrm{O}_{2}$ (balloon) atmosphere, the reaction mixture was concentrated to dryness. The residue was purified by flash chromatography (petroleum ether/ethyl acetate $=40: 1$ ) to afford product $\mathbf{4 u}$ as a colorless oil (52.6 mg, $0.146 \mathrm{mmol}$, yield: $73 \%$ ).

${ }^{1}$ H NMR (500 MHz, $\left.\mathbf{C D C l}_{3}\right) \delta 7.59(\mathrm{ddd}, J=6.2,4.3,2.2 \mathrm{~Hz}, 2 \mathrm{H}), 7.29-7.25(\mathrm{~m}$, $3 \mathrm{H}), 5.31-5.26(\mathrm{~m}, 1 \mathrm{H}), 4.33(\mathrm{dq}, J=13.8,6.9 \mathrm{~Hz}, 1 \mathrm{H}), 3.50-3.44(\mathrm{~m}, 1 \mathrm{H}), 2.17-$ $2.03(\mathrm{~m}, 2 \mathrm{H}), 1.73$ (ddd, $J=14.4,9.3,5.7 \mathrm{~Hz}, 2 \mathrm{H}), 1.66$ (d, $J=7.0 \mathrm{~Hz}, 3 \mathrm{H}), 1.62-$ $1.58(\mathrm{~m}, 2 \mathrm{H}), 1.28(\mathrm{ddd}, J=8.7,7.7,4.4 \mathrm{~Hz}, 1 \mathrm{H}), 1.09$ (dd, $J=6.8,2.3 \mathrm{~Hz}, 3 \mathrm{H})$.

${ }^{13} \mathrm{C}$ NMR (126 MHz, CDCl 3$) \delta$ 169.2, 133.6 (2C), 130.3 (2C), 129.2, 75.7 (2C), 53.6, 53.5, 52.9, 52.8, 31.7, 31.5, 30.7, 30.6, 26.3, 26.1, 21.5(2C), 20.5, 20.4, 20.3.

IR (film): $v\left(\mathrm{~cm}^{-1}\right) 2960,2924,1718,1466,1382,1066,766,721,698$. 
HRMS (ESI, m/z) calcd for $\mathrm{C}_{16} \mathrm{H}_{21} \mathrm{ClNaO}_{2} \mathrm{Se}(\mathrm{M}+\mathrm{Na})^{+}: 383.0288$, found: 383.0284 .

\section{(1S, 2S, 3S)-3-methyl-2-(phenylselanyl)cyclohexyl benzoate (4v)}

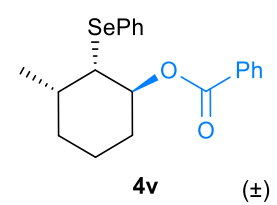

A dried $10 \mathrm{~mL}$ Schlenk tube was charged with $1 \mathrm{a}(24 \mu \mathrm{L}, 19.2 \mathrm{mg}, 0.20 \mathrm{mmol})$, PhCOOH (48.8 mg, 0.40 mmol), 3a (31.2 mg, 0.10 mmol), [Os-Cu] (10.9 mg, 0.01 $\mathrm{mmol})$, and $\mathrm{CH}_{2} \mathrm{Cl}_{2}(4.0 \mathrm{~mL})$. The Schlenk tube was positioned approximately $3 \mathrm{~cm}$ away from a $24 \mathrm{~W}$ blue LEDs lamp $\left(\lambda_{\max }=450 \mathrm{~nm}\right)$. After being stirred at $25^{\circ} \mathrm{C}$ for the $48 \mathrm{~h}$ under an $\mathrm{O}_{2}$ (balloon) atmosphere, the reaction mixture was concentrated to dryness. The residue was purified by flash chromatography (petroleum ether/ethyl acetate $=40: 1)$ to afford product $\mathbf{4 v}$ as a colorless oil $(52.3 \mathrm{mg}, 0.140 \mathrm{mmol}$, yield: $70 \%)$.

${ }^{1} \mathbf{H}$ NMR (500 MHz, CDCl 3$) \delta 8.13-7.96(\mathrm{~m}, 2 \mathrm{H}), 7.69(\mathrm{dd}, J=7.5,1.6 \mathrm{~Hz}, 2 \mathrm{H})$, $7.59(\mathrm{t}, J=7.4 \mathrm{~Hz}, 1 \mathrm{H}), 7.47(\mathrm{t}, J=7.8 \mathrm{~Hz}, 2 \mathrm{H}), 7.34-7.28(\mathrm{~m}, 3 \mathrm{H}), 5.58(\mathrm{dd}, J=$ 4.7, $1.8 \mathrm{~Hz}, 1 \mathrm{H}), 3.67(\mathrm{~s}, 1 \mathrm{H}), 2.35-2.26(\mathrm{~m}, 1 \mathrm{H}), 2.23-2.15(\mathrm{~m}, 1 \mathrm{H}), 1.93-1.86$ (m, 1H), $1.85-1.77(\mathrm{~m}, 1 \mathrm{H}), 1.72(\mathrm{dt}, J=13.4,4.3 \mathrm{~Hz}, 1 \mathrm{H}), 1.68-1.59(\mathrm{~m}, 1 \mathrm{H})$, $1.38(\mathrm{ddd}, J=14.9,11.5,3.8 \mathrm{~Hz}, 1 \mathrm{H}), 1.16(\mathrm{~d}, J=6.8 \mathrm{~Hz}, 3 \mathrm{H})$.

${ }^{13}$ C NMR (126 MHz, CDCl 3$) \delta 165.7,133.6,132.9,130.7,130.6,129.6,129.2,128.4$, $127.2,74.4,54.2,31.9,30.9,26.6,20.7,20.6$.

IR (film): $v\left(\mathrm{~cm}^{-1}\right)$ 2930, 2871, 1718, 1467, 1380, 1066, 770.

HRMS (ESI, m/z) calcd for $\mathrm{C}_{20} \mathrm{H}_{22} \mathrm{NaO}_{2} \mathrm{Se}(\mathrm{M}+\mathrm{Na})^{+}: 397.0677$, found: 397.0678 .

\section{(1S, 2S, 3S)-3-methyl-2-(phenylselanyl)cyclohexyl 1-naphthoate (4w)}

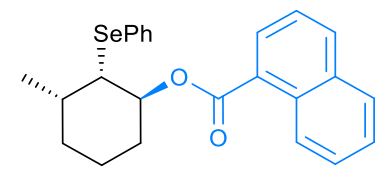

$4 w$ 
A dried $10 \mathrm{~mL}$ Schlenk tube was charged with 1a $(24 \mu \mathrm{L}, 19.2 \mathrm{mg}, 0.20 \mathrm{mmol})$, 1-naphthoic acid (68.9 mg, $0.40 \mathrm{mmol}), 3 \mathbf{3 a}(31.2 \mathrm{mg}, 0.10 \mathrm{mmol})$, [Os-Cu] (10.9 mg, $0.01 \mathrm{mmol})$, and $\mathrm{CH}_{2} \mathrm{Cl}_{2}(4.0 \mathrm{~mL})$. The Schlenk tube was positioned approximately 3 $\mathrm{cm}$ away from a $24 \mathrm{~W}$ blue LEDs lamp $\left(\lambda_{\max }=450 \mathrm{~nm}\right)$. After being stirred at $25{ }^{\circ} \mathrm{C}$ for the $48 \mathrm{~h}$ under an $\mathrm{O}_{2}$ (balloon) atmosphere, the reaction mixture was concentrated to dryness. The residue was purified by flash chromatography (petroleum ether/ethyl acetate $=40: 1)$ to afford product $4 \mathbf{w}$ as a yellow oil $(67.8 \mathrm{mg}, 0.160 \mathrm{mmol}$, yield: $80 \%)$.

${ }^{1} \mathbf{H}$ NMR (400 MHz, CDCl $) \delta 8.99(\mathrm{t}, J=7.2 \mathrm{~Hz}, 1 \mathrm{H}), 8.17(\mathrm{t}, J=6.2 \mathrm{~Hz}, 1 \mathrm{H}), 8.03$ $(\mathrm{d}, J=7.5 \mathrm{~Hz}, 1 \mathrm{H}), 7.90(\mathrm{~d}, J=7.3 \mathrm{~Hz}, 1 \mathrm{H}), 7.74-7.61(\mathrm{~m}, 3 \mathrm{H}), 7.59-7.48(\mathrm{~m}, 2 \mathrm{H})$, $7.28(\mathrm{~d}, J=5.6 \mathrm{~Hz}, 2 \mathrm{H}), 5.67(\mathrm{~d}, J=2.5 \mathrm{~Hz}, 1 \mathrm{H}), 3.72(\mathrm{~s}, 1 \mathrm{H}), 2.40-2.16(\mathrm{~m}, 2 \mathrm{H})$, $1.95-1.61(\mathrm{~m}, 4 \mathrm{H}), 1.41(\mathrm{dd}, J=10.5,6.8 \mathrm{~Hz}, 1 \mathrm{H}), 1.16(\mathrm{~d}, J=6.7 \mathrm{~Hz}, 3 \mathrm{H})$.

${ }^{13}$ C NMR (101 MHz, CDCl 3$) \delta 166.6,133.9,133.6,133.3,131.5,130.6,130.2,129.2$, $128.5,127.7,127.5,127.2,126.2,125.9,124.5,74.6,54.3,32.1,30.9,26.9,20.8$, 20.4

IR (film): $v\left(\mathrm{~cm}^{-1}\right)$ 2929, 1709, 1465, 1380, 1127, 911, 780, 737, 688.

HRMS (ESI, m/z) calcd for $\mathrm{C}_{24} \mathrm{H}_{24} \mathrm{NaO}_{2} \mathrm{Se}(\mathrm{M}+\mathrm{Na})^{+}$: 447.0839 , found: 447.0834 .

\section{(1S, 2S, 3S)-3-methyl-2-(phenylselanyl)cyclohexyl 2-(p-tolyl)acetate (4x)}

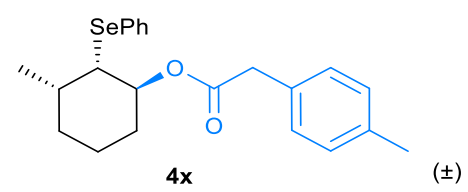

A dried $10 \mathrm{~mL}$ Schlenk tube was charged with 1a $(24 \mu \mathrm{L}, 19.2 \mathrm{mg}, 0.20 \mathrm{mmol})$, 4-methylphenylacetic acid (60.0 mg, $0.40 \mathrm{mmol}), 3 \mathrm{a}(31.2 \mathrm{mg}, 0.10 \mathrm{mmol})$, [Os-Cu] (10.9 $\mathrm{mg}, 0.01 \mathrm{mmol}$ ), and $\mathrm{CH}_{2} \mathrm{Cl}_{2}(4.0 \mathrm{~mL})$. The Schlenk tube was positioned approximately $3 \mathrm{~cm}$ away from a $24 \mathrm{~W}$ blue LEDs lamp $\left(\lambda_{\max }=450 \mathrm{~nm}\right)$. After being stirred at $25{ }^{\circ} \mathrm{C}$ for the $48 \mathrm{~h}$ under an $\mathrm{O}_{2}$ (balloon) atmosphere, the reaction mixture was concentrated to dryness. The residue was purified by flash chromatography (petroleum ether/ethyl acetate $=40: 1)$ to afford product $\mathbf{4} \mathbf{x}$ as a yellow oil $(53.1 \mathrm{mg}$, 0.132 mmol, yield: $66 \%$ ). 


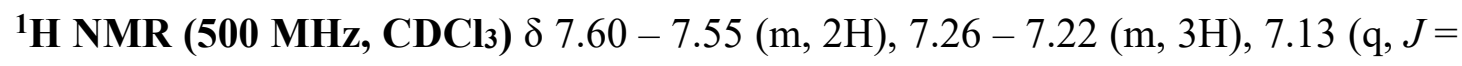
$8.2 \mathrm{~Hz}, 4 \mathrm{H}), 5.24$ (dd, $J=7.4,4.4 \mathrm{~Hz}, 1 \mathrm{H}), 3.53$ (s, 2H), 3.43 (t, $J=4.0 \mathrm{~Hz}, 1 \mathrm{H}), 2.32$ (s, 3H), $2.08-1.92(\mathrm{~m}, 2 \mathrm{H}), 1.54$ (dddd, $J=18.3,12.2,10.1,4.0 \mathrm{~Hz}, 4 \mathrm{H}), 1.27-1.21$ $(\mathrm{m}, 1 \mathrm{H}), 1.04(\mathrm{~d}, J=6.8 \mathrm{~Hz}, 3 \mathrm{H})$.

${ }^{13}$ C NMR (126 MHz, CDCl $) \delta$ 170.9, 136.6, 133.5, 131.1, 130.6, 129.2 (2C), 127.1, $74.3,53.9,41.3,31.6,30.8,26.5,21.1,20.3(2 \mathrm{C})$.

IR (film): $v\left(\mathrm{~cm}^{-1}\right)$ 2929, 2846, 1729, 1466, 1380, 1066, 811, 743.

HRMS (ESI, m/z) calcd for $\mathrm{C}_{22} \mathrm{H}_{26} \mathrm{NaO}_{2} \mathrm{Se}(\mathrm{M}+\mathrm{Na})^{+}$: 425.0996, found: 425.1000 .

(1S, 2S, 3S)-3-methyl-2-(phenylselanyl)cyclohexyl 2-(p-tolyl)acetate (4y)

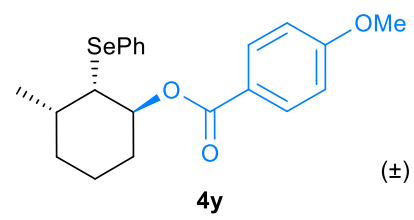

A dried $10 \mathrm{~mL}$ Schlenk tube was charged with 1a $(24 \mu \mathrm{L}, 19.2 \mathrm{mg}, 0.20 \mathrm{mmol})$, 4-methoxybenzoic acid (60.9 mg, $0.40 \mathrm{mmol}), 3 \mathbf{3 a}(31.2 \mathrm{mg}, 0.10 \mathrm{mmol})$, [Os-Cu] (10.9 mg, $0.01 \mathrm{mmol}$ ), and $\mathrm{CH}_{2} \mathrm{Cl}_{2}(4.0 \mathrm{~mL})$. The Schlenk tube was positioned approximately $3 \mathrm{~cm}$ away from a $24 \mathrm{~W}$ blue LEDs lamp $\left(\lambda_{\max }=450 \mathrm{~nm}\right)$. After being stirred at $25{ }^{\circ} \mathrm{C}$ for the $48 \mathrm{~h}$ under an $\mathrm{O}_{2}$ (balloon) atmosphere, the reaction mixture was concentrated to dryness. The residue was purified by flash chromatography (petroleum ether/ethyl acetate $=40: 1)$ to afford product $\mathbf{4 y}$ as a colorless oil (60.3 $\mathrm{mg}$, 0.150 mmol, yield: $75 \%$ ).

${ }^{1}$ H NMR (500 MHz, CDCl $)$ ) $\delta 7.99-7.93(\mathrm{~m}, 2 \mathrm{H}), 7.66-7.60(\mathrm{~m}, 2 \mathrm{H}), 7.26(\mathrm{dd}, J$ $=5.0,1.8 \mathrm{~Hz}, 3 \mathrm{H}), 6.93-6.88(\mathrm{~m}, 2 \mathrm{H}), 5.48(\mathrm{dd}, J=7.1,4.1 \mathrm{~Hz}, 1 \mathrm{H}), 3.85(\mathrm{~s}, 3 \mathrm{H})$, $3.60(\mathrm{t}, J=3.9 \mathrm{~Hz}, 1 \mathrm{H}), 2.28-2.18(\mathrm{~m}, 1 \mathrm{H}), 2.17-2.07(\mathrm{~m}, 1 \mathrm{H}), 1.88-1.79(\mathrm{~m}, 1 \mathrm{H})$, $1.75-1.63(\mathrm{~m}, 2 \mathrm{H}), 1.38-1.25(\mathrm{~m}, 2 \mathrm{H}), 1.10(\mathrm{~d}, J=6.8 \mathrm{~Hz}, 3 \mathrm{H})$.

${ }^{13}$ C NMR (126 MHz, CDCl 3$) \delta 165.4,163.3,133.6,131.6,130.6,129.1,127.2,123.1$, 113.6, 74.0, 55.4, 54.3, 31.9, 30.8, 26.6, 20.7, 20.57.

IR (film): $v\left(\mathrm{~cm}^{-1}\right)$ 2960, 2929, 1739, 1465, 1380, 1293, 1066, 820, 753, 691.

HRMS (ESI, m/z) calcd for $\mathrm{C}_{21} \mathrm{H}_{24} \mathrm{NaO}_{3} \mathrm{Se}(\mathrm{M}+\mathrm{Na})^{+}$: 427.0783, found: 427.0786. 
(1S, 2S, 3S)-3-methyl-2-(phenylselanyl)cyclohexyl 4-cyanobenzoate (4z)

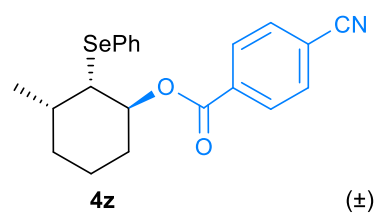

A dried $10 \mathrm{~mL}$ Schlenk tube was charged with 1a $(24 \mu \mathrm{L}, 19.2 \mathrm{mg}, 0.20 \mathrm{mmol})$, 4-cyanobenzoic acid (58.9 mg, $0.40 \mathrm{mmol}), \mathbf{3 a}(31.2 \mathrm{mg}, 0.10 \mathrm{mmol}),[\mathbf{O s}-\mathbf{C u}](10.9$ $\mathrm{mg}, \quad 0.01 \mathrm{mmol})$, and $\mathrm{CH}_{2} \mathrm{Cl}_{2}(4.0 \mathrm{~mL})$. The Schlenk tube was positioned approximately $3 \mathrm{~cm}$ away from a $24 \mathrm{~W}$ blue LEDs lamp $\left(\lambda_{\max }=450 \mathrm{~nm}\right)$. After being stirred at $25{ }^{\circ} \mathrm{C}$ for the $48 \mathrm{~h}$ under an $\mathrm{O}_{2}$ (balloon) atmosphere, the reaction mixture was concentrated to dryness. The residue was purified by flash chromatography (petroleum ether/ethyl acetate $=40: 1$ ) to afford product $4 \mathbf{z}$ as a yellow solid (51.1 mg, 0.128 mmol, yield: 64\%).

${ }^{1}$ H NMR (500 MHz, CDCl 3$) \delta 8.07(\mathrm{~d}, J=8.4 \mathrm{~Hz}, 2 \mathrm{H}), 7.72(\mathrm{~d}, J=8.4 \mathrm{~Hz}, 2 \mathrm{H})$, $7.66-7.59(\mathrm{~m}, 2 \mathrm{H}), 7.28(\mathrm{dd}, J=5.9,2.3 \mathrm{~Hz}, 3 \mathrm{H}), 5.53(\mathrm{dd}, J=7.7,4.7 \mathrm{~Hz}, 1 \mathrm{H})$, $3.61(\mathrm{t}, J=4.1 \mathrm{~Hz}, 1 \mathrm{H}), 2.31-2.15(\mathrm{~m}, 2 \mathrm{H}), 1.89-1.80(\mathrm{~m}, 1 \mathrm{H}), 1.75-1.69(\mathrm{~m}, 2 \mathrm{H})$, $1.61(\mathrm{dt}, J=23.9,9.8 \mathrm{~Hz}, 1 \mathrm{H}), 1.43-1.35(\mathrm{~m}, 1 \mathrm{H}), 1.14(\mathrm{~d}, J=6.8 \mathrm{~Hz}, 3 \mathrm{H})$.

${ }^{13}$ C NMR (126 MHz, CDCl 3$) \delta 164.0,134.4,133.7,132.2,130.4,130.1,129.2,127.4$, $118.0,116.3,75.6,53.8,32.2,30.9,26.9,20.6,20.2$.

IR (film): $v\left(\mathrm{~cm}^{-1}\right)$ 2929, 2850, 2220, 1739, 1460, 1376, 1066, 830, 710.

HRMS (ESI, m/z) calcd for $\mathrm{C}_{21} \mathrm{H}_{21} \mathrm{NNaO}_{2} \mathrm{Se}(\mathrm{M}+\mathrm{Na})^{+}: 422.0635$, found: 422.0629 .

(1S, 2S, 3S)-3-methyl-2-(phenylselanyl)cyclohexyl 3-chlorobenzoate (4za)

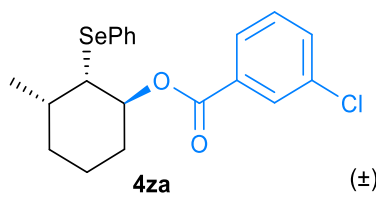

A dried $10 \mathrm{~mL}$ Schlenk tube was charged with $1 \mathrm{a}(24 \mu \mathrm{L}, 19.2 \mathrm{mg}, 0.20 \mathrm{mmol})$, 3-chlorobenzoic acid (62.6 mg, $0.40 \mathrm{mmol}), 3 \mathbf{3}$ (31.2 mg, $0.10 \mathrm{mmol})$, [Os-Cu] (10.9 $\mathrm{mg}, 0.01 \mathrm{mmol})$, and $\mathrm{CH}_{2} \mathrm{Cl}_{2}(4.0 \mathrm{~mL})$. The Schlenk tube was positioned approximately $3 \mathrm{~cm}$ away from a $24 \mathrm{~W}$ blue LEDs lamp $\left(\lambda_{\max }=450 \mathrm{~nm}\right)$. After being stirred at $25{ }^{\circ} \mathrm{C}$ for the $48 \mathrm{~h}$ under an $\mathrm{O}_{2}$ (balloon) atmosphere, the reaction mixture 
was concentrated to dryness. The residue was purified by flash chromatography (petroleum ether/ethyl acetate $=40: 1)$ to afford product $4 \mathbf{z a}$ as a yellow oil $(58.7 \mathrm{mg}$, 0.144 mmol, yield: $72 \%$ ).

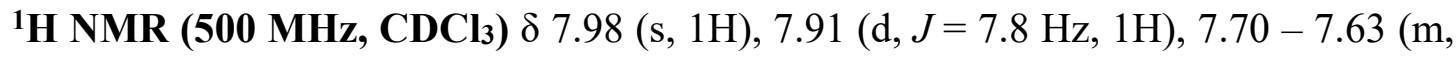
2H), $7.55(\mathrm{~d}, J=8.0 \mathrm{~Hz}, 1 \mathrm{H}), 7.39(\mathrm{t}, J=7.9 \mathrm{~Hz}, 1 \mathrm{H}), 7.32-7.27(\mathrm{~m}, 3 \mathrm{H}), 5.55(\mathrm{dd}$, $J=7.4,4.3 \mathrm{~Hz}, 1 \mathrm{H}), 3.63(\mathrm{t}, J=3.8 \mathrm{~Hz}, 1 \mathrm{H}), 2.33-2.24(\mathrm{~m}, 1 \mathrm{H}), 2.24-2.14(\mathrm{~m}$, 1H), $1.92-1.83(\mathrm{~m}, 1 \mathrm{H}), 1.81-1.69(\mathrm{~m}, 2 \mathrm{H}), 1.67-1.60(\mathrm{~m}, 1 \mathrm{H}), 1.38(\mathrm{dtd}, J=$ $14.8,10.8,4.2 \mathrm{~Hz}, 1 \mathrm{H}), 1.16(\mathrm{~d}, J=6.8 \mathrm{~Hz}, 3 \mathrm{H})$.

${ }^{13}$ C NMR (126 MHz, CDCl 3$) \delta 164.5,134.5,133.7,132.9,132.4,130.5,129.7,129.6$, $129.2,127.8,127.3,75.0,54.0,32.1,30.9,26.8,20.6,20.4$.

IR (film): $v\left(\mathrm{~cm}^{-1}\right)$ 2965, 2930, 1719, 1467, 1381, 1066, 810, 756, 723.

HRMS (ESI, m/z) calcd for $\mathrm{C}_{20} \mathrm{H}_{21} \mathrm{ClNaO}_{2} \mathrm{Se}(\mathrm{M}+\mathrm{Na})^{+}: 431.0288$, found: 431.0288 .

(1S, 2S, 3S)-3-methyl-2-(phenylselanyl)cyclohexyl 2-bromobenzoate (4zb)

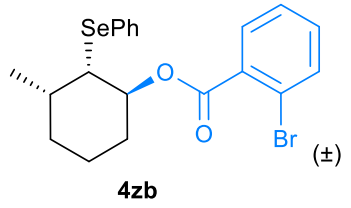

A dried $10 \mathrm{~mL}$ Schlenk tube was charged with 1a $(24 \mu \mathrm{L}, 19.2 \mathrm{mg}, 0.20 \mathrm{mmol})$, 2-bromobenzoic acid (80.4 mg,0.40 mmol), 3a (31.2 mg, $0.10 \mathrm{mmol})$, [Os-Cu] (10.9 $\mathrm{mg}, \quad 0.01 \mathrm{mmol})$, and $\mathrm{CH}_{2} \mathrm{Cl}_{2}(4.0 \mathrm{~mL})$. The Schlenk tube was positioned approximately $3 \mathrm{~cm}$ away from a $24 \mathrm{~W}$ blue LEDs lamp $\left(\lambda_{\max }=450 \mathrm{~nm}\right)$. After being stirred at $25{ }^{\circ} \mathrm{C}$ for the $48 \mathrm{~h}$ under an $\mathrm{O}_{2}$ (balloon) atmosphere, the reaction mixture was concentrated to dryness. The residue was purified by flash chromatography (petroleum ether/ethyl acetate $=40: 1)$ to afford product $\mathbf{4 z b}$ as a colorless oil $(70.5$ mg, 0.156 mmol, yield: 78\%).

${ }^{1}$ H NMR (500 MHz, CDCl $) \delta 7.77-7.73(\mathrm{~m}, 1 \mathrm{H}), 7.67-7.62(\mathrm{~m}, 3 \mathrm{H}), 7.36-7.30$ (m, 2H), 7.25 (ddd, $J=5.8,3.4,1.7 \mathrm{~Hz}, 3 \mathrm{H}), 5.55(\mathrm{dd}, J=7.2,4.1 \mathrm{~Hz}, 1 \mathrm{H}), 3.62(\mathrm{t}, J$ $=3.9 \mathrm{~Hz}, 1 \mathrm{H}), 2.32-2.23(\mathrm{~m}, 1 \mathrm{H}), 2.19-2.10(\mathrm{~m}, 1 \mathrm{H}), 1.91-1.85(\mathrm{~m}, 1 \mathrm{H}), 1.74$ (dddd, $J=21.4,11.8,8.0,4.0 \mathrm{~Hz}, 1 \mathrm{H}), 1.67-1.62(\mathrm{~m}, 1 \mathrm{H}), 1.57$ (ddd, $J=7.6,5.5$, $2.7 \mathrm{~Hz}, 1 \mathrm{H}), 1.36-1.27(\mathrm{~m}, 1 \mathrm{H}), 1.11(\mathrm{~d}, J=6.8 \mathrm{~Hz}, 3 \mathrm{H})$. 
${ }^{13}$ C NMR (126 MHz, CDCl $) \delta 165.48$ (s), 134.3, 133.6, 132.7, 132.4, 131.5, 130.5, 129.2, $127.2(2 \mathrm{C}), 121.5,75.7,54.1,31.8,30.8,26.5,20.6(2 \mathrm{C})$.

IR (film): $v\left(\mathrm{~cm}^{-1}\right)$ 2960, 2929, 1726, 1462, 1380, 1068, 738, 689, 535.

HRMS (ESI, m/z) calcd for $\mathrm{C}_{20} \mathrm{H}_{21} \mathrm{BrNaO}_{2} \mathrm{Se}(\mathrm{M}+\mathrm{Na})^{+}: 474.9788$, found: 474.9788 .

((1S,2S,6S)-2-methoxy-6-methylcyclohexyl)(phenyl)selane (4zc)

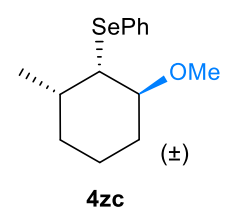

A dried $10 \mathrm{~mL}$ Schlenk tube was charged with 1a $(24 \mu \mathrm{L}, 19.2 \mathrm{mg}, 0.20 \mathrm{mmol}), 3 \mathbf{a}$ (31.2 mg, $0.10 \mathrm{mmol})$, [Os-Cu] (10.9 mg, $0.01 \mathrm{mmol}), 2 \mathrm{a}(0.5 \mathrm{~mL})$, and $\mathrm{CH}_{2} \mathrm{Cl}_{2}(3.5$ $\mathrm{mL}$ ). The Schlenk tube was positioned approximately $3 \mathrm{~cm}$ away from a $24 \mathrm{~W}$ blue LEDs lamp $\left(\lambda_{\max }=450 \mathrm{~nm}\right)$. After being stirred at $25^{\circ} \mathrm{C}$ for the $48 \mathrm{~h}$ under an $\mathrm{O}_{2}$ (balloon) atmosphere, the reaction mixture was concentrated to dryness. The residue was purified by flash chromatography (petroleum ether/ethyl acetate $=50: 1$ ) to afford product 4zc as a yellow oil (49.4 mg, $0.174 \mathrm{mmol}$, yield: 87\%).

${ }^{1}$ H NMR (500 MHz, CDCl3) $\delta 7.63-7.51$ (m, 2H), $7.27-7.23(\mathrm{~m}, 3 \mathrm{H}), 3.65$ (dd, $J$ $=6.9,3.9 \mathrm{~Hz}, 1 \mathrm{H}), 3.49(\mathrm{t}, J=3.7 \mathrm{~Hz}, 1 \mathrm{H}), 3.28(\mathrm{~s}, 3 \mathrm{H}), 2.16-2.07(\mathrm{~m}, 1 \mathrm{H}), 1.92-$ $1.84(\mathrm{~m}, 1 \mathrm{H}), 1.72(\mathrm{ddd}, J=10.0,5.0,3.0 \mathrm{~Hz}, 1 \mathrm{H}), 1.61-1.54(\mathrm{~m}, 1 \mathrm{H}), 1.51-1.42$ (m, 2H), $1.26-1.16(\mathrm{~m}, 1 \mathrm{H}), 1.06(\mathrm{~d}, J=6.8 \mathrm{~Hz}, 3 \mathrm{H})$.

${ }^{13}$ C NMR (126 MHz, CDCl 3$) \delta 133.7,131.1,129.0,127.0,80.2,56.2,55.0,31.0$, $25.8,20.7,20.2$.

IR (film): v(cm $\left.{ }^{-1}\right)$ 2974, 2900, 2871, 1465, 1382, 1273, 1086, 771, 710.

HRMS (ESI, m/z) calcd for $\mathrm{C}_{14} \mathrm{H}_{20} \mathrm{NaOSe}(\mathrm{M}+\mathrm{Na})^{+}$: 307.0572, found: 307.0569 .

((1S, 2S, 6S)-2-ethoxy-6-methylcyclohexyl)(phenyl)selane (4zd)

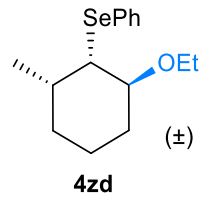


A dried $10 \mathrm{~mL}$ Schlenk tube was charged with $1 \mathbf{a}(24 \mu \mathrm{L}, 19.2 \mathrm{mg}, 0.20 \mathrm{mmol}), \mathrm{EtOH}$ (0.5 mL), 3a (31.2 mg, $0.10 \mathrm{mmol})$, [Os-Cu] (10.9 mg, $0.01 \mathrm{mmol})$, and $\mathrm{CH}_{2} \mathrm{Cl}_{2}(3.5$ $\mathrm{mL}$ ). The Schlenk tube was positioned approximately $3 \mathrm{~cm}$ away from a $24 \mathrm{~W}$ blue LEDs lamp $\left(\lambda_{\max }=450 \mathrm{~nm}\right)$. After being stirred at $25^{\circ} \mathrm{C}$ for the $48 \mathrm{~h}$ under an $\mathrm{O}_{2}$ (balloon) atmosphere, the reaction mixture was concentrated to dryness. The residue was purified by flash chromatography (petroleum ether/ethyl acetate $=50: 1$ ) to afford product 4zd as a colorless oil (38.7 $\mathrm{mg}, 0.130 \mathrm{mmol}$, yield: 65\%).

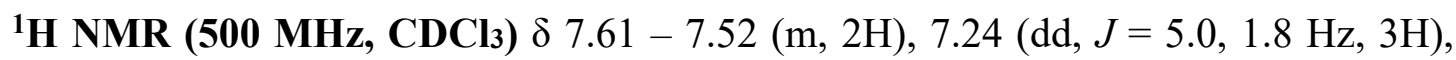
$3.74(\mathrm{dd}, J=7.1,4.0 \mathrm{~Hz}, 1 \mathrm{H}), 3.50-3.40(\mathrm{~m}, 3 \mathrm{H}), 2.16(\mathrm{dtq}, J=13.7,6.8,3.5 \mathrm{~Hz}$, 1H), $1.93-1.82(\mathrm{~m}, 1 \mathrm{H}), 1.71-1.64(\mathrm{~m}, 1 \mathrm{H}), 1.61(\mathrm{ddd}, J=11.8,8.4,4.4 \mathrm{~Hz}, 1 \mathrm{H})$, $1.51-1.42(\mathrm{~m}, 2 \mathrm{H}), 1.24-1.17(\mathrm{~m}, 1 \mathrm{H}), 1.14(\mathrm{t}, J=7.0 \mathrm{~Hz}, 3 \mathrm{H}), 1.05(\mathrm{~d}, J=6.8 \mathrm{~Hz}$, $3 \mathrm{H})$.

${ }^{13}$ C NMR (126 MHz, CDCl $) \delta 133.7,131.2,129.0,126.9,78.2,63.8,55.6,31.1$ (2C), 26.6, 20.5, 20.3, 15.6.

IR (film): $v\left(\mathrm{~cm}^{-1}\right)$ 2972, 2859, 1466, 1382, 1271, 1056, 762, 690.

HRMS (ESI, m/z) calcd for $\mathrm{C}_{15} \mathrm{H}_{22} \mathrm{NaOSe}(\mathrm{M}+\mathrm{Na})^{+}: 321.0734$, found: 321.0729 .

((1S, 2S, 6S)-2-(2-bromoethoxy)-6-methylcyclohexyl)(phenyl)selane (4ze)

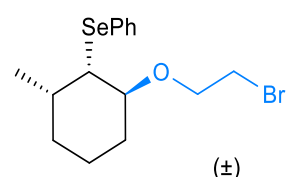

4ze

A dried $10 \mathrm{~mL}$ Schlenk tube was charged with $1 \mathrm{a}(24 \mu \mathrm{L}, 19.2 \mathrm{mg}, 0.20 \mathrm{mmol})$, $\mathrm{BrCH}_{2} \mathrm{CH}_{2} \mathrm{OH}(0.5 \mathrm{~mL}), 3 \mathrm{a}(31.2 \mathrm{mg}, 0.10 \mathrm{mmol})$, [Os-Cu] (10.9 mg, $\left.0.01 \mathrm{mmol}\right)$, and $\mathrm{CH}_{2} \mathrm{Cl}_{2}(3.5 \mathrm{~mL})$. The Schlenk tube was positioned approximately $3 \mathrm{~cm}$ away from a $24 \mathrm{~W}$ blue LEDs lamp $\left(\lambda_{\max }=450 \mathrm{~nm}\right)$. After being stirred at $25{ }^{\circ} \mathrm{C}$ for the 48 $\mathrm{h}$ under an $\mathrm{O}_{2}$ (balloon) atmosphere, the reaction mixture was concentrated to dryness. The residue was purified by flash chromatography (petroleum ether/ethyl acetate = 50:1) to afford product 4ze as a yellow oil (36.8 mg, $0.098 \mathrm{mmol}$, yield: 49\%). 
${ }^{1}$ H NMR (500 MHz, $\left.\mathbf{C D C l}_{3}\right) \delta 7.58(\mathrm{dd}, J=6.5,2.9 \mathrm{~Hz}, 1 \mathrm{H}), 7.27(\mathrm{dd}, J=6.5,4.1$ $\mathrm{Hz}, 1 \mathrm{H}), 3.80(\mathrm{dd}, J=7.0,3.9 \mathrm{~Hz}, 1 \mathrm{H}), 3.76-3.66(\mathrm{~m}, 1 \mathrm{H}), 3.48(\mathrm{t}, J=3.7 \mathrm{~Hz}, 1 \mathrm{H})$, $3.41(\mathrm{t}, J=6.3 \mathrm{~Hz}, 1 \mathrm{H}), 2.27-2.14(\mathrm{~m}, 1 \mathrm{H}), 1.99-1.89(\mathrm{~m}, 1 \mathrm{H}), 1.75-1.62(\mathrm{~m}, 1 \mathrm{H})$, $1.56-1.46(\mathrm{~m}, 1 \mathrm{H}), 1.27-1.18(\mathrm{~m}, 1 \mathrm{H}), 1.09(\mathrm{~d}, J=6.8 \mathrm{~Hz}, 1 \mathrm{H})$.

${ }^{13}$ C NMR (126 MHz, CDCl 3$) \delta 133.7,131.0,129.1,127.1,79.1,68.6,55.2,31.1$, $31.0,26.4,23.7,20.5,20.2$.

IR (film): v (cm-1) 2960, 2854, 1465, 1394, 1241, 1057, 762 ,698, 586.

HRMS (ESI, m/z) calcd for $\mathrm{C}_{15} \mathrm{H}_{21} \mathrm{BrNaOSe}(\mathrm{M}+\mathrm{Na})^{+}:$398.9833, found: 398.9818 .

\section{((1S, 2S, 6S)-2-isopropoxy-6-methylcyclohexyl)(phenyl)selane (4zf)}

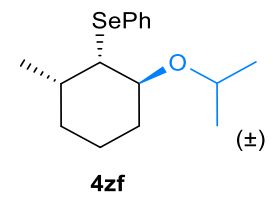

A dried $10 \mathrm{~mL}$ Schlenk tube was charged with 1a $(24 \mu \mathrm{L}, 19.2 \mathrm{mg}, 0.20 \mathrm{mmol})$, isopropyl alcohol (0.5 mL), 3a (31.2 mg, $0.10 \mathrm{mmol})$, [Os-Cu] (10.9 mg, $0.01 \mathrm{mmol})$, and $\mathrm{CH}_{2} \mathrm{Cl}_{2}(3.5 \mathrm{~mL})$. The Schlenk tube was positioned approximately $3 \mathrm{~cm}$ away from a $24 \mathrm{~W}$ blue LEDs lamp $\left(\lambda_{\max }=450 \mathrm{~nm}\right)$. After being stirred at $25^{\circ} \mathrm{C}$ for the 48 $\mathrm{h}$ under an $\mathrm{O}_{2}$ (balloon) atmosphere, the reaction mixture was concentrated to dryness. The residue was purified by flash chromatography (petroleum ether/ethyl acetate = 50:1) to afford product $\mathbf{4 z f}$ as a yellow oil (45.9 mg, $0.122 \mathrm{mmol}$, yield: $61 \%)$.

${ }^{1} \mathbf{H}$ NMR (500 MHz, CDCl 3$) \delta 7.62-7.52(\mathrm{~m}, 2 \mathrm{H}), 7.24(\mathrm{dd}, J=5.0,1.9 \mathrm{~Hz}, 3 \mathrm{H})$, $3.78(\mathrm{dd}, J=5.2,2.0 \mathrm{~Hz}, 1 \mathrm{H}), 3.64-3.52(\mathrm{~m}, 1 \mathrm{H}), 3.42(\mathrm{t}, J=3.8 \mathrm{~Hz}, 1 \mathrm{H}), 2.17$ (dtt, $J=13.7,6.8,3.4 \mathrm{~Hz}, 1 \mathrm{H}), 1.94-1.84(\mathrm{~m}, 1 \mathrm{H}), 1.61(\mathrm{dd}, J=12.5,4.8 \mathrm{~Hz}, 2 \mathrm{H}), 1.52-$ $1.41(\mathrm{~m}, 2 \mathrm{H}), 1.25-1.17(\mathrm{~m}, 1 \mathrm{H}), 1.11-1.03(\mathrm{~m}, 9 \mathrm{H})$.

${ }^{13}$ C NMR (126 MHz, CDCl $) \delta 133.7,131.2,129.0,126.9,75.4,69.2,56.1,31.1$ (2C), 27.4, 22.7 (2C), 20.4 .

IR (film): $v\left(\mathrm{~cm}^{-1}\right)$ 2972, 2851, 1466, 1385, 1374,1270, 1058, 773, 699.

HRMS (ESI, m/z) calcd for $\mathrm{C}_{16} \mathrm{H}_{25} \mathrm{OSe}(\mathrm{M}+\mathrm{Na})^{+}: 313.1065$, found: 313.1070 . 


\section{(1S,2S,3S)-3-methyl-2-(phenylselanyl)cyclohexan-1-ol (4zg)}

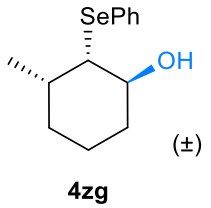

A dried $10 \mathrm{~mL}$ Schlenk tube was charged with $1 \mathbf{z}(24 \mu \mathrm{L}, 19.2 \mathrm{mg}, 0.20 \mathrm{mmol}), \mathrm{H}_{2} \mathrm{O}$ (18 $\mu \mathrm{L}, 18.0 \mathrm{mg}, 1.00 \mathrm{mmol}), 3 \mathbf{a}(31.2 \mathrm{mg}, 0.10 \mathrm{mmol})$, [Os-Cu] (10.9 mg, 0.01 mmol), and $\mathrm{CH}_{2} \mathrm{Cl}_{2}(4.0 \mathrm{~mL})$. The Schlenk tube was positioned approximately $3 \mathrm{~cm}$ away from a $24 \mathrm{~W}$ blue LEDs lamp $\left(\lambda_{\max }=450 \mathrm{~nm}\right)$. After being stirred at $25^{\circ} \mathrm{C}$ for the $48 \mathrm{~h}$ under an $\mathrm{O}_{2}$ (balloon) atmosphere, the reaction mixture was concentrated to dryness. The residue was purified by flash chromatography (petroleum ether/ethyl acetate $=20: 1)$ to afford product 5a as a yellow oil $(22.1 \mathrm{mg}, 0.082 \mathrm{mmol}$, yield: $41 \%)$.

${ }^{1}$ H NMR (500 MHz, CDCl 3$) \delta 7.58(\mathrm{dd}, J=6.3,3.0 \mathrm{~Hz}, 2 \mathrm{H}), 7.25(\mathrm{~d}, J=3.0 \mathrm{~Hz}$, 3H), $3.94(\mathrm{dd}, J=9.9,7.7 \mathrm{~Hz}, 1 \mathrm{H}), 3.26(\mathrm{dd}, J=7.8,4.1 \mathrm{~Hz}, 1 \mathrm{H}), 2.35(\mathrm{ddd}, J=10.6$, 7.0, $3.9 \mathrm{~Hz}, 1 \mathrm{H}), 2.20(\mathrm{~s}, 1 \mathrm{H}), 2.12(\mathrm{ddd}, J=10.8,7.3,4.0 \mathrm{~Hz}, 1 \mathrm{H}), 1.57-1.48$ (m, $3 \mathrm{H}), 1.43(\mathrm{dt}, J=12.5,4.5 \mathrm{~Hz}, 2 \mathrm{H}), 1.10(\mathrm{~d}, J=7.1 \mathrm{~Hz}, 3 \mathrm{H})$.

${ }^{13}$ C NMR (126 MHz, CDCl 3$) \delta 133.8,130.7,129.1,127.2,70.0,60.5,33.4,32.0,29.6$, 19.7 17.7.

IR (film): $v\left(\mathrm{~cm}^{-1}\right)$ 2970, 2856, 1466, 1370, 1355, 1185, 1066, 789, 692.

HRMS (ESI, m/z) calcd for $\mathrm{C}_{13} \mathrm{H}_{18} \mathrm{NaOSe}(\mathrm{M}+\mathrm{Na})^{+}:$293.0415, found: 293.0417.

\subsection{Substrate Scope for Alkenes}

General Procedure. A dried $10 \mathrm{~mL}$ Schlenk tube was charged with 1 (0.20 mmol), 2zc (0.50 mL), 3a (0.10 mmol), [Os-Cu] (10.9 mg, $0.01 \mathrm{mmol})$, and $\mathrm{CH}_{2} \mathrm{Cl}_{2}(3.5 \mathrm{~mL})$. The Schlenk tube was positioned approximately $3 \mathrm{~cm}$ away from a $24 \mathrm{~W}$ blue LEDs lamp $\left(\lambda_{\max }=450 \mathrm{~nm}\right)$. After being stirred at $25{ }^{\circ} \mathrm{C}$ for the $48 \mathrm{~h}$ under an $\mathrm{O}_{2}$ (balloon) atmosphere, the reaction mixture was concentrated to dryness. The residue was purified by flash chromatography (petroleum ether/ethyl acetate) to give the desired product. 

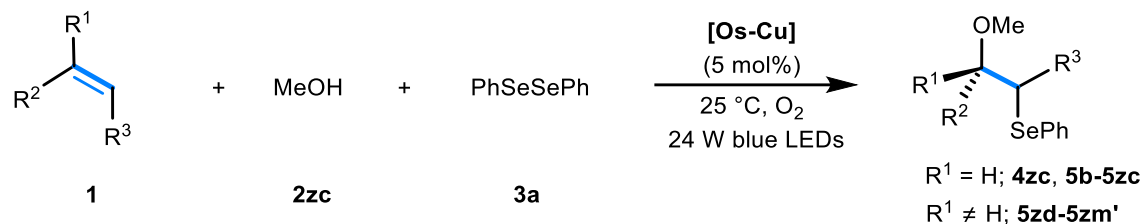

\subsubsection{Experimental details and characterization data of compounds 5} ((1S,2R,6S)-2-benzyl-6-methoxycyclohexyl)(phenyl)selane (5b)

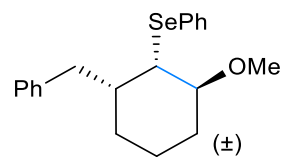

$5 b$

A dried $10 \mathrm{~mL}$ Schlenk tube was charged with $\mathbf{1 b}(34.4 \mathrm{mg}, 0.20 \mathrm{mmol}), \mathbf{3 a}(31.2 \mathrm{mg}$, $0.10 \mathrm{mmol})$, [Os-Cu] (10.9 mg, $0.01 \mathrm{mmol}), \mathbf{2 a}(0.5 \mathrm{~mL})$, and $\mathrm{CH}_{2} \mathrm{Cl}_{2}(3.5 \mathrm{~mL})$. The Schlenk tube was positioned approximately $3 \mathrm{~cm}$ away from a $24 \mathrm{~W}$ blue LEDs lamp $\left(\lambda_{\max }=450 \mathrm{~nm}\right)$. After being stirred at $25{ }^{\circ} \mathrm{C}$ for the $48 \mathrm{~h}$ under an $\mathrm{O}_{2}$ (balloon) atmosphere, the reaction mixture was concentrated to dryness. The residue was purified by flash chromatography (petroleum ether/ethyl acetate $=50: 1$ ) to afford product $\mathbf{5 b}$ as a colorless oil (56.9 $\mathrm{mg}, 0.158 \mathrm{mmol}$, yield: $79 \%)$.

${ }^{1}$ H NMR (500 MHz, CDCl $) \delta 7.49-7.40(\mathrm{~m}, 2 \mathrm{H}), 7.26-7.19(\mathrm{~m}, 5 \mathrm{H}), 7.19-7.14$ (m, 3H), $3.60(\mathrm{dd}, J=6.4,3.3 \mathrm{~Hz}, 1 \mathrm{H}), 3.47(\mathrm{~s}, 1 \mathrm{H}), 3.18(\mathrm{~s}, 3 \mathrm{H}), 2.82(\mathrm{dd}, J=13.6$, $7.6 \mathrm{~Hz}, 1 \mathrm{H}), 2.74(\mathrm{dd}, J=13.6,7.6 \mathrm{~Hz}, 1 \mathrm{H}), 2.35-2.26(\mathrm{~m}, 1 \mathrm{H}), 2.02(\mathrm{dddd}, J=14.4$, 12.1, 5.1, $2.7 \mathrm{~Hz}, 1 \mathrm{H}), 1.77$ (dd, $J=9.4,7.7 \mathrm{~Hz}, 1 \mathrm{H}), 1.58-1.49(\mathrm{~m}, 3 \mathrm{H}), 1.22$ (tdd, $J$ $=17.5,10.5,4.1 \mathrm{~Hz}, 1 \mathrm{H})$.

${ }^{13}$ C NMR (126 MHz, CDCl $) \delta 140.4,133.5,130.6,129.3,129.1,128.2,127.1,125.8$ $80.0,56.0,52.1,41.2,38.0,28.7,25.6,20.4$.

IR (film): v (cm $\left.{ }^{-1}\right)$ 2928, 2870, 1461, 1384, 1273, 1066, 773, 686.

HRMS (ESI, m/z) calcd for $\mathrm{C}_{20} \mathrm{H}_{24} \mathrm{NaOSe}(\mathrm{M}+\mathrm{Na})^{+}: 383.0885$, found: 383.0870 . 


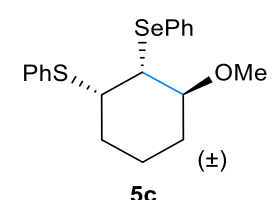

A dried $10 \mathrm{~mL}$ Schlenk tube was charged with 1c (38 mg, $0.20 \mathrm{mmol}), 3 \mathbf{a}(31.2 \mathrm{mg}$, $0.10 \mathrm{mmol})$, [Os-Cu] $(10.9 \mathrm{mg}, 0.01 \mathrm{mmol}), \mathbf{2 a}(0.5 \mathrm{~mL})$, and $\mathrm{CH}_{2} \mathrm{Cl}_{2}(3.5 \mathrm{~mL})$. The Schlenk tube was positioned approximately $3 \mathrm{~cm}$ away from a $24 \mathrm{~W}$ blue LEDs lamp $\left(\lambda_{\max }=450 \mathrm{~nm}\right)$. After being stirred at $25{ }^{\circ} \mathrm{C}$ for the $48 \mathrm{~h}$ under an $\mathrm{O}_{2}$ (balloon) atmosphere, the reaction mixture was concentrated to dryness. The residue was purified by flash chromatography (petroleum ether/ethyl acetate $=50: 1$ ) to afford product $5 \mathbf{c}$ as a yellow oil (55.2 $\mathrm{mg}, 0.146 \mathrm{mmol}$, yield: $73 \%)$.

${ }^{1} \mathbf{H}$ NMR (500 MHz, CDCl 3$) \delta 7.63-7.57$ (m, 2H), $7.41-7.38$ (m, 2H), $7.26-7.18$ (m, 6H), $3.78(\mathrm{dt}, J=10.2,3.7 \mathrm{~Hz}, 1 \mathrm{H}), 3.71(\mathrm{t}, J=4.2 \mathrm{~Hz}, 1 \mathrm{H}), 3.62(\mathrm{dd}, J=7.5,4.5$ $\mathrm{Hz}, 1 \mathrm{H}), 3.25$ (s, 3H), $2.02(\mathrm{dd}, J=6.8,4.6 \mathrm{~Hz}, 1 \mathrm{H}), 1.88-1.80(\mathrm{~m}, 1 \mathrm{H}), 1.69-1.59$ (m, 4H).

${ }^{13}$ C NMR (126 MHz, CDCl 3$) \delta 135.4,134.2,131.6,130.6,129.1,128.9,127.4,126.7$, $80.4,56.4,52.1,30.6,29.7,26.0,20.5$.

IR (film): $v\left(\mathrm{~cm}^{-1}\right)$ 2928, 1465, 1280, 1256, 1066, 777, 686.

HRMS (ESI, m/z) calcd for $\mathrm{C}_{19} \mathrm{H}_{22} \mathrm{NaOSSe}(\mathrm{M}+\mathrm{Na})^{+}: 401.0449$, found: 401.0451 .

\section{((1r,2R,6S)-2,6-dimethoxycyclohexyl)(phenyl)selane (5d)}

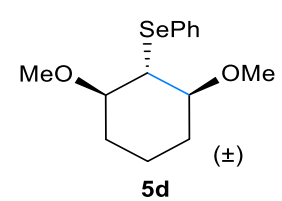

A dried $10 \mathrm{~mL}$ Schlenk tube was charged with 3-bromocyclohexene $(23 \mu \mathrm{L}, 32.2 \mathrm{mg}$, $0.20 \mathrm{mmol}), 3 \mathbf{a}(31.2 \mathrm{mg}, 0.10 \mathrm{mmol})$, [Os-Cu] (10.9 mg, $0.01 \mathrm{mmol}), 2 \mathrm{a}(0.5 \mathrm{~mL})$, and $\mathrm{CH}_{2} \mathrm{Cl}_{2}(3.5 \mathrm{~mL})$. The Schlenk tube was positioned approximately $3 \mathrm{~cm}$ away from a $24 \mathrm{~W}$ blue LEDs lamp $\left(\lambda_{\max }=450 \mathrm{~nm}\right)$. After being stirred at $25^{\circ} \mathrm{C}$ for the 48 $\mathrm{h}$ under an $\mathrm{O}_{2}$ (balloon) atmosphere, the reaction mixture was concentrated to dryness. 
The residue was purified by flash chromatography (petroleum ether/ethyl acetate = 40:1) to afford product $\mathbf{5 d}$ as a yellow oil (48.0 $\mathrm{mg}, 0.160 \mathrm{mmol}$, yield: $80 \%$ ).

${ }^{1}$ H NMR (500 MHz, $\left.\mathbf{C D C l}_{3}\right) \delta 7.64(\mathrm{dd}, J=6.4,3.1 \mathrm{~Hz}, 2 \mathrm{H}), 7.26-7.20(\mathrm{~m}, 3 \mathrm{H})$, $3.69-3.63(\mathrm{~m}, 1 \mathrm{H}), 3.57(\mathrm{td}, J=7.3,3.5 \mathrm{~Hz}, 1 \mathrm{H}), 3.50(\mathrm{dd}, J=7.0,2.9 \mathrm{~Hz}, 1 \mathrm{H}), 3.31$ $(\mathrm{d}, J=8.9 \mathrm{~Hz}, 6 \mathrm{H}), 2.06(\mathrm{ddd}, J=11.2,7.7,4.1 \mathrm{~Hz}, 1 \mathrm{H}), 1.80-1.71(\mathrm{~m}, 1 \mathrm{H}), 1.62-$ $1.49(\mathrm{~m}, 3 \mathrm{H}), 1.42(\mathrm{td}, J=12.6,7.2 \mathrm{~Hz}, 1 \mathrm{H})$.

${ }^{13}$ C NMR (126 MHz, CDCl 3$) \delta 134.2,130.8,128.8,127.1,80.5,78.8,56.7,56.3$, $52.5,28.5,28.2,18.7$.

IR (film): $v\left(\mathrm{~cm}^{-1}\right)$ 2921, 1467, 1278, 1066, 781, 698.

HRMS (ESI, m/z) calcd for $\mathrm{C}_{14} \mathrm{H}_{20} \mathrm{NaO}_{2} \mathrm{Se}(\mathrm{M}+\mathrm{Na})^{+}: 323.0521$, found: 323.0529 .

((2R,3S)-2-methoxy-4-methylpentan-3-yl)(phenyl)selane (5e) ${ }^{20}$

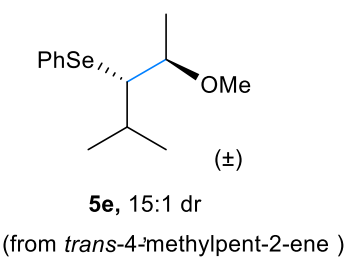

A dried $10 \mathrm{~mL}$ Schlenk tube was charged with $1 \mathrm{e}(25 \mu \mathrm{L}, 16.8 \mathrm{mg}, 0.20 \mathrm{mmol}), 3 \mathbf{a}$ (31.2 mg, $0.10 \mathrm{mmol})$, [Os-Cu] (10.9 mg, $0.01 \mathrm{mmol}), 2 \mathrm{a}(0.5 \mathrm{~mL})$, and $\mathrm{CH}_{2} \mathrm{Cl}_{2}(3.5$ $\mathrm{mL}$ ). The Schlenk tube was positioned approximately $3 \mathrm{~cm}$ away from a $24 \mathrm{~W}$ blue LEDs lamp $\left(\lambda_{\max }=450 \mathrm{~nm}\right)$. After being stirred at $50{ }^{\circ} \mathrm{C}$ for the $48 \mathrm{~h}$ under an $\mathrm{O}_{2}$ (balloon) atmosphere, the reaction mixture was concentrated to dryness. The residue was purified by flash chromatography ( petroleum ether/ethyl acetate $=50: 1$ ) to afford product 5e as a yellow oil (33.7 $\mathrm{mg}, 0.124 \mathrm{mmol}$, yield: 62\%).

${ }^{1} \mathbf{H}$ NMR (500 MHz, CDCl 3$) \delta 7.64-7.59$ (m, 2H), 7.32 - 7.24 (m, 3H), 3.62 - 3.55 (m, 1H), $3.36(\mathrm{~s}, 3 \mathrm{H}), 3.10(\mathrm{dd}, J=7.6,4.1 \mathrm{~Hz}, 1 \mathrm{H}), 2.32(\mathrm{dtd}, J=13.4,6.7,4.1 \mathrm{~Hz}$, $1 \mathrm{H}), 1.33(\mathrm{~d}, J=6.1 \mathrm{~Hz}, 3 \mathrm{H}), 1.13(\mathrm{~d}, J=6.8 \mathrm{~Hz}, 3 \mathrm{H}), 1.03(\mathrm{~d}, J=6.7 \mathrm{~Hz}, 3 \mathrm{H})$.

${ }^{13}$ C NMR (126 MHz, CDCl 3$) \delta 133.8,131.8,129.0,126.8,79.0,62.9,56.6,28.9$, $22.2,19.5,17.9$.

IR (film): $v\left(\mathrm{~cm}^{-1}\right)$ 2958, 2850, 1466, 1384, 1721, 1066, 770, 692.

HRMS (ESI, m/z) calcd for $\mathrm{C}_{13} \mathrm{H}_{20} \mathrm{NaOSe}(\mathrm{M}+\mathrm{Na})^{+}:$295.0572, found: 295.0573. 
$((1 S, 2 S)-2-m e t h o x y c y c l o p e n t y l)\left(\right.$ phenyl)selane $(5 f)^{18}$

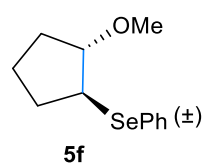

A dried $10 \mathrm{~mL}$ Schlenk tube was charged with $1 \mathbf{f}(18 \mu \mathrm{L}, 13.6 \mathrm{mg}, 0.20 \mathrm{mmol}), 3 \mathbf{a}$ (31.2 mg, $0.10 \mathrm{mmol})$, [Os-Cu] (10.9 mg, $0.01 \mathrm{mmol}), \mathbf{2 a}(0.5 \mathrm{~mL})$, and $\mathrm{CH}_{2} \mathrm{Cl}_{2}(3.5$ $\mathrm{mL}$ ). The Schlenk tube was positioned approximately $3 \mathrm{~cm}$ away from a $24 \mathrm{~W}$ blue LEDs lamp $\left(\lambda_{\max }=450 \mathrm{~nm}\right)$. After being stirred at $25^{\circ} \mathrm{C}$ for the $48 \mathrm{~h}$ under an $\mathrm{O}_{2}$ (balloon) atmosphere, the reaction mixture was concentrated to dryness. The residue was purified by flash chromatography (petroleum ether/ethyl acetate $=50: 1$ ) to afford product $\mathbf{5 f}$ as a colorless oil (33.3 $\mathrm{mg}, 0.130 \mathrm{mmol}$, yield: $65 \%)$.

${ }^{1}$ H NMR (500 MHz, CDCl $) \delta 7.60-7.53(\mathrm{~m}, 2 \mathrm{H}), 7.29-7.26(\mathrm{~m}, 3 \mathrm{H}), 3.74(\mathrm{dt}, J=$ 5.6, $2.7 \mathrm{~Hz}, 1 \mathrm{H}), 3.63-3.57(\mathrm{~m}, 1 \mathrm{H}), 3.18(\mathrm{~s}, 3 \mathrm{H}), 2.21(\mathrm{td}, J=13.7,6.9 \mathrm{~Hz}, 1 \mathrm{H})$, 1.99 (ddd, $J=13.0,8.3,6.3 \mathrm{~Hz}, 1 \mathrm{H}), 1.76-1.64$ (m, 4H).

${ }^{13}$ C NMR (126 MHz, CDCl3) $\delta$ 133.9, 130.2, 129.0, 127.3, 88.2, 56.6, 46.2, 31.7, $30.8,23.1$.

IR (film): $v\left(\mathrm{~cm}^{-1}\right)$ 2921, 1466, 1278, 1066, 763, 695.

HRMS (ESI, m/z) calcd for $\mathrm{C}_{12} \mathrm{H}_{16} \mathrm{NaOSe}(\mathrm{M}+\mathrm{Na})^{+}: 279.0264$, found: 279.0257 .

\section{((1S,2S)-2-methoxycyclohexyl)(phenyl)selane (5g) ${ }^{18}$}

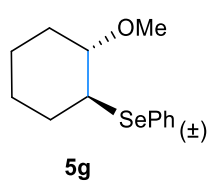

A dried $10 \mathrm{~mL}$ Schlenk tube was charged with $1 \mathrm{~g}(20 \mu \mathrm{L}, 16.4 \mathrm{mg}, 0.20 \mathrm{mmol}), 3 \mathbf{a}$ (31.2 mg, $0.10 \mathrm{mmol})$, [Os-Cu] (10.9 mg, $0.01 \mathrm{mmol}), 2 \mathrm{a}(0.5 \mathrm{~mL})$, and $\mathrm{CH}_{2} \mathrm{Cl}_{2}(3.5$ $\mathrm{mL}$ ). The Schlenk tube was positioned approximately $3 \mathrm{~cm}$ away from a $24 \mathrm{~W}$ blue LEDs lamp $\left(\lambda_{\max }=450 \mathrm{~nm}\right)$. After being stirred at $25^{\circ} \mathrm{C}$ for the $48 \mathrm{~h}$ under an $\mathrm{O}_{2}$ (balloon) atmosphere, the reaction mixture was concentrated to dryness. The residue was purified by flash chromatography (petroleum ether/ethyl acetate $=50: 1$ ) to afford product $\mathbf{5 g}$ as a yellow oil (44.8 $\mathrm{mg}, 0.166 \mathrm{mmol}$, yield: $83 \%)$. 
${ }^{1}$ H NMR (400 MHz, (CD) $\left.)_{2} \mathbf{C O}\right) \delta 7.61-7.55(\mathrm{~m}, 2 \mathrm{H}), 7.31-7.25$ (m, 3H), 3.34 $3.27(\mathrm{~m}, 4 \mathrm{H}), 3.19(\mathrm{td}, J=8.2,3.7 \mathrm{~Hz}, 1 \mathrm{H}), 2.16-2.07(\mathrm{~m}, 1 \mathrm{H}), 2.03-1.94(\mathrm{~m}, 1 \mathrm{H})$, $1.69(\mathrm{tdd}, J=5.9,4.3,2.6 \mathrm{~Hz}, 1 \mathrm{H}), 1.63-1.54(\mathrm{~m}, 1 \mathrm{H}), 1.52-1.43(\mathrm{~m}, 1 \mathrm{H}), 1.34-$ $1.23(\mathrm{~m}, 3 \mathrm{H})$.

${ }^{13}$ C NMR (101 MHz, (CD) 2 CO) $\delta$ 134.7, 129.5, 128.9, 127.2, 81.9, 55.6, 46.9, 31.7, $29.9,25.3,23.1$.

IR (film): $v\left(\mathrm{~cm}^{-1}\right)$ 2926, 1466, 1275, 1071, 760, 691.

HRMS (ESI, m/z) calcd for $\mathrm{C}_{13} \mathrm{H}_{18} \mathrm{NaOSe}(\mathrm{M}+\mathrm{Na})^{+}: 293.0415$, found: 293.0402

((6S,7S)-7-methoxydecahydro-1,4-methanonaphthalen-6-yl)(phenyl)selane (5h)

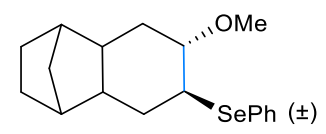

$5 \mathrm{~h}$

A dried $10 \mathrm{~mL}$ Schlenk tube was charged with $\mathbf{1 h}(31 \mu \mathrm{L}, 29.7 \mathrm{mg}, 0.20 \mathrm{mmol}), \mathbf{3 a}$ (31.2 mg, $0.10 \mathrm{mmol})$, [Os-Cu] (10.9 mg, $0.01 \mathrm{mmol}), \mathbf{2 a}(0.5 \mathrm{~mL})$, and $\mathrm{CH}_{2} \mathrm{Cl}_{2}(3.5$ $\mathrm{mL}$ ). The Schlenk tube was positioned approximately $3 \mathrm{~cm}$ away from a $24 \mathrm{~W}$ blue LEDs lamp $\left(\lambda_{\max }=450 \mathrm{~nm}\right)$. After being stirred at $25^{\circ} \mathrm{C}$ for the $48 \mathrm{~h}$ under an $\mathrm{O}_{2}$ (balloon) atmosphere, the reaction mixture was concentrated to dryness. The residue was purified by flash chromatography (petroleum ether/ethyl acetate $=50: 1$ ) to afford product $\mathbf{5 h}$ as a yellow oil (51.7 $\mathrm{mg}, 0.154 \mathrm{mmol}$, yield: $77 \%)$.

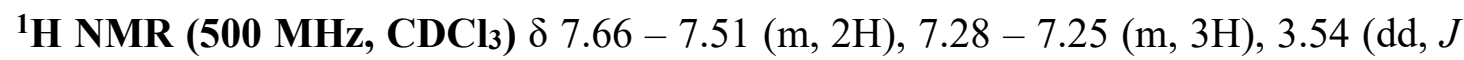
$=5.7,4.4 \mathrm{~Hz}, 1 \mathrm{H}), 3.32(\mathrm{ddd}, J=11.5,5.1,3.5 \mathrm{~Hz}, 1 \mathrm{H}), 3.22(\mathrm{~s}, 3 \mathrm{H}), 1.92(\mathrm{~s}, 1 \mathrm{H})$, $1.86-1.77(\mathrm{~m}, 3 \mathrm{H}), 1.73-1.63(\mathrm{~m}, 2 \mathrm{H}), 1.57-1.49(\mathrm{~m}, 4 \mathrm{H}), 1.23(\mathrm{dd}, J=13.8,7.2$ $\mathrm{Hz}, 2 \mathrm{H}), 1.12-1.02(\mathrm{~m}, 2 \mathrm{H})$.

${ }^{13}$ C NMR (126 MHz, CDCl 3$) \delta 134.2,130.3,129.0,127.3,82.3,56.5,45.3,42.3$, 41.7, 39.7, 38.5, 33.2, 30.3, 29.6, 29.4.

IR (film): $v\left(\mathrm{~cm}^{-1}\right)$ 2921, 2855, 1465, 1274, 1066, 761, 698.

HRMS (ESI, m/z) calcd for $\mathrm{C}_{18} \mathrm{H}_{24} \mathrm{NaOSe}(\mathrm{M}+\mathrm{Na})^{+}: 359.0885$, found: 359.0889 . 
$((1 S, 2 S)-2-m e t h o x y c y c l o h e p t y l)(p h e n y l) s e l a n e ~(5 i){ }^{18}$

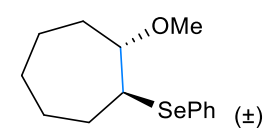

$5 \mathbf{i}$

A dried $10 \mathrm{~mL}$ Schlenk tube was charged with $1 \mathbf{i}(23 \mu \mathrm{L}, 19.2 \mathrm{mg}, 0.20 \mathrm{mmol}), 3 \mathbf{a}$ (31.2 mg, $0.10 \mathrm{mmol})$, [Os-Cu] (10.9 mg, $0.01 \mathrm{mmol}), \mathbf{2 a}(0.5 \mathrm{~mL})$, and $\mathrm{CH}_{2} \mathrm{Cl}_{2}(3.5$ $\mathrm{mL}$ ). The Schlenk tube was positioned approximately $3 \mathrm{~cm}$ away from a $24 \mathrm{~W}$ blue LEDs lamp $\left(\lambda_{\max }=450 \mathrm{~nm}\right)$. After being stirred at $25^{\circ} \mathrm{C}$ for the $48 \mathrm{~h}$ under an $\mathrm{O}_{2}$ (balloon) atmosphere, the reaction mixture was concentrated to dryness. The residue was purified by flash chromatography (petroleum ether/ethyl acetate $=50: 1$ ) to afford product 5i as a yellow oil (36.9 mg, $0.130 \mathrm{mmol}$, yield: $65 \%)$.

${ }^{1} \mathbf{H}$ NMR (500 MHz, CDCl 3$) \delta 7.57(\mathrm{ddd}, J=5.4,3.0,1.5 \mathrm{~Hz}, 2 \mathrm{H}), 7.28-7.24(\mathrm{~m}$, $3 \mathrm{H}), 3.52-3.46(\mathrm{~m}, 1 \mathrm{H}), 3.42(\mathrm{dt}, J=11.0,4.5 \mathrm{~Hz}, 1 \mathrm{H}), 3.26(\mathrm{~s}, 3 \mathrm{H}), 2.08-1.98(\mathrm{~m}$, 1H), $1.85-1.78(\mathrm{~m}, 2 \mathrm{H}), 1.72-1.60(\mathrm{~m}, 4 \mathrm{H}), 1.51-1.41(\mathrm{~m}, 3 \mathrm{H})$.

${ }^{13}$ C NMR (126 MHz, CDCl 3$) \delta 134.6,130.2,128.9,127.3,85.3,56.6,50.2,31.3$, $29.9,28.8,26.6,22.0$.

IR (film): $v\left(\mathrm{~cm}^{-1}\right)$ 2928, 2850, 1468, 1275, 1061, 743, 691.

HRMS (ESI, m/z) calcd for $\mathrm{C}_{14} \mathrm{H}_{20} \mathrm{NaOSe}(\mathrm{M}+\mathrm{Na})^{+}$: 307.0572, found: 307.0574 .

\section{$((1 S, 2 S)-2-m e t h o x y c y c l o o c t y l)\left(\right.$ phenyl)selane $(5 \mathbf{j})^{21}$}

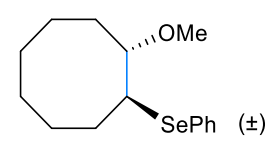

$5 \mathbf{j}$

A dried $10 \mathrm{~mL}$ Schlenk tube was charged with $1 \mathbf{j}(26 \mu \mathrm{L}, 22 \mathrm{mg}, 0.20 \mathrm{mmol}), 3 \mathbf{a}$ (31.2 mg, $0.10 \mathrm{mmol})$, [Os-Cu] (10.9 mg, $0.01 \mathrm{mmol}), 2 \mathrm{a}(0.5 \mathrm{~mL})$, and $\mathrm{CH}_{2} \mathrm{Cl}_{2}(3.5$ $\mathrm{mL}$ ). The Schlenk tube was positioned approximately $3 \mathrm{~cm}$ away from a $24 \mathrm{~W}$ blue LEDs lamp $\left(\lambda_{\max }=450 \mathrm{~nm}\right)$. After being stirred at $25^{\circ} \mathrm{C}$ for the $48 \mathrm{~h}$ under an $\mathrm{O}_{2}$ (balloon) atmosphere, the reaction mixture was concentrated to dryness. The residue was purified by flash chromatography (petroleum ether/ethyl acetate $=50: 1$ ) to afford product $\mathbf{5 j}$ as a yellow oil (42.3 $\mathrm{mg}, 0.142 \mathrm{mmol}$, yield: $71 \%)$. 
${ }^{1}$ H NMR (400 MHz, CDCl $) \delta 7.55-7.47$ (m, 2H), $7.20-7.14(\mathrm{~m}, 3 \mathrm{H}), 3.47$ (ddd, $J$ $=9.7,7.3,2.5 \mathrm{~Hz}, 1 \mathrm{H}), 3.33(\mathrm{ddd}, J=9.2,6.8,2.0 \mathrm{~Hz}, 1 \mathrm{H}), 3.23(\mathrm{~s}, 3 \mathrm{H}), 1.99-1.90$ (m, 1H), $1.83-1.62(\mathrm{~m}, 5 \mathrm{H}), 1.58-1.49(\mathrm{~m}, 2 \mathrm{H}), 1.40-1.25(\mathrm{~m}, 4 \mathrm{H})$.

${ }^{13}$ C NMR (101 MHz, CDCl3) $\delta 135.0,130.3,128.8,127.2,85.4,56.7,50.3,29.5$, 29.1, 26.7, 26.3, 25.6, 24.8.

IR (film): $v\left(\mathrm{~cm}^{-1}\right)$ 2929, 2855, 1461, 1277, 1066, 771, 696.

HRMS (ESI, m/z) calcd for $\mathrm{C}_{15} \mathrm{H}_{22} \mathrm{NaOSe}(\mathrm{M}+\mathrm{Na})^{+}: 321.0728$, found: 321.0742 .

((1S,2S)-2-methoxy-2-methylcyclopentyl)(phenyl)selane (5k)

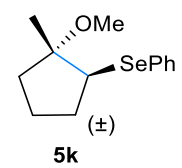

A dried $10 \mathrm{~mL}$ Schlenk tube was charged with 1k $(21 \mu \mathrm{L}, 16.4 \mathrm{mg}, 0.20 \mathrm{mmol}), 3 \mathbf{a}$ (31.2 mg, $0.10 \mathrm{mmol})$, [Os-Cu] (10.9 mg, $0.01 \mathrm{mmol}), 2 \mathrm{a}(0.5 \mathrm{~mL})$, and $\mathrm{CH}_{2} \mathrm{Cl}_{2}(3.5$ $\mathrm{mL}$ ). The Schlenk tube was positioned approximately $3 \mathrm{~cm}$ away from a $24 \mathrm{~W}$ blue LEDs lamp $\left(\lambda_{\max }=450 \mathrm{~nm}\right)$. After being stirred at $25^{\circ} \mathrm{C}$ for the $48 \mathrm{~h}$ under an $\mathrm{O}_{2}$ (balloon) atmosphere, the reaction mixture was concentrated to dryness. The residue was purified by flash chromatography (petroleum ether/ethyl acetate $=50: 1$ ) to afford product 5k as a yellow oil (46.4 mg, $0.172 \mathrm{mmol}$, yield: 86\%).

${ }^{1}$ H NMR (500 MHz, CDCl $) \delta 7.61-7.54(\mathrm{~m}, 2 \mathrm{H}), 7.26-7.23(\mathrm{~m}, 3 \mathrm{H}), 3.67(\mathrm{t}, J=$ $7.3 \mathrm{~Hz}, 1 \mathrm{H}), 3.22$ (s, 3H), 2.27 (tdd, $J=7.2,6.2,4.1 \mathrm{~Hz}, 1 \mathrm{H}), 1.87$ (dt, $J=14.4,5.6$ $\mathrm{Hz}, 1 \mathrm{H}), 1.80-1.66(\mathrm{~m}, 4 \mathrm{H}), 1.35(\mathrm{~s}, 3 \mathrm{H})$.

${ }^{13}$ C NMR (126 MHz, CDCl 3$) \delta 133.7,130.6,128.9,127.0,85.9,51.7,50.3,35.9$, $32.8,21.5,20.8$.

IR (film): $v\left(\mathrm{~cm}^{-1}\right)$ 2970, 1465, 1393, 1251, 1066, 747, 709.

HRMS (ESI, m/z) calcd for $\mathrm{C}_{13} \mathrm{H}_{18} \mathrm{NaOSe}(\mathrm{M}+\mathrm{Na})^{+}: 293.0415$, found: 293.0484 . 


\section{(3-ethyl-3-methoxypentan-2-yl)(phenyl)selane (5l)}

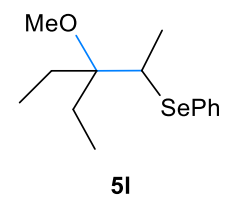

A dried $10 \mathrm{~mL}$ Schlenk tube was charged with 11 (27 $\mu \mathrm{L}, 19.6 \mathrm{mg}, 0.20 \mathrm{mmol}), 3 \mathrm{a}$ (31.2 mg, $0.10 \mathrm{mmol})$, [Os-Cu] (10.9 mg, $0.01 \mathrm{mmol}), 2 \mathrm{a}(0.5 \mathrm{~mL})$, and $\mathrm{CH}_{2} \mathrm{Cl}_{2}(3.5$ $\mathrm{mL}$ ). The Schlenk tube was positioned approximately $3 \mathrm{~cm}$ away from a $24 \mathrm{~W}$ blue LEDs lamp $\left(\lambda_{\max }=450 \mathrm{~nm}\right)$. After being stirred at $25^{\circ} \mathrm{C}$ for the $48 \mathrm{~h}$ under an $\mathrm{O}_{2}$ (balloon) atmosphere, the reaction mixture was concentrated to dryness. The residue was purified by flash chromatography (petroleum ether/ethyl acetate $=50: 1$ ) to afford product $\mathbf{5 l}$ as a yellow oil (37.1 $\mathrm{mg}, 0.130 \mathrm{mmol}$, yield: $65 \%)$.

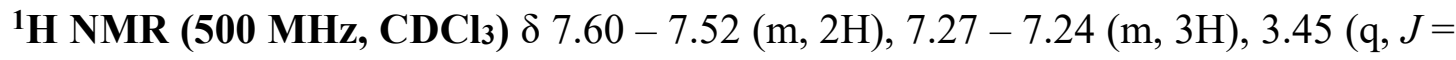
$7.1 \mathrm{~Hz}, 1 \mathrm{H}), 3.25(\mathrm{~s}, 3 \mathrm{H}), 1.80-1.70(\mathrm{~m}, 4 \mathrm{H}), 1.49$ (d, $J=7.1 \mathrm{~Hz}, 3 \mathrm{H}), 0.92$ (q, $J=$ $7.4 \mathrm{~Hz}, 6 \mathrm{H})$.

${ }^{13}$ C NMR (126 MHz, CDCl 3$) \delta 134.4,131.1,129.0,127.2,80.6,50.0,48.0,27.0$, $26.4,18.5,8.7,8.5$.

IR (film): $v\left(\mathrm{~cm}^{-1}\right)$ 2975, 2854, 1471, 1385, 1243, 1066. 765, 710.

HRMS (ESI, m/z) calcd for $\mathrm{C}_{28} \mathrm{H}_{44} \mathrm{NaO}_{2} \mathrm{Se}_{2}(2 \mathrm{M}+\mathrm{Na})^{+}:$595.1564, found: 595.1596.

\section{(2-methoxy-2-methylhexan-3-yl)(phenyl)selane (5m)}

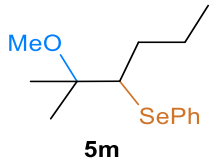

A dried $10 \mathrm{~mL}$ Schlenk tube was charged with 1m $(27 \mu \mathrm{L}, 19.6 \mathrm{mg}, 0.20 \mathrm{mmol}), 3 \mathbf{a}$ (31.2 mg, $0.10 \mathrm{mmol})$, [Os-Cu] (10.9 mg, $0.01 \mathrm{mmol}), 2 \mathrm{a}(0.5 \mathrm{~mL})$, and $\mathrm{CH}_{2} \mathrm{Cl}_{2}(3.5$ $\mathrm{mL}$ ). The Schlenk tube was positioned approximately $3 \mathrm{~cm}$ away from a $24 \mathrm{~W}$ blue LEDs lamp $\left(\lambda_{\max }=450 \mathrm{~nm}\right)$. After being stirred at $25{ }^{\circ} \mathrm{C}$ for the $48 \mathrm{~h}$ under an $\mathrm{O}_{2}$ (balloon) atmosphere, the reaction mixture was concentrated to dryness. The residue was purified by flash chromatography (petroleum ether/ethyl acetate $=50: 1$ ) to afford product $5 \mathrm{~m}$ as a yellow oil (36.1 $\mathrm{mg}, 0.126 \mathrm{mmol}$, yield: $63 \%)$. 
${ }^{1} \mathbf{H}$ NMR (500 MHz, $\left.\mathbf{C D C l}_{3}\right) \delta 7.63-7.53(\mathrm{~m}, 2 \mathrm{H}), 7.24(\mathrm{ddd}, J=8.9,5.7,3.9 \mathrm{~Hz}$, $3 \mathrm{H}), 3.15(\mathrm{~s}, 3 \mathrm{H}), 3.14-3.09(\mathrm{~m}, 1 \mathrm{H}), 1.99-1.89(\mathrm{~m}, 1 \mathrm{H}), 1.85-1.75(\mathrm{~m}, 1 \mathrm{H}), 1.51$ -1.37 (m, 2H), 1.31 (d, $J=8.3 \mathrm{~Hz}, 3 \mathrm{H}), 1.27$ (s, 3H), 0.90 (t, $J=7.2 \mathrm{~Hz}, 3 \mathrm{H})$.

${ }^{13}$ C NMR (126 MHz, CDCl 3$) \delta 134.0,132.1,128.9,127.0,78.4,57.6,49.4,33.5$, 24.1, 23.0, 22.1, 14.0.

IR (film): $v\left(\mathrm{~cm}^{-1}\right)$ 2987, 2927, 1465, 1380, 1251, 1066. 765, 710.

HRMS (ESI, m/z) calcd for $\mathrm{C}_{14} \mathrm{H}_{23} \mathrm{OSe}(\mathrm{M}+\mathrm{H})^{+}:$287.0909, found: 287.0918 .

\section{(2-methoxy-2-methyl-6-phenylhexan-3-yl)(phenyl)selane (5n)}

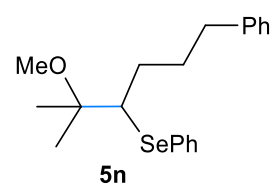

A dried $10 \mathrm{~mL}$ Schlenk tube was charged with 1n (34.6 mg,0.20 mmol), 3a (31.2 mg, $0.10 \mathrm{mmol}),[\mathrm{Os}-\mathrm{Cu}](10.9 \mathrm{mg}, 0.01 \mathrm{mmol}), \mathbf{2 a}(0.5 \mathrm{~mL})$, and $\mathrm{CH}_{2} \mathrm{Cl}_{2}(3.5 \mathrm{~mL})$. The Schlenk tube was positioned approximately $3 \mathrm{~cm}$ away from a $24 \mathrm{~W}$ blue LEDs lamp $\left(\lambda_{\max }=450 \mathrm{~nm}\right)$. After being stirred at $25{ }^{\circ} \mathrm{C}$ for the $48 \mathrm{~h}$ under an $\mathrm{O}_{2}$ (balloon) atmosphere, the reaction mixture was concentrated to dryness. The residue was purified by flash chromatography (petroleum ether/ethyl acetate $=50: 1$ ) to afford product $5 \mathbf{n}$ as a colorless oil (52.9 $\mathrm{mg}, 0.146 \mathrm{mmol}$, yield: $73 \%$ ).

${ }^{1}$ H NMR (500 MHz, CDCl 3$) \delta 7.61-7.55(\mathrm{~m}, 2 \mathrm{H}), 7.28-7.22(\mathrm{~m}, 5 \mathrm{H}), 7.20-7.10$ $(\mathrm{m}, 3 \mathrm{H}), 3.14(\mathrm{~s}, 3 \mathrm{H}), 3.13(\mathrm{~d}, J=2.2 \mathrm{~Hz}, 1 \mathrm{H}), 2.66-2.53(\mathrm{~m}, 2 \mathrm{H}), 2.18-2.08(\mathrm{~m}$, 1H), $2.08-1.99(\mathrm{~m}, 1 \mathrm{H}), 1.81-1.68(\mathrm{~m}, 1 \mathrm{H}), 1.58-1.50(\mathrm{~m}, 1 \mathrm{H}), 1.31(\mathrm{~s}, 3 \mathrm{H}), 1.26$ $(\mathrm{s}, 3 \mathrm{H})$.

${ }^{13}$ C NMR (126 MHz, CDCl 3$) \delta 142.4,134.1,131.9,129.0,128.4,128.3,127.1,125.7$, $78.5,57.7,49.4,35.8,31.0,30.6,24.0,23.0$.

IR (film): $v\left(\mathrm{~cm}^{-1}\right)$ 2988, 2925, 1467, 1390, 1251, 1066. 901, 874, 755, 698 .

HRMS (ESI, m/z) calcd for $\mathrm{C}_{20} \mathrm{H}_{26} \mathrm{NaOSe}(\mathrm{M}+\mathrm{Na})^{+}: 385.1041$, found: 385.1040 . 


\section{(4-methoxy-4-methyl-1-phenylpentan-3-yl)(phenyl)selane (5o)}

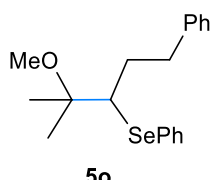

A dried $10 \mathrm{~mL}$ Schlenk tube was charged with $10(32 \mathrm{mg}, 0.20 \mathrm{mmol}), 3 \mathbf{a}(31.2 \mathrm{mg}$, $0.10 \mathrm{mmol})$, [Os-Cu] (10.9 mg, $0.01 \mathrm{mmol}), \mathbf{2 a}(0.5 \mathrm{~mL})$, and $\mathrm{CH}_{2} \mathrm{Cl}_{2}(3.5 \mathrm{~mL})$. The Schlenk tube was positioned approximately $3 \mathrm{~cm}$ away from a $24 \mathrm{~W}$ blue LEDs lamp $\left(\lambda_{\max }=450 \mathrm{~nm}\right)$. After being stirred at $25{ }^{\circ} \mathrm{C}$ for the $48 \mathrm{~h}$ under an $\mathrm{O}_{2}$ (balloon) atmosphere, the reaction mixture was concentrated to dryness. The residue was purified by flash chromatography (petroleum ether/ethyl acetate $=50: 1$ ) to afford product 50 as a colorless oil (49.4 mg, $0.142 \mathrm{mmol}$, yield: 71\%).

${ }^{1} \mathrm{H}$ NMR $\left(500 \mathrm{MHz}, \mathrm{CDCl}_{3}\right) \delta 7.66-7.60(\mathrm{~m}, 2 \mathrm{H}), 7.29(\mathrm{td}, J=4.4,2.1 \mathrm{~Hz}, 5 \mathrm{H})$, $7.20(\mathrm{t}, J=7.4 \mathrm{~Hz}, 1 \mathrm{H}), 7.15(\mathrm{~d}, J=7.1 \mathrm{~Hz}, 2 \mathrm{H}), 3.21-3.02(\mathrm{~m}, 5 \mathrm{H}), 2.72(\mathrm{ddd}, J=$ 13.6, 9.5, 7.4 Hz, 1H), 2.36 (dddd, $J=14.5,9.7,7.3,2.3 \mathrm{~Hz}, 1 \mathrm{H}), 1.90-1.78(\mathrm{~m}, 1 \mathrm{H})$, $1.34(\mathrm{~s}, 3 \mathrm{H}), 1.29(\mathrm{~s}, 3 \mathrm{H})$.

${ }^{13}$ C NMR (126 MHz, CDCl $) \delta$ 142.0, 134.0, 131.8, 129.0, 128.7, 128.3, 127.1, 125.8, $78.4,56.6,49.3,34.8,33.2,24.0,23.1$.

IR (film): $v\left(\mathrm{~cm}^{-1}\right)$ 2978, 2859, 1460, 1387, 1241, 1067, 750, 710.

HRMS (ESI, m/z) calcd for $\mathrm{C}_{19} \mathrm{H}_{24} \mathrm{NaOSe}(\mathrm{M}+\mathrm{Na})^{+}: 371.0890$, found: 371.0902 .

\section{3-methoxy-3-methylheptan-4-yl)(phenyl)selane (5p)}

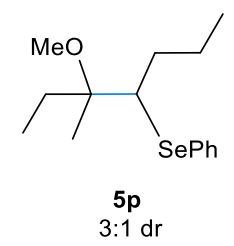

A dried $10 \mathrm{~mL}$ Schlenk tube was charged with $1 p(28 \mu \mathrm{L}, 19.6 \mathrm{mg}, 0.20 \mathrm{mmol}),(31.2$ mg, $0.10 \mathrm{mmol})$, [Os-Cu] (10.9 mg, $0.01 \mathrm{mmol}), \mathbf{2 a}(0.5 \mathrm{~mL})$, and $\mathrm{CH}_{2} \mathrm{Cl}_{2}(3.5 \mathrm{~mL})$. The Schlenk tube was positioned approximately $3 \mathrm{~cm}$ away from a $24 \mathrm{~W}$ blue LEDs lamp $\left(\lambda_{\max }=450 \mathrm{~nm}\right)$. After being stirred at $25{ }^{\circ} \mathrm{C}$ for the $48 \mathrm{~h}$ under an $\mathrm{O}_{2}$ (balloon) atmosphere, the reaction mixture was concentrated to dryness. The residue was 
purified by flash chromatography (petroleum ether/ethyl acetate $=50: 1$ ) to afford product 5p as a yellow oil (40.2 $\mathrm{mg}, 0.134 \mathrm{mmol}$, yield: 67\%).

${ }^{1} \mathbf{H}$ NMR (500 MHz, $\left.\mathbf{C D C l}_{3}\right) \delta 7.63-7.56(\mathrm{~m}, 2 \mathrm{H}), 7.51-7.36(\mathrm{~m}, 1 \mathrm{H}), 7.24(\mathrm{dd}, J$ $=4.9,1.9 \mathrm{~Hz}, 2 \mathrm{H}), 3.48-3.15(\mathrm{~m}, 1 \mathrm{H}), 3.14-3.08(\mathrm{~m}, 3 \mathrm{H}), 1.99-1.60(\mathrm{~m}, 4 \mathrm{H})$, $1.54-1.35(\mathrm{~m}, 2 \mathrm{H}), 1.22(\mathrm{~d}, J=30.8 \mathrm{~Hz}, 3 \mathrm{H}), 0.94-0.72(\mathrm{~m}, 6 \mathrm{H})$.

${ }^{13}$ C NMR (126 MHz, CDCl 3$) \delta 134.0$ (2C), 132.2, 131.9, 128.9 (2C), 127.0 (2C), $79.8,56.6,56.0,49.0,33.8,33.2,28.4,28.2,22.1,20.7,19.9,14.0,7.8$.

IR (film): $v\left(\mathrm{~cm}^{-1}\right)$ 2974, 2925, 2870, 1465, 1382, 1265, 1050, 777, 696.

HRMS (ESI, m/z) calcd for $\mathrm{C}_{15} \mathrm{H}_{24} \mathrm{NaOSe}(\mathrm{M}+\mathrm{Na})^{+}: 323.0885$, found: 323.0894 .

\section{3-methoxy-3-methyl-2-(phenylselanyl)butyl benzoate (5q)}

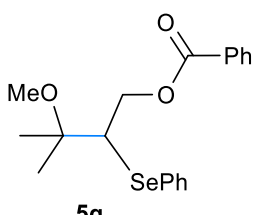

$5 q$

A dried $10 \mathrm{~mL}$ Schlenk tube was charged with $1 \mathbf{q}(38 \mathrm{mg}, 0.20 \mathrm{mmol}), 3 \mathbf{3 a}(31.2 \mathrm{mg}$, $0.10 \mathrm{mmol})$, [Os-Cu] (10.9 mg, $0.01 \mathrm{mmol}), \mathbf{2 a}(0.5 \mathrm{~mL})$, and $\mathrm{CH}_{2} \mathrm{Cl}_{2}(3.5 \mathrm{~mL})$. The Schlenk tube was positioned approximately $3 \mathrm{~cm}$ away from a $24 \mathrm{~W}$ blue LEDs lamp $\left(\lambda_{\max }=450 \mathrm{~nm}\right)$. After being stirred at $25^{\circ} \mathrm{C}$ for the $60 \mathrm{~h}$ under an $\mathrm{O}_{2}$ (balloon) atmosphere, the reaction mixture was concentrated to dryness. The residue was purified by flash chromatography (petroleum ether/ethyl acetate $=20: 1$ ) to afford product 5q as a yellow oil (24.2 $\mathrm{mg}, 0.064 \mathrm{mmol}$, yield: 32\%).

${ }^{1}$ H NMR (500 MHz, CDCl 3$) \delta 7.88(\mathrm{dd}, J=8.3,1.2 \mathrm{~Hz}, 2 \mathrm{H}), 7.64-7.57(\mathrm{~m}, 2 \mathrm{H})$, $7.55-7.50(\mathrm{~m}, 1 \mathrm{H}), 7.38(\mathrm{t}, J=7.8 \mathrm{~Hz}, 2 \mathrm{H}), 7.24-7.17$ (m, 3H), 4.84 (dd, $J=11.8$, $5.5 \mathrm{~Hz}, 1 \mathrm{H}), 4.60(\mathrm{dd}, J=11.8,6.9 \mathrm{~Hz}, 1 \mathrm{H}), 3.61(\mathrm{dd}, J=6.8,5.6 \mathrm{~Hz}, 1 \mathrm{H}), 3.26(\mathrm{~s}$, $3 \mathrm{H}), 1.45$ (s, 3H), $1.42(\mathrm{~s}, 3 \mathrm{H})$.

${ }^{13}$ C NMR (126 MHz, CDCl3) $\delta$ 166.4, 134.3, 132.9, 130.3, 130.1, 129.6, 129.1, 128.3, $127.4,66.0,54.5,49.6,29.7,24.2,24.1$.

IR (film): $v\left(\mathrm{~cm}^{-1}\right)$ 2988, 2853, 1731, 1465, 1382, 1269, 1066, 769, 710.

HRMS (ESI, m/z) calcd for $\mathrm{C}_{19} \mathrm{H}_{22} \mathrm{NaO}_{3} \mathrm{Se}(\mathrm{M}+\mathrm{Na})^{+}$: 401.0626 , found: 401.0628 . 


\section{3-methoxy-3-methyl-2-(phenylselanyl)butyl 4-methoxybenzoate (5r)}

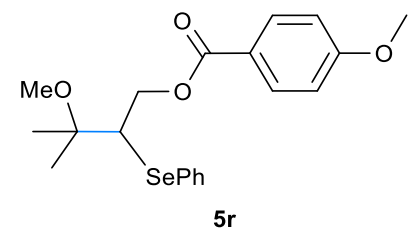

A dried $10 \mathrm{~mL}$ Schlenk tube was charged with 1r (44 mg, $0.20 \mathrm{mmol}), 3 \mathbf{a}$ (31.2 mg, $0.10 \mathrm{mmol})$, [Os-Cu] (10.9 mg, $0.01 \mathrm{mmol}), \mathbf{2 a}(0.5 \mathrm{~mL})$, and $\mathrm{CH}_{2} \mathrm{Cl}_{2}(3.5 \mathrm{~mL})$. The Schlenk tube was positioned approximately $3 \mathrm{~cm}$ away from a $24 \mathrm{~W}$ blue LEDs lamp $\left(\lambda_{\max }=450 \mathrm{~nm}\right)$. After being stirred at $25{ }^{\circ} \mathrm{C}$ for the $48 \mathrm{~h}$ under an $\mathrm{O}_{2}$ (balloon) atmosphere, the reaction mixture was concentrated to dryness. The residue was purified by flash chromatography (petroleum ether/ethyl acetate $=20: 1$ ) to afford product $\mathbf{5 r}$ as a yellow oil (48.9 $\mathrm{mg}, 0.120 \mathrm{mmol}$, yield: $60 \%)$.

${ }^{1} \mathbf{H}$ NMR (500 MHz, CDCl $) \delta 7.90-7.77$ (m, 2H), $7.69-7.53(\mathrm{~m}, 2 \mathrm{H}), 7.26-7.14$ $(\mathrm{m}, 3 \mathrm{H}), 6.89-6.83(\mathrm{~m}, 2 \mathrm{H}), 4.80(\mathrm{dd}, J=11.8,5.5 \mathrm{~Hz}, 1 \mathrm{H}), 4.57(\mathrm{dd}, J=11.8,6.9$ $\mathrm{Hz}, 1 \mathrm{H}), 3.85$ (s, 3H), $3.60(\mathrm{dd}, J=6.7,5.6 \mathrm{~Hz}, 1 \mathrm{H}), 3.25(\mathrm{~s}, 3 \mathrm{H}), 1.44(\mathrm{~s}, 3 \mathrm{H}), 1.41(\mathrm{~s}$, $3 \mathrm{H})$.

${ }^{13}$ C NMR (126 MHz, CDCl 3$) \delta 166.1,163.3,134.3,131.7,130.4,129.1,127.4,122.6$, $113.5,65.7,55.4,54.5,49.6,24.2,24.1$.

IR (film): $v\left(\mathrm{~cm}^{-1}\right)$ 2978, 2900, 1731, 1654, 1386, 1271, 1063, 769, 692.

HRMS (ESI, m/z) calcd for $\mathrm{C}_{19} \mathrm{H}_{22} \mathrm{NaO}_{3} \mathrm{Se}(\mathrm{M}+\mathrm{Na})^{+}: 431.0732$, found: 431.0733 .

\section{3-methoxy-3-methyl-2-(phenylselanyl)butyl 4-(trifluoromethyl)benzoate (5s)}

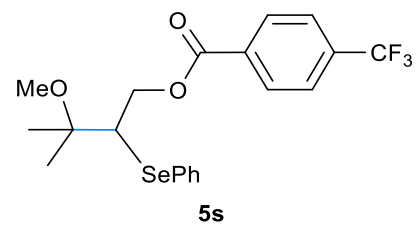

A dried $10 \mathrm{~mL}$ Schlenk tube was charged with $1 \mathrm{~s}(51.6 \mathrm{mg}, 0.20 \mathrm{mmol}), 3 \mathbf{3}(31.2 \mathrm{mg}$, $0.10 \mathrm{mmol})$, [Os-Cu] (10.9 mg, $0.01 \mathrm{mmol}), \mathbf{2 a}(0.5 \mathrm{~mL})$, and $\mathrm{CH}_{2} \mathrm{Cl}_{2}(3.5 \mathrm{~mL})$. The Schlenk tube was positioned approximately $3 \mathrm{~cm}$ away from a $36 \mathrm{~W}$ blue LEDs lamp $\left(\lambda_{\max }=450 \mathrm{~nm}\right)$. After being stirred at $50{ }^{\circ} \mathrm{C}$ for the $48 \mathrm{~h}$ under an $\mathrm{O}_{2}$ (balloon) atmosphere, the reaction mixture was concentrated to dryness. The residue was 
purified by flash chromatography (petroleum ether/ethyl acetate $=20: 1$ ) to afford product $\mathbf{5 s}$ as a white solid (34.8 $\mathrm{mg}, 0.078 \mathrm{mmol}$, yield: $39 \%)$.

${ }^{1}$ H NMR (400 MHz, CDCl 3$) \delta 7.94(\mathrm{~d}, J=8.2 \mathrm{~Hz}, 2 \mathrm{H}), 7.63(\mathrm{~d}, J=8.3 \mathrm{~Hz}, 2 \mathrm{H})$, $7.60-7.46(\mathrm{~m}, 2 \mathrm{H}), 7.25-7.10(\mathrm{~m}, 3 \mathrm{H}), 4.89(\mathrm{dd}, J=11.8,5.2 \mathrm{~Hz}, 1 \mathrm{H}), 4.64(\mathrm{dd}, J$ $=11.8,7.3 \mathrm{~Hz}, 1 \mathrm{H}), 3.62(\mathrm{dd}, J=7.2,5.2 \mathrm{~Hz}, 1 \mathrm{H}), 3.26(\mathrm{~s}, 3 \mathrm{H}), 1.46(\mathrm{~s}, 3 \mathrm{H}), 1.42(\mathrm{~s}$, $3 \mathrm{H})$.

${ }^{13}$ C NMR (101 MHz, CDCl $) \delta 165.1,134.3(\mathrm{q}, J=32.6 \mathrm{~Hz}) 134.1,133.3$ (q, $J=3.7$ $\mathrm{Hz}), 130.3,130.0,129.1,127.4,126.4$ (q, $J=275.0 \mathrm{~Hz}), 125.3$ (q, $J=3.8 \mathrm{~Hz}), 76.9$, $66.6,54.3,49.7,24.2,24.0$.

IR (film): v (cm-1) 2978, 2900, 1741, 1468, 1380, 1271, 1155, 1091, 1063, 781, 672.

HRMS (ESI, m/z) calcd for $\mathrm{C}_{20} \mathrm{H}_{21} \mathrm{~F}_{3} \mathrm{NaO}_{3} \mathrm{Se}(\mathrm{M}+\mathrm{Na})^{+}: 469.0500$, found: 469.0497 .

\section{(2-ethyl-2-methoxybutyl)(phenyl)selane (5t)}

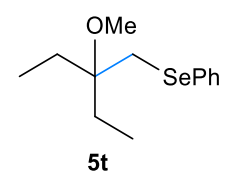

A dried $10 \mathrm{~mL}$ Schlenk tube was charged with $\mathbf{1 t}(24 \mu \mathrm{L}, 16.8 \mathrm{mg}, 0.20 \mathrm{mmol}), 3 \mathbf{a}$ (31.2 mg, $0.10 \mathrm{mmol})$, [Os-Cu] (10.9 mg, $0.01 \mathrm{mmol}), \mathbf{2 a}(0.5 \mathrm{~mL})$, and $\mathrm{CH}_{2} \mathrm{Cl}_{2}(3.5$ $\mathrm{mL}$ ). The Schlenk tube was positioned approximately $3 \mathrm{~cm}$ away from a $24 \mathrm{~W}$ blue LEDs lamp $\left(\lambda_{\max }=450 \mathrm{~nm}\right)$. After being stirred at $25^{\circ} \mathrm{C}$ for the $48 \mathrm{~h}$ under an $\mathrm{O}_{2}$ (balloon) atmosphere, the reaction mixture was concentrated to dryness. The residue was purified by flash chromatography (petroleum ether/ethyl acetate $=50: 1$ ) to afford product $5 \mathrm{t}$ as a yellow oil (34.3 $\mathrm{mg}, 0.126 \mathrm{mmol}$, yield: 63\%).

${ }^{1}$ H NMR (500 MHz, CDCl 3$) \delta 7.58-7.49(\mathrm{~m}, 2 \mathrm{H}), 7.26-7.22(\mathrm{~m}, 3 \mathrm{H}), 3.16(\mathrm{~s}, 3 \mathrm{H})$, $3.09(\mathrm{~s}, 2 \mathrm{H}), 1.62(\mathrm{q}, J=7.5 \mathrm{~Hz}, 4 \mathrm{H}), 0.82(\mathrm{t}, J=7.5 \mathrm{~Hz}, 6 \mathrm{H})$.

${ }^{13}$ C NMR (126 MHz, CDCl 3$) \delta 132.9,131.09,129.0,126.7,79.0,49.0,34.3,26.6$, 7.7 .

IR (film): $v\left(\mathrm{~cm}^{-1}\right)$ 2978, 2922, 1467, 1383, 1273, 1065, 744, 700.

HRMS (ESI, m/z) calcd forC ${ }_{13} \mathrm{H}_{20} \mathrm{NaOSe}(\mathrm{M}+\mathrm{Na})^{+}:$295.0577, found: 295.0572. 


\section{(2-methoxy-2-methylpentyl)(phenyl)selane $(5 u)^{16}$}

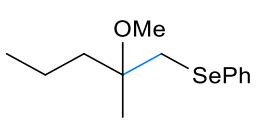

$5 \mathbf{u}$

A dried $10 \mathrm{~mL}$ Schlenk tube was charged with $1 \mathbf{u}(24 \mu \mathrm{L}, 16.8 \mathrm{mg}, 0.20 \mathrm{mmol}), 3 \mathbf{a}$ (31.2 mg, $0.10 \mathrm{mmol})$, [Os-Cu] (10.9 mg, $0.01 \mathrm{mmol}), \mathbf{2 a}(0.5 \mathrm{~mL})$, and $\mathrm{CH}_{2} \mathrm{Cl}_{2}(3.5$ $\mathrm{mL}$ ). The Schlenk tube was positioned approximately $3 \mathrm{~cm}$ away from a $24 \mathrm{~W}$ blue LEDs lamp $\left(\lambda_{\max }=450 \mathrm{~nm}\right)$. After being stirred at $25^{\circ} \mathrm{C}$ for the $48 \mathrm{~h}$ under an $\mathrm{O}_{2}$ (balloon) atmosphere, the reaction mixture was concentrated to dryness. The residue was purified by flash chromatography (petroleum ether/ethyl acetate $=50: 1$ ) to afford product $5 \mathbf{u}$ as a colorless oil (39.7 $\mathrm{mg}, 0.146 \mathrm{mmol}$, yield: $73 \%)$.

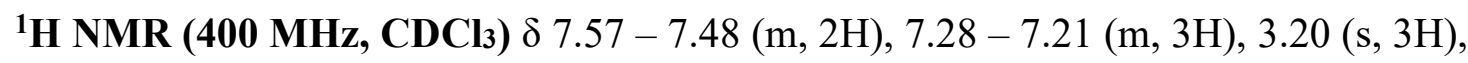
$3.17-3.08(\mathrm{~m}, 2 \mathrm{H}), 1.59(\mathrm{ddd}, J=8.5,5.4,2.4 \mathrm{~Hz}, 2 \mathrm{H}), 1.32(\mathrm{dd}, J=15.5,7.5 \mathrm{~Hz}$, 2H), $1.27(\mathrm{~s}, 3 \mathrm{H}), 0.91(\mathrm{t}, J=7.3 \mathrm{~Hz}, 3 \mathrm{H})$.

${ }^{13}$ C NMR (101 MHz, CDCl 3$) \delta 132.7,131.3,129.0,126.7,76.8,49.4,40.0,37.9$, $22.9,17.0,14.5$.

IR (film): $v\left(\mathrm{~cm}^{-1}\right)$ 2971, 2900, 1467, 1377, 1270, 1066, 774, 696.

HRMS (ESI, m/z) calcd for $\mathrm{C}_{13} \mathrm{H}_{20} \mathrm{NaOSe}(\mathrm{M}+\mathrm{Na})^{+}: 295.0577$, found: 295.0571 .

\section{(2-methoxy-2-methyl-3-phenylpropyl)(phenyl)selane (5v)}

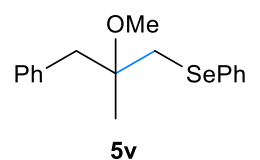

A dried $10 \mathrm{~mL}$ Schlenk tube was charged with $1 \mathbf{v}(28 \mu \mathrm{L}, 24.4 \mathrm{mg}, 0.20 \mathrm{mmol}), 3 \mathbf{a}$ (31.2 mg, $0.10 \mathrm{mmol})$, [Os-Cu] (10.9 mg, $0.01 \mathrm{mmol}), \mathbf{2 a}(0.5 \mathrm{~mL})$, and $\mathrm{CH}_{2} \mathrm{Cl}_{2}(3.5$ $\mathrm{mL}$ ). The Schlenk tube was positioned approximately $3 \mathrm{~cm}$ away from a $24 \mathrm{~W}$ blue LEDs lamp $\left(\lambda_{\max }=450 \mathrm{~nm}\right)$. After being stirred at $25^{\circ} \mathrm{C}$ for the $48 \mathrm{~h}$ under an $\mathrm{O}_{2}$ (balloon) atmosphere, the reaction mixture was concentrated to dryness. The residue was purified by flash chromatography (petroleum ether/ethyl acetate $=40: 1$ ) to afford product 5v as a yellow oil (46.1 mg, $0.144 \mathrm{mmol}$, yield: 72\%). 
${ }^{1} \mathbf{H}$ NMR (500 MHz, CDCl $) \delta 7.57(\mathrm{dt}, J=4.4,2.4 \mathrm{~Hz}, 2 \mathrm{H}), 7.35-7.24(\mathrm{~m}, 8 \mathrm{H})$, 3.37 (s, 3H), 3.16 (d, $J=3.8 \mathrm{~Hz}, 2 \mathrm{H}), 3.03(\mathrm{dd}, J=35.3,13.6 \mathrm{~Hz}, 2 \mathrm{H}), 1.31$ (s, 3H).

${ }^{13}$ C NMR (126 MHz, CDCl 3$) \delta 137.6,132.6,131.3,130.5,129.1,128.1,126.7,126.5$, 49.8, 44.0, 37.4, 22.6 .

IR (film): v (cm $\left.{ }^{-1}\right)$ 2978, 2869, 1465, 1380, 1273, 1066, 774, 699.

HRMS (ESI, m/z) calcd for $\mathrm{C}_{17} \mathrm{H}_{20} \mathrm{NaOSe}(\mathrm{M}+\mathrm{Na})^{+}: 343.0577$, found: 343.0561 .

((1-methoxycyclohexyl)methyl)(phenyl)selane $(5 \mathrm{w})^{17}$

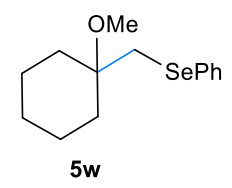

A dried $10 \mathrm{~mL}$ Schlenk tube was charged with $\mathbf{1 w}(24 \mu \mathrm{L}, 19.2 \mathrm{mg}, 0.20 \mathrm{mmol}), 3 \mathbf{a}$ (31.2 mg, $0.10 \mathrm{mmol})$, [Os-Cu] (10.9 mg, $0.01 \mathrm{mmol}), \mathbf{2 a}(0.5 \mathrm{~mL})$, and $\mathrm{CH}_{2} \mathrm{Cl}_{2}(3.5$ $\mathrm{mL}$ ). The Schlenk tube was positioned approximately $3 \mathrm{~cm}$ away from a $24 \mathrm{~W}$ blue LEDs lamp $\left(\lambda_{\max }=450 \mathrm{~nm}\right)$. After being stirred at $25^{\circ} \mathrm{C}$ for the $48 \mathrm{~h}$ under an $\mathrm{O}_{2}$ (balloon) atmosphere, the reaction mixture was concentrated to dryness. The residue was purified by flash chromatography (petroleum ether/ethyl acetate $=50: 1$ ) to afford product $5 \mathrm{w}$ as a yellow oil (37.5 $\mathrm{mg}, 0.132 \mathrm{mmol}$, yield: $66 \%)$.

${ }^{1}$ H NMR (500 MHz, CDCl 3$) \delta 7.56-7.49(\mathrm{~m}, 2 \mathrm{H}), 7.26-7.20(\mathrm{~m}, 3 \mathrm{H}), 3.18(\mathrm{~s}, 3 \mathrm{H})$, $3.13(\mathrm{~s}, 2 \mathrm{H}), 1.89-1.81(\mathrm{~m}, 2 \mathrm{H}), 1.56-1.51(\mathrm{~m}, 2 \mathrm{H}), 1.44(\mathrm{td}, J=12.2,3.7 \mathrm{~Hz}, 4 \mathrm{H})$, $1.33-1.15(\mathrm{~m}, 2 \mathrm{H})$.

${ }^{13}$ C NMR (126 MHz, CDCl 3$) \delta 132.7,131.3,129.0,126.6,75.1,48.6,37.2,34.2$, 25.7, 22.0 .

IR (film): $v\left(\mathrm{~cm}^{-1}\right)$ 2968, 2928, 2854, 1467, 1383, 1273, 1065, 774, 686.

HRMS (ESI, m/z) calcd for $\mathrm{C}_{14} \mathrm{H}_{20} \mathrm{OSe}(\mathrm{M})^{+}:$284.0674, found: 284.0665 .

\section{(2,3-dimethoxy-2-methylpropyl)(phenyl)selane (5x)}

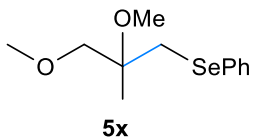


A dried $10 \mathrm{~mL}$ Schlenk tube was charged with 3-chloro-2-methylprop-1-ene (20 $\mu \mathrm{L}$, $18.1 \mathrm{mg}, 0.20 \mathrm{mmol}) \quad(0.20 \mathrm{mmol}), 3 \mathbf{a}(31.2 \mathrm{mg}, 0.10 \mathrm{mmol})$, [Os-Cu] (10.9 mg, $0.01 \mathrm{mmol}), 2 \mathrm{a}(0.5 \mathrm{~mL})$, and $\mathrm{CH}_{2} \mathrm{Cl}_{2}(3.5 \mathrm{~mL})$. The Schlenk tube was positioned approximately $3 \mathrm{~cm}$ away from a $24 \mathrm{~W}$ blue LEDs lamp $\left(\lambda_{\max }=450 \mathrm{~nm}\right)$. After being stirred at $25{ }^{\circ} \mathrm{C}$ for the $48 \mathrm{~h}$ under an $\mathrm{O}_{2}$ (balloon) atmosphere, the reaction mixture was concentrated to dryness. The residue was purified by flash chromatography (petroleum ether/ethyl acetate $=40: 1)$ to afford product $\mathbf{5 x}$ as a yellow oil (38.9 $\mathrm{mg}$, 0.142 mmol, yield: $71 \%$ ).

${ }^{1}$ H NMR (400 MHz, CDCl $) \delta 7.55(\mathrm{~d}, J=6.4 \mathrm{~Hz}, 2 \mathrm{H}), 7.40-7.16(\mathrm{~m}, 3 \mathrm{H}), 3.46(\mathrm{~d}$, $J=9.5 \mathrm{~Hz}, 1 \mathrm{H}), 3.36(\mathrm{~d}, J=9.6 \mathrm{~Hz}, 1 \mathrm{H}), 3.27(\mathrm{~d}, J=5.3 \mathrm{~Hz}, 6 \mathrm{H}), 3.19(\mathrm{t}, J=10.3$ $\mathrm{Hz}, 2 \mathrm{H}), 1.28(\mathrm{~s}, 3 \mathrm{H})$.

${ }^{13}$ C NMR (101 MHz, CDCl 3$) \delta 132.6,131.2,129.0,126.7,76.8,76.3,59.1,50.0$, $34.8,20.3$.

IR (film): $v\left(\mathrm{~cm}^{-1}\right)$ 2970, 2900, 1468, 1389, 1273, 1066, 746, 691.

HRMS (ESI, m/z) calcd for $\mathrm{C}_{12} \mathrm{H}_{18} \mathrm{NaO}_{2} \mathrm{Se}(\mathrm{M}+\mathrm{Na})^{+}: 297.0370$, found: 297.0361 .

\section{(2-methoxy-2-methyl-3-(naphthalen-2-yloxy)propyl)(phenyl)selane (5y)}

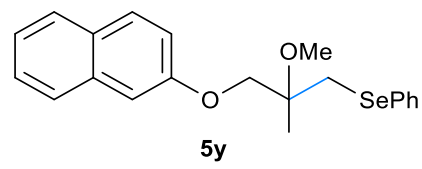

A dried $10 \mathrm{~mL}$ Schlenk tube was charged with 1y (39.6 mg, $0.20 \mathrm{mmol}), \mathbf{3 a}(31.2 \mathrm{mg}$, $0.10 \mathrm{mmol})$, [Os-Cu] (10.9 mg, $0.01 \mathrm{mmol}), \mathbf{2 a}(0.5 \mathrm{~mL})$, and $\mathrm{CH}_{2} \mathrm{Cl}_{2}(3.5 \mathrm{~mL})$. The Schlenk tube was positioned approximately $3 \mathrm{~cm}$ away from a $24 \mathrm{~W}$ blue LEDs lamp $\left(\lambda_{\max }=450 \mathrm{~nm}\right)$. After being stirred at $25{ }^{\circ} \mathrm{C}$ for the $48 \mathrm{~h}$ under an $\mathrm{O}_{2}$ (balloon) atmosphere, the reaction mixture was concentrated to dryness. The residue was purified by flash chromatography (petroleum ether/ethyl acetate $=40: 1$ ) to afford product 5y as a yellow oil (64.1 $\mathrm{mg}, 0.166 \mathrm{mmol}$, yield: $83 \%)$.

${ }^{1}$ H NMR (500 MHz, CDCl 3$) \delta 7.77(\mathrm{~d}, J=8.1 \mathrm{~Hz}, 1 \mathrm{H}), 7.72(\mathrm{dd}, J=8.5,3.4 \mathrm{~Hz}$, 2H), $7.55(\mathrm{~d}, J=6.4 \mathrm{~Hz}, 2 \mathrm{H}), 7.48-7.42(\mathrm{~m}, 1 \mathrm{H}), 7.35(\mathrm{t}, J=7.4 \mathrm{~Hz}, 1 \mathrm{H}), 7.23-$ 
$7.11(\mathrm{~m}, 4 \mathrm{H}), 7.10(\mathrm{~s}, 1 \mathrm{H}), 4.21(\mathrm{~d}, J=9.3 \mathrm{~Hz}, 1 \mathrm{H}), 4.05(\mathrm{~d}, J=9.3 \mathrm{~Hz}, 1 \mathrm{H}), 3.44-$ $3.32(\mathrm{~m}, 5 \mathrm{H}), 1.48(\mathrm{~s}, 3 \mathrm{H})$.

${ }^{13}$ C NMR (126 MHz, $\left.\mathbf{C D C l}_{3}\right) \delta$ 156.6, 134.5, 133.0, 130.8, 129.3, 129.1, 129.0, 128.0, $127.7,126.9,126.8,126.4,123.7,118.9,106.9,76.7,71.3,50.3,35.2,20.6$.

IR (film): $v\left(\mathrm{~cm}^{-1}\right)$ 2976 , 2921, 1460, 1389, 1274, 1066, 943, 878, 786.

HRMS (ESI, m/z) calcd for $\mathrm{C}_{21} \mathrm{H}_{22} \mathrm{NaO}_{2} \mathrm{Se}(\mathrm{M}+\mathrm{Na})^{+}$: 409.0683, found: 409.0678 .

\section{(2-methoxy-2-methyl-3-(phenylselanyl)propyl)(phenyl)sulfane (5z)}

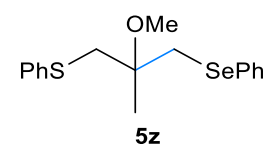

A dried $10 \mathrm{~mL}$ Schlenk tube was charged with $\mathbf{1 z}(32.8 \mathrm{mg}, 0.20 \mathrm{mmol}), \mathbf{3 a}$ (31.2 $\mathrm{mg}$, $0.10 \mathrm{mmol}),[\mathrm{Os}-\mathrm{Cu}](10.9 \mathrm{mg}, 0.01 \mathrm{mmol}), \mathbf{2 a}(0.5 \mathrm{~mL})$, and $\mathrm{CH}_{2} \mathrm{Cl}_{2}(3.5 \mathrm{~mL})$. The Schlenk tube was positioned approximately $3 \mathrm{~cm}$ away from a $24 \mathrm{~W}$ blue LEDs lamp $\left(\lambda_{\max }=450 \mathrm{~nm}\right)$. After being stirred at $25^{\circ} \mathrm{C}$ for the $48 \mathrm{~h}$ under an $\mathrm{O}_{2}$ (balloon) atmosphere, the reaction mixture was concentrated to dryness. The residue was purified by flash chromatography (petroleum ether/ethyl acetate $=40: 1$ ) to afford product $5 \mathbf{z}$ as a colorless oil (49.5 $\mathrm{mg}, 0.136 \mathrm{mmol}$, yield: $68 \%$ ).

${ }^{1}$ H NMR (500 MHz, CDCl 3$) \delta 7.56-7.47(\mathrm{~m}, 2 \mathrm{H}), 7.34(\mathrm{dt}, J=8.4,1.7 \mathrm{~Hz}, 2 \mathrm{H})$, 7.23 (ddt, $J=7.6,4.4,2.2 \mathrm{~Hz}, 5 \mathrm{H}), 7.18-7.10$ (m, 1H), $3.31-3.23$ (m, 4H), 3.21 (s, 1H), 1.37 (s, 3H).

${ }^{13}$ C NMR (126 MHz, CDCl 3$) \delta 137.0,132.9,130.8,129.6,129.1,128.9,127.0,126.1$, $77.6,50.1,42.5,37.0,22.5$.

IR (film): $v\left(\mathrm{~cm}^{-1}\right) 2978$, 2901, 1644, 1389, 1272, 1081, 1066, 768, 713.

HRMS (ESI, m/z) calcd for $\mathrm{C}_{17} \mathrm{H}_{20} \mathrm{NaOSSe}(\mathrm{M}+\mathrm{Na})^{+}: 375.0298$, found: 375.0299 . 


\section{2-methoxy-2-methyl-3-(phenylselanyl)propyl benzoate (5za)}

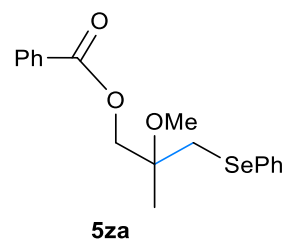

A dried $10 \mathrm{~mL}$ Schlenk tube was charged with 1 za $(35.2 \mathrm{mg}, 0.20 \mathrm{mmol}), 3 \mathbf{a}(31.2 \mathrm{mg}$, $0.10 \mathrm{mmol})$, [Os-Cu] $(10.9 \mathrm{mg}, 0.01 \mathrm{mmol}), \mathbf{2 a}(0.5 \mathrm{~mL})$, and $\mathrm{CH}_{2} \mathrm{Cl}_{2}(3.5 \mathrm{~mL})$. The Schlenk tube was positioned approximately $3 \mathrm{~cm}$ away from a $24 \mathrm{~W}$ blue LEDs lamp $\left(\lambda_{\max }=450 \mathrm{~nm}\right)$. After being stirred at $25{ }^{\circ} \mathrm{C}$ for the $48 \mathrm{~h}$ under an $\mathrm{O}_{2}$ (balloon) atmosphere, the reaction mixture was concentrated to dryness. The residue was purified by flash chromatography (petroleum ether/ethyl acetate $=20: 1$ ) to afford product 5za as a yellow oil (59.0 $\mathrm{mg}, 0.162 \mathrm{mmol}$, yield: $81 \%)$.

${ }^{1}$ H NMR (500 MHz, CDCl3) $\delta 7.97(\mathrm{~d}, J=8.0 \mathrm{~Hz}, 2 \mathrm{H}), 7.55$ (t, $\left.J=7.0 \mathrm{~Hz}, 3 \mathrm{H}\right), 7.42$ (t, $J=7.6 \mathrm{~Hz}, 2 \mathrm{H}), 7.18(\mathrm{~d}, J=5.3 \mathrm{~Hz}, 3 \mathrm{H}), 4.46(\mathrm{~d}, J=11.6 \mathrm{~Hz}, 1 \mathrm{H}), 4.36(\mathrm{~d}, J=$ $11.6 \mathrm{~Hz}, 1 \mathrm{H}), 3.30$ (s, 3H), $3.26(\mathrm{~s}, 2 \mathrm{H}), 1.38(\mathrm{~s}, 3 \mathrm{H})$.

${ }^{13}$ C NMR (126 MHz, CDCl $) \delta 166.1,133.1(2 \mathrm{C}), 130.7,129.9,129.7,129.1,128.3$, $127.0,76.3,67.1,50.3,35.5,20.5$.

IR (film): $v\left(\mathrm{~cm}^{-1}\right)$ 2980, 2859, 1746, 1463, 1378, 1270, 1066, 772, 699.

HRMS (ESI, m/z) calcd for $\mathrm{C}_{18} \mathrm{H}_{20} \mathrm{NaO}_{3} \mathrm{Se}(\mathrm{M}+\mathrm{Na})^{+}: 387.0475$, found: 387.0474 .

\section{2-methoxy-2-methyl-3-(phenylselanyl)propyl 1-naphthoate (5zb)}

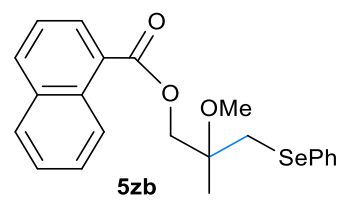

A dried $10 \mathrm{~mL}$ Schlenk tube was charged with 1zb $(45.2 \mathrm{mg}, 0.20 \mathrm{mmol}), 3 \mathbf{a}(31.2$ mg, $0.10 \mathrm{mmol}),[\mathbf{O s}-\mathbf{C u}$ (10.9 mg, $0.01 \mathrm{mmol}), \mathbf{2 a}(0.5 \mathrm{~mL})$, and $\mathrm{CH}_{2} \mathrm{Cl}_{2}(3.5 \mathrm{~mL})$. The Schlenk tube was positioned approximately $3 \mathrm{~cm}$ away from a $24 \mathrm{~W}$ blue LEDs lamp $\left(\lambda_{\max }=450 \mathrm{~nm}\right)$. After being stirred at $25{ }^{\circ} \mathrm{C}$ for the $48 \mathrm{~h}$ under an $\mathrm{O}_{2}$ (balloon) atmosphere, the reaction mixture was concentrated to dryness. The residue was 
purified by flash chromatography (petroleum ether/ethyl acetate $=20: 1$ ) to afford product 5zb as a yellow oil (69.6 mg, $0.168 \mathrm{mmol}$, yield: 84\%).

${ }^{1}$ H NMR (500 MHz, CDCl 3$) \delta 8.91(\mathrm{~d}, J=8.4 \mathrm{~Hz}, 1 \mathrm{H}), 8.08(\mathrm{dd}, J=7.2,1.2 \mathrm{~Hz}$, 1H), $8.02(\mathrm{~d}, J=8.2 \mathrm{~Hz}, 1 \mathrm{H}), 7.89(\mathrm{~d}, J=8.0 \mathrm{~Hz}, 1 \mathrm{H}), 7.62-7.52(\mathrm{~m}, 4 \mathrm{H}), 7.49-$ $7.45(\mathrm{~m}, 1 \mathrm{H}), 7.20-7.13(\mathrm{~m}, 3 \mathrm{H}), 4.56(\mathrm{~d}, J=11.6 \mathrm{~Hz}, 1 \mathrm{H}), 4.46(\mathrm{~d}, J=11.6 \mathrm{~Hz}$, 1H), 3.34 (s, 3H), $3.30(\mathrm{~s}, 2 \mathrm{H}), 1.43(\mathrm{~s}, 3 \mathrm{H})$.

${ }^{13}$ C NMR (126 MHz, CDCl $) \delta$ 167.0, 133.8, 133.5, 133.1, 131.4, 130.7, 130.3, 129.1, $128.5,127.8,127.1,126.8,126.3,125.8,124.5,76.4,67.1,50.3,35.5,20.5$.

IR (film): v (cm $\left.{ }^{-1}\right)$ 2988, 2900, 1755, 1470,1380, 1271, 1066, 941, 879, 772, 693.

HRMS (ESI, m/z) calcd for $\mathrm{C}_{22} \mathrm{H}_{22} \mathrm{NaO}_{3} \mathrm{Se}^{+}(\mathrm{M}+\mathrm{Na})^{+}: 437.0632$, found: 437.0631 .

\section{2-methoxy-2-methyl-3-(phenylselanyl)propyl 2-(p-tolyl)acetate (5zc)}

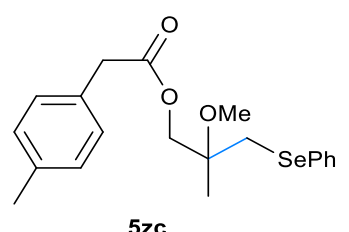

A dried $10 \mathrm{~mL}$ Schlenk tube was charged with $\mathbf{1 z c}(40.8 \mathrm{mg}, 0.20 \mathrm{mmol}), \mathbf{3 a}(31.2 \mathrm{mg}$, $0.10 \mathrm{mmol})$, [Os-Cu] $(10.9 \mathrm{mg}, 0.01 \mathrm{mmol}), \mathbf{2 a}(0.5 \mathrm{~mL})$, and $\mathrm{CH}_{2} \mathrm{Cl}_{2}(3.5 \mathrm{~mL})$. The Schlenk tube was positioned approximately $3 \mathrm{~cm}$ away from a $24 \mathrm{~W}$ blue LEDs lamp $\left(\lambda_{\max }=450 \mathrm{~nm}\right)$. After being stirred at $25{ }^{\circ} \mathrm{C}$ for the $48 \mathrm{~h}$ under an $\mathrm{O}_{2}$ (balloon) atmosphere, the reaction mixture was concentrated to dryness. The residue was purified by flash chromatography (petroleum ether/ethyl acetate $=20: 1$ ) to afford product 5zc as a yellow oil (61.9 $\mathrm{mg}, 0.158 \mathrm{mmol}$, yield: 79\%).

${ }^{1}$ H NMR (500 MHz, CDCl 3$) \delta 7.55-7.47(\mathrm{~m}, 2 \mathrm{H}), 7.24(\mathrm{dd}, J=4.9,1.7 \mathrm{~Hz}, 3 \mathrm{H})$, $7.11(\mathrm{q}, J=8.2 \mathrm{~Hz}, 4 \mathrm{H}), 4.22(\mathrm{~d}, J=11.6 \mathrm{~Hz}, 1 \mathrm{H}), 4.12(\mathrm{~d}, J=11.6 \mathrm{~Hz}, 1 \mathrm{H}), 3.53(\mathrm{~d}$, $J=2.8 \mathrm{~Hz}, 2 \mathrm{H}), 3.19(\mathrm{~s}, 3 \mathrm{H}), 3.09(\mathrm{~s}, 2 \mathrm{H}), 2.31(\mathrm{~s}, 3 \mathrm{H}), 1.23(\mathrm{~s}, 3 \mathrm{H})$.

${ }^{13}$ C NMR (126 MHz, CDCl3) $\delta$ 171.2, 136.7, 133.0, 130.8 (2C), 129.2, 129.1 (2C), $127.0,76.1,67.1,50.2,40.8,35.1,21.1,20.3$.

IR (film): $v\left(\mathrm{~cm}^{-1}\right)$ 2986, 2859, 1759, 1261, 1386, 1079, 1066, 811,753, 648.

HRMS (ESI, $\mathbf{m} / \mathbf{z})$ calcd for $\mathrm{C}_{20} \mathrm{H}_{24} \mathrm{NaO}_{3} \mathrm{Se}(\mathrm{M}+\mathrm{Na})^{+}: 415.0788$, found: 415.0787 


\section{(2-(4-fluorophenyl)-2-methoxyethyl)(phenyl)selane (5zd)}<smiles>COC(C[Sb]c1ccccc1)c1ccc(F)cc1</smiles>

A dried $10 \mathrm{~mL}$ Schlenk tube was charged with 1zd (24 $\mu \mathrm{L}, 24.4 \mathrm{mg}, 0.20 \mathrm{mmol}), 3 \mathbf{a}$ (31.2 mg, $0.10 \mathrm{mmol})$, [Os-Cu] (10.9 mg, $0.01 \mathrm{mmol}), 2 \mathrm{a}(0.5 \mathrm{~mL})$, and $\mathrm{CH}_{2} \mathrm{Cl}_{2}(3.5$ $\mathrm{mL}$ ). The Schlenk tube was positioned approximately $3 \mathrm{~cm}$ away from a $24 \mathrm{~W}$ blue LEDs lamp $\left(\lambda_{\max }=450 \mathrm{~nm}\right)$. After being stirred at $25^{\circ} \mathrm{C}$ for the $48 \mathrm{~h}$ under an $\mathrm{O}_{2}$ (balloon) atmosphere, the reaction mixture was concentrated to dryness. The residue was purified by flash chromatography (petroleum ether/ethyl acetate $=40: 1$ ) to afford product 5zd as a colorless oil (32.8 $\mathrm{mg}, 0.106 \mathrm{mmol}$, yield: 53\%).

${ }^{1}$ H NMR (400 MHz, CDCl $) \delta 7.41-7.30$ (m, 2H), 7.20 - 7.08 (m, 5H), $6.92(\mathrm{ddd}, J$ $=8.7,5.8,2.4 \mathrm{~Hz}, 2 \mathrm{H}), 4.23(\mathrm{dd}, J=7.9,5.5 \mathrm{~Hz}, 1 \mathrm{H}), 3.20(\mathrm{dd}, J=12.3,8.0 \mathrm{~Hz}, 1 \mathrm{H})$, $3.12(\mathrm{~s}, 3 \mathrm{H}), 2.97(\mathrm{dd}, J=12.3,5.5 \mathrm{~Hz}, 1 \mathrm{H})$.

${ }^{13}$ C NMR (101 MHz, CDCl $) \delta 162.5(\mathrm{~d}, J=246.1 \mathrm{~Hz}), 136.7(\mathrm{~d}, J=3.1 \mathrm{~Hz}), 132.7$, 130.5, 129.1, $128.4(\mathrm{~d}, J=8.1 \mathrm{~Hz}), 126.9,115.4(\mathrm{~d}, J=21.4 \mathrm{~Hz}), 82.6,57.0,35.3$.

IR (film): $v\left(\mathrm{~cm}^{-1}\right)$ 2970, 2866, 1460, 1385, 1260, 1135, 1066, 828, 736.

HRMS (ESI, m/z) calcd for $\mathrm{C}_{15} \mathrm{H}_{15} \mathrm{FNaOSe}(\mathrm{M}+\mathrm{Na})^{+}: 333.0170$, found: 333.0179 .

\section{phenyl(2,3,3-trimethoxypropyl)selane (5ze)}

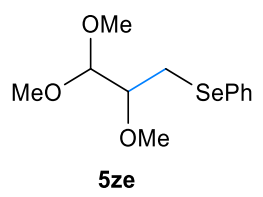

A dried $10 \mathrm{~mL}$ Schlenk tube was charged with 1ze ( $24 \mu \mathrm{L}, 20.4 \mathrm{mg}, 0.20 \mathrm{mmol}), 3 \mathbf{a}$ (31.2 mg, $0.10 \mathrm{mmol})$, [Os-Cu] (10.9 mg, $0.01 \mathrm{mmol}), 2 \mathrm{a}(0.5 \mathrm{~mL})$, and $\mathrm{CH}_{2} \mathrm{Cl}_{2}(3.5$ $\mathrm{mL}$ ). The Schlenk tube was positioned approximately $3 \mathrm{~cm}$ away from a $24 \mathrm{~W}$ blue LEDs lamp $\left(\lambda_{\max }=450 \mathrm{~nm}\right)$. After being stirred at $25^{\circ} \mathrm{C}$ for the $48 \mathrm{~h}$ under an $\mathrm{O}_{2}$ (balloon) atmosphere, the reaction mixture was concentrated to dryness. The residue 
was purified by flash chromatography (petroleum ether/ethyl acetate $=40: 1$ ) to afford product 5ze as a yellow oil (40.0 mg, $0.138 \mathrm{mmol}$, yield: 69\%).

${ }^{1}$ H NMR (500 MHz, CDCl 3$) \delta 7.68-7.60(\mathrm{~m}, 2 \mathrm{H}), 7.27(\mathrm{dd}, J=4.8,2.0 \mathrm{~Hz}, 3 \mathrm{H})$, $4.60(\mathrm{~d}, J=4.6 \mathrm{~Hz}, 1 \mathrm{H}), 3.65(\mathrm{qd}, J=10.0,6.0 \mathrm{~Hz}, 2 \mathrm{H}), 3.46(\mathrm{dd}, J=9.8,4.5 \mathrm{~Hz}$, $7 \mathrm{H}), 3.33(\mathrm{~s}, 3 \mathrm{H})$.

${ }^{13}$ C NMR (126 MHz, CDCl 3$) ~ \delta 134.6,129.4,128.9,127.5,105.4,72.5,58.8,56.2$, $55.6,47.7$.

IR (film): $v\left(\mathrm{~cm}^{-1}\right)$ 2942, 1465, 1135, 1066, 770, 696.

HRMS (ESI, m/z) calcd for $\mathrm{C}_{12} \mathrm{H}_{18} \mathrm{NaO}_{3} \mathrm{Se}(\mathrm{M}+\mathrm{Na})^{+}: 313.0313$, found: 313.0321 .

\section{(2-methoxy-2-(naphthalen-2-yl)ethyl)(phenyl)selane (5zf)}

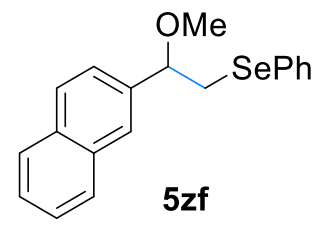

A dried $10 \mathrm{~mL}$ Schlenk tube was charged with $\mathbf{1 z f}(30.8 \mathrm{mg}, 0.20 \mathrm{mmol}), \mathbf{3 a}(31.2 \mathrm{mg}$, $0.10 \mathrm{mmol}),[\mathrm{Os}-\mathrm{Cu}](10.9 \mathrm{mg}, 0.01 \mathrm{mmol}), \mathbf{2 a}(0.5 \mathrm{~mL})$, and $\mathrm{CH}_{2} \mathrm{Cl}_{2}(3.5 \mathrm{~mL})$. The Schlenk tube was positioned approximately $3 \mathrm{~cm}$ away from a $24 \mathrm{~W}$ blue LEDs lamp $\left(\lambda_{\max }=450 \mathrm{~nm}\right)$. After being stirred at $25{ }^{\circ} \mathrm{C}$ for the $48 \mathrm{~h}$ under an $\mathrm{O}_{2}$ (balloon) atmosphere, the reaction mixture was concentrated to dryness. The residue was purified by flash chromatography (petroleum ether/ethyl acetate $=40: 1$ ) to afford product 5zf as a yellow oil (48.4 mg, $0.144 \mathrm{mmol}$, yield: $72 \%)$.

${ }^{1} \mathbf{H}$ NMR (400 MHz, CDCl $) \delta 7.92(\mathrm{~d}, J=8.1 \mathrm{~Hz}, 3 \mathrm{H}), 7.84(\mathrm{~s}, 1 \mathrm{H}), 7.63-7.51(\mathrm{~m}$, 5H), $7.35-7.24(\mathrm{~m}, 3 \mathrm{H}), 4.62(\mathrm{dd}, J=8.3,5.2 \mathrm{~Hz}, 1 \mathrm{H}), 3.53(\mathrm{dd}, J=12.3,8.3 \mathrm{~Hz}$, $1 \mathrm{H}), 3.38(\mathrm{~s}, 3 \mathrm{H}), 3.27(\mathrm{~s}, 1 \mathrm{H})$.

${ }^{13}$ C NMR (101 MHz, CDCl $) \delta$ 138.4, 133.4, 133.3, 132.7, 130.8, 129.1, 128.7, 128.0, $127.9,126.9,126.4,126.3,126.2,124.2,83.5,57.2,35.3$.

IR (film): $v\left(\mathrm{~cm}^{-1}\right) 2935,1465,1220,1066,780$.

HRMS (ESI, m/z) calcd for $\mathrm{C}_{19} \mathrm{H}_{18} \mathrm{NaOSe}(\mathrm{M}+\mathrm{Na})^{+}: 365.0421$, found: 365.0419 . 


\section{(1-methoxy-2-(phenylselanyl)ethyl)trimethylsilane (5zg)}

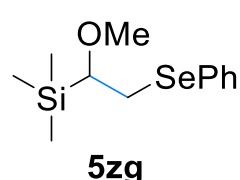

A dried $10 \mathrm{~mL}$ Schlenk tube was charged with $1 \mathrm{zg}(29 \mu \mathrm{L}, 20.0 \mathrm{mg}, 0.20 \mathrm{mmol}), 3 \mathbf{a}$ (31.2 mg, $0.10 \mathrm{mmol})$, [Os-Cu] (10.9 mg, $0.01 \mathrm{mmol}), 2 \mathrm{a}(0.5 \mathrm{~mL})$, and $\mathrm{CH}_{2} \mathrm{Cl}_{2}(3.5$ $\mathrm{mL}$ ). The Schlenk tube was positioned approximately $3 \mathrm{~cm}$ away from a $24 \mathrm{~W}$ blue LEDs lamp $\left(\lambda_{\max }=450 \mathrm{~nm}\right)$. After being stirred at $25^{\circ} \mathrm{C}$ for the $48 \mathrm{~h}$ under an $\mathrm{O}_{2}$ (balloon) atmosphere, the reaction mixture was concentrated to dryness. The residue was purified by flash chromatography (petroleum ether) to afford product $\mathbf{5 z g}$ as a colorless oil (32.3 mg, $0.112 \mathrm{mmol}$, yield: 56\%).

${ }^{1}$ H NMR (400 MHz, (CD) $)_{2}$ CO) $\delta 7.59-7.54(\mathrm{~m}, 2 \mathrm{H}), 7.30-7.23(\mathrm{~m}, 3 \mathrm{H}), 3.73(\mathrm{dd}$, $J=5.6,1.6 \mathrm{~Hz}, 2 \mathrm{H}), 3.25(\mathrm{~s}, 3 \mathrm{H}), 2.74(\mathrm{t}, J=5.6 \mathrm{~Hz}, 1 \mathrm{H}), 0.14(\mathrm{~s}, 9 \mathrm{H})$.

${ }^{13}$ C NMR (101 MHz, (CD) 2 2CO) $\delta 134.8,133.8,131.3,129.0,77.1,60.0,33.5,0.0$.

IR (film): $v\left(\mathrm{~cm}^{-1}\right)$ 2970, 2880, 1465, 1380, 1270, 1066, 843, 750.

HRMS (ESI, m/z) calcd for $\mathrm{C}_{12} \mathrm{H}_{20} \mathrm{NaOSeSi}(\mathrm{M}+\mathrm{Na})^{+}: 311.0346$, found: 311.0341 .

\section{2-(1-methoxy-2-(phenylselanyl)ethyl)thiophene (5zh)}

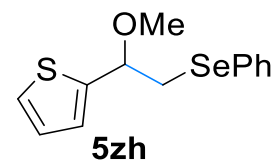

A dried $10 \mathrm{~mL}$ Schlenk tube was charged with 1zh $(21 \mu \mathrm{L}, 22.0 \mathrm{mg}, 0.20 \mathrm{mmol}), \mathbf{3 a}$ (31.2 mg, $0.10 \mathrm{mmol})$, [Os-Cu] (10.9 mg, $0.01 \mathrm{mmol}), 2 \mathrm{a}(0.5 \mathrm{~mL})$, and $\mathrm{CH}_{2} \mathrm{Cl}_{2}(3.5$ $\mathrm{mL}$ ). The Schlenk tube was positioned approximately $3 \mathrm{~cm}$ away from a $24 \mathrm{~W}$ blue LEDs lamp $\left(\lambda_{\max }=450 \mathrm{~nm}\right)$. After being stirred at $25^{\circ} \mathrm{C}$ for the $48 \mathrm{~h}$ under an $\mathrm{O}_{2}$ (balloon) atmosphere, the reaction mixture was concentrated to dryness. The residue was purified by flash chromatography (petroleum ether) to afford product $\mathbf{5 z h}$ as a colorless oil (41.6 mg, $0.140 \mathrm{mmol}$, yield: 70\%). 
${ }^{1}$ H NMR (400 MHz, CDCl $)$ ) $\delta 7.55(\mathrm{ddd}, J=5.8,3.2,2.0 \mathrm{~Hz}, 2 \mathrm{H}), 7.34-7.26(\mathrm{~m}$, 4H), $7.07-7.00(\mathrm{~m}, 2 \mathrm{H}), 4.67(\mathrm{dd}, J=7.8,5.7 \mathrm{~Hz}, 1 \mathrm{H}), 3.47(\mathrm{dd}, J=12.3,7.8 \mathrm{~Hz}$, 1H), 3.34 (s, 3H), 3.25 (dd, $J=12.3,5.6 \mathrm{~Hz}, 1 \mathrm{H})$.

${ }^{13}$ C NMR (101 MHz, CDCl $) \delta$ 144.6, 132.8, 130.58, 129.18, 127.0, 126.6, 125.9, 125.4, 78.9, 57.0, 35.4.

IR (film): v ( $\left.\mathrm{cm}^{-1}\right)$ 2920, 2882, 1633, 1465, 1240, 1066, 920, 760, 684.

HRMS (ESI, m/z) calcd for $\mathrm{C}_{13} \mathrm{H}_{14} \mathrm{NaOSSe}(\mathrm{M}+\mathrm{Na})^{+}:$320.9828, found:320.9825.

$((1 S, 6 S)-6-m e t h o x y c y c l o h e x-3-e n-1-y l)(\text { phenyl)selane }(5 z i))^{20}$

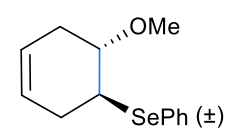

$5 z i$

A dried $10 \mathrm{~mL}$ Schlenk tube was charged with 1zi (19 $\mu \mathrm{L}, 16.0 \mathrm{mg}, 0.20 \mathrm{mmol}), 3 \mathbf{a}$ (31.2 mg, $0.10 \mathrm{mmol})$, [Os-Cu] (10.9 mg, $0.01 \mathrm{mmol}), \mathbf{2 a}(0.5 \mathrm{~mL})$, and $\mathrm{CH}_{2} \mathrm{Cl}_{2}(3.5$ $\mathrm{mL}$ ). The Schlenk tube was positioned approximately $3 \mathrm{~cm}$ away from a $24 \mathrm{~W}$ blue LEDs lamp $\left(\lambda_{\max }=450 \mathrm{~nm}\right)$. After being stirred at $25^{\circ} \mathrm{C}$ for the $48 \mathrm{~h}$ under an $\mathrm{O}_{2}$ (balloon) atmosphere, the reaction mixture was concentrated to dryness. The residue was purified by flash chromatography (petroleum ether/ethyl acetate $=50: 1$ ) to afford product 5zi as a yellow oil (32.7 mg, $0.122 \mathrm{mmol}$, yield: $61 \%)$.

${ }^{1}$ H NMR (500 MHz, CDCl $) \delta 7.59(\mathrm{ddd}, J=4.7,3.2,2.0 \mathrm{~Hz}, 2 \mathrm{H}), 7.30-7.26(\mathrm{~m}$, $3 \mathrm{H}), 5.65-5.55(\mathrm{~m}, 2 \mathrm{H}), 3.60(\mathrm{dt}, 1 \mathrm{H}), 3.52(\mathrm{dt}, 1 \mathrm{H}), 3.39(\mathrm{~s}, 3 \mathrm{H}), 2.67-2.60(\mathrm{~m}$, $1 \mathrm{H}), 2.59-2.52(\mathrm{~m}, 1 \mathrm{H}), 2.33-2.24(\mathrm{~m}, 1 \mathrm{H}), 2.18-2.09(\mathrm{~m}, 1 \mathrm{H})$.

${ }^{13}$ C NMR (126 MHz, CDCl3) $\delta$ 135.0, 128.90 (2C), 127.5, 125.3, 124.0, 78.2, 56.5, 42.0, 30.7, 29.6.

IR (film): $v\left(\mathrm{~cm}^{-1}\right)$ 2926, 2900, 1466, 1276, 1068, 990, 771, 698.

HRMS ESI, m/z) calcd for $\mathrm{C}_{13} \mathrm{H}_{16} \mathrm{OSe}(\mathrm{M})^{+}:$268.0366, found: 268.0307 . 
$((1 S, 8 S, Z)-8-m e t h o x y c y c l o o c t-4-e n-1-y l)(p h e n y l) s e l a n e ~(5 z j))^{18}$

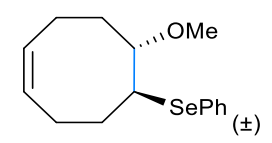

$5 \mathbf{z j}$

A dried $10 \mathrm{~mL}$ Schlenk tube was charged with 1zj ( $24 \mu \mathrm{L}, 21.6 \mathrm{mg}, 0.20 \mathrm{mmol}), 3 \mathbf{a}$ (31.2 mg, $0.10 \mathrm{mmol})$, [Os-Cu] (10.9 mg, $0.01 \mathrm{mmol}), \mathbf{2 a}(0.5 \mathrm{~mL})$, and $\mathrm{CH}_{2} \mathrm{Cl}_{2}(3.5$ $\mathrm{mL}$ ). The Schlenk tube was positioned approximately $3 \mathrm{~cm}$ away from a $24 \mathrm{~W}$ blue LEDs lamp $\left(\lambda_{\max }=450 \mathrm{~nm}\right)$. After being stirred at $25^{\circ} \mathrm{C}$ for the $60 \mathrm{~h}$ under an $\mathrm{O}_{2}$ (balloon) atmosphere, the reaction mixture was concentrated to dryness. The residue was purified by flash chromatography (petroleum ether/ethyl acetate $=50: 1$ ) to afford product 5zj as a yellow oil (32.6 mg, $0.110 \mathrm{mmol}$, yield: 55\%).

${ }^{1}$ H NMR (500 MHz, CDCl $) \delta 7.59-7.52(\mathrm{~m}, 2 \mathrm{H}), 7.27-7.23(\mathrm{~m}, 3 \mathrm{H}), 5.67(\mathrm{dt}, J=$ 10.8, $6.9 \mathrm{~Hz}, 1 \mathrm{H}), 5.54(\mathrm{dt}, J=10.7,7.7 \mathrm{~Hz}, 1 \mathrm{H}), 3.74(\mathrm{ddd}, J=10.0,8.2,3.7 \mathrm{~Hz}$, 1H), $3.42(\mathrm{td}, J=8.2,3.2 \mathrm{~Hz}, 1 \mathrm{H}), 3.37(\mathrm{~s}, 3 \mathrm{H}), 2.43-2.29(\mathrm{~m}, 2 \mathrm{H}), 2.15$ (tt, $J=11.7$, $3.6 \mathrm{~Hz}, 2 \mathrm{H}), 2.07-1.93(\mathrm{~m}, 2 \mathrm{H}), 1.82-1.69(\mathrm{~m}, 2 \mathrm{H})$.

${ }^{13}$ C NMR (126 MHz, CDCl3) $\delta$ 135.0, 130.6, 130.2, 128.8, 128.5, 127.2, 83.8, 58.0, 48.9, 33.1, 31.1, 25.6, 23.0.

IR (film): $v\left(\mathrm{~cm}^{-1}\right)$ 2920, 2850, 1465, 1273, 1065, 990, 771, 694.

HRMS (ESI, m/z) calcd for $\mathrm{C}_{15} \mathrm{H}_{20} \mathrm{NaOSe}(\mathrm{M}+\mathrm{Na})^{+}: 319.0572$, found: 319.0574 .

((1S,2S)-2-methoxycyclohepta-3,5-dien-1-yl)(phenyl)selane (5zk)

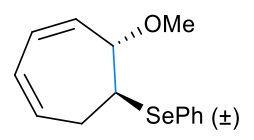

$5 \mathbf{z k}$

A dried $10 \mathrm{~mL}$ Schlenk tube was charged with 1zk $(21 \mu \mathrm{L}, 18.4 \mathrm{mg}, 0.20 \mathrm{mmol}), 3 \mathbf{a}$ (31.2 mg, $0.10 \mathrm{mmol})$, [Os-Cu] (10.9 mg, $0.01 \mathrm{mmol}), \mathbf{2 a}(0.5 \mathrm{~mL})$, and $\mathrm{CH}_{2} \mathrm{Cl}_{2}(3.5$ $\mathrm{mL}$ ). The Schlenk tube was positioned approximately $3 \mathrm{~cm}$ away from a $24 \mathrm{~W}$ blue LEDs lamp $\left(\lambda_{\max }=450 \mathrm{~nm}\right)$. After being stirred at $25^{\circ} \mathrm{C}$ for the $72 \mathrm{~h}$ under an $\mathrm{O}_{2}$ (balloon) atmosphere, the reaction mixture was concentrated to dryness. The residue 
was purified by flash chromatography (petroleum ether/ethyl acetate $=40: 1$ ) to afford product 5zk as a yellow oil (17.9 $\mathrm{mg}, 0.064 \mathrm{mmol}$, yield: 32\%).

${ }^{1}$ H NMR (500 MHz, CDCl $) \delta 7.60-7.57$ (m, 2H), $7.31-7.27$ (m, 3H), 6.07 (dd, $J$ $=11.6,6.7 \mathrm{~Hz}, 1 \mathrm{H}), 6.02(\mathrm{dt}, J=9.3,4.6,1.7 \mathrm{~Hz}, 1 \mathrm{H}), 5.95(\mathrm{dd}, J=11.7,5.2 \mathrm{~Hz}, 1 \mathrm{H})$, $5.90(\mathrm{dt}, J=11.0,7.1,3.8 \mathrm{~Hz}, 1 \mathrm{H}), 4.01(\mathrm{dd}, J=5.2 \mathrm{~Hz}, 1 \mathrm{H}), 3.78(\mathrm{dd}, J=8.4,5.2$ $\mathrm{Hz}, 1 \mathrm{H}), 3.38(\mathrm{~s}, 3 \mathrm{H}), 2.92-2.84(\mathrm{~m}, 1 \mathrm{H}), 2.75-2.66(\mathrm{~m}, 1 \mathrm{H})$.

${ }^{13}$ C NMR (126 MHz, CDCl $) \delta$ 134.5, 133.0, 130.2, 129.7, 129.1, 127.6, 127.5, 126.4, 81.6, 56.3, 46.0, 31.6.

IR (film): $v\left(\mathrm{~cm}^{-1}\right)$ 1468, 1274, 1088, 992, 773, 690.

HRMS (ESI, m/z) calcd for $\mathrm{C}_{28} \mathrm{H}_{32} \mathrm{KO}_{2} \mathrm{Se}_{2}(2 \mathrm{M}+\mathrm{K})^{+}$: 599.0364, found: 599.0349 . ’

\section{(2-methoxy-2,3-dimethylbut-3-en-1-yl)(phenyl)selane (5zl) ${ }^{21}$}

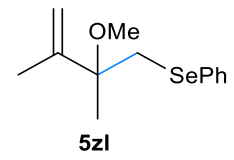

A dried $10 \mathrm{~mL}$ Schlenk tube was charged with 1zl $(23 \mu \mathrm{L}, 16.4 \mathrm{mg}, 0.20 \mathrm{mmol}), 3 \mathrm{a}$ $31.2 \mathrm{mg}, 0.10 \mathrm{mmol})$, [Os-Cu] (10.9 mg, $0.01 \mathrm{mmol}), \mathbf{2 a}(0.5 \mathrm{~mL})$, and $\mathrm{CH}_{2} \mathrm{Cl}_{2}(3.5$ $\mathrm{mL}$ ). The Schlenk tube was positioned approximately $3 \mathrm{~cm}$ away from a $24 \mathrm{~W}$ blue LEDs lamp $\left(\lambda_{\max }=450 \mathrm{~nm}\right)$. After being stirred at $25^{\circ} \mathrm{C}$ for the $28 \mathrm{~h}$ under an $\mathrm{O}_{2}$ (balloon) atmosphere, the reaction mixture was concentrated to dryness. The residue was purified by flash chromatography (petroleum ether/ethyl acetate $=40: 1$ ) to afford product $5 z \mathbf{z}$ as a yellow oil (42.7 $\mathrm{mg}, 0.158 \mathrm{mmol}$, yield: $79 \%)$.

${ }^{1}$ H NMR (500 MHz, CDCl 3$) \delta 7.55-7.51(\mathrm{~m}, 2 \mathrm{H}), 7.28-7.21(\mathrm{~m}, 3 \mathrm{H}), 5.06(\mathrm{~s}, 1 \mathrm{H})$, $5.01(\mathrm{~s}, 1 \mathrm{H}), 3.29$ (d, $J=11.5 \mathrm{~Hz}, 1 \mathrm{H}), 3.15$ (d, $J=13.4 \mathrm{~Hz}, 4 \mathrm{H}), 1.73(\mathrm{~s}, 3 \mathrm{H}), 1.46(\mathrm{~s}$, $3 \mathrm{H})$.

${ }^{13}$ C NMR (126 MHz, CDCl 3$) \delta 146.3,132.6,131.3,129.0,126.7,114.4,79.3,50.6$, $37.9,21.8,18.5$.

IR (film): $v\left(\mathrm{~cm}^{-1}\right)$ 2978, 2850, 1467, 1383, 1721, 1063, 889, 771, 693.

HRMS (ESI, m/z) calcd for $\mathrm{C}_{26} \mathrm{H}_{36} \mathrm{KO}_{2} \mathrm{Se}_{2}(2 \mathrm{M}+\mathrm{K})^{+}:$579.0677, found: 579.0697 . 


\section{(2-methoxy-2,5-dimethylhex-5-en-1-yl)(phenyl)selane (5zm)}

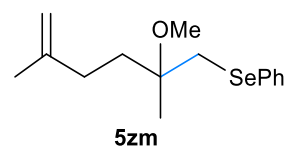

A dried $10 \mathrm{~mL}$ Schlenk tube was charged with $\mathbf{1 z m}(30 \mu \mathrm{L}, 22.0 \mathrm{mg}, 0.20 \mathrm{mmol}), 3 \mathbf{a}$ (31.2 mg, $0.10 \mathrm{mmol})$, [Os-Cu] (10.9 mg, $0.01 \mathrm{mmol}), \mathbf{2 a}(0.5 \mathrm{~mL})$, and $\mathrm{CH}_{2} \mathrm{Cl}_{2}(3.5$ $\mathrm{mL}$ ). The Schlenk tube was positioned approximately $3 \mathrm{~cm}$ away from a $24 \mathrm{~W}$ blue LEDs lamp $\left(\lambda_{\max }=450 \mathrm{~nm}\right)$. After being stirred at $25^{\circ} \mathrm{C}$ for the $30 \mathrm{~h}$ under an $\mathrm{O}_{2}$ (balloon) atmosphere, the reaction mixture was concentrated to dryness. The residue was purified by flash chromatography (petroleum ether/ethyl acetate $=40: 1$ ) to afford product 5zm as a yellow oil (24.4 $\mathrm{mg}, 0.082 \mathrm{mmol}$, yield: 41\%).

${ }^{1}$ H NMR (500 MHz, CDCl 3$) \delta 7.55-7.51(\mathrm{~m}, 2 \mathrm{H}), 7.27-7.20(\mathrm{~m}, 3 \mathrm{H}), 4.69(\mathrm{~s}, 1 \mathrm{H})$, $4.66(\mathrm{~s}, 1 \mathrm{H}), 3.20(\mathrm{~s}, 3 \mathrm{H}), 3.17(\mathrm{~d}, J=11.9 \mathrm{~Hz}, 1 \mathrm{H}), 3.10(\mathrm{~d}, J=11.9 \mathrm{~Hz}, 1 \mathrm{H}), 1.99$ (t, $J=8.2 \mathrm{~Hz}, 2 \mathrm{H}), 1.76(\mathrm{t}, J=8.3 \mathrm{~Hz}, 2 \mathrm{H}), 1.72(\mathrm{~s}, 3 \mathrm{H}), 1.29(\mathrm{~s}, 3 \mathrm{H})$

${ }^{13}$ C NMR (126 MHz, CDCl $) \delta 145.8$ 132.8, 131.2, 129.0, 126.8, 109.7, 76.7, 49.4, $37.835 .7,31.7,22.9,22.7$.

IR (film): $v\left(\mathrm{~cm}^{-1}\right)$ 2940, 2859, 1467, 1374, 1720, 1063, 886, 771, 697.

HRMS (ESI, m/z) calcd for $\mathrm{C}_{15} \mathrm{H}_{22} \mathrm{NaOSe}(\mathrm{M}+\mathrm{Na})^{+}: 321.0728$, found: 321.0727 .

\section{(2,5-dimethoxy-2,5-dimethylhexane-1,6-diyl)bis(phenylselane) (5zm')}

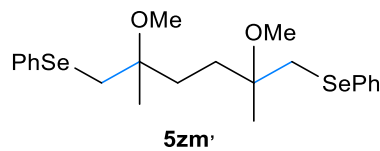

A dried $10 \mathrm{~mL}$ Schlenk tube was charged with $\mathbf{1 z m}(30 \mu \mathrm{L}, 22.0 \mathrm{mg}, 0.20 \mathrm{mmol}), 3 \mathbf{a}$ (31.2 mg, $0.10 \mathrm{mmol})$, [Os-Cu] (10.9 mg, $0.01 \mathrm{mmol}), \mathbf{2 a}(0.5 \mathrm{~mL})$, and $\mathrm{CH}_{2} \mathrm{Cl}_{2}(3.5$ $\mathrm{mL}$ ). The Schlenk tube was positioned approximately $3 \mathrm{~cm}$ away from a $24 \mathrm{~W}$ blue LEDs lamp $\left(\lambda_{\max }=450 \mathrm{~nm}\right)$. After being stirred at $25{ }^{\circ} \mathrm{C}$ for the $72 \mathrm{~h}$ under an $\mathrm{O}_{2}$ (balloon) atmosphere, the reaction mixture was concentrated to dryness. The residue 
was purified by flash chromatography (petroleum ether/ethyl acetate $=20: 1$ ) to afford product 5zm' as a yellow oil (67.1 $\mathrm{mg}, 0.138 \mathrm{mmol}$, yield: $69 \%)$.

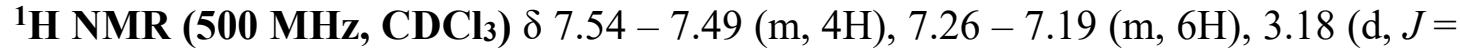
$1.4 \mathrm{~Hz}, 6 \mathrm{H}), 3.09$ (dd, $J=33.3,11.9 \mathrm{~Hz}, 4 \mathrm{H}), 1.68-1.59$ (m, 4H), 1.25 (s, 6H).

${ }^{13}$ C NMR (126 MHz, CDCl 3$) \delta 132.7,131.2,129.0,126.8,76.6,49.5,37.8,31.4$, 23.0.

IR (film): $v\left(\mathrm{~cm}^{-1}\right)$ 2964, 2919, 1467, 1378, 1271, 1075, , 774, 690.

HRMS (ESI, m/z) calcd for $\mathrm{C}_{22} \mathrm{H}_{30} \mathrm{NaO}_{2} \mathrm{Se}_{2}(\mathrm{M}+\mathrm{Na})^{+}:$509.0468, found: 509.0474 .

\subsection{Synthetic Utility of the Method}

\subsubsection{Synthesis of L-menthol derivative 6a}
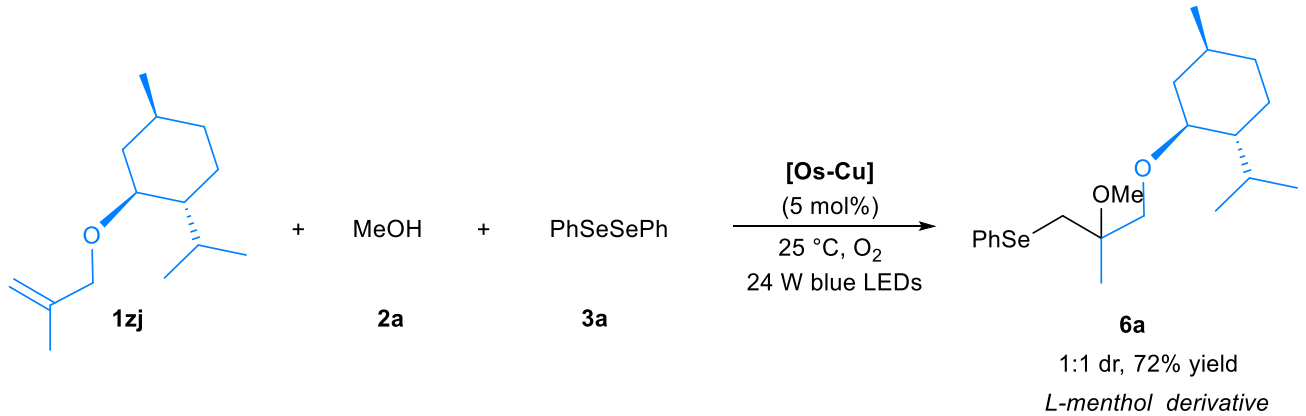

A dried $10 \mathrm{~mL}$ Schlenk tube was charged with 1zj (42.0 mg, $0.20 \mathrm{mmol}), \mathbf{2 a}(0.5 \mathrm{~mL})$, 3a (31.2 mg, $0.10 \mathrm{mmol})$, [Os-Cu] (10.9 mg, $0.01 \mathrm{mmol})$, and $\mathrm{CH}_{2} \mathrm{Cl}_{2}(3.5 \mathrm{~mL})$. The Schlenk tube was positioned approximately $3 \mathrm{~cm}$ away from a $24 \mathrm{~W}$ blue LEDs lamp $\left(\lambda_{\max }=450 \mathrm{~nm}\right)$. After being stirred at $25{ }^{\circ} \mathrm{C}$ for the $48 \mathrm{~h}$ under an $\mathrm{O}_{2}$ (balloon) atmosphere, the reaction mixture was concentrated to dryness. The residue was purified by flash chromatography (petroleum ether/ethyl acetate $=40: 1$ ) to afford product 6a as a colorless oil (57.3 $\mathrm{mg}, 0.144 \mathrm{mmol}$, yield: 72\%).

${ }^{1}$ H NMR (500 MHz, CDCl3) $\delta 7.56-7.48(\mathrm{~m}, 2 \mathrm{H}), 7.26-7.17$ (m, 3H), $3.66(\mathrm{dd}, J$ $=90.6,9.2 \mathrm{~Hz}, 1 \mathrm{H}), 3.46-3.11(\mathrm{~m}, 7 \mathrm{H}), 3.03(\mathrm{tt}, J=10.5,3.6 \mathrm{~Hz}, 1 \mathrm{H}), 2.21(\mathrm{dqd}, J$ $=13.6,6.9,2.7 \mathrm{~Hz}, 1 \mathrm{H}), 2.12-2.01(\mathrm{~m}, 1 \mathrm{H}), 1.66-1.57(\mathrm{~m}, 2 \mathrm{H}), 1.28(\mathrm{~d}, J=6.1 \mathrm{~Hz}$, $3 \mathrm{H}), 0.95(\mathrm{ddd}, J=12.9,7.8,2.6 \mathrm{~Hz}, 1 \mathrm{H}), 0.89(\mathrm{~d}, J=6.2 \mathrm{~Hz}, 3 \mathrm{H}), 0.87-0.83(\mathrm{~m}$, $3 \mathrm{H}), 0.83-0.77(\mathrm{~m}, 2 \mathrm{H}), 0.76-0.71(\mathrm{~m}, 3 \mathrm{H})$. 
${ }^{13}$ C NMR (126 MHz, $\left.\mathbf{C D C l}_{3}\right) \delta 132.5$ (2C), 131.5, 131.4, 128.9, 126.5, 79.7, 79.6, $77.0(2 \mathrm{C}), 71.8,50.0,48.2,40.1,40.0,35.3,35.2,34.6,31.5,25.6,25.5,23.2(2 \mathrm{C})$, $22.4(2 \mathrm{C}), 21.1,21.0,20.8(2 \mathrm{C}), 16.1,16.0$.

IR (film): $v\left(\mathrm{~cm}^{-1}\right)$ 2975, 2900, 1452, 1383, 1267, 1049, 763, 690.

HRMS (ESI, m/z) calcd for $\mathrm{C}_{21} \mathrm{H}_{34} \mathrm{NaO}_{2} \mathrm{Se}(\mathrm{M}+\mathrm{Na})^{+}: 421.1616$, found: 421.1627 .

\subsubsection{Synthesis of piperonylic acid derivative $6 \mathrm{~b}$}
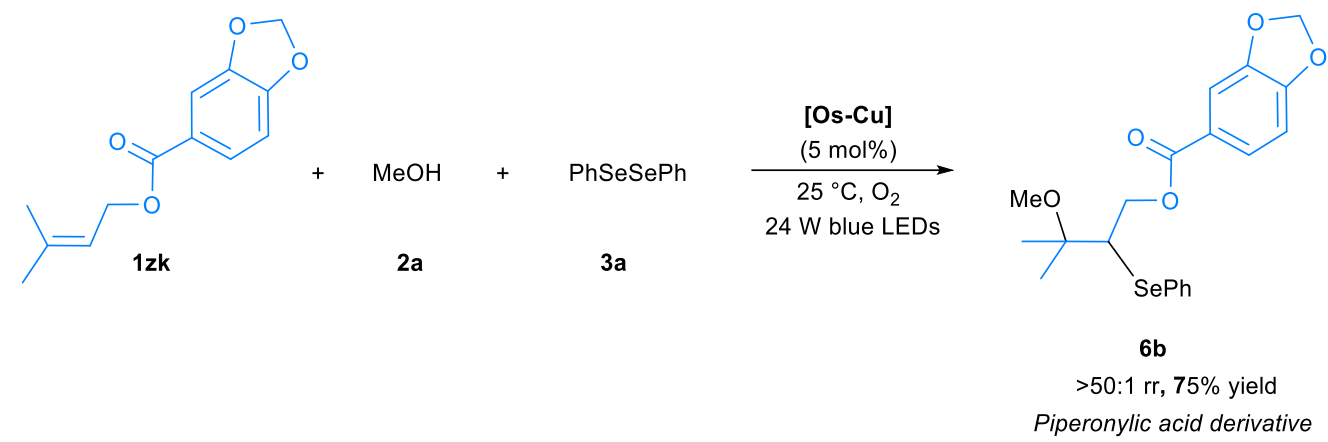

A dried $10 \mathrm{~mL}$ Schlenk tube was charged with 1 zk (46.8 mg, $0.20 \mathrm{mmol}), 2 \mathbf{a}(0.5 \mathrm{~mL})$, 3a (31.2 mg, $0.10 \mathrm{mmol})$, [Os-Cu] (10.9 mg, $0.01 \mathrm{mmol})$, and DCM (3.5 mL). The Schlenk tube was positioned approximately $3 \mathrm{~cm}$ away from a $24 \mathrm{~W}$ blue LEDs lamp $\left(\lambda_{\max }=450 \mathrm{~nm}\right)$. After being stirred at $25{ }^{\circ} \mathrm{C}$ for the $48 \mathrm{~h}$ under an $\mathrm{O}_{2}$ (balloon) atmosphere, the reaction mixture was concentrated to dryness. The residue was purified by flash chromatography (petroleum ether/ethyl acetate $=40: 1$ ) to afford product $\mathbf{6 b}$ as a colorless oil (63.3 $\mathrm{mg}, 0.150 \mathrm{mmol}$, yield: $75 \%)$.

${ }^{1} \mathbf{H}$ NMR (500 MHz, CDCl 3$) ~ \delta 7.60(\mathrm{dd}, J=6.2,2.7 \mathrm{~Hz}, 2 \mathrm{H}), 7.47(\mathrm{dd}, J=8.2,1.1$ $\mathrm{Hz}, 1 \mathrm{H}), 7.29(\mathrm{~s}, 1 \mathrm{H}), 7.22$ (dd, $J=9.2,5.6 \mathrm{~Hz}, 3 \mathrm{H}), 6.77$ (d, $J=8.2 \mathrm{~Hz}, 1 \mathrm{H}), 6.02(\mathrm{~s}$, 2H), 4.79 (dd, $J=11.8,5.4 \mathrm{~Hz}, 1 \mathrm{H}), 4.56(\mathrm{dd}, J=11.8,6.9 \mathrm{~Hz}, 1 \mathrm{H}), 3.59$ (t, $J=5.7$ $\mathrm{Hz}, 1 \mathrm{H}), 3.25$ (s, 3H), 1.43 (s, 3H), $1.41(\mathrm{~s}, 3 \mathrm{H})$.

${ }^{13}$ C NMR (126 MHz, CDCl $) \delta 165.7,151.6,147.6,134.3,130.3,129.1,127.5,125.4$, 124.1, 109.6, 107.9, 101.7, 65.9, 54.5, 49.6, $24.2(2 \mathrm{C})$.

IR (film): $v\left(\mathrm{~cm}^{-1}\right)$ 2977, 2920, 1459, 1383, 1179, 1049, 763, 690.

HRMS (ESI, m/z) calcd for $\mathrm{C}_{20} \mathrm{H}_{22} \mathrm{NaO}_{5} \mathrm{Se}(\mathrm{M}+\mathrm{Na})^{+}: 445.0525$, found: 445.0528 . 


\subsubsection{Synthesis of naproxen derivative 6c}
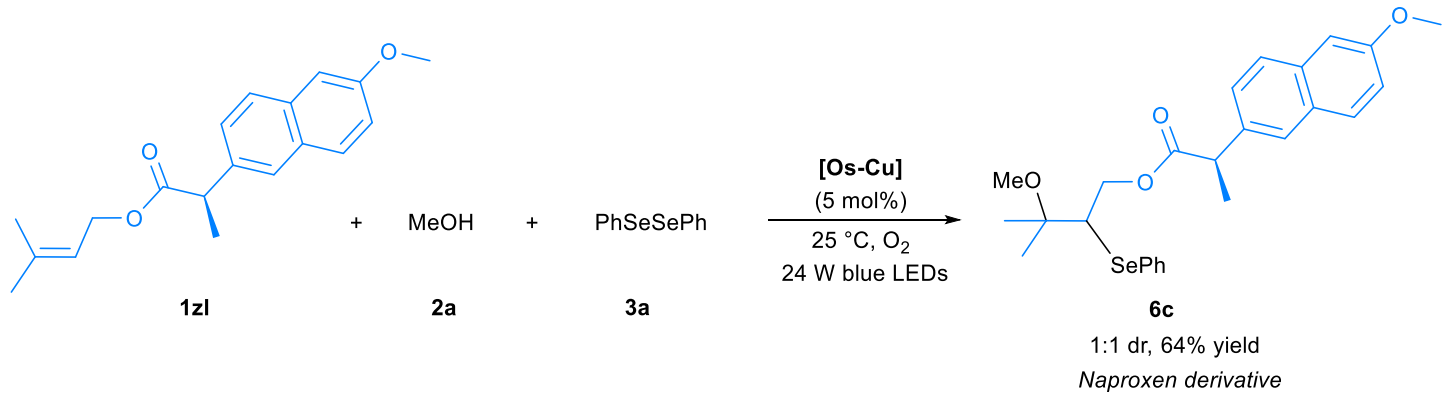

A dried $10 \mathrm{~mL}$ Schlenk tube was charged with $\mathbf{1 z l}(60 \mathrm{mg}, 0.20 \mathrm{mmol}), \mathbf{2 a}(0.5 \mathrm{~mL})$, 3a (31.2 mg, $0.10 \mathrm{mmol})$, [Os-Cu] (10.9 mg, $0.01 \mathrm{mmol})$, and $\mathrm{CH}_{2} \mathrm{Cl}_{2}(3.5 \mathrm{~mL})$. The Schlenk tube was positioned approximately $3 \mathrm{~cm}$ away from a $24 \mathrm{~W}$ blue LEDs lamp $\left(\lambda_{\max }=450 \mathrm{~nm}\right)$. After being stirred at $25{ }^{\circ} \mathrm{C}$ for the $48 \mathrm{~h}$ under an $\mathrm{O}_{2}$ (balloon) atmosphere, the reaction mixture was concentrated to dryness. The residue was purified by flash chromatography (petroleum ether/ethyl acetate $=10: 1$ ) to afford product $6 \mathbf{c}$ as a white solid (62.2 $\mathrm{mg}, 0.128 \mathrm{mmol}$, yield: 64\%).

${ }^{1}$ H NMR (500 MHz, $\left.\left(\mathbf{C D}_{3}\right)_{2} \mathbf{C O}\right) \delta 7.75(\mathrm{td}, J=8.7,3.2 \mathrm{~Hz}, 1 \mathrm{H}), 7.68(\mathrm{~d}, J=7.5 \mathrm{~Hz}$, 1H), $7.58-7.48(\mathrm{~m}, 1 \mathrm{H}), 7.37$ (ddd, $J=8.1,5.9,1.8 \mathrm{~Hz}, 1 \mathrm{H}), 7.31-7.19(\mathrm{~m}, 2 \mathrm{H})$, $7.14(\mathrm{dt}, J=8.9,2.6 \mathrm{~Hz}, 1 \mathrm{H}), 4.55$ (ddd, $J=44.3,11.8,5.5 \mathrm{~Hz}, 1 \mathrm{H}), 4.32$ (ddd, $J=$ 31.5, 11.8, $6.7 \mathrm{~Hz}, 1 \mathrm{H}), 3.90(\mathrm{~d}, J=2.2 \mathrm{~Hz}, 1 \mathrm{H}), 3.76(\mathrm{dq}, J=14.4,7.2 \mathrm{~Hz}, 1 \mathrm{H}), 3.47$ (ddd, $J=6.6,5.6,4.0 \mathrm{~Hz}, 1 \mathrm{H}), 3.14(\mathrm{~d}, J=8.5 \mathrm{~Hz}, 2 \mathrm{H}), 1.27(\mathrm{dd}, J=17.6,8.7 \mathrm{~Hz}$, $3 \mathrm{H})$.

${ }^{13}$ C NMR (126 MHz, (CD) $)$ CO) $\delta$ 173.9, 173.8, 170.4, 157.8, 135.9, 133.9, 133.6, $130.7,130.6,129.2,129.1$ (2C), 129.0, 127.3, 127.2, 127.1 (2C), 126.3, 126.2, 125.9, 118.8, 105.6, 76.7 (2C), 67.2, 65.3, 65.2, 59.8, 54.8, 54.6, 54.5, 54.1, 48.8, 45.1 (2C), 25.2, 23.4(2C), 23.3, 23.2, 20.0, $18.0(2 \mathrm{C}), 13.6$.

IR (film): $v\left(\mathrm{~cm}^{-1}\right)$ 2973, 2926, 1732, 1506, 1370, 1176, 1067, 841, 766.

HRMS (ESI, m/z) calcd for $\mathrm{C}_{26} \mathrm{H}_{30} \mathrm{NaO}_{4} \mathrm{Se}(\mathrm{M}+\mathrm{Na})^{+}:$509.1202, found: 509.1205. 


\subsubsection{Synthesis of indomethacin derivative 6d}
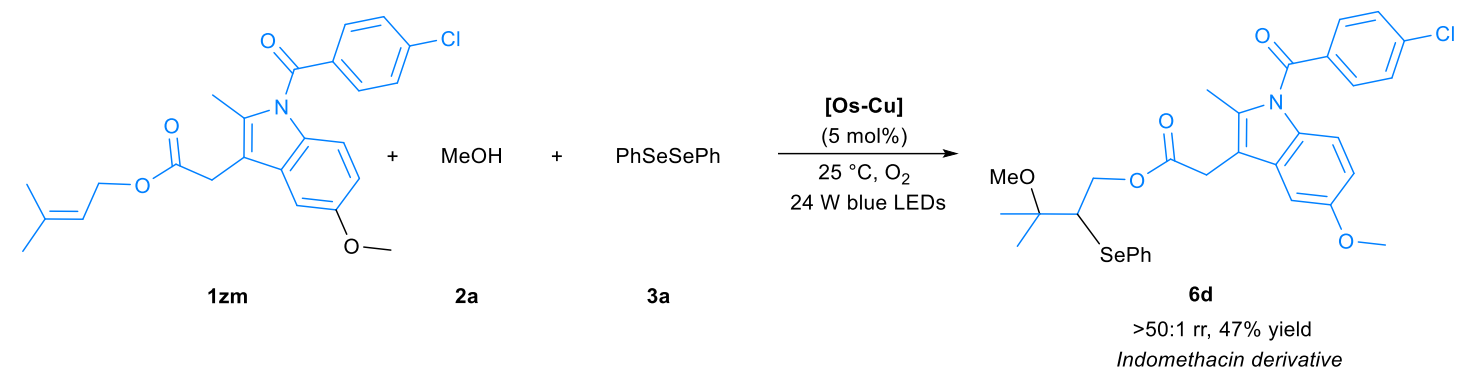

A dried $10 \mathrm{~mL}$ Schlenk tube was charged with $\mathbf{1 z m}(85 \mathrm{mg}, 0.20 \mathrm{mmol}), \mathbf{2 a}(0.5 \mathrm{~mL})$, 3a (31.2 mg, $0.10 \mathrm{mmol})$, [Os-Cu] (10.9 mg, $0.01 \mathrm{mmol})$, and $\mathrm{CH}_{2} \mathrm{Cl}_{2}(3.5 \mathrm{~mL})$. The Schlenk tube was positioned approximately $3 \mathrm{~cm}$ away from a $24 \mathrm{~W}$ blue LEDs lamp $\left(\lambda_{\max }=450 \mathrm{~nm}\right)$. After being stirred at $25{ }^{\circ} \mathrm{C}$ for the $48 \mathrm{~h}$ under an $\mathrm{O}_{2}$ (balloon) atmosphere, the reaction mixture was concentrated to dryness. The residue was purified by flash chromatography (petroleum ether/ethyl acetate $=10: 1$ ) to afford product $\mathbf{6 d}$ as a white solid (57.6 $\mathrm{mg}, 0.094 \mathrm{mmol}$, yield: 47\%).

${ }^{1} \mathrm{H}$ NMR $\left(500 \mathrm{MHz}, \mathrm{CDCl}_{3}\right) \delta 7.61(\mathrm{~d}, J=8.4 \mathrm{~Hz}, 2 \mathrm{H}), 7.55-7.48(\mathrm{~m}, 2 \mathrm{H}), 7.43(\mathrm{~d}$, $J=8.5 \mathrm{~Hz}, 2 \mathrm{H}), 7.25-7.17(\mathrm{~m}, 3 \mathrm{H}), 6.93(\mathrm{~d}, J=2.4 \mathrm{~Hz}, 1 \mathrm{H}), 6.86(\mathrm{~d}, J=9.0 \mathrm{~Hz}$, $1 \mathrm{H}), 6.66(\mathrm{dd}, J=9.0,2.4 \mathrm{~Hz}, 1 \mathrm{H}), 4.62(\mathrm{dd}, J=11.8,5.2 \mathrm{~Hz}, 1 \mathrm{H}), 4.36(\mathrm{dd}, J=11.8$, $7.2 \mathrm{~Hz}, 1 \mathrm{H}), 3.81(\mathrm{~s}, 3 \mathrm{H}), 3.55(\mathrm{q}, J=15.8 \mathrm{~Hz}, 2 \mathrm{H}), 3.41(\mathrm{t}, 1 \mathrm{H}), 3.18(\mathrm{~s}, 3 \mathrm{H}), 2.33(\mathrm{~s}$, $3 \mathrm{H}), 1.30(\mathrm{~s}, 3 \mathrm{H}), 1.27(\mathrm{~s}, 3 \mathrm{H})$.

${ }^{13} \mathrm{C}$ NMR $\left(126 \mathrm{MHz}, \mathrm{CDCl}_{3}\right) \delta 170.6,168.3,156.1,139.2,135.9,134.1,133.9,131.2$, $130.8,130.7,130.5,129.1$ (2C), , 127.5, 115.0, 112.5, 111.7, 101.3, 77.1, 65.5, 55.7, $54.5,49.6,30.3,24.1,23.7,13.4$.

IR (film): $v\left(\mathrm{~cm}^{-1}\right)$ 2951, 2925, 1735, 1485, 1380, 1257, 1066, 805, 738, 710.

HRMS (ESI, m/z) calcd for $\mathrm{C}_{31} \mathrm{H}_{32} \mathrm{ClNNaO}_{5} \mathrm{Se}(\mathrm{M}+\mathrm{Na})^{+}:$636.1026, found: 636.1027 . 


\subsubsection{Synthesis of indomethacin derivative $6 \mathrm{e}$}
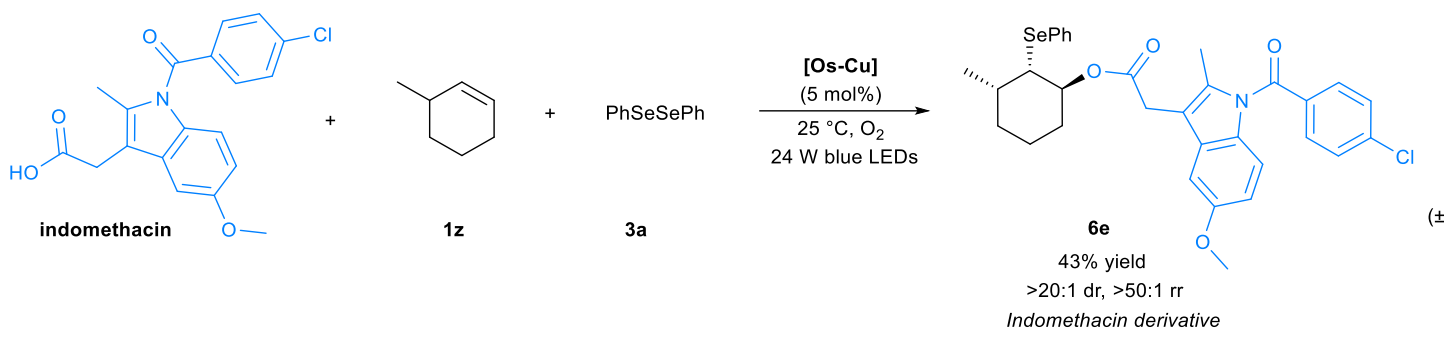

A dried $10 \mathrm{~mL}$ Schlenk tube was charged with $\mathbf{1 z}(24 \mu \mathrm{L}, 19.2 \mathrm{mg}, 0.20 \mathrm{mmol})$, indomethacin (142.8 mg, $0.40 \mathrm{mmol}), 3 \mathbf{a}(31.2 \mathrm{mg}, 0.10 \mathrm{mmol})$, [Os-Cu] (10.9 mg, $0.01 \mathrm{mmol})$, and $\mathrm{CH}_{2} \mathrm{Cl}_{2}(4.0 \mathrm{~mL})$. The Schlenk tube was positioned approximately 3 cm away from a $24 \mathrm{~W}$ blue LEDs lamp $\left(\lambda_{\max }=450 \mathrm{~nm}\right)$. After being stirred at $25{ }^{\circ} \mathrm{C}$ for the $48 \mathrm{~h}$ under an $\mathrm{O}_{2}$ (balloon) atmosphere, the reaction mixture was concentrated to dryness. The residue was purified by flash chromatography (petroleum ether/ethyl acetate $=20: 1)$ to afford product $\mathbf{6 e}$ as a colorless oil $(52.4 \mathrm{mg}, 0.86 \mathrm{mmol}$, yield: $43 \%)$.

${ }^{1}$ H NMR (500 MHz, CDCl 3$) \delta 7.63(\mathrm{~d}, J=8.4 \mathrm{~Hz}, 2 \mathrm{H}), 7.56(\mathrm{dd}, J=6.3,2.8 \mathrm{~Hz}$, 2H), $7.45(\mathrm{~d}, J=8.4 \mathrm{~Hz}, 2 \mathrm{H}), 7.25-7.20(\mathrm{~m}, 3 \mathrm{H}), 6.94(\mathrm{~d}, J=2.2 \mathrm{~Hz}, 1 \mathrm{H}), 6.86(\mathrm{~d}, J$ $=9.0 \mathrm{~Hz}, 1 \mathrm{H}), 6.66(\mathrm{dd}, J=9.0,2.2 \mathrm{~Hz}, 1 \mathrm{H}), 5.25(\mathrm{~d}, J=3.0 \mathrm{~Hz}, 1 \mathrm{H}), 3.79(\mathrm{~s}, 3 \mathrm{H})$, $3.62(\mathrm{~d}, J=1.1 \mathrm{~Hz}, 2 \mathrm{H}), 3.38(\mathrm{~s}, 1 \mathrm{H}), 2.37(\mathrm{~s}, 3 \mathrm{H}), 2.01-1.89(\mathrm{~m}, 2 \mathrm{H}), 1.56-1.51$ (m, 1H), $1.45(\mathrm{ddd}, J=13.7,11.2,5.5 \mathrm{~Hz}, 2 \mathrm{H}), 1.26-1.13(\mathrm{~m}, 2 \mathrm{H}), 0.98(\mathrm{~d}, J=6.8$ $\mathrm{Hz}, 3 \mathrm{H})$.

${ }^{13}$ C NMR (126 MHz, CDCl 3$) \delta 170.0,168.3,156.1,139.3,135.7,133.9,133.5,131.2$, $130.8,130.6,130.5,129.1,127.2,115.0,112.8,111.8,101.3,74.4,55.7,54.0,31.7$, $30.8,30.7,29.7,26.7,20.3,13.3$.

IR (film): $v\left(\mathrm{~cm}^{-1}\right)$ 2988, 1735, 1603, 1463, 1393, 1257, 1176, 1066, 845,760, 738.

HRMS (ESI, m/z) calcd for $\mathrm{C}_{32} \mathrm{H}_{32} \mathrm{ClNNaO}_{4} \mathrm{Se}(\mathrm{M}+\mathrm{Na})^{+}:$632.1077, found: 632.1080 . 


\subsubsection{Synthesis of indomethacin derivative $6 f$}

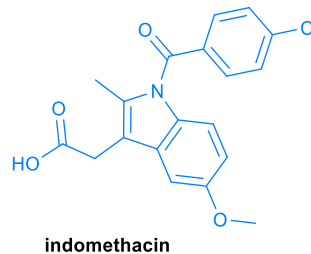

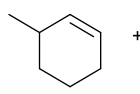

$1 z$

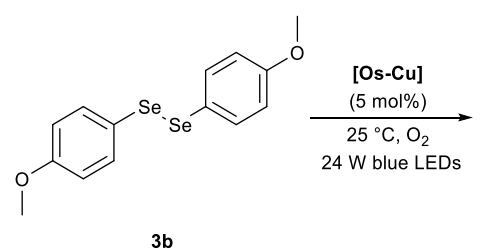

$3 \mathbf{b}$

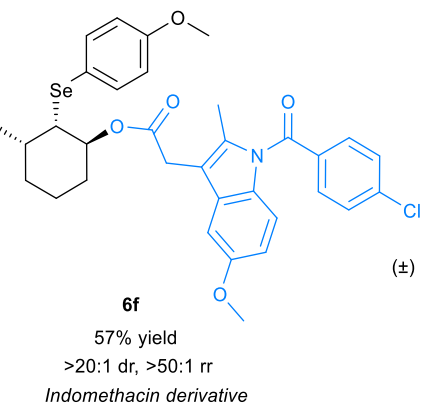

$( \pm)$

A dried $10 \mathrm{~mL}$ Schlenk tube was charged with $\mathbf{1 z}(24 \mu \mathrm{L}, 19.2 \mathrm{mg}, 0.20 \mathrm{mmol})$, indomethacin (0.40 mmol), (4-OMe)PhSeSePh(4-OMe) (37.2 mg, $0.10 \mathrm{mmol})$, [Os-Cu] (10.9 mg, $0.01 \mathrm{mmol})$, and $\mathrm{CH}_{2} \mathrm{Cl}_{2}(4.0 \mathrm{~mL})$. The Schlenk tube was positioned approximately $3 \mathrm{~cm}$ away from a $24 \mathrm{~W}$ blue LEDs lamp $\left(\lambda_{\max }=450 \mathrm{~nm}\right)$. After being stirred at $25{ }^{\circ} \mathrm{C}$ for the $48 \mathrm{~h}$ under an $\mathrm{O}_{2}$ (balloon) atmosphere, the reaction mixture was concentrated to dryness. The residue was purified by flash chromatography (petroleum ether/ethyl acetate $=40: 1$ ) to afford product $\mathbf{6 f}$ as a yellow oil (72.8 mg, $0.114 \mathrm{mmol}$, yield: 57\%).

${ }^{1}$ H NMR (500 MHz, CDCl 3$) \delta 7.64-7.60(\mathrm{~m}, 2 \mathrm{H}), 7.49-7.43(\mathrm{~m}, 4 \mathrm{H}), 6.93(\mathrm{~d}, J=$ $2.4 \mathrm{~Hz}, 1 \mathrm{H}), 6.86(\mathrm{~d}, J=9.0 \mathrm{~Hz}, 1 \mathrm{H}), 6.80-6.77(\mathrm{~m}, 2 \mathrm{H}), 6.66(\mathrm{dd}, J=9.0,2.5 \mathrm{~Hz}$, $1 \mathrm{H}), 5.23(\mathrm{dd}, J=7.1,4.2 \mathrm{~Hz}, 1 \mathrm{H}), 3.79$ (s, 3H), 3.78 (s, 3H), 3.62 (s, 2H), 3.23 (s, 1H), $2.36(\mathrm{~s}, 3 \mathrm{H}), 2.03-1.82(\mathrm{~m}, 3 \mathrm{H}), 1.55-1.49(\mathrm{~m}, 1 \mathrm{H}), 1.46-1.38(\mathrm{~m}, 2 \mathrm{H}), 1.20$ (dd, $J=15.4,8.6 \mathrm{~Hz}, 1 \mathrm{H}), 0.99(\mathrm{~d}, J=6.8 \mathrm{~Hz}, 3 \mathrm{H})$.

${ }^{13}$ C NMR (126 MHz, $\left.\mathbf{C D C l}_{3}\right) \delta 170.0,168.3,159.4,156.1,139.3,136.2,135.7,133.9$, 131.2, 130.8, 130.6, 129.1, 120.3, 115.0, 114.8, 112.8, 111.8, 101.2, 74.4, 55.7, 55.3, $54.9,31.6,30.8,30.6,29.7,26.5,20.4,20.2,13.3$.

IR (film): $v\left(\mathrm{~cm}^{-1}\right)$ 2971, 2850, 1736, 1685, 1394, 1249, 1066, 898, 738, 649.

HRMS (ESI, m/z) calcd for $\mathrm{C}_{33} \mathrm{H}_{34} \mathrm{ClNNaO}_{5} \mathrm{Se}(\mathrm{M}+\mathrm{Na})^{+}:$662.1183, found: 662.1186. 


\subsubsection{Synthesis of oxaprozin derivative $6 \mathrm{~g}$}

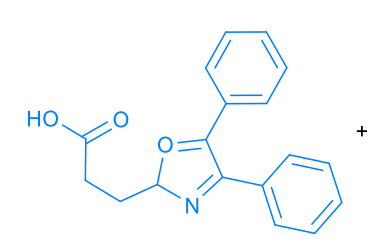

Oxaprozin

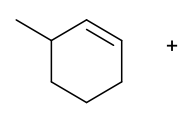

$1 z$
$\mathrm{PhSeSePh}$

3a

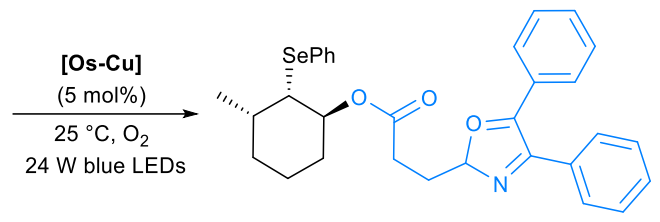

$( \pm)$

A dried $10 \mathrm{~mL}$ Schlenk tube was charged with $\mathbf{1 z}(24 \mu \mathrm{L}, 19.2 \mathrm{mg}, 0.20 \mathrm{mmol})$, oxaprozin (117.6 mg, $0.40 \mathrm{mmol}), 3 \mathbf{a}$ (31.2 mg, $0.10 \mathrm{mmol})$, [Os-Cu] (10.9 mg, 0.01 mmol), and $\mathrm{CH}_{2} \mathrm{Cl}_{2}(4.0 \mathrm{~mL})$. The Schlenk tube was positioned approximately $3 \mathrm{~cm}$ away from a $24 \mathrm{~W}$ blue LEDs lamp $\left(\lambda_{\max }=450 \mathrm{~nm}\right)$. After being stirred at $25^{\circ} \mathrm{C}$ for the $48 \mathrm{~h}$ under an $\mathrm{O}_{2}$ (balloon) atmosphere, the reaction mixture was concentrated to dryness. The residue was purified by flash chromatography (petroleum ether/ethyl acetate $=10: 1)$ to afford product $\mathbf{6 g}$ as a colorless oil $(61.2 \mathrm{mg}, 0.112 \mathrm{mmol}$, yield: $56 \%)$.

${ }^{1}$ H NMR (500 MHz, CDCl 3$) \delta 7.63-7.54(\mathrm{~m}, 6 \mathrm{H}), 7.37$ - $7.30(\mathrm{~m}, 6 \mathrm{H}), 7.26$ - 7.23 (m, 3H), $5.31(\mathrm{dd}, J=7.8,4.9 \mathrm{~Hz}, 1 \mathrm{H}), 3.45(\mathrm{t}, J=3.9 \mathrm{~Hz}, 1 \mathrm{H}), 3.16(\mathrm{td}, J=7.4,2.3$ $\mathrm{Hz}, 2 \mathrm{H}), 2.89$ (td, $J=7.4,5.5 \mathrm{~Hz}, 2 \mathrm{H}), 2.09-1.96$ (m, 2H), $1.73-1.66$ (m, 1H), 1.61 - 1.52 (m, 2H), 1.49 - 1.41 (m, 1H), $1.25-1.18$ (m, 1H), 0.99 (d, J=6.8 Hz, 3H).

${ }^{13}$ C NMR (126 MHz, CDCl 3$) \delta 171.0,161.8,145.4,135.2,133.5,132.4,130.6,129.1$, 129.0, 128.7, 128.5 (2C), 128.1, 127.9, 127.2, 126.5, 74.4, 54.0, 31.6, 31.5, 30.7, 26.5, 23.6, 20.3 .

IR (film): $v\left(\mathrm{~cm}^{-1}\right)$ 2927, 2866, 1733, 1673, 1467, 1378, 1167, 1062, 962, 763, 738, 692.

HRMS (ESI, m/z) calcd for $\mathrm{C}_{31} \mathrm{H}_{31} \mathrm{NNaO}_{3} \mathrm{Se}(\mathrm{M}+\mathrm{H})^{+}:$: 546.1542, found: 546.1543 . 


\subsubsection{Synthesis of aspirin derivative $6 \mathrm{~h}$}

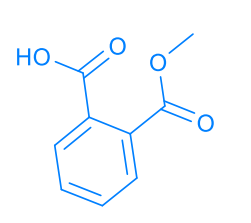

Aspirin

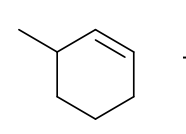

12
$\mathrm{PhSeSePh}$

3a

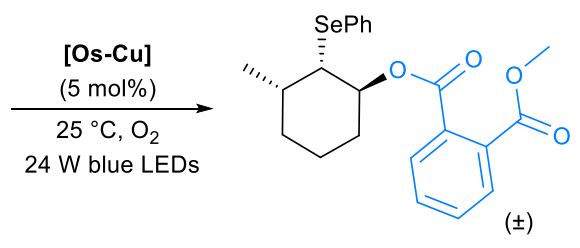

$6 \mathrm{~h}$

$70 \%$ yield

$>20: 1 \mathrm{dr},>20: 1 \mathrm{rr}$

Aspirin derivative

A dried $10 \mathrm{~mL}$ Schlenk tube was charged with $\mathbf{1 z}(24 \mu \mathrm{L}, 19.2 \mathrm{mg}, 0.20 \mathrm{mmol})$, aspirin (72 mg, $0.40 \mathrm{mmol}), \mathbf{3 a}(31.2 \mathrm{mg}, 0.10 \mathrm{mmol})$, [Os-Cu] (10.9 mg, $0.01 \mathrm{mmol})$, and $\mathrm{CH}_{2} \mathrm{Cl}_{2}(4.0 \mathrm{~mL})$. The Schlenk tube was positioned approximately $3 \mathrm{~cm}$ away from a $24 \mathrm{~W}$ blue LEDs lamp $\left(\lambda_{\max }=450 \mathrm{~nm}\right)$. After being stirred at $25{ }^{\circ} \mathrm{C}$ for the 48 $\mathrm{h}$ under an $\mathrm{O}_{2}$ (balloon) atmosphere, the reaction mixture was concentrated to dryness. The residue was purified by flash chromatography (petroleum ether/ethyl acetate $=$ 20:1) to afford product $\mathbf{6 h}$ as a yellow oil $(60.5 \mathrm{mg}, 0.140 \mathrm{mmol}$, yield: $70 \%)$.

${ }^{1}$ H NMR (500 MHz, CDCl 3$) ~ \delta 7.91(\mathrm{dd}, J=7.8,1.1 \mathrm{~Hz}, 1 \mathrm{H}), 7.61(\mathrm{dd}, J=6.4,2.9$ $\mathrm{Hz}, 2 \mathrm{H}), 7.54(\mathrm{td}, J=8.0,1.5 \mathrm{~Hz}, 1 \mathrm{H}), 7.28-7.21(\mathrm{~m}, 4 \mathrm{H}), 7.09(\mathrm{~d}, J=8.1 \mathrm{~Hz}, 1 \mathrm{H})$, $5.45(\mathrm{dd}, J=7.1,4.0 \mathrm{~Hz}, 1 \mathrm{H}), 3.57(\mathrm{~s}, 1 \mathrm{H}), 2.33(\mathrm{~s}, 3 \mathrm{H}), 2.24-2.16(\mathrm{~m}, 1 \mathrm{H}), 2.14-$ $2.07(\mathrm{~m}, 1 \mathrm{H}), 1.83-1.78(\mathrm{~m}, 1 \mathrm{H}), 1.70-1.63(\mathrm{~m}, 2 \mathrm{H}), 1.57-1.53(\mathrm{~m}, 1 \mathrm{H}), 1.34-$ $1.27(\mathrm{~m}, 1 \mathrm{H}), 1.09(\mathrm{~d}, J=6.8 \mathrm{~Hz}, 3 \mathrm{H})$.

${ }^{13}$ C NMR (126 MHz, CDCl $) \delta$ 169.7, 163.5, 150.8, 133.7, 133.5, 131.4, 130.6, 129.1, $127.1,125.9,123.7(2 \mathrm{C}), 74.7,54.0,31.9,30.8,26.5,21.1,20.6,20.5$.

IR (film): $v\left(\mathrm{~cm}^{-1}\right)$ 2960, 2850, 1733, 1460, 1389, 1238, 1066, 787, 656.

HRMS (ESI, m/z) calcd for $\mathrm{C}_{22} \mathrm{H}_{24} \mathrm{NaO}_{4} \mathrm{Se}(\mathrm{M}+\mathrm{Na})^{+}:$: 455.0732, found: 455.0735 . 


\subsubsection{Synthesis of isoxepac derivative 6i}

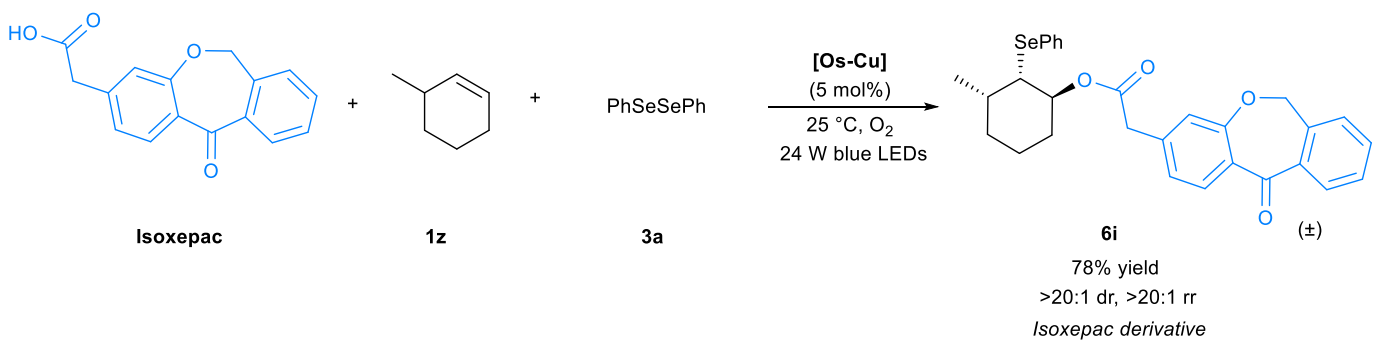

A dried $10 \mathrm{~mL}$ Schlenk tube was charged with $\mathbf{1 z}(24 \mu \mathrm{L}, 19.2 \mathrm{mg}, 0.20 \mathrm{mmol})$, isoxepac (107.2 mg, $0.40 \mathrm{mmol}), 3 \mathrm{a}(31.2 \mathrm{mg}, 0.10 \mathrm{mmol})$, [Os-Cu] (10.9 mg, 0.01 mmol), and $\mathrm{CH}_{2} \mathrm{Cl}_{2}(4.0 \mathrm{~mL})$. The Schlenk tube was positioned approximately $3 \mathrm{~cm}$ away from a $24 \mathrm{~W}$ blue LEDs lamp $\left(\lambda_{\max }=450 \mathrm{~nm}\right)$. After being stirred at $25^{\circ} \mathrm{C}$ for the $48 \mathrm{~h}$ under an $\mathrm{O}_{2}$ (balloon) atmosphere, the reaction mixture was concentrated to dryness. The residue was purified by flash chromatography (petroleum ether/ethyl acetate $=20: 1)$ to afford product $\mathbf{6 i}$ as a yellow oil $(81.1 \mathrm{mg}, 0.156 \mathrm{mmol}$, yield: $78 \%)$.

${ }^{1}$ H NMR (500 MHz, CDCl 3$) \delta 8.12(\mathrm{~d}, J=2.3 \mathrm{~Hz}, 1 \mathrm{H}), 7.89(\mathrm{dd}, J=7.7,1.0 \mathrm{~Hz}$, $1 \mathrm{H}), 7.57(\mathrm{ddd}, J=12.7,5.6,4.1 \mathrm{~Hz}, 3 \mathrm{H}), 7.47(\mathrm{td}, J=7.6,1.1 \mathrm{~Hz}, 1 \mathrm{H}), 7.41(\mathrm{dd}, J=$ 8.4, $2.4 \mathrm{~Hz}, 1 \mathrm{H}), 7.36$ (d, $J=7.4 \mathrm{~Hz}, 1 \mathrm{H}), 7.27-7.23(\mathrm{~m}, 3 \mathrm{H}), 7.02(\mathrm{~d}, J=8.4 \mathrm{~Hz}$, $1 \mathrm{H}), 5.27(\mathrm{dd}, J=7.5,4.5 \mathrm{~Hz}, 1 \mathrm{H}), 5.18(\mathrm{~s}, 2 \mathrm{H}), 3.59(\mathrm{~s}, 2 \mathrm{H}), 3.45(\mathrm{t}, J=3.9 \mathrm{~Hz}, 1 \mathrm{H})$, $2.10-1.97(\mathrm{~m}, 2 \mathrm{H}), 1.71-1.66(\mathrm{~m}, 1 \mathrm{H}), 1.59-1.53(\mathrm{~m}, 2 \mathrm{H}), 1.53-1.46(\mathrm{~m}, 1 \mathrm{H})$, $1.28-1.23(\mathrm{~m}, 1 \mathrm{H}), 1.06(\mathrm{~d}, J=6.8 \mathrm{~Hz}, 3 \mathrm{H})$.

${ }^{13}$ C NMR (126 MHz, CDCl $) \delta$ 190.8, 170.5, 160.4, 140.5, 136.4, 135.6, 133.5, 132.8 $132.5,130.6,129.5,129.3,129.1,127.9,127.8,127.2,125.1,121.0,74.5,73.6,53.9$, $40.6,31.7,30.8,26.6,20.3$.

IR (film): $v\left(\mathrm{~cm}^{-1}\right)$ 2988, 2863, 1735, 1673, 1389, 1243, 1112, 1057, 883, 752, 629.

HRMS (ESI, m/z) calcd for $\mathrm{C}_{29} \mathrm{H}_{28} \mathrm{NaO}_{4} \mathrm{Se}(\mathrm{M}+\mathrm{Na})^{+}: 543.1045$, found: 543.1047 . 


\subsection{Transformation of product}

\subsubsection{Removal of the phenylseleno group}

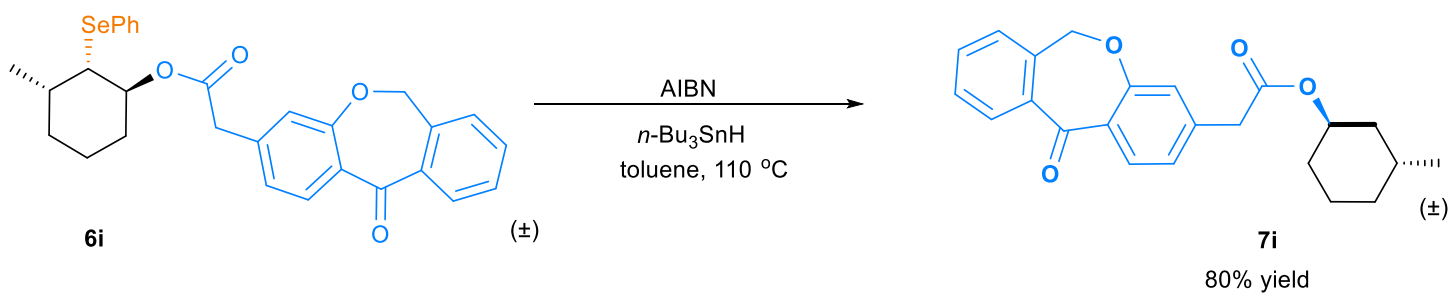

A mixture of $6 \mathbf{i}(100 \mathrm{mg}, 0.20 \mathrm{mmol})$, tri- $n$-butyltin hydride $(116.4 \mathrm{mg}, 0.40 \mathrm{mmol})$ and AIBN (0.6 mg, $0.004 \mathrm{mmol})$ in $2 \mathrm{~mL}$ toluene was refluxed under nitrogen for 2.0 hours. After cooled to ambient temperature all the volatiles were removed under reduced pressure. The resultant residue was purified by flash silica gel column chromatography (petroleum ether/ethyl acetate $=5: 1$ ), to afford product $7 \mathbf{i}$ as a yellow solid (58.2 mg, $0.16 \mathrm{mmol}$, yield: 80\%).

${ }^{1}$ H NMR (500 MHz, CDCl3) $\delta 8.14(\mathrm{~d}, J=1.9 \mathrm{~Hz}, 1 \mathrm{H}), 7.89(\mathrm{~d}, J=7.7 \mathrm{~Hz}, 1 \mathrm{H})$, $7.58-7.53(\mathrm{~m}, 1 \mathrm{H}), 7.49-7.42(\mathrm{~m}, 2 \mathrm{H}), 7.37(\mathrm{~d}, J=7.4 \mathrm{~Hz}, 1 \mathrm{H}), 7.03(\mathrm{~d}, J=8.4 \mathrm{~Hz}$, 1H), 5.19 (s, 2H), 5.08 (d, $J=2.6 \mathrm{~Hz}, 1 \mathrm{H}), 3.63$ (s, 2H), 1.78 (dd, $J=16.4,15.0 \mathrm{~Hz}$, 2H), $1.71-1.63(\mathrm{~m}, 2 \mathrm{H}), 1.52(\mathrm{dd}, J=11.1,7.6 \mathrm{~Hz}, 2 \mathrm{H}), 1.45-1.31(\mathrm{~m}, 2 \mathrm{H}), 1.18-$ $1.11(\mathrm{~m}, 1 \mathrm{H}), 0.86(\mathrm{~d}, J=6.5 \mathrm{~Hz}, 3 \mathrm{H})$.

${ }^{13}$ C NMR (126 MHz, CDCl 3$) \delta 190.9,170.8,160.4,140.5,136.4,135.6,132.7,132.5$, $129.5,129.3,128.3,127.8,125.1,120.9,73.7,71.1,40.8,38.3,34.1,29.8,27.0,22.1$, 20.6 .

IR (film): $v\left(\mathrm{~cm}^{-1}\right)$ 2988, 2863, 1735, 1653, 1380, 1241, 1057, 882, 741.

HRMS (ESI, m/z) calcd for $\mathrm{C}_{23} \mathrm{H}_{24} \mathrm{NaO}_{4}(\mathrm{M}+\mathrm{Na})^{+}: 387.1567$, found: 387.1567 .

\subsubsection{Synthesis of selenoxide 80}

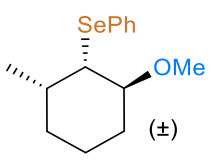

4zc

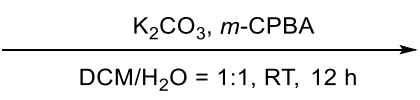

8a, $79 \%$ yield $>20: 1 \mathrm{dr},>50: 1 \mathrm{rr}$ 
A dried $50 \mathrm{~mL}$ round-bottom flask was charged with 40 (310 mg, $1.10 \mathrm{mmol}), \mathrm{K}_{2} \mathrm{CO}_{3}$ aq (11mL, sat.), and $\mathrm{CH}_{2} \mathrm{Cl}_{2}(11 \mathrm{~mL})$. After that, $m$-CPBA (246.8 mg, $1.40 \mathrm{mmol}, 77 \%$ purity, 1.3 equiv.) was added portionwise, and allowed to stir for $4 \mathrm{~h}$ at $25{ }^{\circ} \mathrm{C} . \mathrm{H}_{2} \mathrm{O}$ $(50 \mathrm{~mL})$ was added, phases were separated, aqueous phase was extracted with dichloromethane $(2 \times 50 \mathrm{~mL})$, combined organic phases were washed with $\mathrm{H}_{2} \mathrm{O}(100$ $\mathrm{mL}$ ), and dried over anhydrous $\mathrm{Na}_{2} \mathrm{SO}_{4}$. Mixture was filtered, evaporated, and separated with column chromatography (ethyl acetate) to afford product $\mathbf{8 0}$ as white crystal ( $260 \mathrm{mg}, 0.87 \mathrm{mmol}$, yield: 79\%).

${ }^{1}$ H NMR (500 MHz, CDCl $) \delta 7.78-7.72(\mathrm{~m}, 2 \mathrm{H}), 7.51-7.41(\mathrm{~m}, 3 \mathrm{H}), 3.76(\mathrm{td}, J=$ 10.7, $4.3 \mathrm{~Hz}, 1 \mathrm{H}), 3.38(\mathrm{~s}, 3 \mathrm{H}), 2.96(\mathrm{dd}, J=11.0,4.3 \mathrm{~Hz}, 1 \mathrm{H}), 2.42(\mathrm{pd}, J=7.0,4.0$ $\mathrm{Hz}, 1 \mathrm{H}), 2.31-2.23(\mathrm{~m}, 1 \mathrm{H}), 1.62-1.50(\mathrm{~m}, 3 \mathrm{H}), 1.37$ (ddd, $J=17.6,9.1,4.3 \mathrm{~Hz}$, $1 \mathrm{H}), 1.16(\mathrm{~d}, J=7.1 \mathrm{~Hz}, 3 \mathrm{H}), 1.10(\mathrm{tdd}, J=12.5,10.6,5.0 \mathrm{~Hz}, 1 \mathrm{H})$.

${ }^{13}$ C NMR (126 MHz, CDCl 3$) \delta 141.8,130.5,129.2,126.7,75.8,71.0,56.1,33.2$, $32.0,29.7,18.9,14.5$.

IR (film): $v\left(\mathrm{~cm}^{-1}\right)$ 2988, 2920, 1451, 1380, 1241, 1077, 1046, 752.

HRMS (ESI, m/z) calcd for $\mathrm{C}_{28} \mathrm{H}_{41} \mathrm{NaO}_{4} \mathrm{Se}_{2}(2 \mathrm{M}+\mathrm{H})^{+}: 601.1335$, found: 601.1344.

\subsection{Reaction at a gram scale}
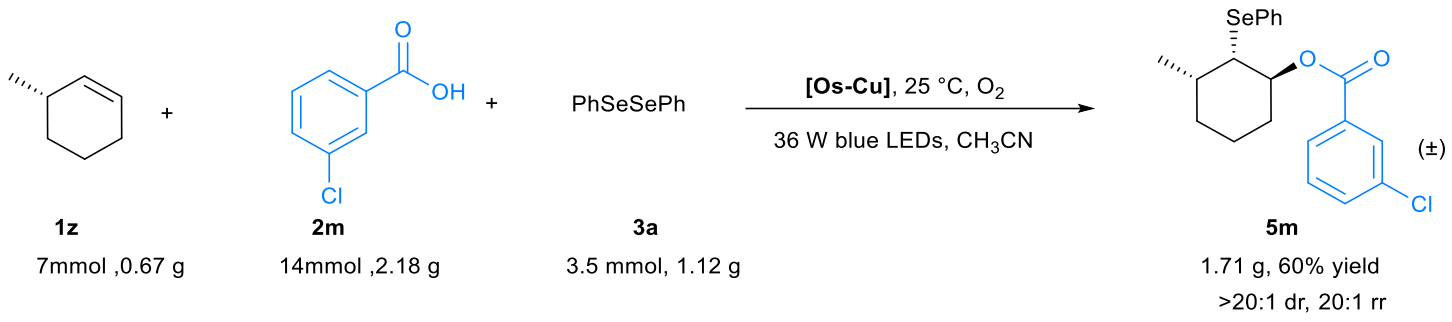

A dried $250 \mathrm{~mL}$ Schlenk tube was charged with $\mathbf{1 z}(0.67 \mathrm{~g}, 7.00 \mathrm{mmol}), \mathbf{2 n}(2.18 \mathrm{~g}$, $14.00 \mathrm{mmol}), 3 \mathbf{a}(1.12 \mathrm{~g}, 3.50 \mathrm{mmol})$, [Os-Cu] (763 mg, $0.70 \mathrm{mmol})$, and $\mathrm{CH}_{3} \mathrm{CN}$ (70 $\mathrm{mL}$ ). The Schlenk tube was positioned approximately $3 \mathrm{~cm}$ away from a $24 \mathrm{~W}$ blue LEDs lamp $\left(\lambda_{\max }=450 \mathrm{~nm}\right)$. After being stirred at $25{ }^{\circ} \mathrm{C}$ for the $120 \mathrm{~h}$ under an $\mathrm{O}_{2}$ (balloon) atmosphere, the reaction mixture was concentrated to dryness. The residue 
was purified by flash chromatography (petroleum ether/ethyl acetate $=40: 1$ ) to afford product $\mathbf{5 m}$ as a yellow oil (1.71g, $4.19 \mathrm{mmol}$, yield: 60\%).

\section{Mechanistic Investigations}

\subsection{Variations from the standard conditions (Table S2)}

Table S2. Variations from the standard conditions

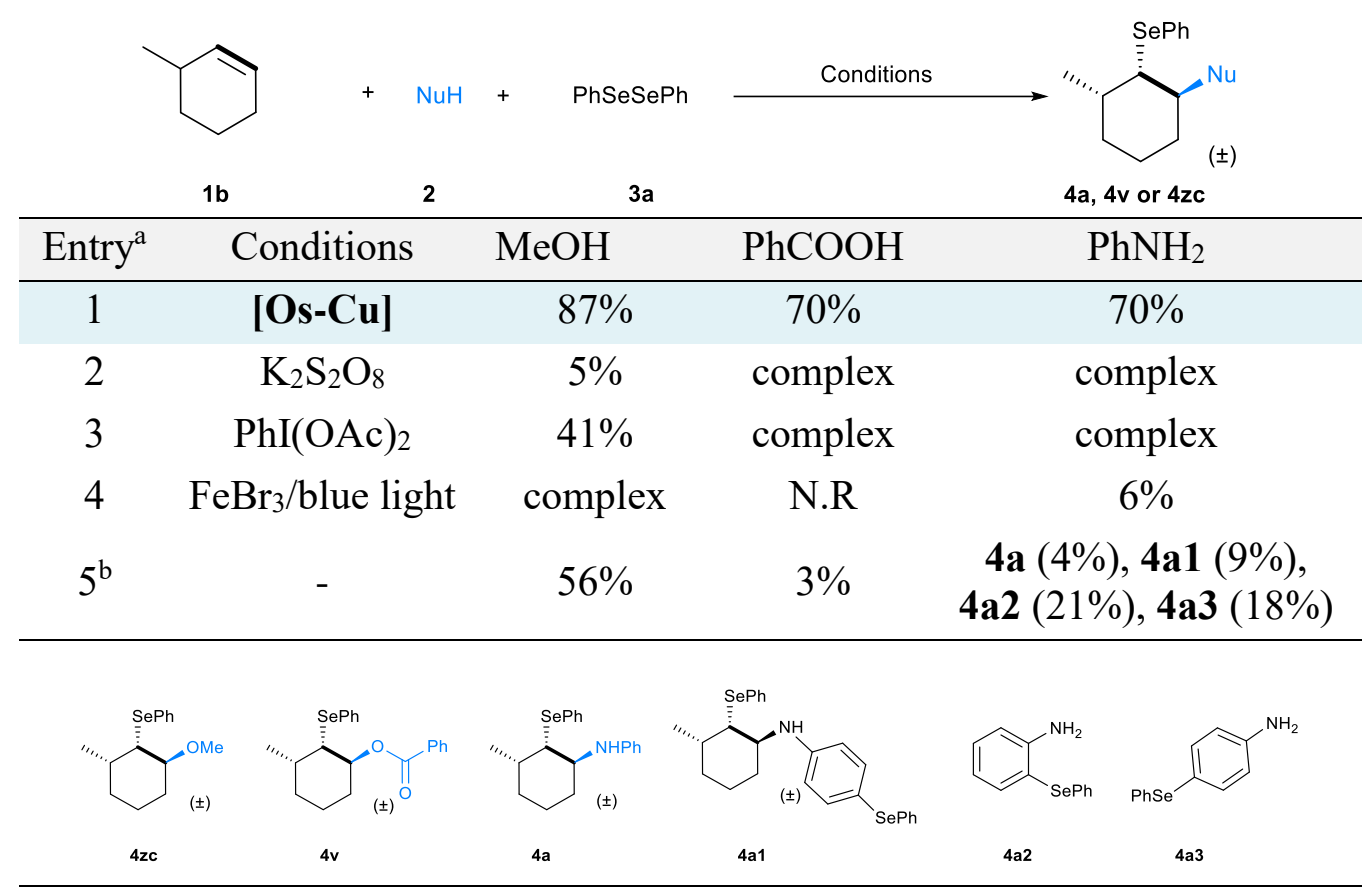

${ }^{a}$ Isolated yield. ${ }^{b}$ Replacement of $\mathrm{PhSeSePh}$ with $\mathrm{PhSeCl}$. ${ }^{\mathrm{c}}$ 1:1 ratio of 3-methylcyclohex-1-ene and nucleophile complex $=$ more than four species mixture.

Previously, two classical methods have been employed for vicinal difunctionalized organoselenium compounds, i.e. in situ generation of free selenonium in the presence of oxidants (e.g., $\mathrm{K}_{2} \mathrm{~S}_{2} \mathrm{O}_{8}, \mathrm{PhI}(\mathrm{OAc})_{2}$ ) or direct make use of ArSeX reagents (e.g., $\mathrm{PhSeCl})$. In order to gain insight of [Os-Cu] catalyst involvement in the reaction, a series of control experiments have been performed. The seleno-difunctionalization of 3-methyl-cyclohexene with three types of nucleophiles under the reported reaction conditions were conducted (Table S2). Using $\mathrm{K}_{2} \mathrm{~S}_{2} \mathrm{O}_{8}$ or $\mathrm{PhI}(\mathrm{OAc})_{2}$ as the oxidant and $\mathrm{MeOH}$ as nucleophile, product $4 \mathrm{zc}$ could be obtained in $5 \%$ or $41 \%$ yields. However, the reactions were carried with $\mathrm{PhCOOH}$ or $\mathrm{PhNH}_{2}$ as the nucleophiles, gave only a 
complex reaction mixture (entries 2 and 3). The reaction run under the condition of $\mathrm{FeBr}_{3}$ and visible-light irradiation afforded a complex mixture for $\mathrm{MeOH}$, while $\mathrm{PhCOOH}$ or $\mathrm{PhNH}_{2}$ led to only no reaction or a negligible amount of $4 \mathbf{a}$ (6\% yield) (entry 4). Replacing $\mathrm{PhSeSePh}$ with $\mathrm{PhSeCl}$ as the selenation reagent in the reaction with $\mathrm{MeOH}$ can give rise to product $4 \mathbf{z c}$ in $56 \%$ yield. Nevertheless, the reactions with $\mathrm{PhCOOH}$ or $\mathrm{PhNH}_{2}$ led to the formation of products (4a or $\mathbf{4 u}$ ) in very low yields $(3 \%$ or $4 \%$ ), inversely affording the undesired mixtures $4 \mathbf{a} 1,4 \mathbf{a} 2$ and $\mathbf{4 a 3}$ (entry 5). These results demonstrate the title [Os-Cu] system exhibits significantly enhanced catalytic performance for the reaction efficiency and selectivity as well as substrate expansion.

General Procedure for entry 1 (MeOH as a nucleophile):A dried $10 \mathrm{~mL}$ Schlenk tube was charged with 1a $(24 \mu \mathrm{L}, 19.2 \mathrm{mg}, 0.20 \mathrm{mmol}), \mathrm{CH}_{3} \mathrm{OH}(0.5 \mathrm{ml}), 3 \mathbf{a}(31.2 \mathrm{mg}$, $0.10 \mathrm{mmol})$, [Os-Cu] (10.9 mg, $0.01 \mathrm{mmol})$, and $\mathrm{CH}_{2} \mathrm{Cl}_{2}(3.5 \mathrm{~mL})$. The Schlenk tube was positioned approximately $3 \mathrm{~cm}$ away from a $24 \mathrm{~W}$ blue LEDs lamp $\left(\lambda_{\max }=450\right.$ nm). After being stirred at $25{ }^{\circ} \mathrm{C}$ for the $48 \mathrm{~h}$ under an $\mathrm{O}_{2}$ (balloon) atmosphere, the reaction mixture was concentrated to dryness. The residue was purified by flash chromatography (petroleum ether/ethyl acetate $=50: 1$ ) to afford pure product.

General Procedure for entry 1 (PhCOOH as a nucleophile): A dried $10 \mathrm{~mL}$ Schlenk tube was charged with 1a $(24 \mu \mathrm{L}, 19.2 \mathrm{mg}, 0.20 \mathrm{mmol})$, PhCOOH (48.8 mg, 0.40 mmol), 3a (31.2 mg, $0.10 \mathrm{mmol})$, [Os-Cu] (10.9 mg, $0.01 \mathrm{mmol})$, and $\mathrm{CH}_{2} \mathrm{Cl}_{2}$ (4.0 $\mathrm{mL}$ ). The Schlenk tube was positioned approximately $3 \mathrm{~cm}$ away from a $24 \mathrm{~W}$ blue LEDs lamp $\left(\lambda_{\max }=450 \mathrm{~nm}\right)$. After being stirred at $25^{\circ} \mathrm{C}$ for the $48 \mathrm{~h}$ under an $\mathrm{O}_{2}$ (balloon) atmosphere, the reaction mixture was concentrated to dryness. The residue was purified by flash chromatography (petroleum ether/ethyl acetate $=40: 1$ ) to afford pure product.

General Procedure for entry 1 ( $\mathrm{PhNH}_{2}$ as a nucleophile): A dried $10 \mathrm{~mL}$ Schlenk tube was charged with 1a $(24 \mu \mathrm{L}, 19.2 \mathrm{mg}, 0.20 \mathrm{mmol})$, phenylamine $(37 \mu \mathrm{L}, 37.3 \mathrm{mg}$, 
$0.40 \mathrm{mmol}), 3 \mathbf{a}(31.2 \mathrm{mg}, 0.10 \mathrm{mmol})$, [Os-Cu] (10.9 mg, $0.01 \mathrm{mmol})$, and $\mathrm{CH}_{2} \mathrm{Cl}_{2}$ $(4.0 \mathrm{~mL})$. The Schlenk tube was positioned approximately $3 \mathrm{~cm}$ away from a $24 \mathrm{~W}$ blue LEDs lamp $\left(\lambda_{\max }=450 \mathrm{~nm}\right)$. After being stirred at $25^{\circ} \mathrm{C}$ for the $48 \mathrm{~h}$ under an $\mathrm{O}_{2}$ (balloon) atmosphere, the reaction mixture was concentrated to dryness. The residue was purified by flash chromatography (petroleum ether/ethyl acetate $=40: 1$ ) to afford pure product.

General Procedure for entry 2: A dried $10 \mathrm{~mL}$ Schlenk tube was charged with 1a (0.5 mmol), 2 (0.55 mmol), $\mathrm{K}_{2} \mathrm{~S}_{2} \mathrm{O}_{8}(1.0 \mathrm{mmol}), 3 \mathrm{a}(0.5 \mathrm{mmol})$ in $2 \mathrm{~mL}$ THF, stirred at 80 ${ }^{\circ} \mathrm{C}$ for $12 \mathrm{~h}$. The reaction mixture was concentrated to dryness. The residue was purified by flash chromatography (petroleum ether/ethyl acetate) to afford pure product.

General Procedure for entry 3: A dried $10 \mathrm{~mL}$ Schlenk tube was charged with 1a (0.25 mmol), 3a (0.25 mmol), $\mathrm{PhI}(\mathrm{OAc})_{2}(0.25 \mathrm{mmol}), 2(1.0 \mathrm{mmol})$, and $\mathrm{CH}_{2} \mathrm{Cl}_{2}(2.0$ $\mathrm{mL}$ ) in an open flask at room temperature for $20 \mathrm{~h}$. The reaction mixture was concentrated to dryness. The residue was purified by flash chromatography (petroleum ether/ethyl acetate) to afford pure product.

General Procedure for entry 4: A dried $10 \mathrm{~mL}$ Schlenk tube was charged with 1a (0.1 $\mathrm{mmol}), 2$ (0.15 mmol), 3a (0.06 mmol), $\mathrm{FeBr}_{3}(10 \mathrm{~mol} \%)$, and EtOAc (1.0 mL), in air, at room temperature, under the irradiation of $24 \mathrm{~W}$ blue LEDs for $24 \mathrm{~h}$. The reaction mixture was concentrated to dryness. The residue was purified by flash chromatography (petroleum ether/ethyl acetate) to afford pure product.

General Procedure for entry 5: A dried $10 \mathrm{~mL}$ Schlenk tube was charged with 1a (0.5 mmol), 2 (0.55 mmol), $\mathrm{PhSeCl}(0.5 \mathrm{mmol})$ in $2 \mathrm{~mL} \mathrm{CH}_{2} \mathrm{Cl}_{2}$, stirred at $25{ }^{\circ} \mathrm{C}$ for $24 \mathrm{~h}$. The reaction mixture was concentrated to dryness. The residue was purified by flashchromatography (petroleum ether/ethyl acetate) to afford pure product 


\subsection{Control experiments}

A. Evidence of interaction between nucleophile and catalyst

(i)

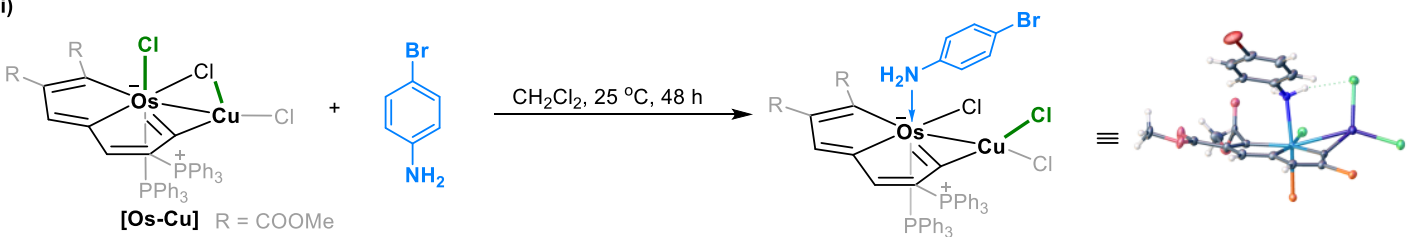

[Os-Cu-L1], 92\%

$92 \%\left(24 \mathrm{~W}\right.$ blue LEDs, $\mathrm{O}_{2}$ ) $90 \%\left(24 \mathrm{~W}\right.$ blue LEDs, $\mathrm{N}_{2}$ )

(ii) $90 \%$ (dark, $\mathrm{O}_{2}$.)

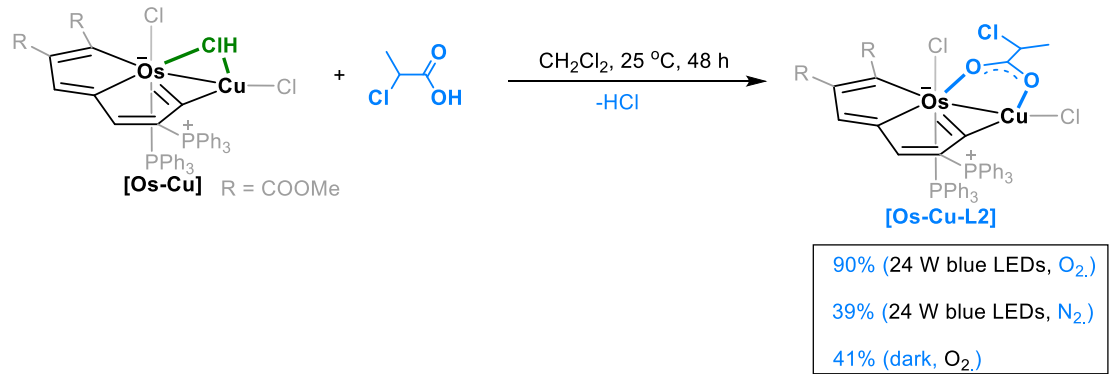

B. Stoichiometric reactions with or without $\mathrm{O}_{2}$ and light

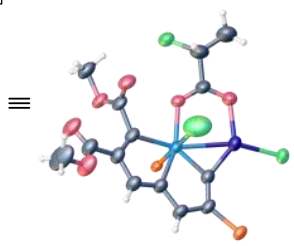

(i)

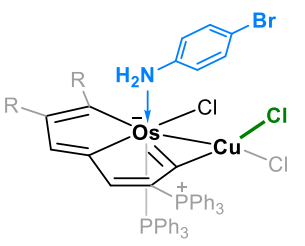

[Os-Cu-L1]<smiles>CC1C=C[C+]CC1</smiles>

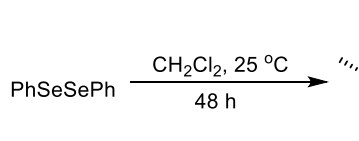

$3 a$

(ii)

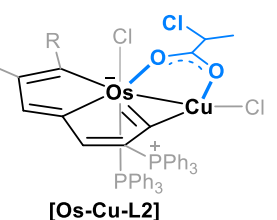<smiles>CC1C=C[CH+]CC1</smiles>

$\underset{48 \mathrm{~h}}{\stackrel{\mathrm{CH}_{2} \mathrm{Cl}_{2}, 25^{\circ} \mathrm{C}}{\longrightarrow}}$

$3 a$

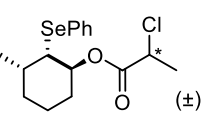

$( \pm$

$4 u, 1: 1 d r$
$0 \%$ (dark, $\mathrm{O}_{2}$ )

$65 \%\left(24 \mathrm{~W}\right.$ blue LEDs, $\mathrm{O}_{2}$ )

$63 \%\left(24 \mathrm{~W}\right.$ blue LEDs, $\mathrm{N}_{2}$ )

C. The isolated intermediate species as the catalyst

(i)<smiles>CC1C=CCCC1</smiles><smiles>Nc1ccc(Br)cc1</smiles>

$\mathrm{PhSeSePh}$

$3 a$

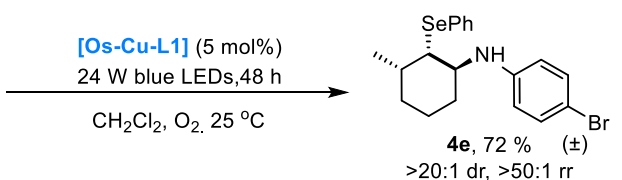

(ii)<smiles>CC1C=CCCC1</smiles>

1a<smiles>CC(O)C(C)Cl</smiles>

$\mathrm{OH}$
PhSeSePh

$3 a$

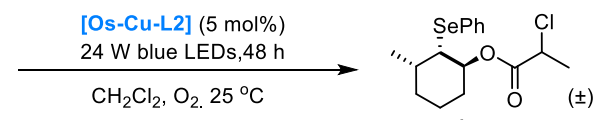

$4 u, 69 \%$ $1: 1 \mathrm{dr}$

Figure S1. Control experiments.

The stoichiometric reactions of the [Os-Cu] catalyst with amine or carboxylic acid generate [Os-Cu-L1] and [Os-Cu-L2] in high yields under the optimized reaction conditions (Figure S1, A). Further experiments showed that the formation of 
[Os-Cu-L1] can be also proceeded under a nitrogen atmosphere or in dark. In contrast, parallel experiments carried under a nitrogen atmosphere or in dark provided significantly decreased yields of [Os-Cu-L2] with 39\% and 41\%, respectively (Figure S1, A). The results infer both light and oxygen are essential for the formation of [Os-Cu-L2], which may play an important role in the deprotonation of carboxylic acid.

Next, stoichiometric reaction of [Os-Cu-L1] in oxygen atmosphere under the irradiation of $24 \mathrm{~W}$ blue LEDs led to the formation of $4 \mathbf{e}$ in $68 \%$ yield but the similar reaction carried without oxygen led to significantly decrease the yield to $17 \%$ (Figure $\mathrm{S} 1, \mathrm{~B})$. In contrast, since the proton on the carboxylic acid has been removed in [Os-Cu-L2], $4 \mathbf{u}$ can be produced in comparable yields $(65 \%$ and $63 \%)$ under the irradiation of $24 \mathrm{~W}$ blue LEDs with or without $\mathrm{O}_{2}$ in the stoichiometric reaction (Figure S1, B). The outcomes infer the proton of the nucleophile can be abstracted by oxygen enabled by the light.

Procedure for (Ai): A dried $10 \mathrm{~mL}$ Schlenk tube was charged with 4-bromoaniline (68.8 mg, $0.4 \mathrm{mmol})$, [Os-Cu] $(87.2 \mathrm{mg}, 0.08 \mathrm{mmol})$ and $\mathrm{CH}_{2} \mathrm{Cl}_{2}(4.0 \mathrm{~mL})$. The mixture was stirred at $25{ }^{\circ} \mathrm{C}$ under the irradiation of $24 \mathrm{~W}$ blue LEDs for $48 \mathrm{~h}$ in $\mathrm{O}_{2}$ (balloon) atmosphere. Then the solution was evaporated was evaporated under vacuum to a volume of approximately $2.0 \mathrm{~mL}$, and diethyl ether $(20 \mathrm{~mL})$ was added to the solution. The precipitate was collected by filtration, washed with diethyl ether $(2 \times 10 \mathrm{~mL})$ and dried under vacuum to give a yellow solid (yield: $92 \%)$. Recrystallization from a solution of $\mathrm{CH}_{2} \mathrm{Cl}_{2}$ layered with $n$-hexane and was further confirmed by a single crystal structure of [Os-Cu-L1].

Procedure for (Aii): A dried $10 \mathrm{~mL}$ Schlenk tube was charged with 2-chloropropanoic acid (42.8 mg, $0.40 \mathrm{mmol})$, [Os-Cu] $(87.2 \mathrm{mg}, 0.08 \mathrm{mmol})$ and $\mathrm{CH}_{2} \mathrm{Cl}_{2}(4.0 \mathrm{~mL})$. The mixture was stirred at $25{ }^{\circ} \mathrm{C}$ under the irradiation of $24 \mathrm{~W}$ blue LEDs for $48 \mathrm{~h}$ in $\mathrm{O}_{2}$ (balloon) atmosphere. Then the solution was evaporated under vacuum to a volume of approximately $2.0 \mathrm{~mL}$, and diethyl ether $(20 \mathrm{~mL})$ was added to the solution. The 
precipitate was collected by filtration, washed with diethyl ether $(2 \times 10 \mathrm{~mL})$ and dried under vacuum to give a yellow solid (yield: 90\%). Recrystallization from a solution of $\mathrm{CH}_{2} \mathrm{Cl}_{2}$ layered with $n$-hexane and was further confirmed by a single crystal structure of [Os-Cu-L2].

Procedure for (Bi, dark, $\left.\mathrm{O}_{2}\right)$ : A dried $10 \mathrm{~mL}$ Schlenk tube was charged with $1 \mathrm{a}(12 \mu \mathrm{L}$, $9.6 \mathrm{mg}, 0.10 \mathrm{mmol}), 3 \mathbf{a}(15.6 \mathrm{mg}, 0.05 \mathrm{mmol})$, [Os-Cu-L1] (125 mg, $0.1 \mathrm{mmol})$, and $\mathrm{CH}_{2} \mathrm{Cl}_{2}(4.0 \mathrm{~mL})$. The reaction mixture was stirred at $25^{\circ} \mathrm{C}$ for the $48 \mathrm{~h}$ under an $\mathrm{O}_{2}$ (balloon) atmosphere in dark.

Procedure for (Bi, $24 \mathrm{~W}$ blue LEDs, $\left.\mathrm{O}_{2}\right)$ : A dried $10 \mathrm{~mL}$ Schlenk tube was charged with 1a $(12 \mu \mathrm{L}, 9.6 \mathrm{mg}, 0.10 \mathrm{mmol}), \mathbf{3 a}(15.6 \mathrm{mg}, 0.05 \mathrm{mmol})$, [Os-Cu-L1] (125 mg, $0.1 \mathrm{mmol})$, and $\mathrm{CH}_{2} \mathrm{Cl}_{2}(4.0 \mathrm{~mL})$. The Schlenk tube was positioned approximately 3 $\mathrm{cm}$ away from a $24 \mathrm{~W}$ blue LEDs lamp $\left(\lambda_{\max }=450 \mathrm{~nm}\right)$. After being stirred at $25^{\circ} \mathrm{C}$ for the $48 \mathrm{~h}$ under an $\mathrm{O}_{2}$ (balloon) atmosphere, the reaction mixture was concentrated to dryness. The residue was purified by flash chromatography (petroleum ether/ethyl acetate $=40: 1)$ to afford product $4 \mathbf{e}$ as a brown oil $(28.6 \mathrm{mg}, 0.068 \mathrm{mmol}$, yield: $68 \%$ ).

Procedure for (Bi, $24 \mathrm{~W}$ blue LEDs, $\left.N_{2}\right)$ : A dried $10 \mathrm{~mL}$ Schlenk tube was charged with 1a (12 $\mu \mathrm{L}, 9.6 \mathrm{mg}, 0.10 \mathrm{mmol}), \mathbf{3 a}(15.6 \mathrm{mg}, 0.05 \mathrm{mmol})$, [Os-Cu-L1] (125 mg, $0.1 \mathrm{mmol})$, and $\mathrm{CH}_{2} \mathrm{Cl}_{2}(4.0 \mathrm{~mL})$. The Schlenk tube was positioned approximately 3 $\mathrm{cm}$ away from a $24 \mathrm{~W}$ blue LEDs lamp $\left(\lambda_{\max }=450 \mathrm{~nm}\right)$. After being stirred at $25^{\circ} \mathrm{C}$ for the $48 \mathrm{~h}$ under an $\mathrm{N}_{2}$ (balloon) atmosphere, the reaction mixture was concentrated to dryness. The residue was purified by flash chromatography (petroleum ether/ethyl acetate $=40: 1)$ to afford product $4 \mathbf{e}$ as a brown oil $(7.18 \mathrm{mg}, 0.017 \mathrm{mmol}$, yield: $17 \%)$.

Procedure for (Bii, dark, $\mathrm{O}_{2}$ ): A dried $10 \mathrm{~mL}$ Schlenk tube was charged with 1a (12 $\mu \mathrm{L}, 9.6 \mathrm{mg}, 0.10 \mathrm{mmol})$, 2-chloropropionic acid (37 $\mu \mathrm{L}, 43.2 \mathrm{mg}, 0.40 \mathrm{mmol}), 3 \mathbf{a}$ (15.6 mg, $0.05 \mathrm{mmol}$ ), [Os-Cu-L2] (116 mg, $0.1 \mathrm{mmol}$ ), and $\mathrm{CH}_{2} \mathrm{Cl}_{2}(4.0 \mathrm{~mL})$. The reaction mixture was stirred at $25^{\circ} \mathrm{C}$ for the $48 \mathrm{~h}$ under an $\mathrm{O}_{2}$ (balloon) atmosphere in dark. 
Procedure for (Bii, $24 \mathrm{~W}$ blue LEDs, $\mathrm{O}_{2}$ ): A dried $10 \mathrm{~mL}$ Schlenk tube was charged with 1a $(12 \mu \mathrm{L}, 9.6 \mathrm{mg}, 0.10 \mathrm{mmol}), 3 \mathbf{a}(15.6 \mathrm{mg}, 0.05 \mathrm{mmol})$, [Os-Cu-L2] (11.6 mg, $0.01 \mathrm{mmol})$, and $\mathrm{CH}_{2} \mathrm{Cl}_{2}(4.0 \mathrm{~mL})$. The Schlenk tube was positioned approximately 3 $\mathrm{cm}$ away from a $24 \mathrm{~W}$ blue LEDs lamp $\left(\lambda_{\max }=450 \mathrm{~nm}\right)$. After being stirred at $25^{\circ} \mathrm{C}$ for the $48 \mathrm{~h}$ under an $\mathrm{O}_{2}$ (balloon) atmosphere, the reaction mixture was concentrated to dryness. The residue was purified by flash chromatography (petroleum ether/ethyl acetate $=40: 1)$ to afford product $\mathbf{4} \mathbf{u}$ as a colorless oil $(23.5 \mathrm{mg}, 0.065 \mathrm{mmol}$, yield: $65 \%$ ).

Procedure for (Bii, $24 \mathrm{~W}$ blue LEDs, $N_{2}$ ): A dried $10 \mathrm{~mL}$ Schlenk tube was charged with $1 \mathrm{a}(12 \mu \mathrm{L}, 9.6 \mathrm{mg}, 0.10 \mathrm{mmol})$, 2-chloropropionic acid (37 $\mu \mathrm{L}, 43.2 \mathrm{mg}, 0.40$ mmol), 3a (15.6 mg, $0.05 \mathrm{mmol})$, [Os-Cu-L2] (11.6 mg, $0.01 \mathrm{mmol})$, and $\mathrm{CH}_{2} \mathrm{Cl}_{2}$ $(4.0 \mathrm{~mL})$. The Schlenk tube was positioned approximately $3 \mathrm{~cm}$ away from a $24 \mathrm{~W}$ blue LEDs lamp $\left(\lambda_{\max }=450 \mathrm{~nm}\right)$. After being stirred at $25{ }^{\circ} \mathrm{C}$ for the $48 \mathrm{~h}$ under an $\mathrm{O}_{2}$ (balloon) atmosphere, the reaction mixture was concentrated to dryness. The residue was purified by flash chromatography (petroleum ether/ethyl acetate $=40: 1$ ) to afford product $\mathbf{4} \mathbf{u}$ as a colorless oil (22.6 $\mathrm{mg}, 0.063 \mathrm{mmol}$, yield: 63\%).

Procedure for (Ci): A dried $10 \mathrm{~mL}$ Schlenk tube was charged with 1a $(24 \mu \mathrm{L}, 19.2 \mathrm{mg}$, $0.20 \mathrm{mmol}$ ), 4-bromoaniline (68 $\mathrm{mg}, 0.40 \mathrm{mmol}), 3 \mathbf{a}(31.2 \mathrm{mg}, 0.10 \mathrm{mmol})$, [Os-Cu-L1] (12.5 mg, $0.01 \mathrm{mmol})$, and $\mathrm{CH}_{2} \mathrm{Cl}_{2}(4.0 \mathrm{~mL})$. The Schlenk tube was positioned approximately $3 \mathrm{~cm}$ away from a $24 \mathrm{~W}$ blue LEDs lamp ( $\lambda_{\max }=450 \mathrm{~nm}$ ). After being stirred at $25{ }^{\circ} \mathrm{C}$ for the $48 \mathrm{~h}$ under an $\mathrm{O}_{2}$ (balloon) atmosphere, the reaction mixture was concentrated to dryness. The residue was purified by flash chromatography (petroleum ether/ethyl acetate $=40: 1$ ) to afford product $4 \mathbf{e}$ as a brown oil (60.5 mg, $0.143 \mathrm{mmol}$, yield: $72 \%)$.

Procedure for (Cii): A dried $10 \mathrm{~mL}$ Schlenk tube was charged with 1a $(24 \mu \mathrm{L}, 19.2$ $\mathrm{mg}, 0.20 \mathrm{mmol}), 2$-chloropropionic acid $(37 \mu \mathrm{L}, 43.2 \mathrm{mg}, 0.40 \mathrm{mmol}), 3 \mathrm{a}(31.2 \mathrm{mg}$, $0.10 \mathrm{mmol})$, [Os-Cu-L2] (11.6 mg, $0.01 \mathrm{mmol})$, and $\mathrm{CH}_{2} \mathrm{Cl}_{2}(4.0 \mathrm{~mL})$. The Schlenk 
tube was positioned approximately $3 \mathrm{~cm}$ away from a $24 \mathrm{~W}$ blue LEDs lamp $\left(\lambda_{\max }=\right.$ $450 \mathrm{~nm}$ ). After being stirred at $25^{\circ} \mathrm{C}$ for the $48 \mathrm{~h}$ under an $\mathrm{O}_{2}$ (balloon) atmosphere, the reaction mixture was concentrated to dryness. The residue was purified by flash chromatography (petroleum ether/ethyl acetate $=40: 1$ ) to afford product $\mathbf{4 u}$ as a colorless oil (49.3 mg, $0.137 \mathrm{mmol}$, yield: 69\%).

\subsection{Proposed catalytic cycle.}

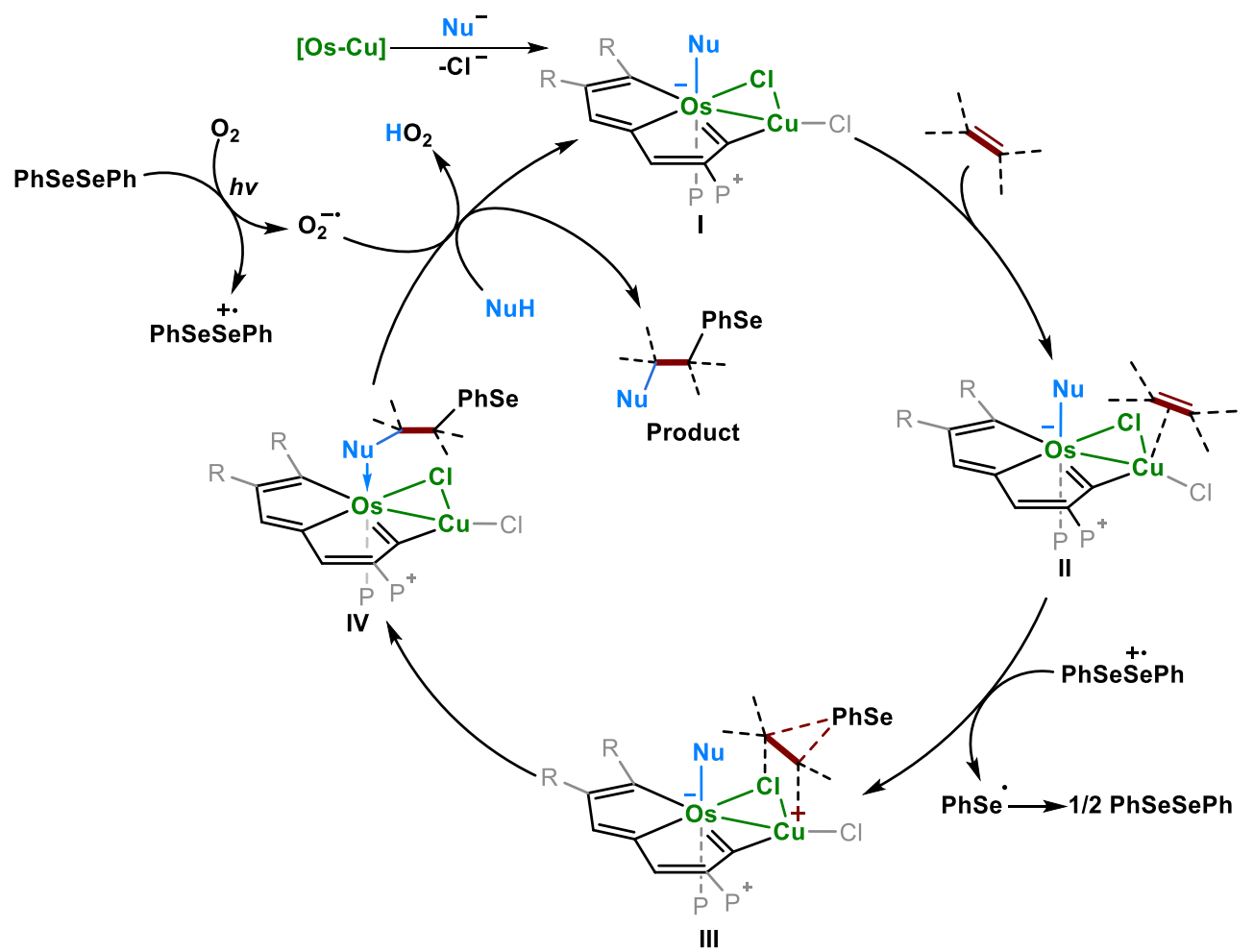

Figure S2. Proposed catalytic cycle.

A possible reaction mechanism was proposed. Under the aerobic and visible light conditions, the selenium radical species $[\mathrm{PhSeSePh}]^{+\bullet}$ was formed from the diphenyl diselenide. The catalyst [Os-Cu] undergoes fast ligand exchange with the $\mathrm{N}$ - or O-nucleophile to generate an intermediate complex (I). The $\mathrm{Cu}$ in I interacts with the alkene and brings the two substrates into close proximity, leading to complex II. Then the electrophile $[\mathrm{PhSeSePh}]^{+\bullet}$ attacks the coordinated alkene to generate intermediate 
III which bears a seleniranium cation and interacts with the chloride bridge. With the electronic and steric effects, the nucleophile will selectively interact with one side of the seleniranium cation to give intermediate IV. The final ligand exchange leads to release of the product and regeneration of I. Specifically, the metallaaromatic feature of $[\mathbf{O s}-\mathbf{C u}]$ permits electronic delocalization within the metallacyclic core. In this context, the charge of two metal centers may be better viewed integrally during the catalytic process.

\section{Single-Crystal X-Ray Diffraction}

Single-crystal X-ray diffraction data were collected on a Rigaku XtaLAB Synergy, Dualflex,Rigaku XtaLAB Synergy-S diffractometer coupled to a RigakuHypix detector with $\mathrm{Cu} \mathrm{K} \alpha$ radiation $(\lambda=1.54184 \AA$ ). All data were corrected for absorption effects using the multi-scan techniqueSingle. crystals suitable for X-ray diffraction were obtained by recrystallization from a solution of $\mathrm{CH}_{2} \mathrm{Cl}_{2}$ layered with $n$-hexane. The crystal was kept at a steady $\mathrm{T}=100 \mathrm{~K}$ during the data collection. The structures were solved with the SHELXT solution program by using Olex 2 (Dolomanov et al., 2009) as the graphical interface. The model was refined using Least Squares minimisation with the $2018 / 3$ version of the program SHELXL. ${ }^{22}$ Non-H atoms were refined anisotropically unless otherwise stated. The hydrogen atoms were introduced at their geometric positions and refined as riding atoms unless otherwise stated. The disordered solvents were removed from the dataset using the SOLVENT MASK routine of Olex 2 which was reported in the CIF. CCDC 2092430 (4c), 2092421 (4z), 2092432 (5s), 2092431 (8a), 2112115 ([Os-Cu-L1]), 2092433 ([Os-Cu-L2]), contain the supplementary crystallographic data for this paper. Further details on the crystal data, data collection, and refinements are provided in Table S3, S6 and S9. These data

can be obtained free of charge from the Cambridge Crystallographic Data Centre via https://www.ccdc.cam.ac.uk/structures/?access=referee 

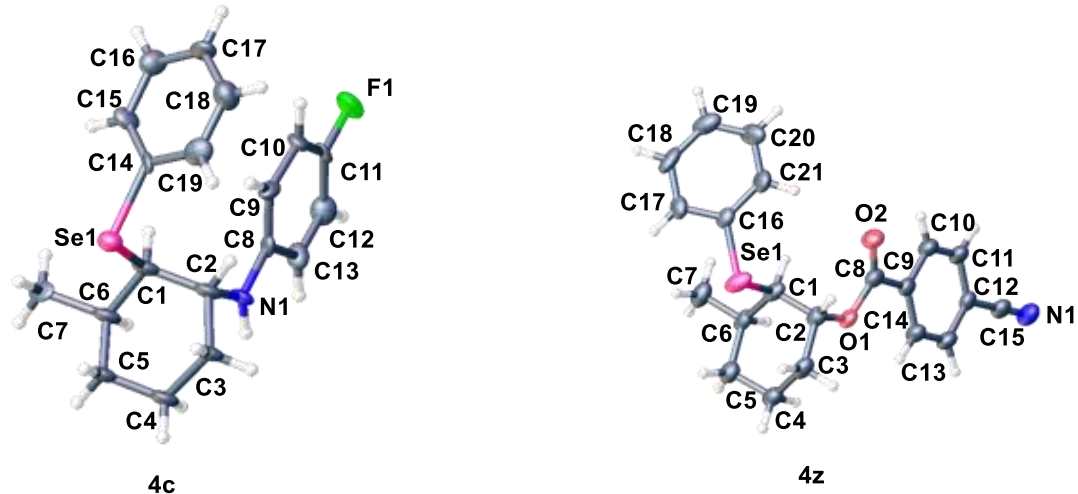

Figure S3. X-ray molecular structure of $\mathbf{4 c}$ and $\mathbf{4 z}$ (ellipsoids set at 50\% probability).

Table S3. Crystal data and structure refinement for $\mathbf{4 c}$ and $\mathbf{4 z}$.

\begin{tabular}{|c|c|c|}
\hline Identification code & $4 c$ & $4 z$ \\
\hline Empirical formula & $\mathrm{C}_{19} \mathrm{H}_{22} \mathrm{FNSe}$ & $\mathrm{C}_{21} \mathrm{H}_{21} \mathrm{NO}_{2} \mathrm{Se}$ \\
\hline Formula weight & 363.09 & 399.07 \\
\hline Temperature/K & $100.00(10)$ & 100 \\
\hline Crystal system & triclinic & monoclinic \\
\hline Space group & P-1 & $\mathrm{P} 21 / \mathrm{n}$ \\
\hline $\mathrm{a} / \AA \AA$ & $9.4793(2)$ & $7.668(7)$ \\
\hline $\mathrm{b} / \AA$ & $12.9986(3)$ & $12.768(15)$ \\
\hline $\mathrm{c} / \AA \AA$ & $14.6161(3)$ & $18.69(2)$ \\
\hline$\alpha /^{\circ}$ & $83.377(2)$ & 90 \\
\hline$\beta /{ }^{\circ}$ & $75.477(2)$ & 90 \\
\hline$\gamma /{ }^{\circ}$ & $80.393(2)$ & 90 \\
\hline Volume $/ \AA^{3}$ & $1713.86(7)$ & $1830(3)$ \\
\hline $\mathrm{Z}$ & 1 & 2 \\
\hline$\rho c a l c g / \mathrm{cm}^{3}$ & 1.404 & 1.446 \\
\hline$\mu / \mathrm{mm}-1$ & 3.009 & 2.882 \\
\hline $\mathrm{F}(000)$ & 744 & 816 \\
\hline Crystal size $/ \mathrm{mm}^{3}$ & $0.1 \times 0.1 \times 0.05$ & $0.1 \times 0.1 \times 0.1$ \\
\hline Radiation & $\mathrm{Cu} \mathrm{K} \mathrm{K}_{\alpha}(\lambda=1.54184)$ & $\mathrm{Cu} \mathrm{K}_{\alpha}(\lambda=1.54184)$ \\
\hline $2 \Theta$ range for data collection $/{ }^{\circ}$ & 6.266 to 119.982 & 8.388 to 124.966 \\
\hline
\end{tabular}


Index ranges

Reflections collected

Independent reflections

Data/restraints/parameters

Goodness-of-fit on $\mathrm{F}^{2}$

Final $\mathrm{R}$ indexes $[\mathrm{I}>=2 \sigma(\mathrm{I})]$

Data/restraints/parameters
Goodness-of-fit on $\mathrm{F}^{2}$
Final R indexes $[\mathrm{I}>=2 \sigma(\mathrm{I})]$

$$
\begin{array}{ll}
-9 \leq \mathrm{h} \leq 10,-14 \leq \mathrm{k} \leq & -8 \leq \mathrm{h} \leq 8,-14 \leq \mathrm{k} \leq 14, \\
14,-16 \leq 1 \leq 16 & -21 \leq 1 \leq 21
\end{array}
$$

32471

32320

$9792\left[\mathrm{R}_{\text {int }}=0.0357, \quad 2817\left[\mathrm{R}_{\text {int }}=0.0561\right.\right.$,

$$
\left.\left.\mathrm{R}_{\text {sigma }}=0.0339\right] \quad \mathrm{R}_{\text {sigma }}=0.0187\right]
$$

$9792 / 3 / 677$

$2817 / 0 / 227$

1.084

1.068

$\mathrm{R} 1=0.0487, \mathrm{wR} 2=$

$\mathrm{R} 1=0.0465, \mathrm{wR} 2=$

0.1255

0.1044

Final R indexes [all data]

$$
\mathrm{R} 1=0.0542, \mathrm{wR} 2=
$$

$\mathrm{R} 1=0.0482, \mathrm{wR} 2=$

0.1302

0.1053

Largest diff. peak/hole / e $\AA^{-3} \quad 3.93 /-0.78$

$0.96 /-0.62$

Table S4. Selected bond distances and angles for $\mathbf{4 c}$.

\begin{tabular}{cl|ll}
\hline \multicolumn{5}{c}{ Bond Distances $(\AA)$} \\
Se1-C8 & $1.939(16)$ & $\mathrm{C} 9-\mathrm{C} 10$ & $1.40(3)$ \\
$\mathrm{Se} 1-\mathrm{C} 2$ & $2.011(18)$ & $\mathrm{C} 13-\mathrm{C} 12$ & $1.38(3)$ \\
F1-C17 & $1.39(2)$ & $\mathrm{C} 12-\mathrm{C} 11$ & $1.38(3)$ \\
C8-C13 & $1.44(2)$ & $\mathrm{N} 1-\mathrm{C} 1$ & $1.47(2)$ \\
C17-C18 & $1.32(2)$ & $\mathrm{C} 1-\mathrm{C} 2$ & $1.48(2)$ \\
C17-C16 & $1.35(3)$ & $\mathrm{C} 1-\mathrm{C} 6$ & $1.56(2)$ \\
C14-C19 & $1.41(3)$ & $\mathrm{C} 2-\mathrm{C} 3$ & $1.52(2)$ \\
C14-C15 & $1.36(2)$ & $\mathrm{C} 6-\mathrm{C} 5$ & $1.61(2)$ \\
C14-N1 & $1.46(2)$ & $\mathrm{C} 3-\mathrm{C} 4$ & $1.49(2)$ \\
C18-C19 & $1.42(2)$ & $\mathrm{C} 3-\mathrm{C} 7$ & $1.58(2)$ \\
\hline \multicolumn{5}{|c}{$\mathrm{B} 7 \mathrm{Angles}\left({ }^{\circ}\right)$} & & \\
C8-Se1-C2 & $94.7(6)$ & $\mathrm{N} 1-\mathrm{C} 1-\mathrm{C} 2$ & $111.2(14)$ \\
C9-C8-Se1 & $118.2(12)$ & $\mathrm{N} 1-\mathrm{C} 1-\mathrm{C} 6$ & $107.7(13)$ \\
C13-C8-Se1 & $121.4(11)$ & $\mathrm{C} 2-\mathrm{C} 1-\mathrm{C} 6$ & $113.6(14)$ \\
C13-C8-C9 & $120.4(16)$ & $\mathrm{C} 1-\mathrm{C} 2-\mathrm{Se} 1$ & $109.2(12)$ \\
F1-C17-C16 & $118.6(15)$ & $\mathrm{C} 1-\mathrm{C} 2-\mathrm{C} 3$ & $110.6(13)$ \\
C18-C17-F1 & $119.6(15)$ & $\mathrm{C} 3-\mathrm{C} 2-\mathrm{Se} 1$ & $110.8(11)$ \\
C18-C17-C16 & $121.8(16)$ & $\mathrm{C} 1-\mathrm{C} 6-\mathrm{C} 5$ & $108.5(13)$ \\
C19-C14-C15 & $118.9(16)$ & $\mathrm{C} 2-\mathrm{C} 3-\mathrm{C} 7$ & $112.4(12)$ \\
C19-C14-N1 & $124.1(15)$ & $\mathrm{C} 4-\mathrm{C} 3-\mathrm{C} 2$ & $116.5(13)$ \\
N1-C14-C15 & $116.8(15)$ & $\mathrm{C} 4-\mathrm{C} 3-\mathrm{C} 7$ & $110.9(13)$ \\
C17-C18-C19 & $118.7(16)$ & $\mathrm{C} 4-\mathrm{C} 5-\mathrm{C} 6$ & $112.8(13)$ \\
& & &
\end{tabular}




\begin{tabular}{|c|c|c|c|}
\hline C15-C16-C17 & $119.8(17)$ & C8-Se1-C2 & $94.7(6)$ \\
\hline C10-C9-C8 & $118.6(17)$ & C9-C8-Se1 & $118.2(12)$ \\
\hline
\end{tabular}

Table S5. Selected bond distances and angles for $\mathbf{4 z}$.

\begin{tabular}{|c|c|c|c|}
\hline \multicolumn{4}{|c|}{ Bond Distances $(\AA)$} \\
\hline Se1-C16 & $1.861(4)$ & $\mathrm{C} 5-\mathrm{C} 4$ & $1.476(5)$ \\
\hline Se1-C1 & $1.871(3)$ & C15-C12 & $1.332(5)$ \\
\hline $\mathrm{O} 1-\mathrm{C} 2$ & $1.365(4)$ & C15-N1 & $1.059(5)$ \\
\hline $\mathrm{O} 1-\mathrm{C} 8$ & $1.371(4)$ & $\mathrm{C} 3-\mathrm{C} 4$ & $1.720(5)$ \\
\hline C16-C21 & $1.447(5)$ & $\mathrm{C} 6-\mathrm{C} 7$ & $1.469(5)$ \\
\hline $\mathrm{C} 16-\mathrm{C} 17$ & $1.345(5)$ & $\mathrm{C} 11-\mathrm{C} 10$ & $1.274(5)$ \\
\hline $\mathrm{O} 2-\mathrm{C} 8$ & $1.232(4)$ & $\mathrm{C} 11-\mathrm{C} 12$ & $1.374(5)$ \\
\hline $\mathrm{C} 17-\mathrm{C} 18$ & $1.361(6)$ & C10-C9 & $1.373(5)$ \\
\hline $\mathrm{C} 2-\mathrm{C} 1$ & $1.479(5)$ & C9-C14 & $1.377(5)$ \\
\hline $\mathrm{C} 2-\mathrm{C} 3$ & $1.525(5)$ & C14-C13 & $1.268(5)$ \\
\hline $\mathrm{C} 8-\mathrm{C} 9$ & $1.379(5)$ & $\mathrm{C} 13-\mathrm{C} 12$ & $1.374(5)$ \\
\hline $\mathrm{C} 1-\mathrm{C} 6$ & $1.717(5)$ & C19-C18 & $1.440(6)$ \\
\hline $\mathrm{C} 5-\mathrm{C} 6$ & $1.537(5)$ & $\mathrm{C} 19-\mathrm{C} 20$ & $1.331(6)$ \\
\hline \multicolumn{4}{|c|}{ Bond Angles $\left({ }^{\circ}\right)$} \\
\hline $\mathrm{C} 16-\mathrm{Se} 1-\mathrm{C} 1$ & $96.50(15)$ & $\mathrm{C} 2-\mathrm{C} 3-\mathrm{C} 4$ & $115.7(3)$ \\
\hline $\mathrm{C} 2-\mathrm{O} 1-\mathrm{C} 8$ & $114.2(3)$ & C5-C6-C1 & $115.1(3)$ \\
\hline C21-C16-Se1 & $123.5(2)$ & C7-C6-C1 & $115.9(3)$ \\
\hline C17-C16-Se1 & $114.7(3)$ & C7-C6-C5 & $109.5(3)$ \\
\hline $\mathrm{O} 1-\mathrm{C} 2-\mathrm{C} 1$ & $104.2(3)$ & $\mathrm{C} 10-\mathrm{C} 11-\mathrm{C} 12$ & $117.1(3)$ \\
\hline $\mathrm{O} 1-\mathrm{C} 2-\mathrm{C} 3$ & $104.6(3)$ & C11-C10-C9 & $118.3(3)$ \\
\hline $\mathrm{C} 1-\mathrm{C} 2-\mathrm{C} 3$ & $108.3(3)$ & C10-C9-C8 & $116.5(3)$ \\
\hline $\mathrm{O} 1-\mathrm{C} 8-\mathrm{C} 9$ & $107.5(3)$ & C10-C9C-14 & $124.0(3)$ \\
\hline $\mathrm{O} 2-\mathrm{C} 8-\mathrm{O} 1$ & $130.8(3)$ & C14-C9-C8 & $119.5(3)$ \\
\hline $\mathrm{O} 2-\mathrm{C} 8-\mathrm{C} 9$ & $121.6(3)$ & C13-C14-C9 & $118.1(3)$ \\
\hline C2-C1-Se1 & $100.4(2)$ & C14-C13-C12 & $117.5(3)$ \\
\hline $\mathrm{C} 2-\mathrm{C} 1-\mathrm{C} 6$ & $117.2(3)$ & $\mathrm{C} 15-\mathrm{C} 12-\mathrm{C} 11$ & $117.5(3)$ \\
\hline C6-C1-Se1 & $114.2(2)$ & $\mathrm{C} 15-\mathrm{C} 12-\mathrm{C} 13$ & $117.4(3)$ \\
\hline C4-C5-C6 & $108.2(3)$ & C13-C12-C11 & $125.0(3)$ \\
\hline N1-C15-C12 & $178.8(5)$ & $\mathrm{C} 5-\mathrm{C} 4-\mathrm{C} 3$ & $116.4(3)$ \\
\hline
\end{tabular}




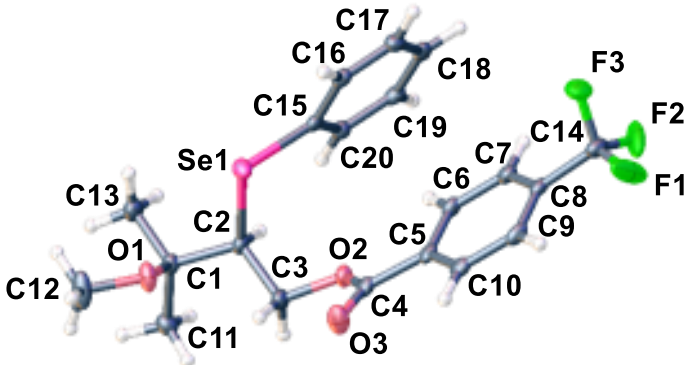

5s

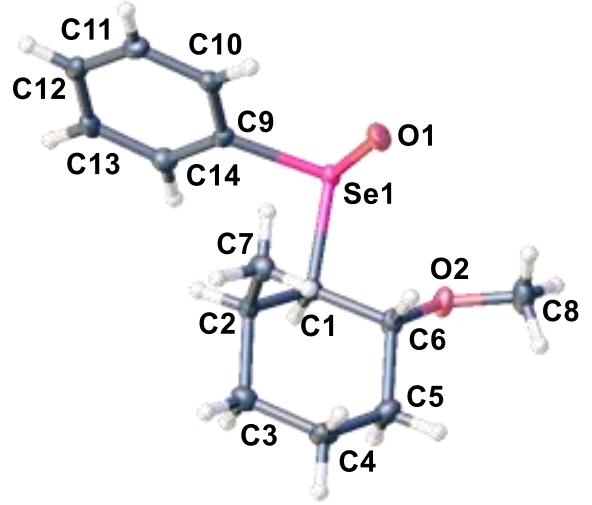

$8 \mathbf{a}$

Figure S4. X-ray molecular structure of $5 \mathbf{s}$ and $8 \mathbf{a}$ (ellipsoids set at 50\% probability).

Table S6. Crystal data and structure refinement for $\mathbf{5 s}$ and $\mathbf{8 a}$.

\begin{tabular}{|c|c|c|}
\hline Identification code & $5 s$ & $8 \mathbf{a}$ \\
\hline Empirical formula & $\mathrm{C}_{20} \mathrm{H}_{21} \mathrm{~F}_{3} \mathrm{O}_{3} \mathrm{Se}$ & $\mathrm{C}_{14} \mathrm{H}_{20} \mathrm{O}_{2} \mathrm{Se}$ \\
\hline Formula weight & 445.33 & 598.52 \\
\hline Temperature/K & $100.00(10)$ & $100.00(10)$ \\
\hline Crystal system & monoclinic & triclinic \\
\hline Space group & $\mathrm{P} 21 / \mathrm{n}$ & $\mathrm{P}-1$ \\
\hline $\mathrm{a} / \AA$ & $5.6806(3)$ & $5.90940(10)$ \\
\hline $\mathrm{b} / \AA$ & $7.2301(3)$ & $15.06560(10)$ \\
\hline $\mathrm{c} / \AA$ & $45.4938(19)$ & $15.1636(2)$ \\
\hline$\alpha /^{\circ}$ & 90.1 & $87.3430(10)$ \\
\hline$\beta /{ }^{\circ}$ & 90 & $85.4970(10)$ \\
\hline$\gamma /{ }^{\circ}$ & 90 & $82.3230(10)$ \\
\hline Volume $/ \AA^{3}$ & $1868.49(15)$ & $1332.89(3)$ \\
\hline Z & 4 & 2 \\
\hline$\rho$ calcg $/ \mathrm{cm}^{3}$ & 1.583 & 1.491 \\
\hline$\mu / \mathrm{mm}^{-1}$ & 3.151 & 3.715 \\
\hline $\mathrm{F}(000)$ & 904 & 616 \\
\hline Crystal size $/ \mathrm{mm}^{3}$ & $0.15 \times 0.10 \times 0.10$ & $0.15 \times 0.1 \times 0.1$ \\
\hline Radiation & $\mathrm{Cu} \mathrm{K} \alpha(\lambda=1.54184)$ & $\mathrm{Cu} \mathrm{K} \alpha(\lambda=1.54184)$ \\
\hline $2 \Theta$ range for data collection ${ }^{\circ}$ & 7.774 to 149.816 & 5.85 to 129.976 \\
\hline
\end{tabular}




\begin{tabular}{lll} 
Index ranges & $-7 \leq \mathrm{h} \leq 6,-3 \leq \mathrm{k} \leq 8$, & $-6 \leq \mathrm{h} \leq 6,-17 \leq \mathrm{k} \leq 17$, \\
& $-54 \leq 1 \leq 56$ & $-17 \leq 1 \leq 17$ \\
Reflections collected & 10003 & 24911 \\
Independent reflections & $3624\left[\mathrm{R}_{\text {int }}=0.0290\right.$, & $4518\left[\mathrm{R}_{\text {int }}=0.0294\right.$, \\
& $\left.\mathrm{R}_{\text {sigma }}=0.0281\right]$ & $\left.\mathrm{R}_{\text {sigma }}=0.0141\right]$ \\
Data/restraints/parameters & $3624 / 39 / 275$ & $4518 / 0 / 311$ \\
Goodness-of-fit on $\mathrm{F}^{2}$ & 1.272 & 1.133 \\
Final R indexes [I $>=2 \sigma(\mathrm{I})]$ & $\mathrm{R} 1=0.0612, \mathrm{wR} 2=$ & $\mathrm{R} 1=0.0250, \mathrm{wR} 2=$ \\
& 0.1442 & 0.0615 \\
Final R indexes [all data] & $\mathrm{R} 1=0.0626, \mathrm{wR} 2=$ & $\mathrm{R} 1=0.0254, \mathrm{wR} 2=$ \\
& 0.1448 & 0.0616 \\
\hline Largest diff. peak/hole $/ \mathrm{e} \AA^{-3}$ & $1.19 /-1.00$ & $0.41 /-0.52$ \\
\hline
\end{tabular}

Table S7. Selected bond distances and angles for $\mathbf{5 s .}$

\begin{tabular}{|c|c|c|c|}
\hline \multicolumn{4}{|c|}{ Bond Distances $(\AA)$} \\
\hline $\mathrm{Se} 1-\mathrm{C} 2$ & $1.968(5)$ & C19-C20 & $1.387(7)$ \\
\hline Se1-C15 & $1.917(5)$ & $\mathrm{C} 2-\mathrm{C} 3$ & $1.522(7)$ \\
\hline F1-C14 & $1.314(12)$ & $\mathrm{C} 20-\mathrm{C} 15$ & $1.383(7)$ \\
\hline $\mathrm{O} 1-\mathrm{C} 1$ & $1.436(6)$ & $\mathrm{C} 15-\mathrm{C} 16$ & $1.404(7)$ \\
\hline $\mathrm{O} 1-\mathrm{C} 13$ & $1.417(7)$ & $\mathrm{C} 7-\mathrm{C} 8$ & $1.395(7)$ \\
\hline $\mathrm{O} 3-\mathrm{C} 4$ & $1.207(6)$ & C7-C6 & $1.399(7)$ \\
\hline $\mathrm{O} 2-\mathrm{C} 3$ & $1.457(6)$ & $\mathrm{C} 8-\mathrm{C} 9$ & $1.370(7)$ \\
\hline $\mathrm{O} 2-\mathrm{C} 4$ & $1.336(6)$ & $\mathrm{C} 8-\mathrm{C} 14$ & $1.508(7)$ \\
\hline $\mathrm{C} 11-\mathrm{C} 1$ & $1.524(7)$ & C9-C10 & $1.394(7)$ \\
\hline C17-C18 & $1.373(7)$ & $\mathrm{C} 10-\mathrm{C} 5$ & $1.395(7)$ \\
\hline $\mathrm{C} 17-\mathrm{C} 16$ & $1.389(7)$ & $\mathrm{C} 5-\mathrm{C} 6$ & $1.381(7)$ \\
\hline C12-C1 & $1.533(7)$ & $\mathrm{C} 5-\mathrm{C} 4$ & $1.506(7)$ \\
\hline C18-C19 & $1.397(7)$ & $\mathrm{C} 1-\mathrm{C} 2$ & $1.548(7)$ \\
\hline \multicolumn{4}{|c|}{ Bond Angles $\left({ }^{\circ}\right)$} \\
\hline C15-Se1-C2 & $101.2(2)$ & $\mathrm{C} 10-\mathrm{C} 5-\mathrm{C} 4$ & $120.4(5)$ \\
\hline C13-O1-C1 & $116.5(4)$ & C6-C5-C10 & $120.4(5)$ \\
\hline $\mathrm{C} 4-\mathrm{O} 2-\mathrm{C} 3$ & $117.8(4)$ & $\mathrm{C} 6-\mathrm{C} 5-\mathrm{C} 4$ & $119.1(5)$ \\
\hline $\mathrm{O} 1-\mathrm{C} 1-\mathrm{C} 11$ & $112.8(4)$ & C5-C6-C7 & $120.4(5)$ \\
\hline O1-C1-C12 & $111.1(4)$ & $\mathrm{O} 3-\mathrm{C} 4-\mathrm{O} 2$ & $124.8(5)$ \\
\hline $\mathrm{O} 1-\mathrm{C} 1-\mathrm{C} 2$ & $102.6(4)$ & $\mathrm{O} 3-\mathrm{C} 4-\mathrm{C} 5$ & $122.9(5)$ \\
\hline C11-C1-C12 & $108.6(4)$ & $\mathrm{O} 2-\mathrm{C} 4-\mathrm{C} 5$ & $112.3(4)$ \\
\hline
\end{tabular}




\begin{tabular}{ll|ll}
$\mathrm{C} 11-\mathrm{C} 1-\mathrm{C} 2$ & $111.1(4)$ & $\mathrm{C} 1-\mathrm{C} 2-\mathrm{Se} 1$ & $108.8(3)$ \\
$\mathrm{C} 12-\mathrm{C} 1-\mathrm{C} 2$ & $110.5(4)$ & $\mathrm{F} 1-\mathrm{C} 14-\mathrm{F} 2$ & $107.0(9)$ \\
\hline
\end{tabular}

Table S8. Selected bond distances and angles for $\mathbf{8 a}$.

\begin{tabular}{cl|cl}
\hline \multicolumn{4}{c}{ Bond Distances $(\AA)$} \\
\hline Se1-O1 & $1.6643(17)$ & C2-C7 & $1.521(3)$ \\
Se1-C9 & $1.944(2)$ & C12-C11 & $1.386(4)$ \\
Se1-C1 & $2.000(2)$ & C12-C13 & $1.387(4)$ \\
O2-C6 & $1.434(3)$ & C14-C13 & $1.393(3)$ \\
O2-C8 & $1.419(3)$ & C6-C5 & $1.533(3)$ \\
C9-C14 & $1.389(3)$ & C10-C11 & $1.391(3)$ \\
C9-C10 & $1.383(3)$ & C5-C4 & $1.529(3)$ \\
C1-C2 & $1.531(3)$ & C4-C3 & $1.529(3)$ \\
C1-C6 & $1.519(3)$ & C2-C3 & $1.540(3)$ \\
\hline \multicolumn{5}{|c}{ Bond Angles $\left({ }^{\circ}\right)$} \\
O1-Se1-C9 & $103.30(9)$ & O2-C6-C1 & $106.36(17)$ \\
O1-Se1-C1 & $106.59(9)$ & O2-C6-C5 & $111.25(18)$ \\
C9-Se1-C1 & $98.49(9)$ & C1-C6-C5 & $110.16(19)$ \\
C8-O2-C6 & $112.94(18)$ & C9-C10-C11 & $119.2(2)$ \\
C14-C9-Se1 & $120.04(17)$ & O4-C15-C16 & $111.24(19)$ \\
C10-C9-Se1 & $118.38(17)$ & C12-C11-C10 & $120.1(2)$ \\
C10-C9-C14 & $121.4(2)$ & C4-C5-C6 & $111.21(19)$ \\
C2-C1-Se1 & $117.85(15)$ & C5-C4-C3 & $110.8(2)$ \\
\hline
\end{tabular}
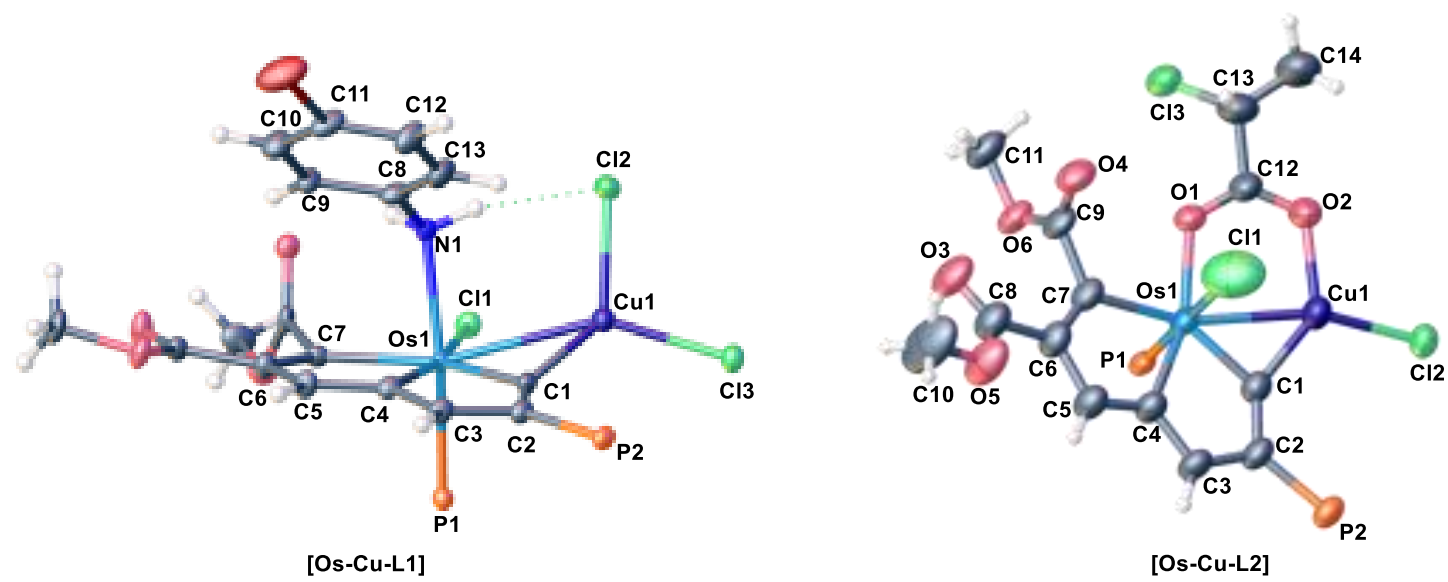

Figure S5. X-ray molecular structure of [Os-Cu-L1] and [Os-Cu-L2] (ellipsoids set at $50 \%$ probability). The phenylgroups in the $\mathrm{PPh}_{3}$ moieties are omitted for clarity. 
Table S9. Crystal data and structure refinement for [Os-Cu-L1] and [Os-Cu-L2].

\begin{tabular}{|c|c|c|}
\hline Identification code & {$[\mathrm{Os}-\mathrm{Cu}-\mathrm{L} 1] \cdot 2 \mathrm{CH}_{2} \mathrm{Cl}_{2}$} & [Os-Cu-L2] \\
\hline Empirical formula & $\mathrm{C}_{55} \mathrm{H}_{48} \mathrm{BrCl}_{7} \mathrm{CuNO}_{4} \mathrm{OsP}_{2}$ & $\mathrm{C}_{50} \mathrm{H}_{42} \mathrm{Cl}_{3} \mathrm{CuO}_{6} \mathrm{OsP}_{2}$ \\
\hline Formula weight & 1430.68 & 1160.86 \\
\hline Temperature/K & $99.9(8)$ & $100.0(2)$ \\
\hline Crystal system & triclinic & triclinic \\
\hline Space group & P-1 & $\mathrm{P}-1$ \\
\hline $\mathrm{a} / \AA$ & $10.4803(2)$ & $10.8062(2)$ \\
\hline $\mathrm{b} / \AA$ & $13.0925(2)$ & $13.1879(3)$ \\
\hline $\mathrm{c} / \AA$ & $20.2701(4)$ & $20.1787(4)$ \\
\hline$\alpha /^{\circ}$ & $92.9790(10)$ & $97.721(2)$ \\
\hline$\beta /{ }^{\circ}$ & $93.0570(10)$ & $96.207(2)$ \\
\hline$\gamma /{ }^{\circ}$ & $91.2700(10)$ & $105.232(2)$ \\
\hline Volume $/ \AA^{3}$ & $2772.75(9)$ & $2718.40(10)$ \\
\hline Z & 2 & 2 \\
\hline pcalcg $/ \mathrm{cm}^{3}$ & 1.714 & 1.418 \\
\hline$\mu / \mathrm{mm}^{-1}$ & 3.834 & 7.085 \\
\hline $\mathrm{F}(000)$ & 1412.0 & 1152 \\
\hline Crystal size $/ \mathrm{mm}^{3}$ & $0.2 \times 0.2 \times 0.2$ & $0.2 \times 0.1 \times 0.1$ \\
\hline Radiation & $\operatorname{Mo~K\alpha }(\lambda=0.71073)$ & $\mathrm{Cu} \mathrm{K} \alpha(\lambda=1.54184)$ \\
\hline $2 \Theta$ range for data collection $/^{\circ}$ & 3.62 to 62.41 & 7.05 to 146.442 \\
\hline Index ranges & $\begin{array}{l}-14 \leq \mathrm{h} \leq 13,-18 \leq \mathrm{k} \leq 18 \\
-28 \leq 1 \leq 28\end{array}$ & $\begin{array}{l}-13 \leq \mathrm{h} \leq 13,-16 \leq \mathrm{k} \leq 15 \\
-24 \leq 1 \leq 24\end{array}$ \\
\hline Reflections collected & 46162 & 33336 \\
\hline Independent reflections & $\begin{array}{l}15084\left[R_{\text {int }}=0.0408, R_{\text {sigma }}\right. \\
=0.0468]\end{array}$ & $\begin{array}{l}10434\left[\mathrm{R}_{\text {int }}=0.0368,\right. \\
\left.\mathrm{R}_{\text {sigma }}=0.0357\right]\end{array}$ \\
\hline Data/restraints/parameters & $15084 / 78 / 705$ & $10434 / 402 / 591$ \\
\hline Goodness-of-fit on $\mathrm{F}^{2}$ & 1.025 & 1.034 \\
\hline Final $\mathrm{R}$ indexes $[\mathrm{I}>=2 \sigma(\mathrm{I})]$ & $\mathrm{R}_{1}=0.0361, \mathrm{wR}_{2}=0.0783$ & $\begin{array}{l}\mathrm{R} 1=0.0447, \mathrm{wR} 2= \\
0.1163\end{array}$ \\
\hline
\end{tabular}


Final $\mathrm{R}$ indexes [all data]

$$
\begin{array}{ll}
\mathrm{R}_{1}=0.0459, \mathrm{wR}_{2}=0.0811 & \mathrm{R} 1=0.0491, \mathrm{wR} 2= \\
0.1218
\end{array}
$$

Largest diff. peak/hole / e $\AA^{-3} \quad 1.75 /-1.14$

$2.52 /-1.33$

\begin{tabular}{|c|c|c|c|}
\hline \multicolumn{4}{|c|}{ Bond Distances $(\AA)$} \\
\hline Os1-Cul & $2.7899(4)$ & C8 -C9 & $1.448(10)$ \\
\hline Os1-Cl1 & $2.4286(8)$ & C8- C13 & $1.346(14)$ \\
\hline Os1-P1 & $2.3743(8)$ & C9-C10 & $1.380(12)$ \\
\hline Os1-N1 & $2.209(3)$ & C10- C11 & $1.387(9)$ \\
\hline Os1-C7 & $2.058(3)$ & C13- C12 & $1.384(13)$ \\
\hline Os1-C4 & $2.075(3)$ & $\mathrm{C} 11-\mathrm{C} 12$ & $1.380(9)$ \\
\hline Os1-C1 & $1.890(3)$ & $\mathrm{C} 7-\mathrm{C} 6$ & $1.381(4)$ \\
\hline $\mathrm{Cu} 1-\mathrm{Cl} 3$ & $2.2303(8)$ & $\mathrm{C} 5-\mathrm{C} 6$ & $1.413(4)$ \\
\hline $\mathrm{Cu} 1-\mathrm{Cl} 2$ & $2.2617(9)$ & $\mathrm{C} 5-\mathrm{C} 4$ & $1.386(4)$ \\
\hline $\mathrm{Cu} 1-\mathrm{C} 1$ & $1.891(3)$ & $\mathrm{C} 3-\mathrm{C} 4$ & $1.413(4)$ \\
\hline Br1-C11 & $1.907(7)$ & $\mathrm{C} 3-\mathrm{C} 2$ & $1.386(4)$ \\
\hline Br1-C15 & $2.099(19)$ & $\mathrm{C} 2-\mathrm{C} 1$ & $1.415(4)$ \\
\hline $\mathrm{P} 2-\mathrm{C} 2$ & $1.786(3)$ & C14-C16 & $1.35(3)$ \\
\hline N1-C8 & $1.440(4)$ & & \\
\hline \multicolumn{4}{|c|}{ Bond Angles $\left({ }^{\circ}\right)$} \\
\hline Cl1-Os1-Cul & $80.99(2)$ & Cl3-Cu1-Os1 & $148.33(3)$ \\
\hline P1-Os1-Cu1 & $104.58(2)$ & $\mathrm{Cl} 3-\mathrm{Cu} 1-\mathrm{Cl} 2$ & $105.19(3)$ \\
\hline N1-Os1-Cu1 & $81.13(7)$ & Cl2-Cu1-Os1 & $104.67(3)$ \\
\hline N1-Os1-P1 & 166.92(7) & C1-Cu1-Os1 & $42.44(9)$ \\
\hline C7-Os1-Cu1 & $162.49(9)$ & $\mathrm{C} 1-\mathrm{Cu} 1-\mathrm{Cl} 3$ & $117.21(9)$ \\
\hline C7-Os1-C11 & $92.28(9)$ & $\mathrm{C} 1-\mathrm{Cu} 1-\mathrm{Cl} 2$ & $132.66(9)$ \\
\hline C7-Os1-P1 & $91.29(9)$ & C8-N1-Os1 & $121.1(2)$ \\
\hline C7-Os1-N1 & $81.84(11)$ & C6-C7-Os1 & $119.6(2)$ \\
\hline
\end{tabular}

Table S10. Selected bond distances and angles for [Os-Cu-L1]. 


\begin{tabular}{lc|cc} 
C7-Os1-C4 & $74.74(12)$ & C5-C4-Os1 & $118.9(2)$ \\
C4-Os1-Cu1 & $110.95(9)$ & C5-C4-C3 & $122.3(3)$ \\
C4-Os1-C11 & $166.89(9)$ & C3-C4-Os1 & $118.8(2)$ \\
C4-Os1-P1 & $93.44(9)$ & C3-C2-P2 & $124.3(3)$ \\
C4-Os1-N1 & $95.44(11)$ & C3-C2-C1 & $108.6(3)$ \\
C1-Os1-Cu1 & $42.46(10)$ & C1-C2-P2 & $127.0(2)$ \\
C1-Os1-C11 & $120.14(10)$ & Os1-C1-Cu1 & $95.11(14)$ \\
C1-Os1-P1 & $88.98(9)$ & C2-C1-Os1 & $127.6(2)$ \\
C1-Os1-N1 & $102.80(11)$ & C2-C1-Cu1 & $133.4(2)$ \\
C1-Os1-C7 & $147.57(13)$ & N1-C8-C9 & $117.0(4)$ \\
C1-Os1-C4 & $72.88(13)$ & C13-Cu1-Os1 & $148.33(3)$ \\
\hline
\end{tabular}

Table S11. Selected bond distances and angles for [Os-Cu-L2].

\begin{tabular}{|c|c|c|c|c|c|}
\hline \multicolumn{6}{|c|}{ Bond Distances $(\AA)$} \\
\hline $\mathrm{Os}(1)-\mathrm{Cu}(1)$ & $2.5954(8)$ & $C(1)-C(2)$ & $1.412(7)$ & $\mathrm{O}(1)-\mathrm{C}(12)$ & $1.242(6)$ \\
\hline Os(1)-P(1) & $2.3449(12)$ & $C(2)-C(3)$ & $1.394(7)$ & $\mathrm{O}(6)-\mathrm{C}(9)$ & $1.326(7)$ \\
\hline $\mathrm{Os}(1)-\mathrm{Cl}(1)$ & $2.360(3)$ & $C(9)-C(7)$ & $1.503(7)$ & $\mathrm{O}(6)-\mathrm{C}(11)$ & $1.446(6)$ \\
\hline Os(1)-O(1) & $2.144(3)$ & $C(6)-C(7)$ & $1.389(7)$ & $\mathrm{O}(4)-\mathrm{C}(9)$ & $1.206(7)$ \\
\hline Os(1)-C(1) & $1.886(5)$ & $C(6)-C(5)$ & $1.412(7)$ & $\mathrm{O}(2)-\mathrm{C}(12)$ & $1.254(6)$ \\
\hline $\operatorname{Os}(1)-\mathrm{C}(7)$ & $2.076(5)$ & $C(6)-C(8)$ & $1.483(7)$ & $\mathrm{O}(3)-\mathrm{C}(8)$ & $1.189(7)$ \\
\hline Os(1)-C(4) & $2.093(5)$ & $C(4)-C(5)$ & $1.360(7)$ & $\mathrm{O}(5)-\mathrm{C}(8)$ & $1.327(7)$ \\
\hline $\mathrm{Cu}(1)-\mathrm{Cl}(2)$ & $2.2195(13)$ & $C(4)-C(3)$ & $1.411(6)$ & $\mathrm{O}(5)-\mathrm{C}(10)$ & $1.462(8)$ \\
\hline $\mathrm{Cu}(1)-\mathrm{O}(2)$ & $2.034(4)$ & $C(12)-C(13)$ & $1.533(8)$ & $P(2)-C(2)$ & $1.781(5)$ \\
\hline $\mathrm{Cu}(1)-\mathrm{C}(1)$ & $1.897(5)$ & $\mathrm{C}(13)-\mathrm{Cl}(3)$ & $1.777(9)$ & $C(13)-C(14)$ & $1.41(3)$ \\
\hline \multicolumn{6}{|c|}{ Bond Angles $\left({ }^{\circ}\right)$} \\
\hline \multicolumn{2}{|c|}{$\mathrm{C}(2)-\mathrm{C}(1-) \mathrm{Cu}(1)$} & $140.8(4)$ & \multicolumn{2}{|c|}{$\mathrm{C}(2)-\mathrm{C}(3)-\mathrm{C}(4)$} & $111.2(4)$ \\
\hline \multicolumn{2}{|c|}{$C(3)-C(2)-C(1)$} & $109.0(4)$ & \multicolumn{2}{|c|}{$\mathrm{O}(1)-\mathrm{C}(12)-\mathrm{O}(2)$} & $126.6(5)$ \\
\hline \multicolumn{2}{|c|}{$C(7)-C(6)-C(5)$} & $13.8(4)$ & \multicolumn{2}{|c|}{$\mathrm{C}(2)-\mathrm{C}(1)-\mathrm{Cu}(1)$} & $140.8(4)$ \\
\hline \multicolumn{2}{|c|}{$C(5)-C(4)-C(3)$} & $22.2(5)$ & \multicolumn{2}{|c|}{$\mathrm{C}(3)-\mathrm{C}(2)-\mathrm{C}(1)$} & $109.0(4)$ \\
\hline
\end{tabular}




\begin{tabular}{cc|cc}
$\mathrm{C}(3)-\mathrm{C}(4)-\mathrm{Os}(1)$ & $118.9(4)$ & $\mathrm{C}(7)-\mathrm{C}(6)-\mathrm{C}(5)$ & $113.8(4)$ \\
$\mathrm{C}(4)-\mathrm{C}(5)-\mathrm{C}(6)$ & $114.3(5)$ & $\mathrm{C}(6)-\mathrm{C}(7)-\mathrm{Os}(1)$ & $118.1(3)$ \\
$\mathrm{O}(1)-\mathrm{C}(12)-\mathrm{C}(13)$ & $113.9(5)$ & $\mathrm{C}(5)-\mathrm{C}(4)-\mathrm{Os}(1)$ & $118.5(4)$ \\
$\mathrm{C}(2)-\mathrm{C}(1)-\mathrm{Cu}(1)$ & $140.8(4)$ & $\mathrm{C}(3)-\mathrm{C}(4)-\mathrm{Os}(1)$ & $118.9(4)$ \\
\hline
\end{tabular}




\section{8. ${ }^{1} \mathrm{H}$ and ${ }^{13} \mathrm{C}$ NMR Spectrum}

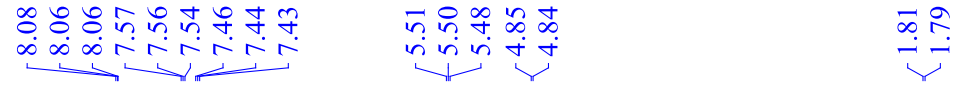

\begin{tabular}{l}
${ }^{1} \mathrm{H} \mathrm{NMR} \mathrm{NDCl}_{3}$ \\
\hline
\end{tabular}<smiles>CC(C)=CCOC(=O)c1ccccc1</smiles>

$1 q$
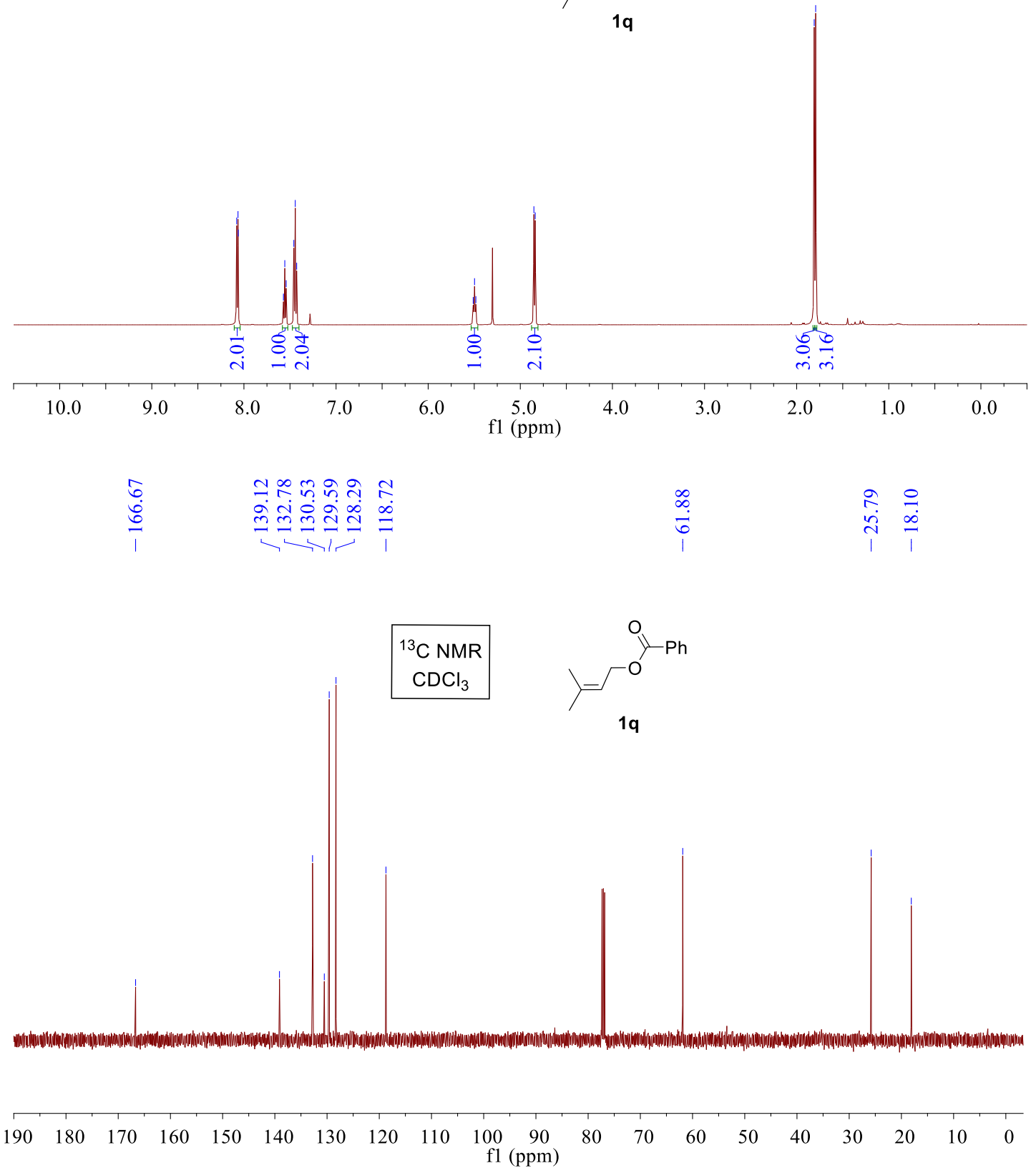


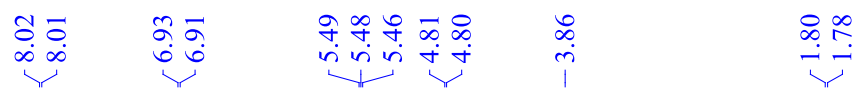

${ }^{1} \mathrm{H}$ NMR

$\mathrm{CDCl}_{3}$
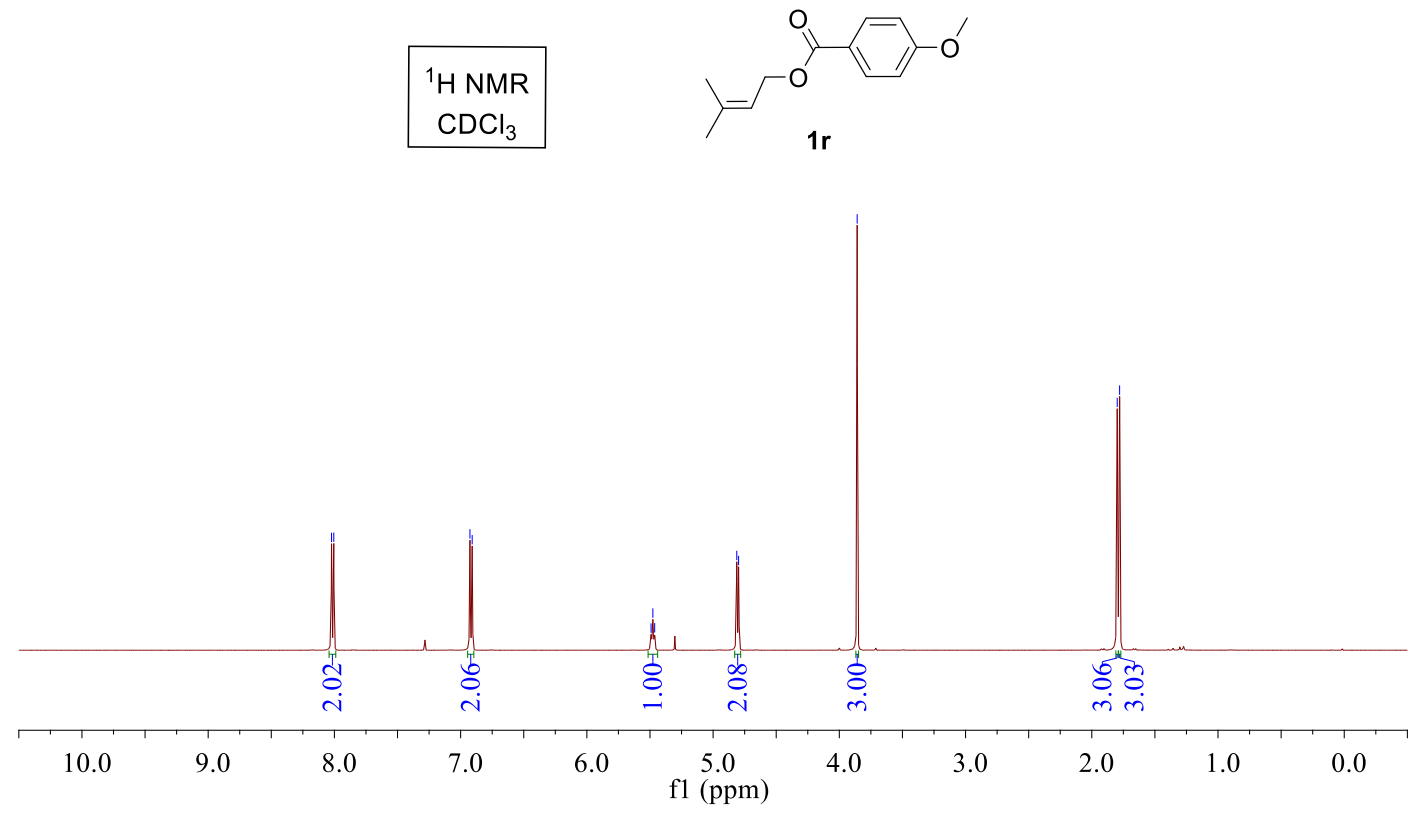

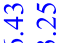

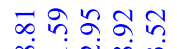

:

百

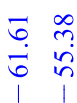

$\begin{array}{ll}\infty & 8 \\ \stackrel{0}{n} & \infty \\ i & 1\end{array}$

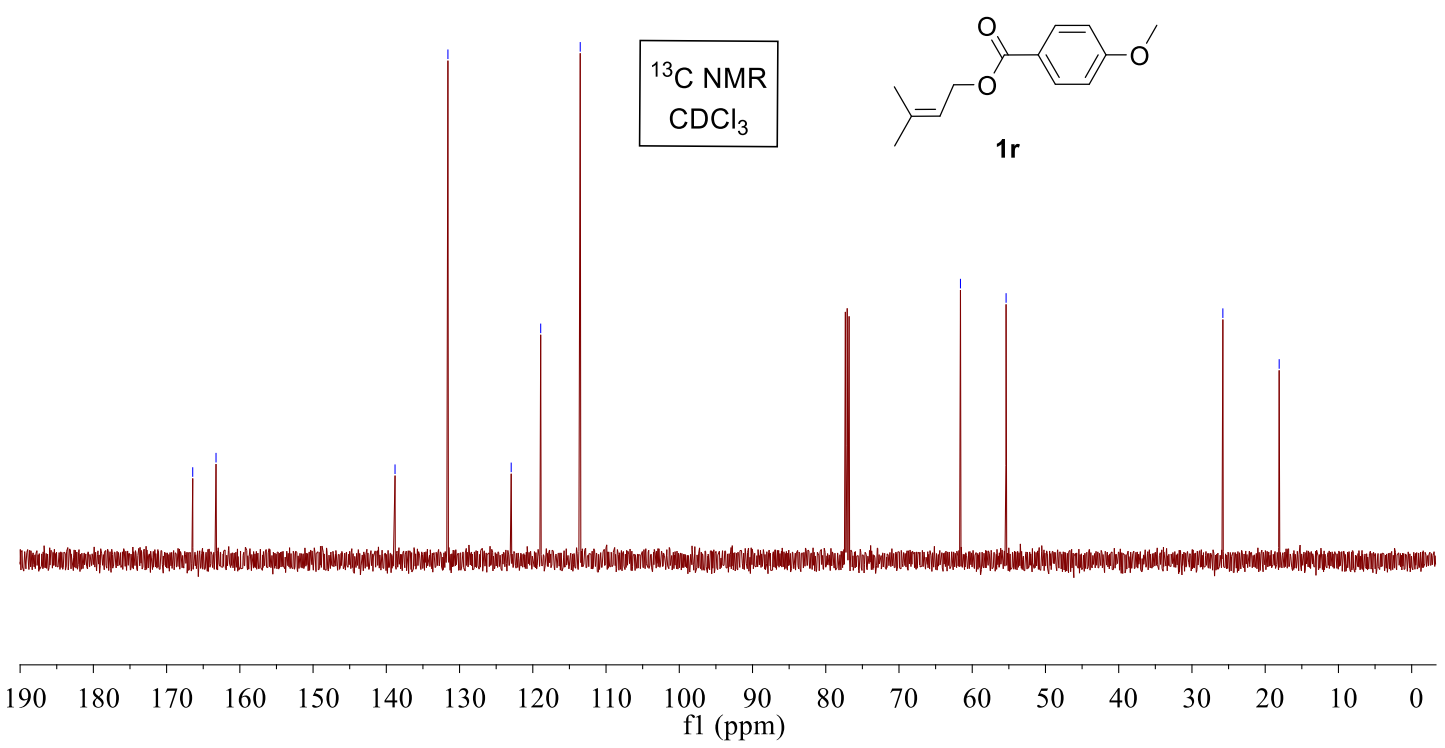




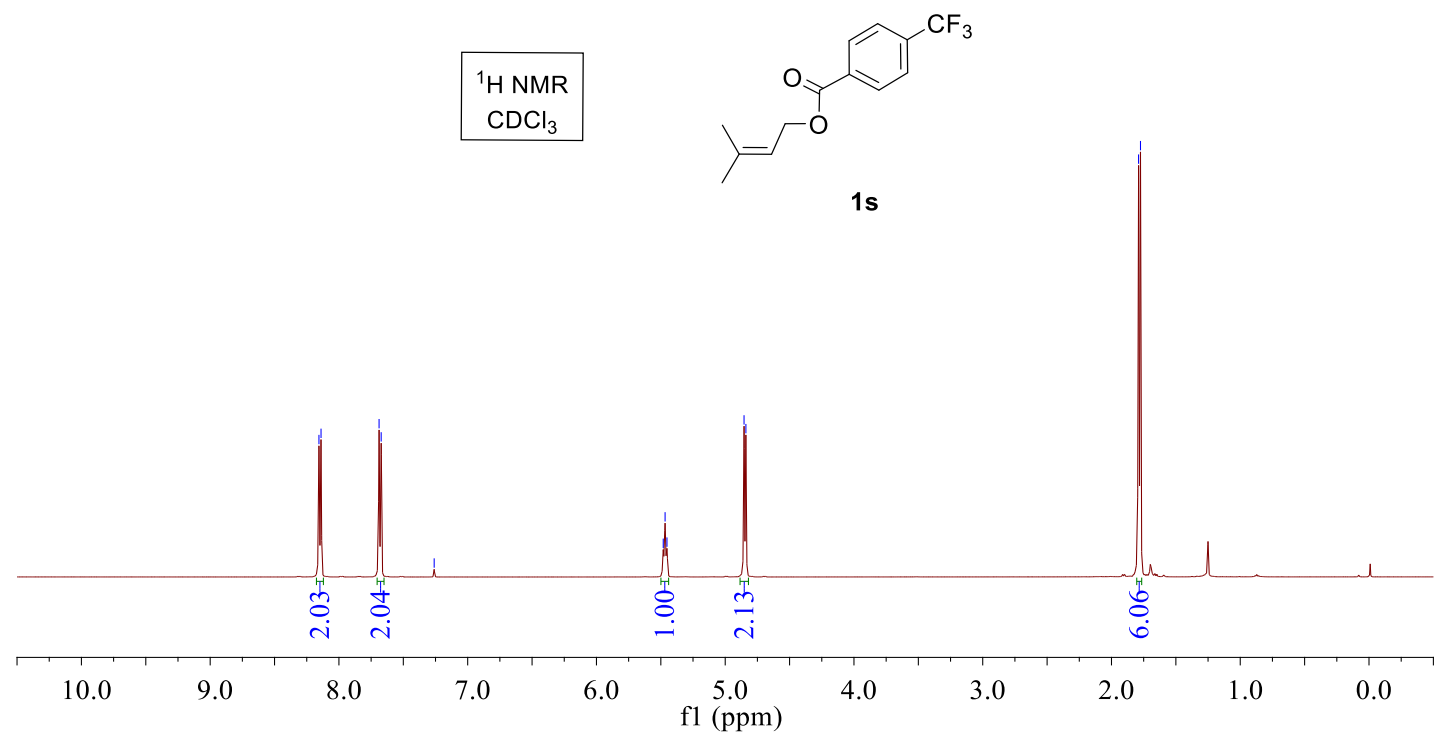

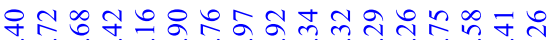

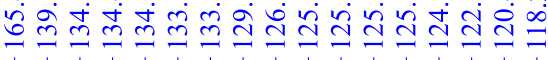

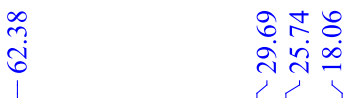

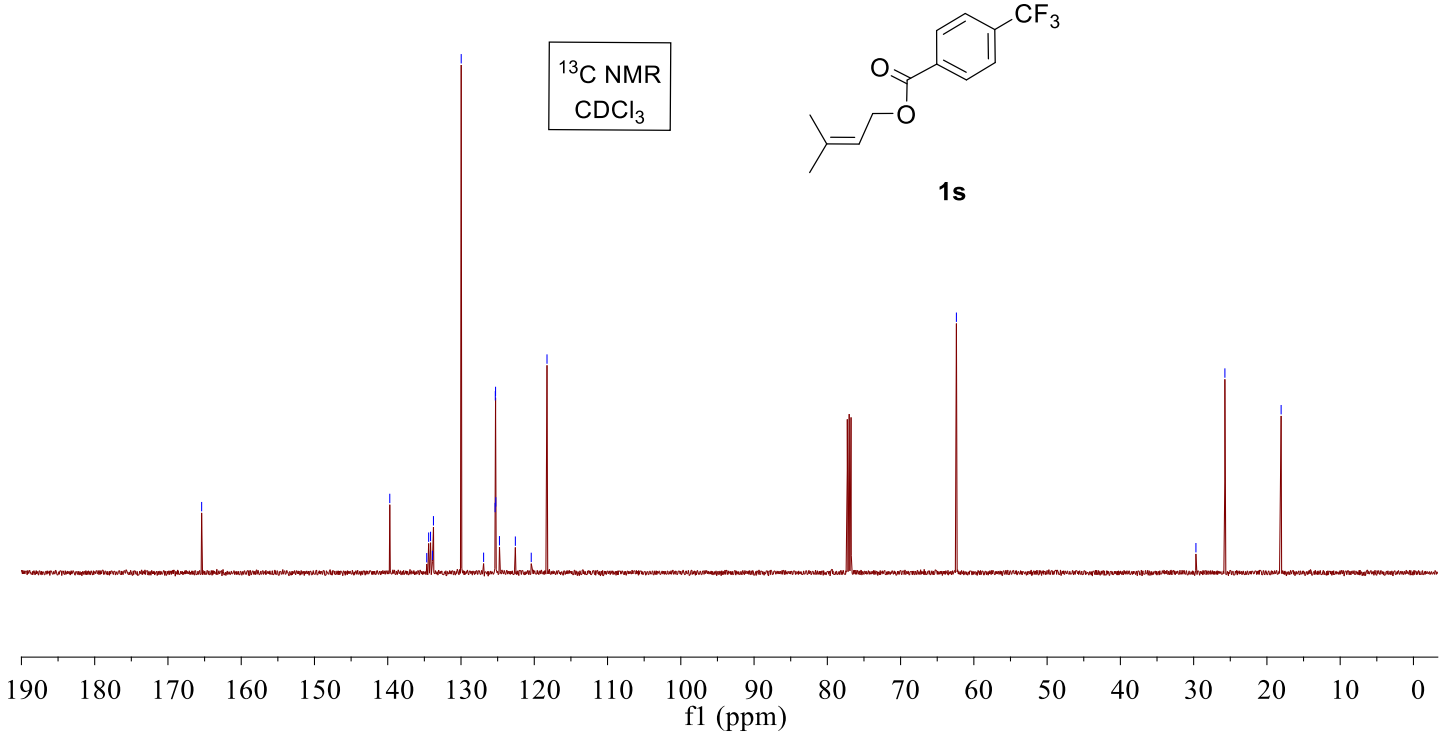



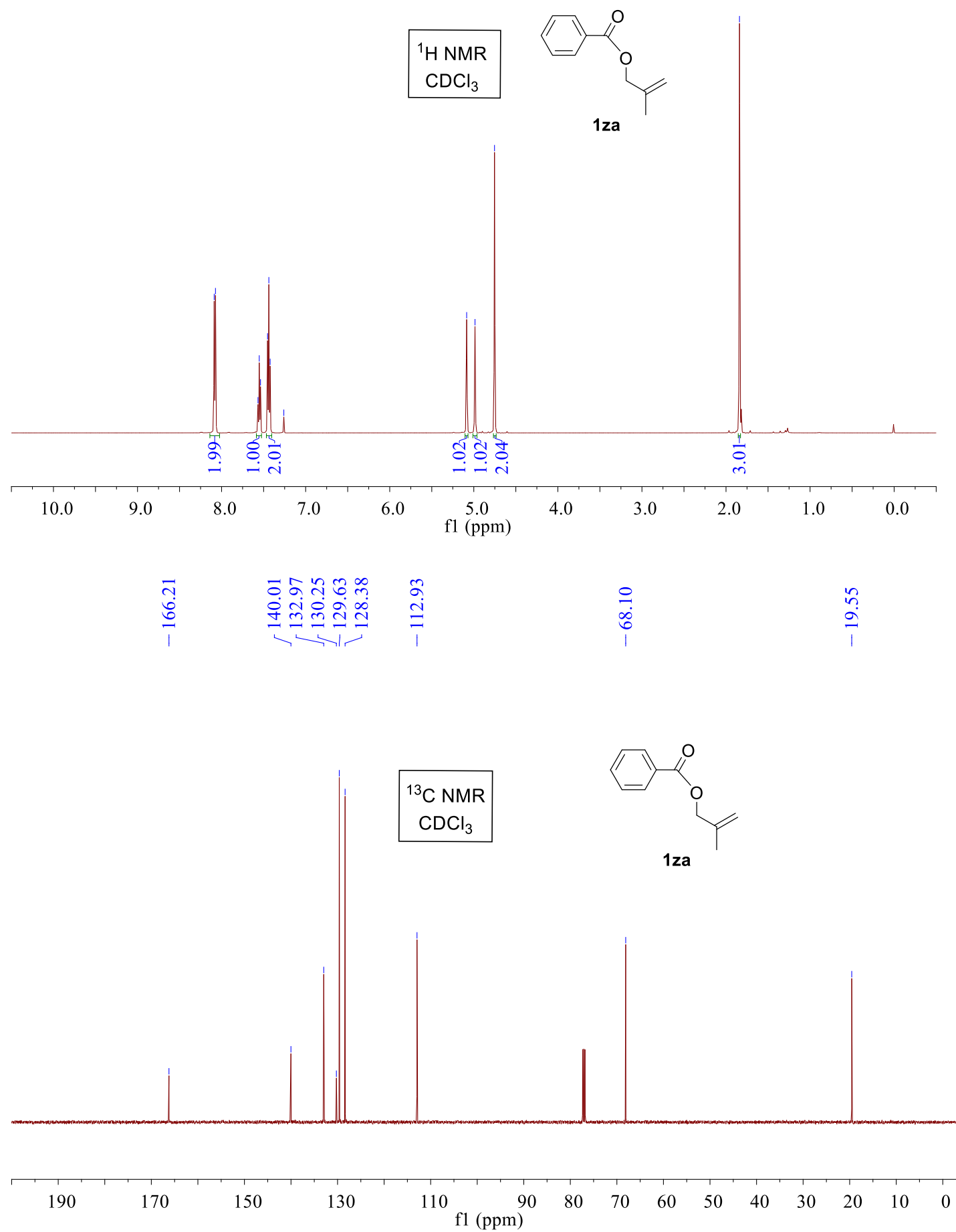


\begin{tabular}{|l|}
${ }^{1} \mathrm{H} \mathrm{NMR}$ \\
$\mathrm{CDCl}_{3}$ \\
\hline
\end{tabular}

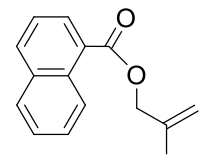

1zb
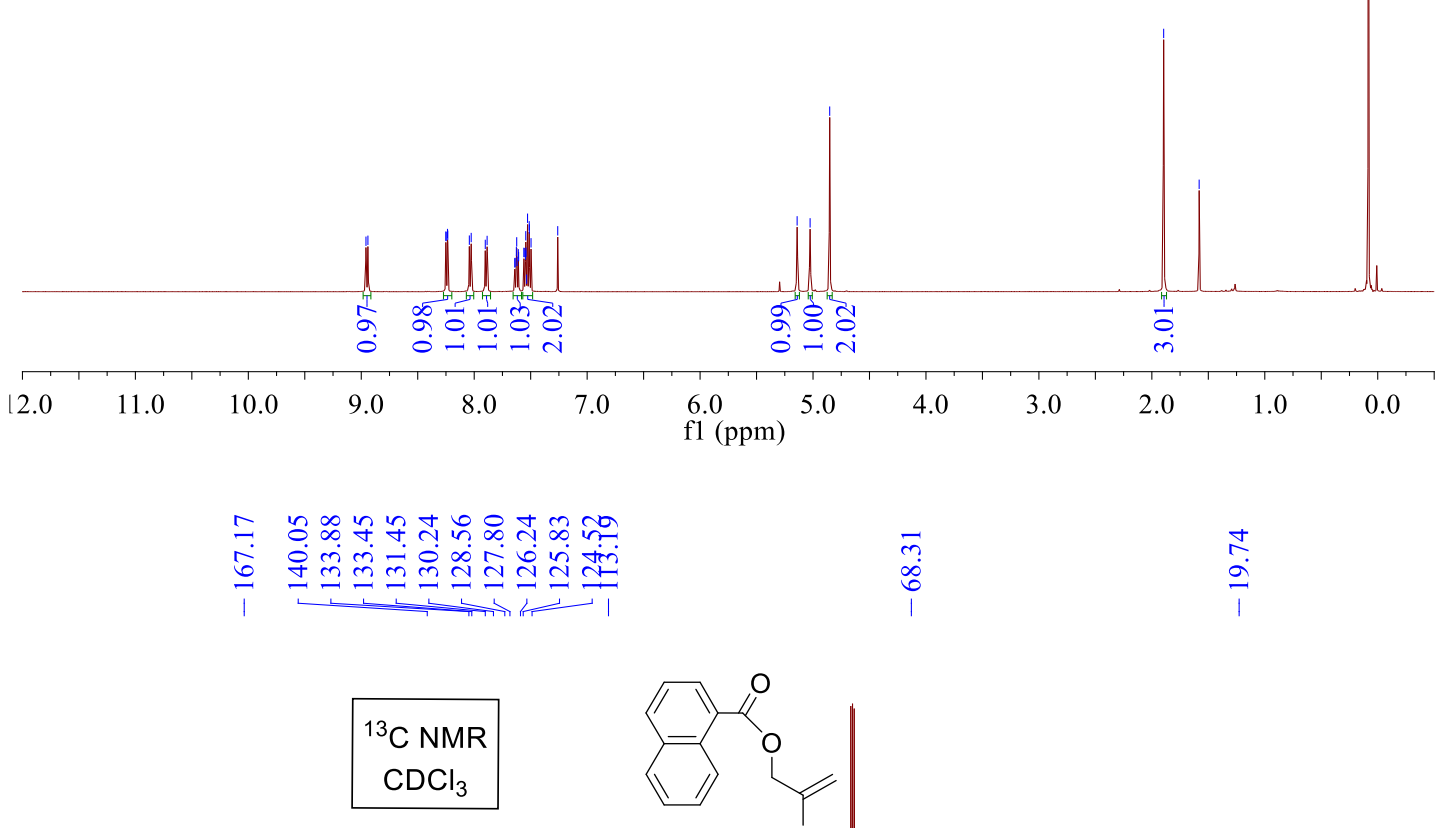

$1 \mathrm{zb}$

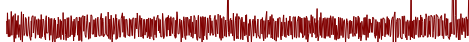

\begin{tabular}{llllllllllllllll}
\hline 190 & 170 & 150 & 130 & 110 & $\begin{array}{r}90 \\
\mathrm{f} 1(\mathrm{ppm})\end{array}$ & 80 & 70 & 60 & 50 & 40 & 30 & 20 & 10 & 0 & -11 \\
\hline
\end{tabular}



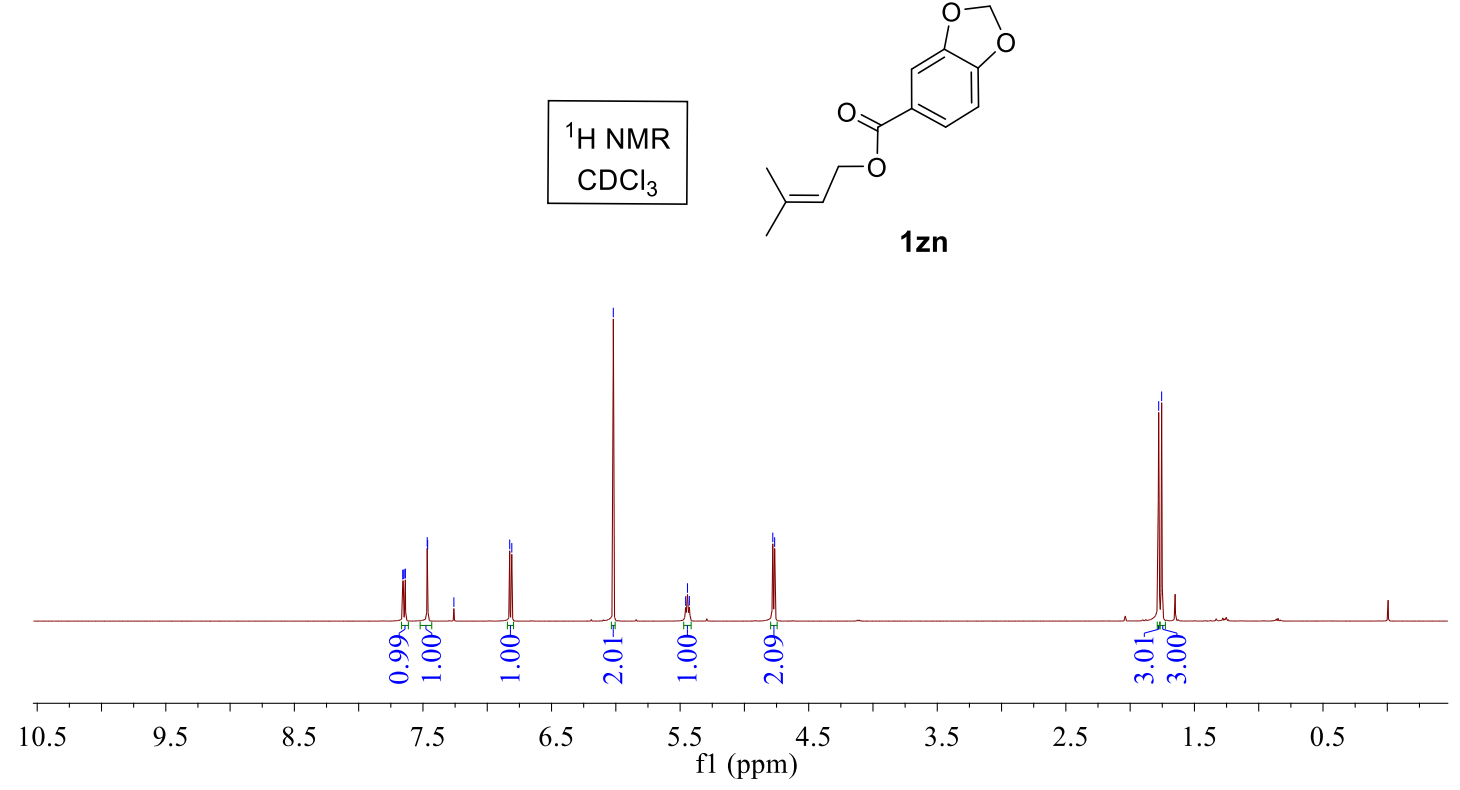

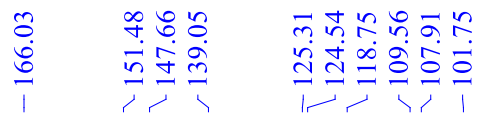
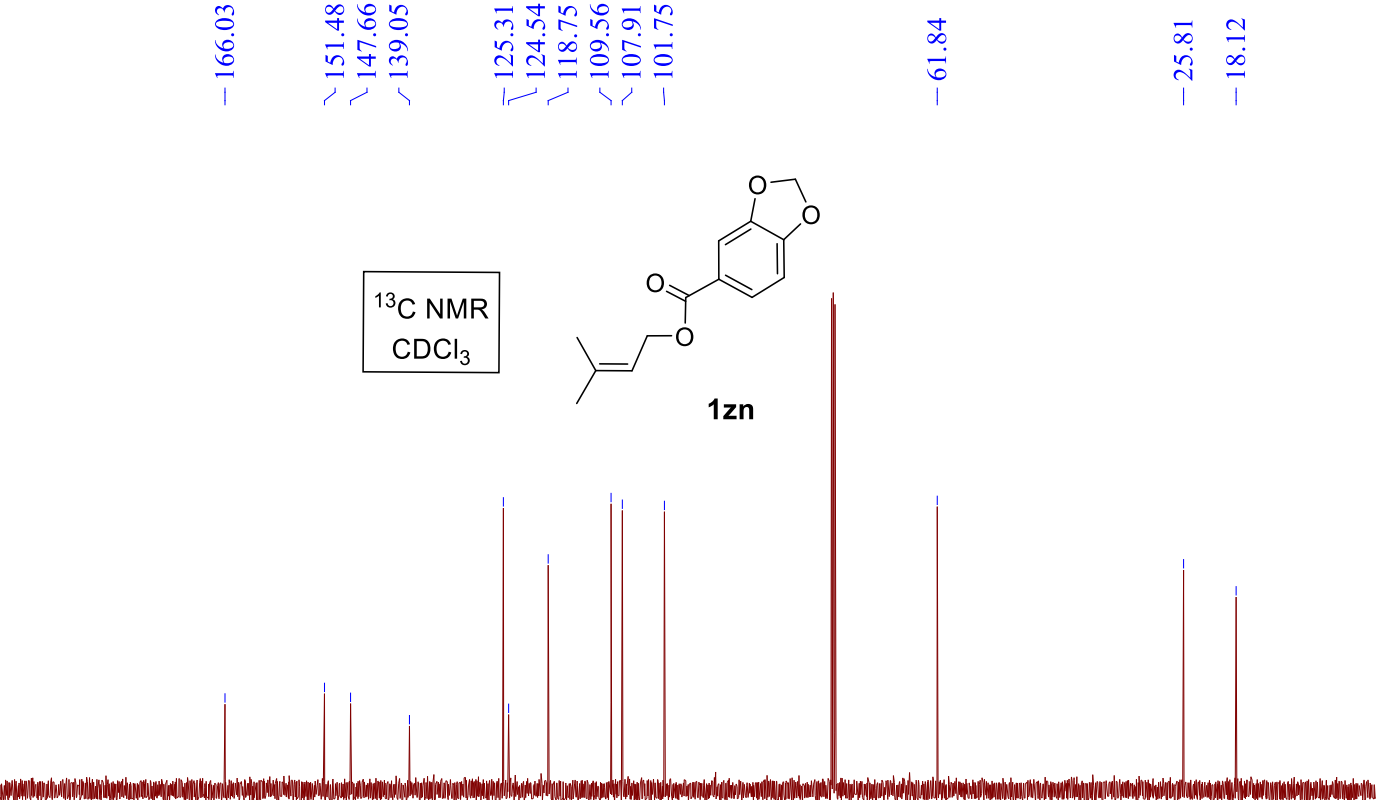

190

170

150

130

110 f1 (ppm) 


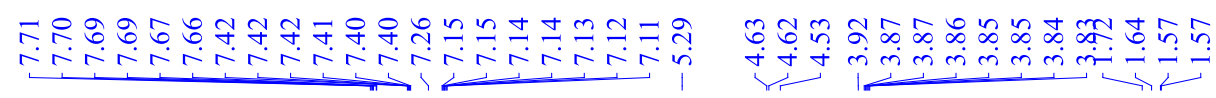
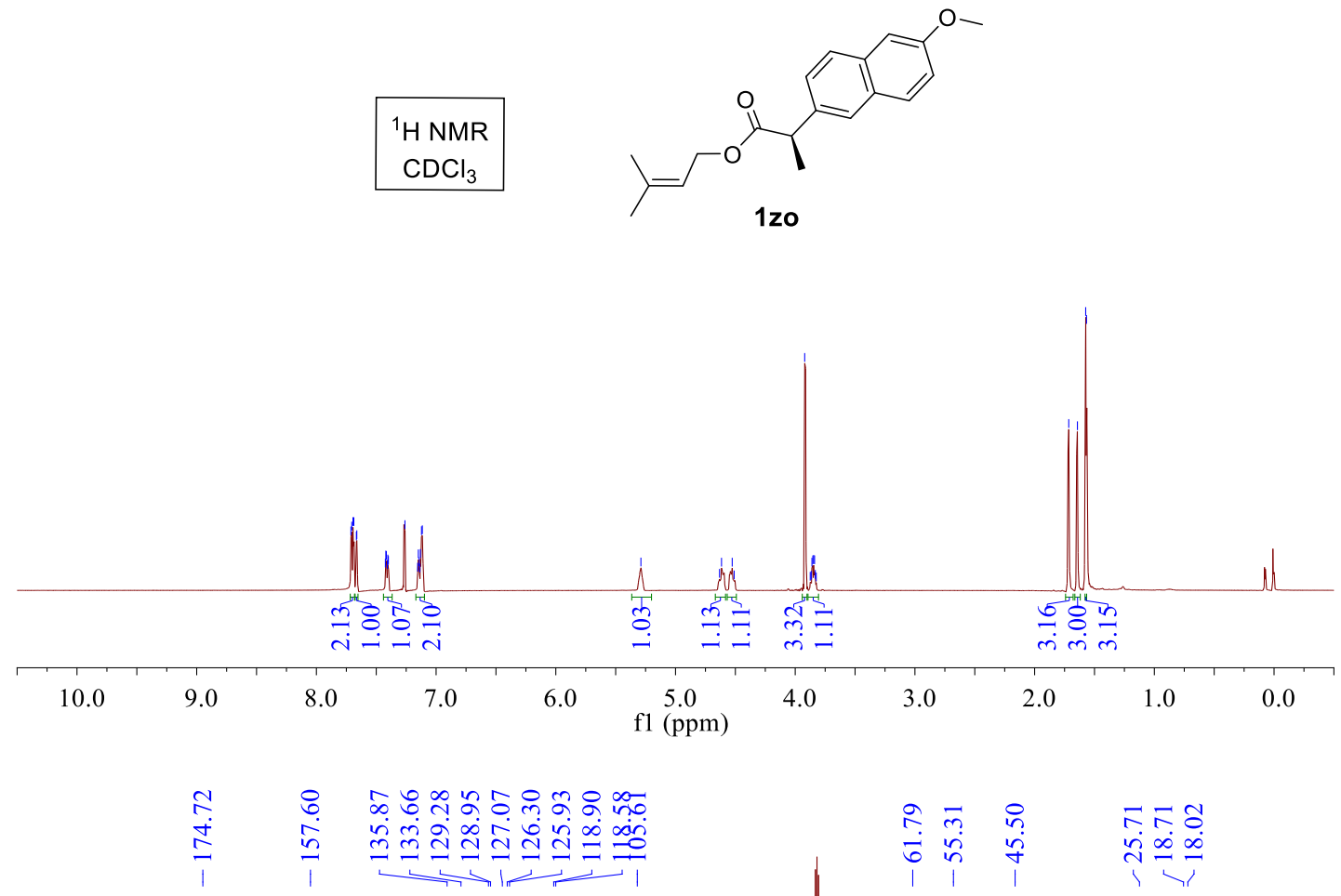

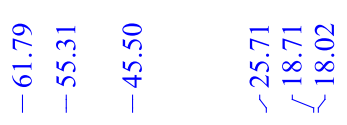

${ }^{13} \mathrm{C} \mathrm{NMR}$
$\mathrm{CDCl}_{3}$<smiles>COc1ccc2cc(C(C)C(=O)OCC=C(C)C)ccc2c1</smiles>

$1 z o$

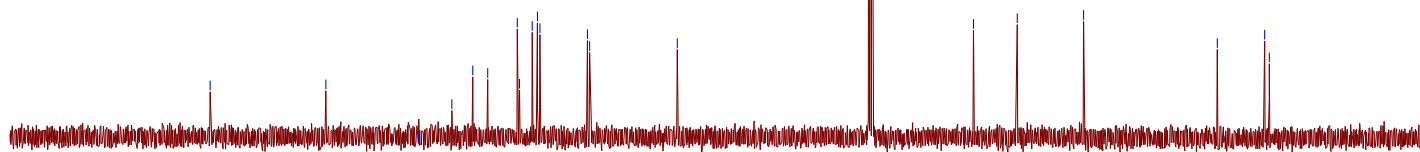

190

170

150

130

110

90

80

$\begin{array}{llllll}50 & 40 & 30 & 20 & 10 & 0\end{array}$ 


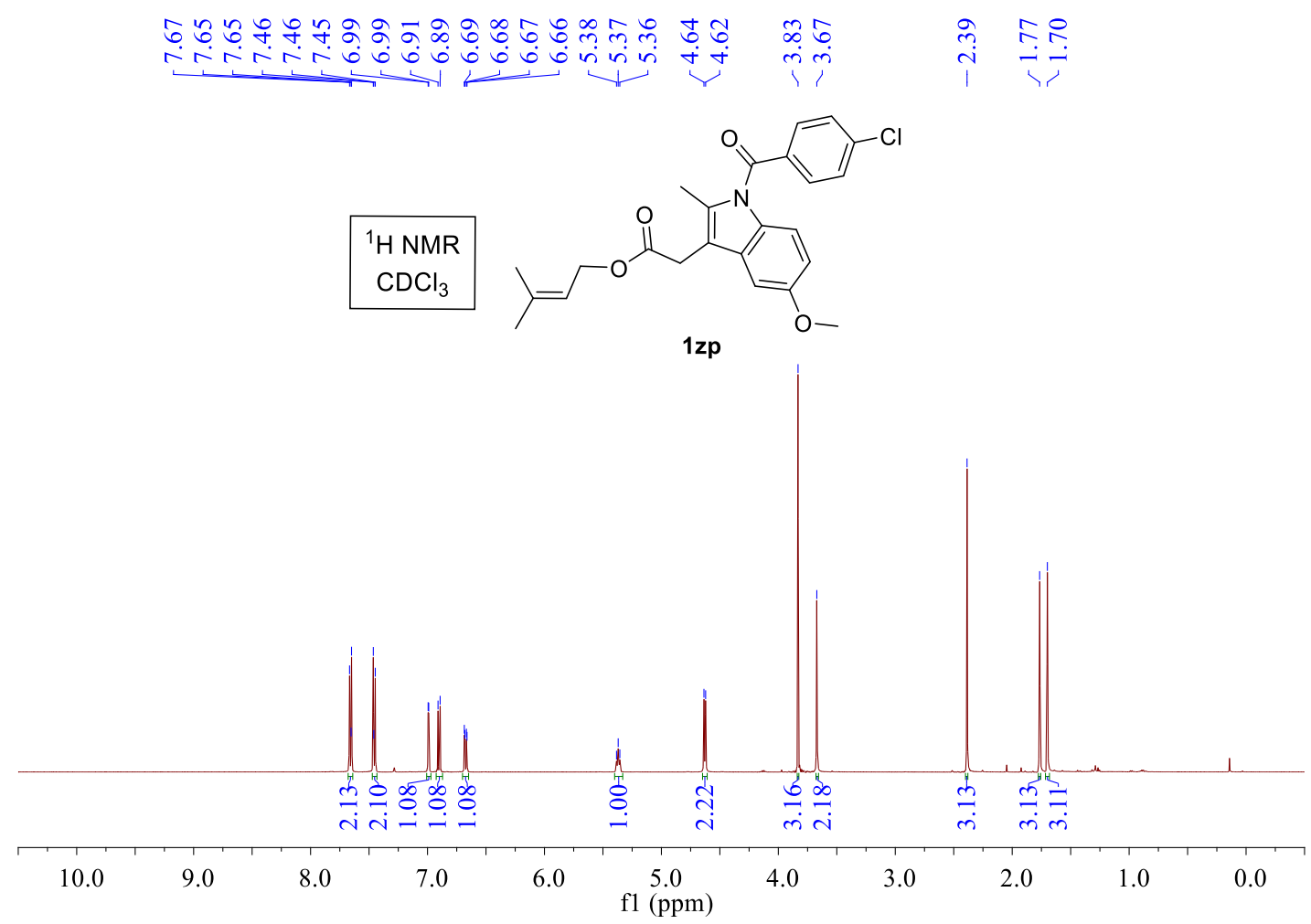

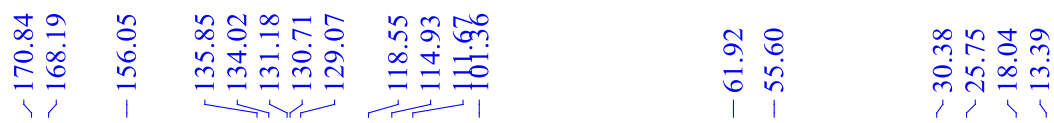

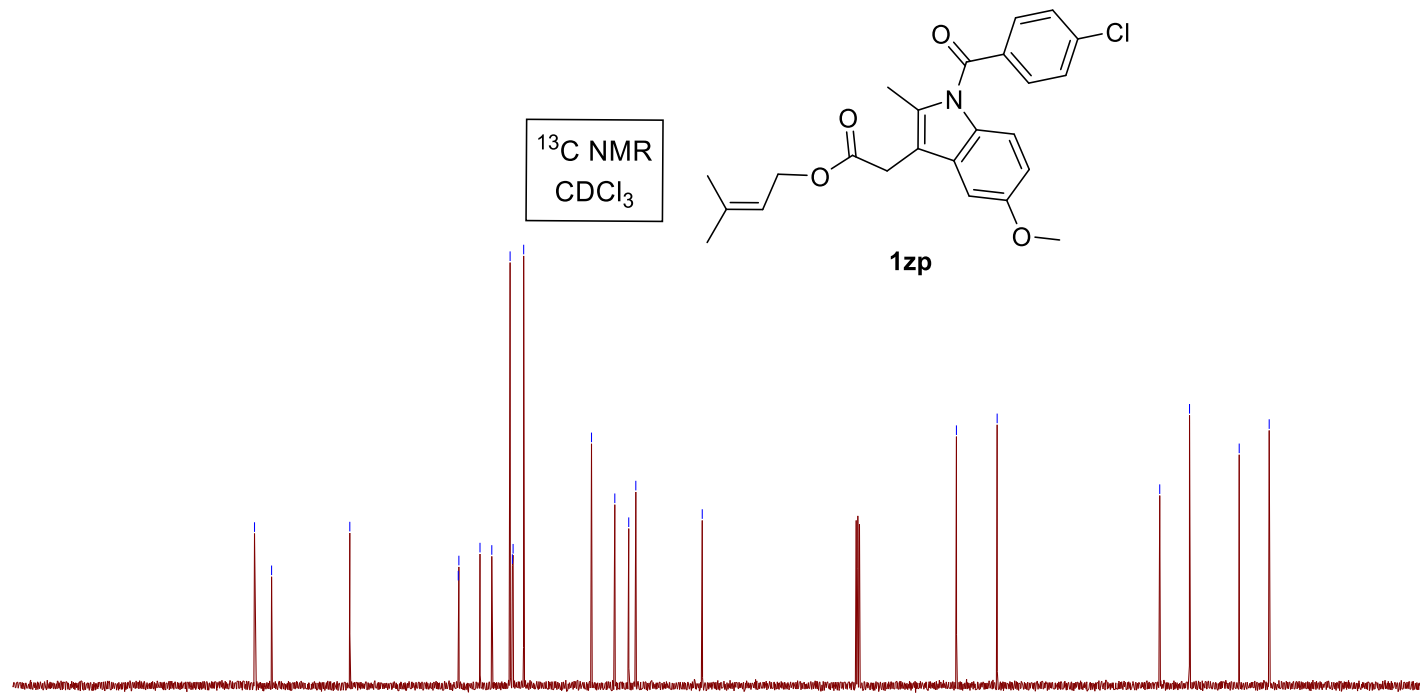

190

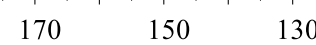

110

$\begin{array}{llllllllll}90 & 80 & 70 & 60 & 50 & 40 & 30 & 20 & 10 & 0\end{array}$ 

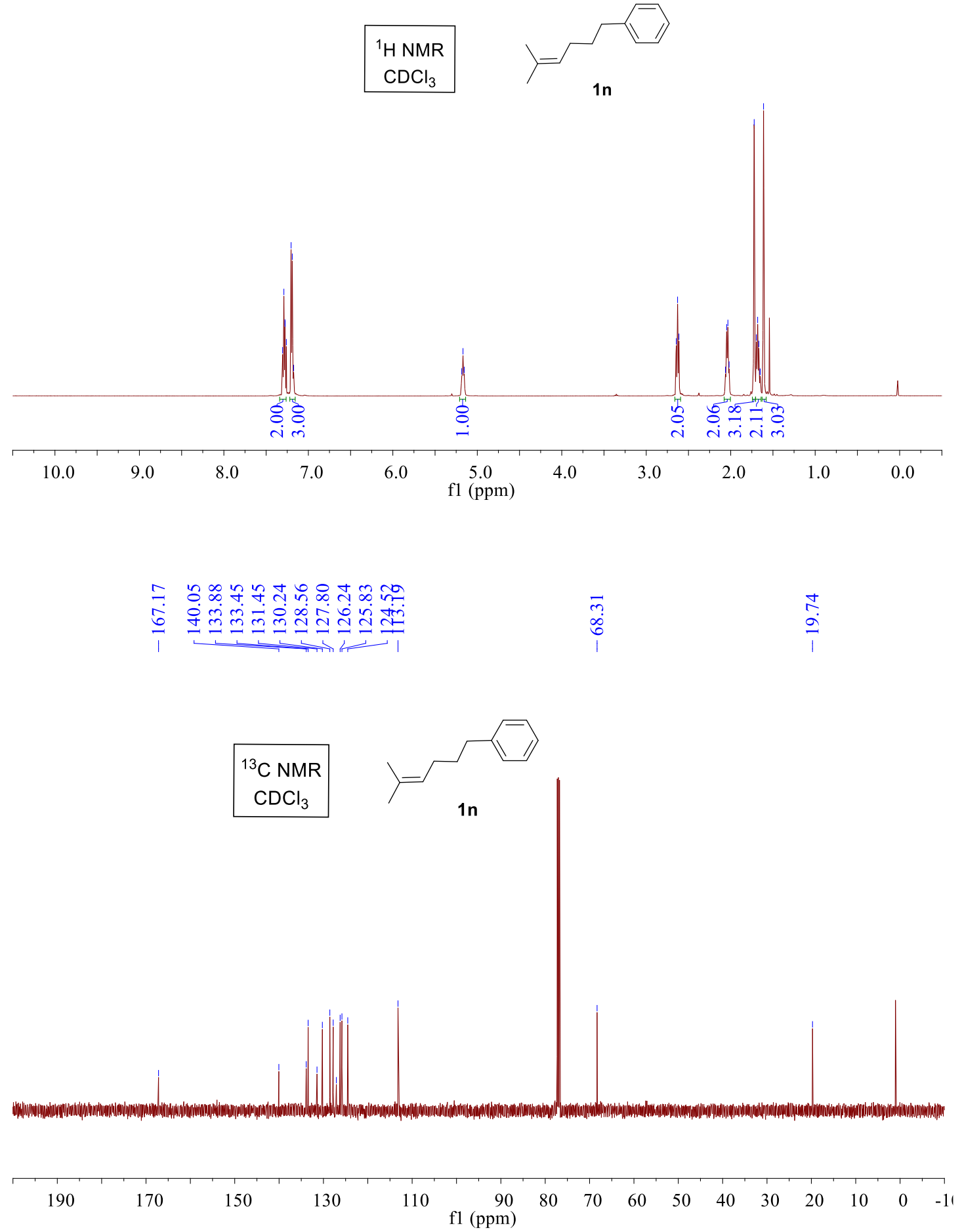

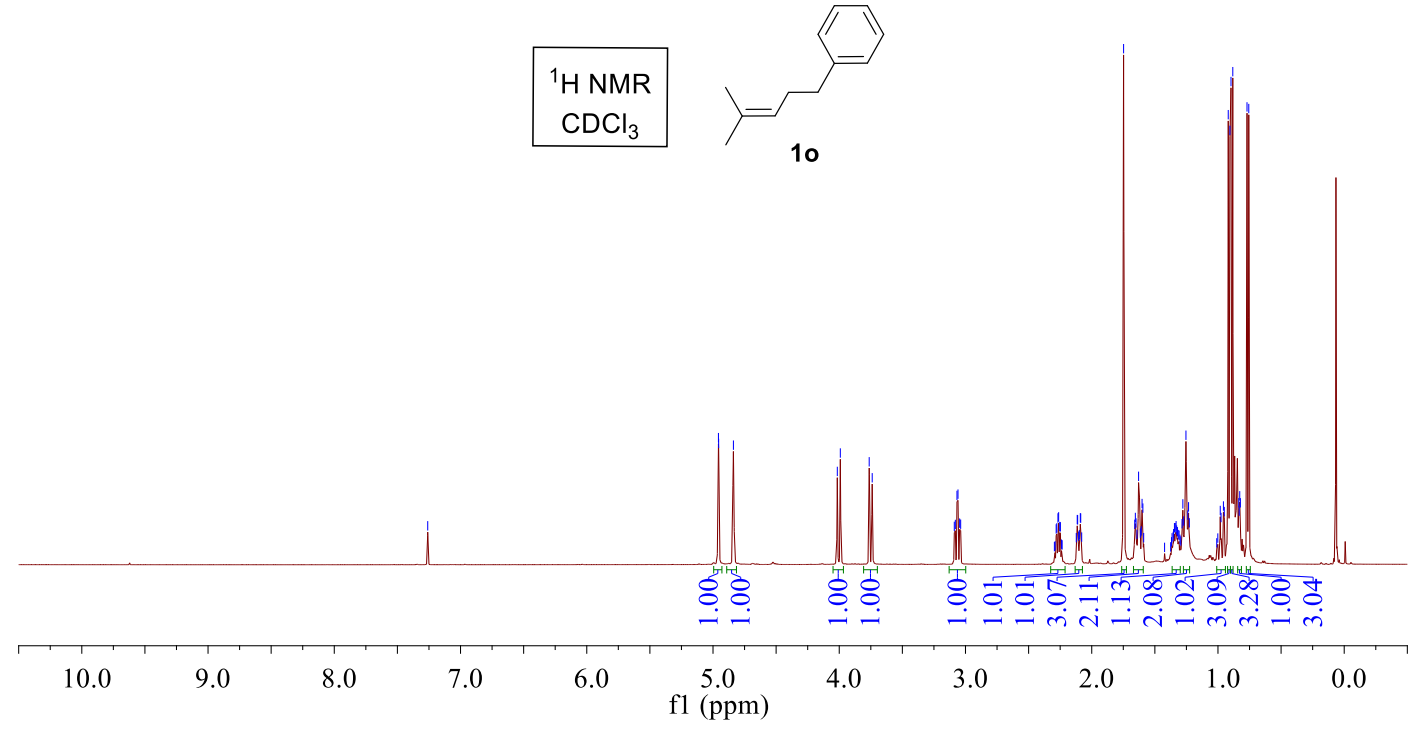

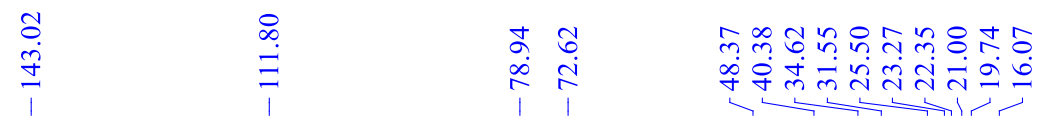

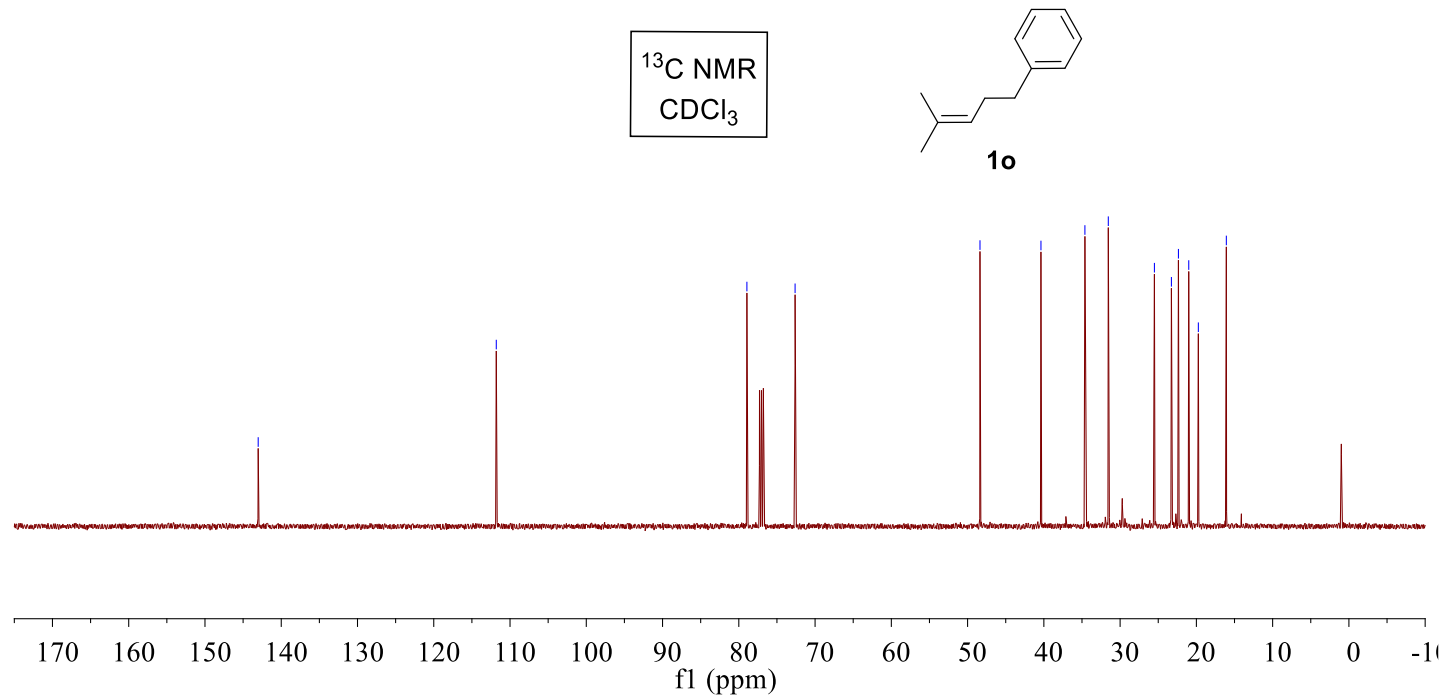



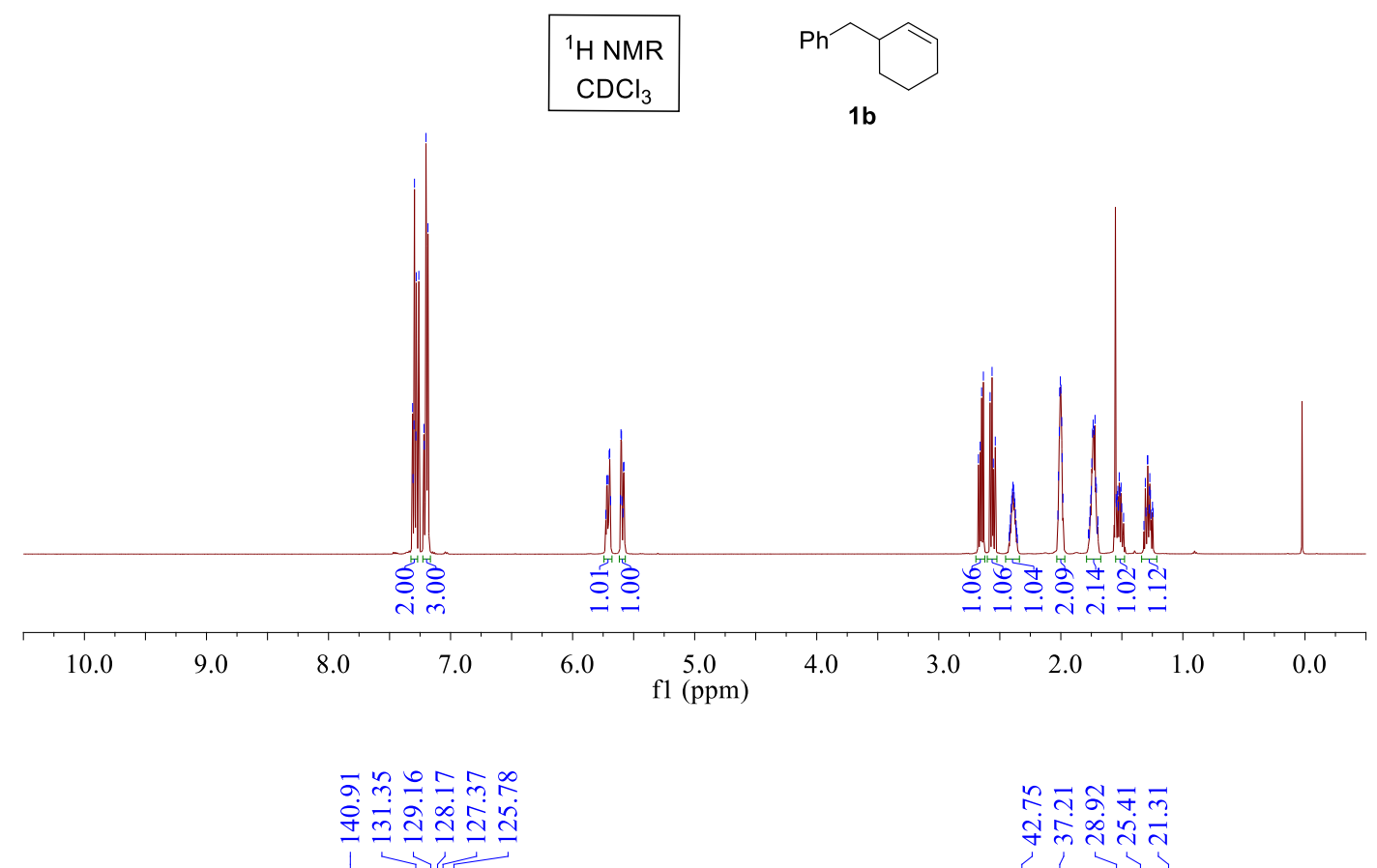

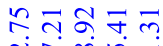

ํํำ

\begin{tabular}{|c|}
${ }^{13} \mathrm{C} \mathrm{NMR}$ \\
$\mathrm{CDCl}_{3}$ \\
\hline
\end{tabular}<smiles>C1=CC(Cc2ccccc2)CCC1</smiles>

1b

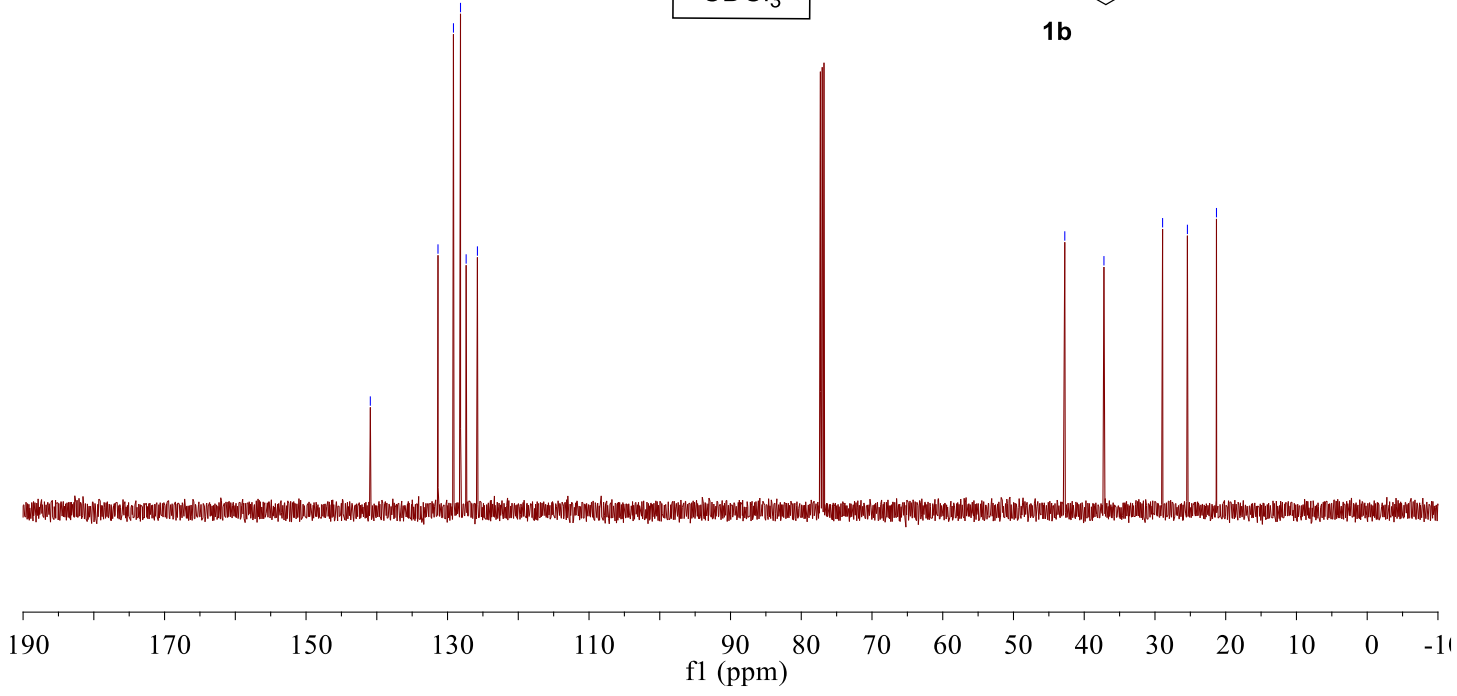




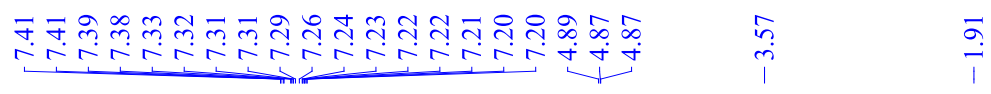

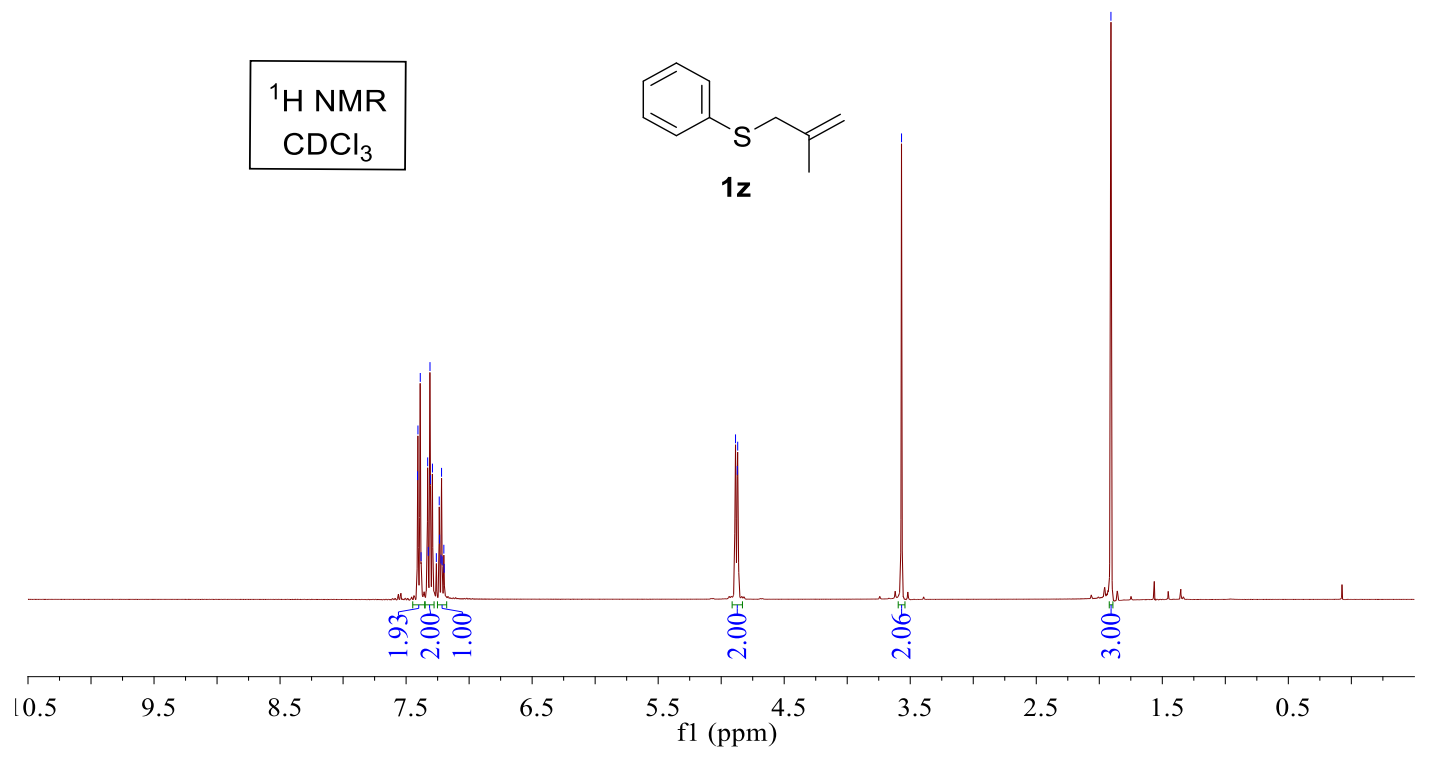

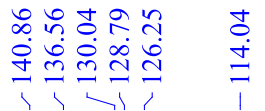

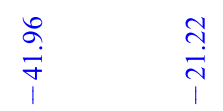

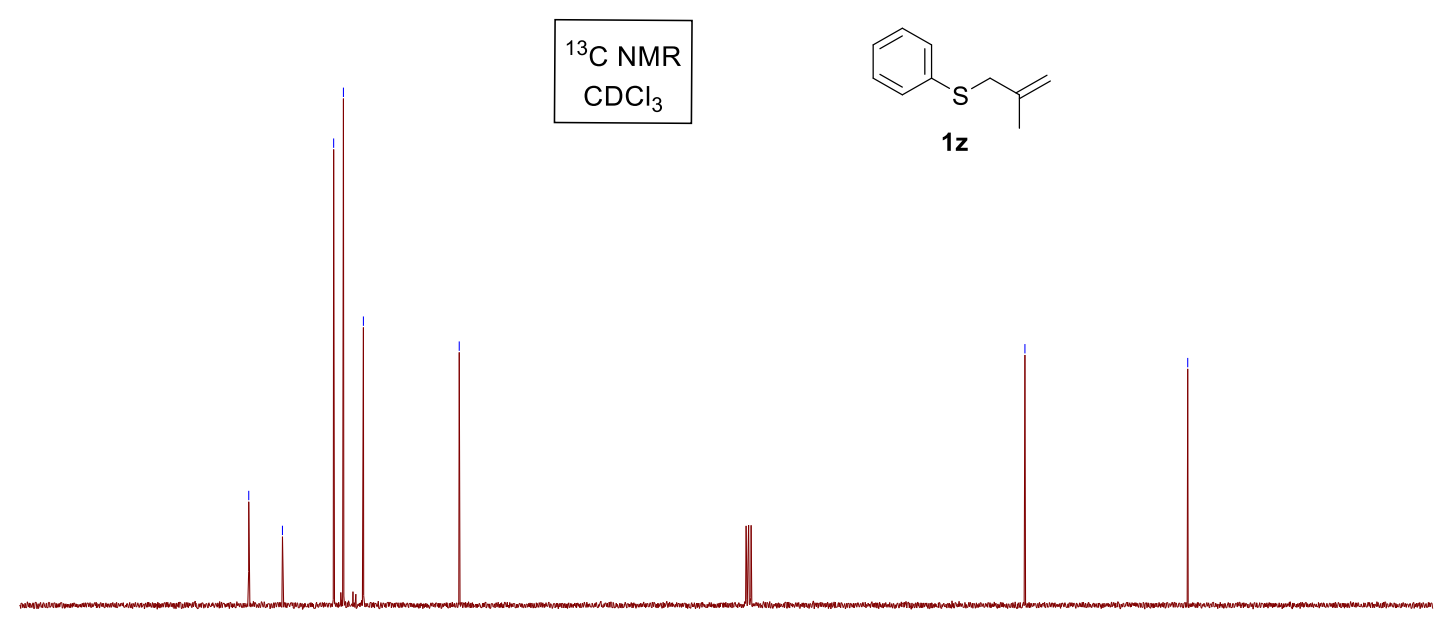

$170 \quad 160 \quad 150$

$\begin{array}{lll}130 & 120\end{array}$

$\begin{array}{llll}110 & 100 & 90\end{array}$

$\begin{array}{llllllllll}80 & 70 & 60 & 50 & 40 & 30 & 20 & 10 & 0 & -11\end{array}$

f1 (ppm) 


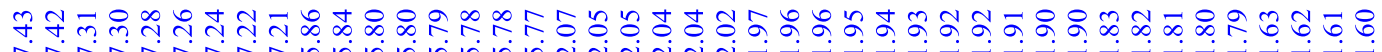

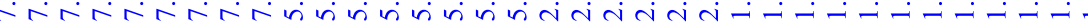
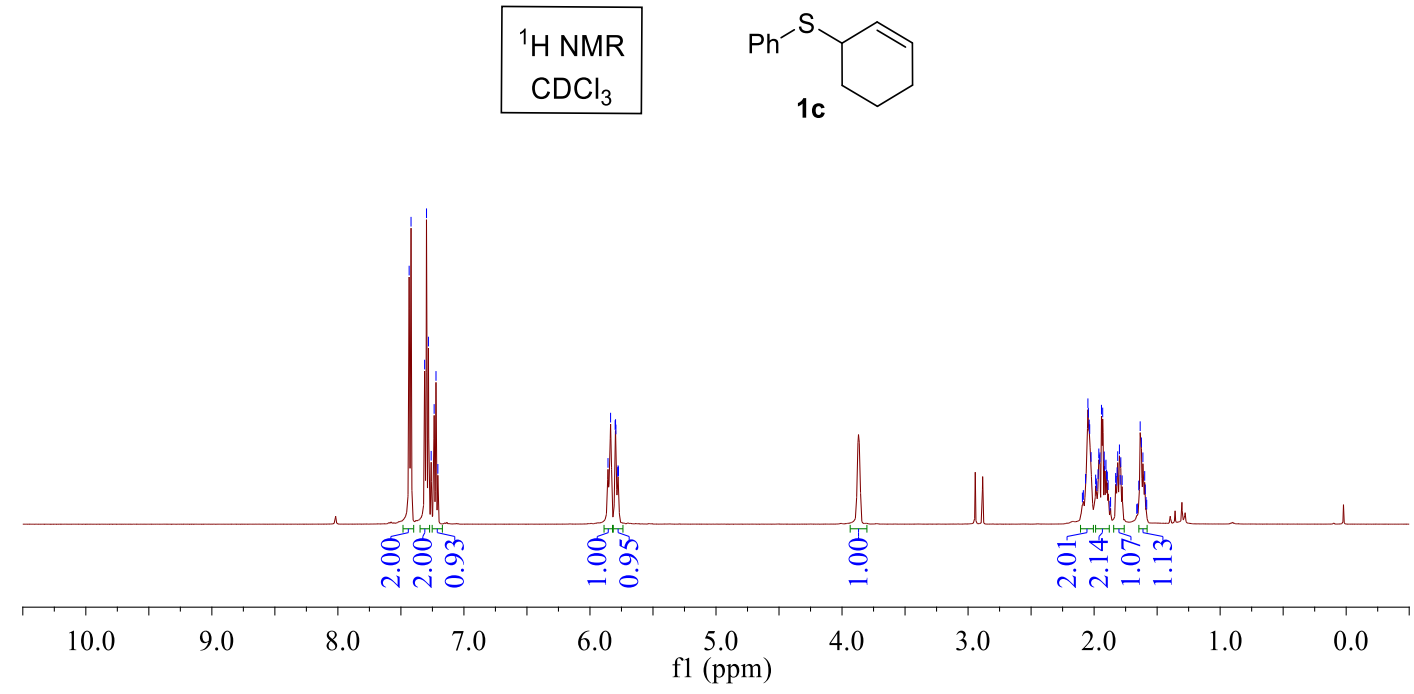

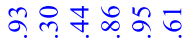

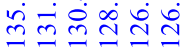

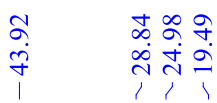
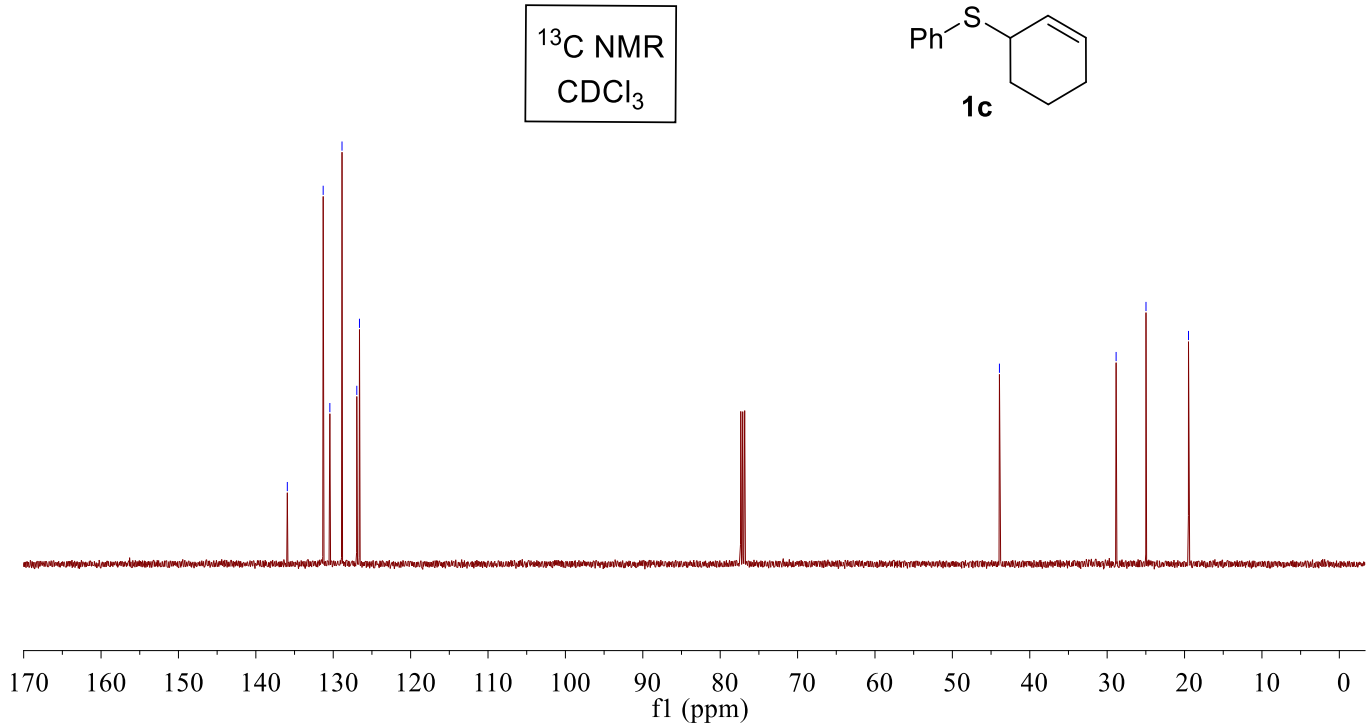


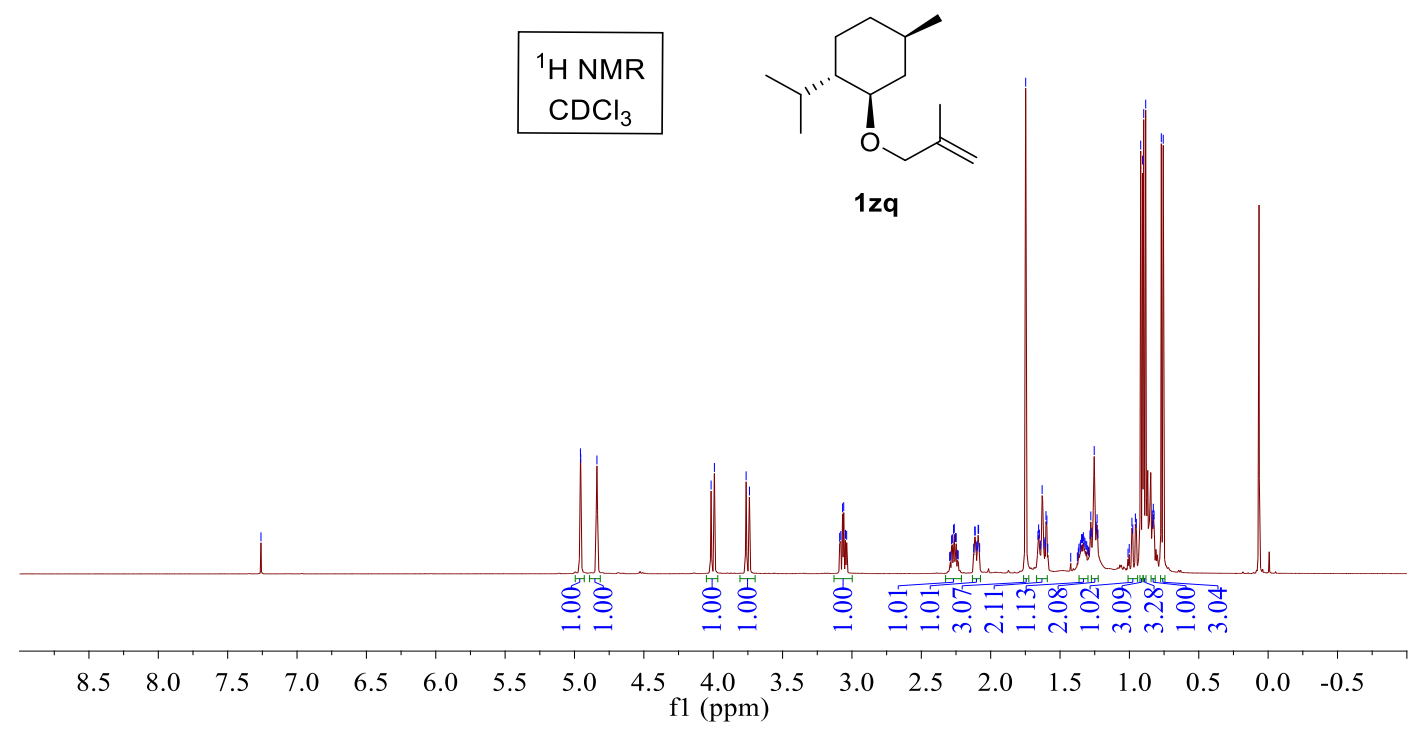

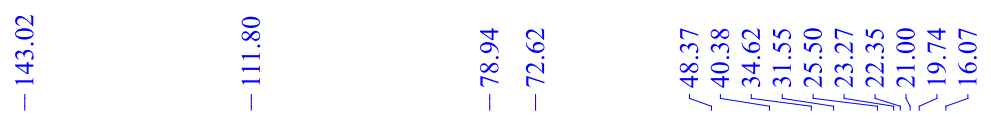
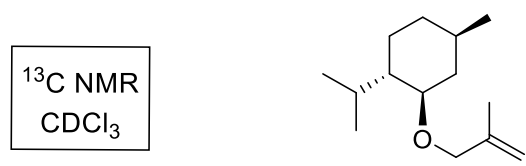

$1 z q$

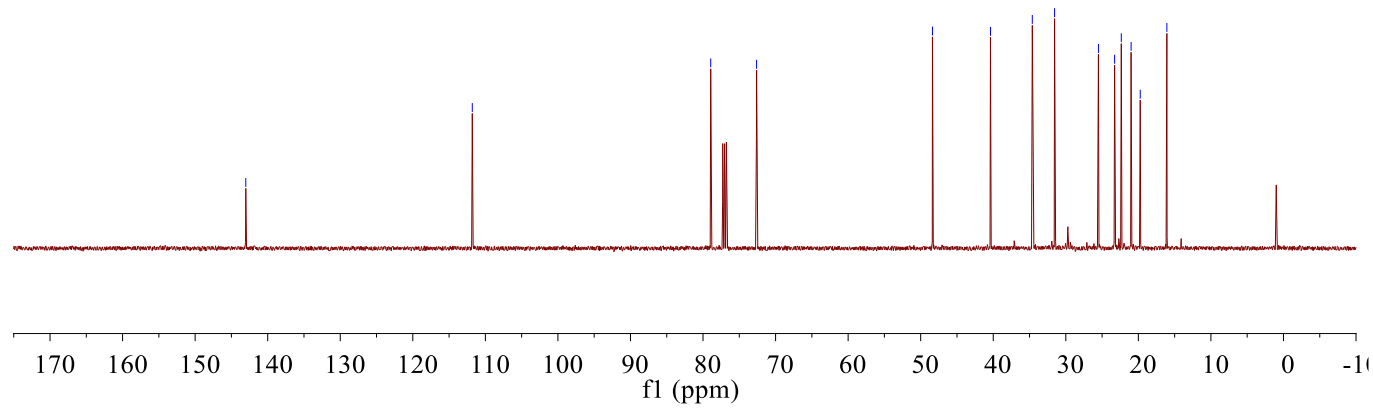




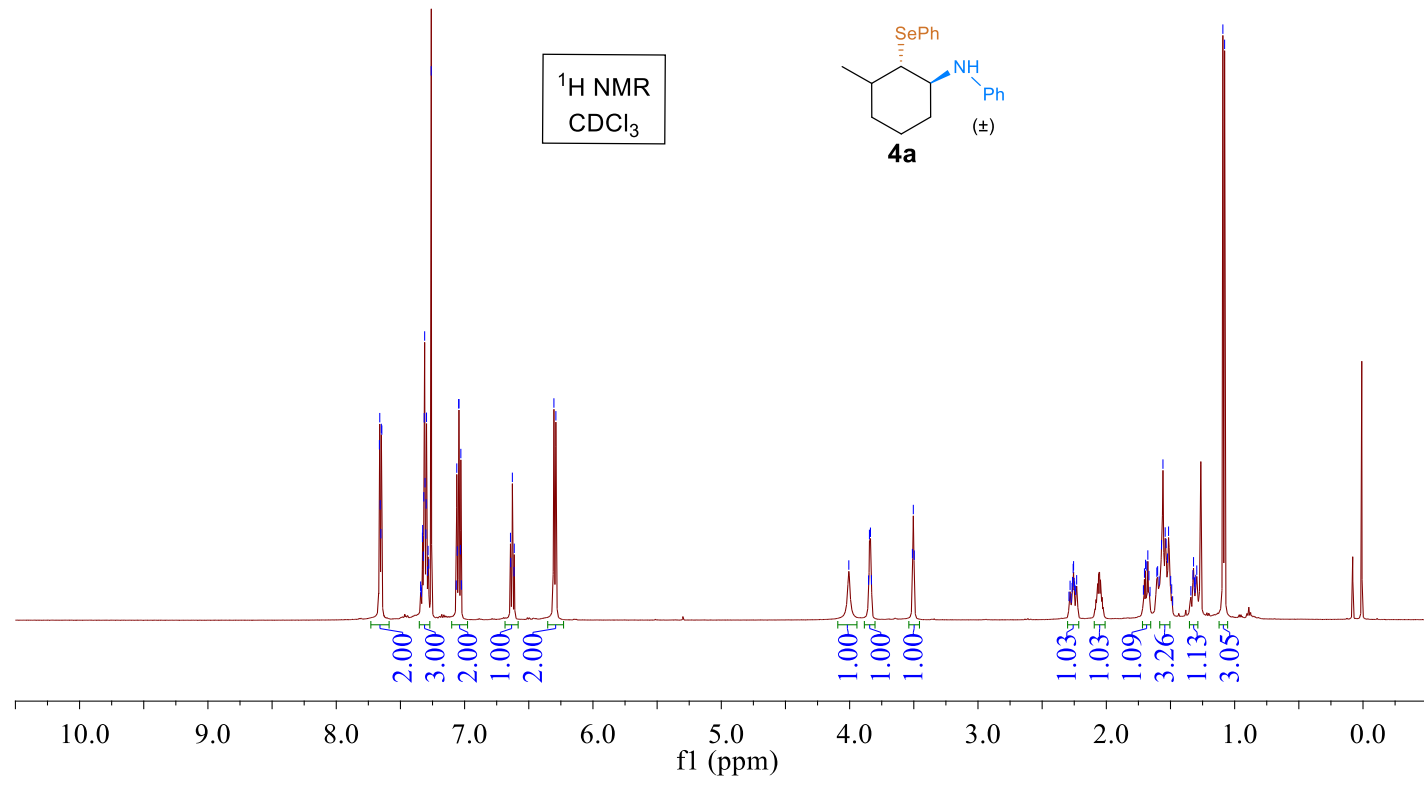

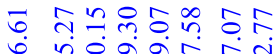
药

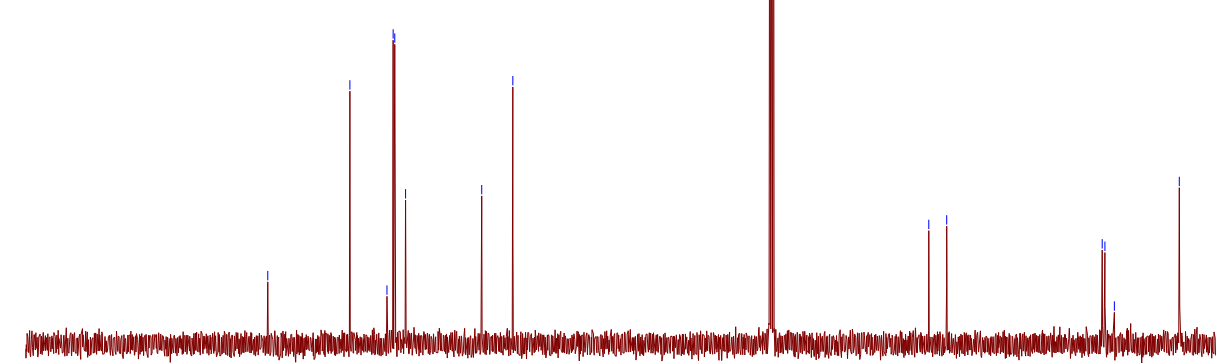



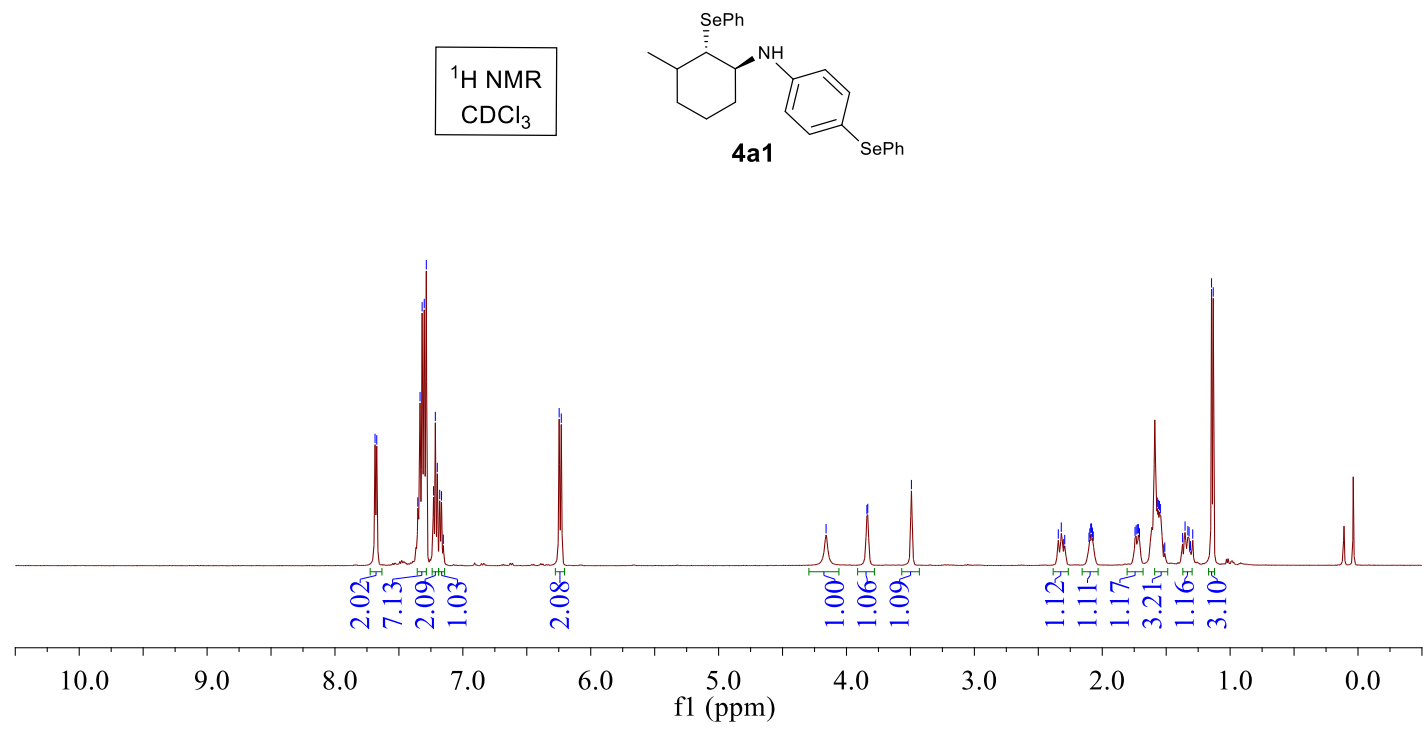

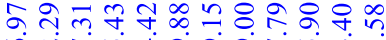

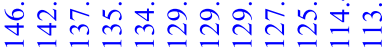
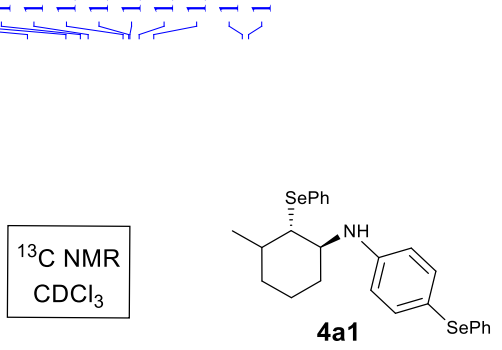

$4 a 1$

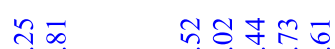

in

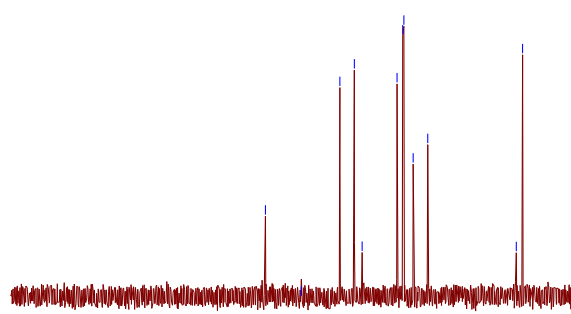

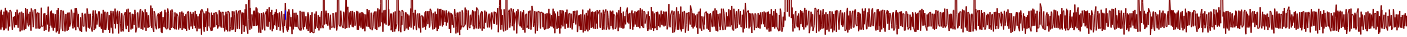



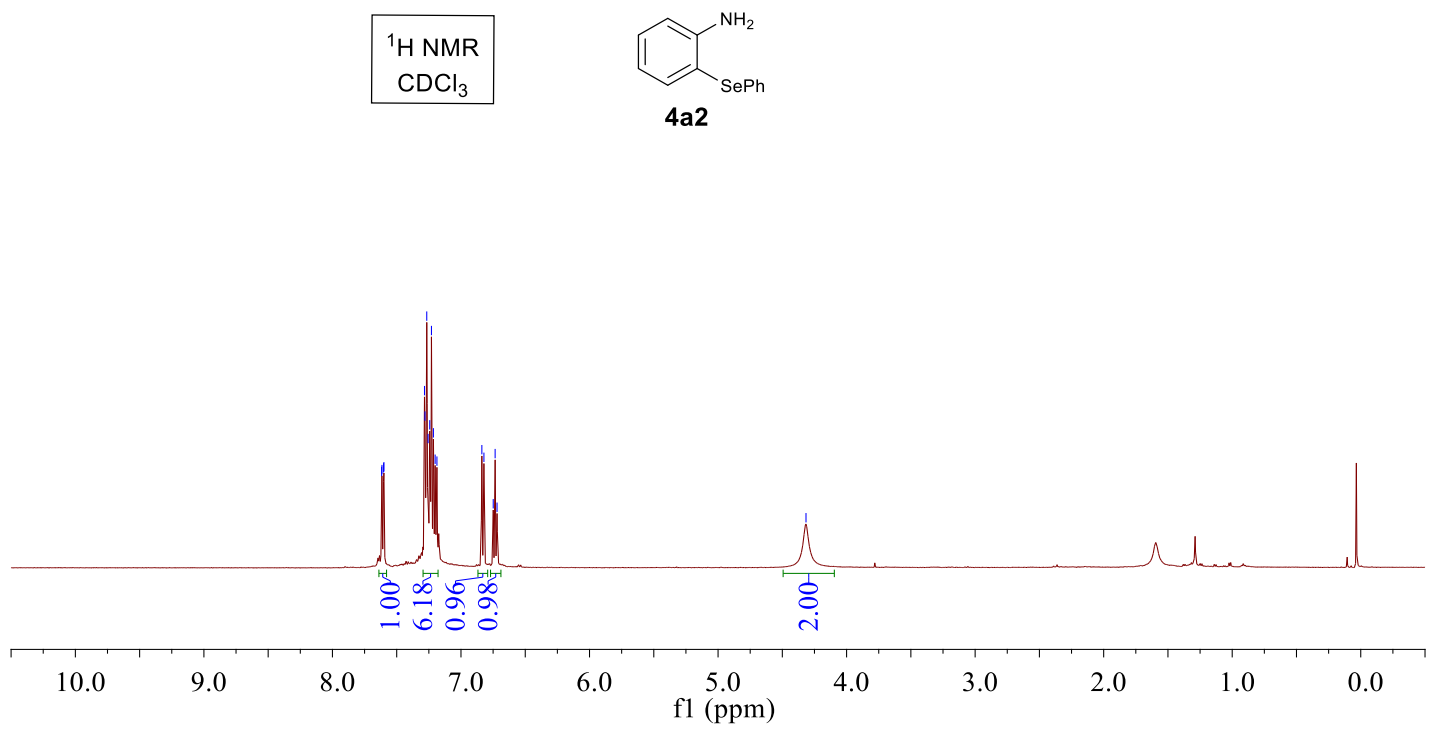

ㄴ.

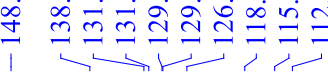

$$
{ }^{13} \mathrm{C} \mathrm{NMR}
$$

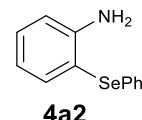

$4 \mathrm{a} 2$

$\begin{array}{lllllllllllllllll}170 & 160 & 150 & 140 & 130 & 120 & 110 & 100 \underset{\mathrm{f} 1(\mathrm{ppm})}{90} & 80 & 70 & 60 & 50 & 40 & 30 & 20 & 10 & 0\end{array}$




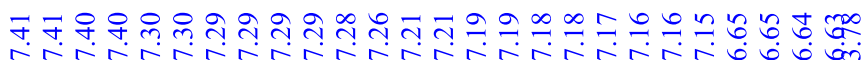
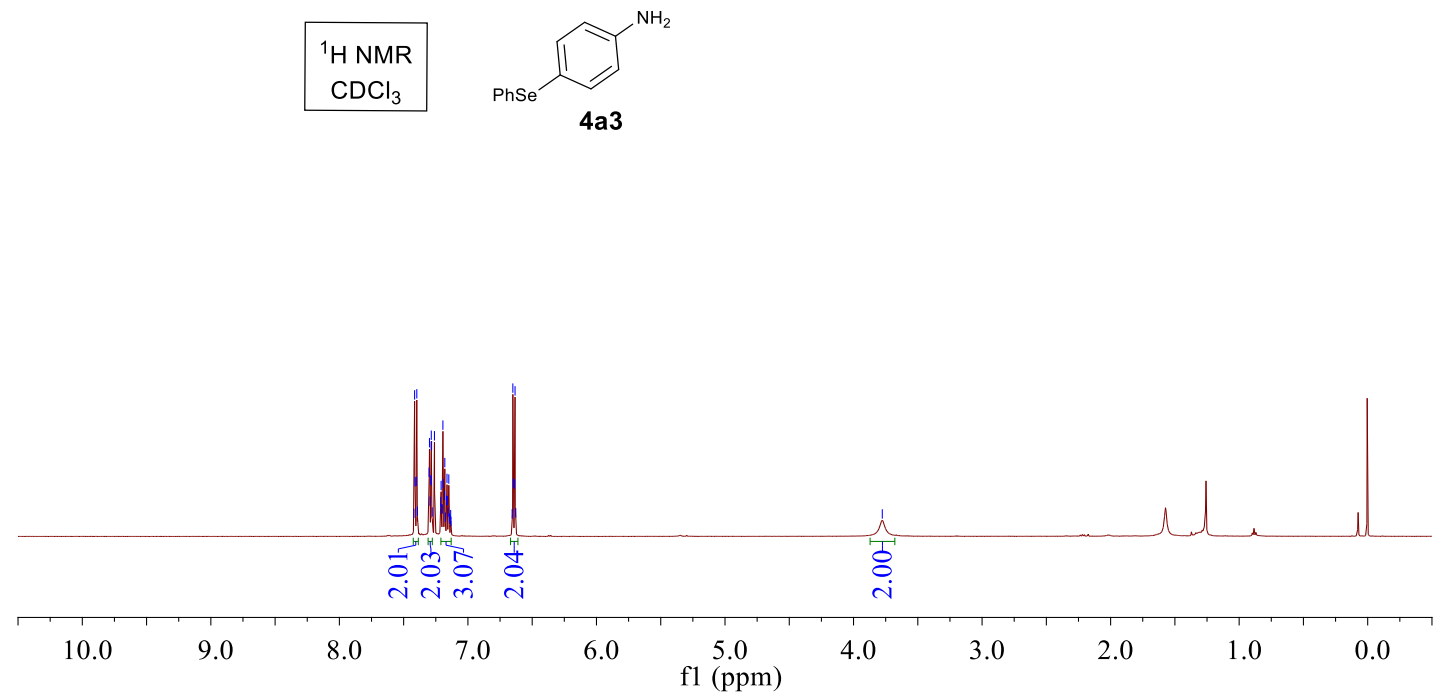

+

守
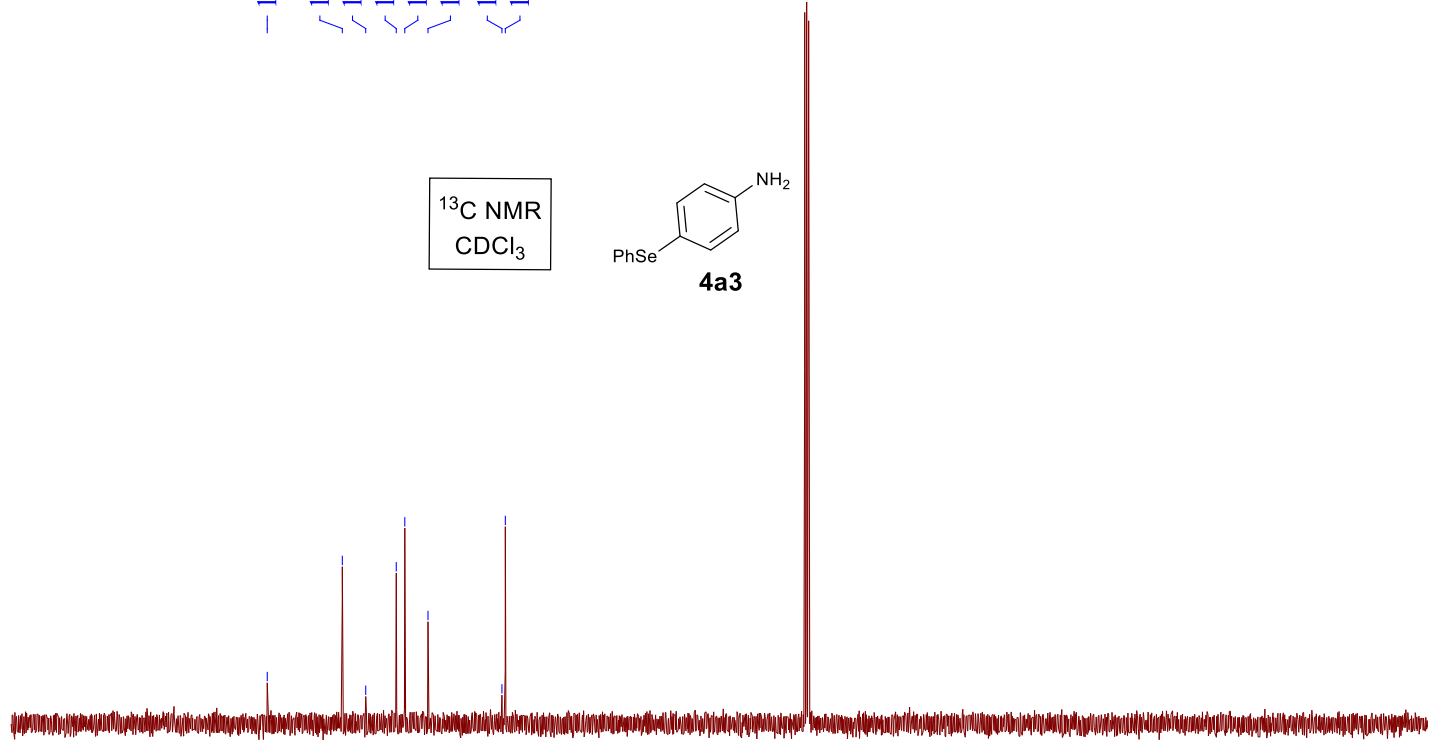

$00 \quad 90 \quad 80$

70

60

$50 \quad 40$

30

$20 \quad 10$

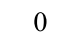




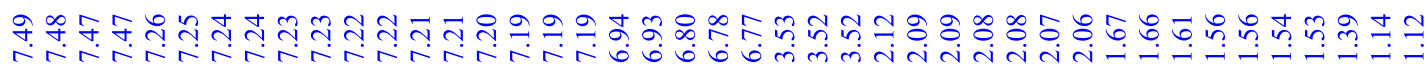

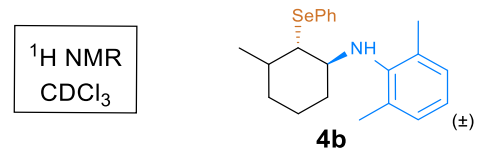

$4 b$

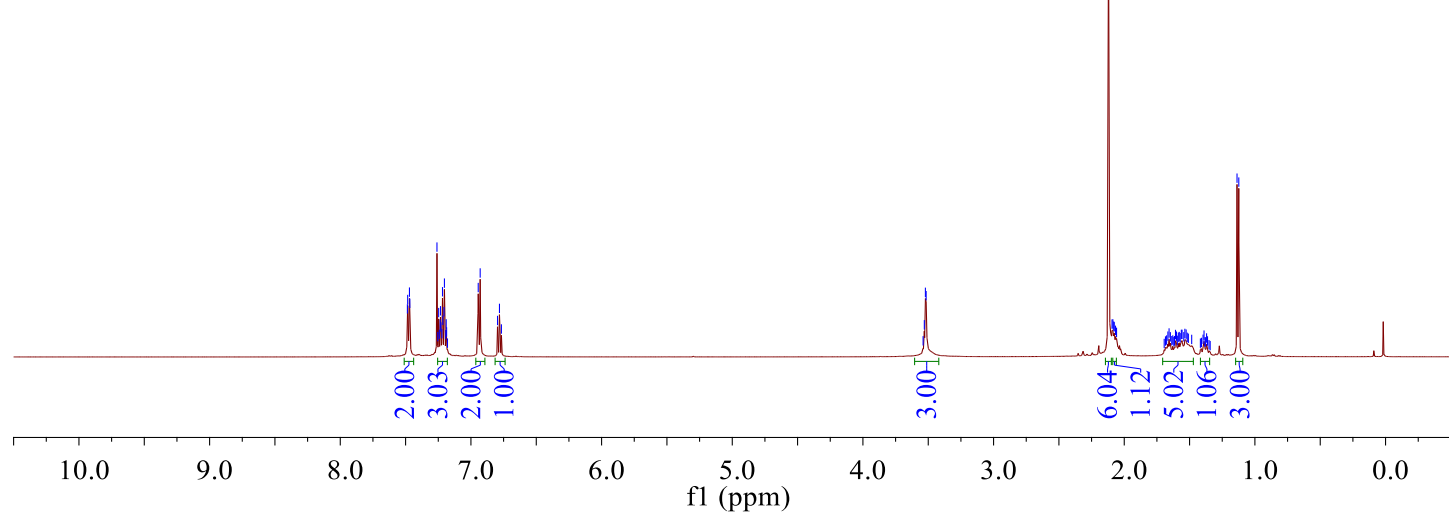

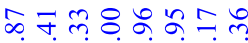

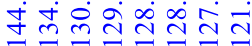

I

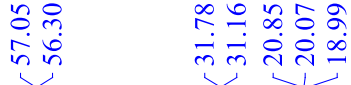

\section{${ }^{13} \mathrm{C}$ NMR}

$\mathrm{CDCl}_{3}$

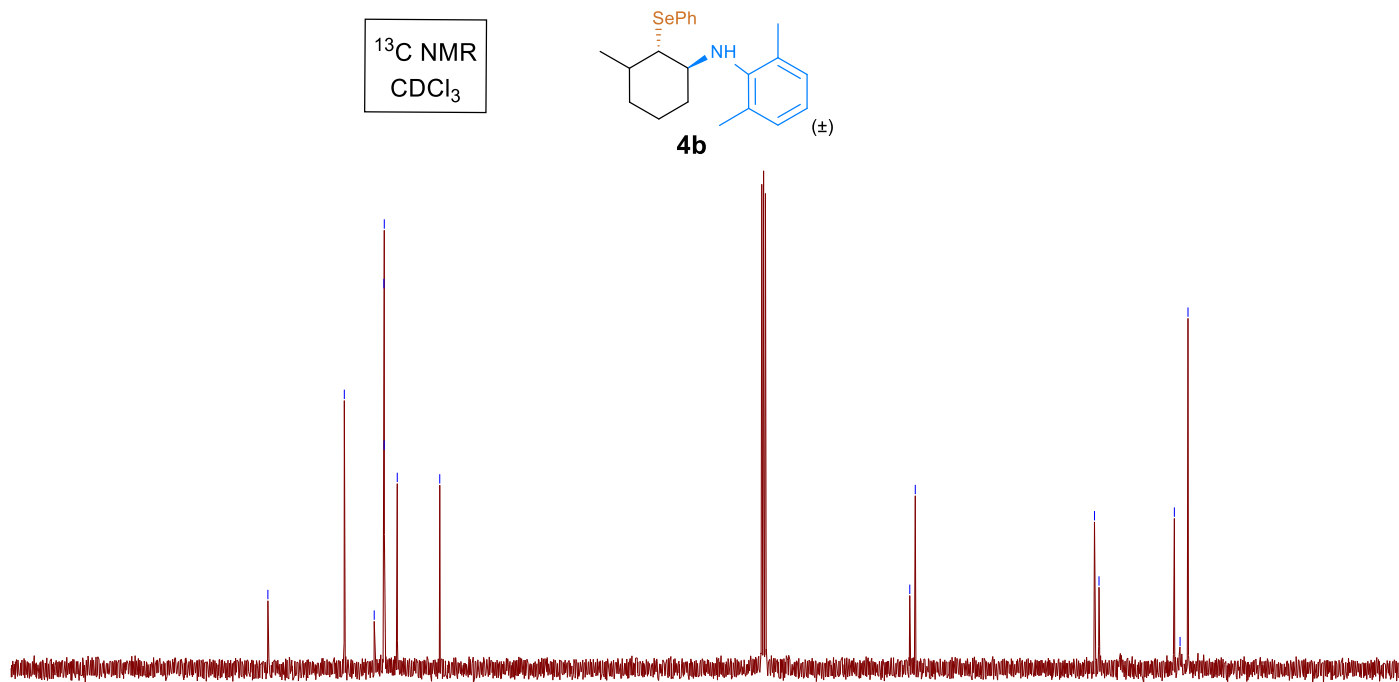

$\begin{array}{llllllllll}180 & 170 & 160 & 150 & 140 & 130 & 120 & 110 & 100 & 90 \quad 80\end{array}$

$\begin{array}{lllllllll}70 & 60 & 50 & 40 & 30 & 20 & 10 & 0 & -11\end{array}$



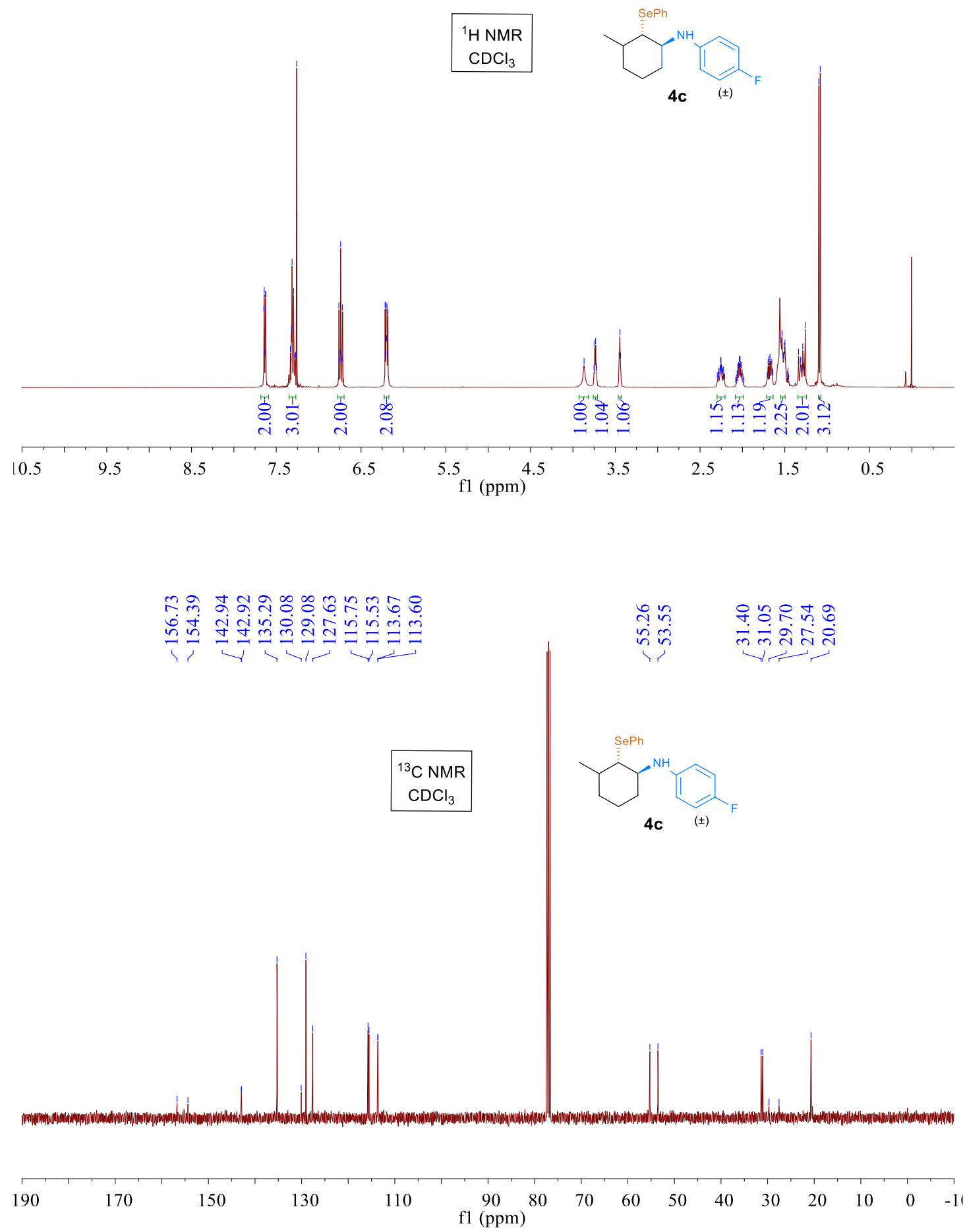

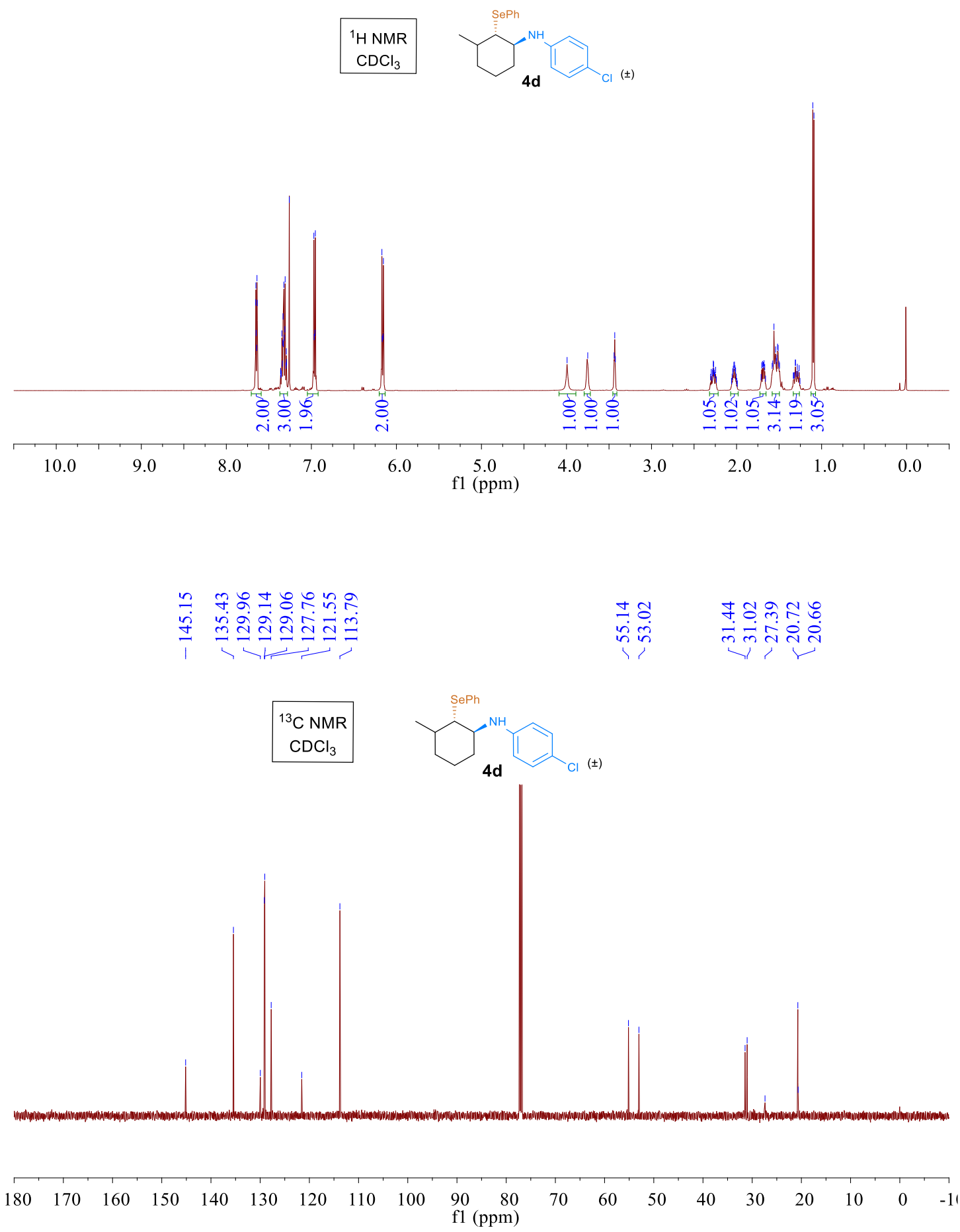


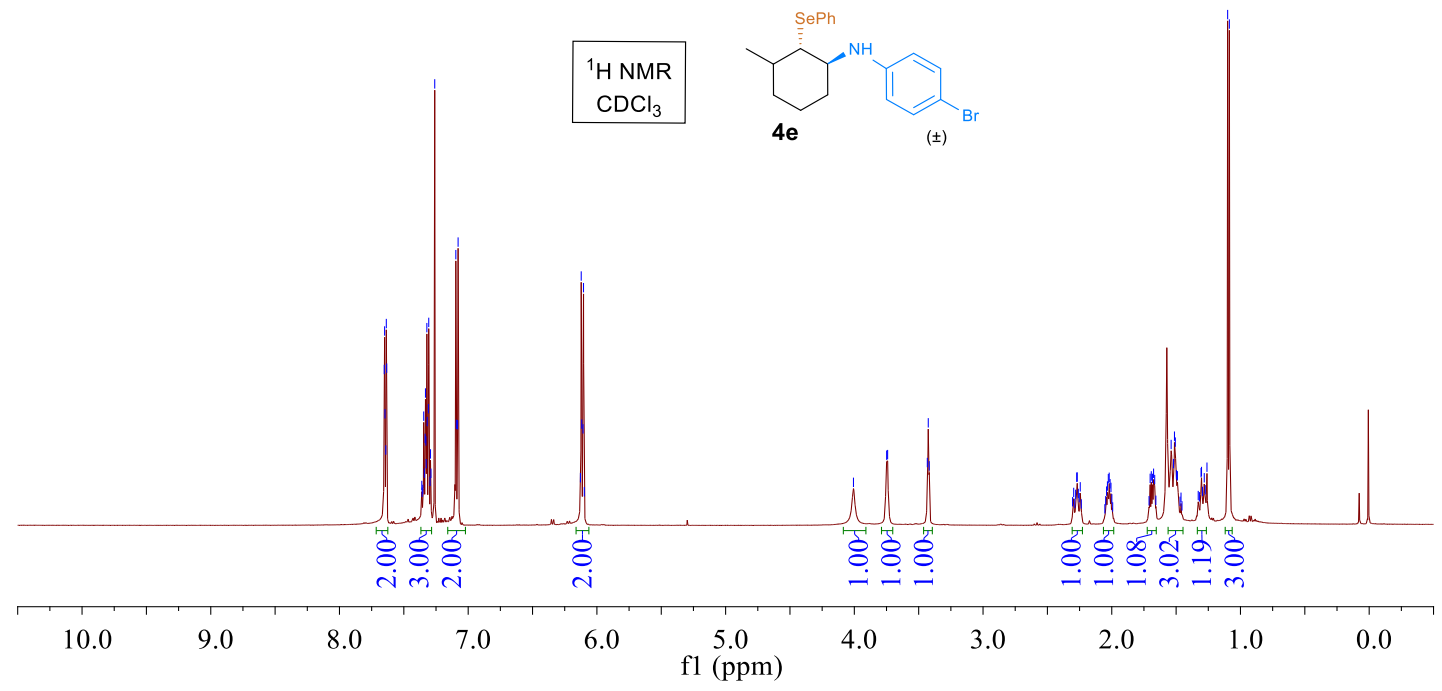

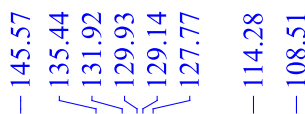

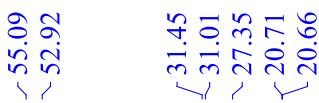
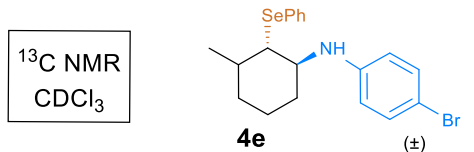

( $)$

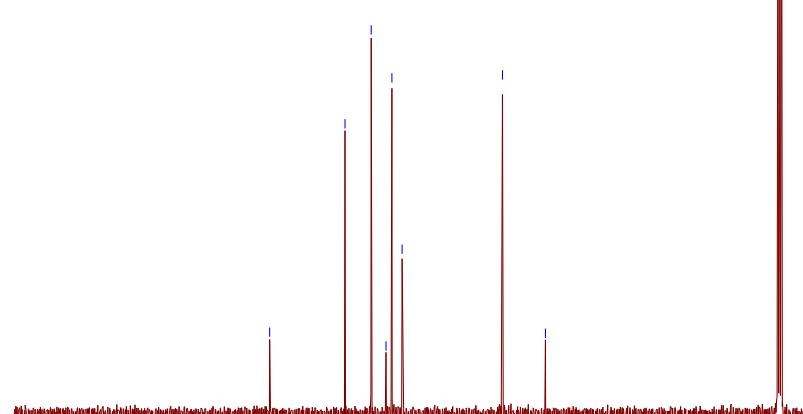

$\begin{array}{llllllllllllllllllll}180 & 170 & 160 & 150 & 140 & 130 & 120 & 110 & 100 & \begin{array}{l}90 \\ \mathrm{f} 1(\mathrm{ppm})\end{array} & 70 & 60 & 50 & 40 & 30 & 20 & 10 & 0 & -1\end{array}$ 

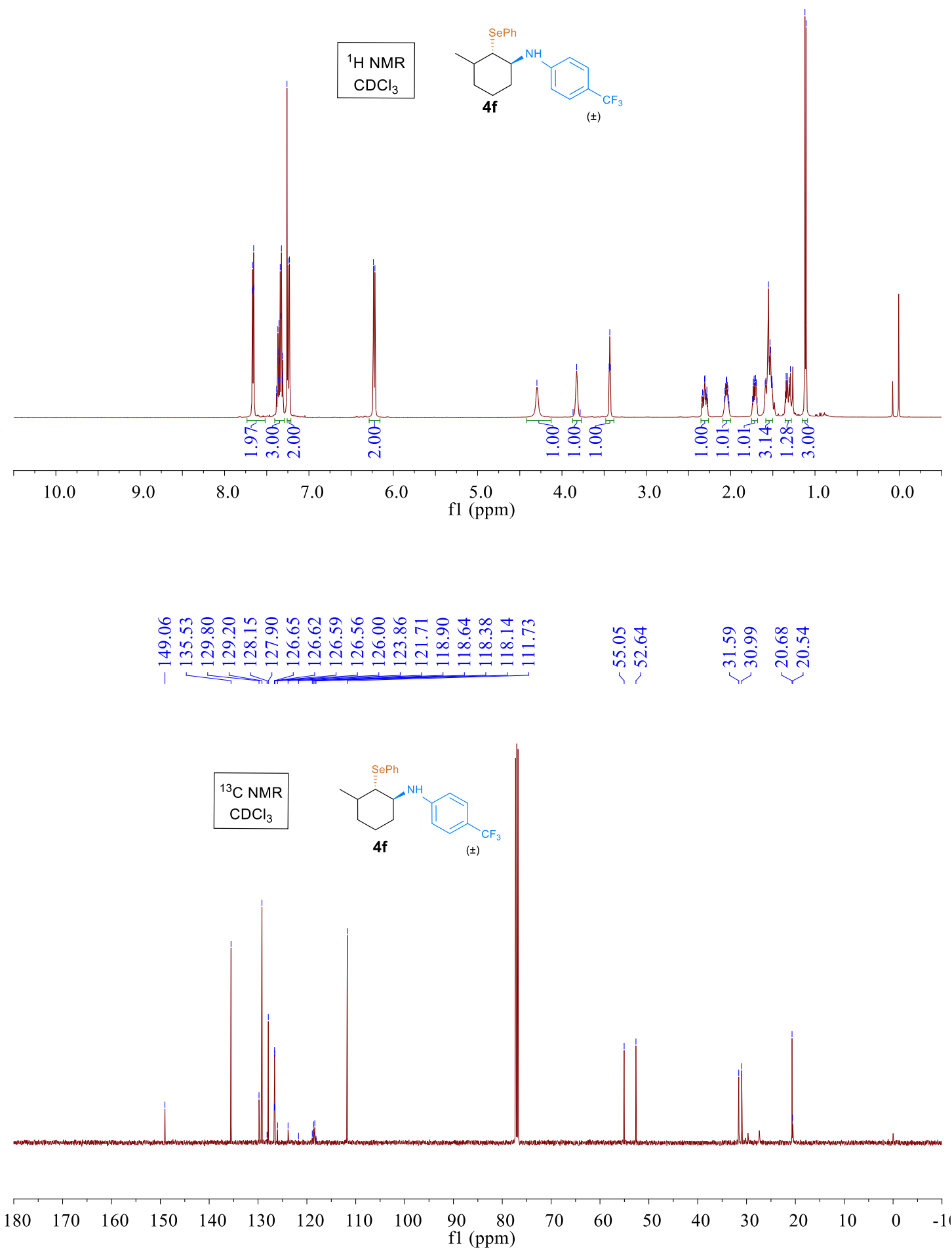

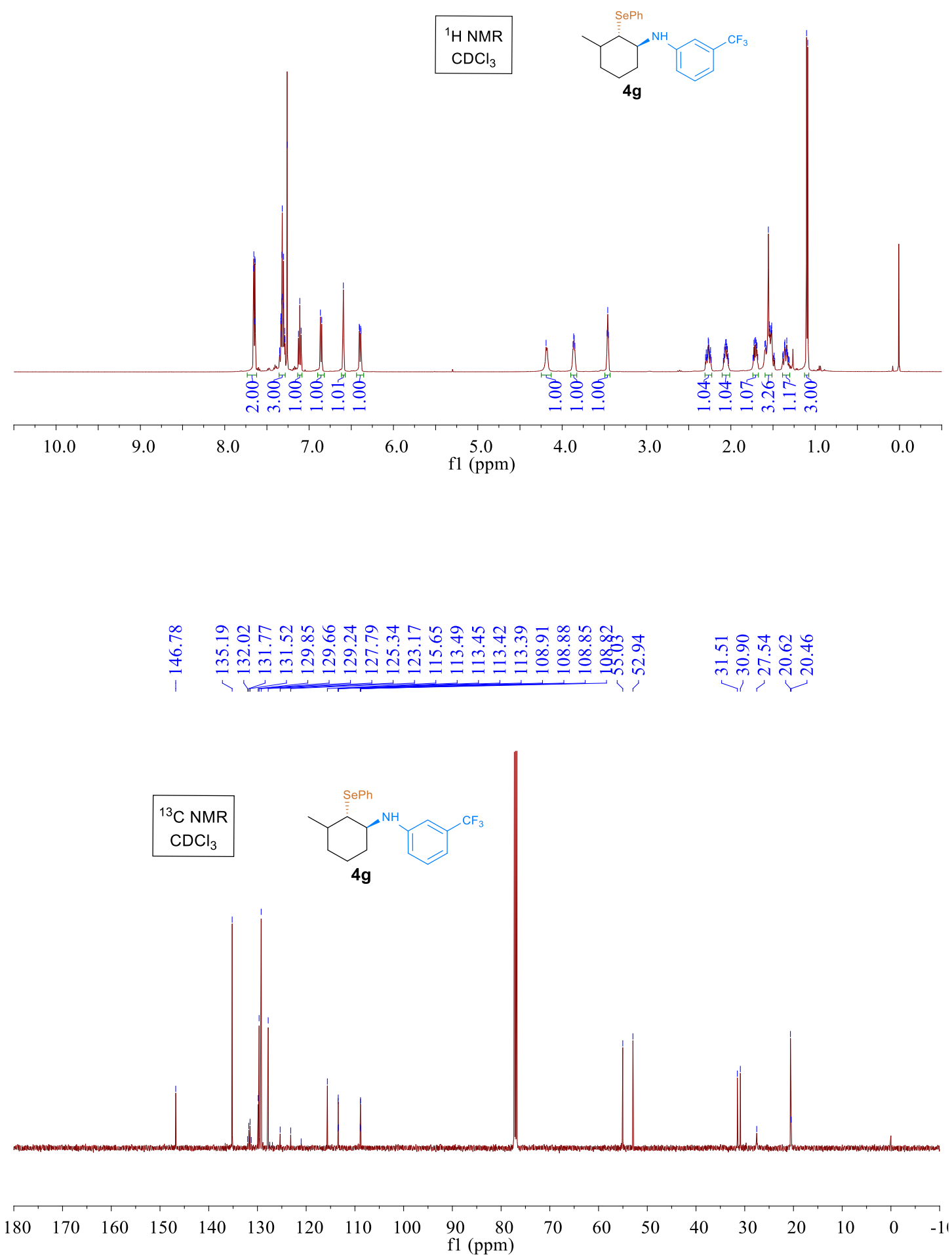


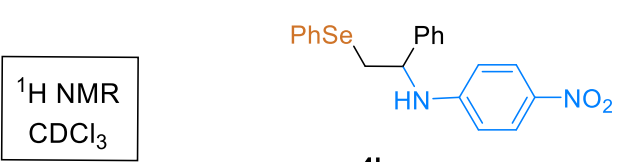

$4 h$
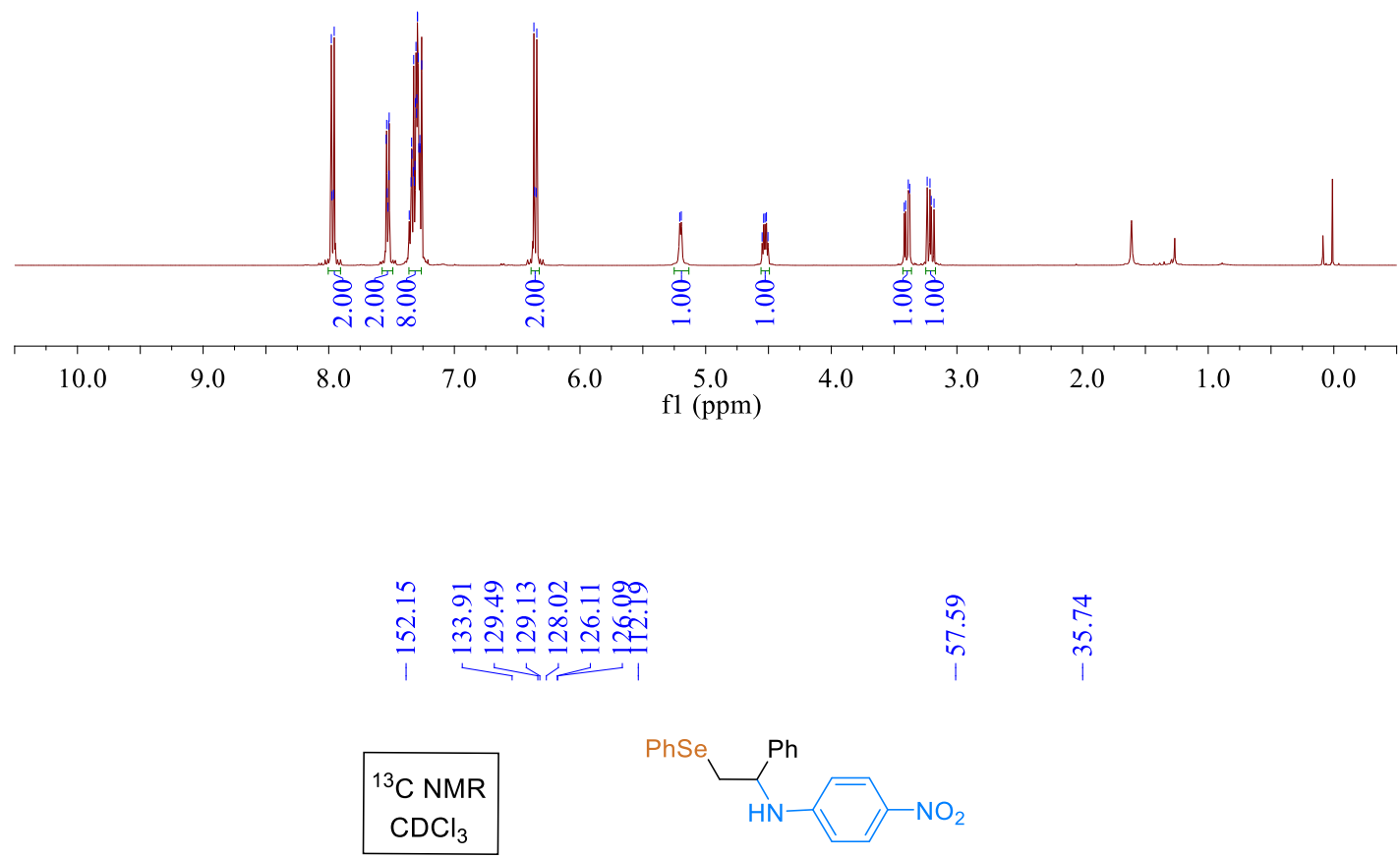

4h

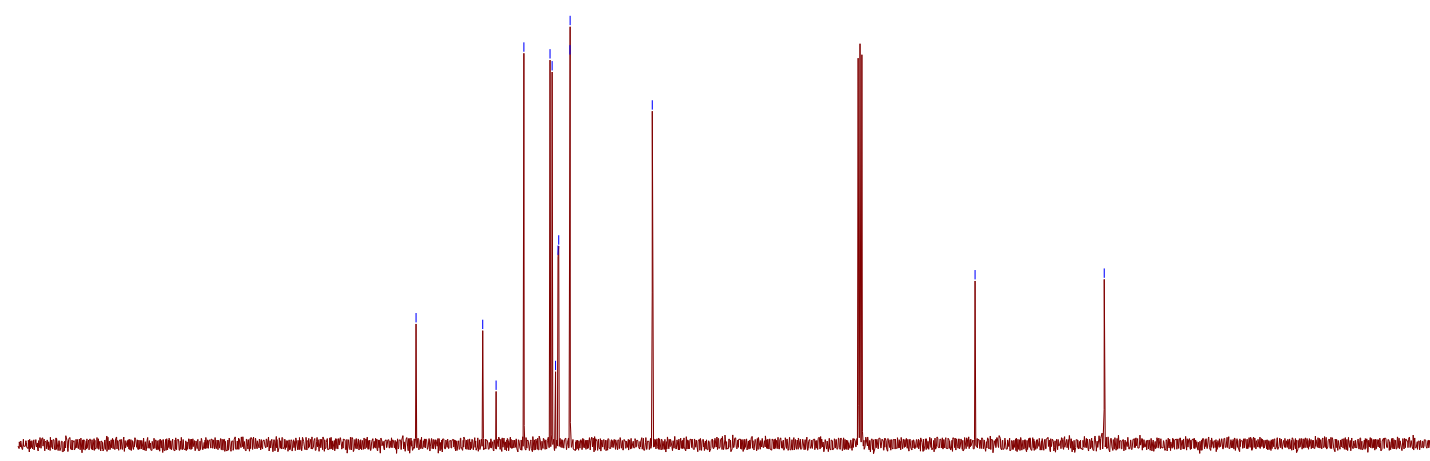

$\begin{array}{llllllllllllllll}210 & 190 & 170 & 150 & 130 & \begin{array}{c}110 \\ \mathrm{fl}(\mathrm{ppm})\end{array} & 90 & 70 & 60 & 50 & 40 & 30 & 20 & 10 & 0\end{array}$




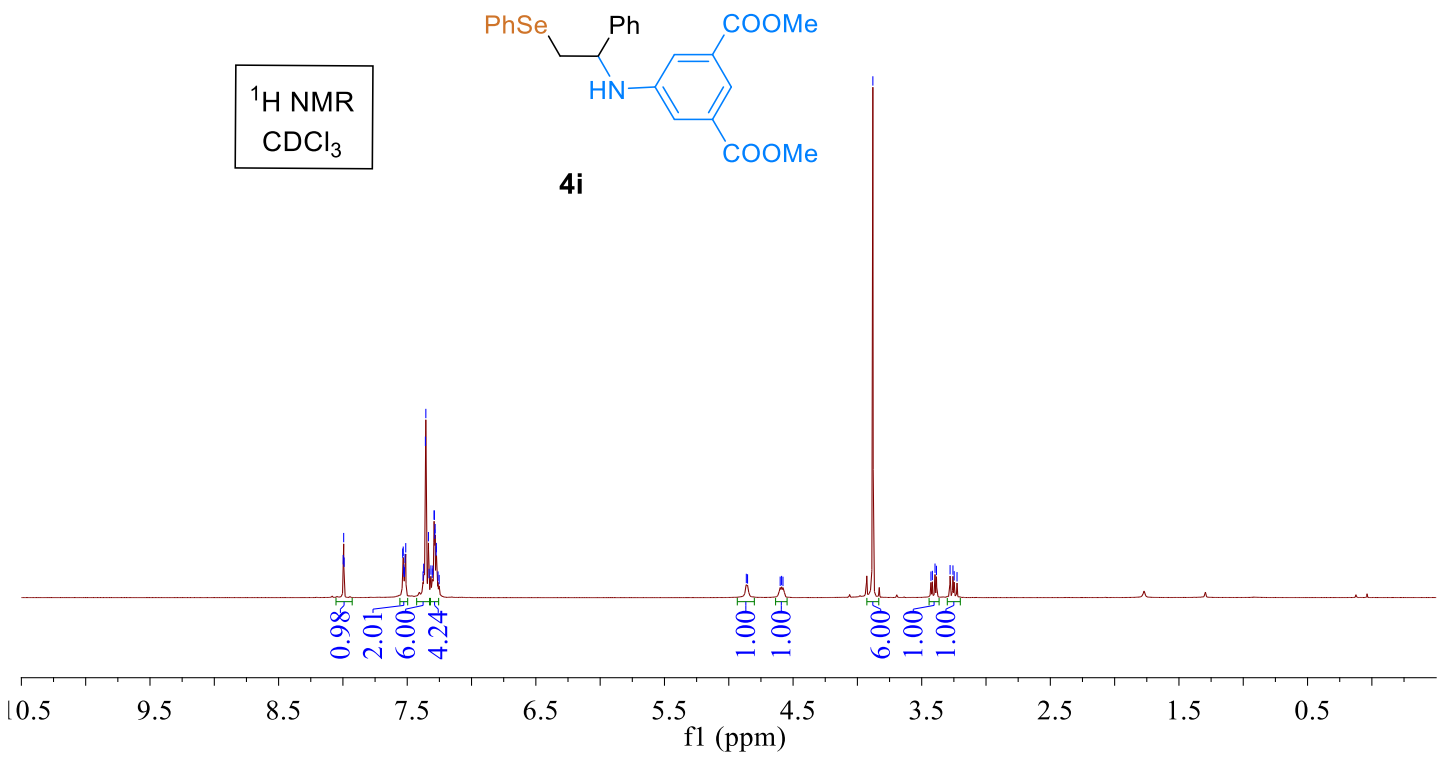

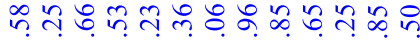

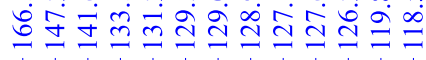

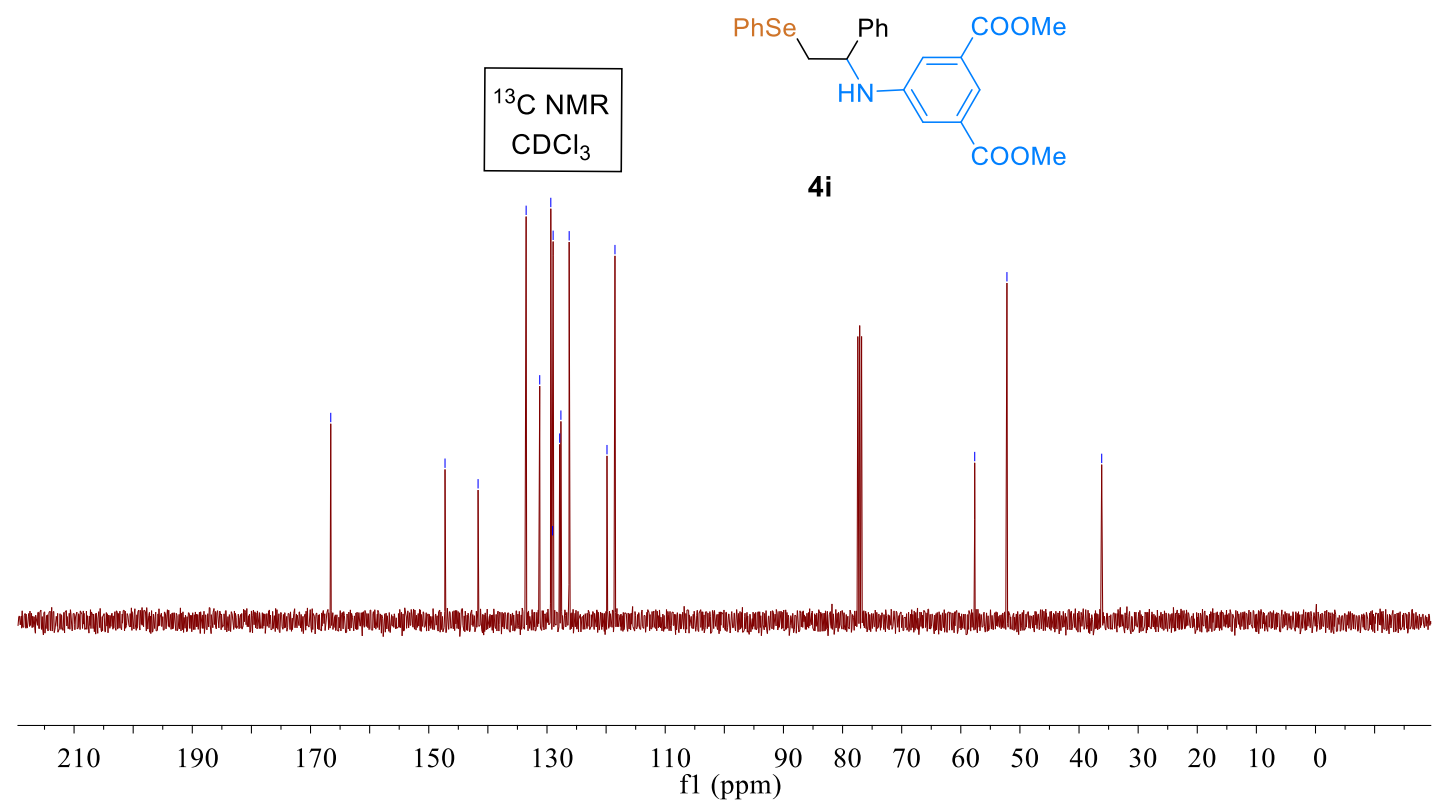



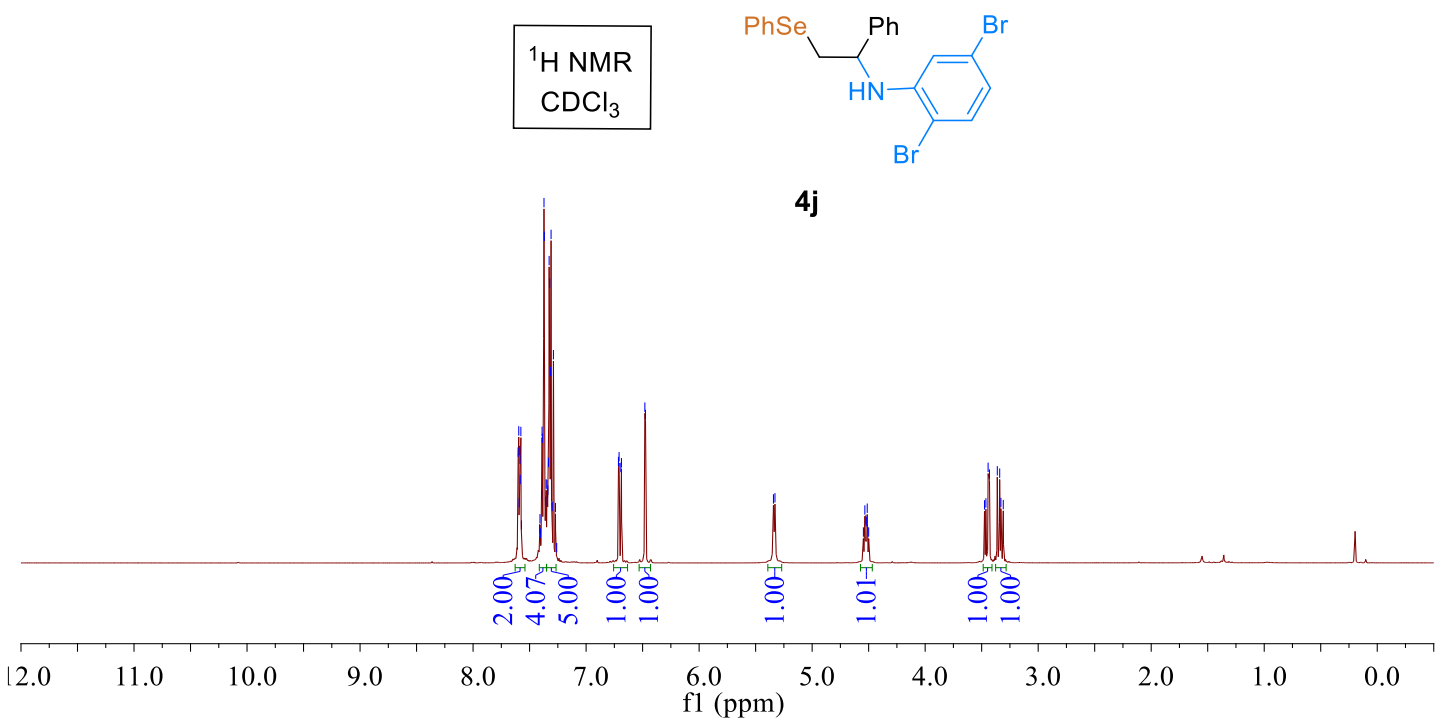

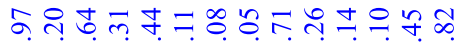

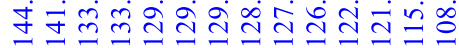

$\begin{array}{ll}\text { n. } & \text { in } \\ \text { in } & \text { n } \\ 1 & 1\end{array}$

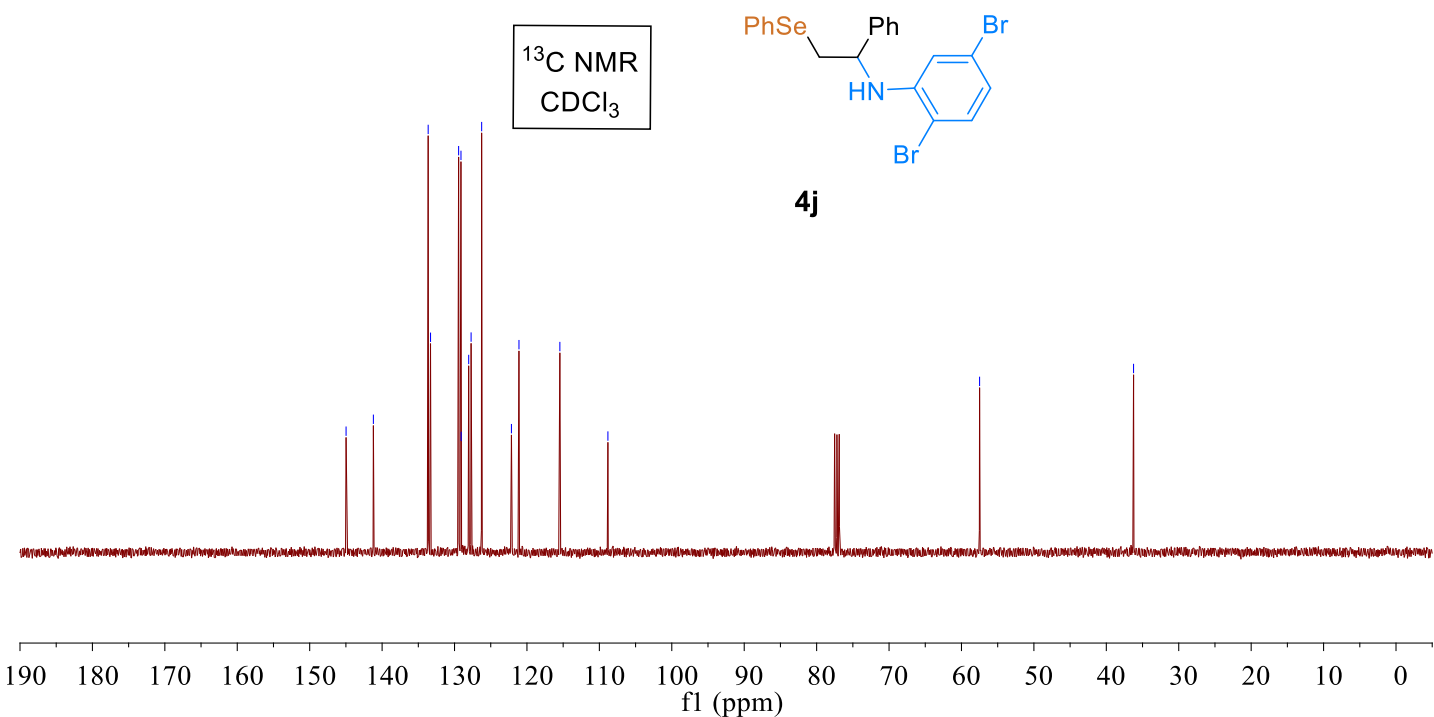




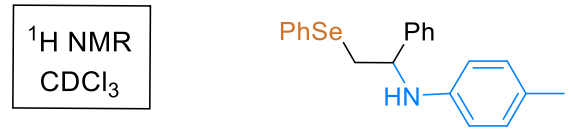

4k

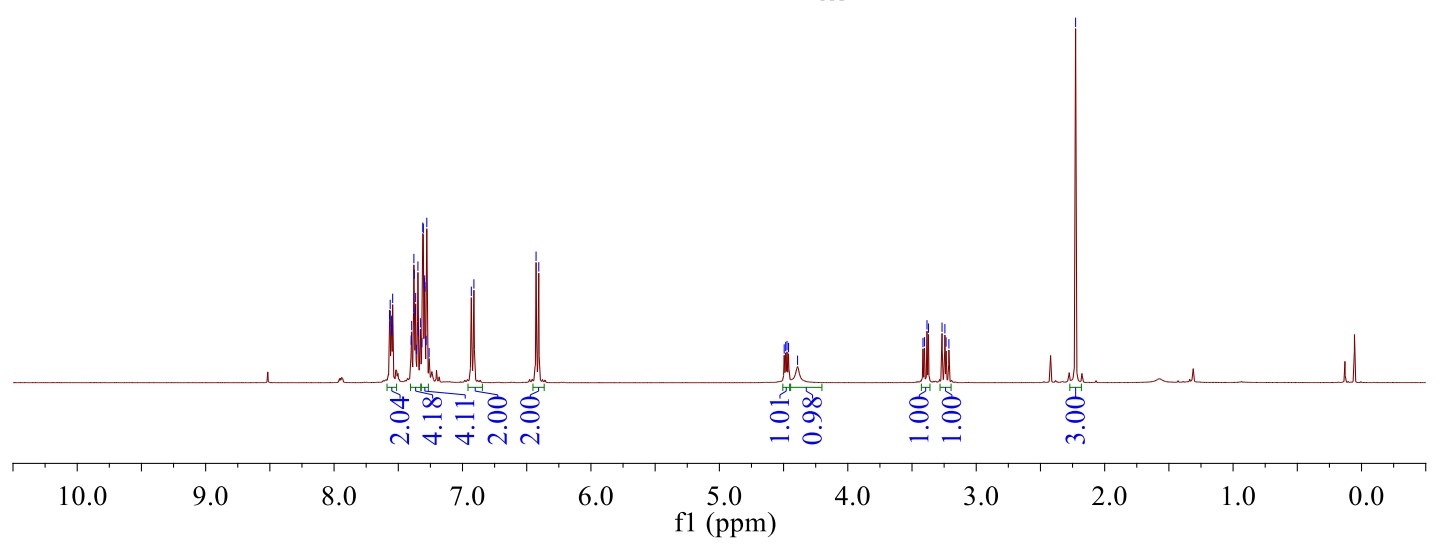

का

过

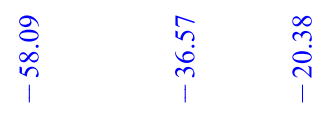

\begin{tabular}{|c|}
${ }^{13} \mathrm{C} \mathrm{NMR}$ \\
$\mathrm{CDCl}_{3}$
\end{tabular}

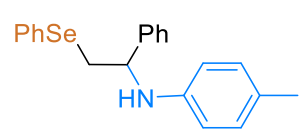

4k

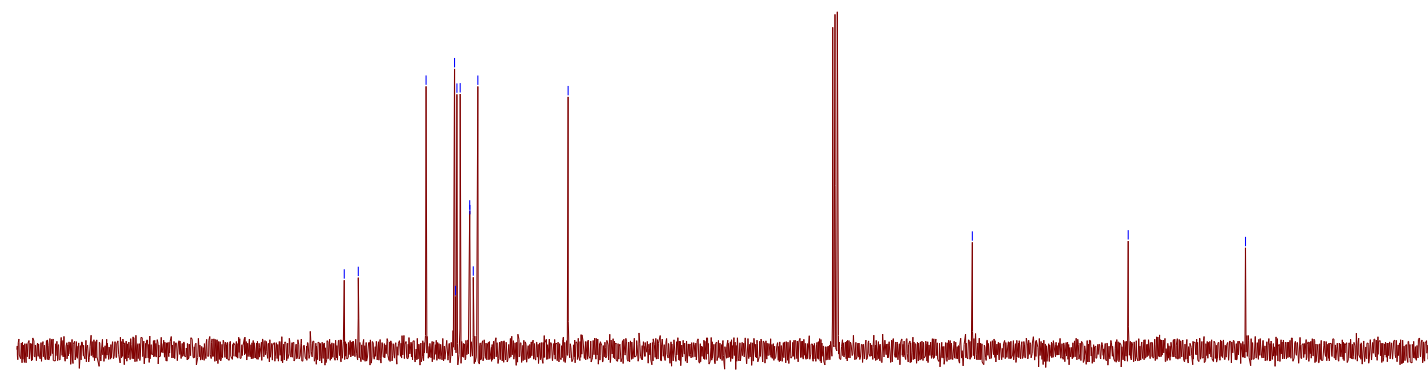

$\begin{array}{lllllllllll}190 & 180 & 170 & 160 & 150 & 140 & 130 & 120 & 110 & 100 & 90 \\ 9\end{array}$ f1 (ppm) 

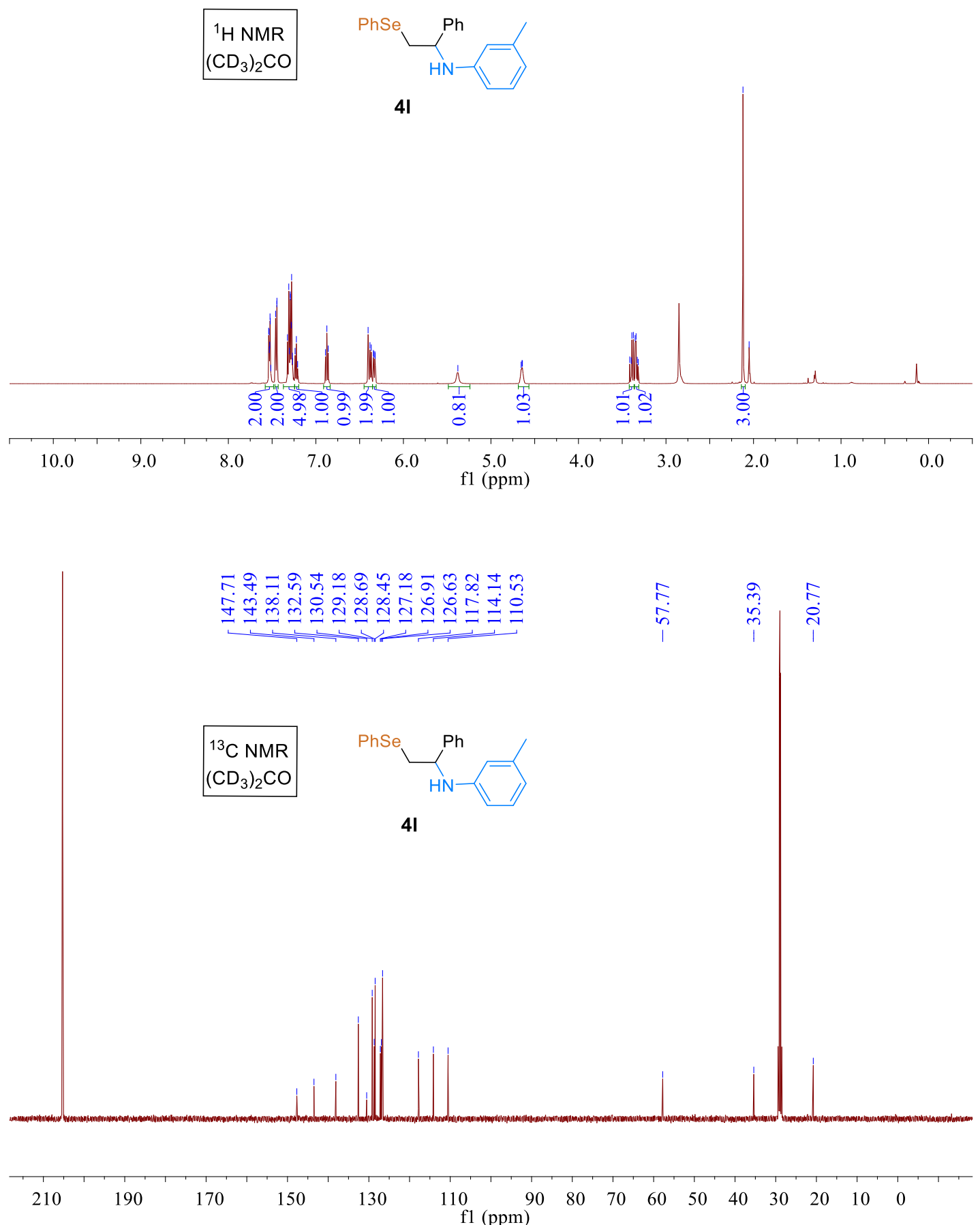


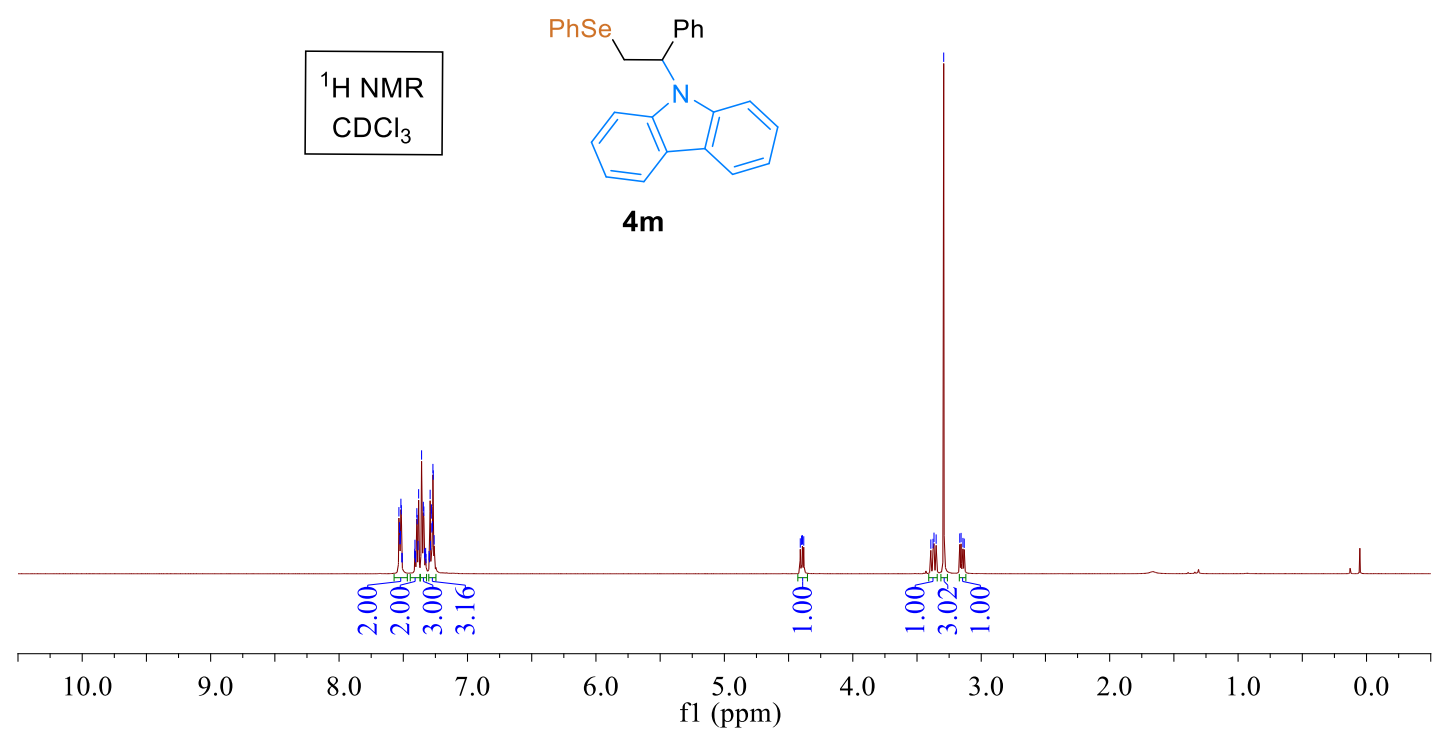

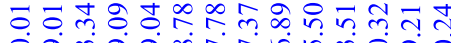

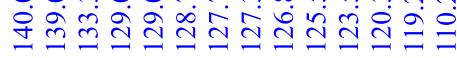
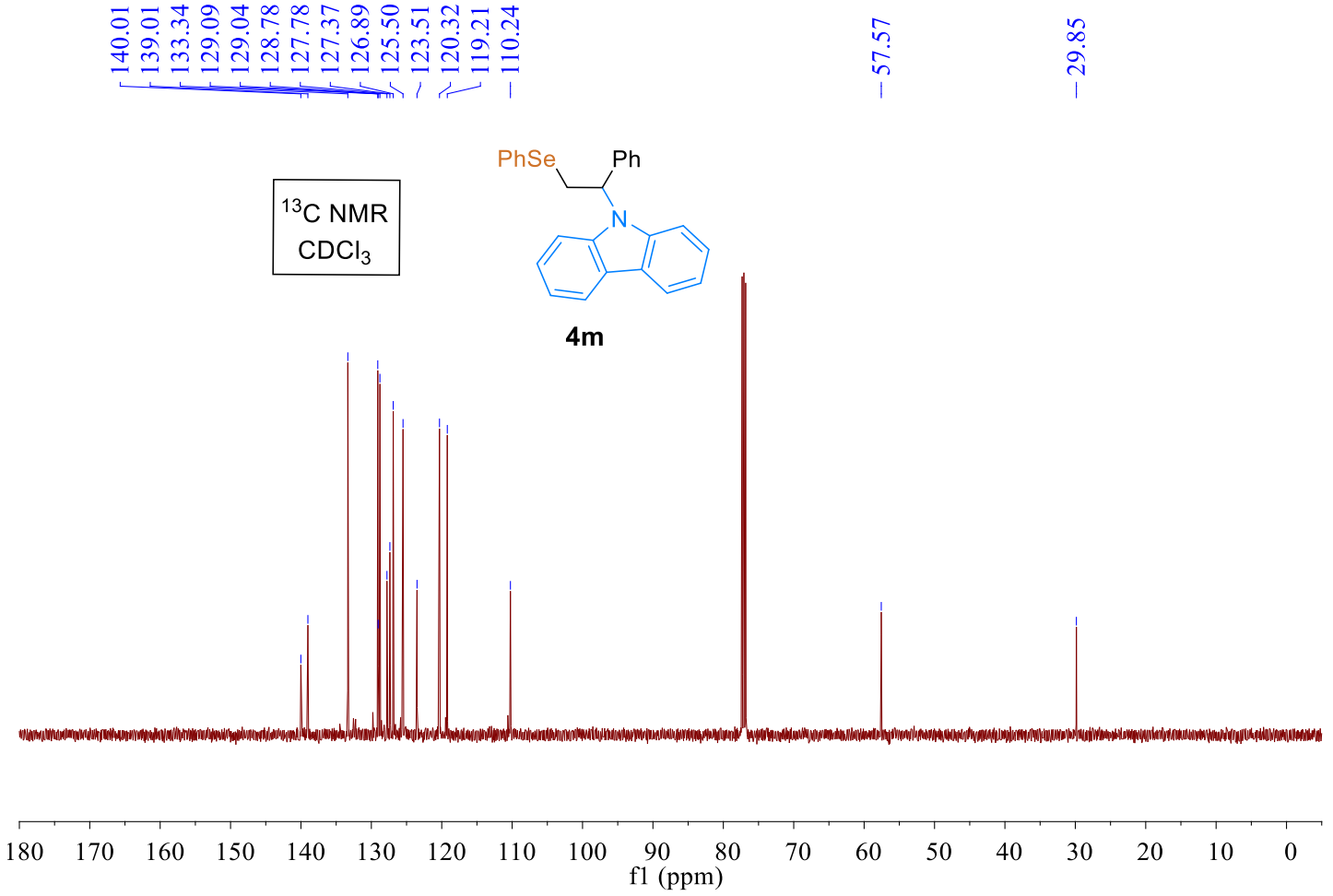


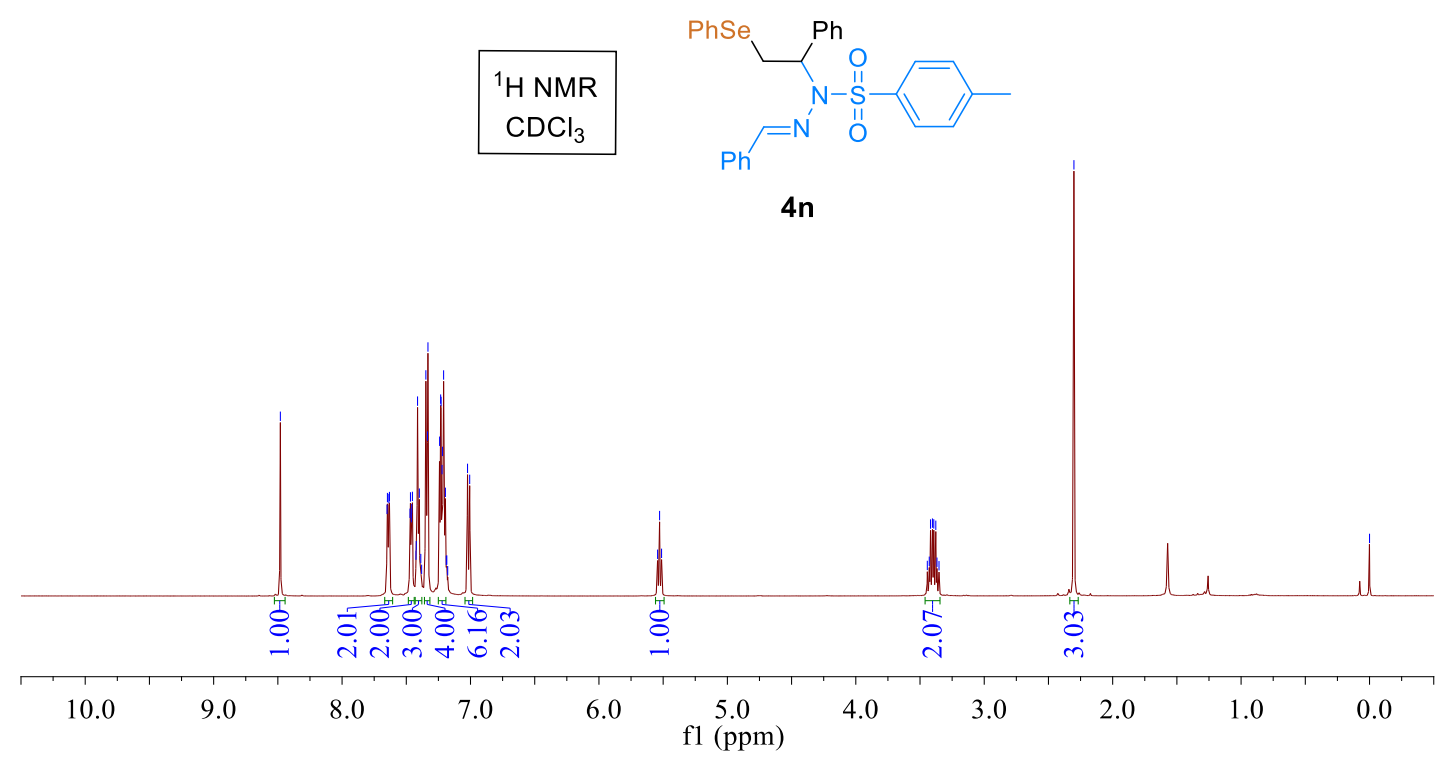

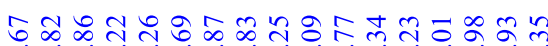

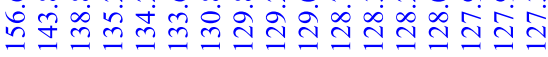

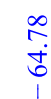

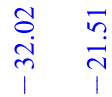

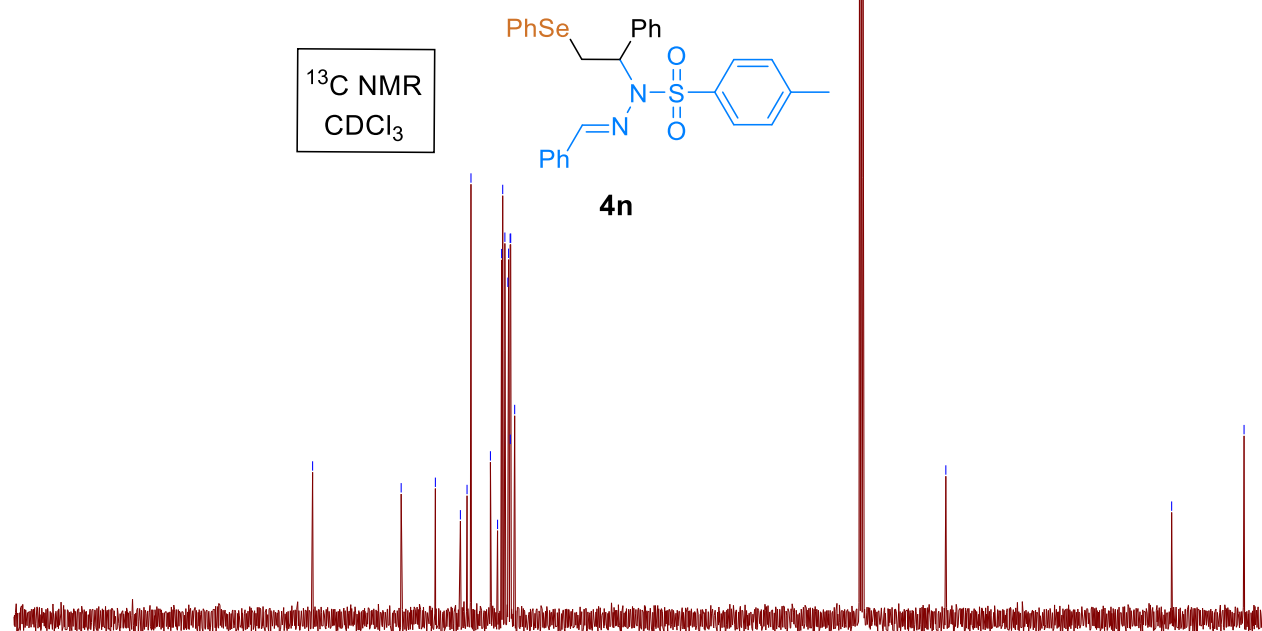

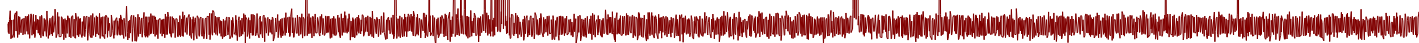

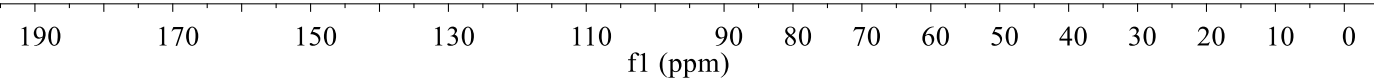




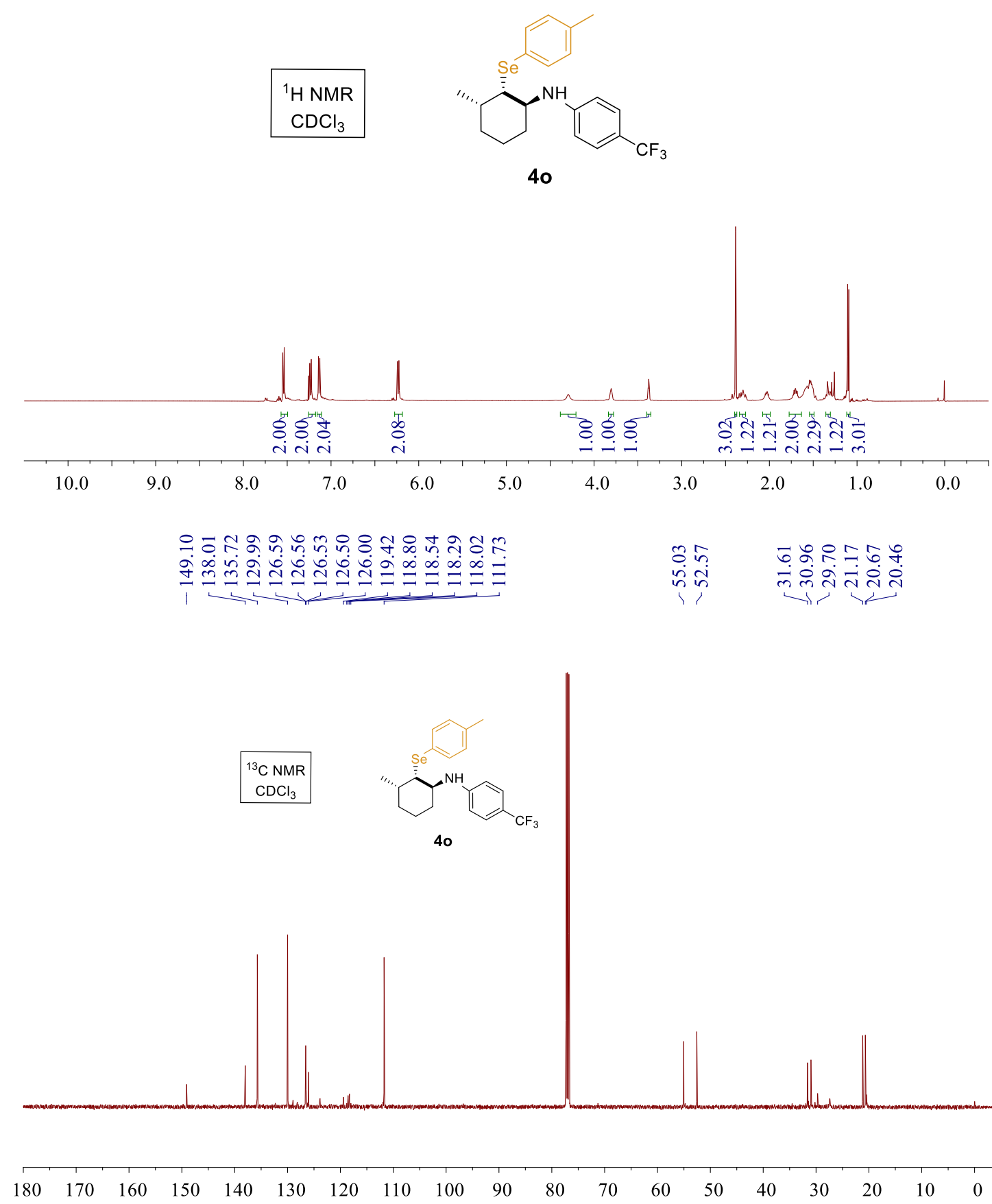




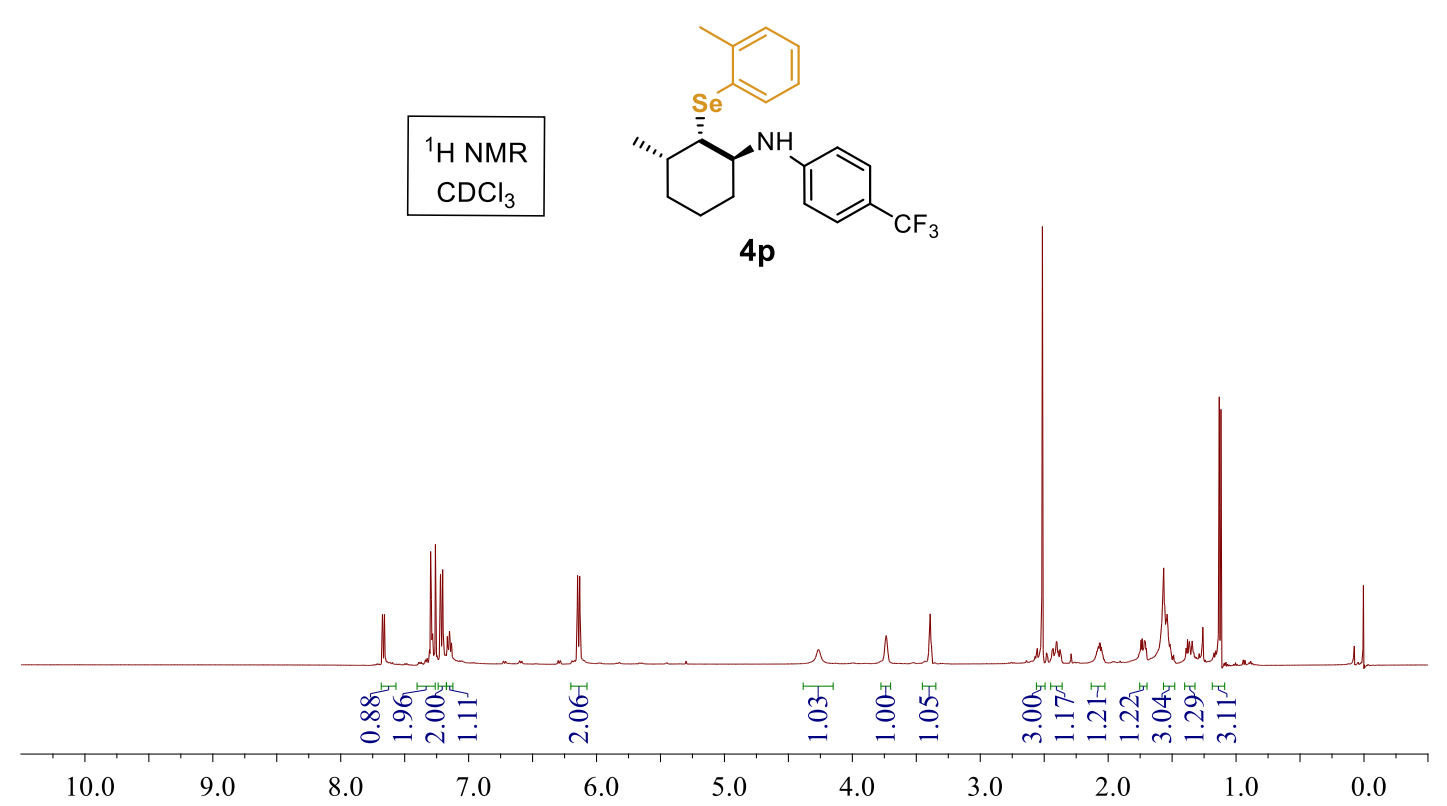

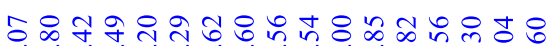

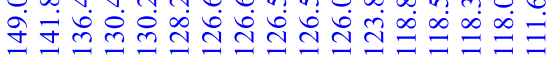

ำ

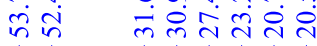

${ }^{13} \mathrm{C} \mathrm{NMR}^{\mathrm{CDCl}_{3}}$

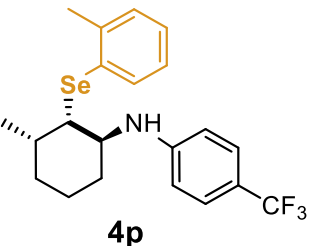

$4 p$

i

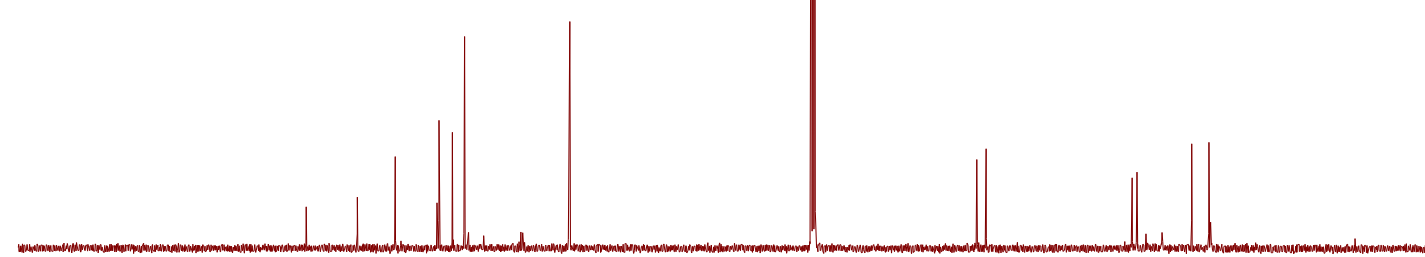

190

170

150

130

110

90

$\begin{array}{llllllllll}80 & 70 & 60 & 50 & 40 & 30 & 20 & 10 & 0 & -1\end{array}$ 

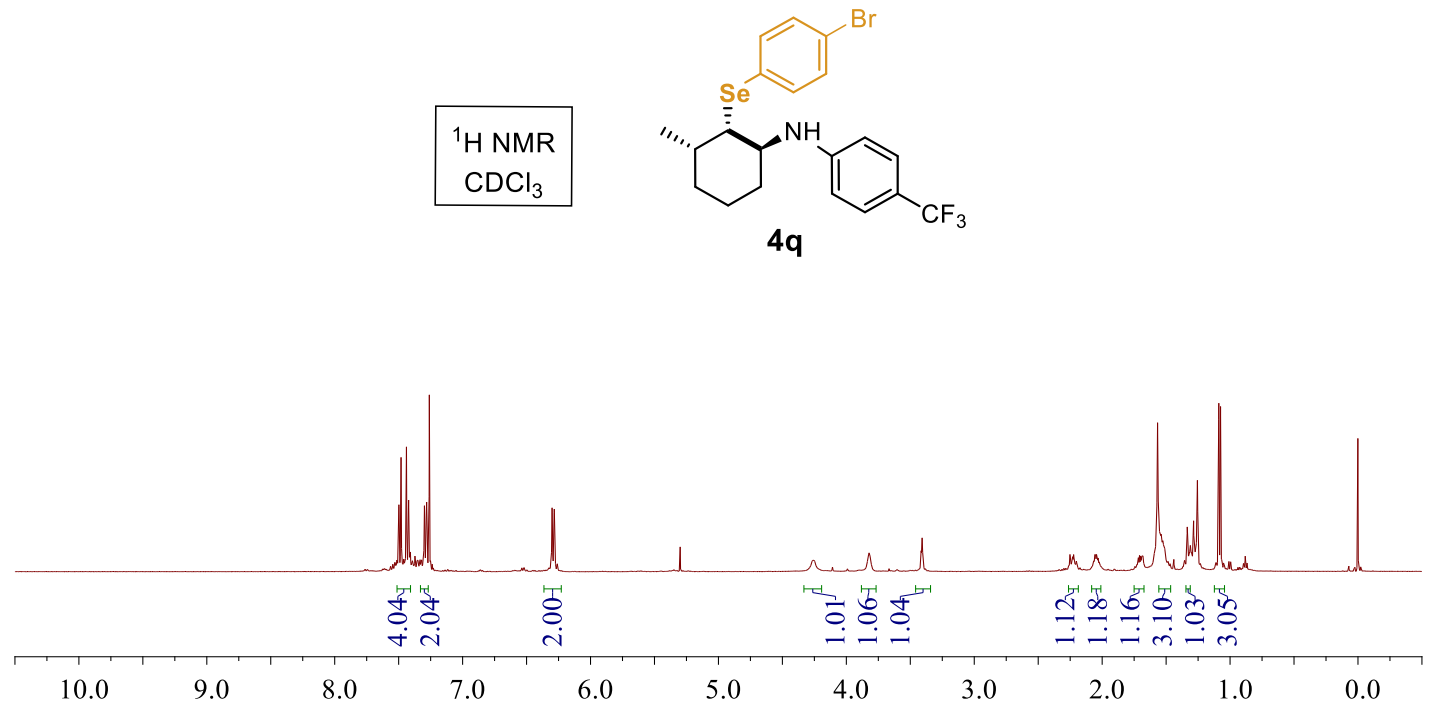

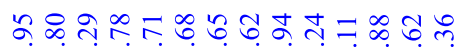

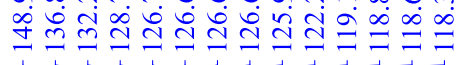

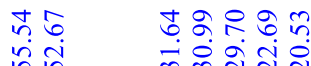

थี

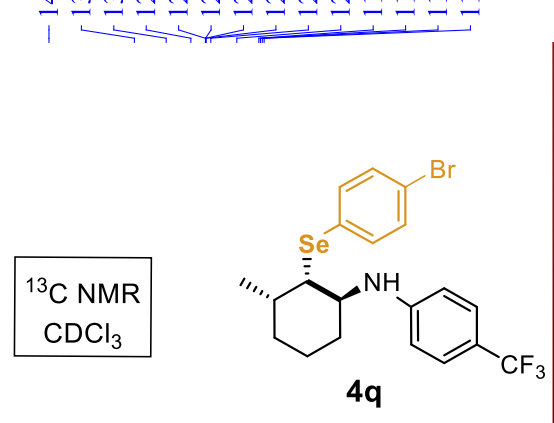

तl ir

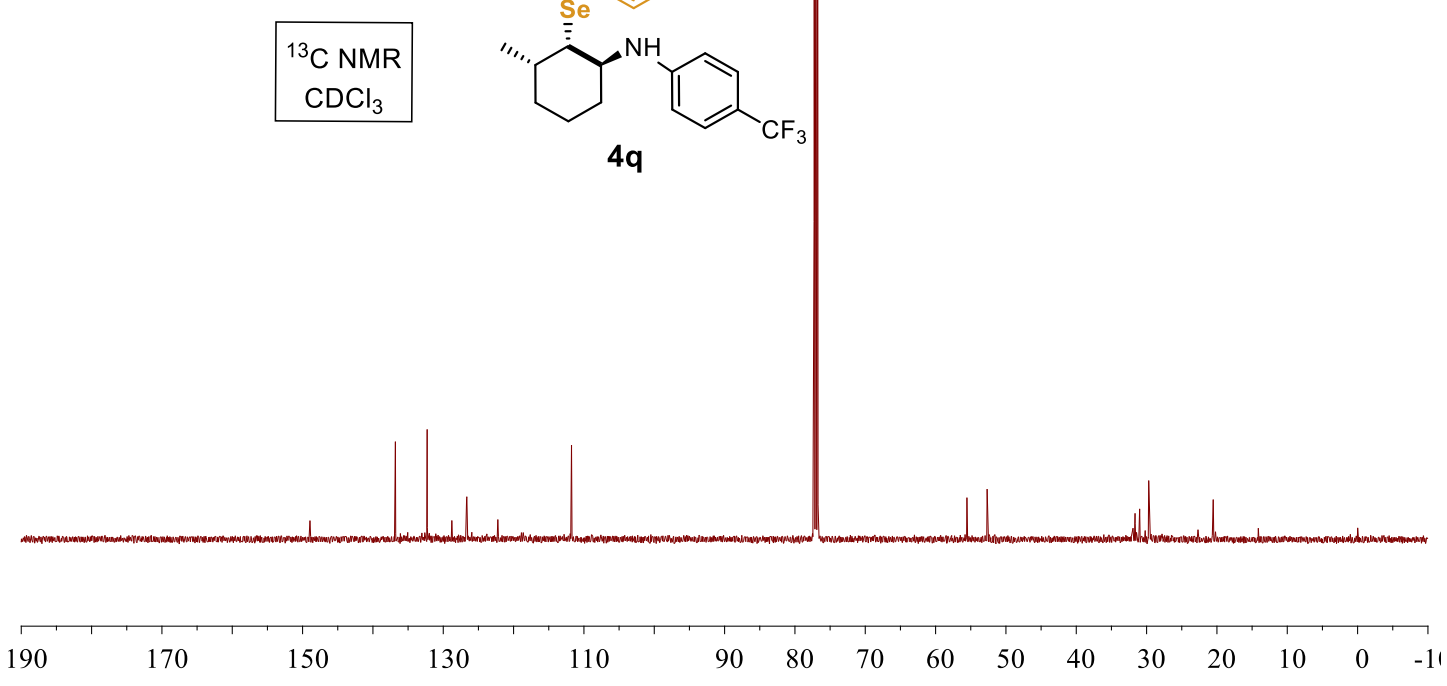



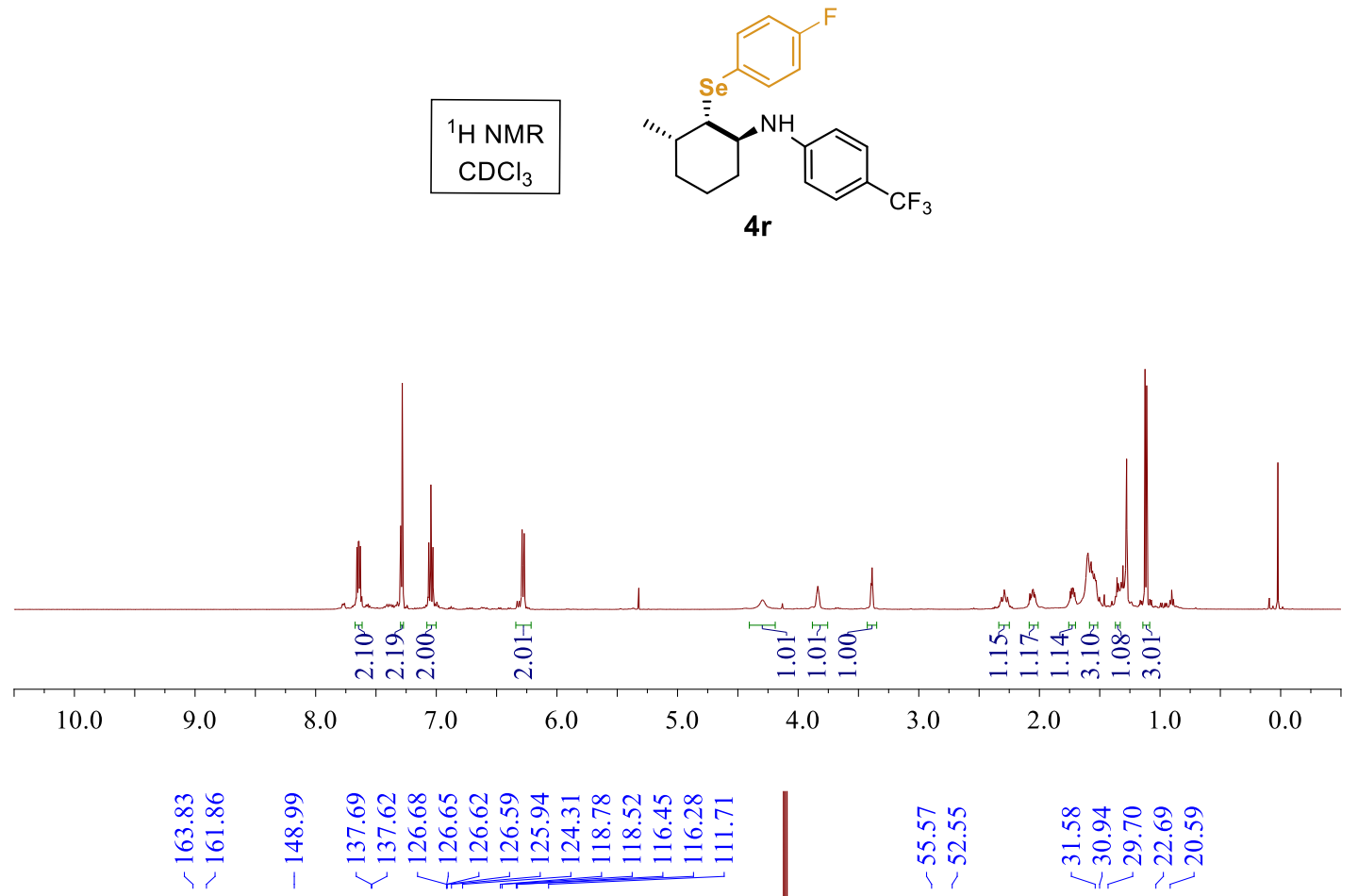

華负

in का ment
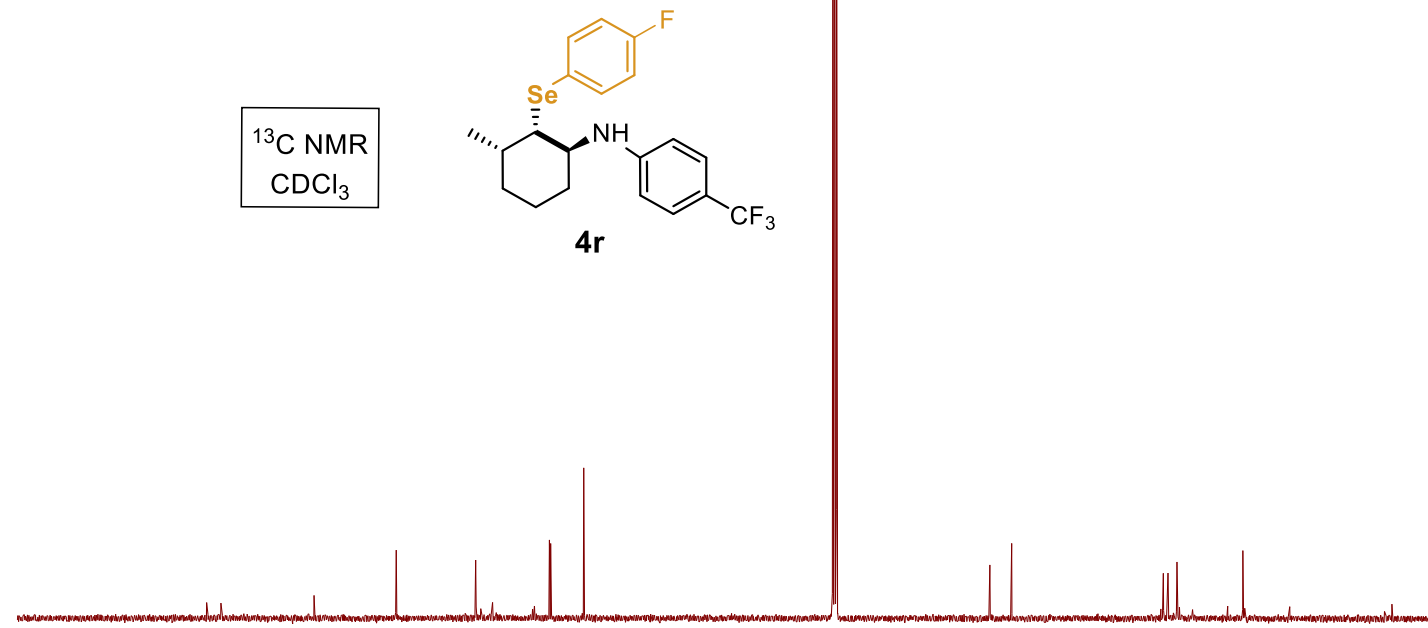

$\begin{array}{llllllllllllllllllll}190 & 180 & 170 & 160 & 150 & 140 & 130 & 120 & 110 & 100 & 90 & 80 & 70 & 60 & 50 & 40 & 30 & 20 & 10 & 0\end{array}$ 

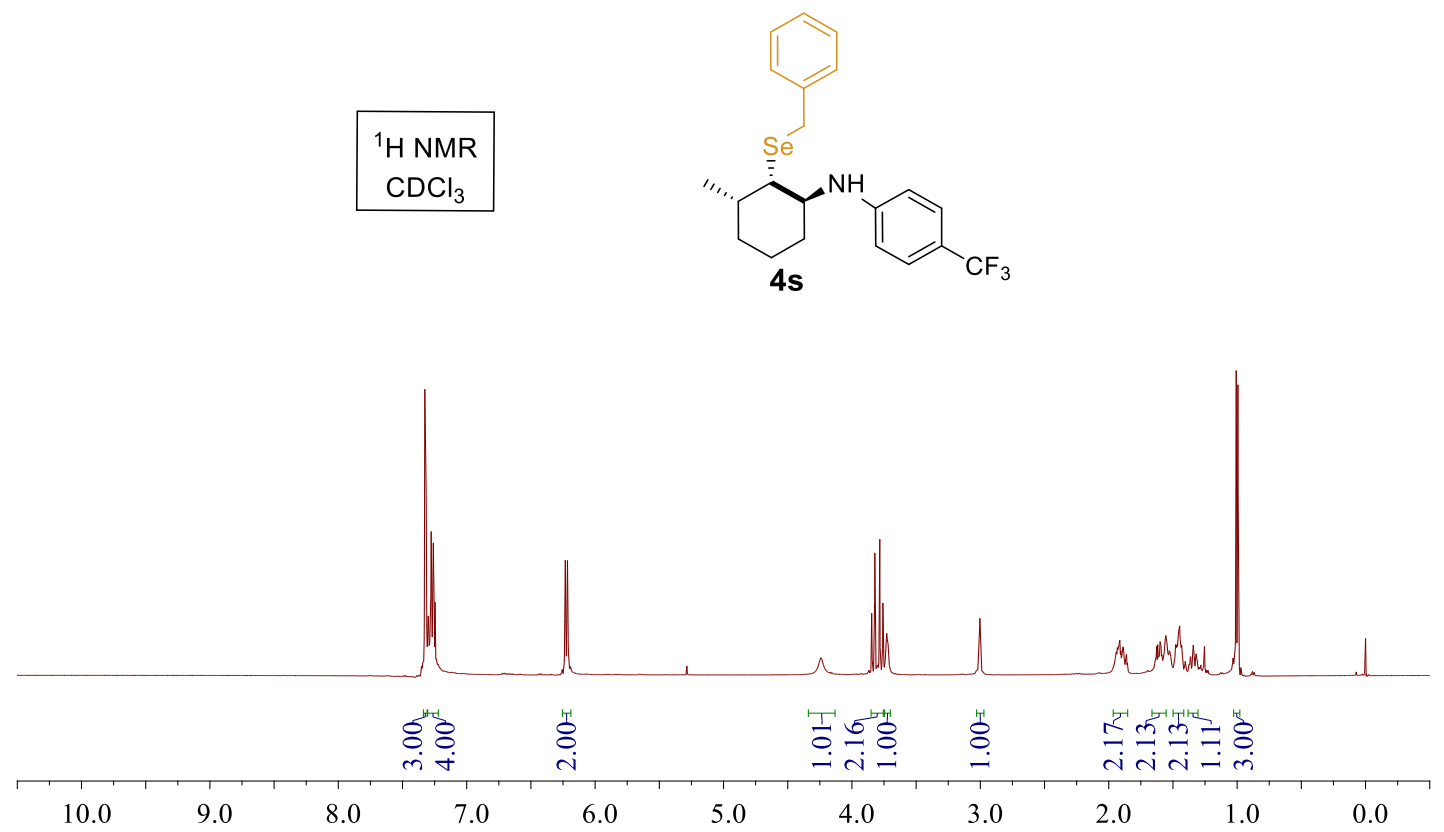

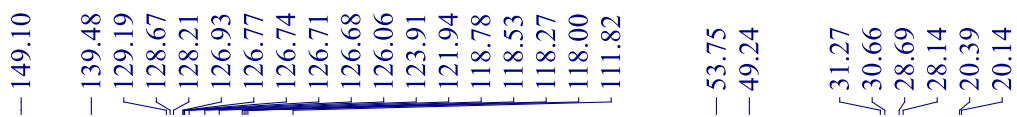

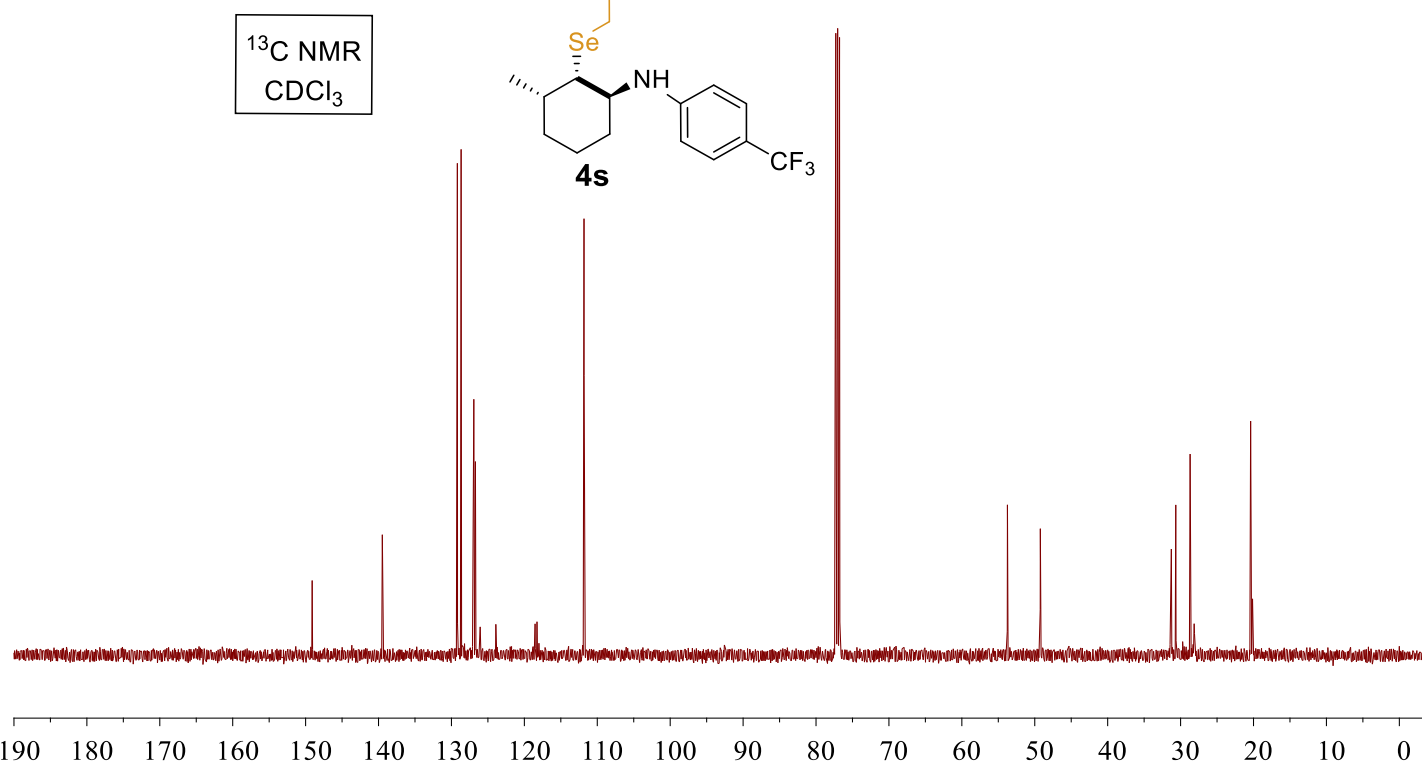




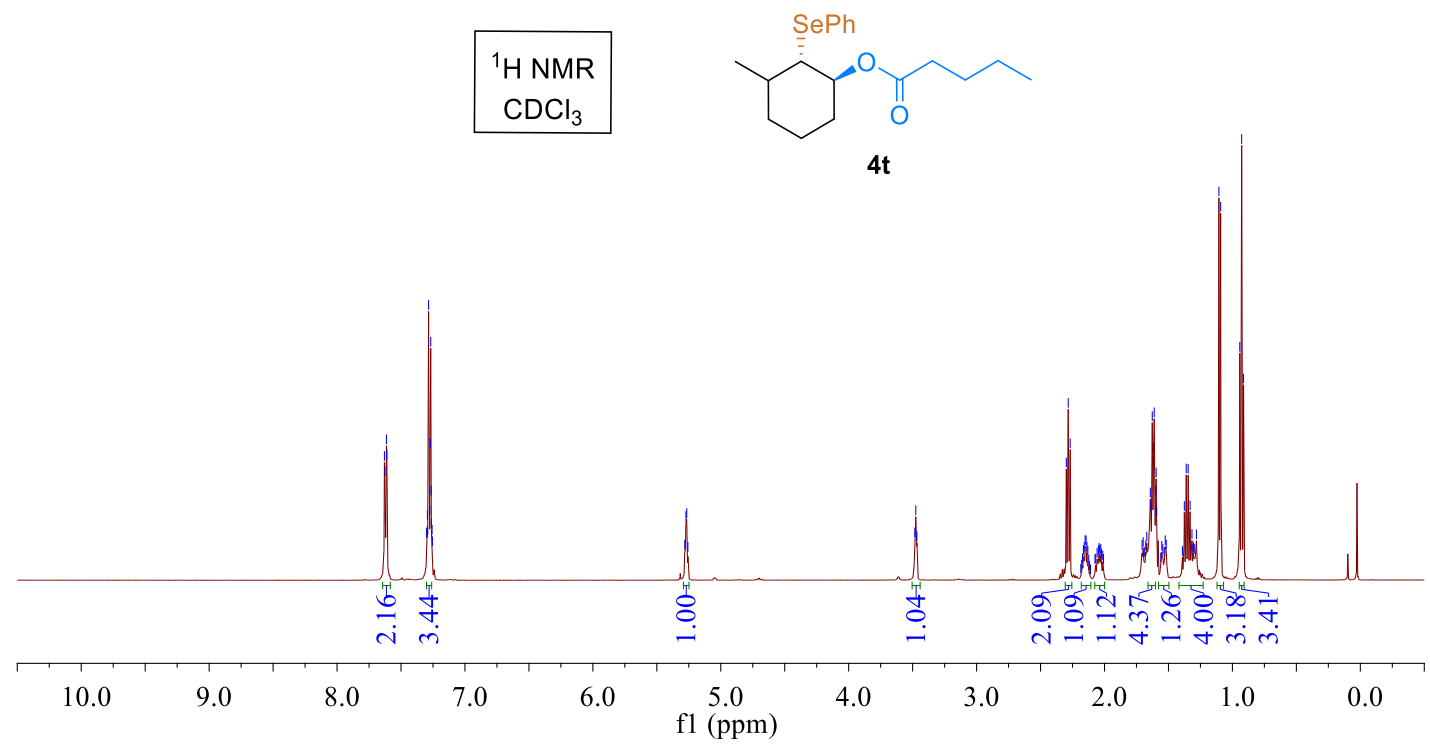

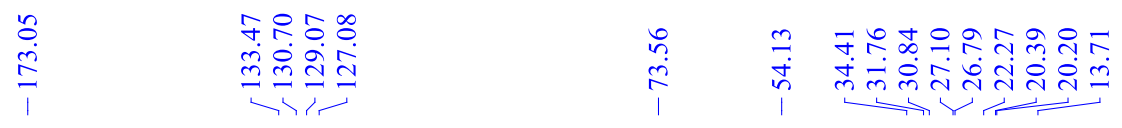

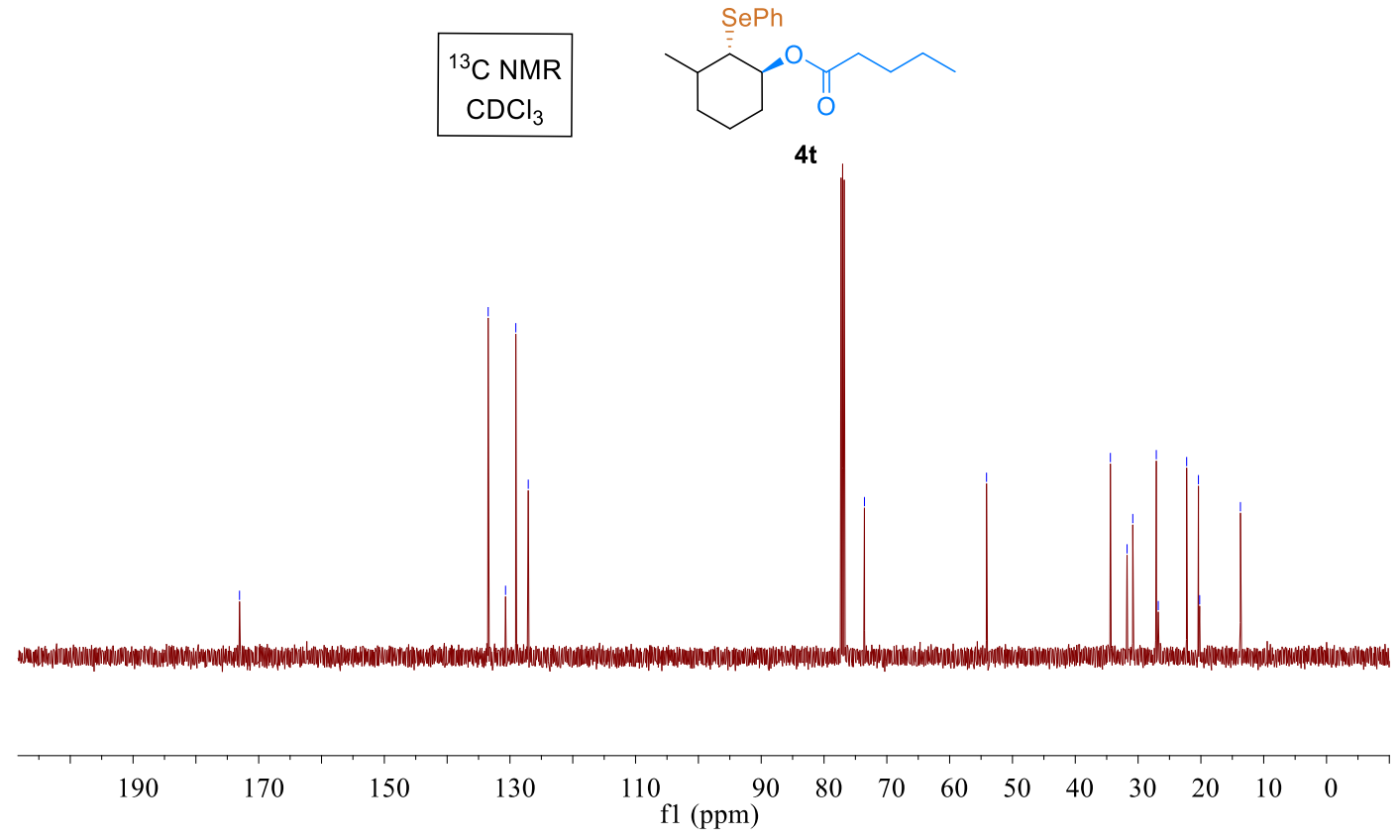




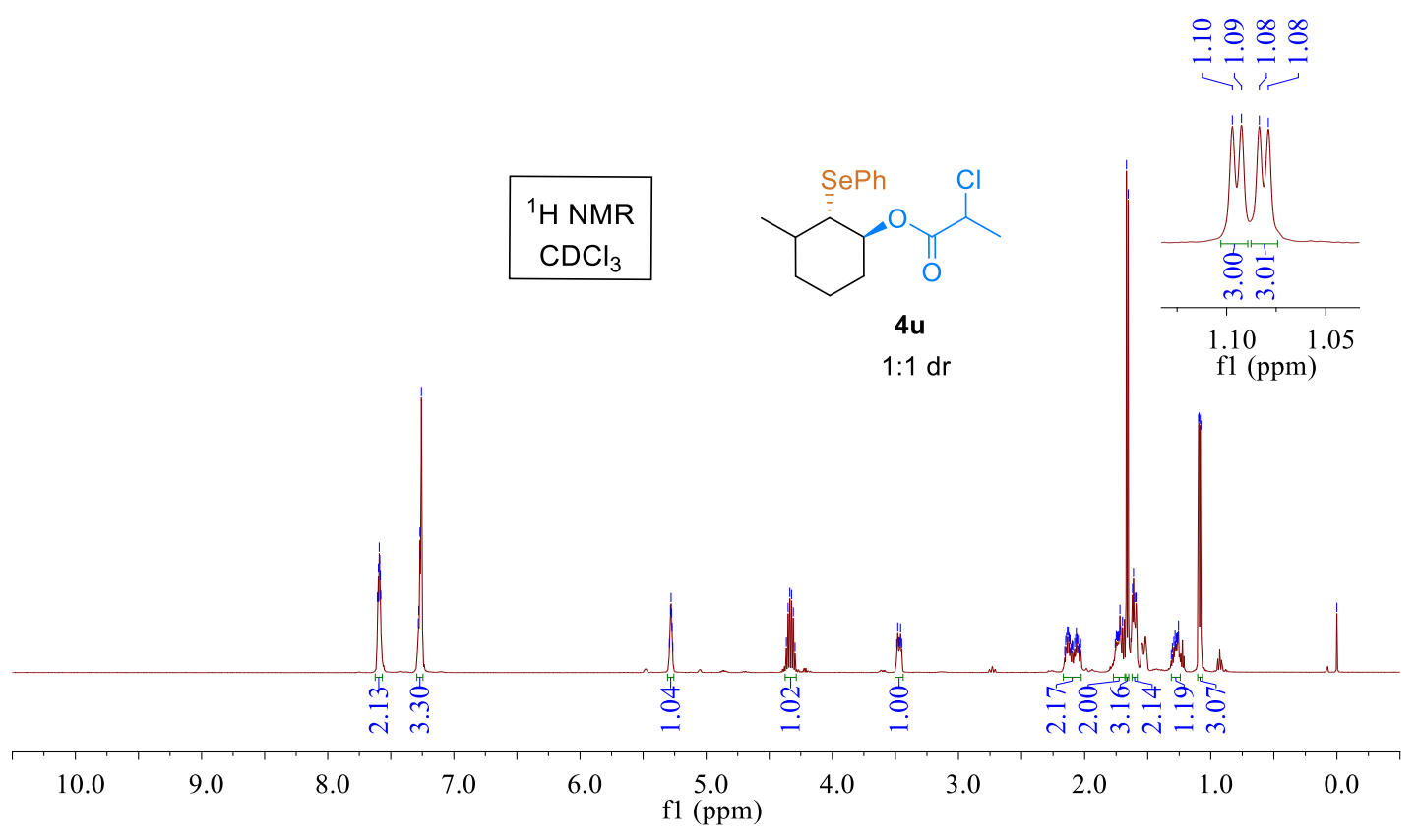

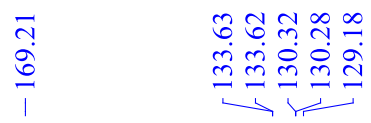

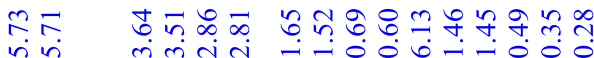

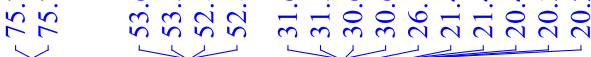
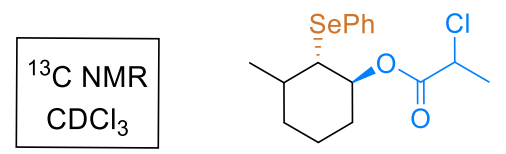

$4 u$

$1: 1 \mathrm{dr}$

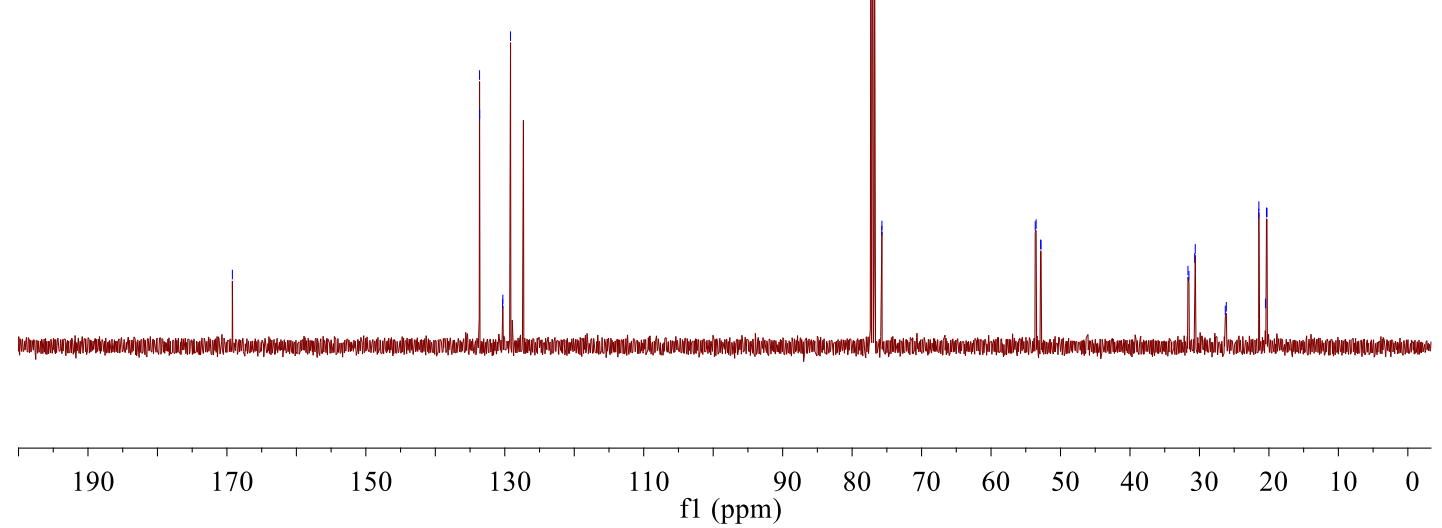



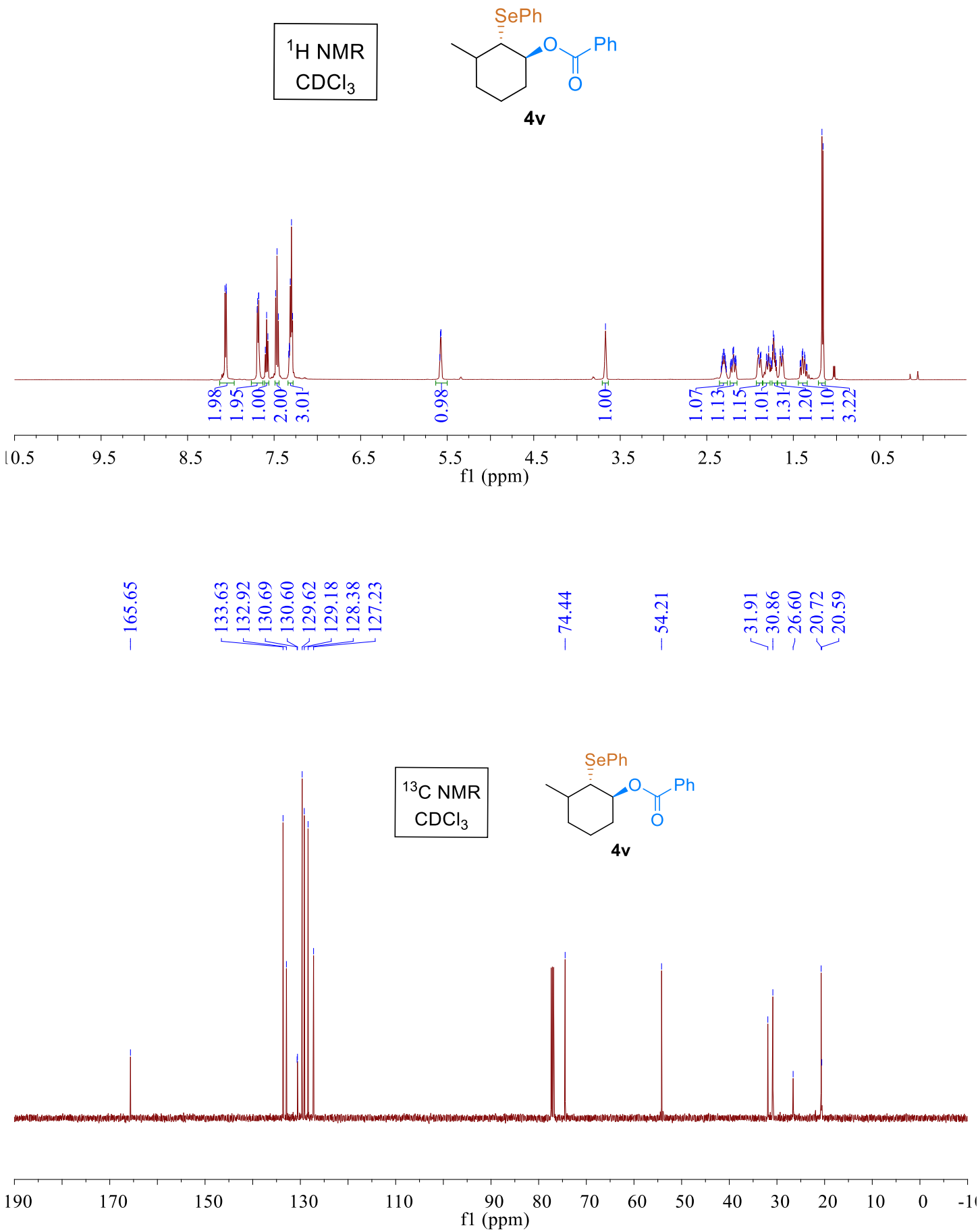


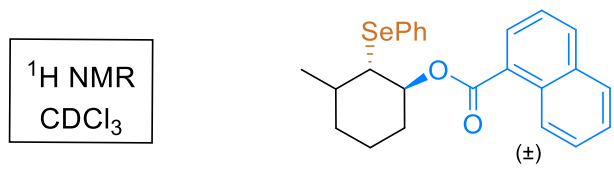

$4 w$

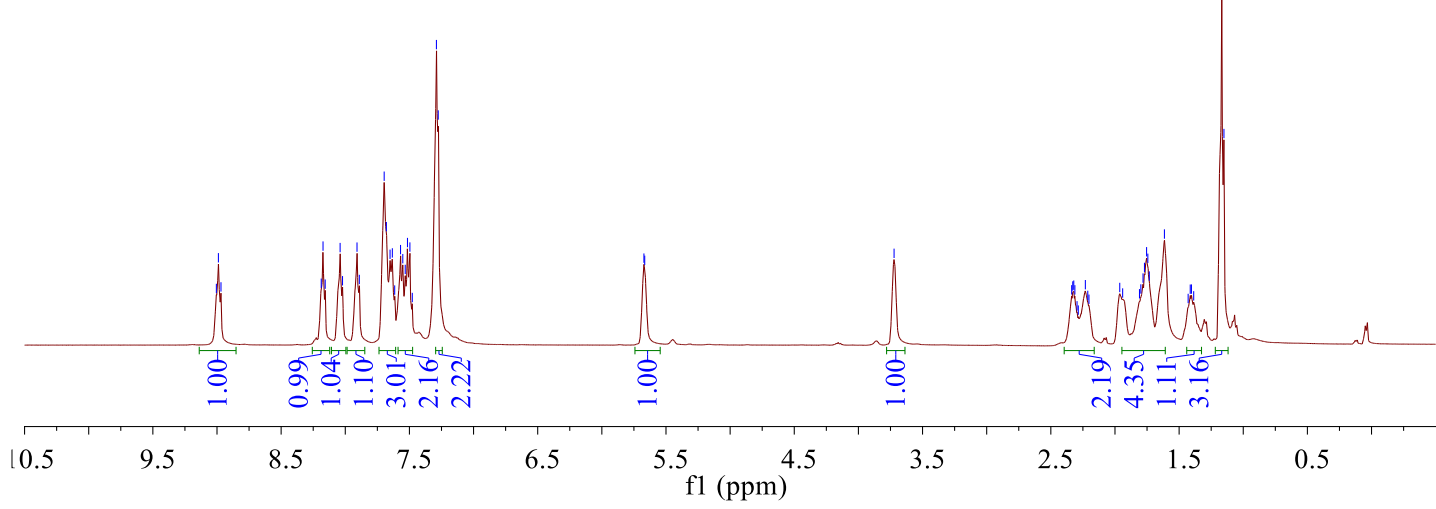

$4 w$

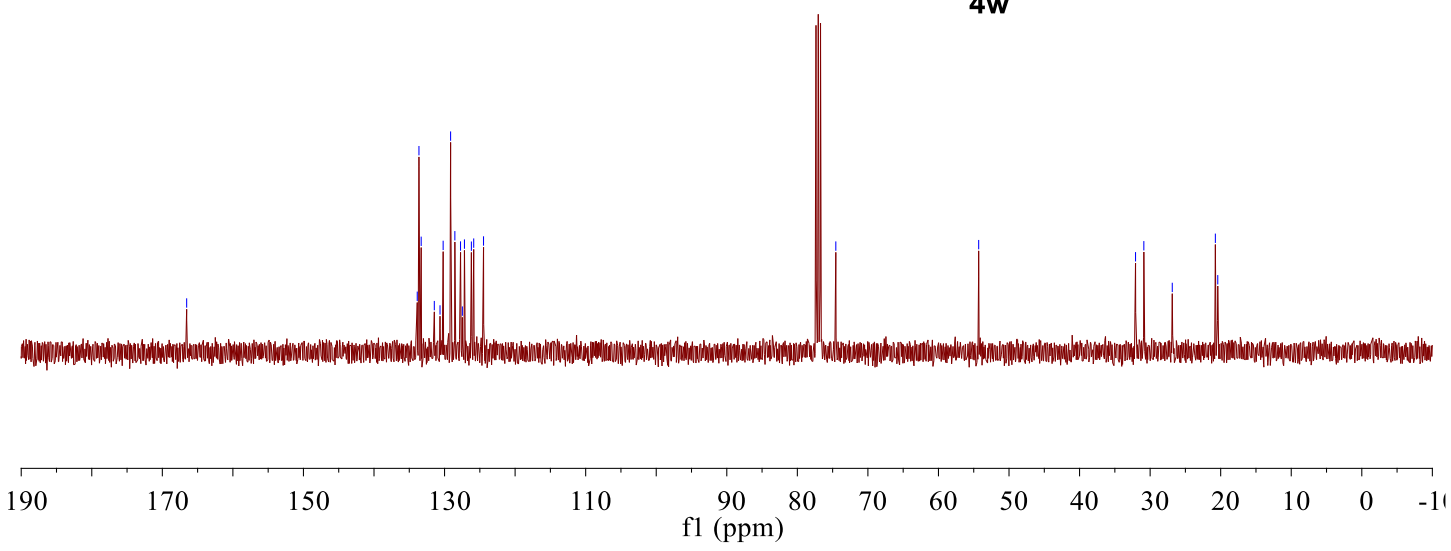




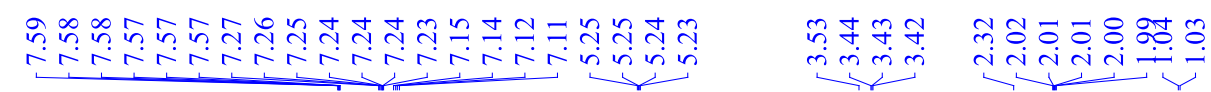

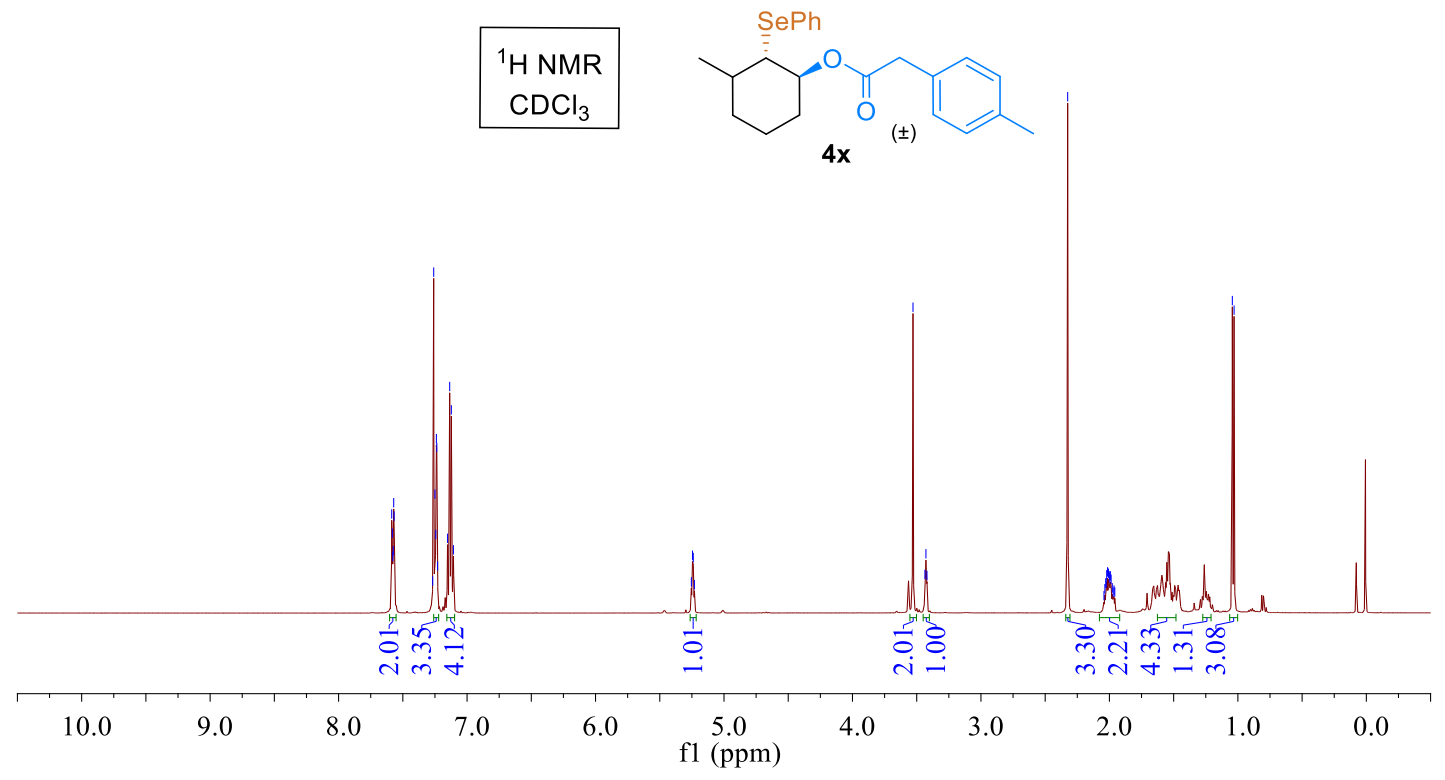

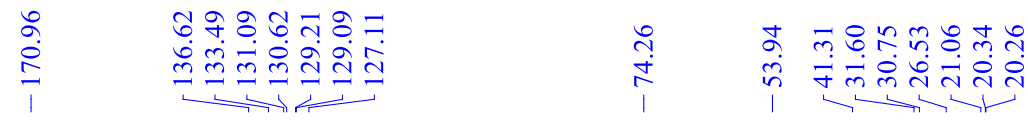

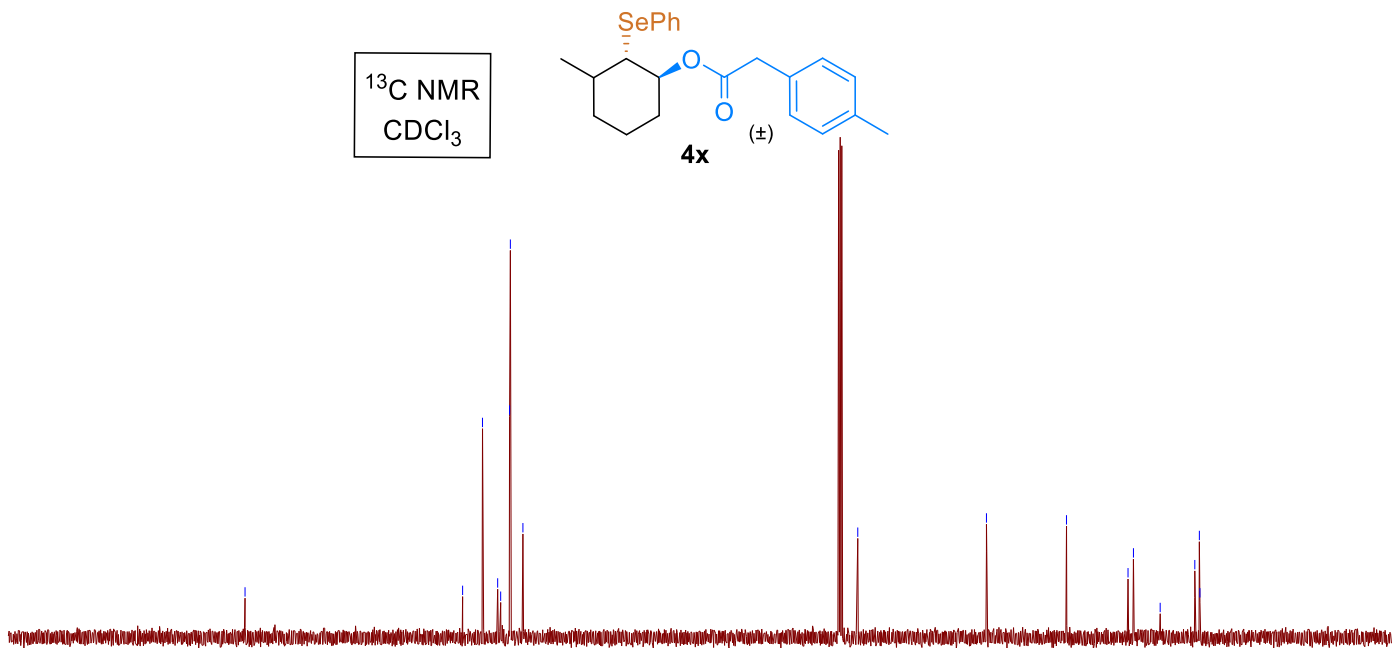

190

150

130

$\begin{array}{llllllllll}90 & 80 & 70 & 60 & 50 & 40 & 30 & 20 & 10 & 0\end{array}$

f1 (ppm) 

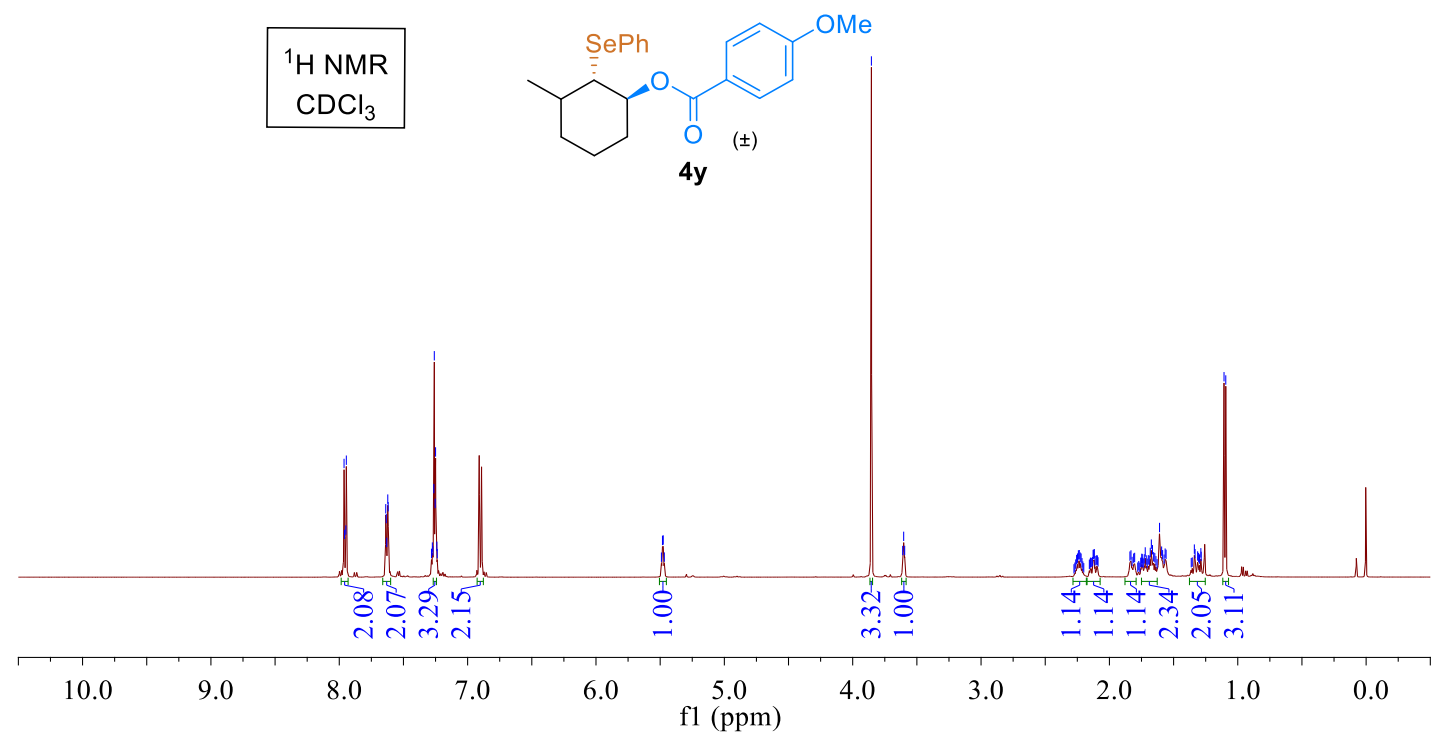

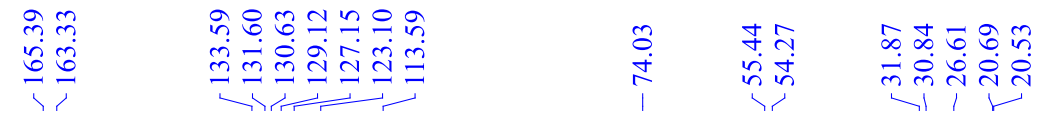
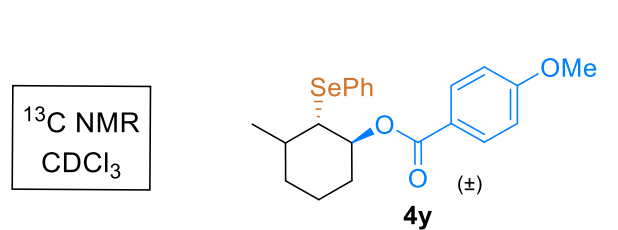

4y

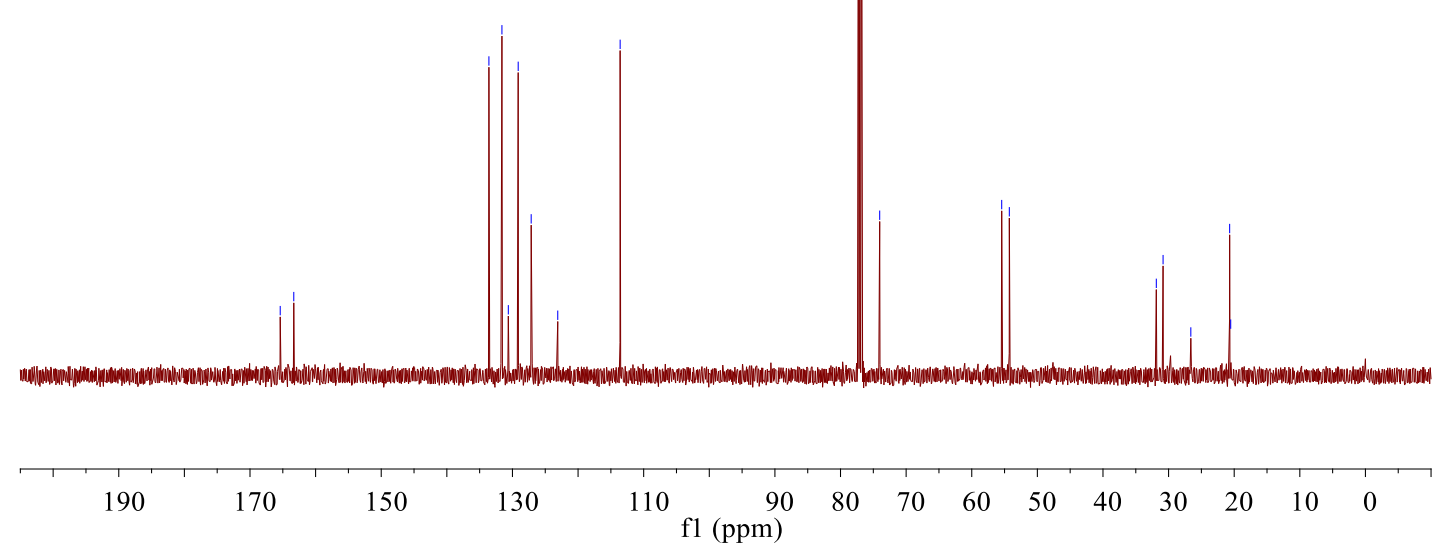



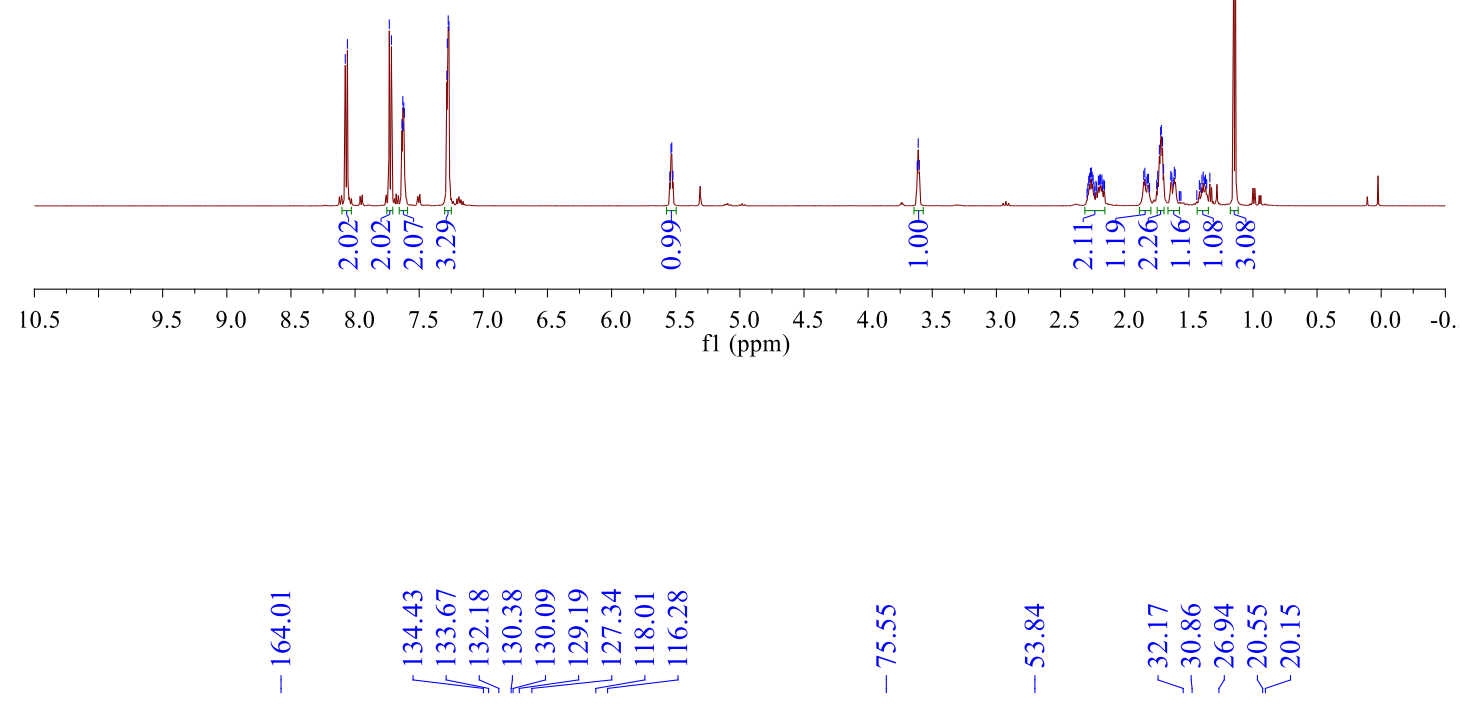

${ }^{13} \mathrm{C} \mathrm{NMR}$
$\mathrm{CDCl}_{3}$

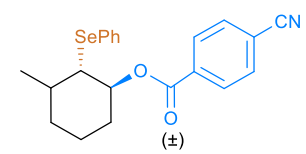

$4 z$

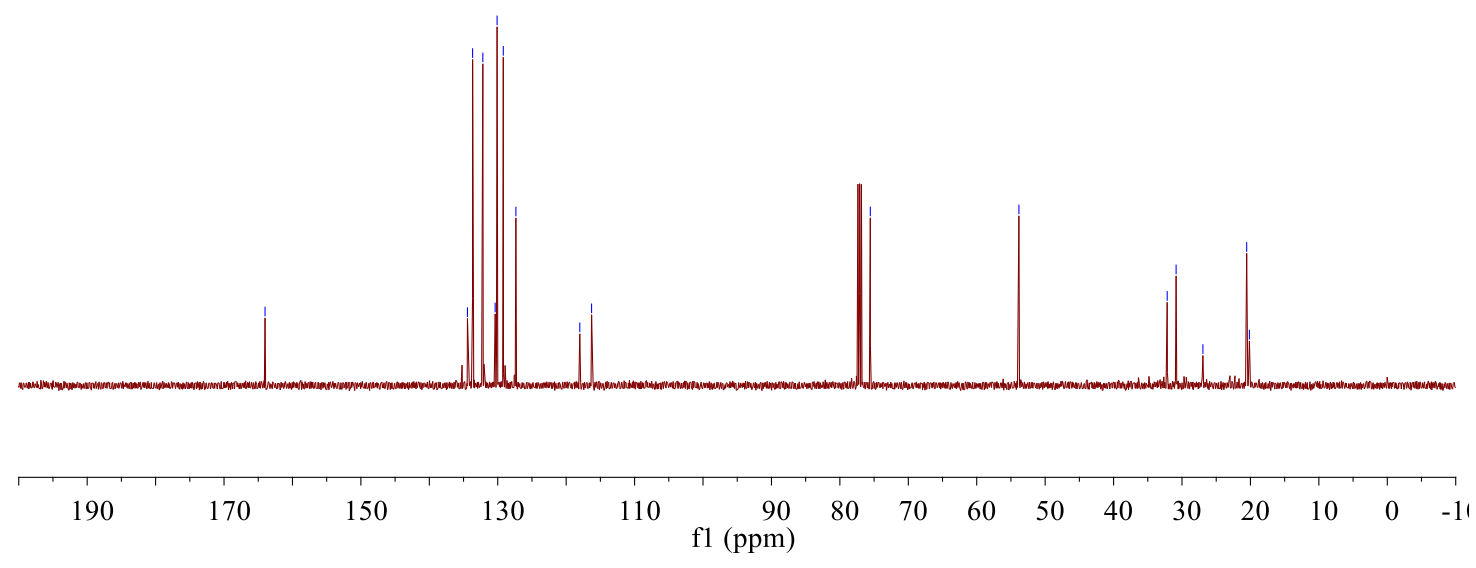



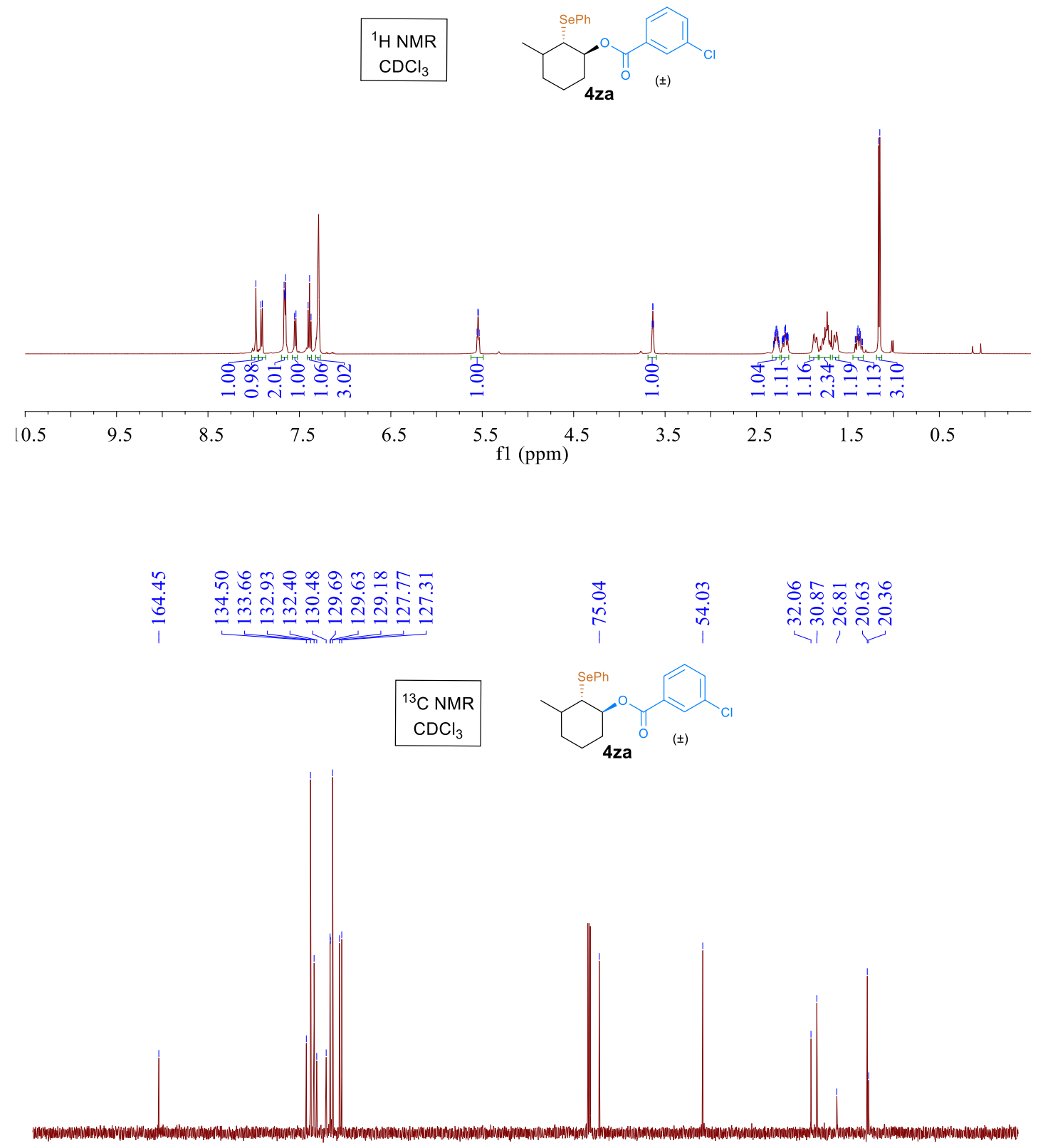

170 

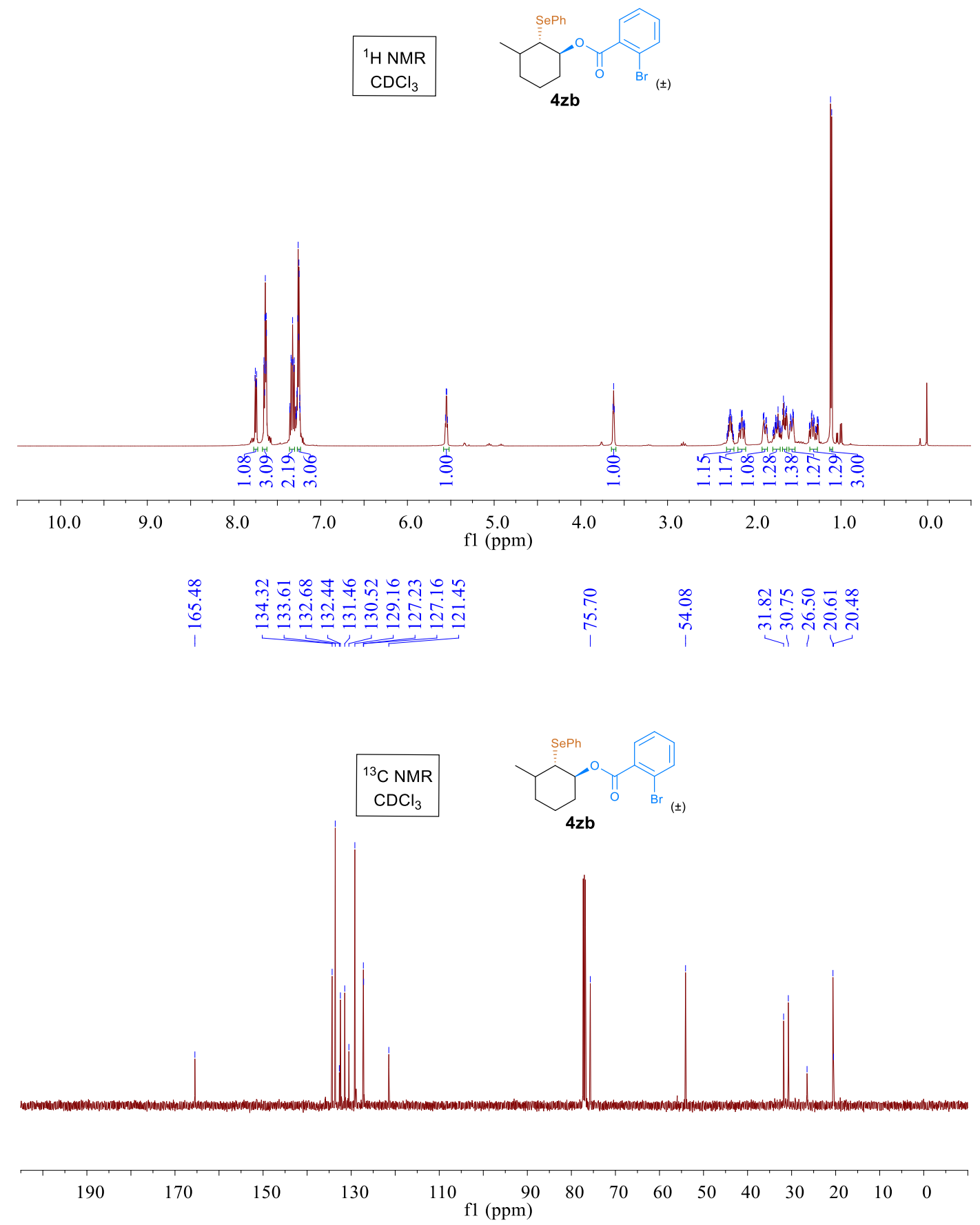

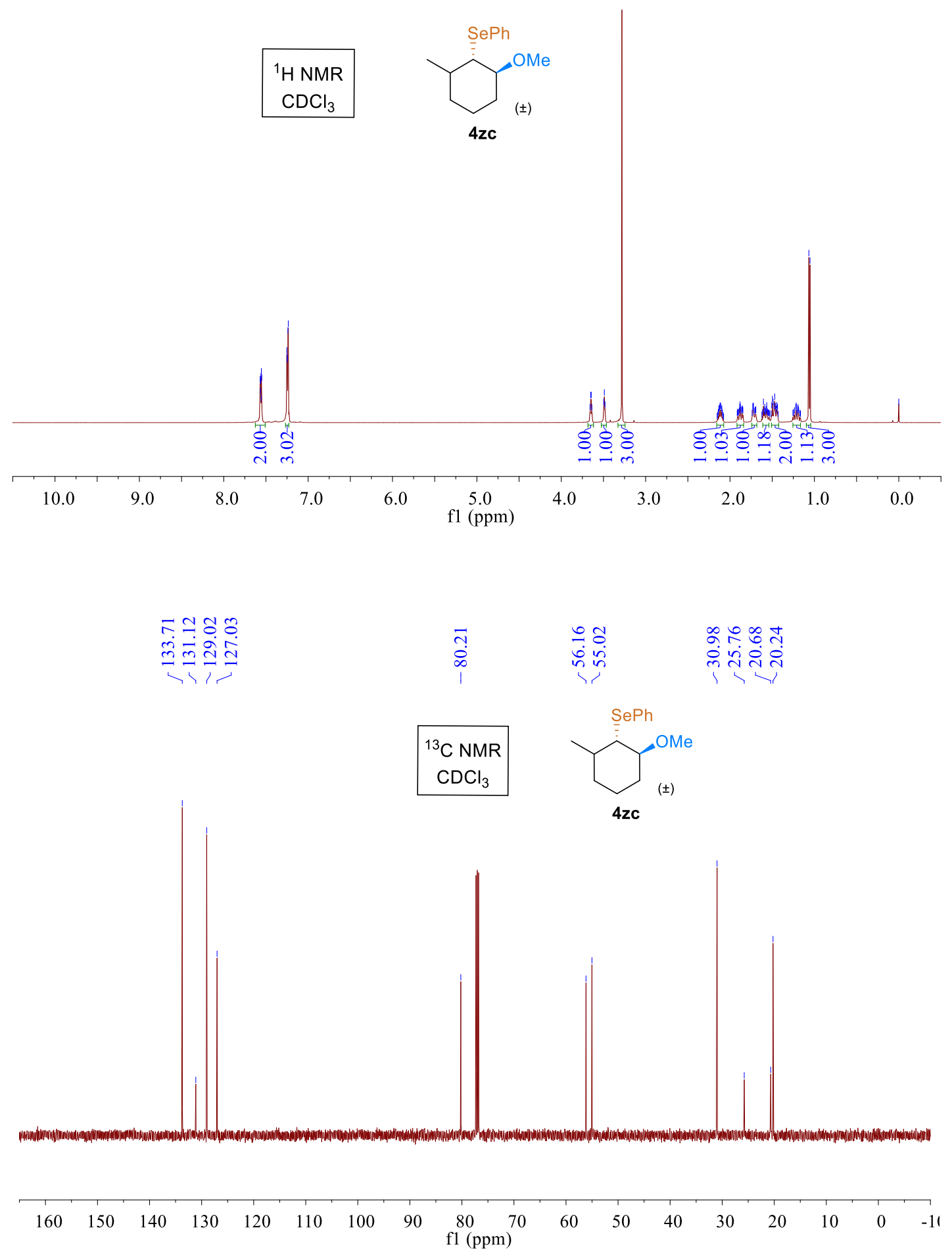


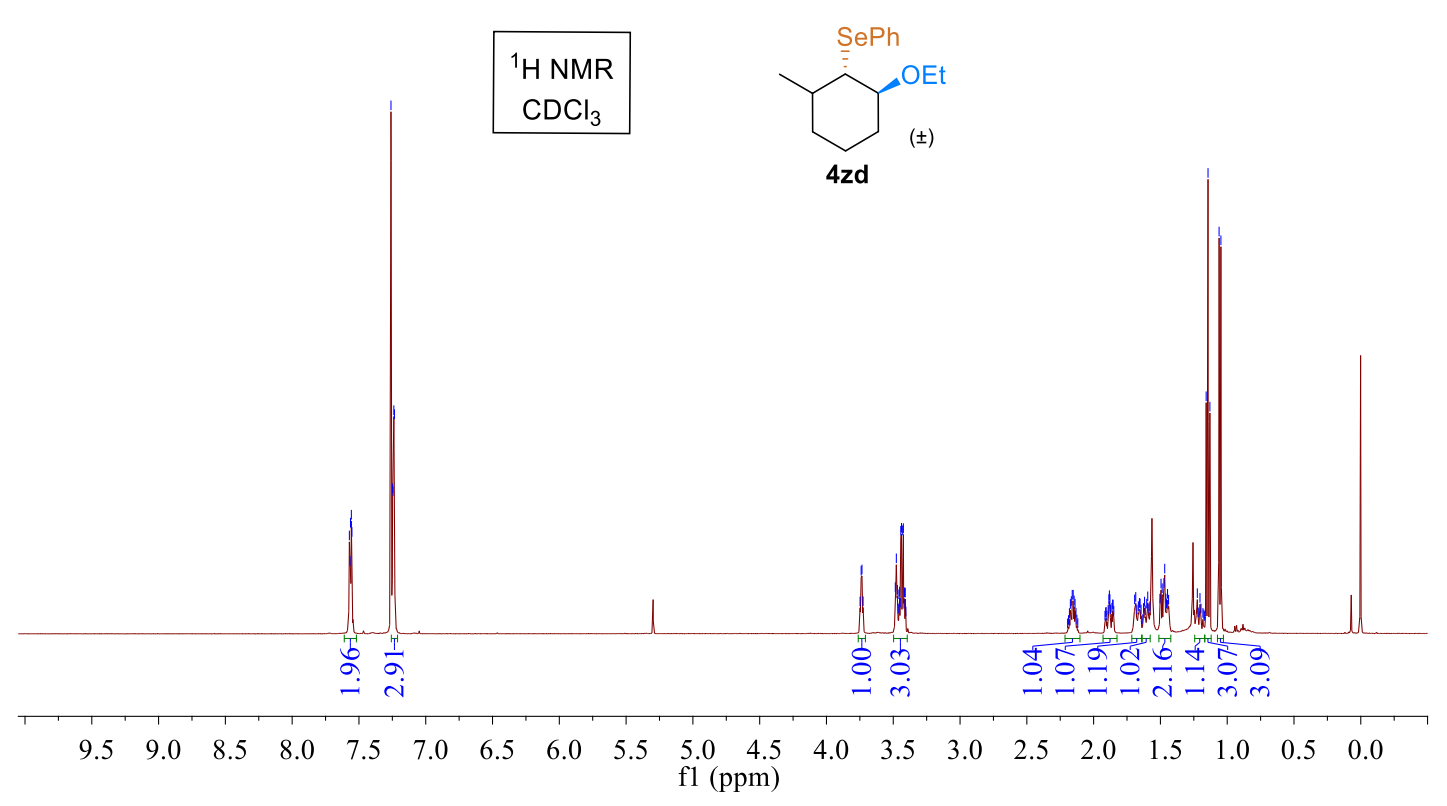

ㄷำ

mim

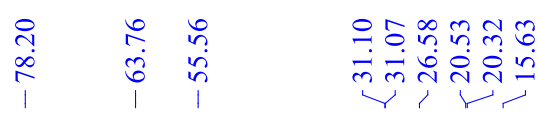

人仿

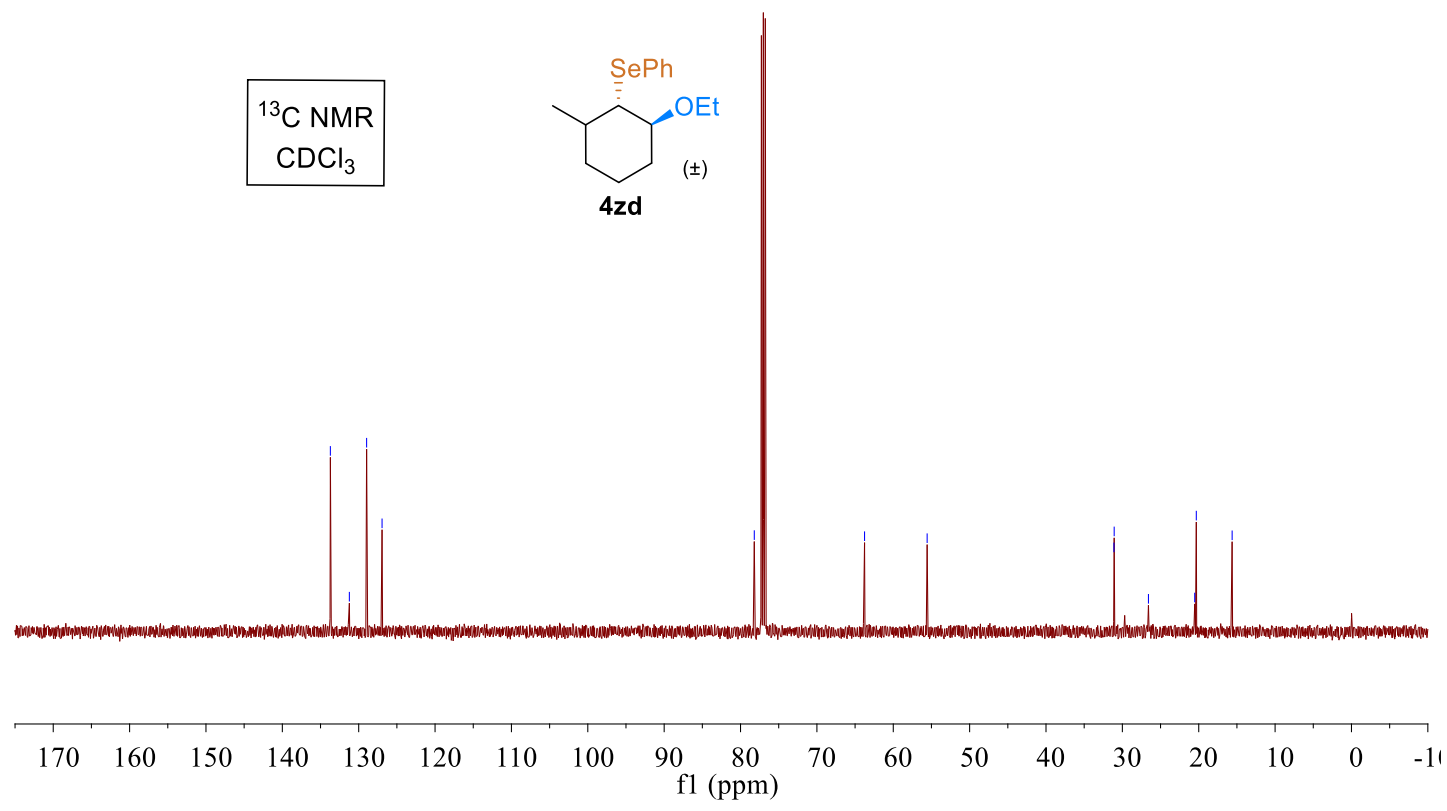




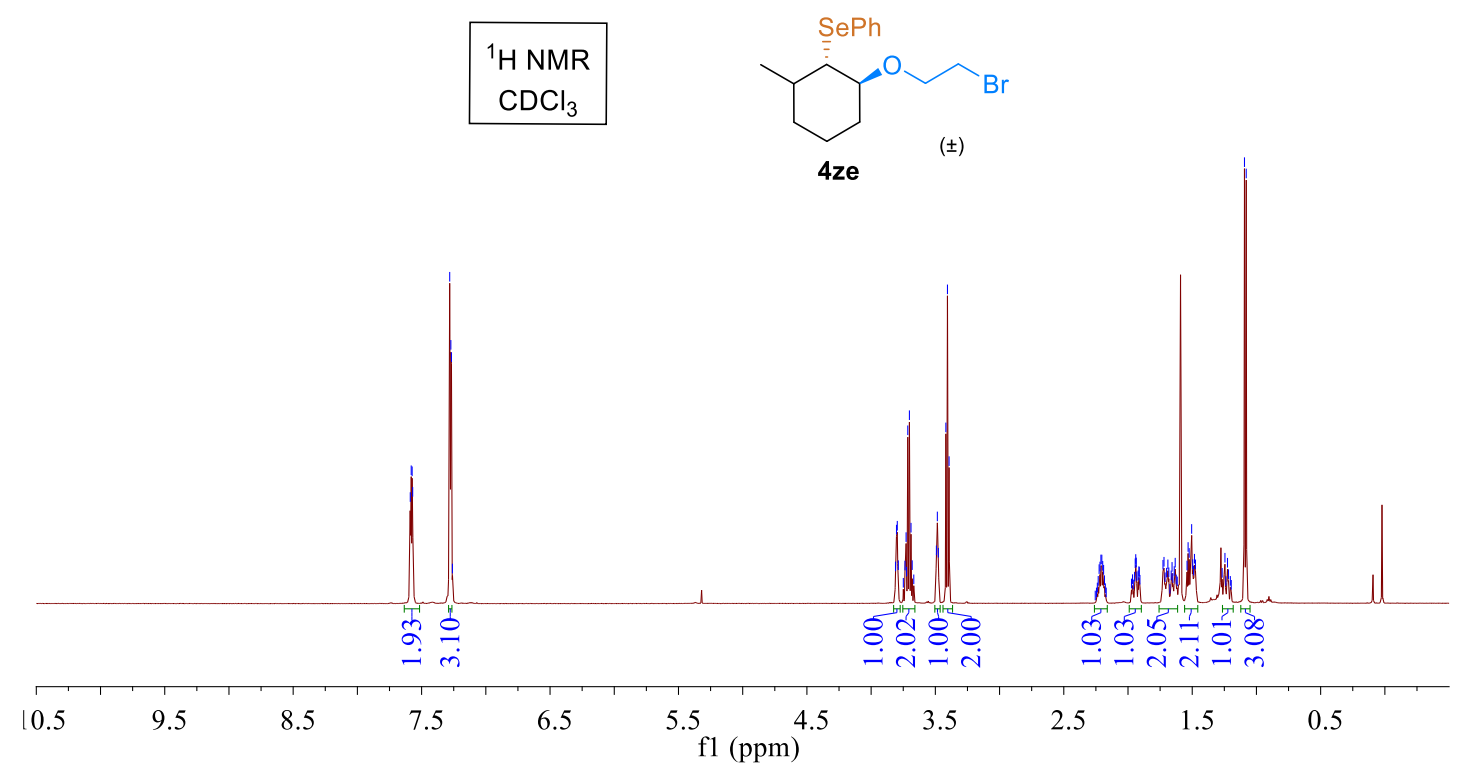

\section{ㄷํำㅇำ}

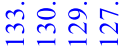

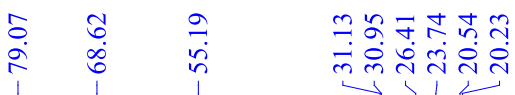

${ }^{13} \mathrm{C} \mathrm{NMR}^{\mathrm{CDCl}}$
$\mathrm{CD}_{3}$<smiles>CC1CCCC(OCCBr)C1[SeH]</smiles>

$( \pm)$

4ze

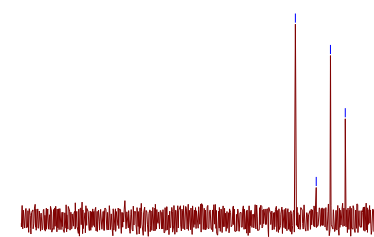

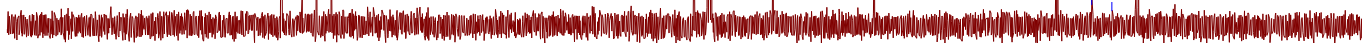

$\begin{array}{lllllllllllllllllll}170 & 160 & 150 & 140 & 130 & 120 & 110 & 100 & 90 & 80 & 70 & 60 & 50 & 40 & 30 & 20 & 10 & 0 & -1\end{array}$ 

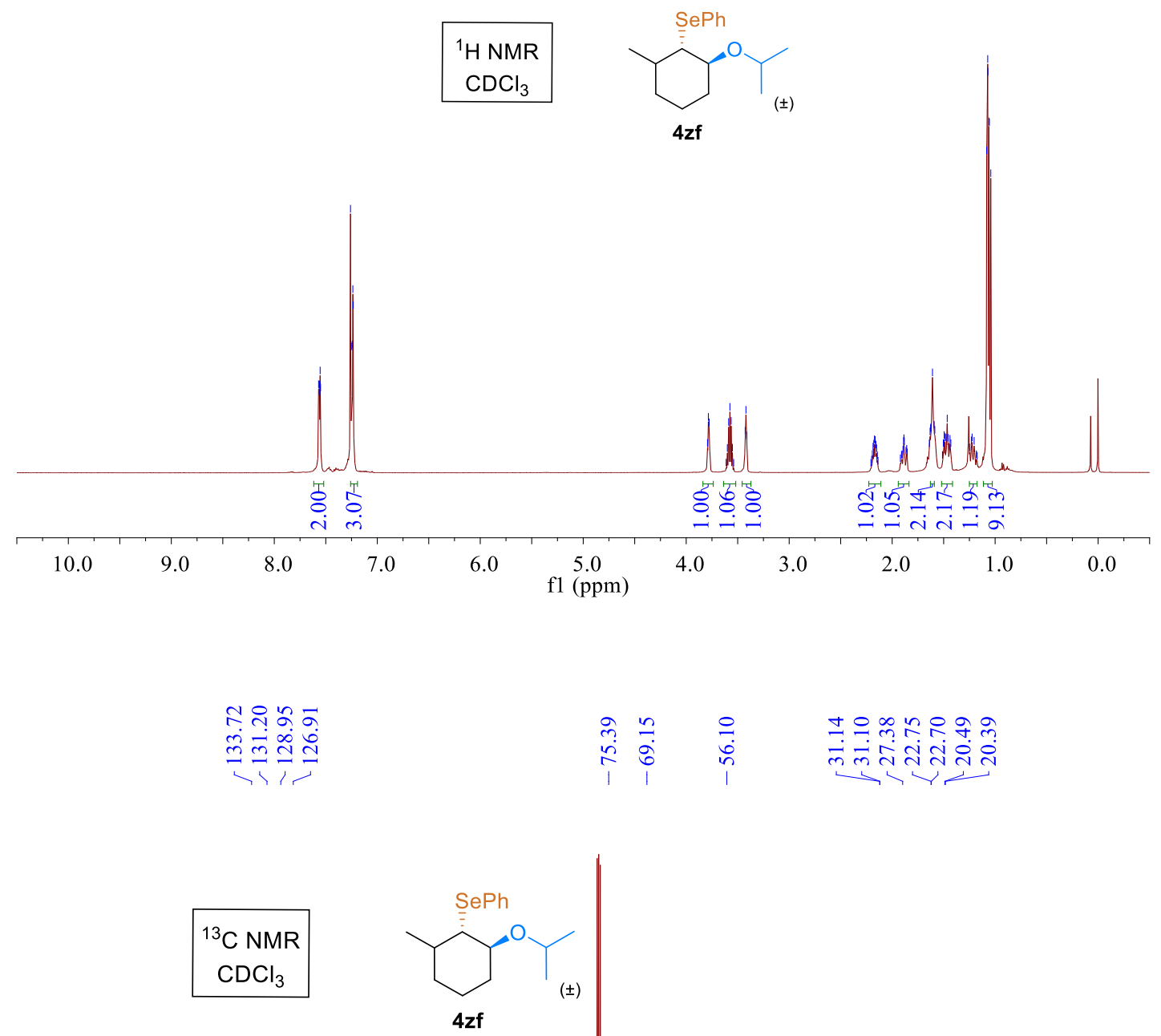

$( \pm)$

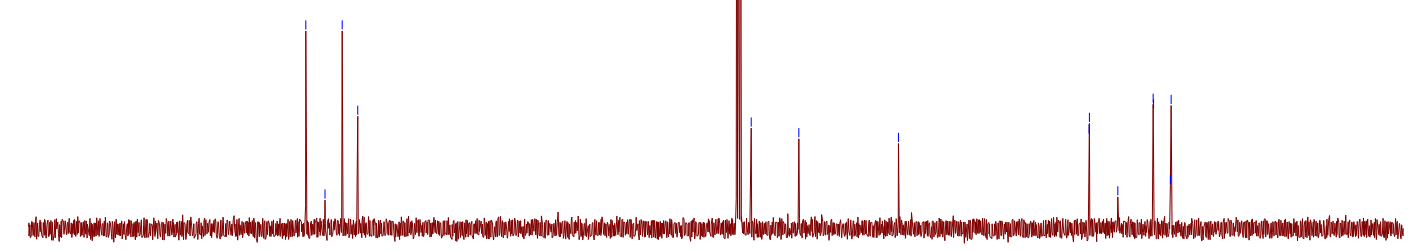

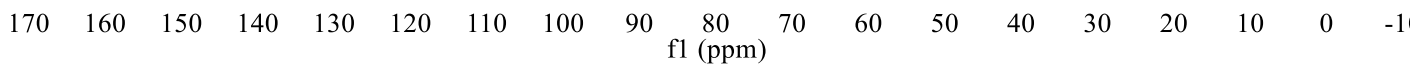




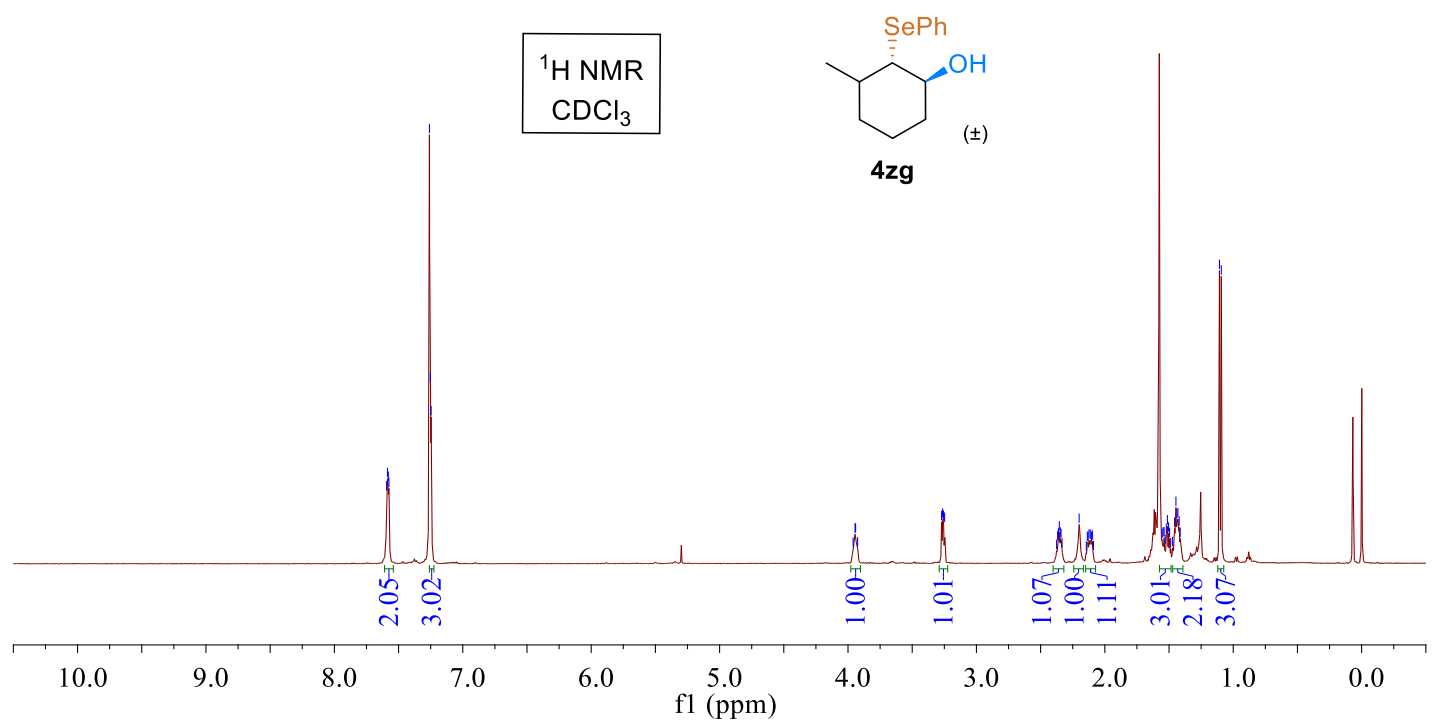

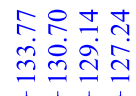

${ }^{13} \mathrm{C}$ NMR $\mathrm{CDCl}_{3}$ $\stackrel{\infty}{\circ}+\frac{0}{0}$

m

लेंì

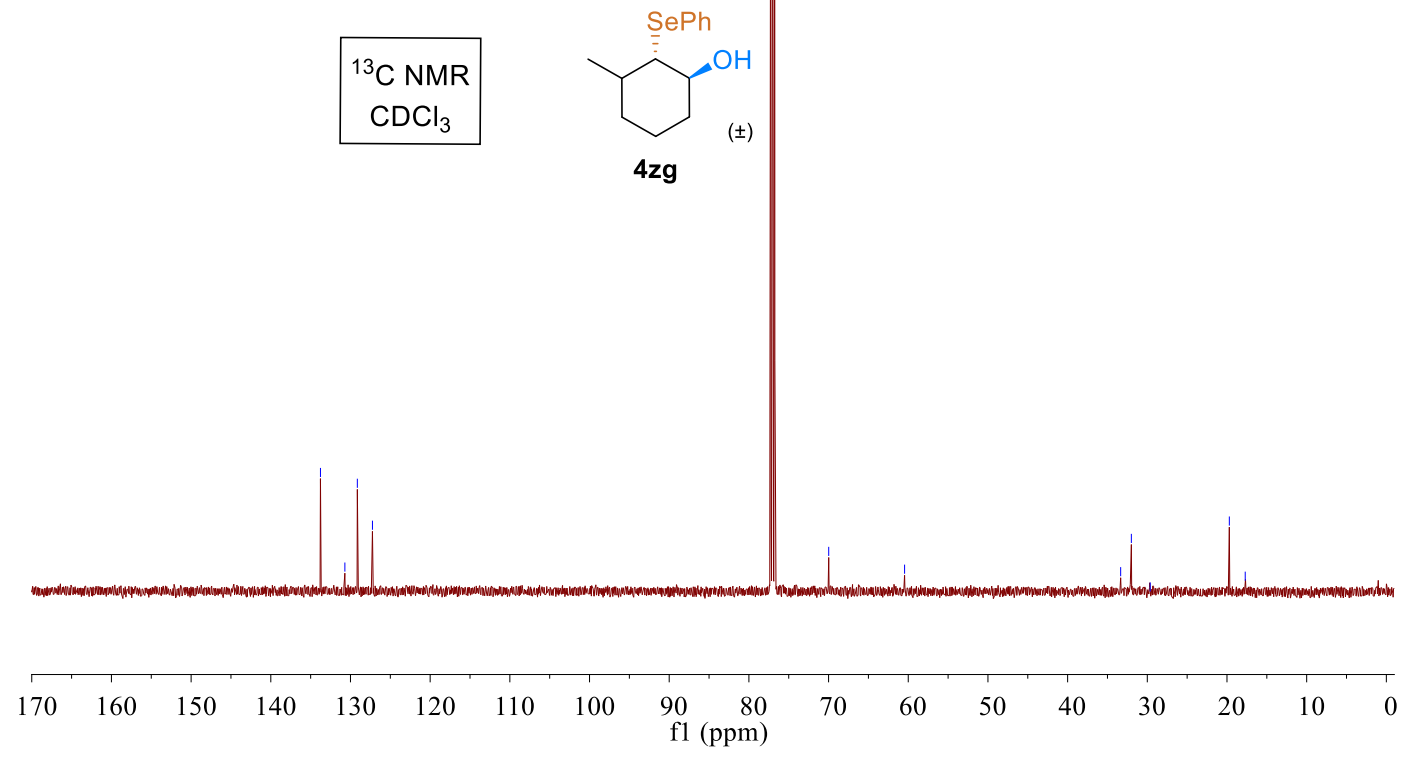



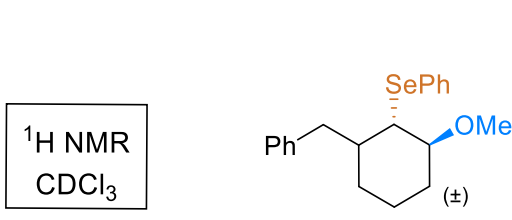

$5 b$
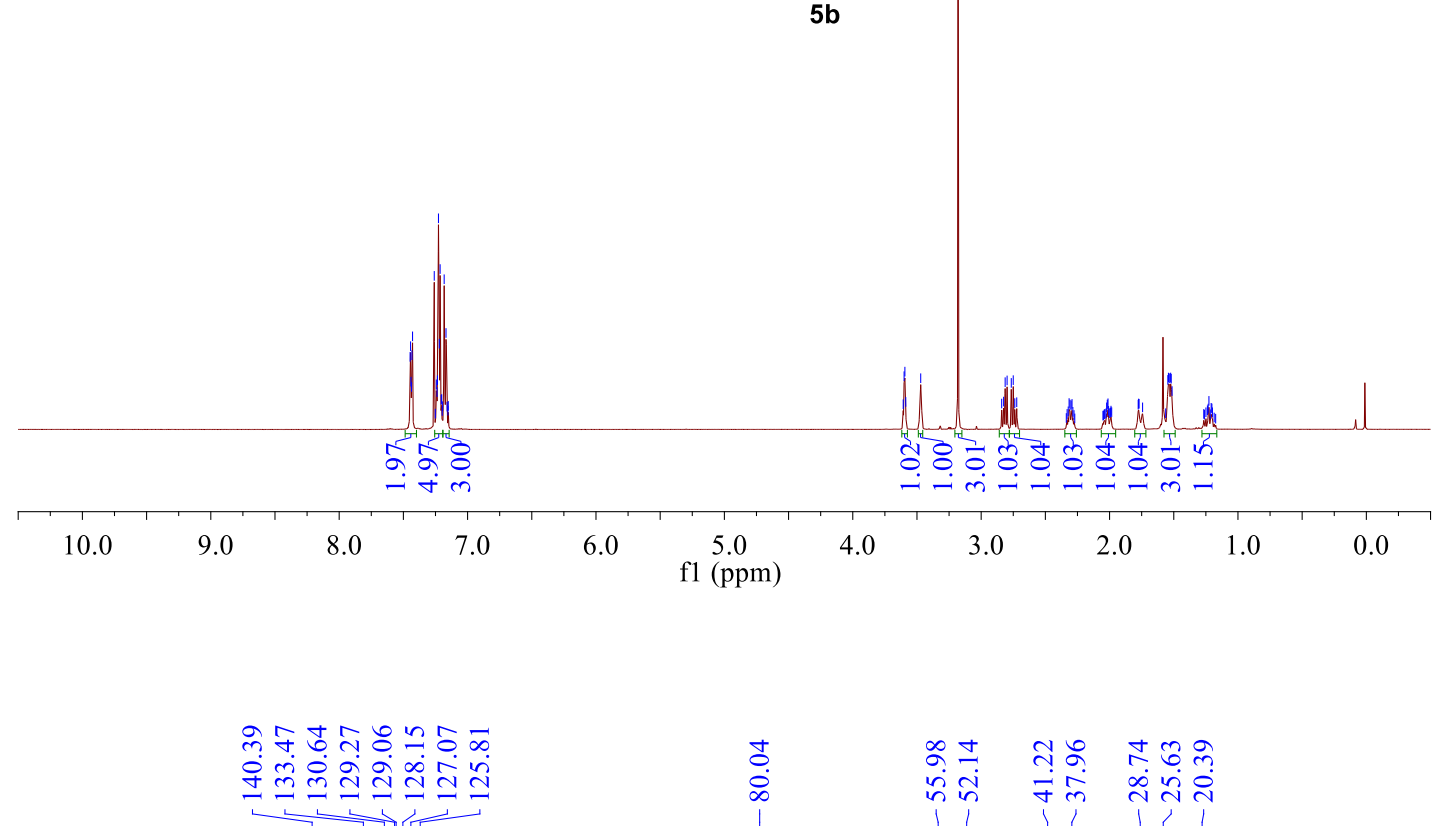

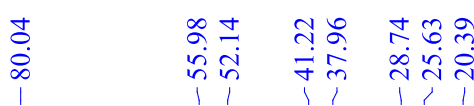

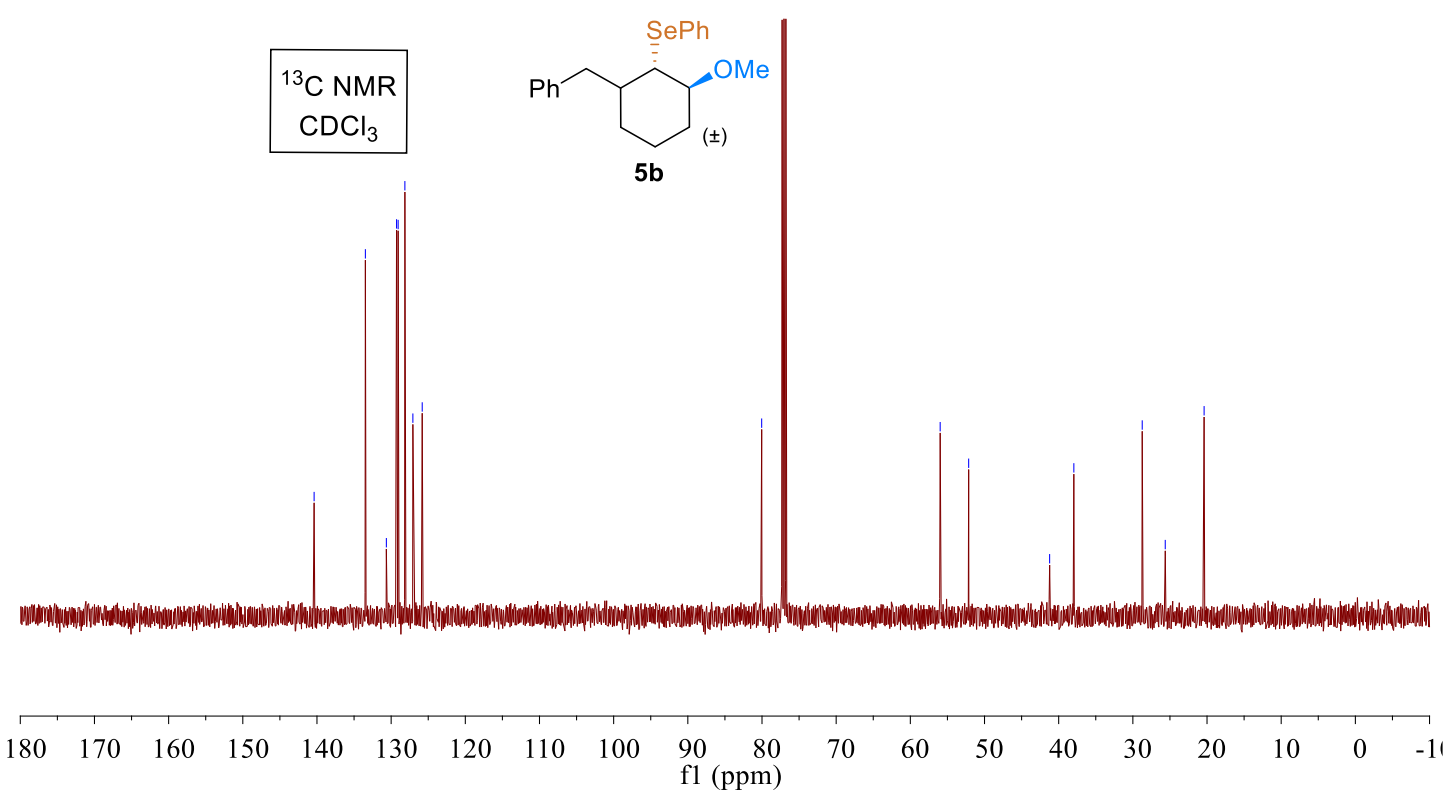




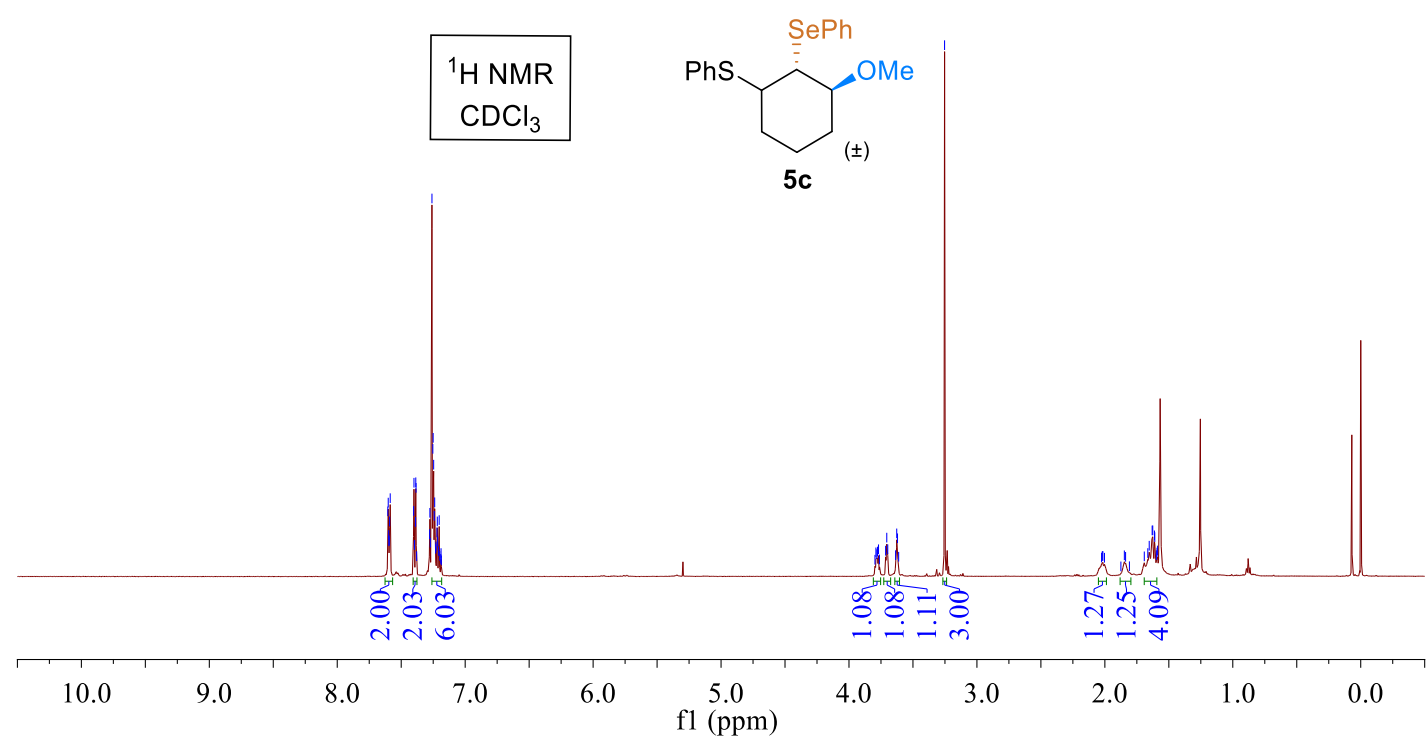

mิ

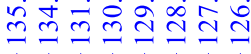

\begin{tabular}{c}
${ }^{13} \mathrm{C} \mathrm{NMR}^{\mathrm{CDCl}_{3}}$ \\
\hline
\end{tabular}

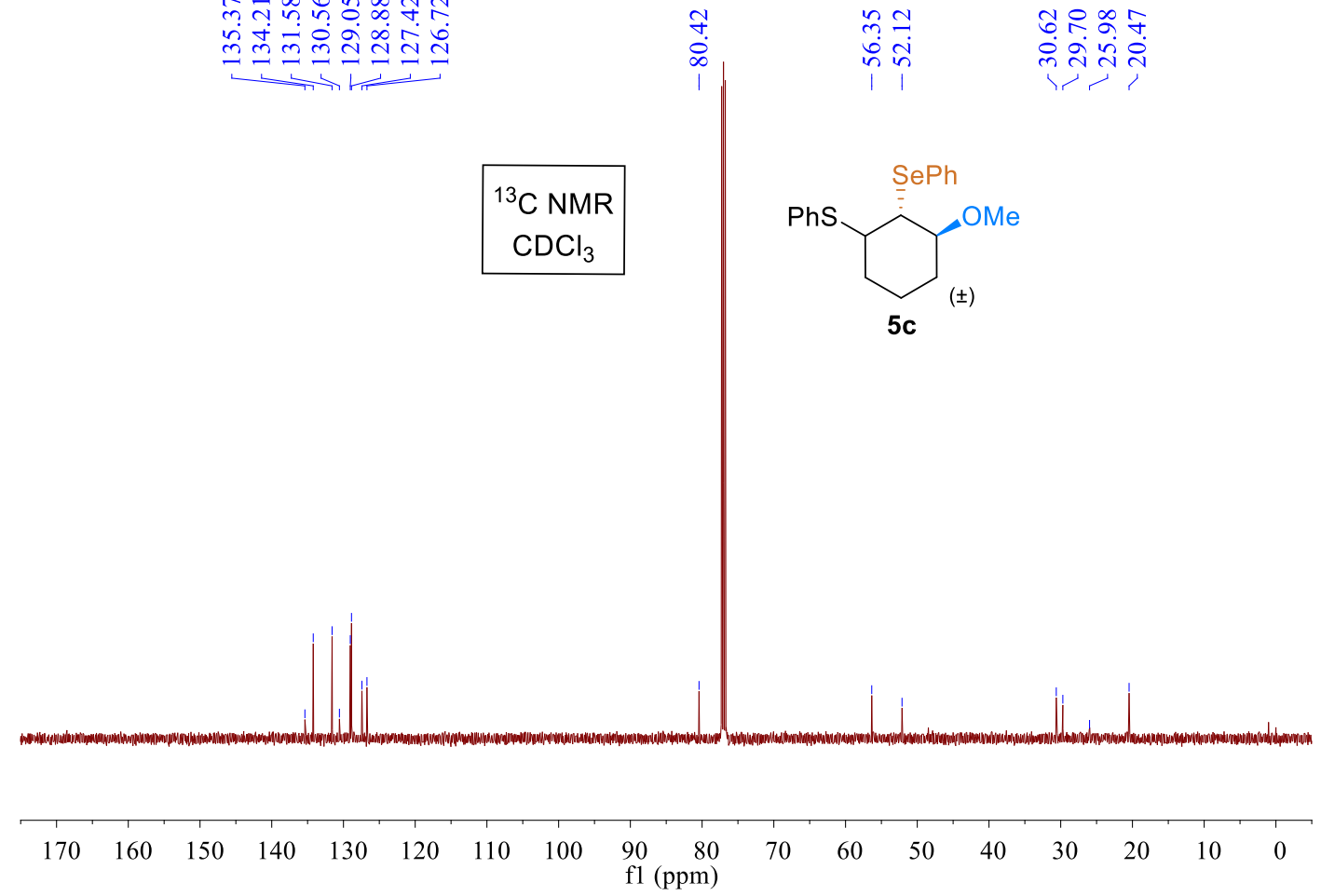

눙용ำ

कंतोंते 


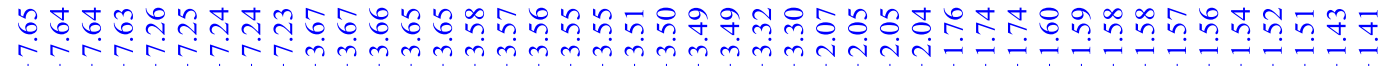
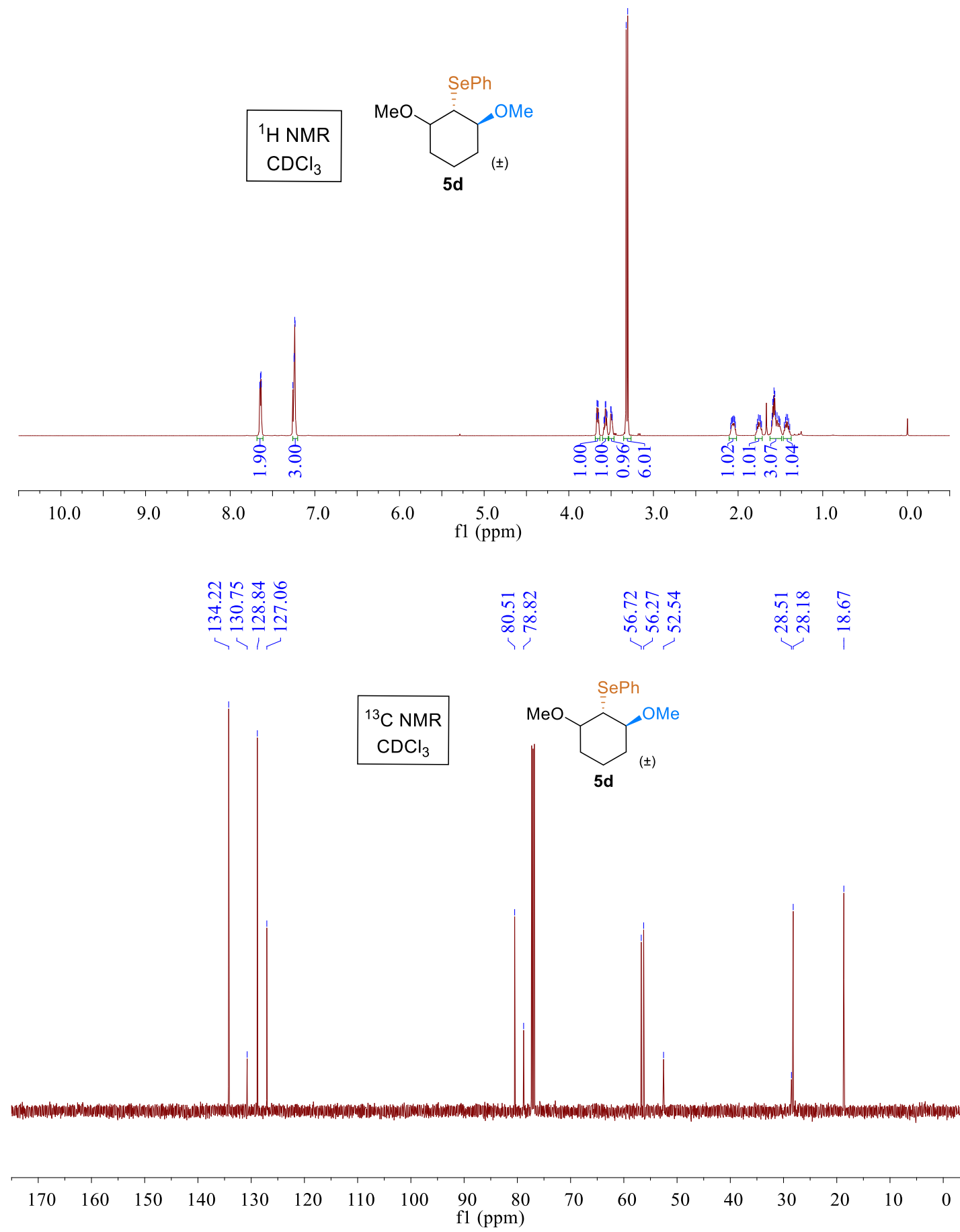


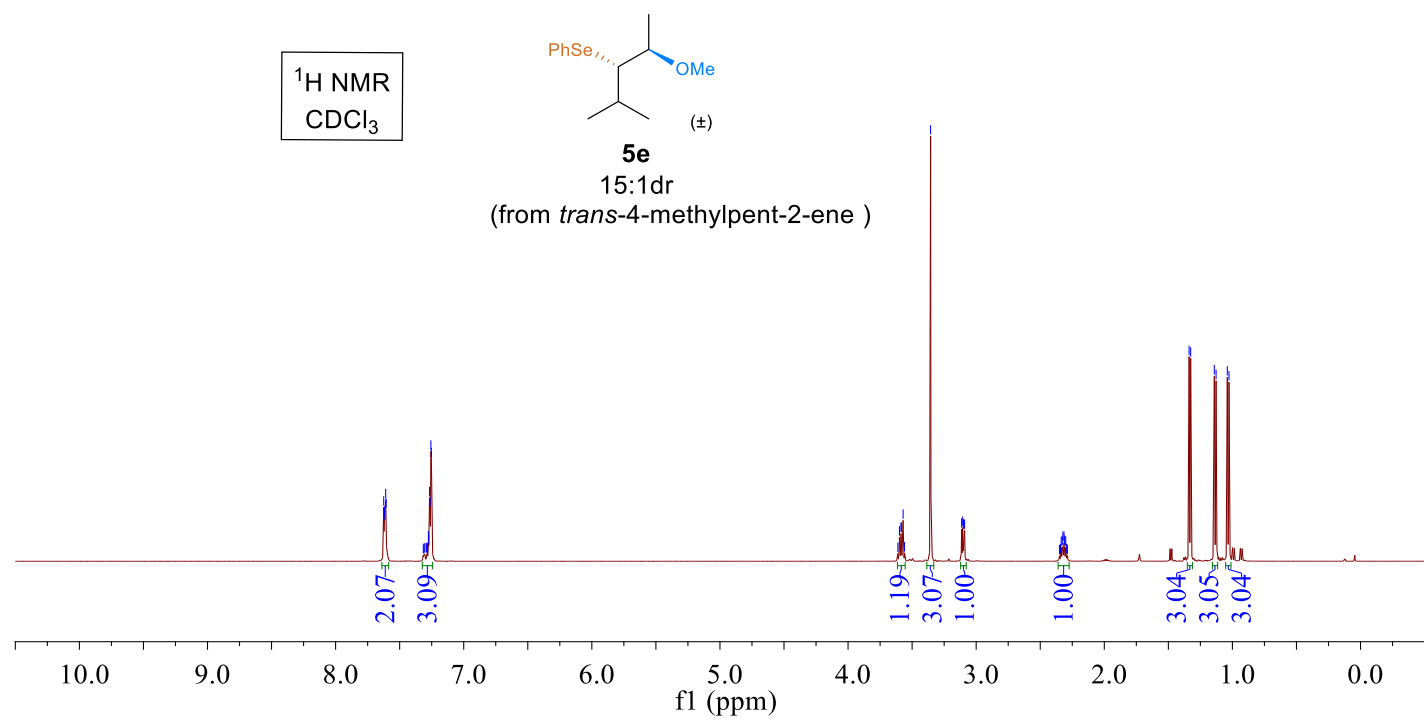

$\therefore$ 동

$\dot{m} \dot{m} \dot{i}$

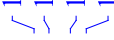

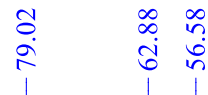

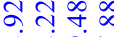

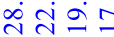
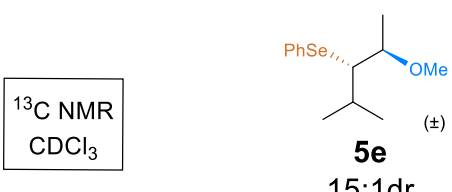

$15: 1 d r$

(from trans-4-Methyl-2-pentene)

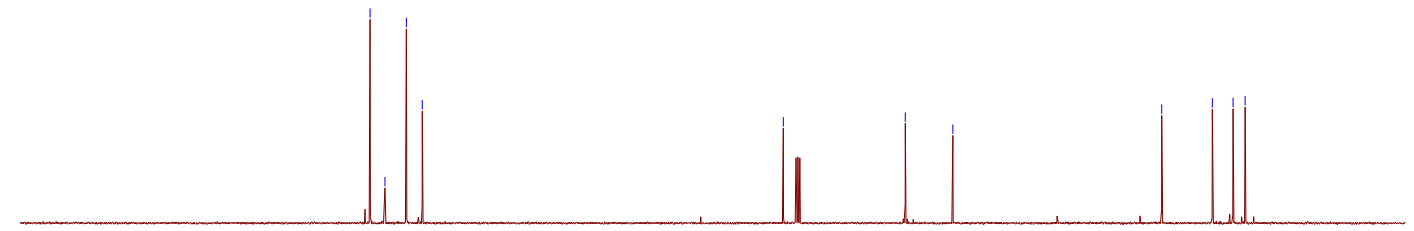

$\begin{array}{llllllllllllllllll}180 & 170 & 160 & 150 & 140 & 130 & 120 & 110 & 100 \underset{\mathrm{fl}(\mathrm{ppm})}{90} \mathbf{8 0} & 70 & 60 & 50 & 40 & 30 & 20 & 10 & 0\end{array}$ 


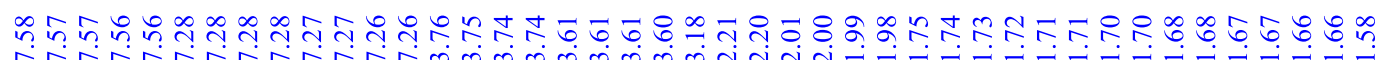

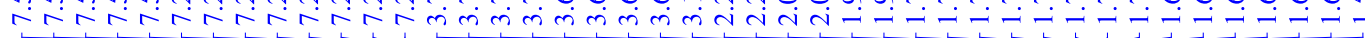

\begin{tabular}{c}
${ }^{1} \mathrm{H} \mathrm{NMR}$ \\
$\mathrm{CDCl}_{3}$ \\
\hline
\end{tabular}

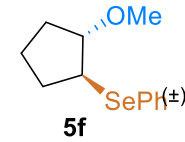

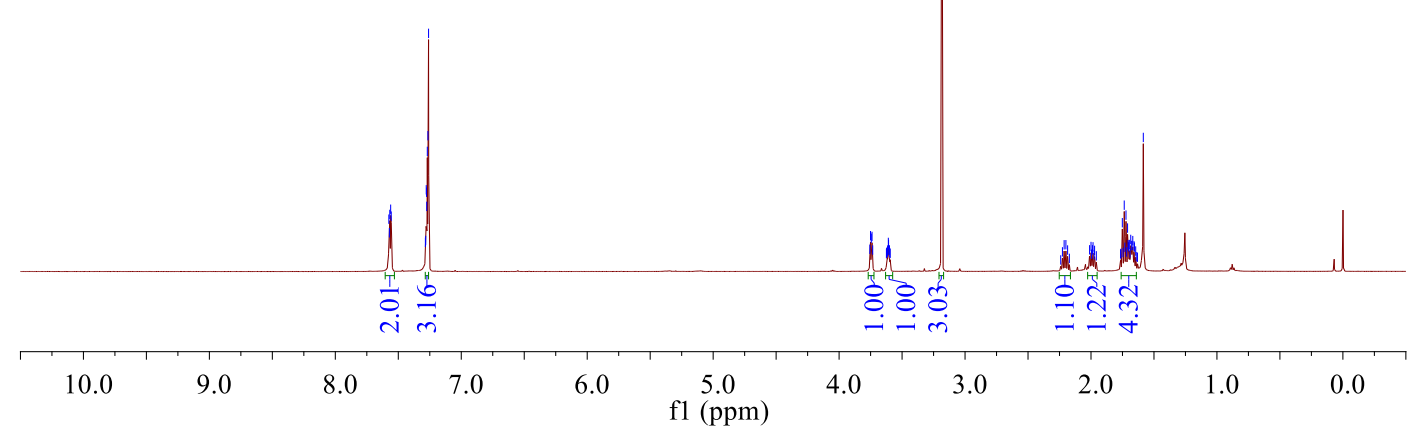

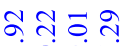

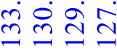

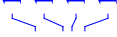

$\underset{\substack{1 \\ \infty \\ \infty}}{1}$

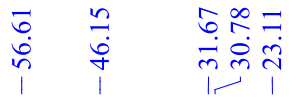

${ }^{13} \mathrm{C} \mathrm{NMR}^{\mathrm{CDCl}} 3$
$\mathrm{CDCl}_{3}$
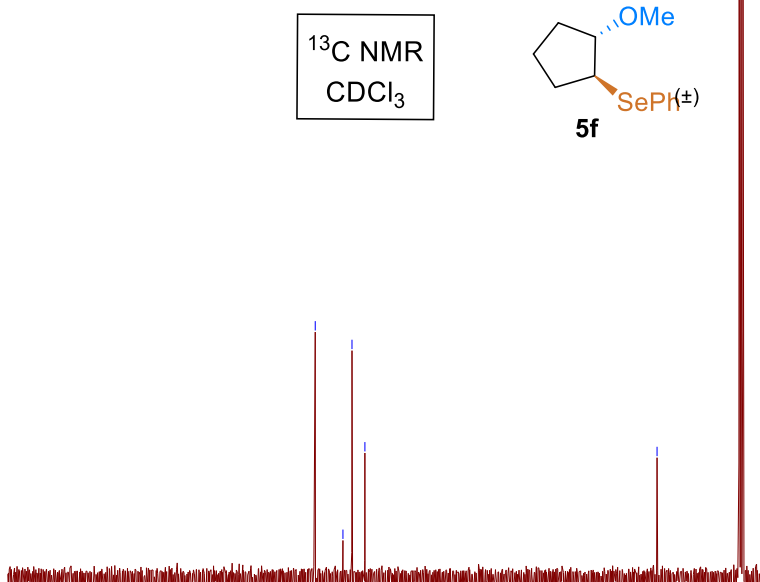

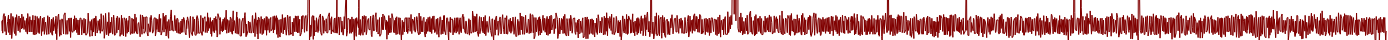

$\begin{array}{lllllllllllllllllll}170 & 160 & 150 & 140 & 130 & 120 & 110 & 100 & \begin{array}{c}90 \\ \mathrm{f} 1(\mathrm{ppm})\end{array} & 70 & 60 & 50 & 40 & 30 & 20 & 10 & 0 & -1\end{array}$




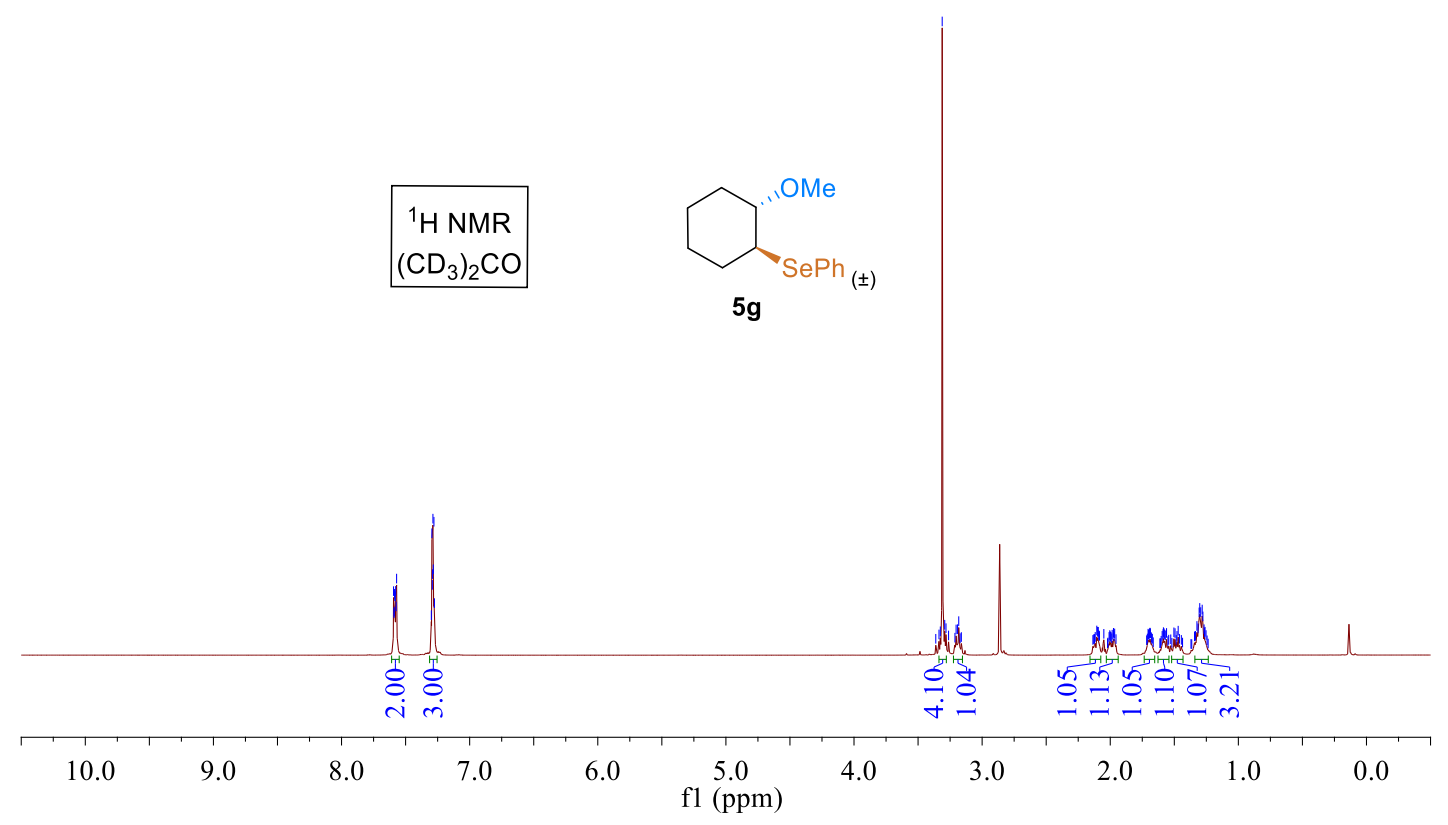

ㄷำ $\bar{\infty} \bar{c}$

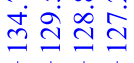
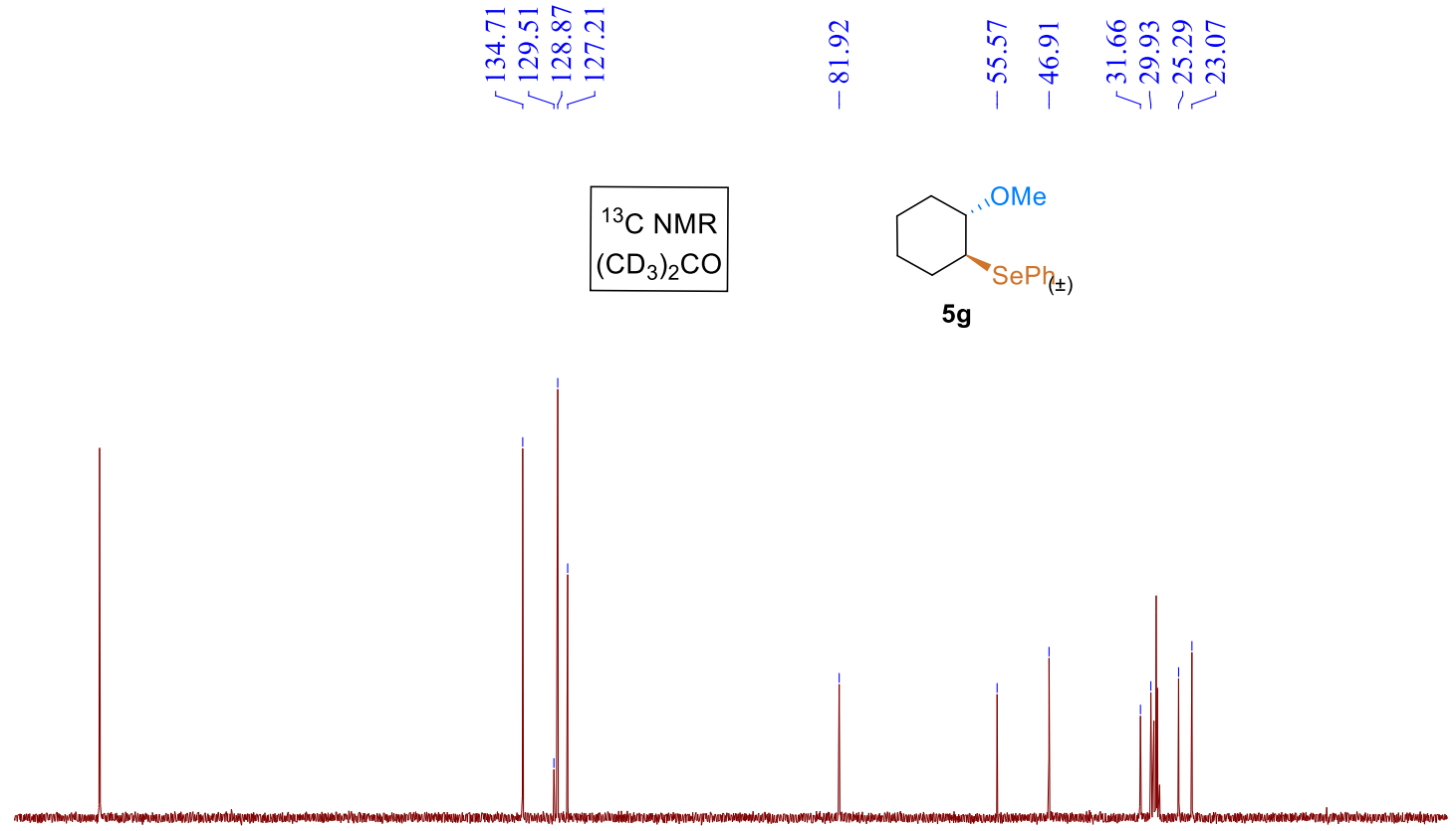

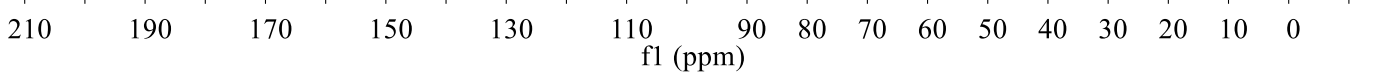



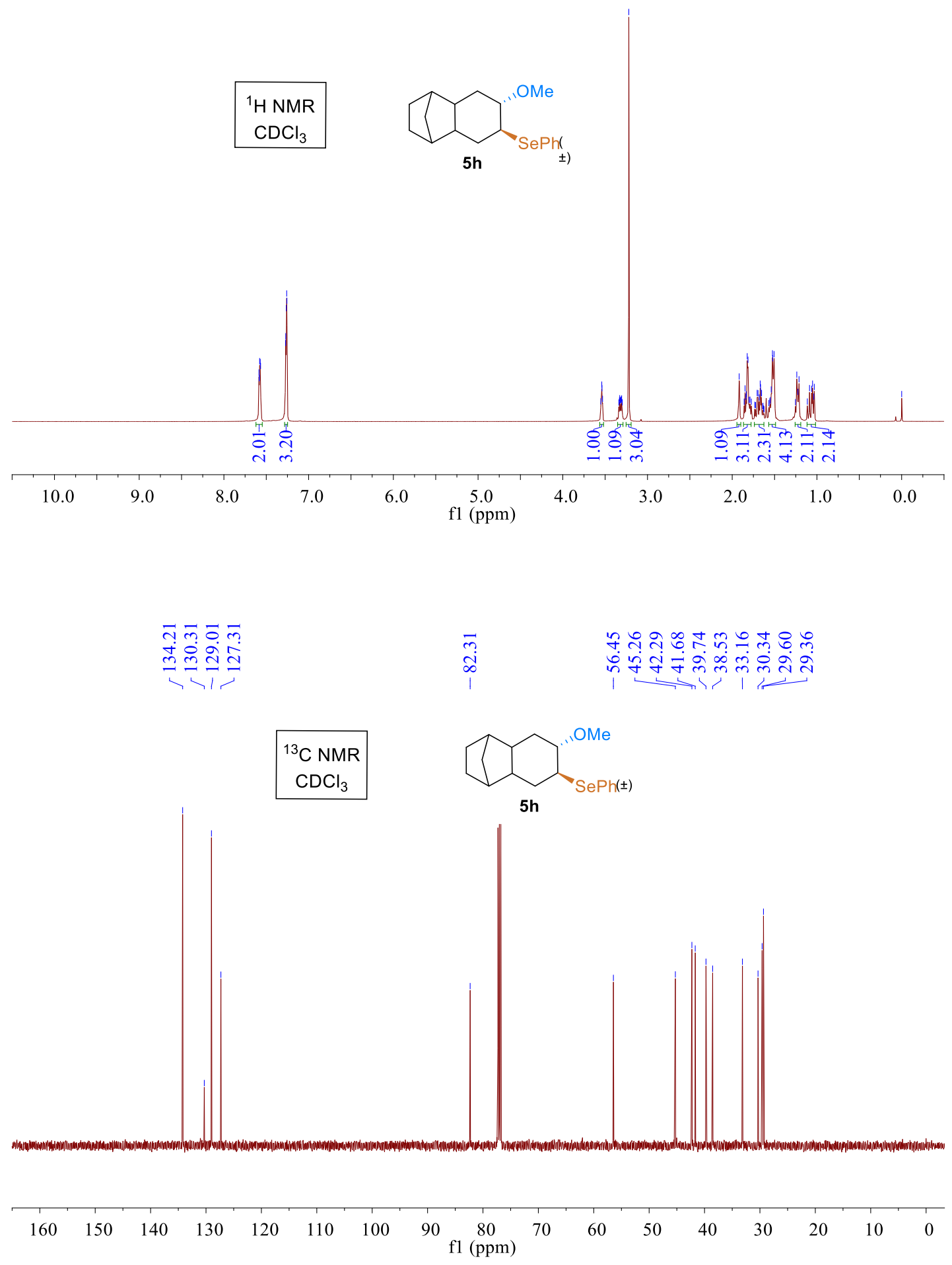


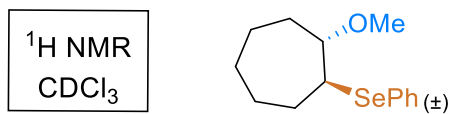

$5 \mathbf{i}$

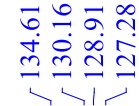

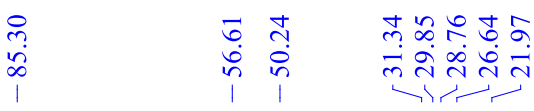

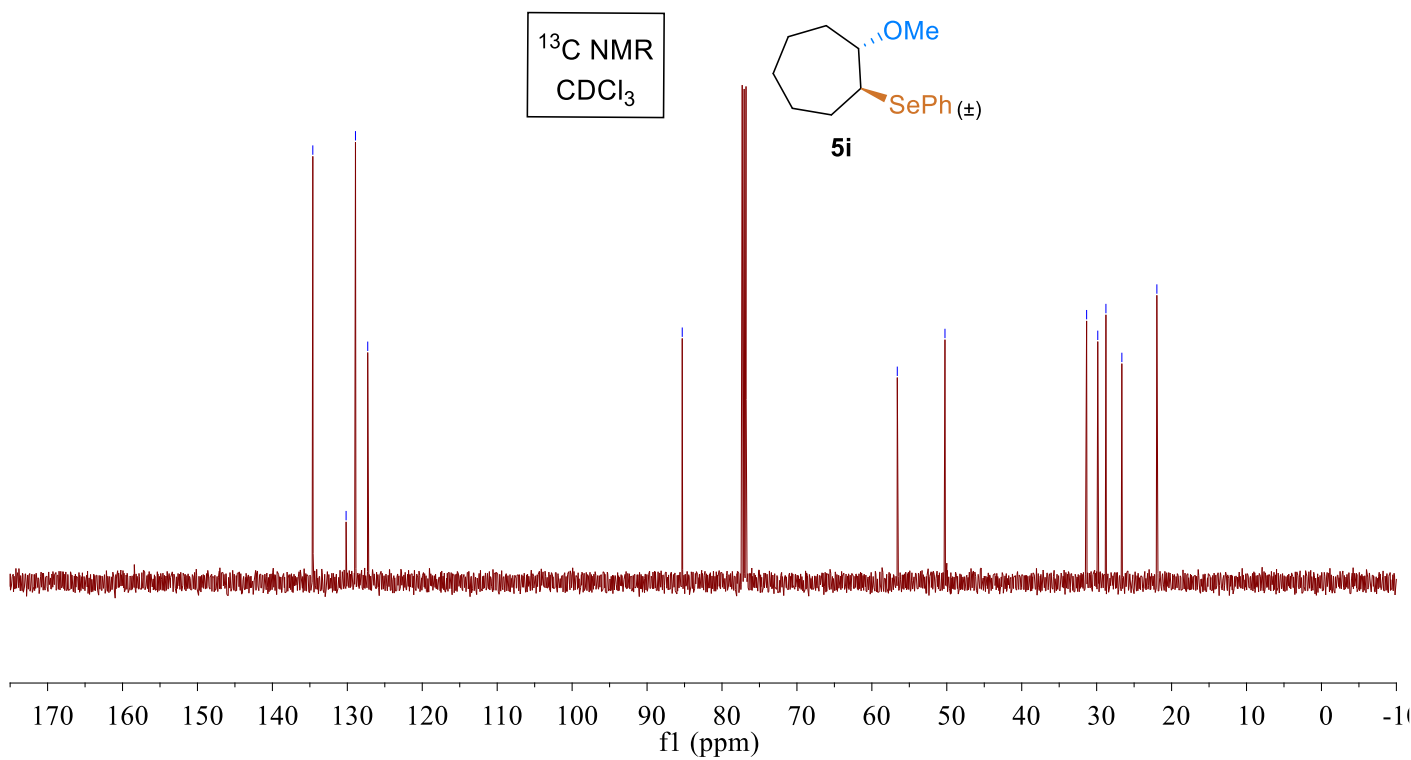



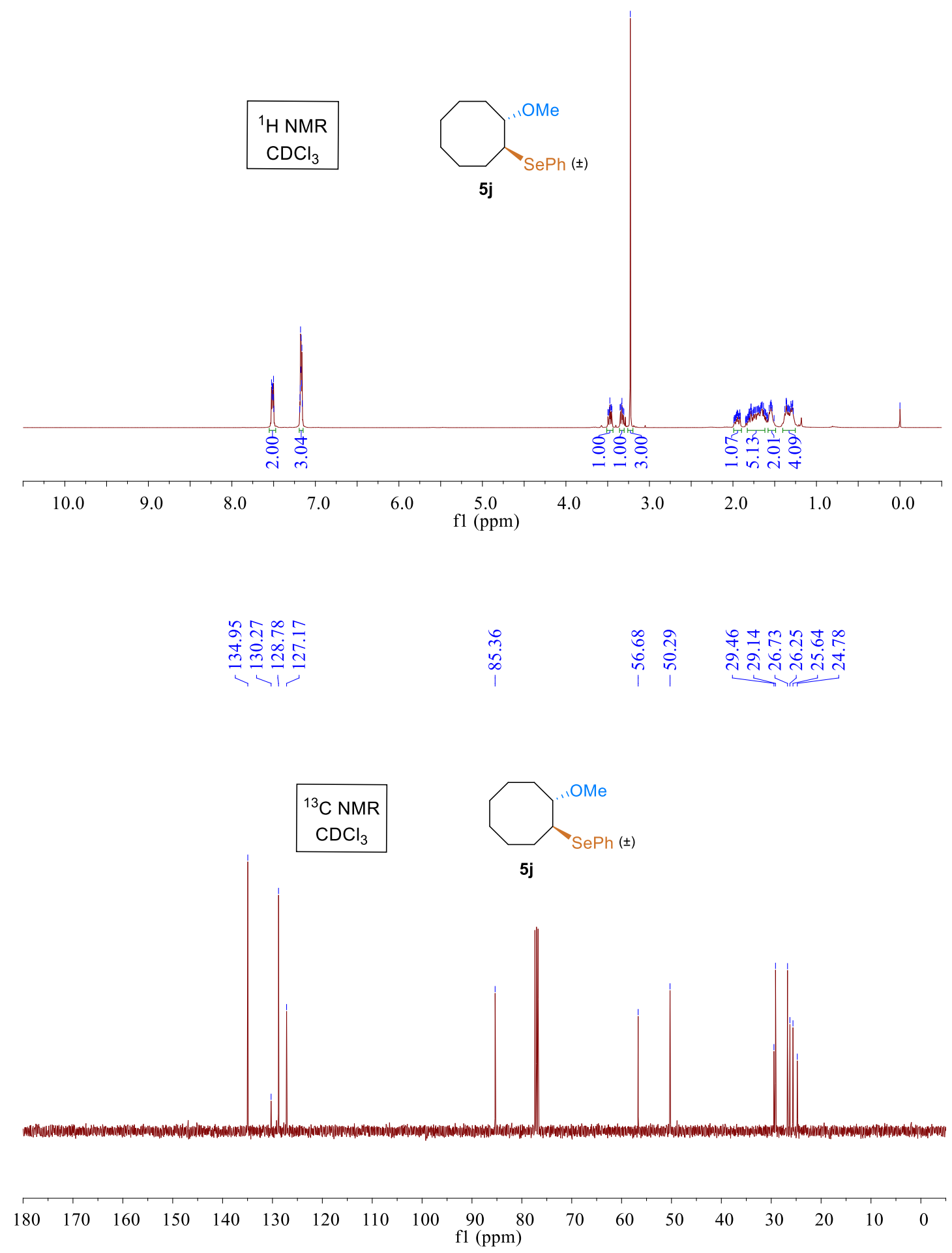


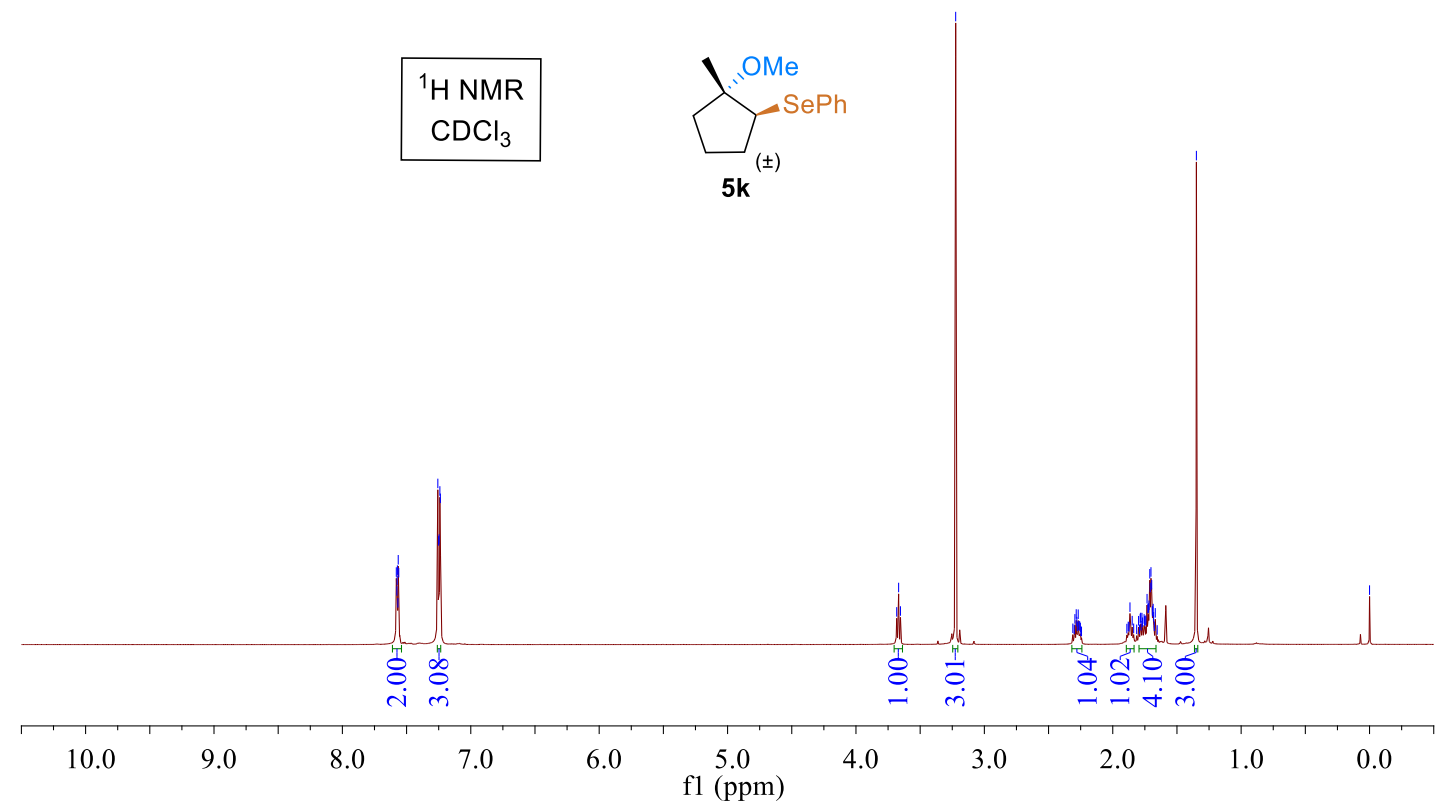

लिखे

ल요웡

$\checkmark<1$

$\curvearrowright$
$\infty$
1

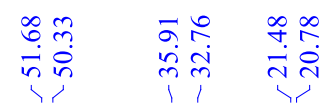

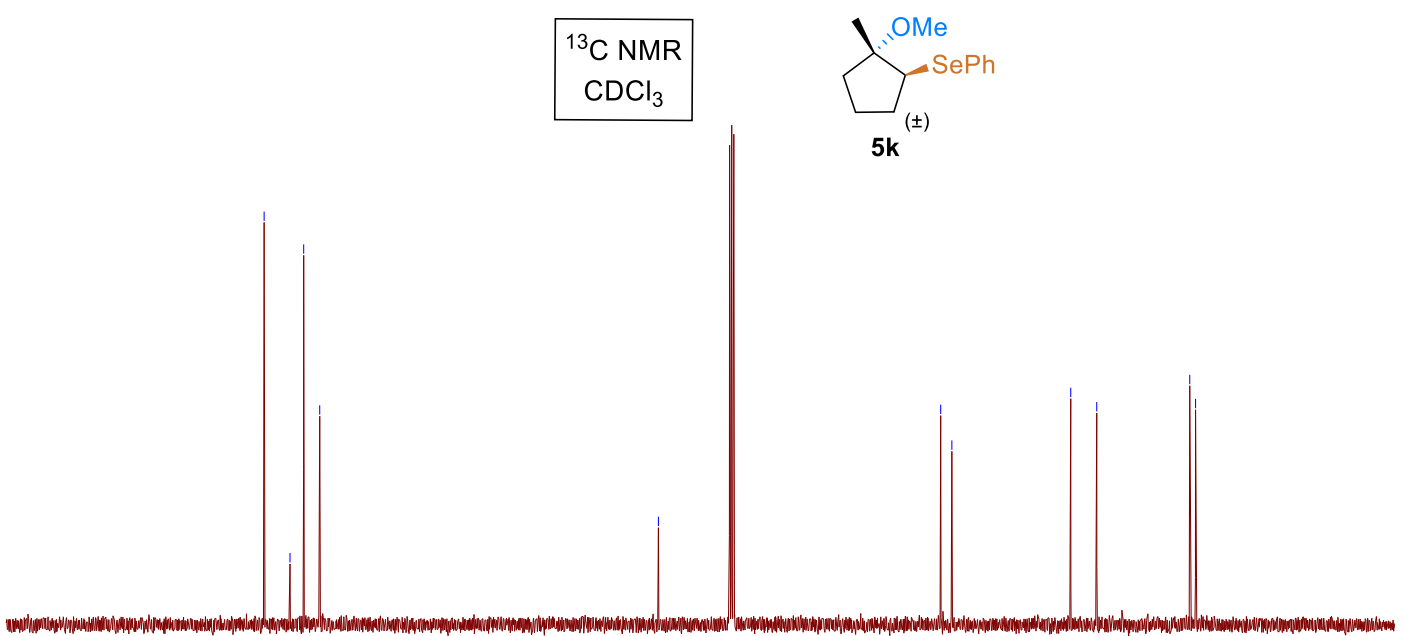

$90 \quad 80$

60

$30 \quad 20$

10

0 


\begin{tabular}{c}
${ }^{1} \mathrm{H} \mathrm{NMR}$ \\
$\mathrm{CDCl}_{3}$ \\
\hline
\end{tabular}

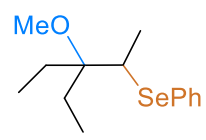

51

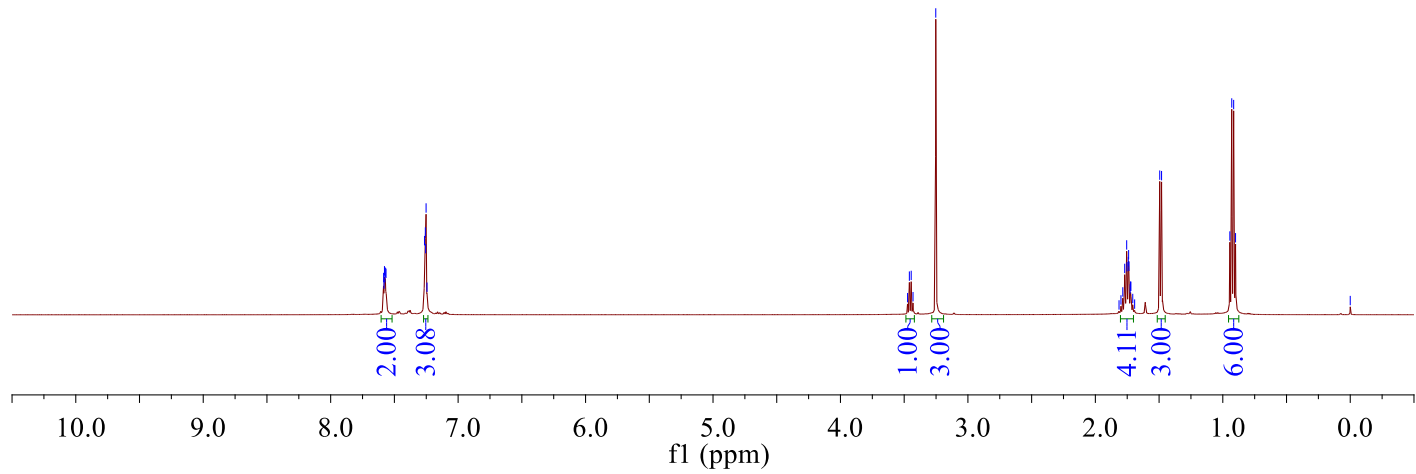

F⿻ำ

菂㐫

$\underset{\infty}{\infty}$

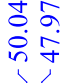

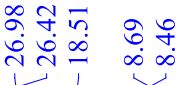

${ }^{13} \mathrm{C} \mathrm{NMR}$
$\mathrm{CDCl}_{3}$

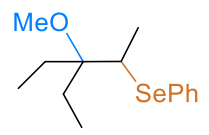

$5 \mathbf{1}$

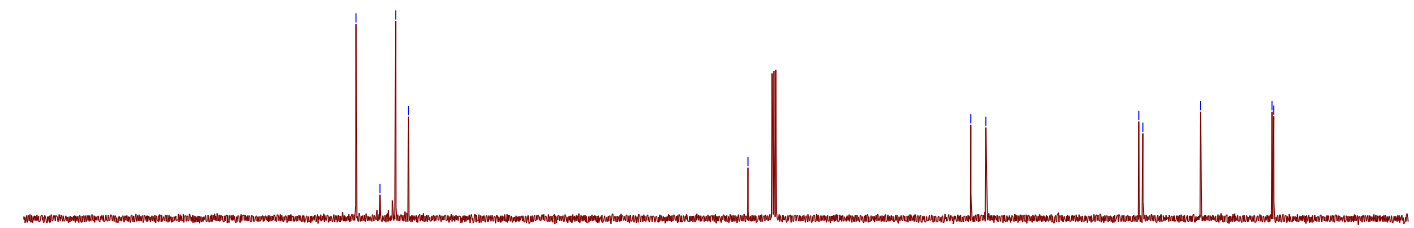

$\begin{array}{lllllllllll}180 & 170 & 160 & 150 & 140 & 130 & 120 & 110 & 100 & 90 & 80\end{array}$ f1 (ppm) 


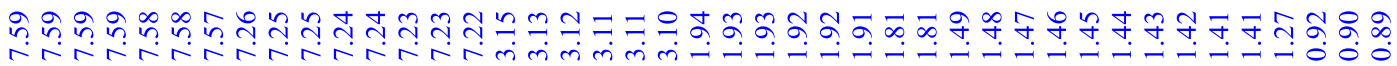

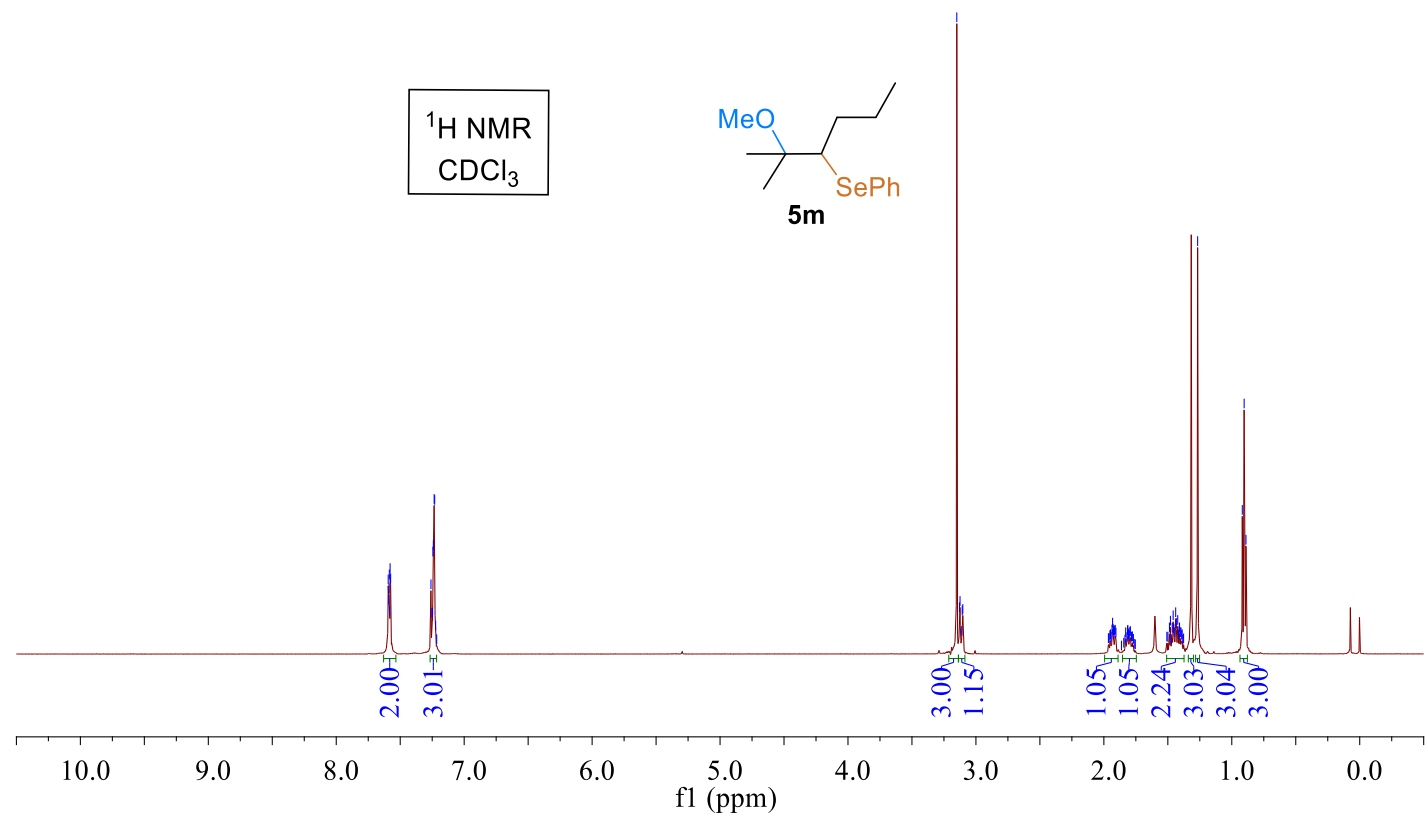

ธूेจ

m워

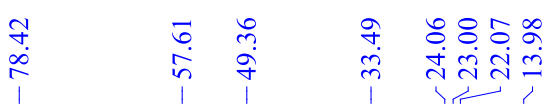
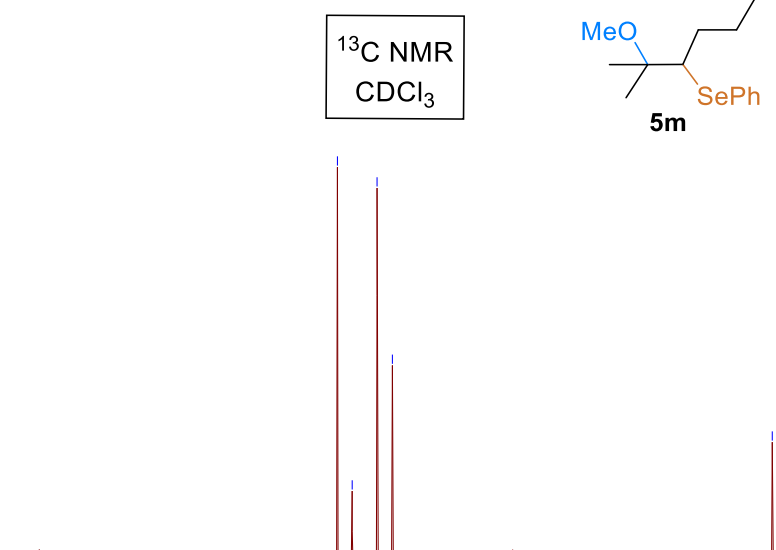

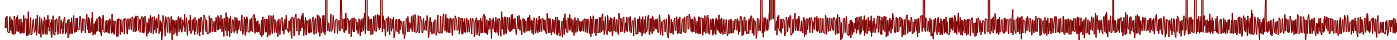

$\begin{array}{lllllllllllllllll}170 & 160 & 150 & 140 & 130 & 120 & 110 & 100 & \begin{array}{c}90 \\ \mathrm{f} 1(\mathrm{ppm})\end{array} & 70 & 60 & 50 & 40 & 30 & 20 & 10 & 0\end{array}$ 


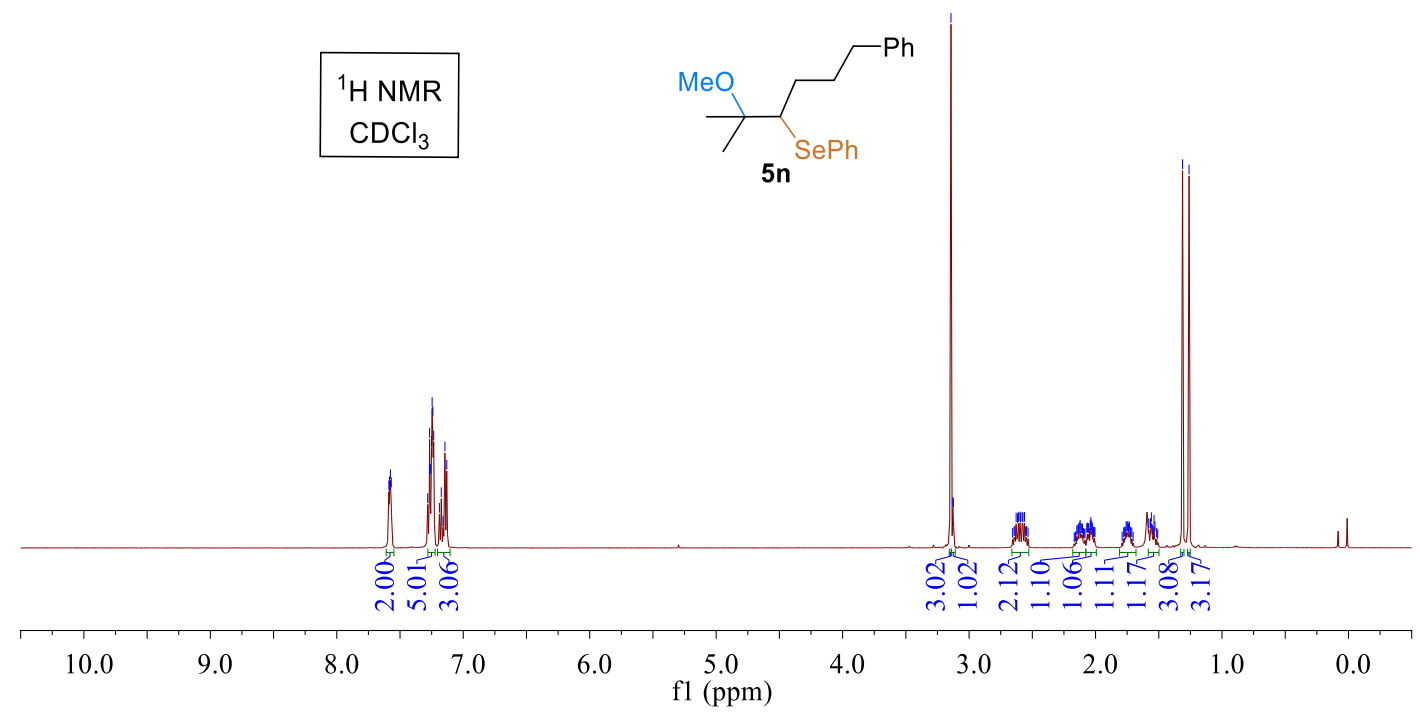

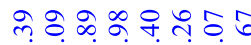

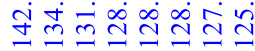

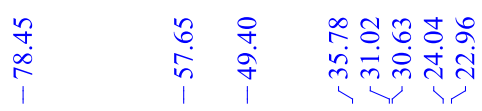
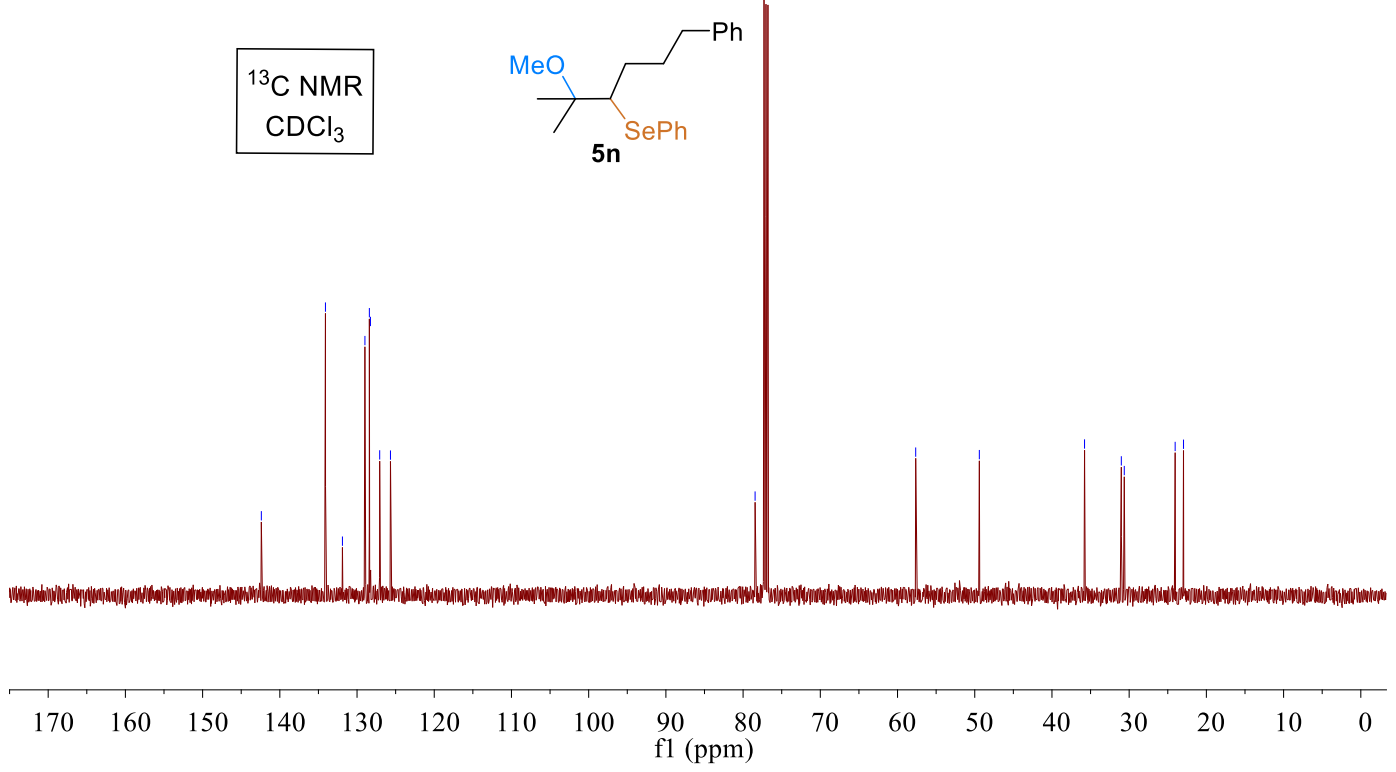

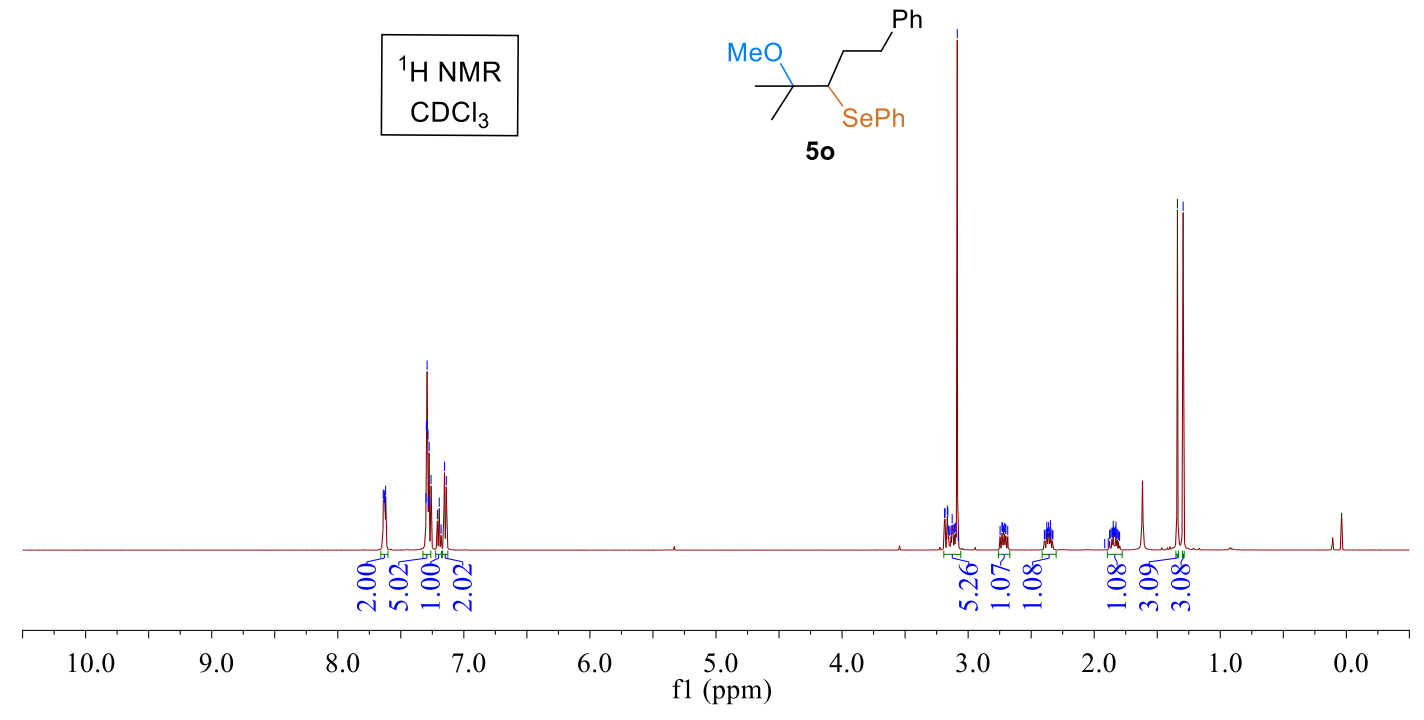

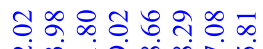

ป઼்

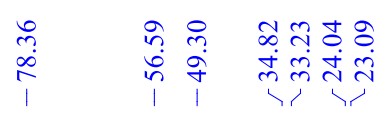

${ }^{13} \mathrm{C} \mathrm{NMR}$
$\mathrm{CDCl}_{3}$
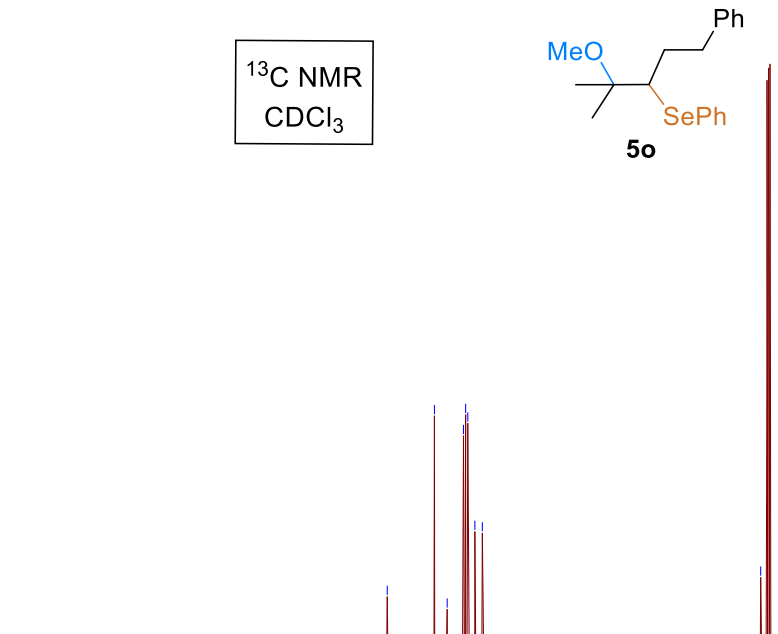

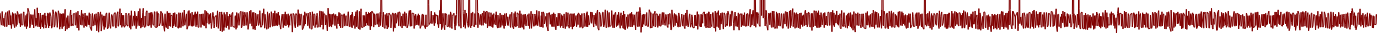

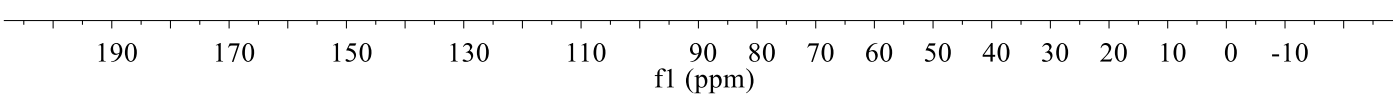



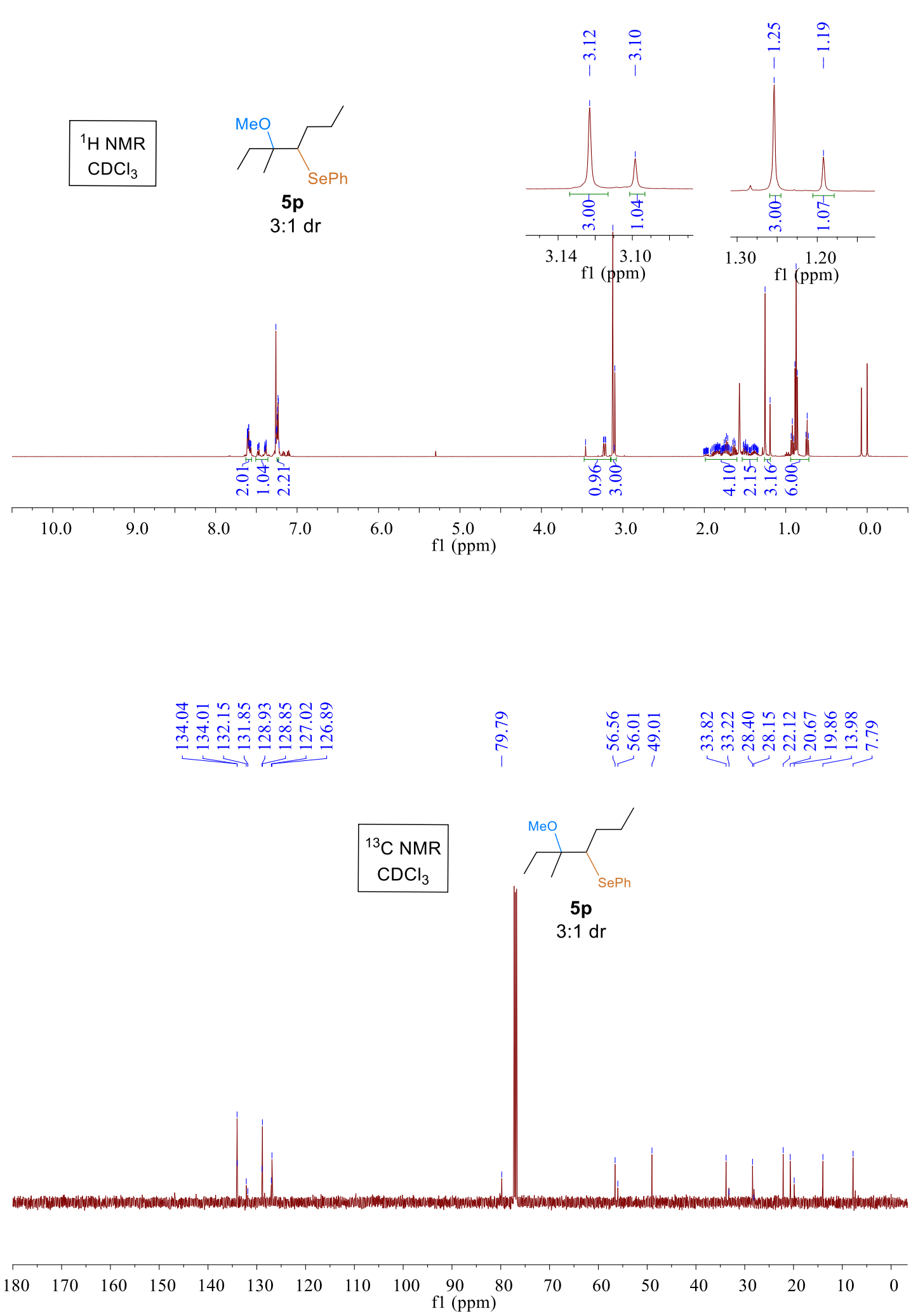

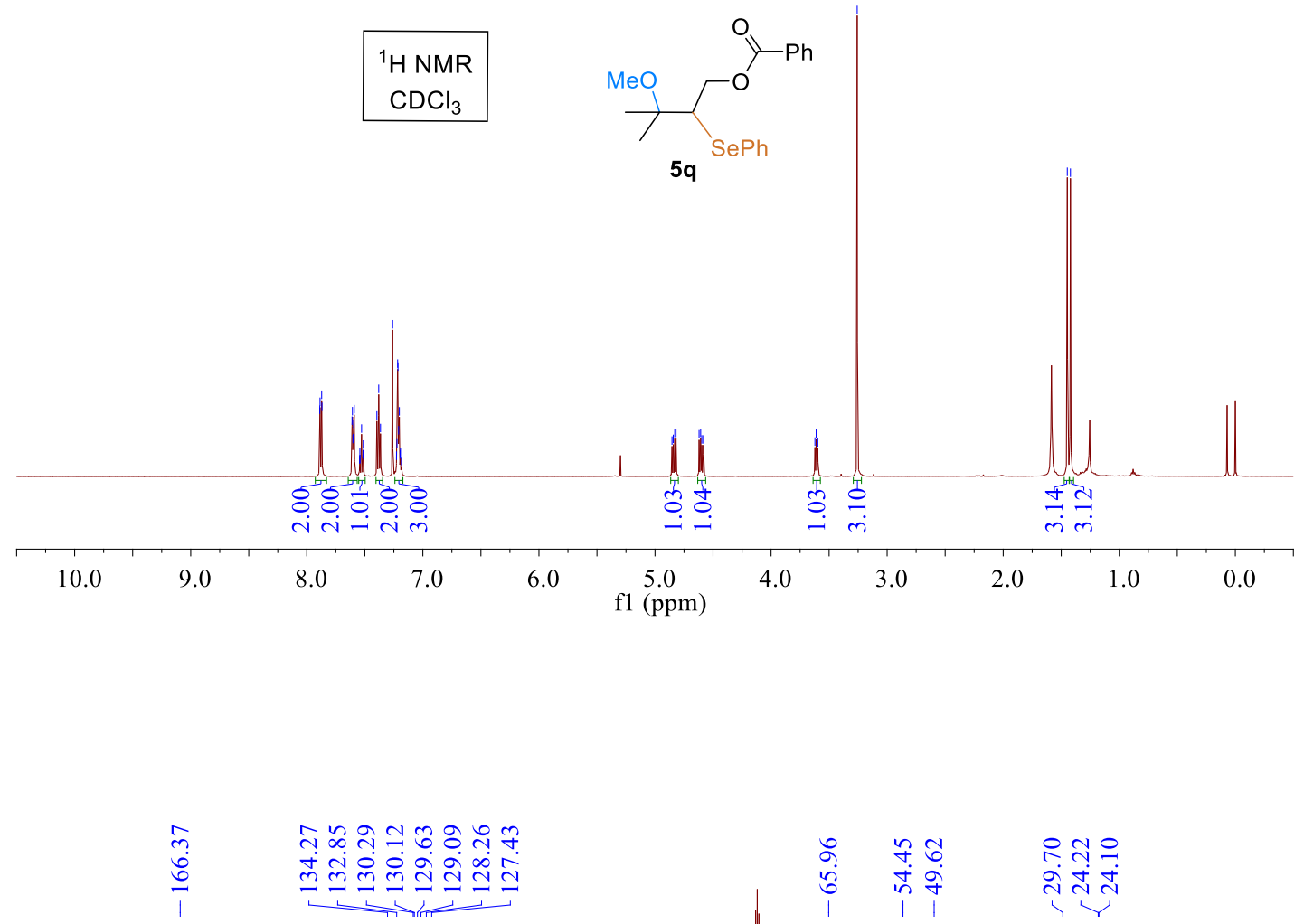

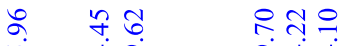
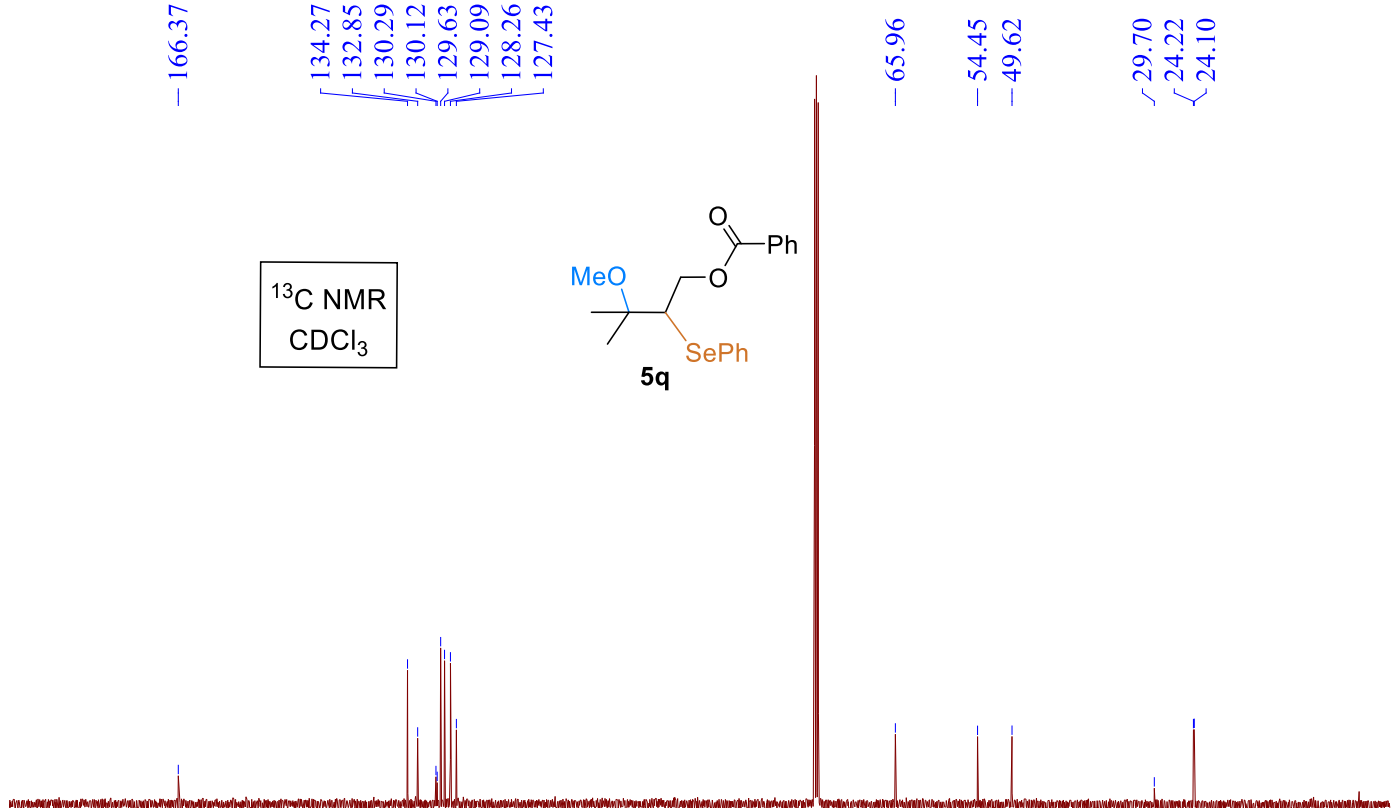

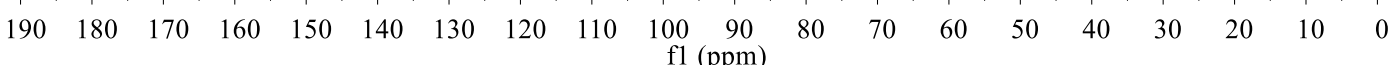




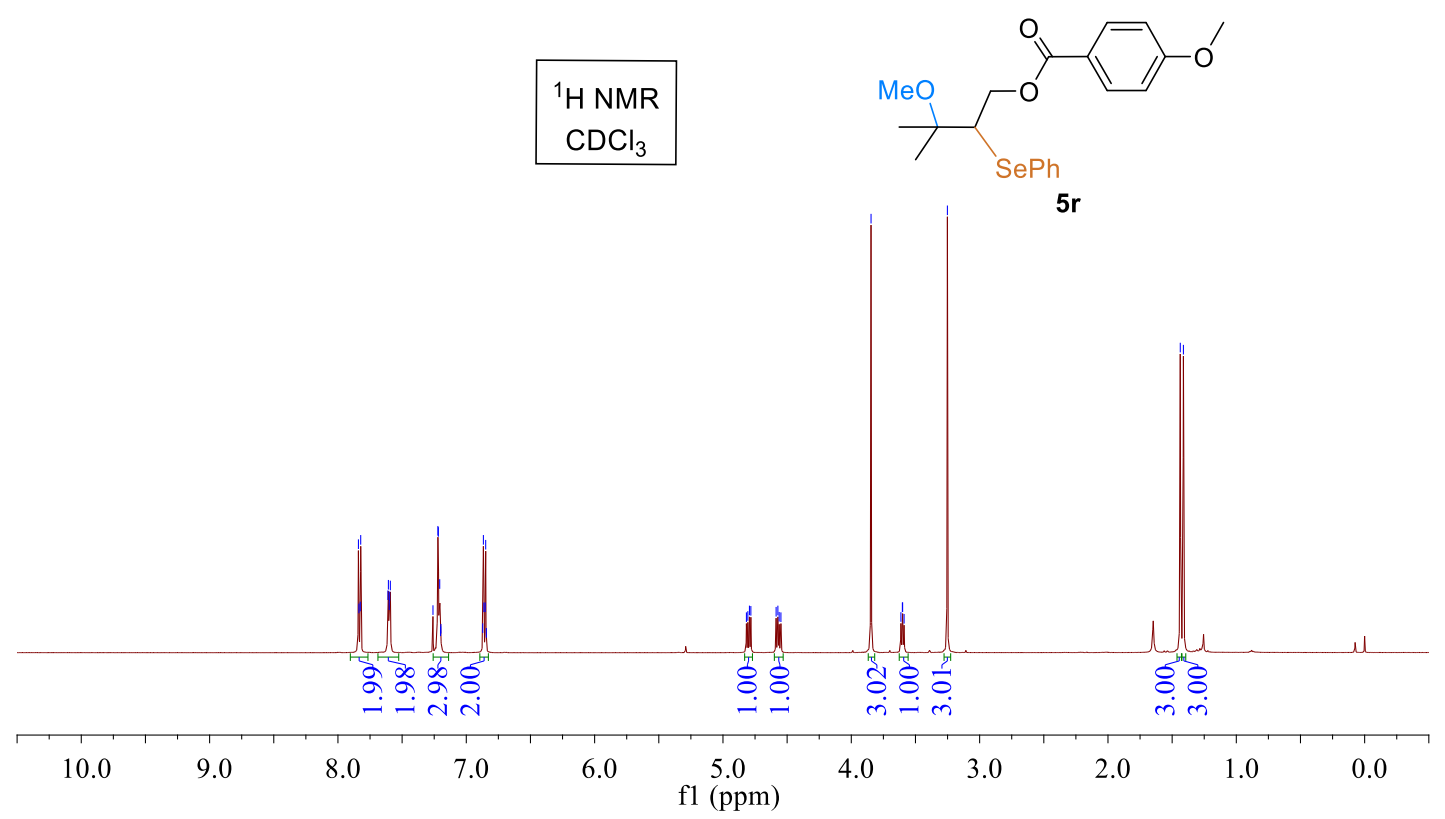

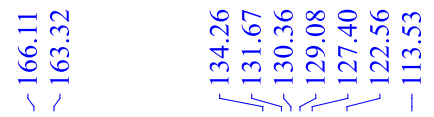
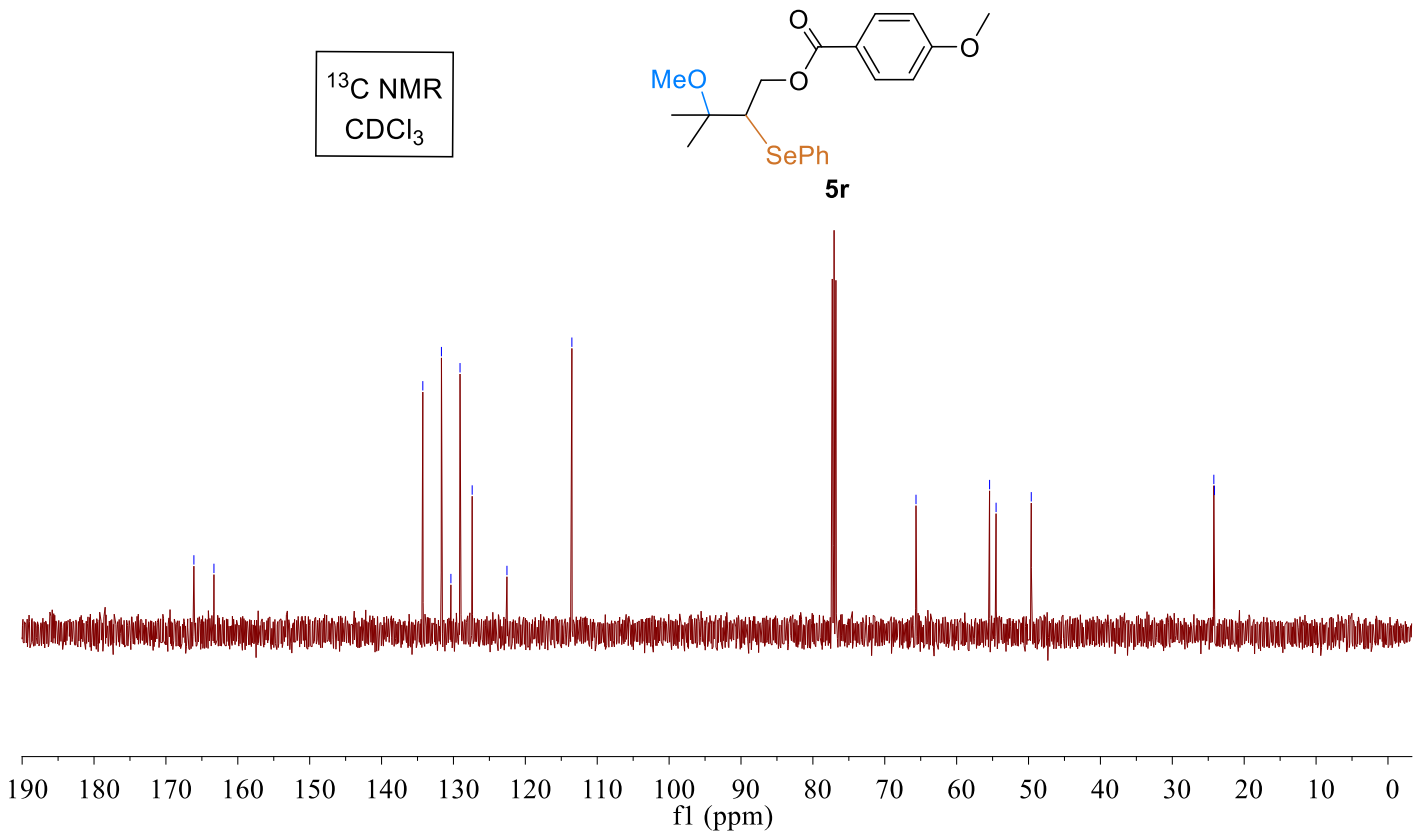

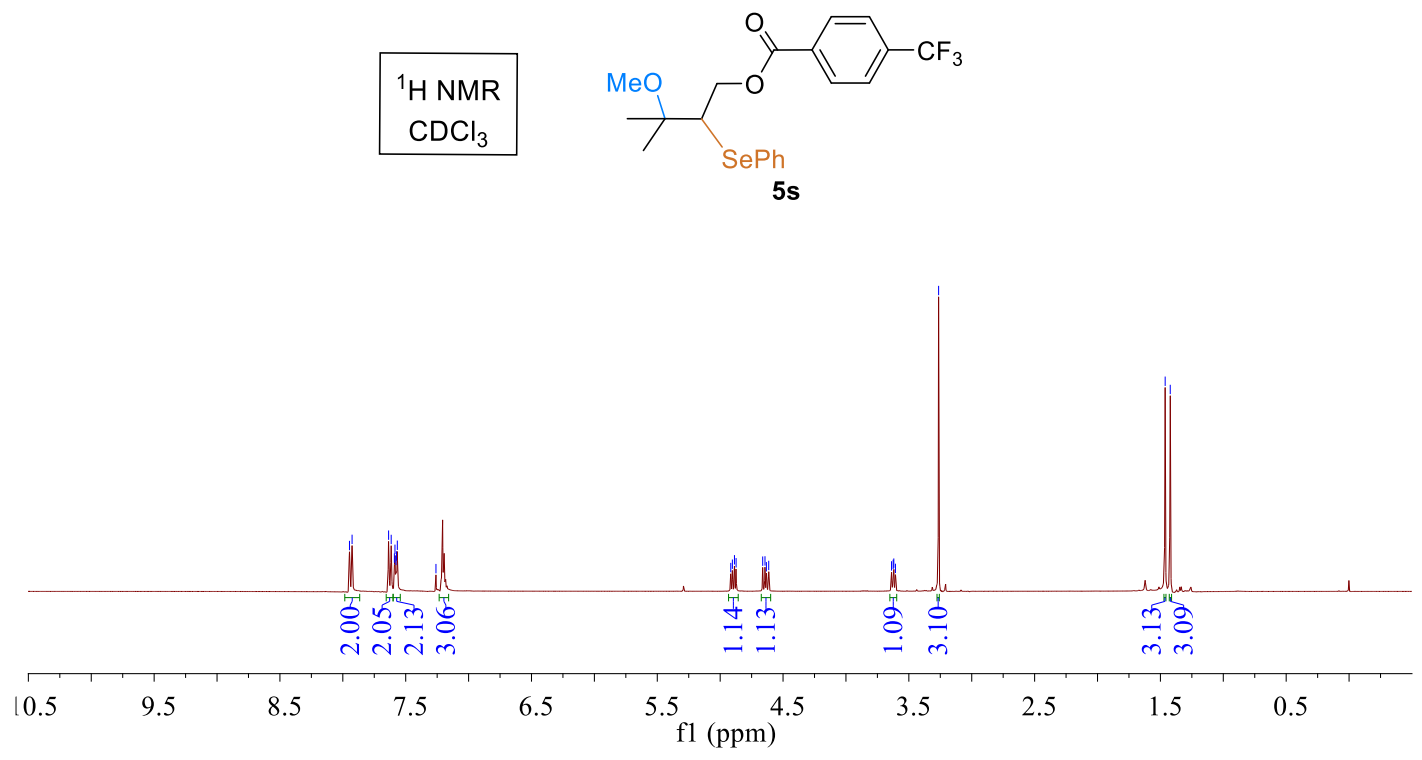

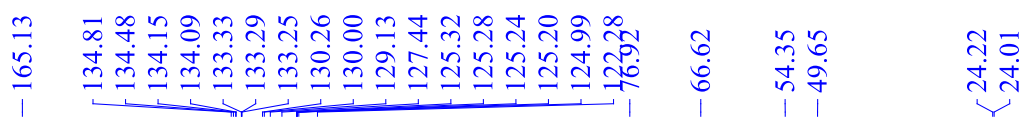
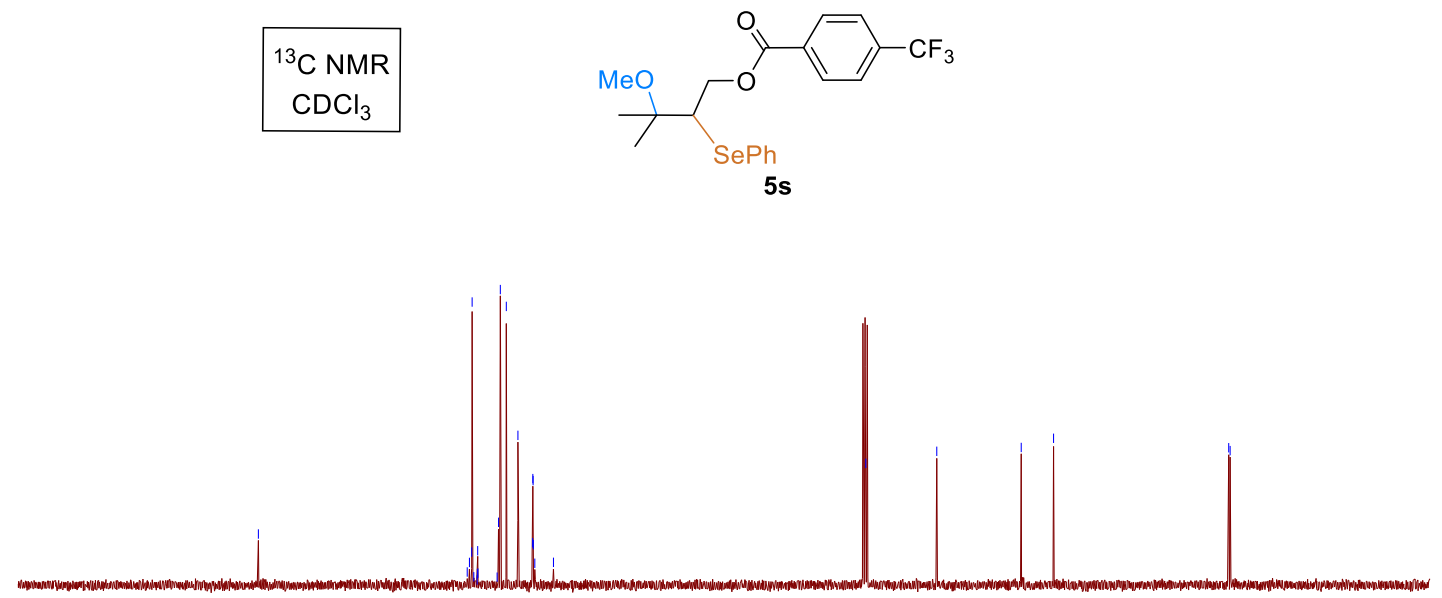

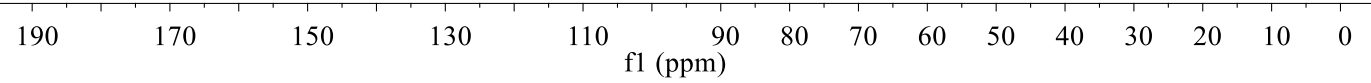



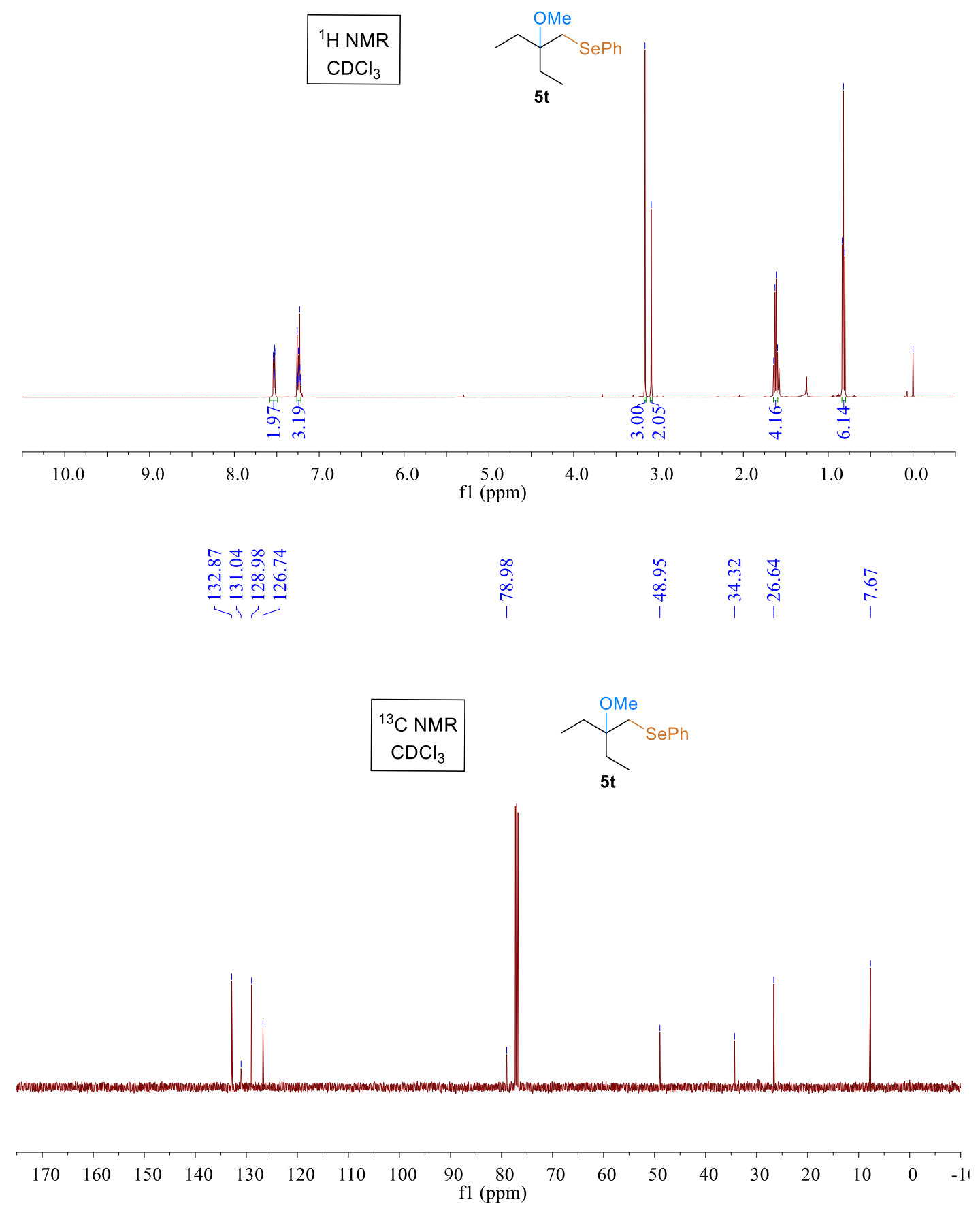


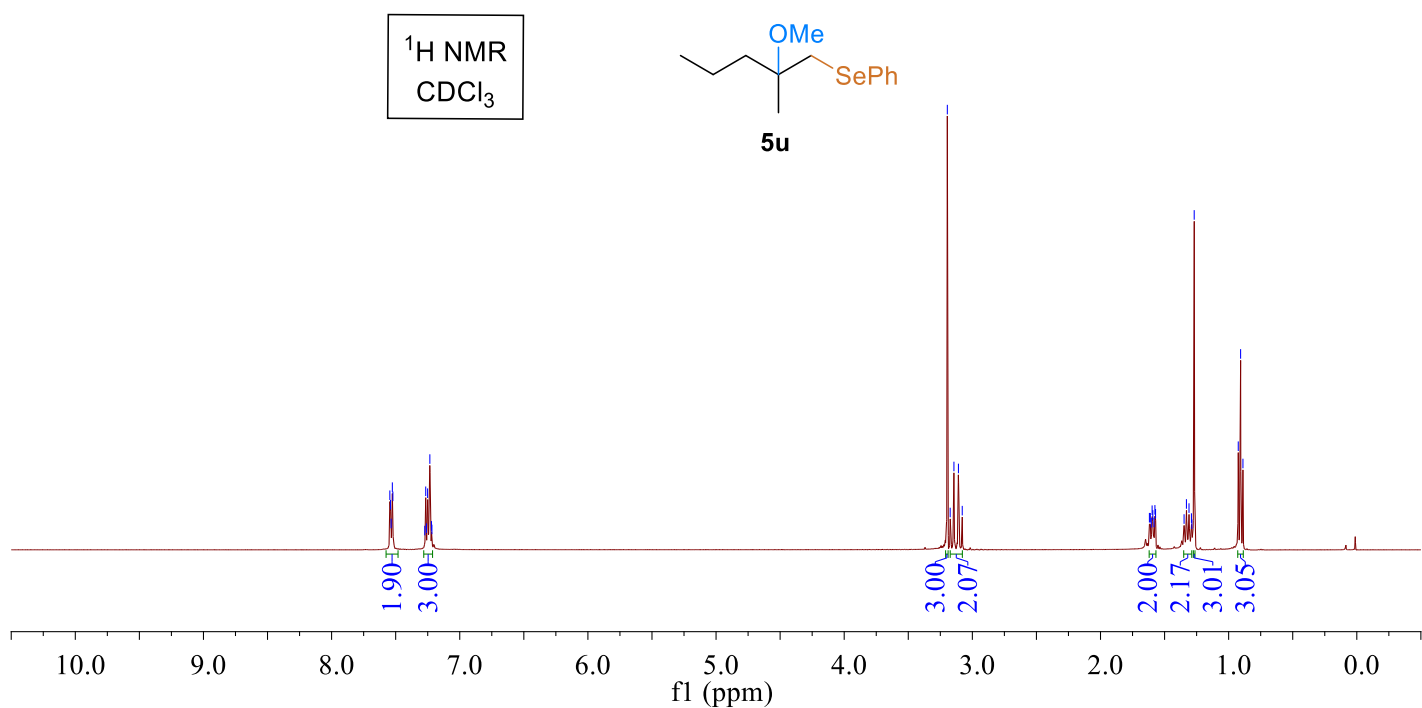

\begin{tabular}{|c|c|c|c|}
\hline 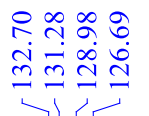 & $\stackrel{i}{i}$ & $\begin{array}{c}\infty \\
\stackrel{\infty}{a} \\
\stackrel{\sigma}{+}\end{array}$ & $\begin{array}{l}m \\
0 \\
0 \\
\dot{+} \\
\dot{9} \\
1\end{array}$ \\
\hline
\end{tabular}

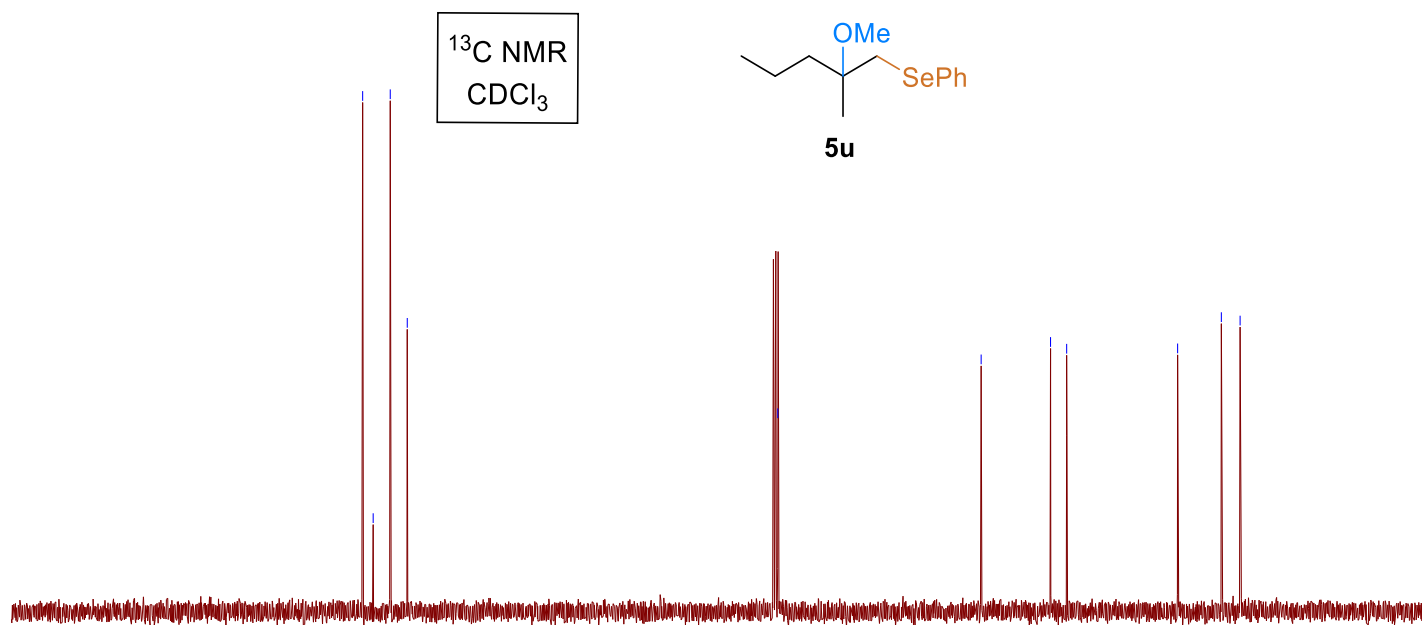

$\begin{array}{llllllllllr}180 & 170 & 160 & 150 & 140 & 130 & 120 & 110 & 100 & 90 & 80\end{array}$

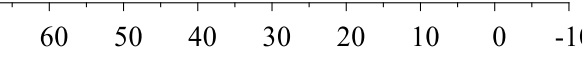




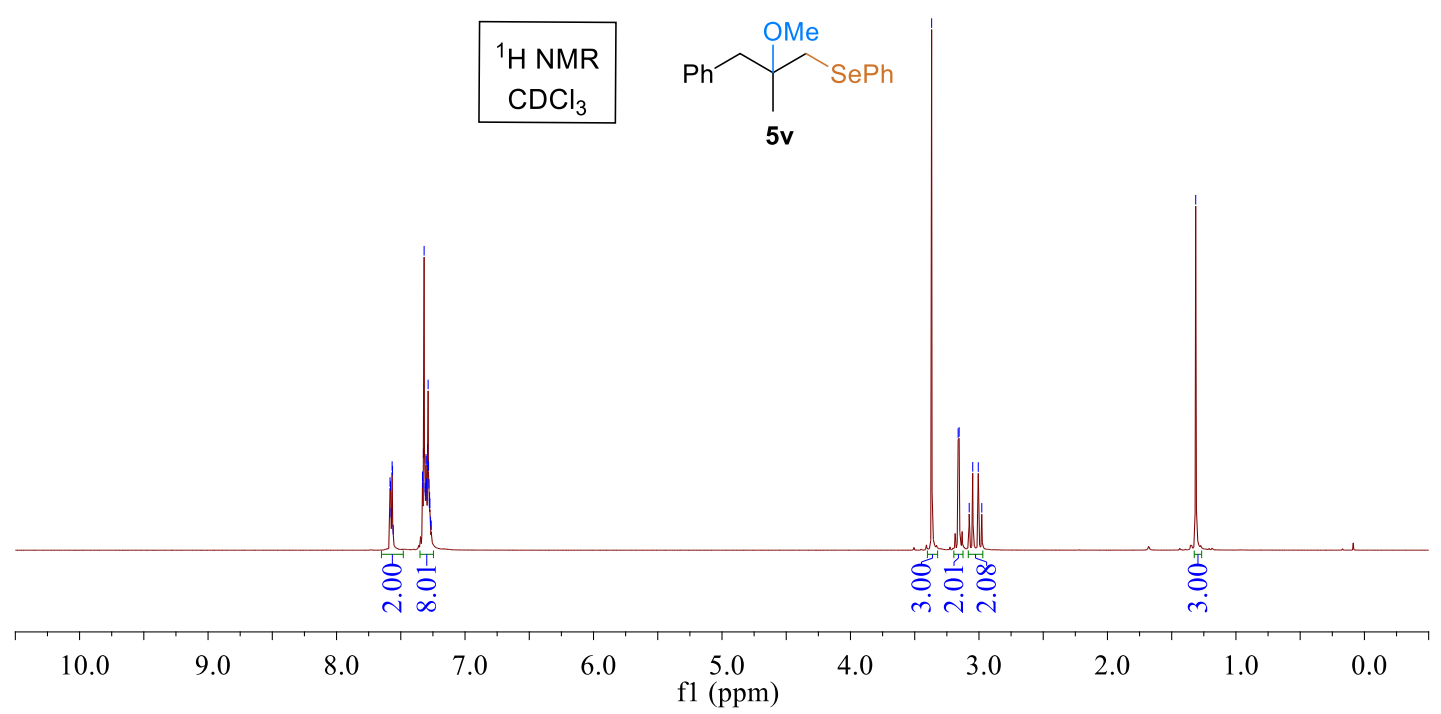

잉

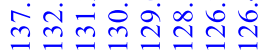

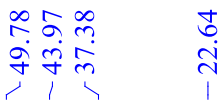
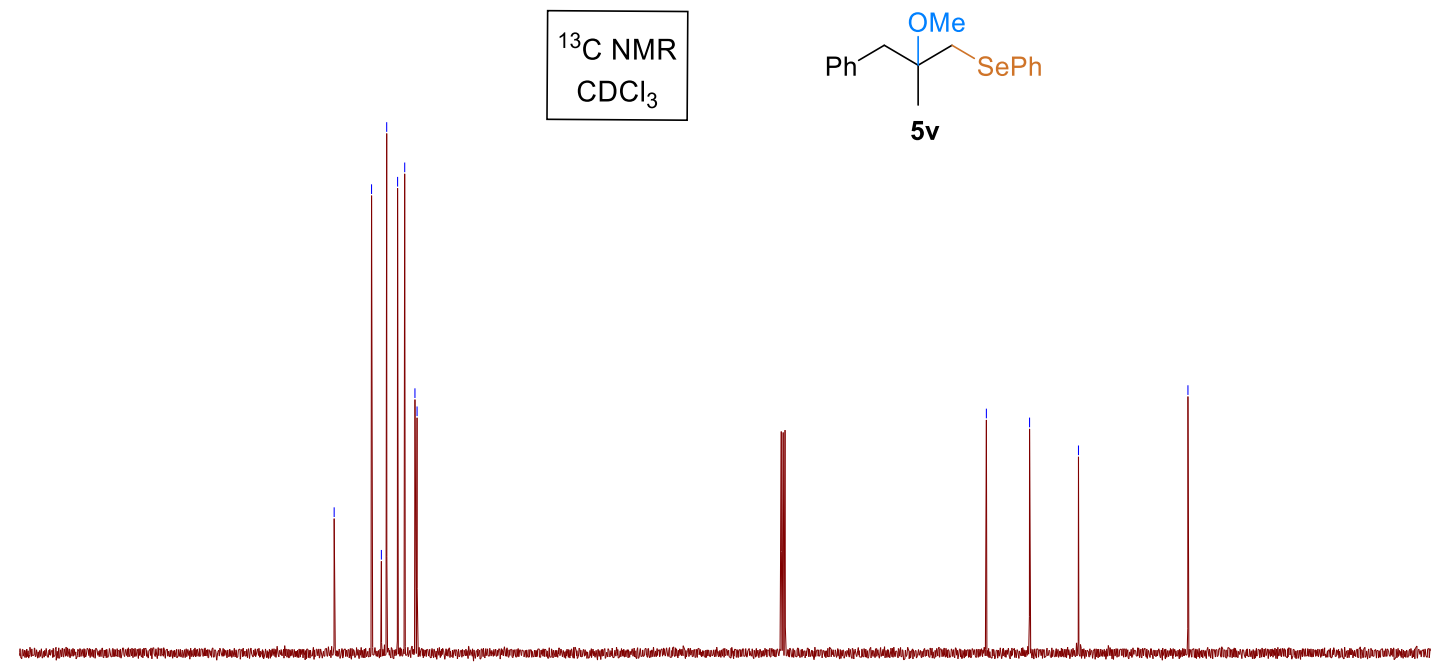

$\begin{array}{llllllllllllllllllll}180 & 170 & 160 & 150 & 140 & 130 & 120 & 110 & 100 & 90 & 80 & 70 & 60 & 50 & 40 & 30 & 20 & 10 & 0 & -1\end{array}$ f1 (ppm) 


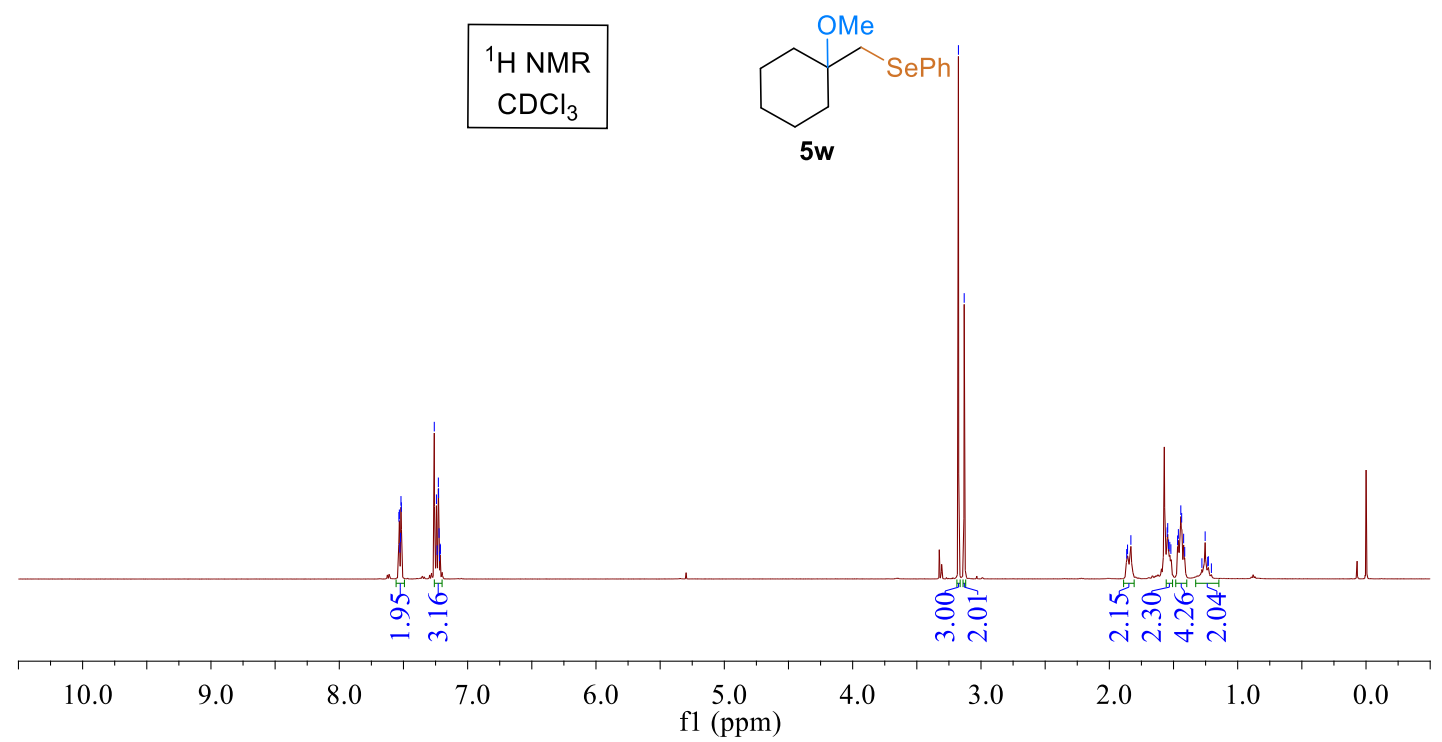

뭉ํㅇ

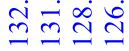

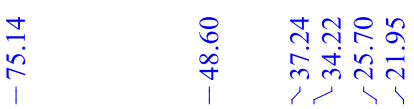

${ }^{13} \mathrm{C}$ NMR

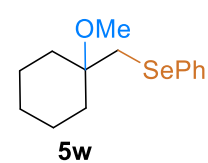

$\mathrm{CDCl}_{3}$

$5 w$

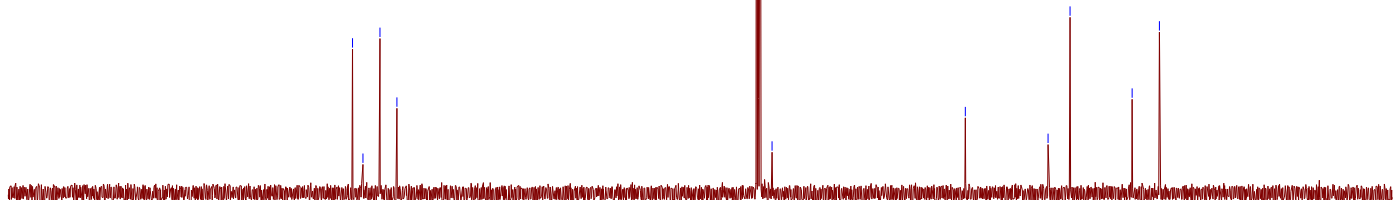

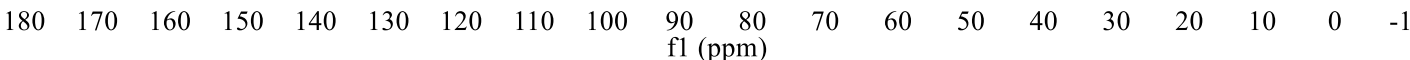




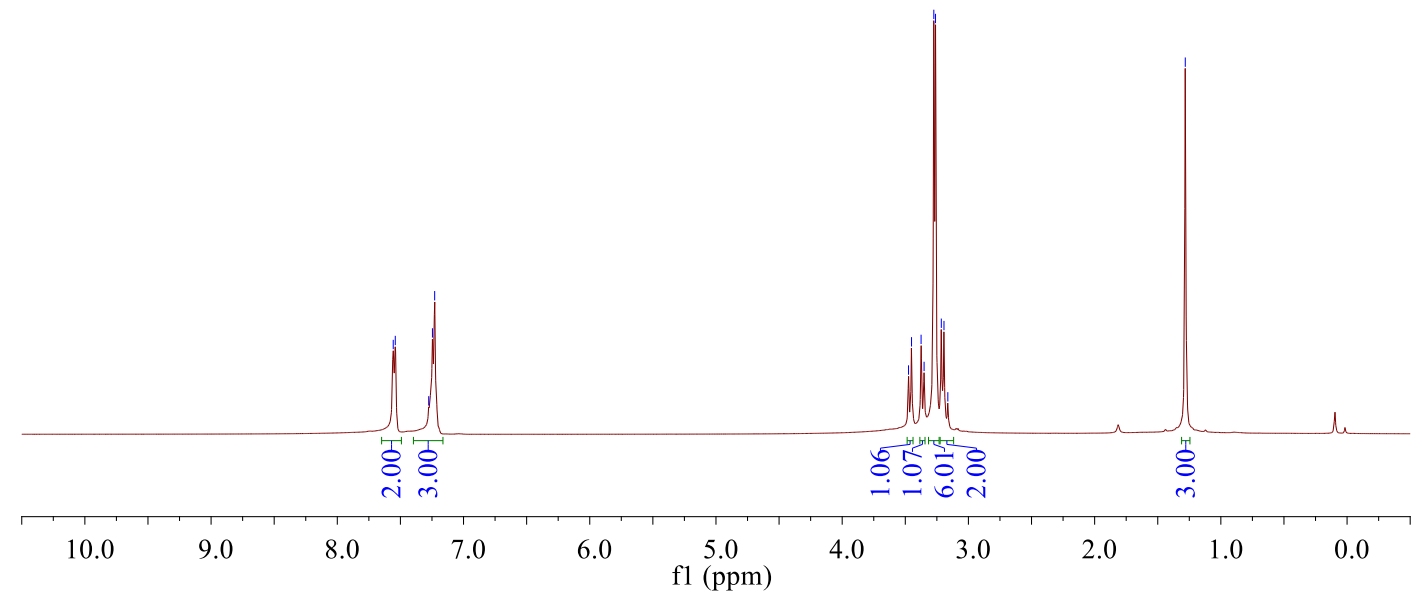

no nूo

르롱

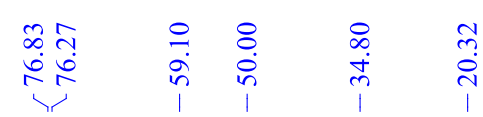

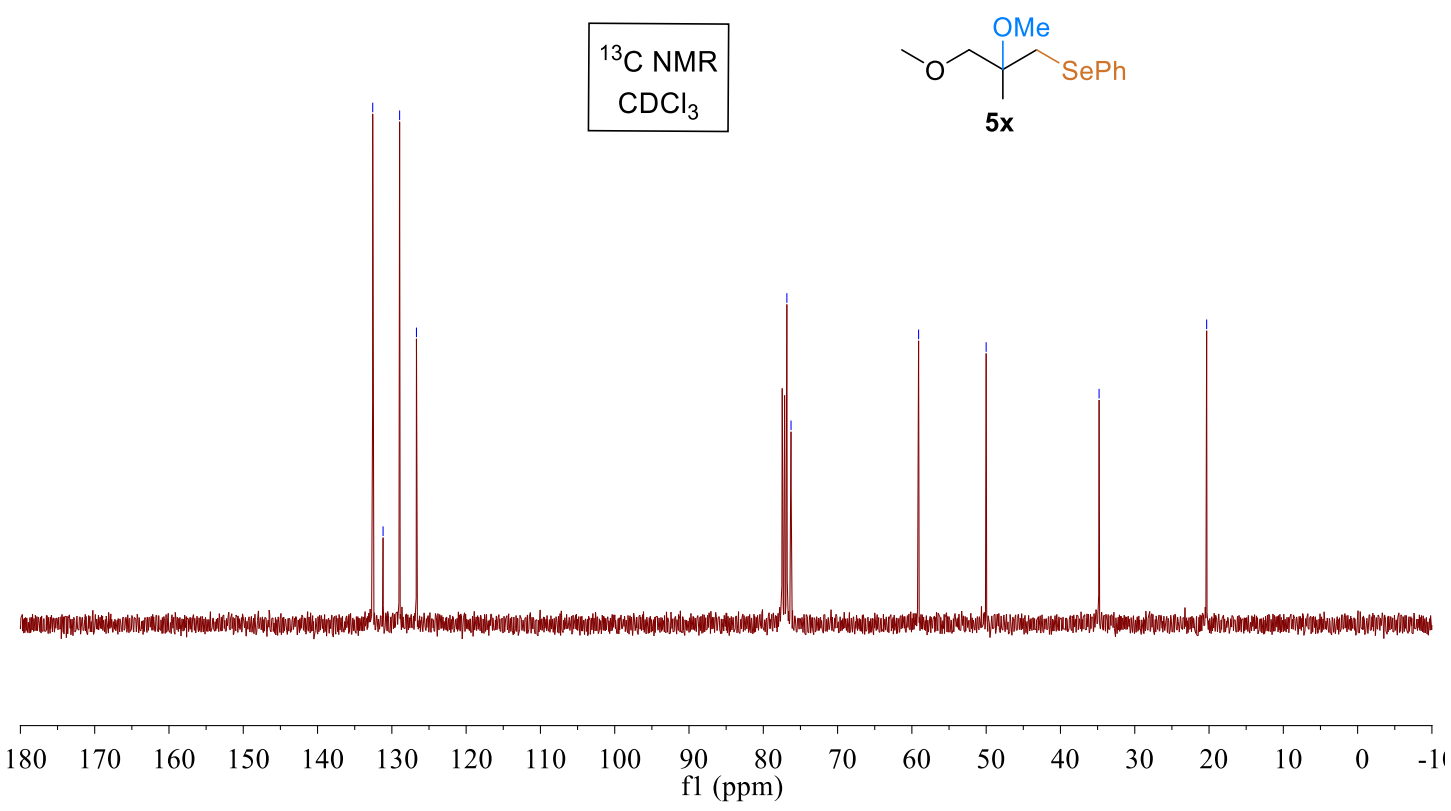



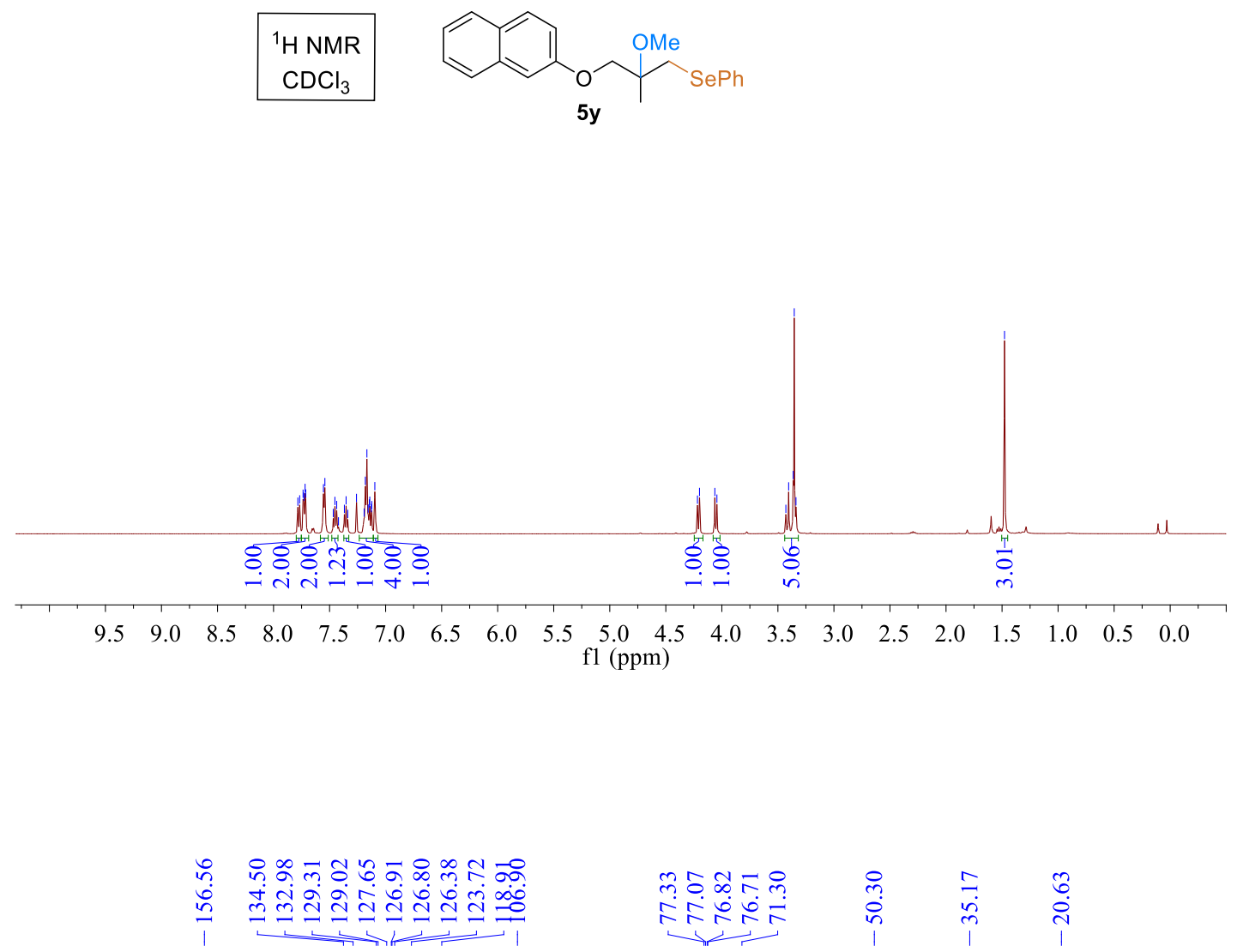

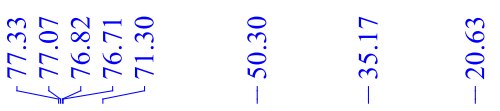
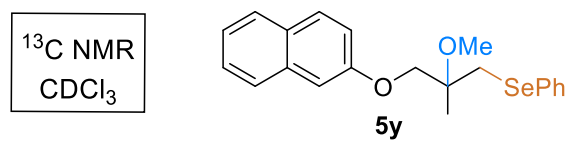

$5 y$

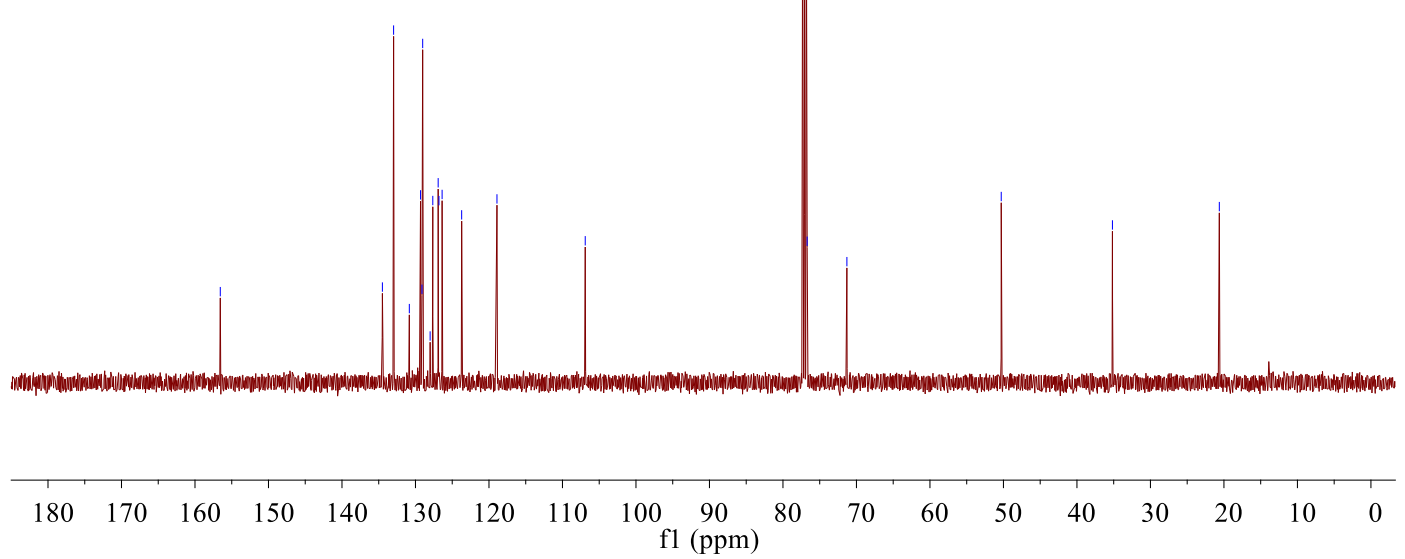



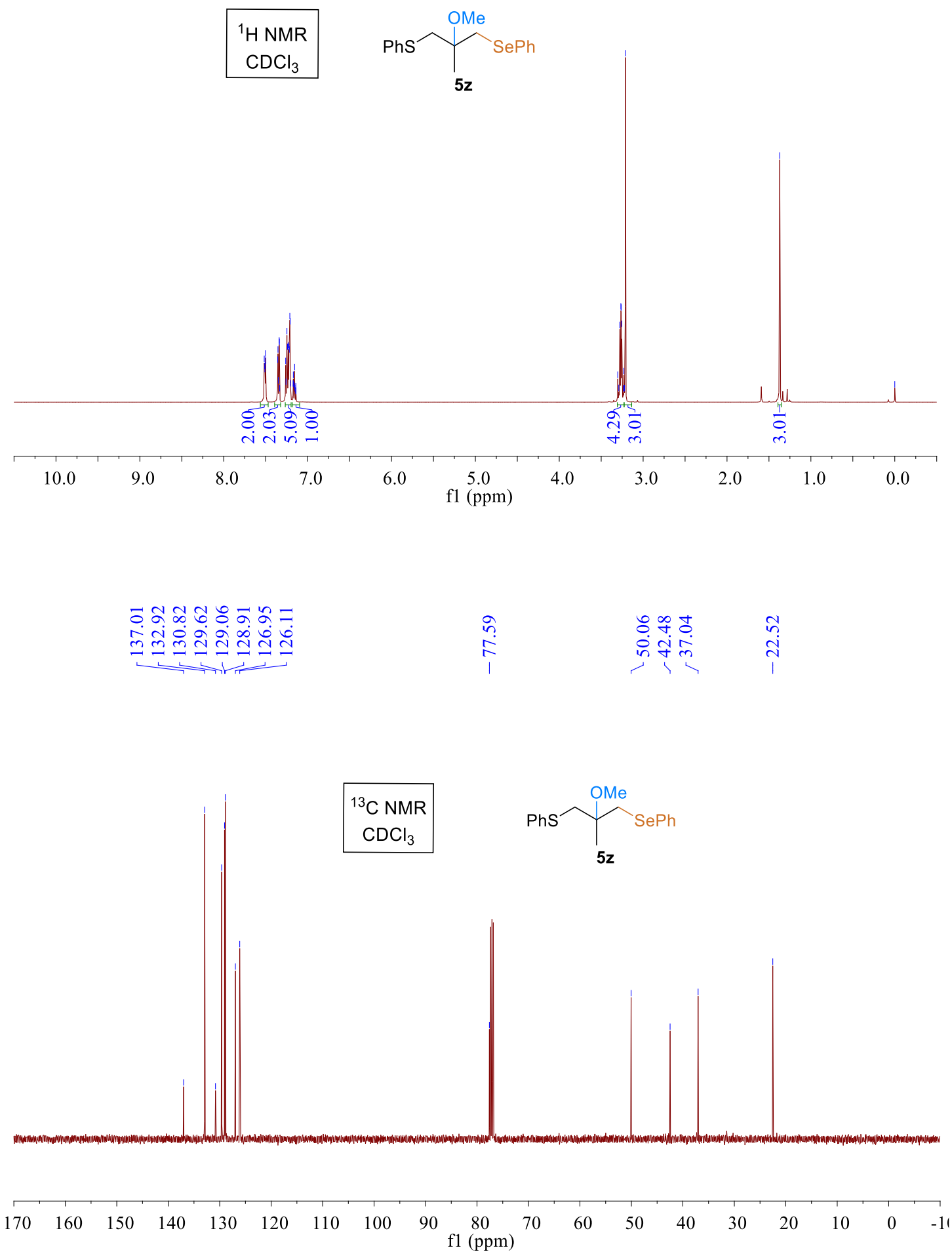

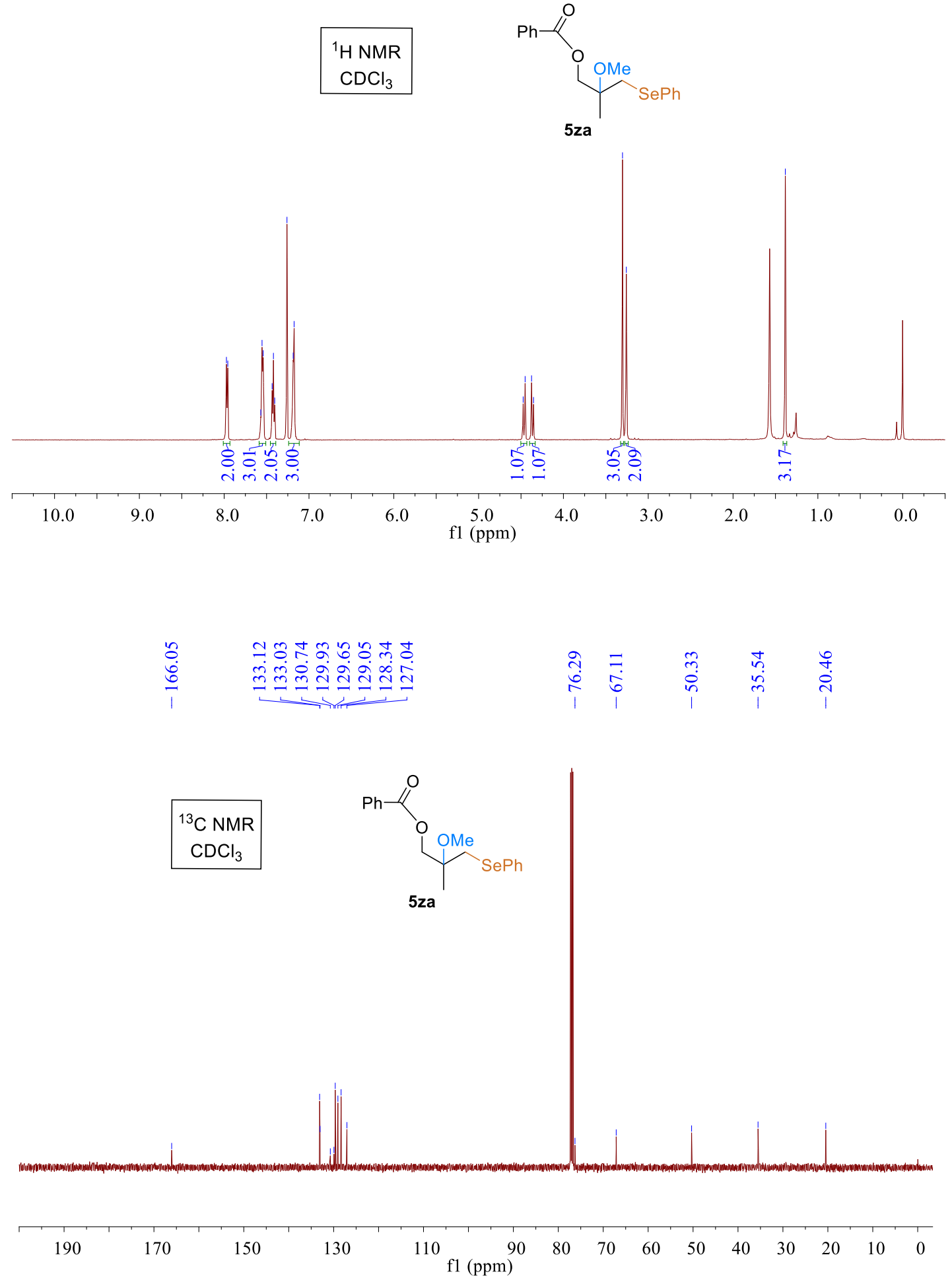

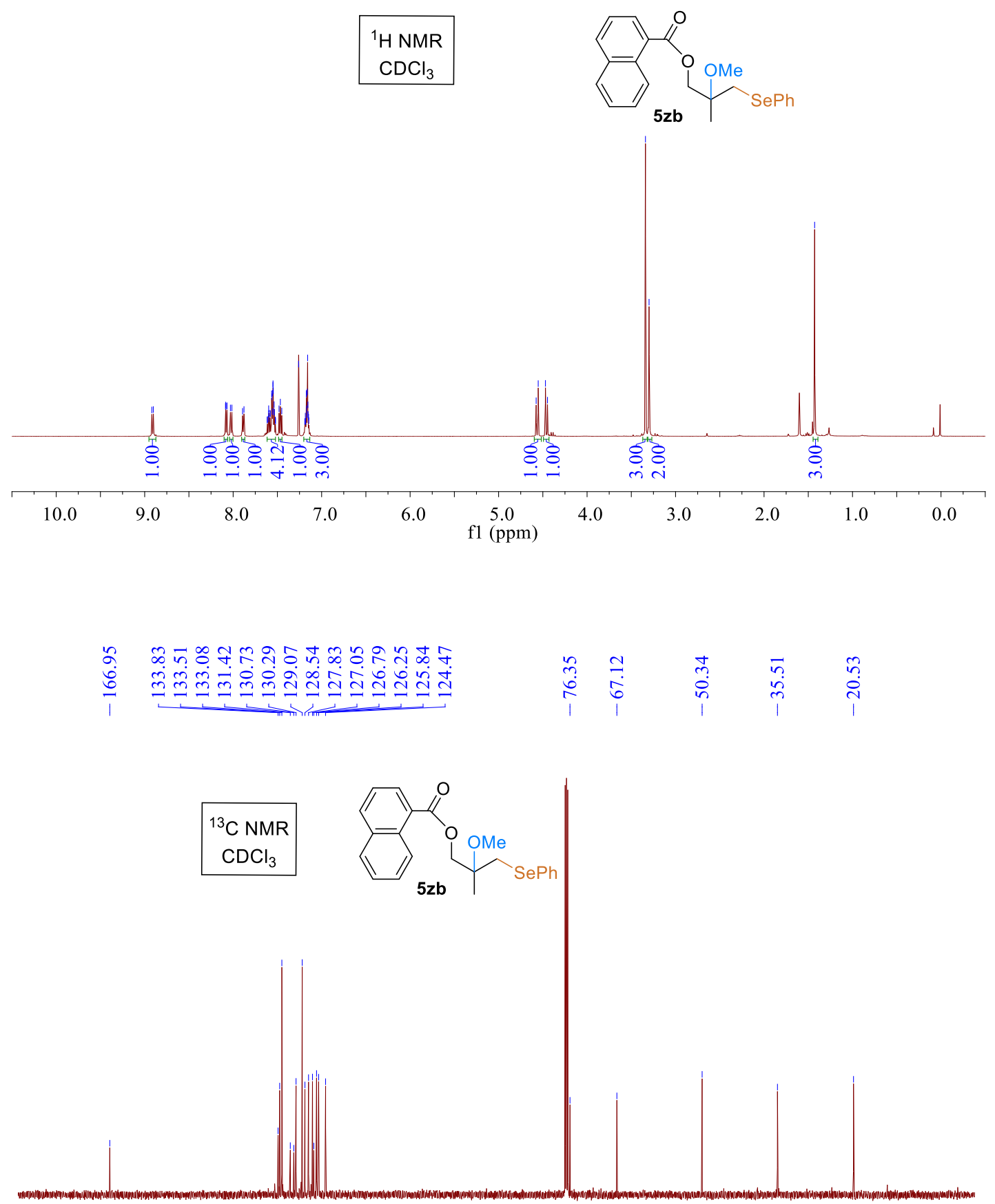

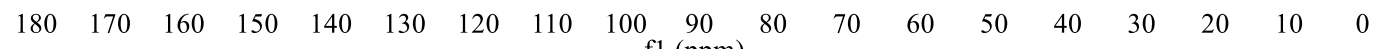




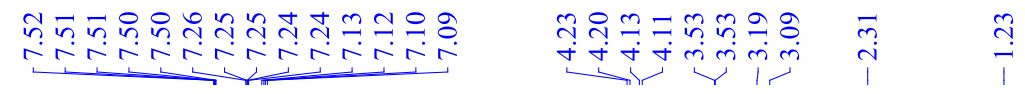

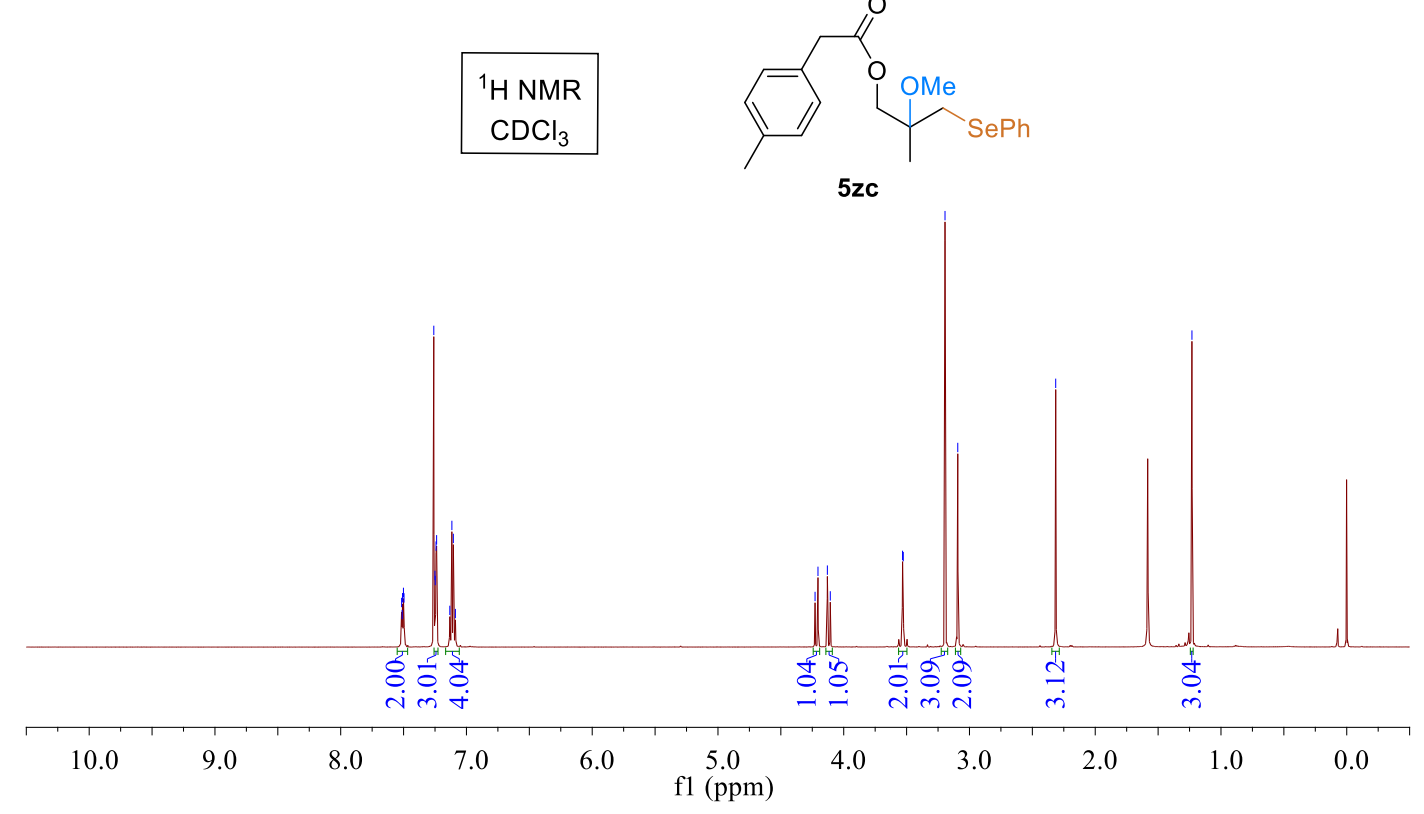

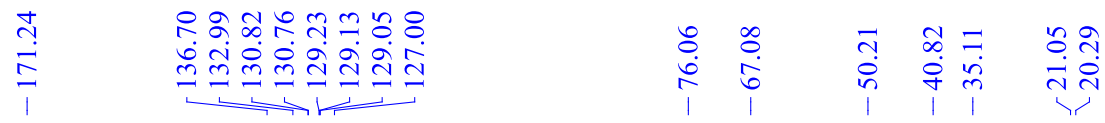

${ }^{13} \mathrm{C}$ NMR

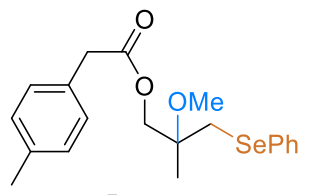

$5 z c$

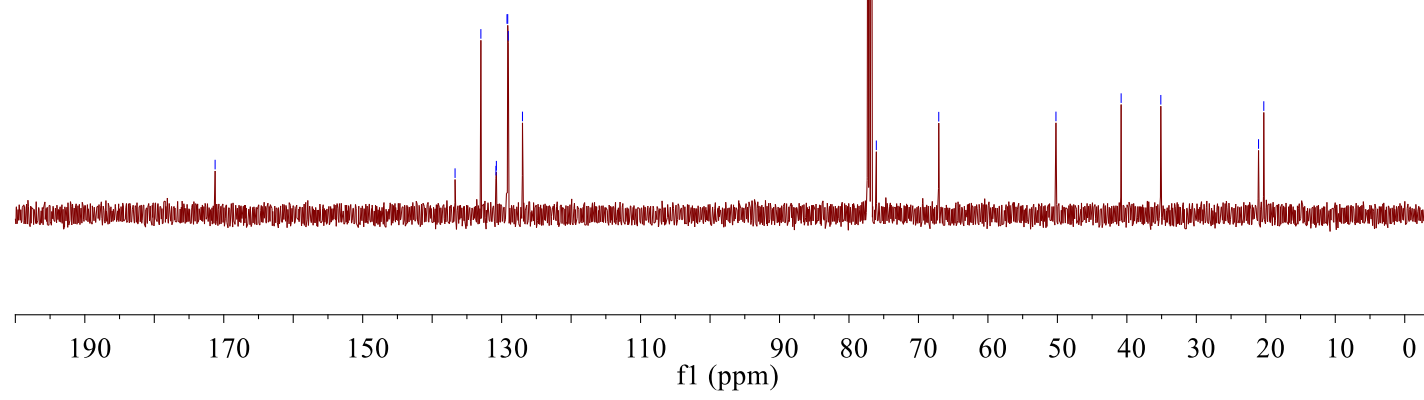




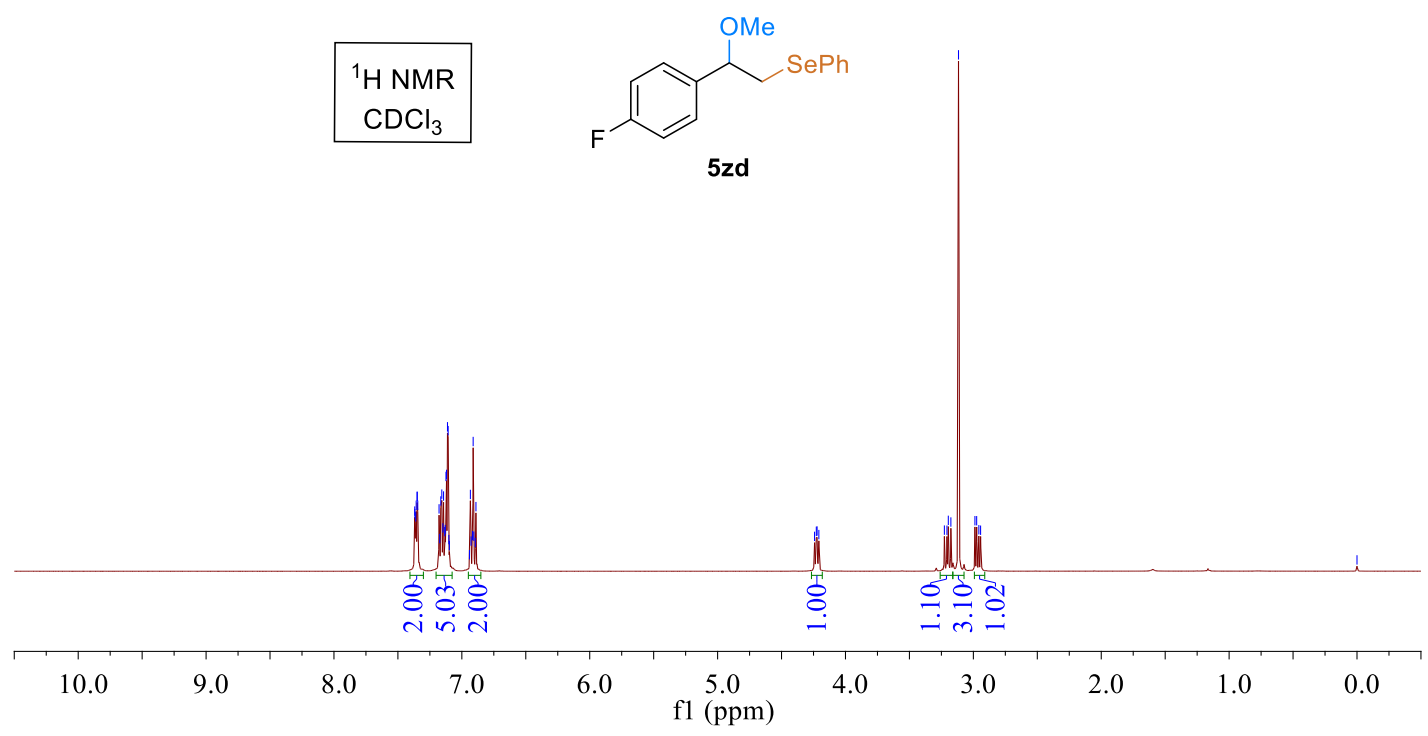

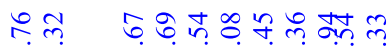

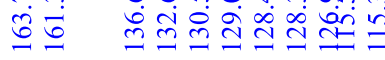
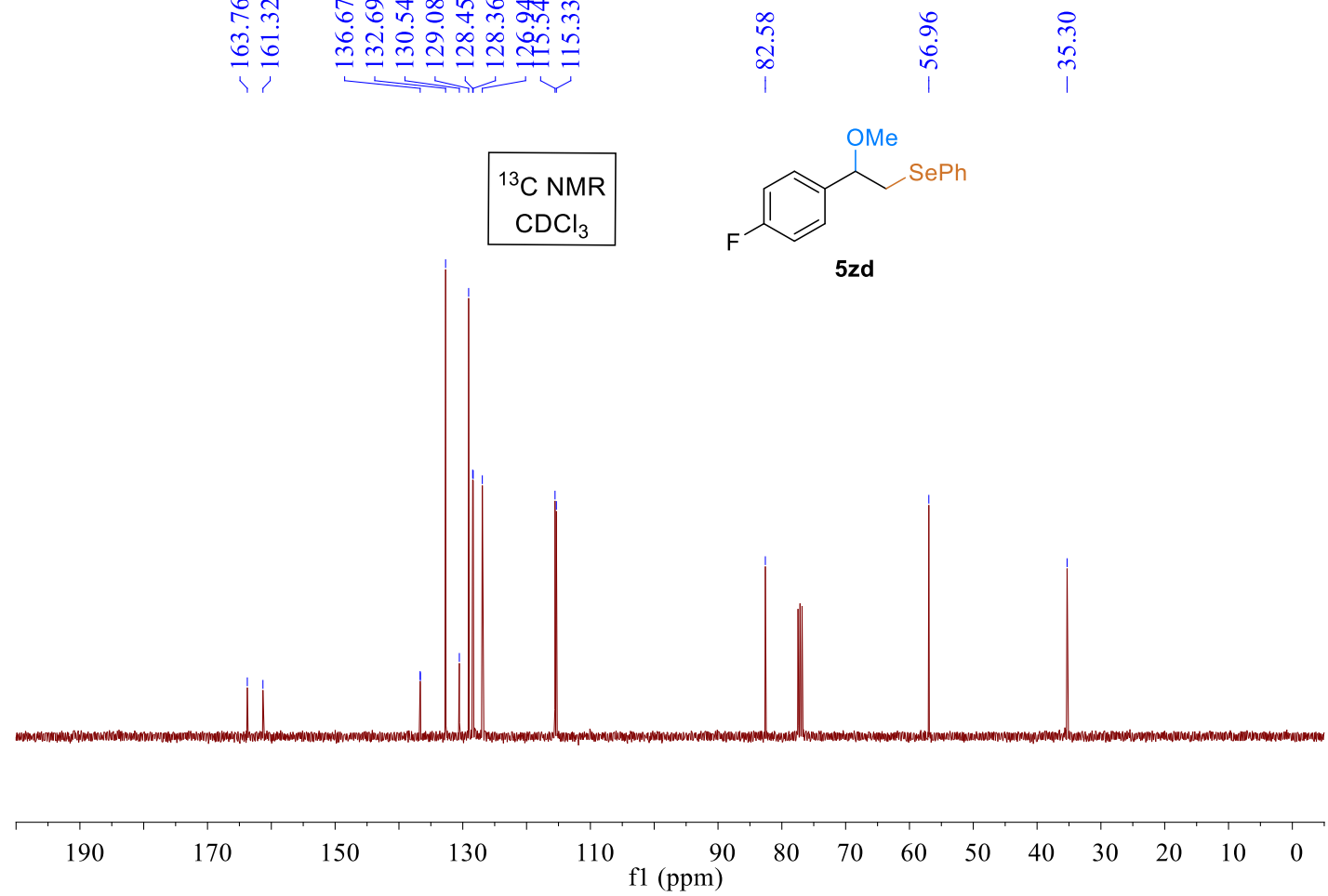


\begin{tabular}{|l|}
${ }^{1} \mathrm{H} \mathrm{NMR}$ \\
$\mathrm{CDCl}_{3}$
\end{tabular}
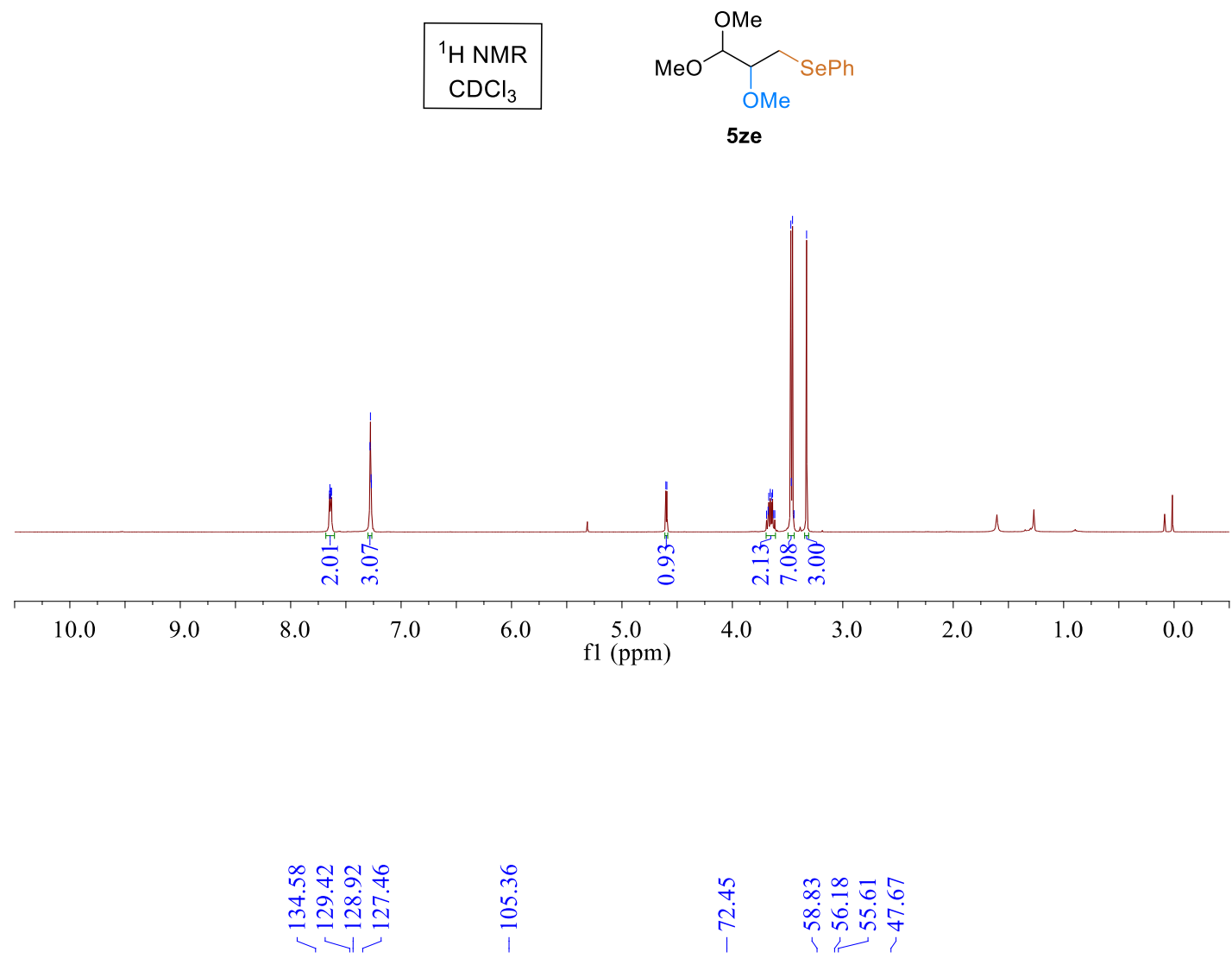

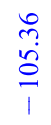

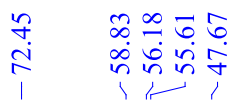

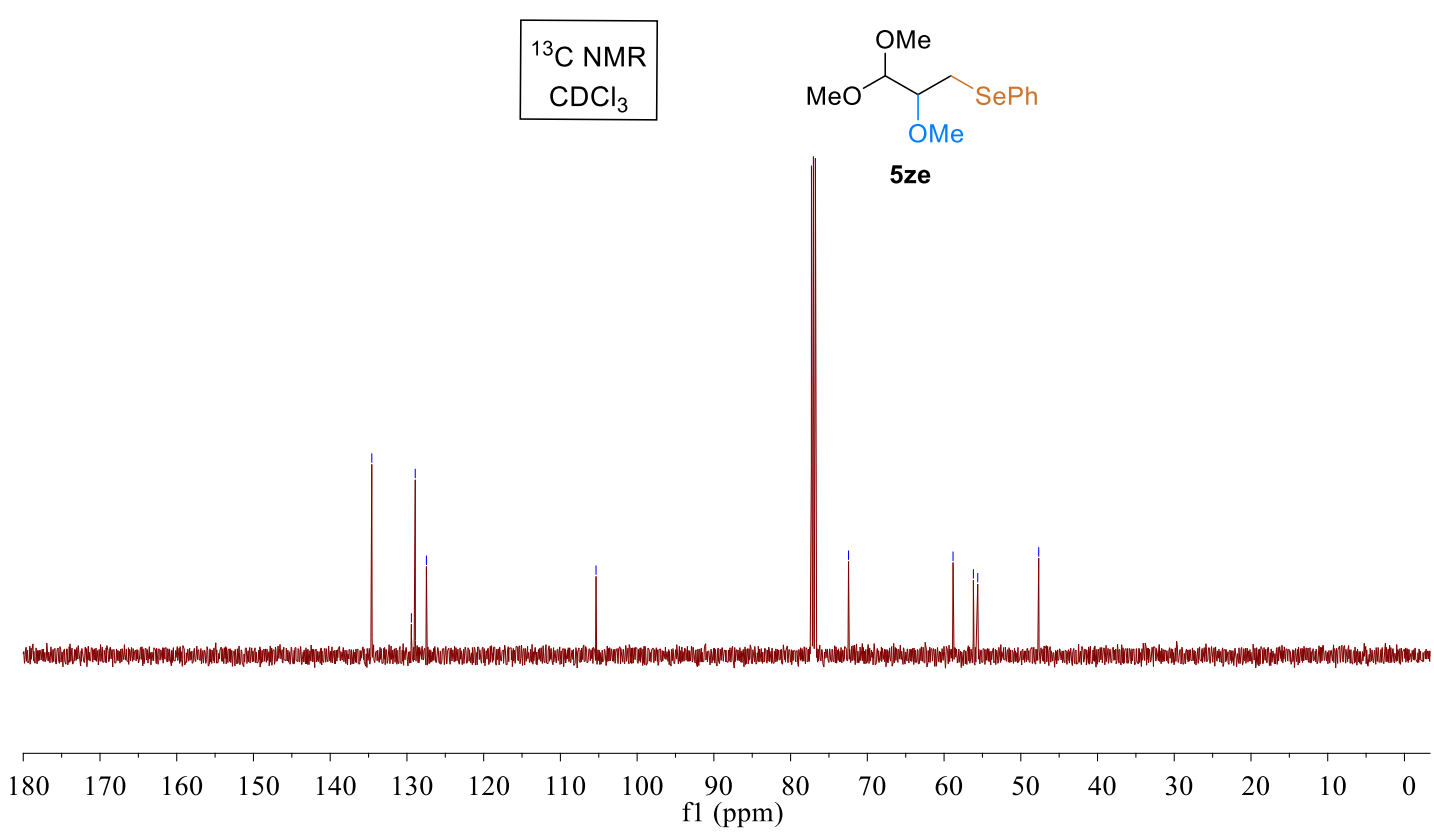



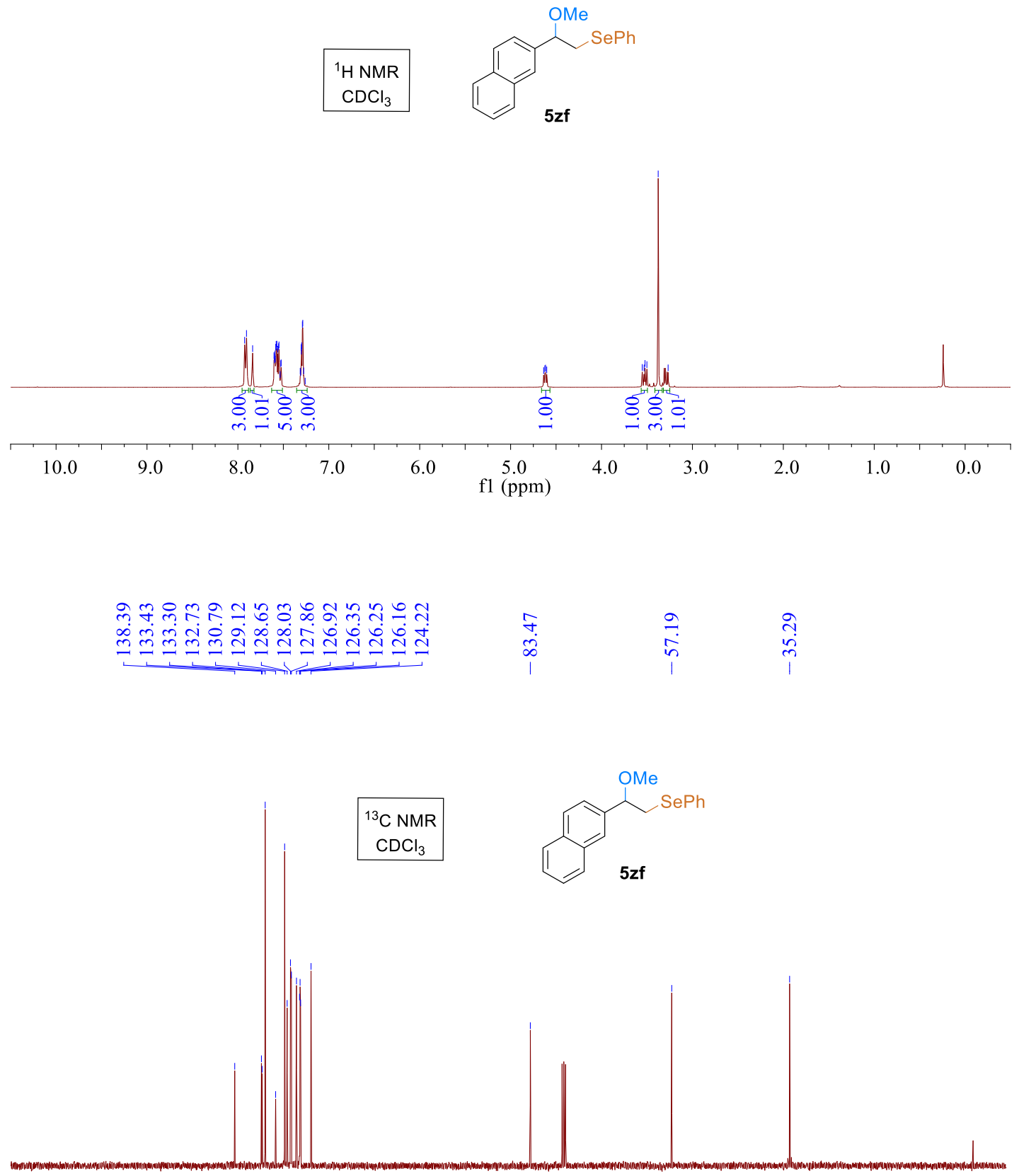


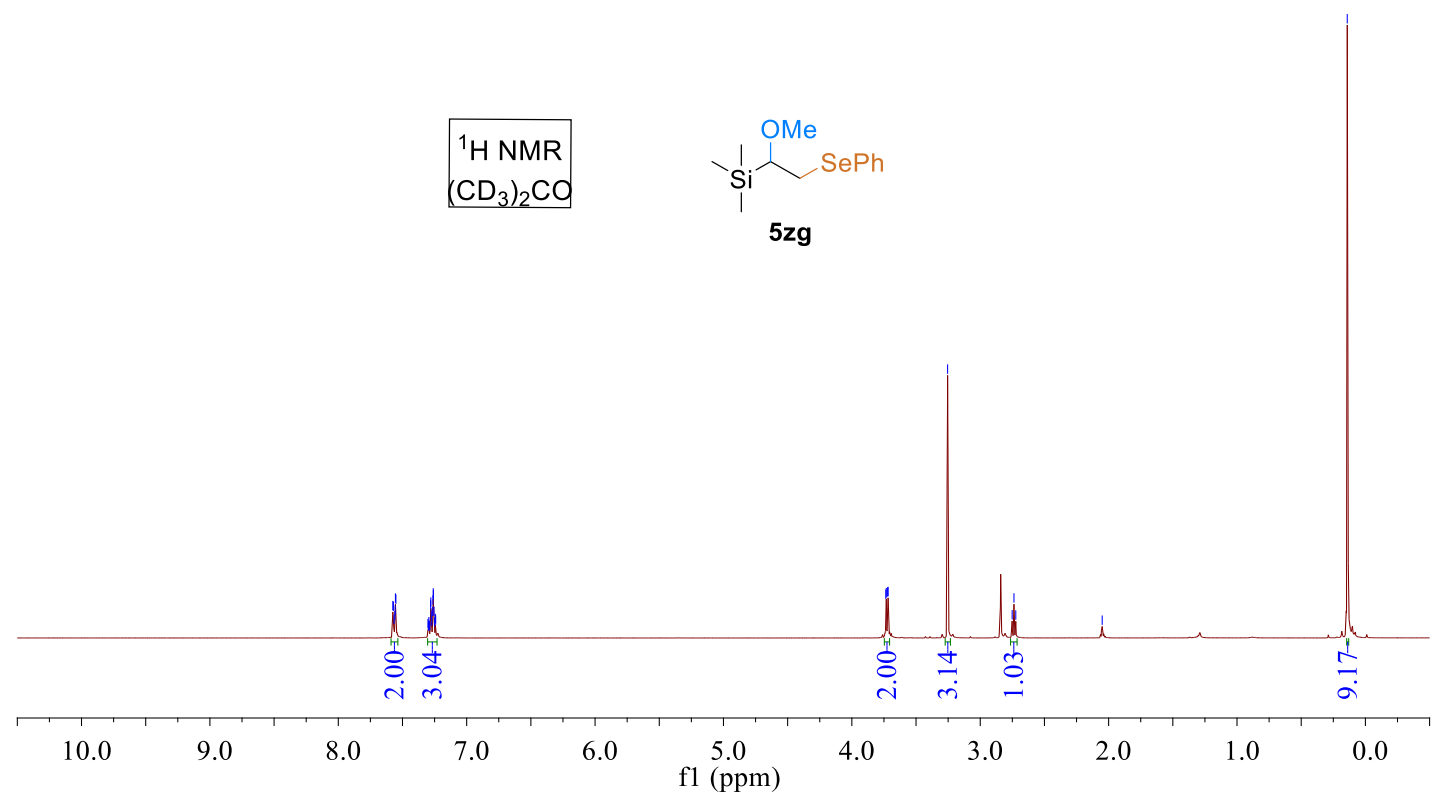

유유

मलㅛㅇㅝ

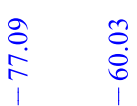

in

8
0
0

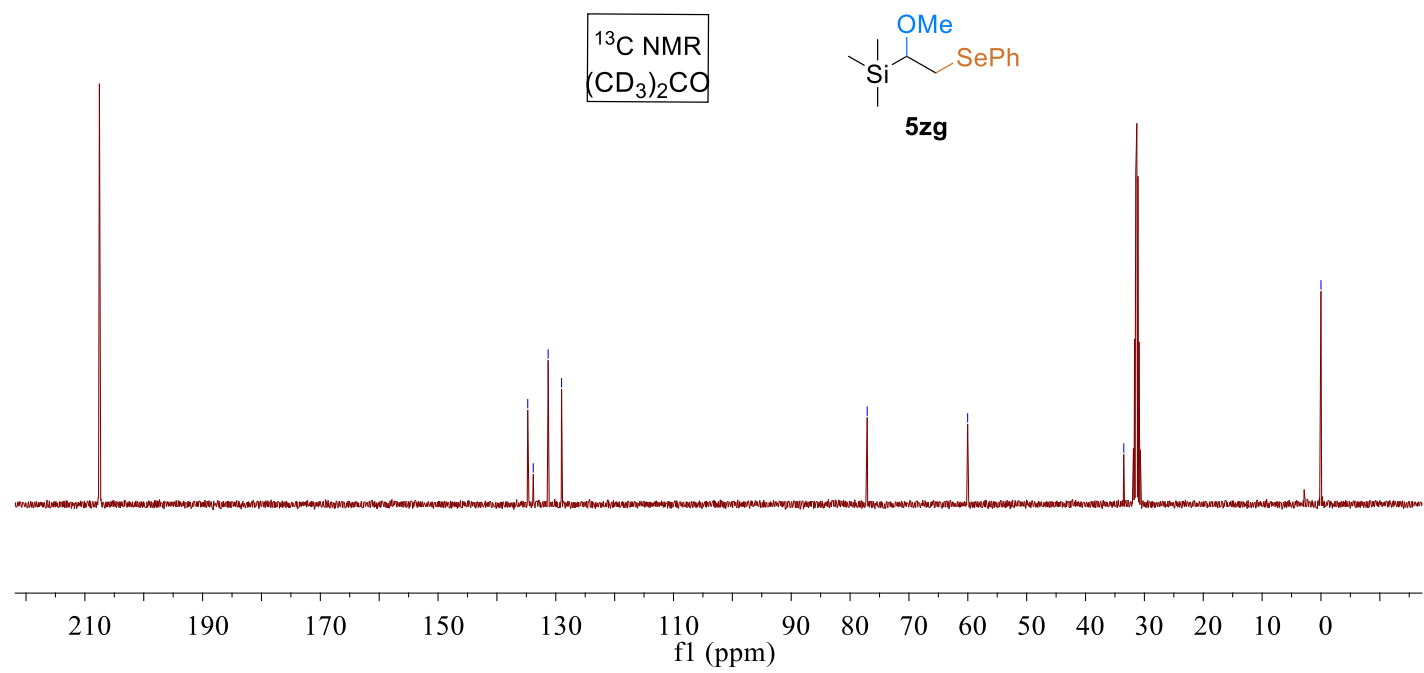




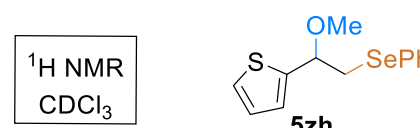

$5 z h$

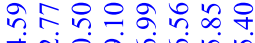

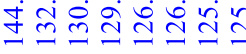

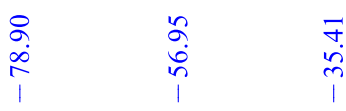

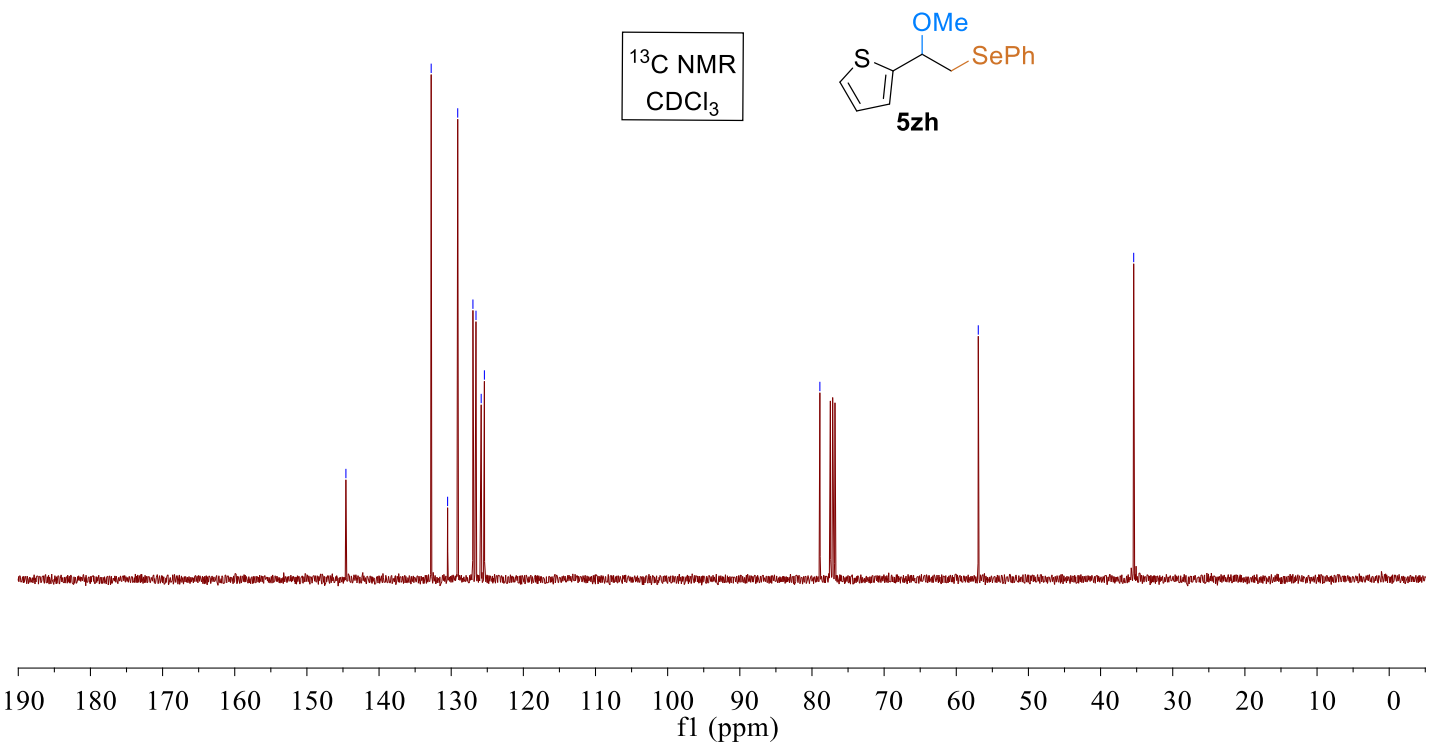




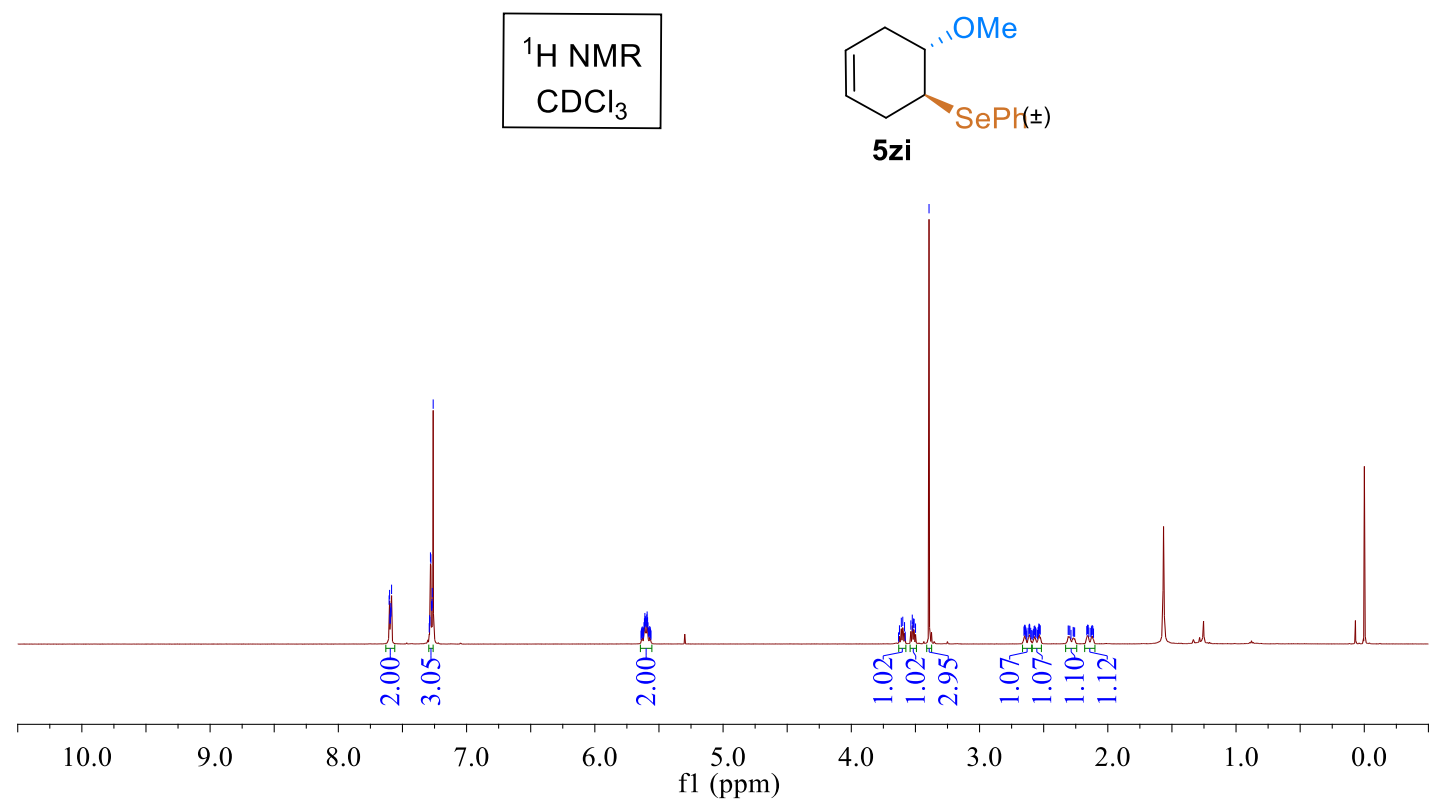

${ }^{13} \mathrm{C} \mathrm{NMR}$
$\mathrm{CDCl}_{3}$<smiles>CO[C@H]1CC=CCC1[SeH]c1ccccc1</smiles>

5zi $\quad( \pm$ 

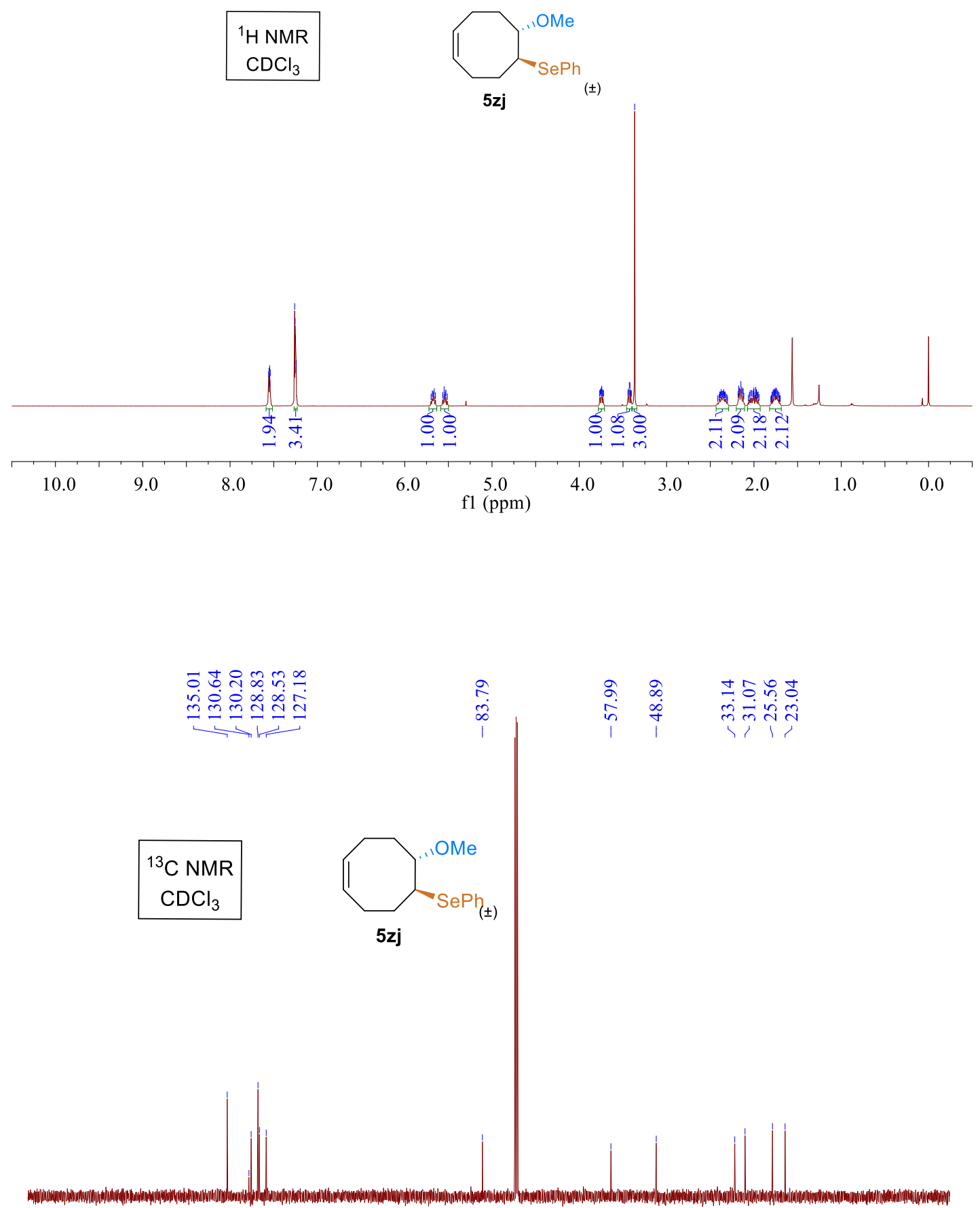

$\begin{array}{lllllllllllllllllll}170 & 160 & 150 & 140 & 130 & 120 & 110 & 100 & 90 & \underset{\mathrm{f} 1(\mathrm{ppm})}{80} & 70 & 60 & 50 & 40 & 30 & 20 & 10 & 0 & -11\end{array}$ 

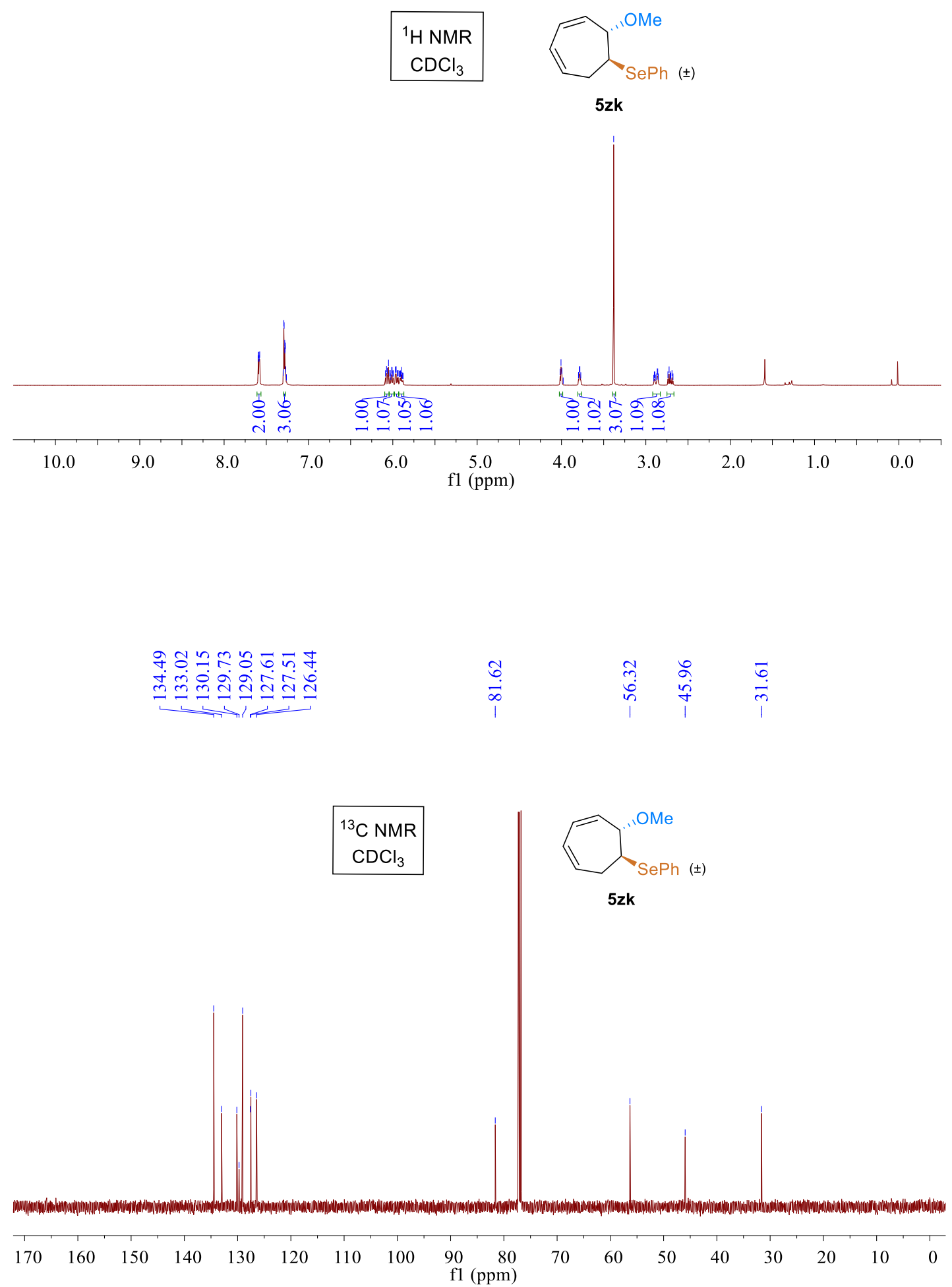


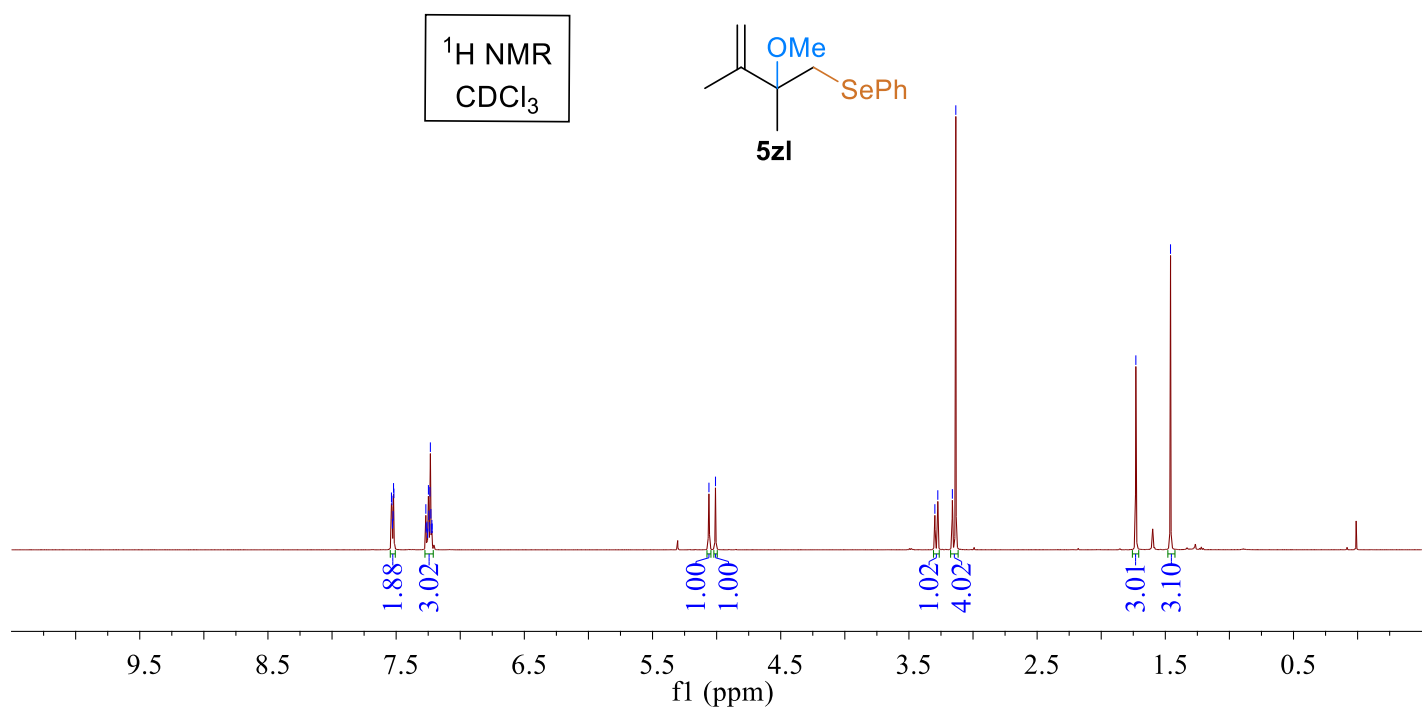

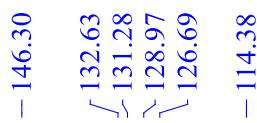

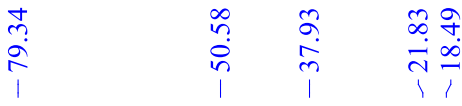

\begin{tabular}{c|}
${ }^{13} \mathrm{C} \mathrm{NMR}$ \\
$\mathrm{CDCl}_{3}$ \\
\hline
\end{tabular}<smiles>C=C(C)C([13CH])([14CH3])C[SeH]c1ccccc1</smiles>

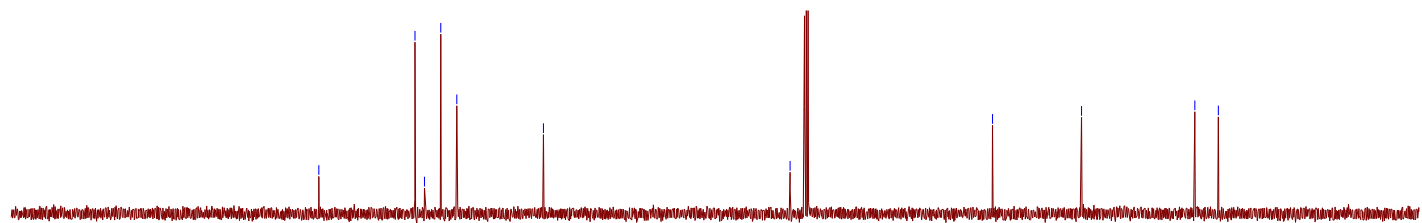

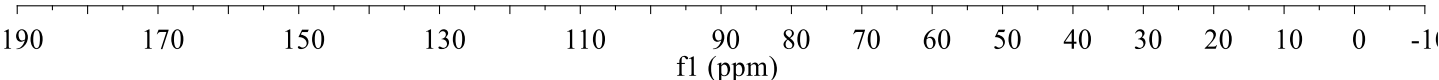




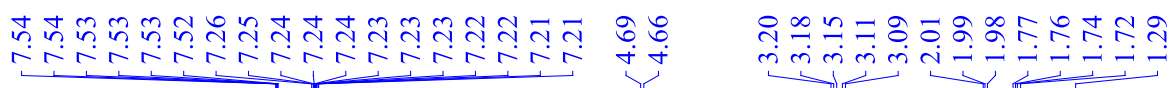
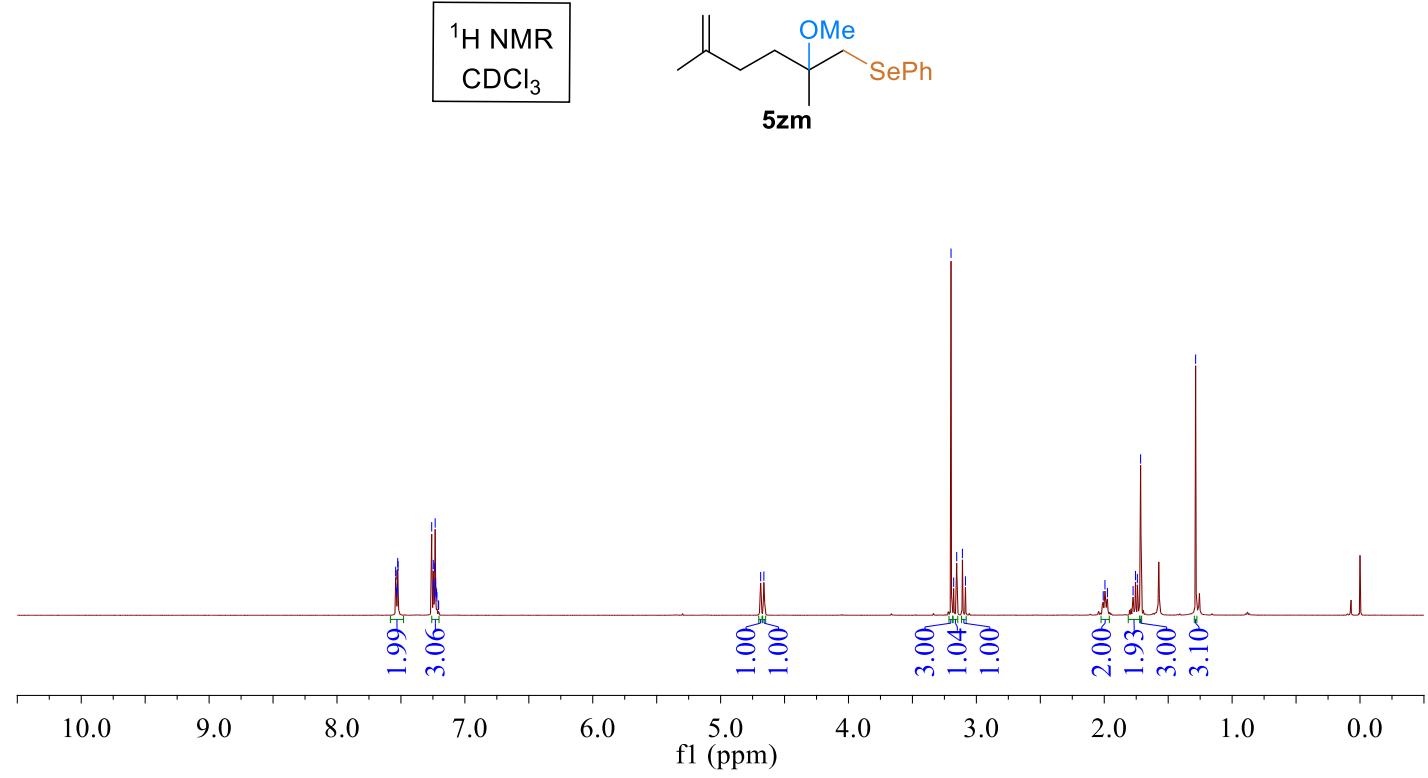

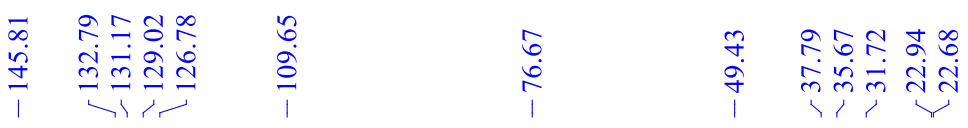

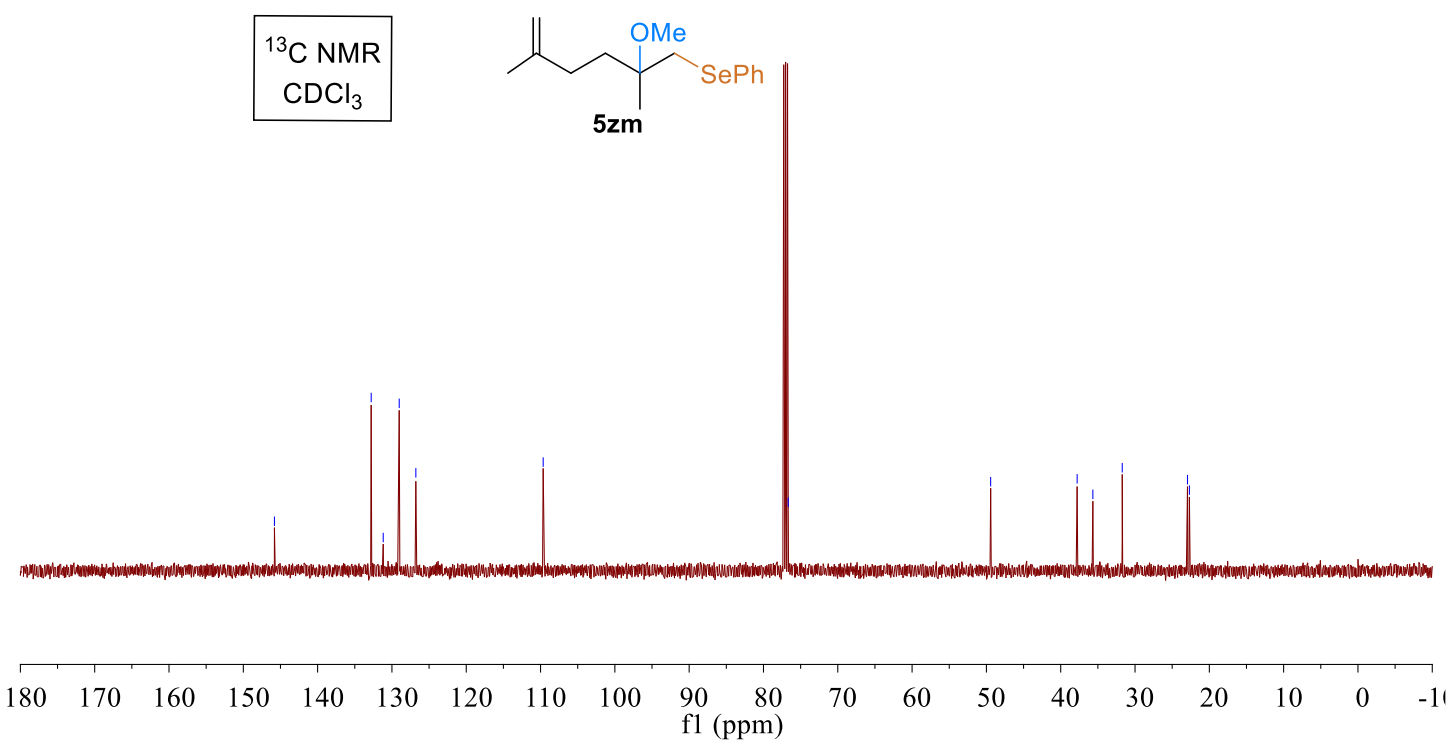




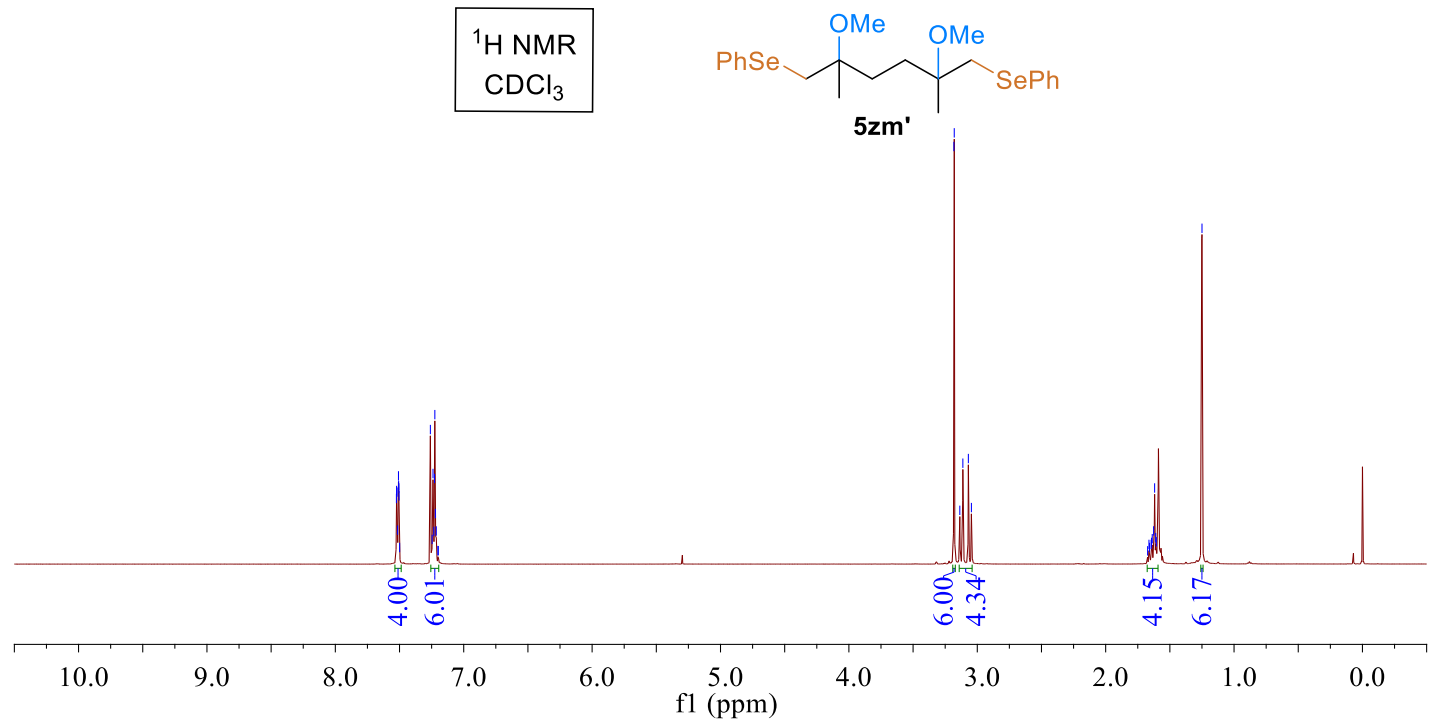

눈

तुल

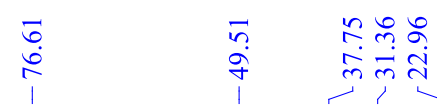

${ }^{13} \mathrm{C} \mathrm{NMR}$
$\mathrm{CDCl}_{3}$
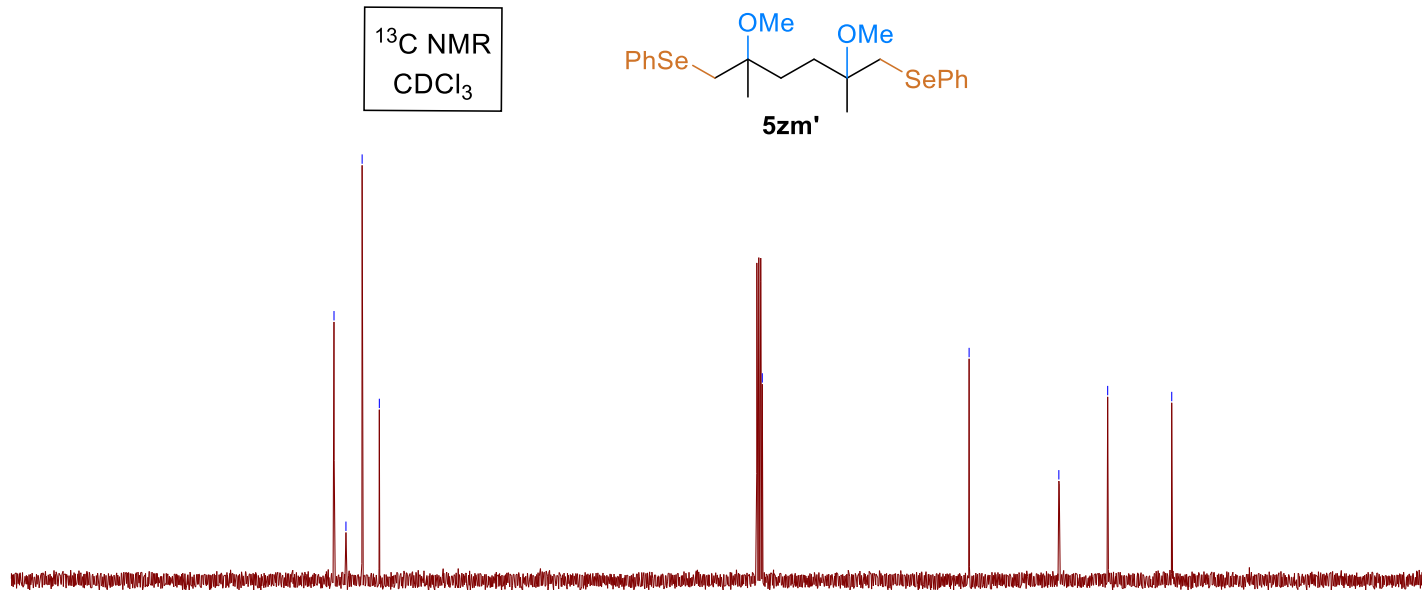

$\begin{array}{llllll}170 & 160 & 150 & 140 & 130 & 120\end{array}$ 


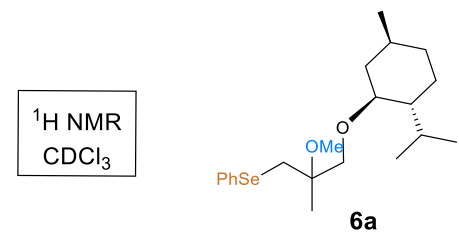

$1: 1 \mathrm{dr}$
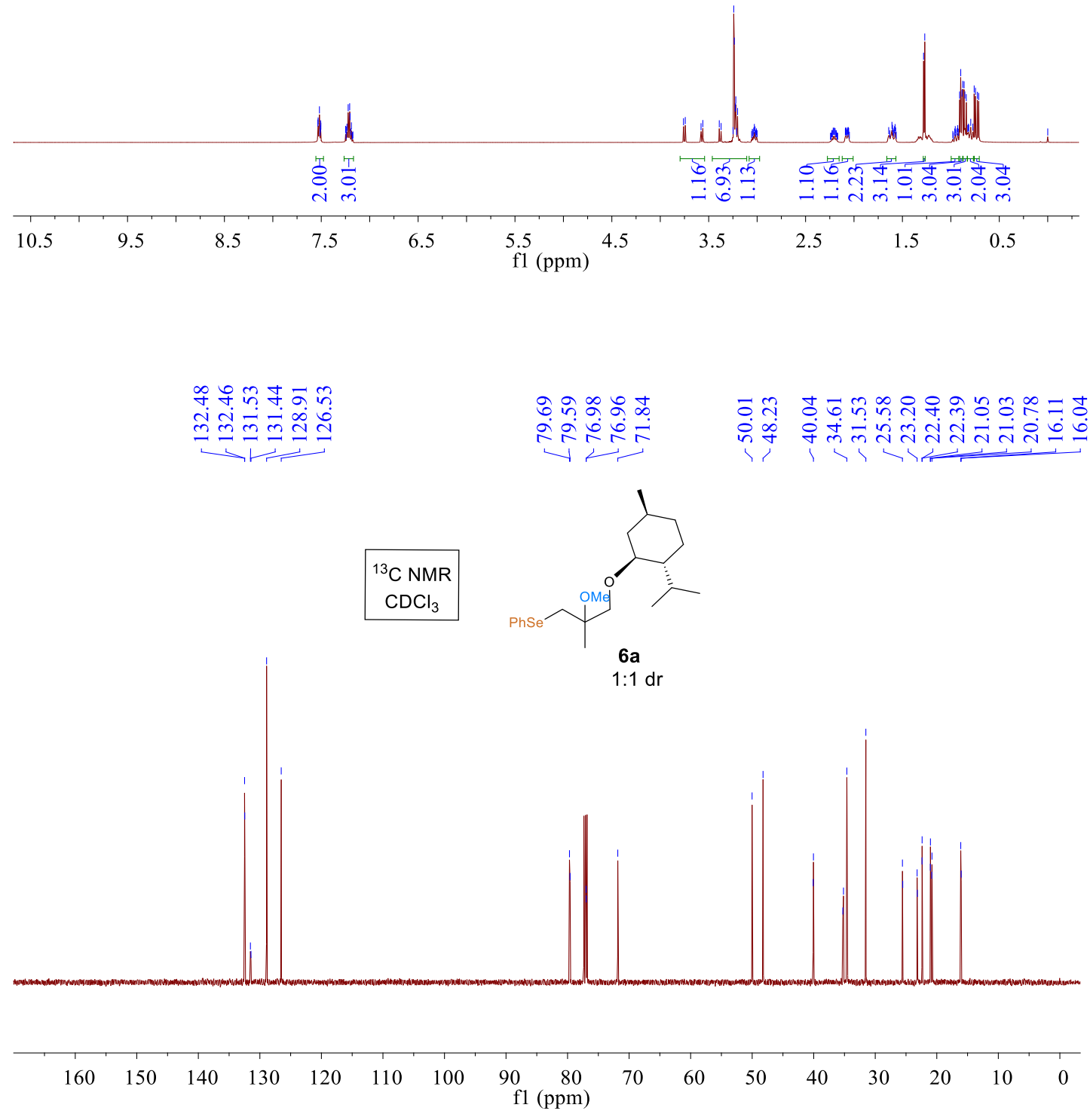


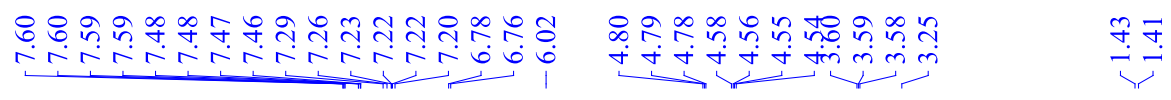

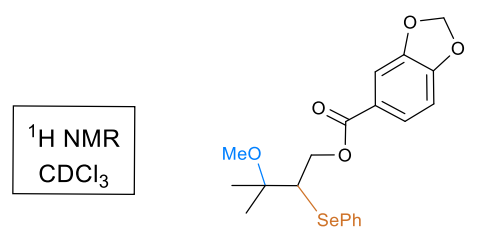

$6 b$
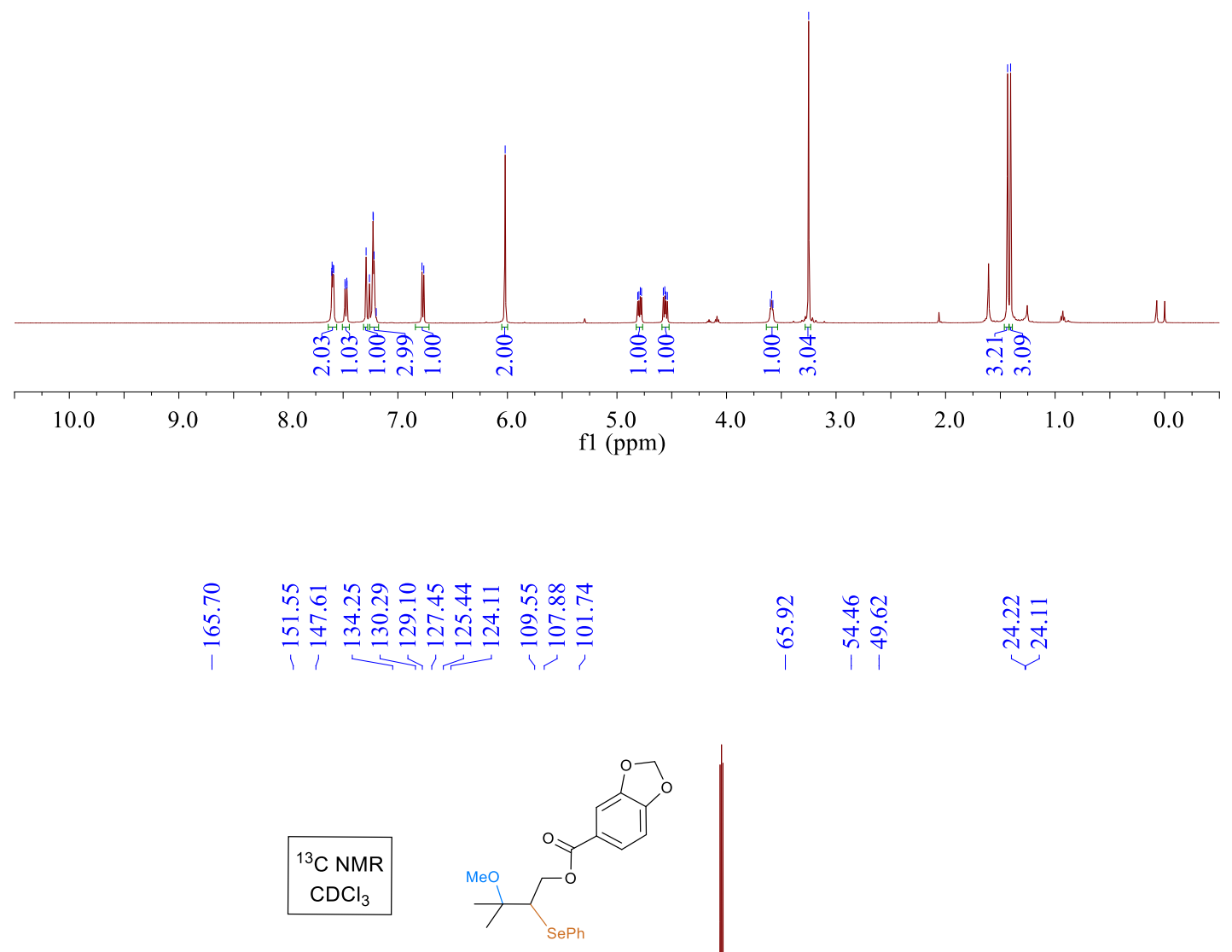

$6 b$

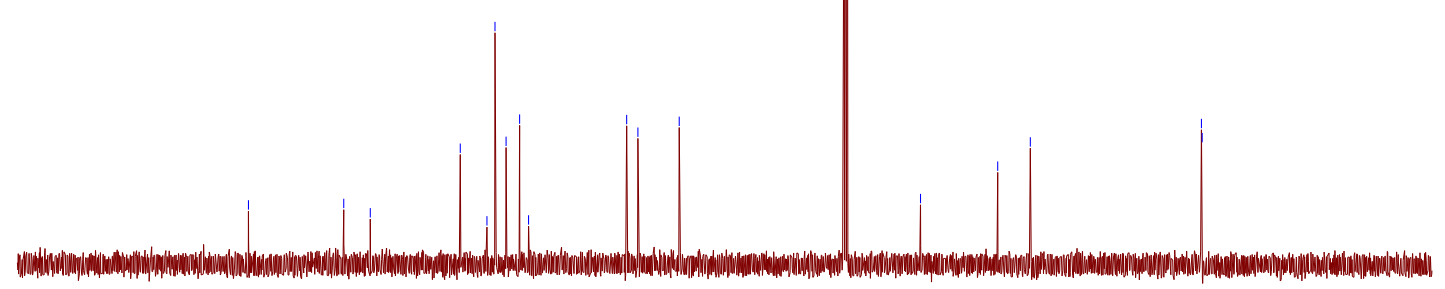

190

170

150

130

110

$\begin{array}{llllllllll}80 & 70 & 60 & 50 & 40 & 30 & 20 & 10 & 0 & -11\end{array}$ 


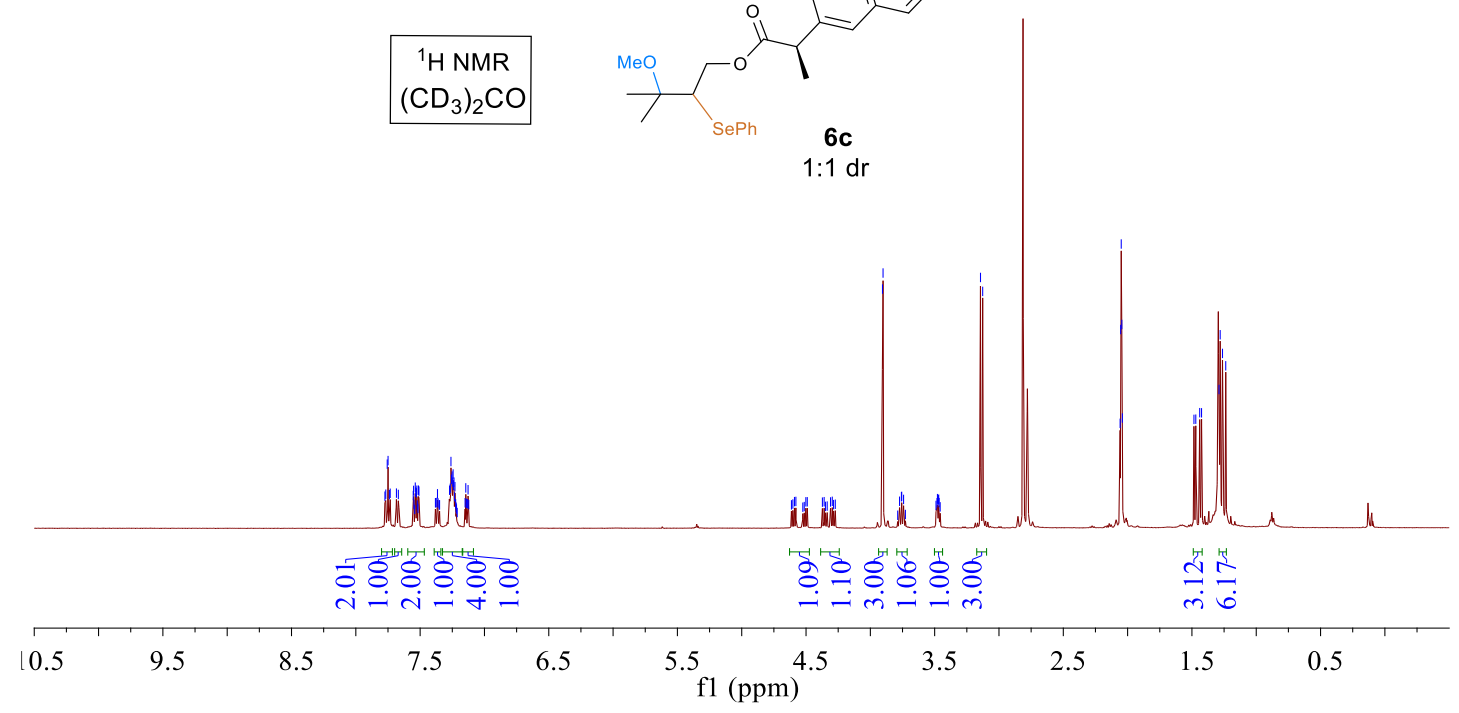

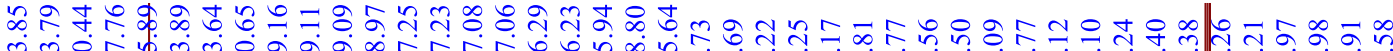

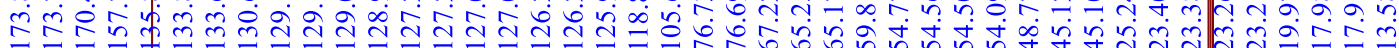

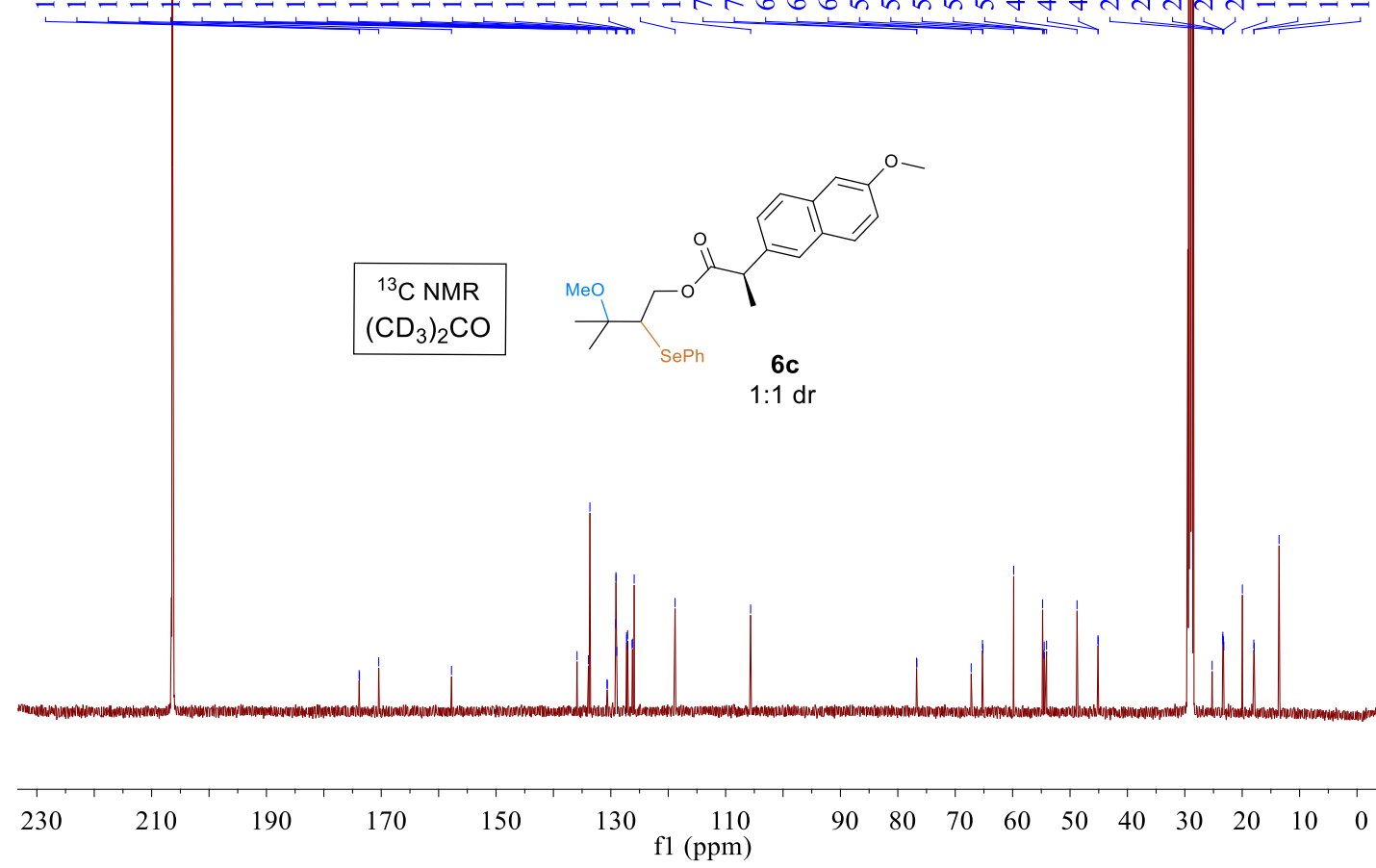




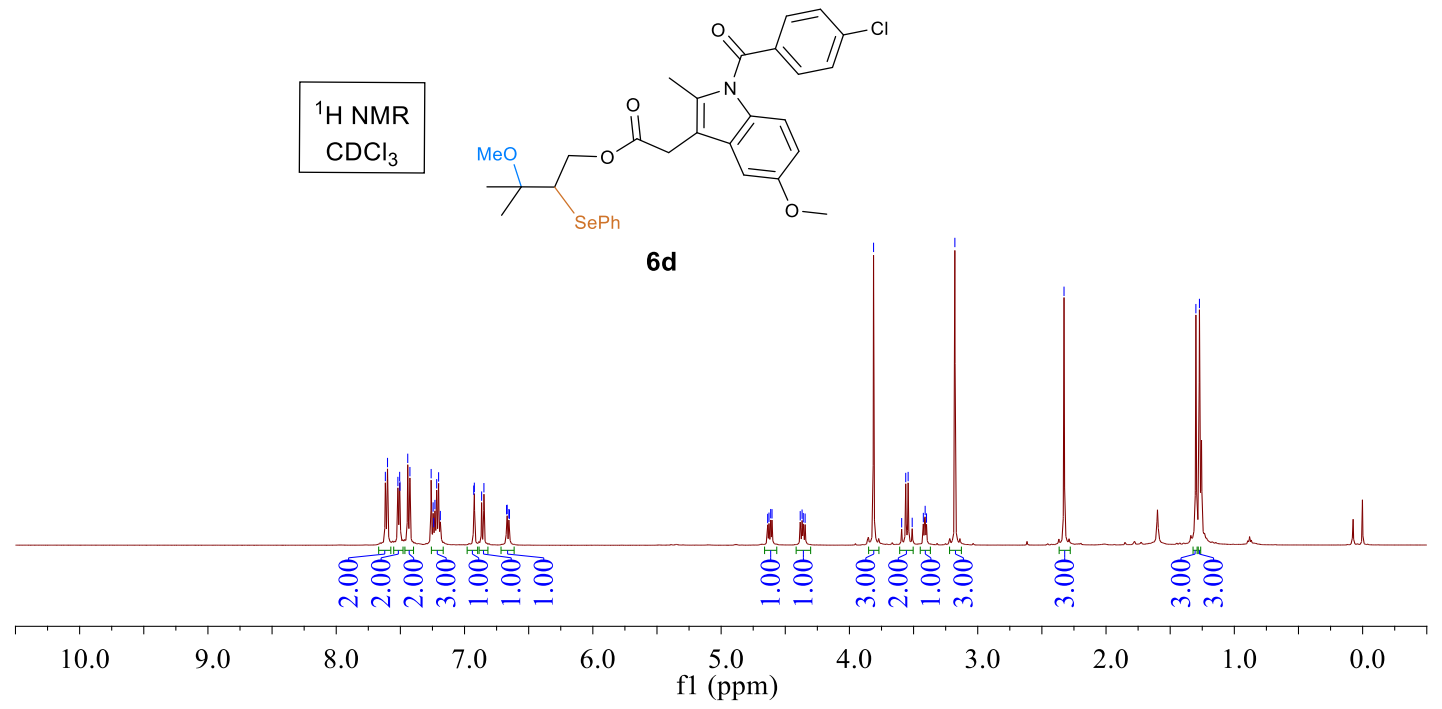

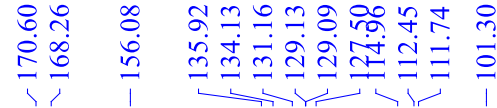

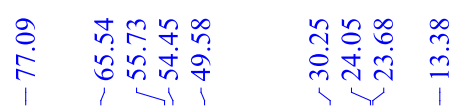

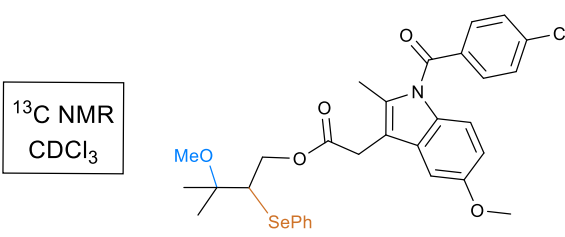

$6 d$

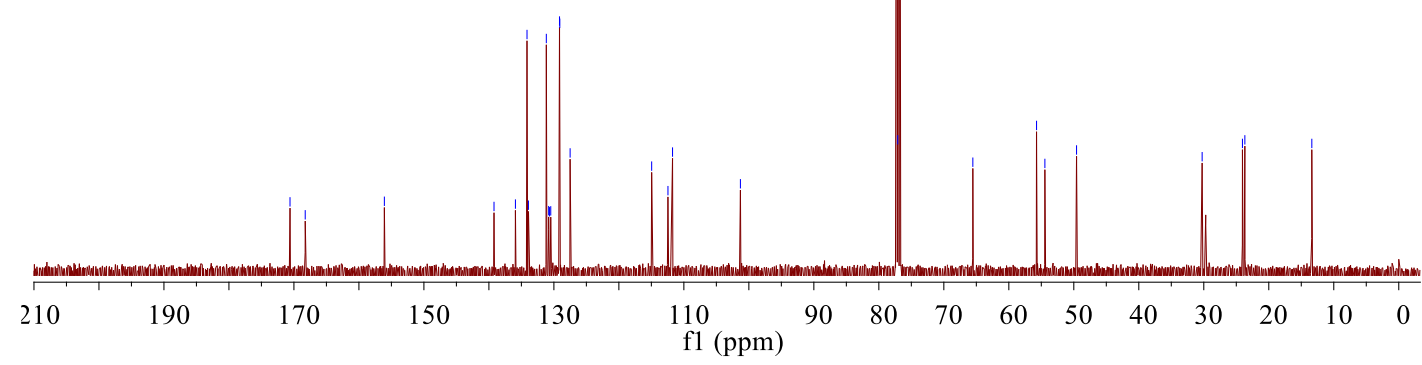



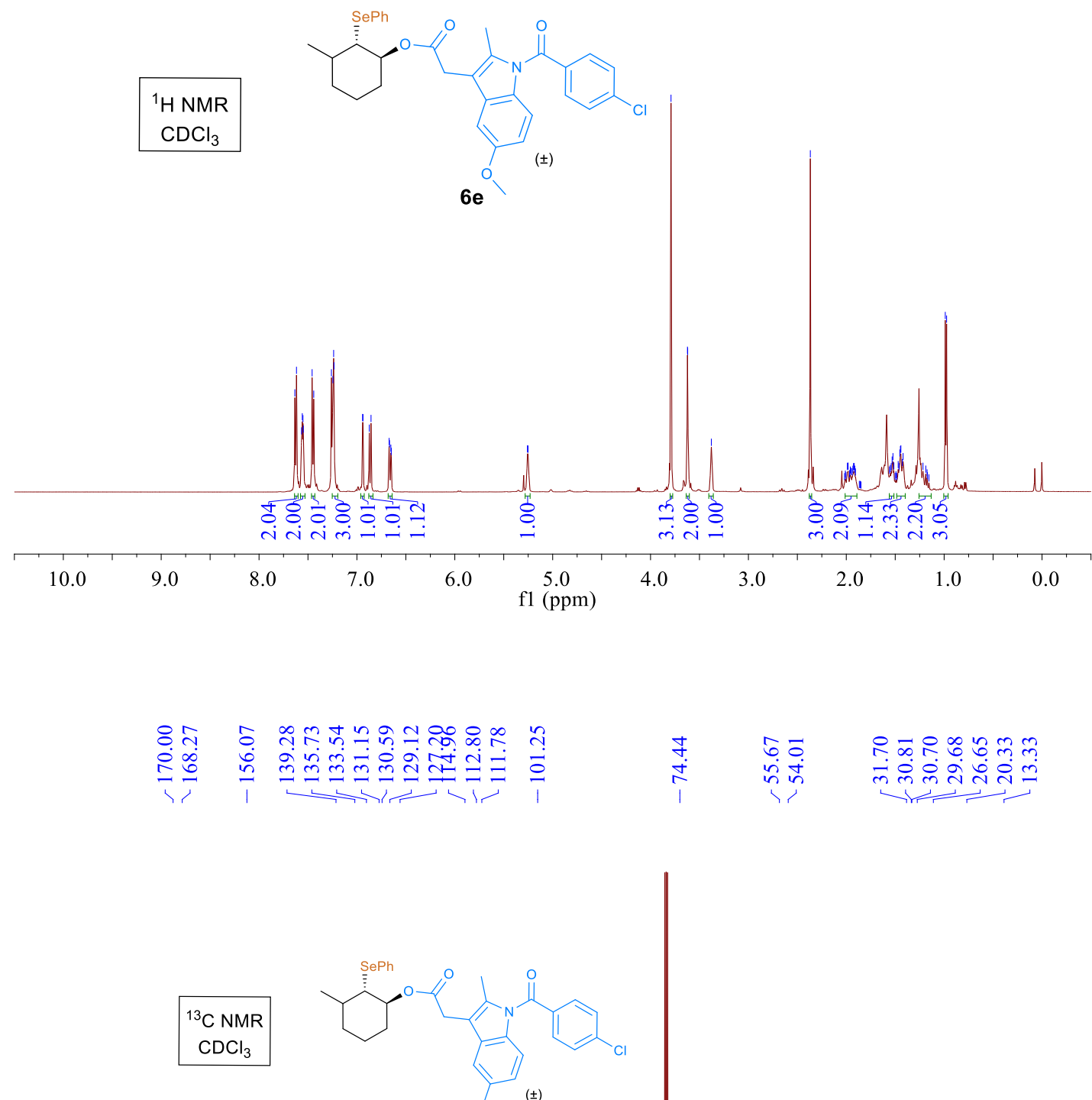

$6 \mathrm{e}$

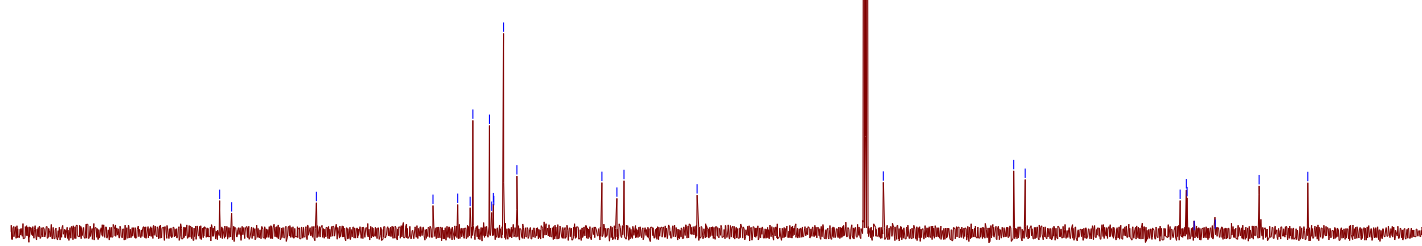

190

170

150

130

110

$\begin{array}{llllllllll}90 & 80 & 70 & 60 & 50 & 40 & 30 & 20 & 10 & 0\end{array}$ f1 (ppm) 


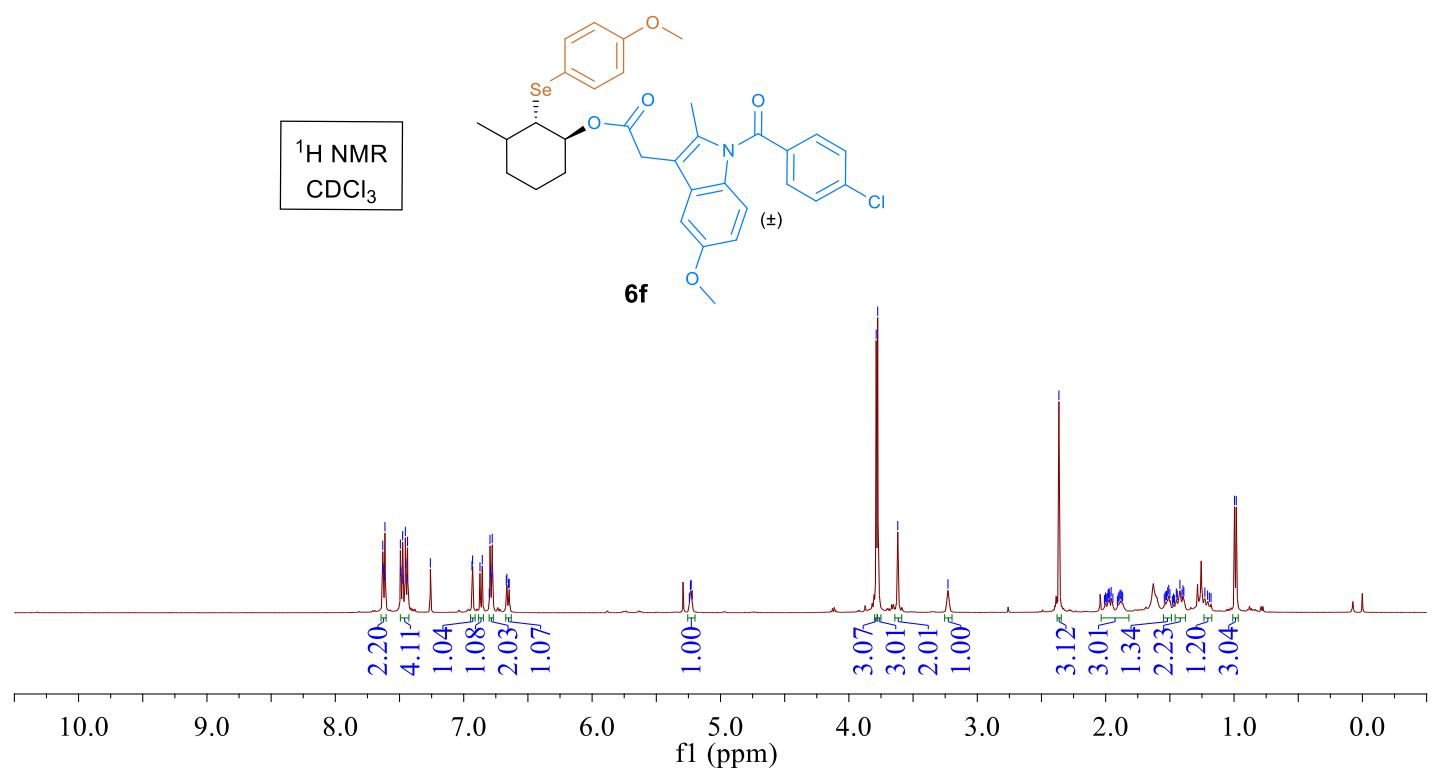

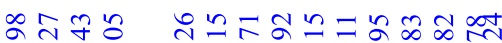

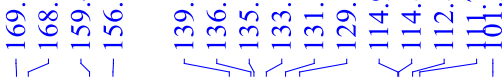

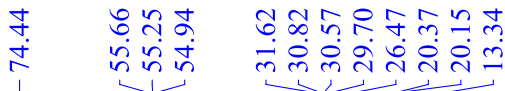
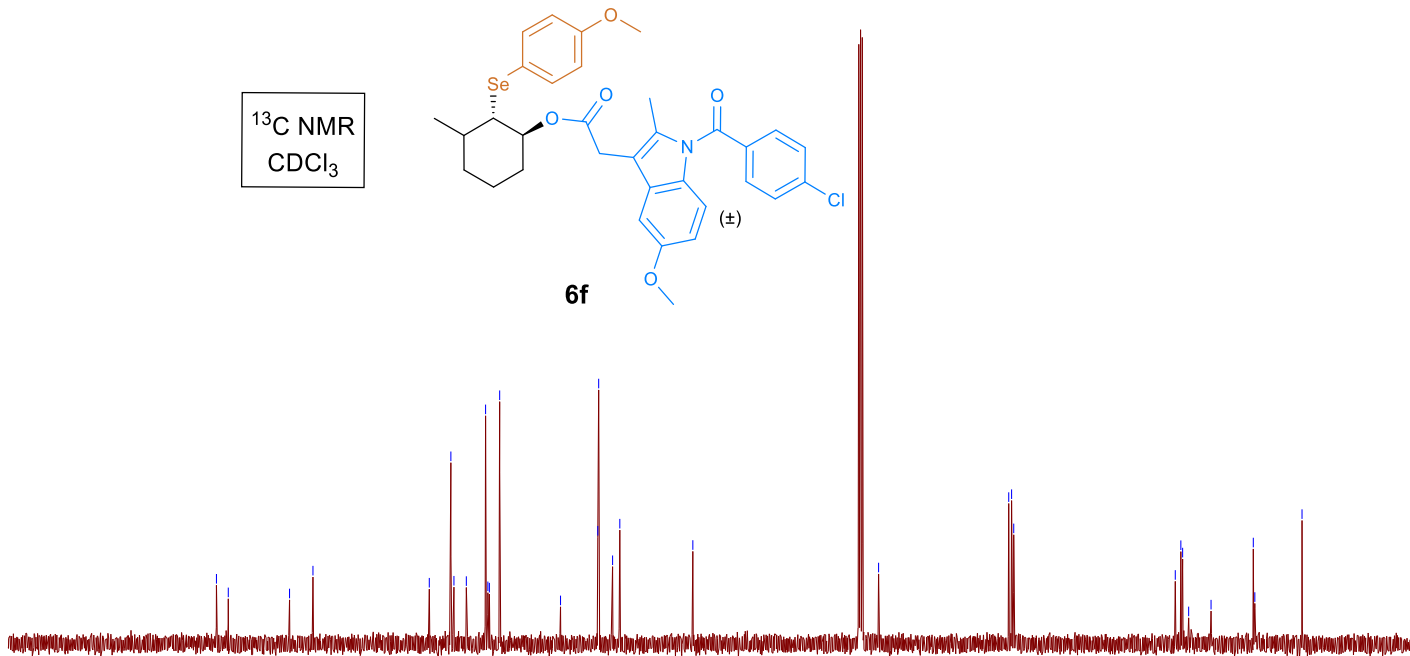

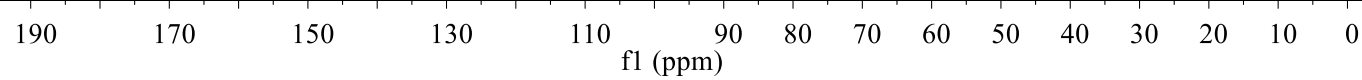




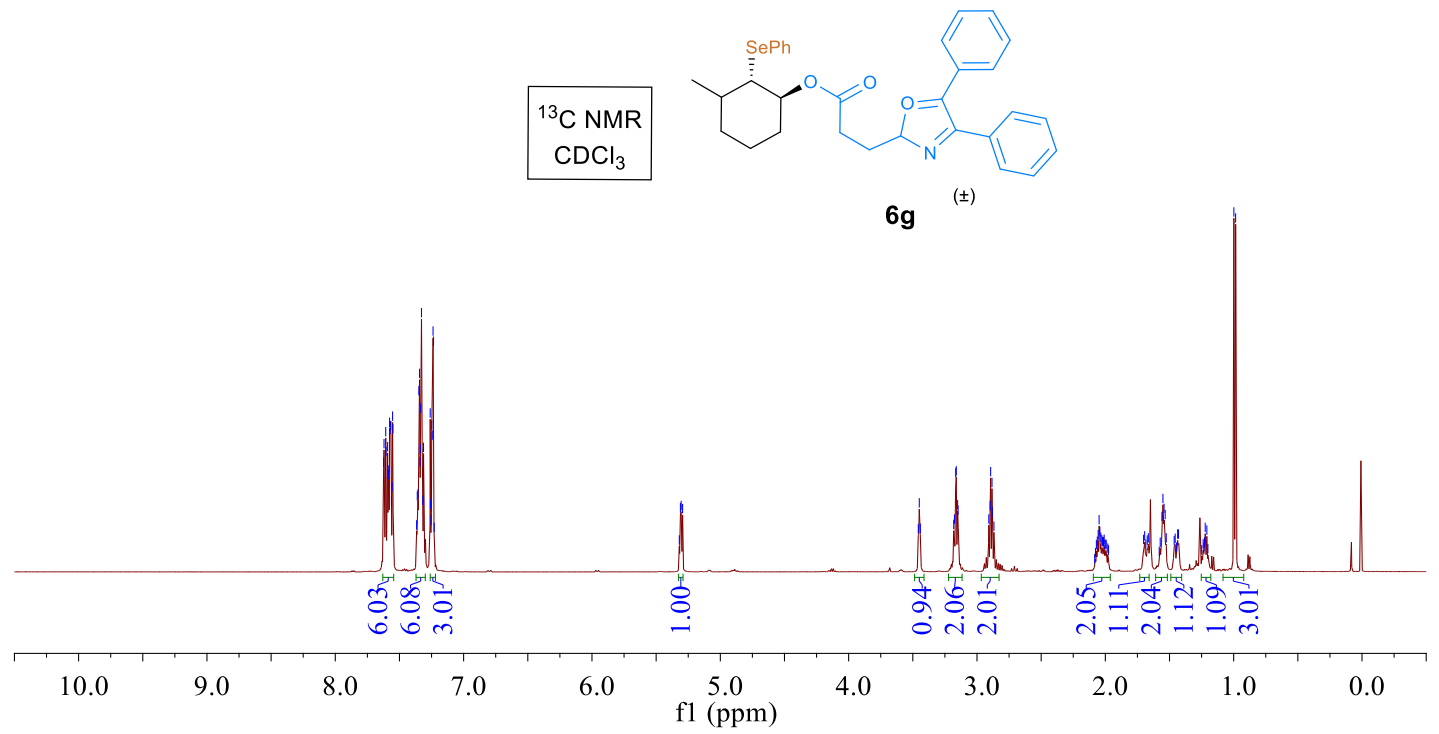

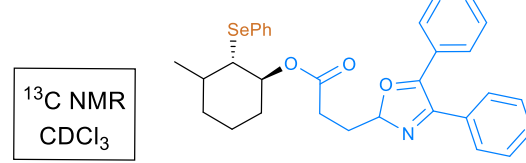

$6 \mathrm{~g}$

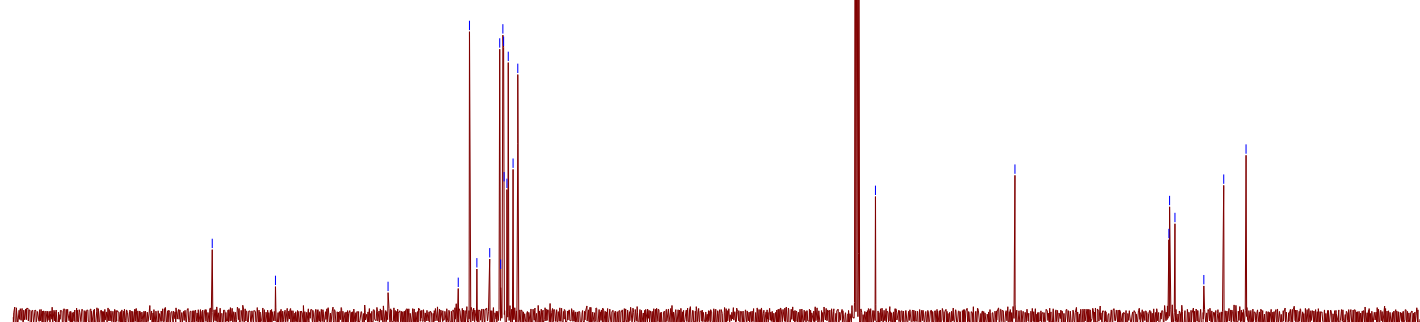




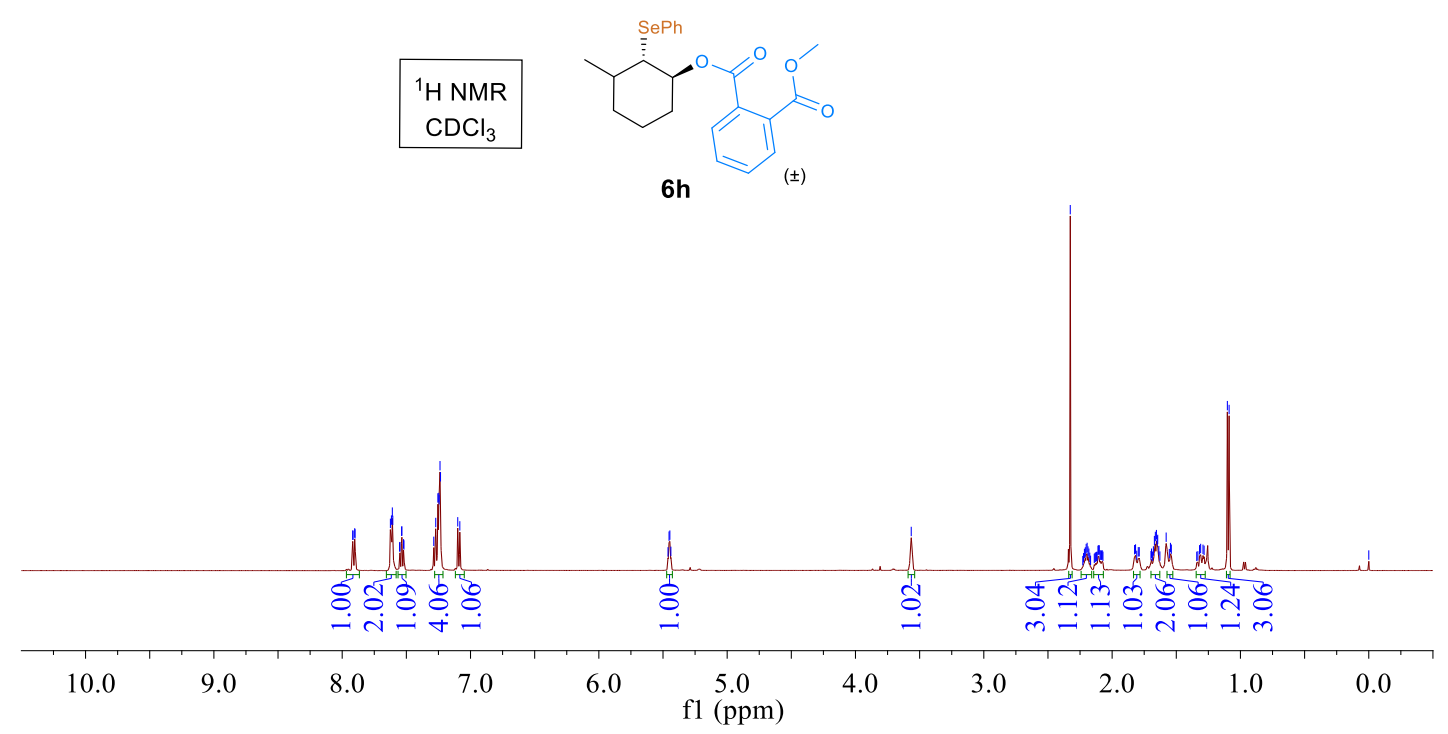

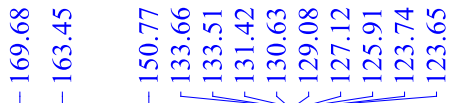

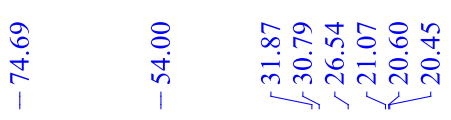
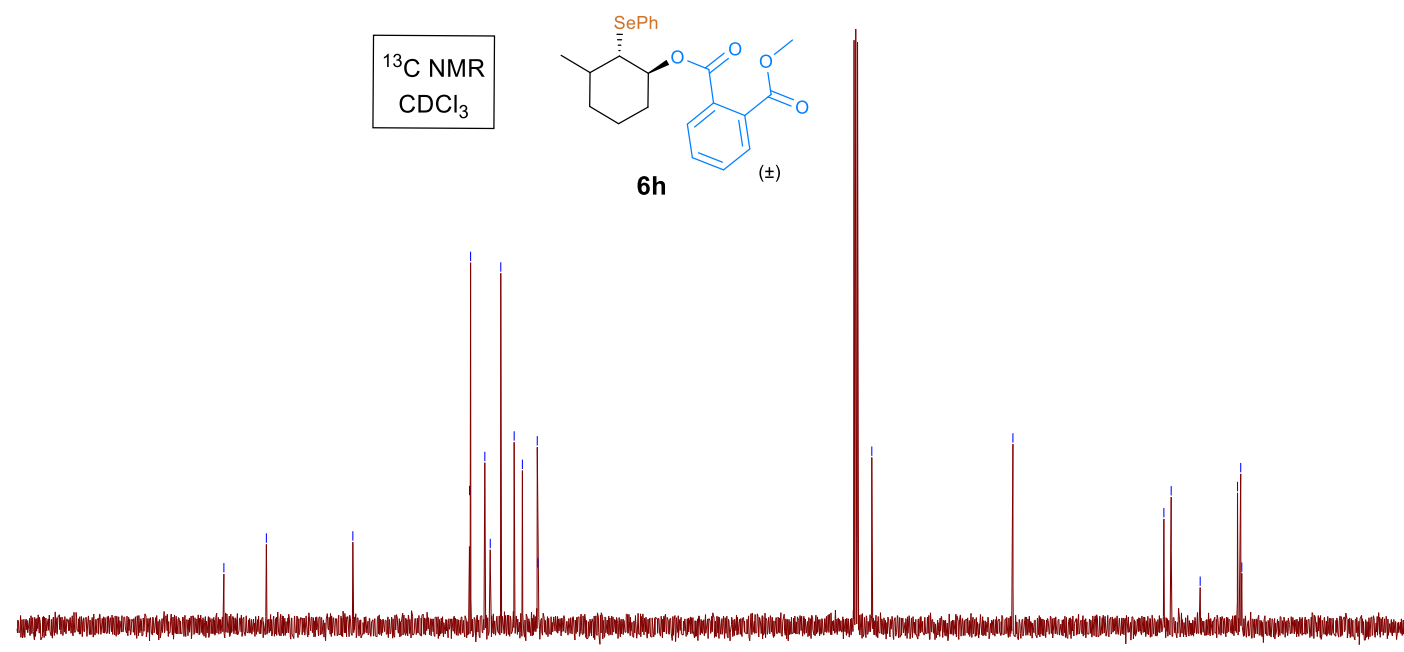

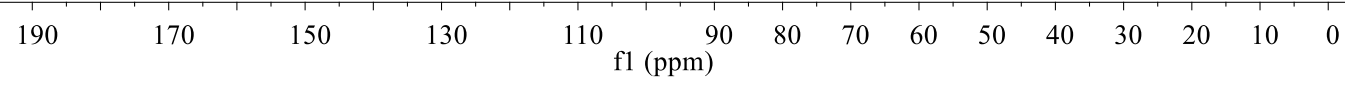



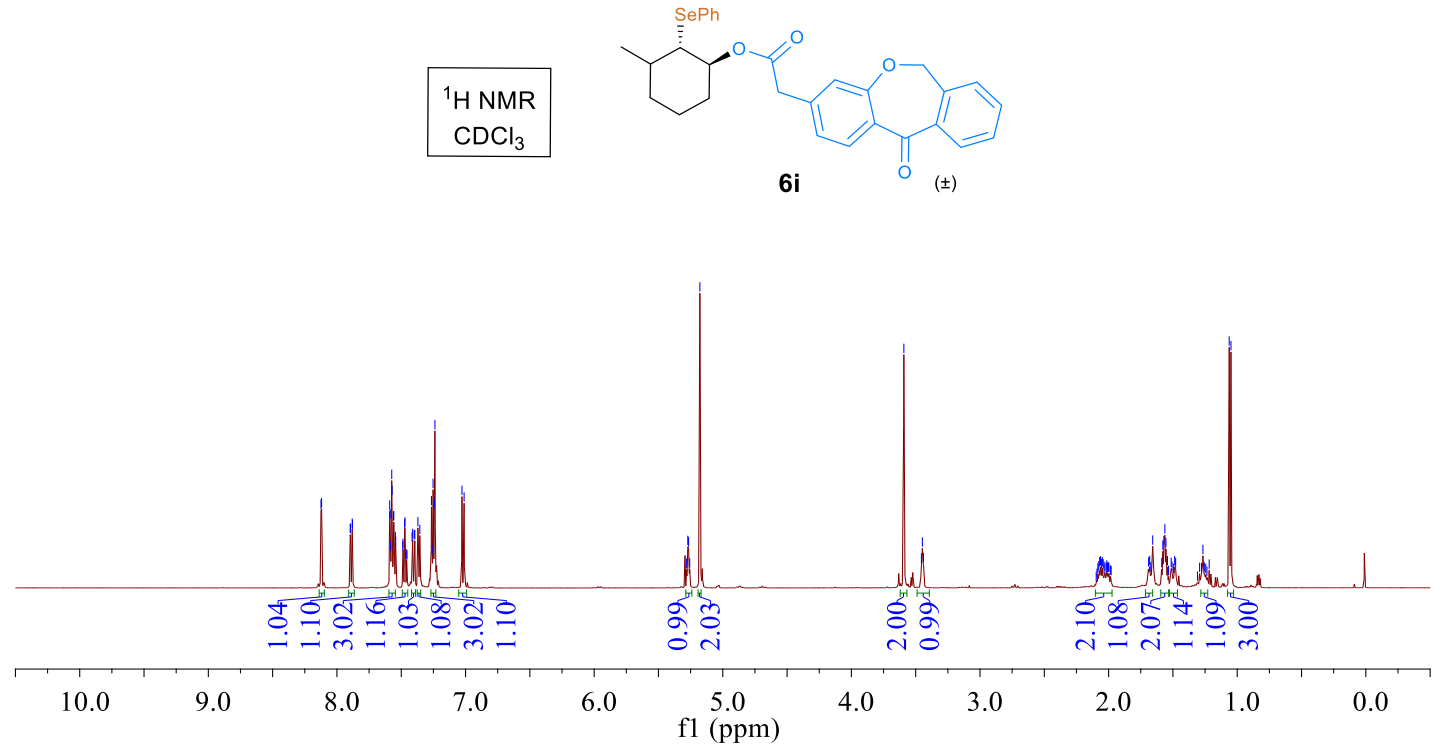

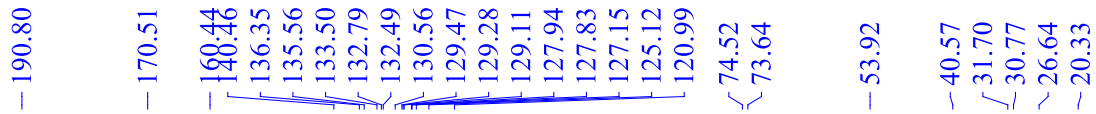
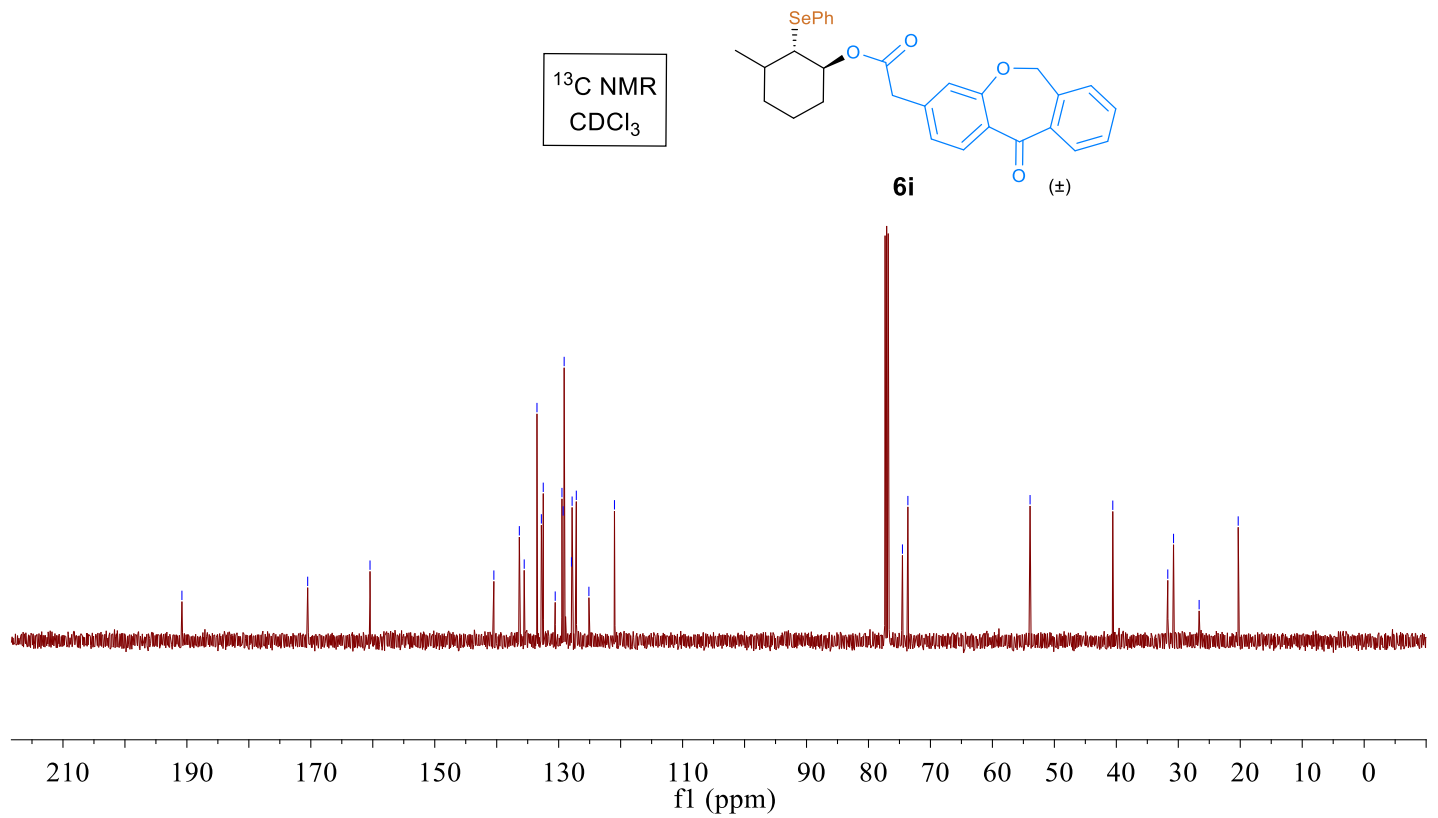


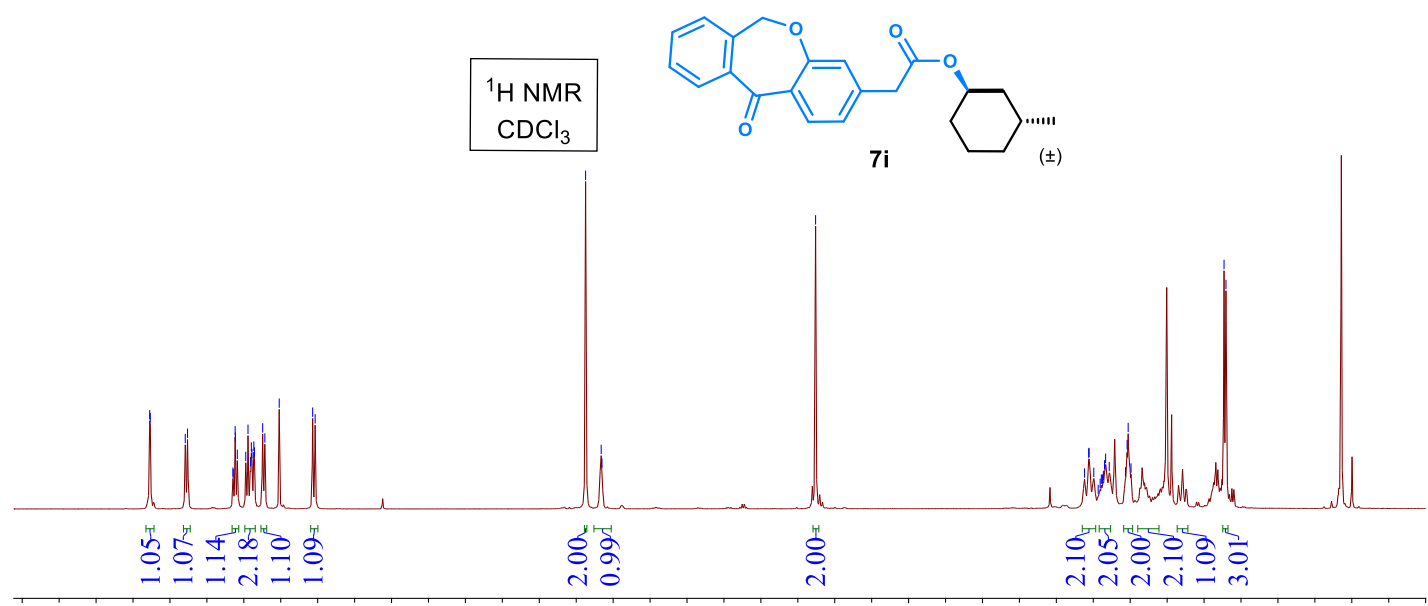

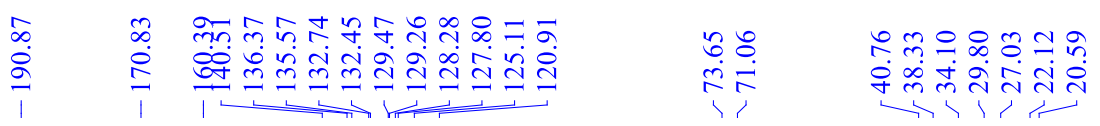
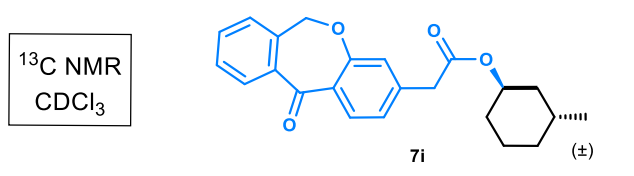

230

210

190

170

150

130

110

$\begin{array}{llllllllll}90 & 80 & 70 & 60 & 50 & 40 & 30 & 20 & 10 & 0\end{array}$ 


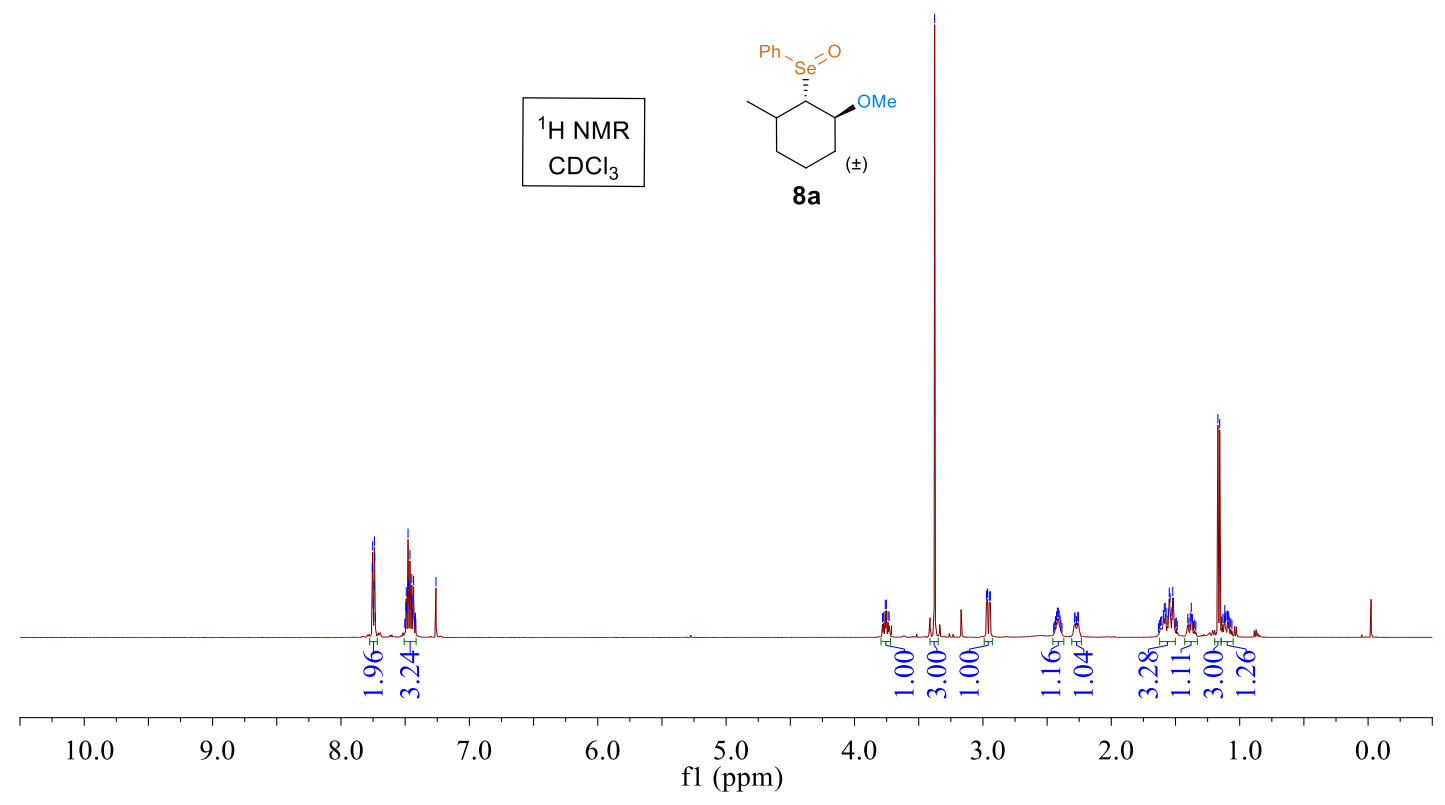

\begin{tabular}{|c|c|c|c|}
\hline 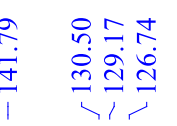 & 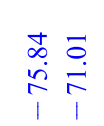 & $\begin{array}{l}m \\
\dot{0} \\
i\end{array}$ & 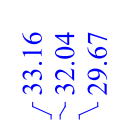 \\
\hline
\end{tabular}

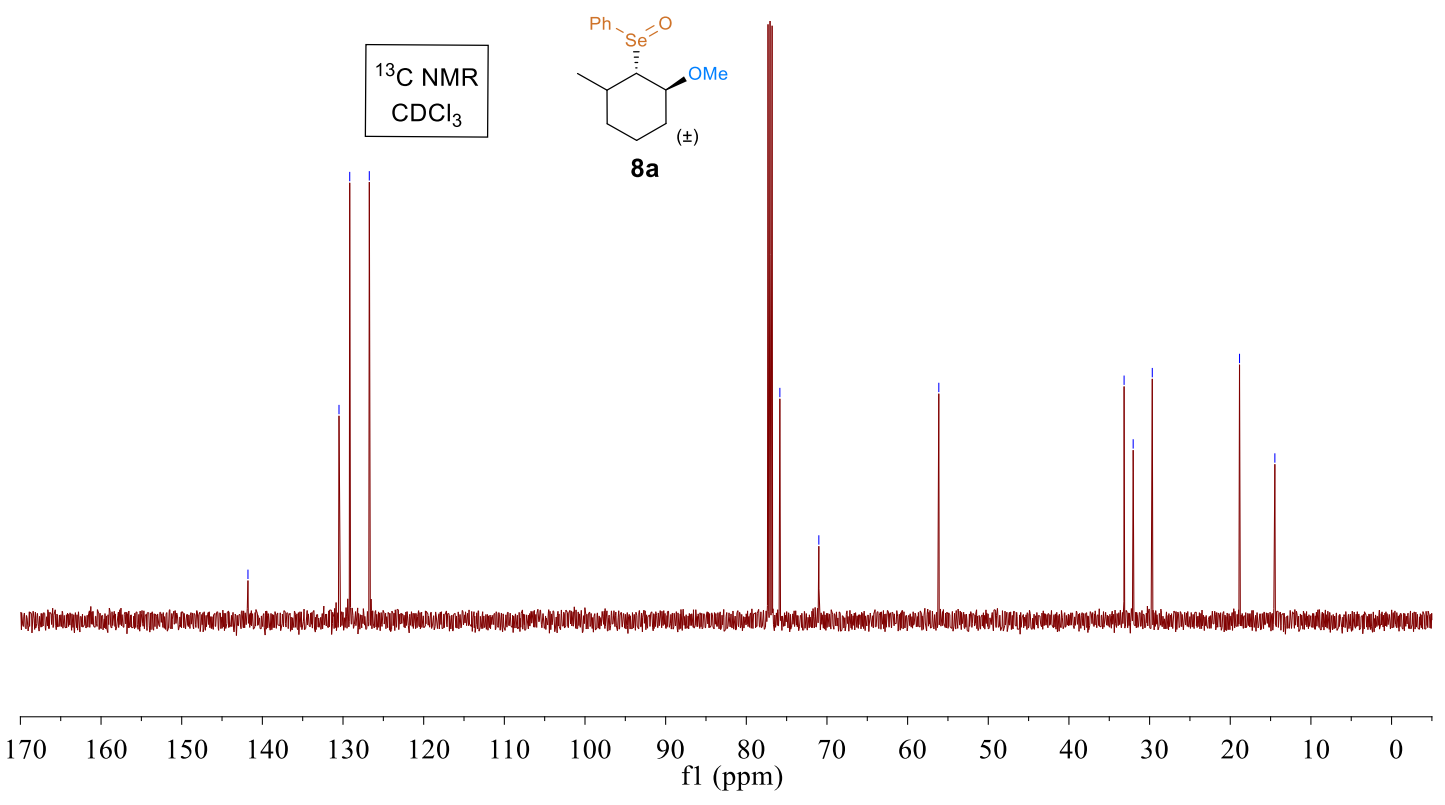




\section{Supplementary References}

1.Zhu, C; Zhu, J; Zhou, X; Zhu, Q; Yang, Y; Wen, T. B; Xia, H. Isolation of an eleven-atom polydentate carbon-chain chelate obtained by cycloaddition of a cyclic osmium carbyne with an alkyne. Angew. Chem. Int. Ed. 2018, 57, 3154-3157.

2. Zhu, C.; Li, S.; Luo, M.; Zhou, X.; Niu, Y.; Lin, M.; Zhu, J.;Cao, Z.; Lu, X.; Wen, T.; Xie, Z.; Schleyer, P. v. R.; Xia, H. Stabilization of anti-aromatic and strained five-membered rings with a transition metal. Nat. Chem. 2013, 5, 698-703. 47.

3.Zhuo, Q., Lin, J., Hua, Y., Zhou, X., Shao, Y., Chen, S., Chen, Z., Zhu, J., Zhang, H., and Xia, H. (2017). Multiyne chains chelating osmium via three metal-carbon $\sigma$ bonds. Nat. Commun. 8, 1912.

4.Zhou, X; Li, Y; Shao, Y; Hua, Y; Zhang, H; Lin, Y. M; Xia, H. Reactions of cyclic osmacarbyne with coinage metal complexes. Organometallics 2018, 37, 1788-1794. 5.Hayrapetyan, D; Rit, R. K; Kratz, M; Tschulik, K.; Gooßen, L. J. Electrochemical C-H cyanation of electron-rich (hetero)arenes. Chem. Eur. J. 2018, 24, 11288-11291.

6. Al-Amin, M; Roth, K. E.; Blum, S. A. Mechanistic studies of gold and palladium cooperative dual-catalytic cross-coupling systems. ACS Catal. 2014, 4, 622-629.

7.Lu, Z; Hennis, O; Gentry, J; Xu, B; Hammond, G. B. Base-promoted radical azofluoromethylation of unactivated alkenes. Org. Lett. 2020, 22, 4383-4388.

8.Ma, X.; Herzon, S. B. Cobalt bis(acetylacetonate)-tert-butyl hydroperoxidetriethyl-silane: a general reagent combination for the Markovnikov-selective hydrofunctionalization of alkenes by hydrogen atom transfer. Beilstein $\mathrm{J}$. Org. Chem. 2018, 14, 2259-2265.

9.Bertrand, X; Paquin, J. F. Direct hydrofluorination of methallyl alkenes using a methanesulfonic acid/triethylamine trihydrofluoride combination. Org. Lett. 2019, 21, 9759-9762.

10.Nagasawa, S; Sasano, Y; Iwabuchi, Y. Catalytic oxygenative allylic transposition of alkenes into enones with an azaadamantane-type oxoammonium salt catalyst. Chem. Eur. J.2017, 23, 10276-10279. 
11.Xiao, J; He, Y; Ye, F; Zhu, S. Remote sp3 C-H amination of alkenes with Nitroarenes. Chem 2018, 4, 1645-1657.

12.Lv, L; Zhu, D; Qiu, Z; Li, J; Li, C. J. Nickel-catalyzed regioselective hydrobenzylation of 1,3-dienes with Hydrazones. ACS Catal. 2019, 9, 9199-9205.

13.Yan, X; Li, C; Xu, X; Zhao, Xi; Pan, Y. Hemin catalyzed dealkylative intercepted $[2,3]$-sigmatropic rearrangement reactions of sulfoniu trifluorodiazoethanem ylides with 2, 2, 2- trifluorodiazoethane. Adv. Synth. Catal. 2020, 362, 2005-2011.

14.Kim, J; Kang, B; Hong, S. H. Direct allylic C(sp3)-H thiolation with disulfides via visible light photoredox catalysis. ACS Catal. 2020, 10, 6013-6022.

15.Edwards, J. T. et al; Decarboxylative alkenylation. Nature 2017, 545, 213-218.

16.Uemura S; Fukuzawa, S. Oxidation of alkyl phenyl selenides, tellurides, and telluroxides with meta-chloroperbenzoic acid for a facile and novel transformation of C-Se and C-Te bonds to C-P bonds. J. Chem. Soc., Perkin Trans. 1985, 1, 471-480. 17.Bosman, C; D’Annibalq, A; Resta, S; Trogolo, C. Oxidation of diphenyl diselenide with ceric ammonium nitrate: a novel route for functlonalization of olefins . Tetrahedron Letters ,1994, 35, 6525-6528.

18.Krief, A. (2001). Phenyl Selenocyanate-Copper(II) Chloride. e-EROS Encyclopedia of Reagents for Organic Synthesis. 10.1002/047084289X.rp097.

19.Tingoli, M; Diana, R; Panunzi, B. N-Phenylselenosaccharin (NPSSac): a new electrophilic selenium-containing reagent. Tetrahedron Letters, 2006, 47, 7529-7531. 20.GARRATT, D. G; KABO, A. Factors influencing the nature of seleniranium ions in selenenyl chloride additions to alkenes: the use of methanol as solvent. Can. J. Chem. 1979, 58, 1030-1041.

21.Toshimitsu, A; Uemura, S; Okano, M. Synthesis of 1-Phenylselenobuta-1,3-diene and its methyl-substituted homologues via oxyselenation of conjugated dienes. $J$. Chem. Soc., Chem. Commun. 1982, 1, 965-966.

22.Sheldrick, G. M. SHELXTL; Siemens Analytical X-ray Systems: Madison, Wisconsin, USA. 